\title{
Atmospheric Photochemistry Studies of Pollutant Emissions from Transportation Vehicles Operating on Alternative Fuels
}

\author{
H. Jeffries, K. Sexton, and J. Yu
}

University of North Carolina

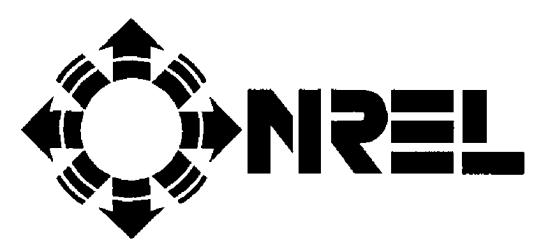

Alternative Fuels Hotline: 1-800-423-1DOE

Alternative Fuels Data Center World Wide Web Site: http://www.afdc.doe.gov

\author{
National Renewable Energy Laboratory \\ 1617 Cole Boulevard \\ Golden, Colorado 80401-3393 \\ A national laboratory of the \\ U.S. Department of Energy \\ Managed by the Midwest Research Institute \\ For the U.S. Department of Energy \\ Under Contract No. DE-AC36-83CH10093
}




\section{Atmospheric Photochemistry Studies of Pollutant Emissions from Transportation Vehicles Operating on Alternative Fuels}

H. Jeffries, K. Sexton, and J. Yu

University of North Carolina

NREL Technical Monitors: Brent Bailey, Michelle Bergin, and Paul Bergeron

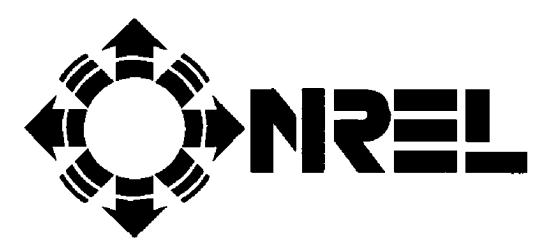

Alternative Fuels Hotline: 1-800-423-1DOE

Alternative Fuels Data Center World Wide Web Site: http://www.afdc.doe.gov

National Renewable Energy Laboratory

1617 Cole Boulevard

Golden, Colorado 80401-3393

A national laboratory of the

U.S. Department of Energy

Managed by the Midwest Research Institute

For the U.S. Department of Energy

Under Contract No. DE-AC36-83CH10093

Prepared under Subcontract Number XAT-3-11153-01

July 1998 


\section{NOTICE}

This report was prepared as an account of work sponsored by an agency of the United States government. Neither the United States government nor any agency thereof, nor any of their employees, makes any warranty, express or implied, or assumes any legal liability or responsibility for the accuracy, completeness, or usefulness of any information, apparatus, product, or process disclosed, or represents that its use would not infringe privately owned rights. Reference herein to any specific commercial product, process, or service by trade name, trademark, manufacturer, or otherwise does not necessarily constitute or imply its endorsement, recommendation, or favoring by the United States govemment or any agency thereof. The views and opinions of authors expressed herein do not necessarily state or reflect those of the United States government or any agency thereof.

Available to DOE and DOE contractors from:

Office of Scientific and Technical Information (OSTI)

P.O. Box 62

Oak Ridge, TN 37831

Prices available by calling (423) 576-8401

Available to the public from:

National Technical Information Service (NTIS)

U.S. Department of Commerce

5285 Port Royal Road

Springfield, VA 22161

(703) $487-4650$ 


\section{Contents}

1 Executive Summary 1

1.1 Purpose .......................... 1

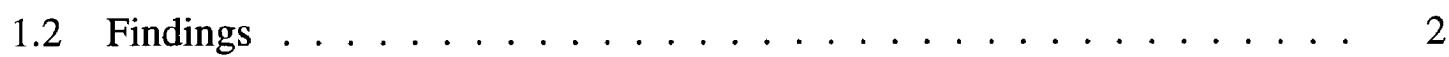

1.3 Background ........................ 4

1.3.1 Summary of Background . . . . . . . . . . . 7

1.4 Experimental Design . . . . . . . . . . . . . . . . 8

1.4.1 VOC Mixtures . . . . . . . . . . . . . . . 8

1.4.2 Experimental Design ............... 8

1.5 Experimental Results . . . . . . . . . . . . . . . . . . . . 9

1.5.1 Block 6: Chamber Characterization Tests . . . . . . . . . . 9

1.5.2 Block 1: Base CNG Tests . . . . . . . . . . . . . . 11

1.5.3 Block 5: Base LPG Tests . . . . . . . . . . . . . 11

1.5.4 Block 2: Base E85 Tests . . . . . . . . . . . . . . 12

1.5.5 Block 3: Single Mixture E85 Tests . . . . . . . . . . . . 12

1.5.6 Block 4: E85 Mixture Variations Tests . . . . . . . . . . . . . . 12

1.6 Simulation Results . . . . . . . . . . . . . . . . . . . 16

1.6.1 Temperature Effects . . . . . . . . . . . . . 16

1.6.2 Block 6: Chamber Characterization Tests . . . . . . . . . . 18

1.6.3 Alternative Fuel Simulations . . . . . . . . . . . . . . . . 18

1.7 New Analytical Methods . . . . . . . . . . . . . . . . . . . . . 23

1.8 Aromatics Mechanism . . . . . . . . . . . . . . . . . . 24

1.9 UAM Simulations and Analysis . . . . . . . . . . . . . . . 27

1.9.1 Emissions Inventory Improvements . . . . . . . . . . . . . 27

1.9 .2 Process Analysis . . . . . . . . . . . . . . . . . . . 29

1.9.3 UAM Simulation Results ... . . . . . . . . . 30

2 Experimental Design 31

2.1 VOC Mixture Composition .................. . 31

2.2 Chamber Experimental Conditions . . . . . . . . . . . . . 38 
3 Experimental Results $\quad \mathbf{4 0}$

3.1 Outdoor Smog Chamber Results . . . . . . . . . . . . . . . . . . . . 40

3.1.1 Block 1: Urb/IAG versus Urb/CNG . . . . . . . . . . . . . . 42

3.1.2 Block 5: Urb/LAG versus Urb/LPG . . . . . . . . . . . . . 51

3.1.3 Block 2 - Urb/LAG versus Urb/E85 . . . . . . . . . . . . . 56

3.1.4 Block 3: Single Mixture Experiments . . . . . . . . . . . . 76

3.1.5 Block 4: Mixture Variations . . . . . . . . . . . . . 83

3.1.6 Block 6: Characterization . . . . . . . . . . . 95

3.2 Relevant CRC Methanol Study Results . . . . . . . . . . . . . 106

4 Modeling Results $\quad 115$

4.1 The Simulation Process . . . . . . . . . . . . . . . . 115

4.2 Temperature Effects . . . . . . . . . . . . . . . . . 117

4.2.1 Effect of Temperature on Ozone . . . . . . . . . . . . . . 117

4.2.2 Modeled Temperature Effect . . . . . . . . . . . . . . 127

4.2.3 Temperature Effect Conclusions . . . . . . . . . . . . . . 136

4.3 Characterization Run Simulations . . . . . . . . . . . . . . . 137

4.3.1 Block 6 . . . . . . . . . . . . . . . . . . . 137

4.4 Alternative Fuels Runs Simulations . . . . . . . . . . . . . . . . . 141

4.4.1 Block 1 ..................... 141

4.4 .2 Block 5 ...................... 149

4.4 .3 Block $2 \ldots \ldots \ldots \ldots 15 \ldots \ldots \ldots$

4.4 .4 Block $3 \ldots \ldots \ldots \ldots$

4.4.5 Block $4 \ldots \ldots \ldots 16 \ldots \ldots$

5 New Analytical Methods $\quad 169$

5.1 Detection and Identification of Carbonyls . . . . . . . . . . . . . . . 169

5.1.1 The Derivatization Procedure . . . . . . . . . . . . . . 169

5.1 .2 Ion Trap Mass Spectra . . . . . . . . . . . . . . . . 170

6 Aromatic Reaction Mechanisms 175

6.1 Experimental Results . . . . . . . . . . . . . . . . 175

$6.1 .1 \quad$ Aromatic Aldehydes . . . . . . . . . . . . . . . 209

6.1 .2 Quinones................... 209

6.1.3 Di-unsaturated 1,6-Dicarbonyls ... . . . . . . . . 212

6.1.4 Unsaturated 1,4-Dicarbonyls . . . . . . . . . . . . . . 213

6.1.5 Unsaturated 1,4-dicarbonyls . . . . . . . . . . . . . 214

6.1 .6 Saturated Dicarbonyls . . . . . . . . . . . . . 223 
6.1 .7 Hydroxy Dicarbonyls ................. 223

6.1 .8 Triones ... . . . . . . . . . . . . . . 228

6.1.9 Glycolaldehyde and Hydroxyacetone ........... . . 229

6.1 .10 Unknown Carbonyls . . . . . . . . . . . . . . . 232

6.2 Epoxy Carbonyls . . . . . . . . . . . . . . . . 233

6.2.1 Epoxide Toxicity . . . . . . . . . . . . . . . 233

6.2.2 Theoretical Considerations . . . . . . . . . . . . . . 234

6.2 .3 Model Epoxides . . . . . . . . . . . . . . 235

6.2.4 Epoxy Carbonyls in Experiments . . . . . . . . . . . 237

6.2 .5 Conclusions ....................... 241

7 Airshed Modeling 250

7.1 Emissions Inventory Improvements . . . . . . . . . . . . . . . . 250

7.1.1 Area Source Emissions . . . . . . . . . . . . . 250

7.1.2 Mobile Source Emissions . . . . . . . . . . . . . 251

7.2 Process Analysis . . . . . . . . . . . . . . . . . 252

7.2.1 The Model's Processes . . . . . . . . . . . . . . 253

7.2 .2 The Code Changes . . . . . . . . . . . . . . . . 254

7.2.3 The Process Analyses . . . . . . . . . . . . . . . . 257

7.2.4 Example Analyses Results . . . . . . . . . . . . . . 258

$\begin{array}{ll}\text { A Chamber Facilities } & 277\end{array}$

A.1 The UNC Outdoor Chamber . . . . . . . . . . . . . . . . . . . . 277

A.2 The Teflon Bag Reactor Chamber . . . . . . . . . . . . . 278

B UAM Ozone Sensitivities $\quad 279$ 


\section{List of Figures}

1.1 Schematic of the many steps needed to make inferences about the effect of alternative fuels on ozone production in urban areas. . . . . . . . . . 5

1.2 A legend for the experimental plan. . . . . . . . . . . . . . 9

1.3 The original DOE experimental plan. . . . . . . . . . . . . 10

1.4 A chamber characterization test example showing matched chamber performance. . . . . . . . . . . . . . . 11

1.5 Base CNG and LPG test examples. . . . . . . . . . . . . . . . . 13

1.6 Base E85 test examples. . . . . . . . . . . . . . . . . . . . . . . . . 14

1.7 Single mixture E85 test examples. . . . . . . . . . . . . . . . . . . 15

1.8 Comparison of SynURB/SynIAG experiments from 1991 and 1994 . . . . . 17

1.9 Simulation results with the CB4 mechanism for JL0992 using different year's temperatures. . . . . . . . . . . . . . . . . . . . . . 19

1.10 Run 3-9:1 SynUrban/SynIAG:NO versus 9:1 SynUrban/SynCNG:NO Carbon Bond Four Simulation . . . . . . . . . . . . . . 20

1.11 Run 10-6:1 SynUrban/SynIAG:NO versus 6:1 SynUrban/SynE85E:NO ${ }_{\mathrm{x}} \quad 21$

1.12 Run 12-6:1 SynUrban/SynIAG:NO versus 6:1 SynUrban/SynE85B:NO Carbon Bond Four Simulation . . . . . . . . . . . . . . . . . . 22

1.13 The O-(2,3,4,5,6)-pentafluorobenzyl oxime of of acetaldehyde. . . . . . . 24

1.14 Reconstructed $\mathrm{m} / \mathrm{z} 181$ ion chromatogram of the batch sample collected from an outdoor smog chamber toluene/ $\mathrm{NO}_{\mathrm{x}}$ daytime experiment. . . . . . 26

1.15 Ion trap methane CI mass of the PFBHA derivative of 2,3-epoxy-2,4dimethyl-6-oxo-4-heptenal. . . . . . . . . . . . . . . 28

2.1 Summary composition of the CRC VOC mixtures. The NMOC bars are the distribution of the non-methanol fraction of the mixtures. . . . . . . . . 35

2.2 Summary composition of the compressed natural gas VOC mixtures. . . . 36

2.3 Summary composition of the ethanol VOC mixtures. . . . . . . . . . 37

2.4 DOE Experimental Plan ... . . . . . . . . . . . . . . . . 39

3.1 Run 2-6:1 SynUrban/SynIAG:NO ${ }_{x}$ versus 6:1 SynUrban/SynCNGNO $\ldots$. 44

3.2 Run 3-9:1 SynUrban/SynIAG:NO ${ }_{x}$ versus 9:1 SynUrban/SynCNG:NO ${ }_{x} \quad$. 46 
3.3 Run 5-6:1 SynUrban/SynIAG:NO versus 6:1 SynUrban/SynCNG:NO $\ldots 48$

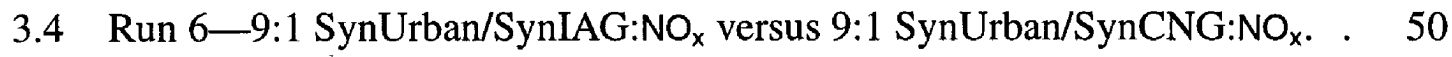

3.5 Run 7-6:1 SynUrban/SynIAG:NO ${ }_{x}$ versus 6:1 SynUrban/SynLPG:NO . $\quad 53$

3.6 Run 7-6:1 SynUrban/SynIAG:NO ${ }_{x}$ versus 6:1 SynUrban/SynLPG:NO . . $^{2} 55$

3.7 Run 9-4.5:1 SynUrban/SynIAG: $\mathrm{NO}_{\mathrm{x}}$ versus 4.5:1 SynUrban/SynE85E:NO ${ }_{\mathrm{x}} 59$

3.8 Run 9-4.5:1 SynUrban/SynIAG: $\mathrm{NO}_{\mathrm{x}}$ versus 4.5:1 SynUrban/SynE85E:NO ${ }_{x} 61$

3.9 Run 10-6:1 SynUrban/SynIAG:NO versus 6:1 SynUrban/SynE85E:NO.$\quad 63$

3.10 Run 10-6:1 SynUrban/SynIAG:NO versus 6:1 SynUrban/SynE85E:NO.$\quad 65$

3.11 Run 10-6:1 SynUrban/SynIAG:NO versus 6:1 SynUrban/SynE85E:NO.$\quad 67$

3.12 Run 11-9:1 SynUrban/SynIAG:NO versus 9:1 SynUrban/SynE85E:NO . $\quad 69$

3.13 Run 11-9:1 SynUrban/SynIAG:NO versus 9:1 SynUrban/SynE85E:NO . $\quad 71$

3.14 Run 12-6:1 SynUrban/SynIAG:NO versus 6:1 SynUrban/SynE85B:NO 73

3.15 Run 12-6:1 SynUrban/SynIAG:NO versus 6:1 SynUrban/SynE85B:NO 75

3.16 Run 14-6:1 SynUrban: $\mathrm{NO}_{\mathrm{x}}$ versus 6:1 SynE85E: $\mathrm{NO}_{\mathrm{x}} \ldots \ldots \ldots$. . . . . . 78

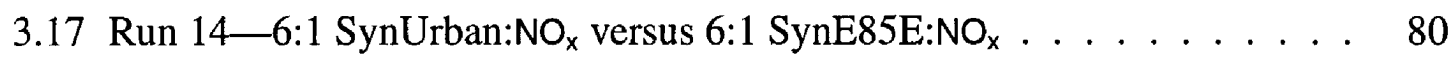

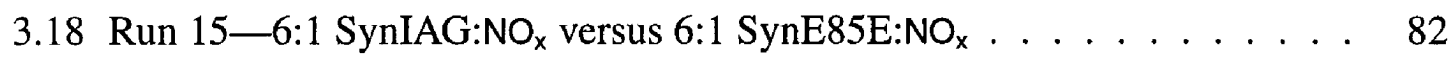

3.19 Run 17-6:1 SynUrban:NO $\mathrm{NO}_{\mathrm{x}}$ versus 6:1 SynUrban/SynE85E:NO $\mathrm{NO}_{\mathrm{x}} \ldots$. . . . 86

3.20 Run 18-6:1 SynUrban:NO ${ }_{x}$ versus 6:1 SynUrban/SynE85E(no ETOH): $\mathrm{NO}_{x} \quad 88$

3.21 Run 19-6:1 SynUrban/SynIAG:NO versus 6:1 SynUrban/SynE85E (xt $\mathrm{EtOH}): \mathrm{NO}_{\mathrm{x}} \ldots \ldots \ldots$. . . . . . . . . . . . . . . . . 90

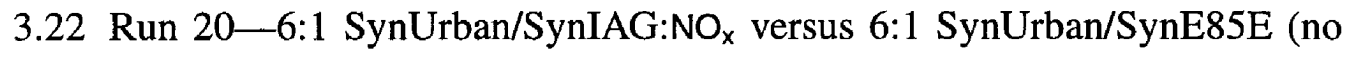
aldehyde) $: \mathrm{NO}_{\mathrm{x}} . \ldots \ldots . \ldots . \ldots . . \ldots 2$

3.23 Run 21-6:1 SynUrban/SynIAG:NO versus SynUrban/SynE85E (equal mass) $: \mathrm{NO}_{\mathrm{x}} \ldots \ldots \ldots \ldots$. . . . . . . . . . . . . . . . . 94

3.24 Run C2-100 ppm CO:NO versus 250 ppm CO:NO $\ldots$. . . . . . . . . . . 97

3.25 Run C2-100 ppm CO:NO versus 250 ppm CO:NO $\ldots$. . . . . . . . . . . 99

3.26 Run C3-250 ppm CO:NO versus 250 ppm CO:NO $\ldots$. . . . . . . . . . . 101

3.27 Run C4-6:1 propene: $\mathrm{NO}_{\mathrm{x}}$ versus 6:1 propene: $\mathrm{NO}_{\mathrm{x}} \ldots \ldots$. . . . . . . . . . 103

3.28 Run C5-6:1 SynUrban:NO $\mathrm{NO}_{\mathrm{x}}$ versus 6:1 SynUrban:NO $\ldots$. . . . . . . . . 105

3.29 Run CRC-2-6:1 SynUrban/SynIAG:NO versus 6:1 SynUrban/SynM85:NO 108

3.30 Run CRC-11-6:1 SynUrban/SynIAG:NO versus 6:1 SynUrban/SynM85/NOx110

3.31 Run C5-6:1 SynUrban:NO $\mathrm{NO}_{\mathrm{x}}$ versus 6:1 SynUrban: $\mathrm{NO}_{\mathbf{x}} \ldots \ldots$. . . . . . . . 112

3.32 Run CRC-C1-250 ppm $\mathrm{CH}_{4}: \mathrm{NO}_{\mathrm{x}}$ versus $500 \mathrm{ppm} \mathrm{CH} \mathrm{CHO}_{4}$. . . . . . . . 114

4.1 The Chamber simulation process. . . . . . . . . . . . . . . 116

4.2 Comparison of SynURB-only experiments from 1991 and 1994. . . . . . . 119

4.3 Comparison of SynURB/SynIAG experiments from 1991 and 1994 . . . . . 121

4.4 Comparison of SynURB/SynIAG experiments from 1992 and 1994. . . . . . 122 
4.5 Comparison of SynURB/SynIAG experiments from 1992 and 1994 . . . . . 123

4.6 Comparison of SynURB/SynIAG experiments from 1992 and 1994 . . . . . 125

4.7 Comparison of SynURB/SynIAG experiments from 1992 and 1994 . . . . . 126

4.8 Solar radiation simulation for JL3092. . . . . . . . . . . . . . . . . . . . 127

4.9 Carbon Bond Four simulation of methane/ $\mathrm{NO}_{\mathrm{x}}$ experiment. . . . . . . . . 128

4.10 Carbon Bond Four simulation of full urban and alkane/alkene-only experiment 130

4.11 Comparison of in chamber temperature in 1992 and 1994 . . . . . . . . 131

4.12 Solar radiation simulation for JL0992. . . . . . . . . . . . . . . . 131

4.13 Carbon Bond Four simulation of JL0992 at different temperatures. . . . . 132

4.14 Solar radiation simulation for JL1092 . . . . . . . . . . . . . . . 133

4.15 Solar radiation simulation for $\mathrm{JL} 1592 \ldots \ldots \ldots$

4.16 Carbon Bond Four simulation of JL1092 at different temperatures. . . . . 134

4.17 Carbon Bond Four simulation of JL1592 at different temperatures. . . . . 135

4.18 Run C2-100 ppm CO:NO ${ }_{x}$ verses 250 ppm CO:NO ; Carbon Bond Four

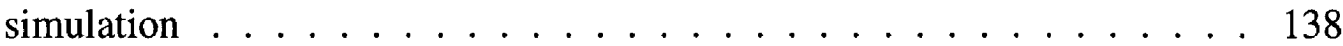

4.19 Run C2-Solar radiation simulation . . . . . . . . . . . . . . . . . . . 139

4.20 Run C5-Solar radiation simulation . . . . . . . . . . . . . . . . . . 139

4.21 Run C5-6:1 SynUrban: $\mathrm{NO}_{x}$ verses 6:1 SynUrban:NO $\mathrm{N}_{x}$ Carbon Bond Four Simulation . . . . . . . . . . . . . . . . . . . 140

4.22 Run 2-6:1 SynUrban/SynIAG:NO $\mathrm{NO}_{\mathrm{x}}$ verses 6:1 SynUrban/SynCNGNO${ }_{\mathrm{x}}$; Carbon Bond Four simulation . . . . . . . . . . . . . . . . . 143

4.23 Run 2-Solar radiation simulation . . . . . . . . . . . . . . . . . 144

4.24 Run 3-Solar radiation simulation . . . . . . . . . . . . . . . . . . 144

4.25 Run 3-9:1 SynUrban/SynIAG:NOx verses 9:1 SynUrban/SynCNG:NO ; Carbon Bond Four simulation . . . . . . . . . . . . . . . . . 145

4.26 Run 5-6:1 SynUrban/SynIAG:NO ${ }_{x}$ verses 6:1 SynUrban/SynCNG:NO ${ }_{x}$; Carbon Bond Four simulation . . . . . . . . . . . . . . . . 146

4.27 Run 5-Solar radiation simulation . . . . . . . . . . . . . . . . . 147

4.28 Run 6-Solar radiation simulation . . . . . . . . . . . . . . . . . . 147

4.29 Run 6-9:1 SynUrban/SynIAG:NO ${ }_{x}$ verses 9:1 SynUrban/SynCNG:NO ${ }_{x}$. 148

4.30 Run 7-Solar radiation simulation . . . . . . . . . . . . . . . . . . . . . 149

4.31 Run 7—9:1 SynUrban/SynIAG:NO ${ }_{x}$ verses 9:1 SynUrban/SynLPG:NO Carbon Bond Four simulation . . . . . . . . . . . . . . . . 150

4.32 Run 10-6:1 SynUrban/SynIAG:NO ${ }_{x}$ verses 6:1 SynUrban/SynEevE:NO ${ }_{x} .153$

4.33 Run 10 -Solar radiation simulation . . . . . . . . . . . . . . . 154

4.34 Run 10-Solar radiation simulation . . . . . . . . . . . . . . . . 154

4.35 Run 10—6:1 SynUrban/SynIAG:NO ${ }_{x}$ verses 6:1 SynUrban/SynEevE:NO ${ }_{x} . \quad 155$ 
4.36 Run 11-9:1 SynUrban/SynIAG:NO verses 9:1 SynUrban/SynE85E:NO Carbon Bond Four simulation . . . . . . . . . . . . . . 156

4.37 Run 11 -Solar radiation simulation . . . . . . . . . . . . . . . 157

4.38 Run 12-Solar radiation simulation . . . . . . . . . . . . . . . . 157

4.39 Run 12-6:1 SynUrban/SynIAG:NOx verses 6:1 SynUrban/SynE85B:NO Carbon Bond Four simulation . . . . . . . . . . . . . . . 158

4.40 Run $14-$ Solar radiation simulation . . . . . . . . . . . . . . . . . 159

4.41 Run 14-6:1 SynUrban:NO ${ }_{x}$ verses 6:1 SynE85E:NO ${ }_{x}$; Carbon Bond Four

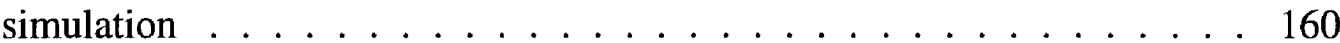

4.42 Run 17-6:1 SynUrban: $\mathrm{NO}_{x}$ verses 6:1 SynUrban/SynE85E:NO Bond Four simulation . . . . . . . . . . . . . . . . 163

4.43 Run 17 -Solar radiation simulation $\ldots \ldots \ldots$

4.44 Run 18-Solar radiation simulation . . . . . . . . . . . . . . . . . 164

4.45 Run 18-6:1 SynUrban: $\mathrm{NO}_{\mathrm{x}}$ verses 6:1 SynUrban/SynE85E(noETOH):NO Carbon Bond Four simulation . . . . . . . . . . . . . . . 165

4.46 Run 19-6:1 SynUrban/SynIAG:NO ${ }_{x}$ verses 6:1 SynUrban/SynE85E (xt EtOH) $: \mathrm{NO}_{x}$; Carbon Bond Four simulation . . . . . . . . . 166

4.47 Run 19-Solar radiation simulation . . . . . . . . . . . . . . . 167

4.48 Run 20 -Solar radiation simulation . . . . . . . . . . . . . . . . . 167

4.49 Run 20-6:1 SynUrban/ SynIAG:NO ${ }_{x}$ verses 6:1 SynUrban/ SynE85E (no aldehyde) $: \mathrm{NO}_{x}$; Carbon Bond Four simulation . . . . . . . . . 168

5.1 The reaction of O- $(2,3,4,5,6)$-pentafluorobenzyl) hydroxylamine with a carbonyl to produce two isomers for non-symmetrical carbonyls. . . . . . . . 170

5.2 A schematic of the Saturn II capillary column gas chromatograph with ion trap mass spectrometer detector. . . . . . . . . . . . . . . . . . 171

5.3 Cross section of the Saturn II ion trap mass spectrometer detector. . . . . . 172

6.1 Run Arom-1 Toluene: $\mathrm{NO}_{\mathrm{x}}$ verses m-Xylene: $\mathrm{NO}_{\mathrm{x}} . \quad \ldots \ldots$. . . . . . . 177

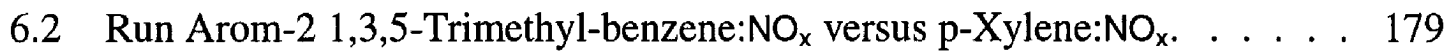

6.3 Run Arom-3 1,2,4-Trimethyl-benzene: $\mathrm{NO}_{x}$ versus o-Xylene: $\mathrm{NO}_{x}$. . . . . . 181

6.4 Reconstructed $\mathrm{m} / \mathrm{z} 181$ ion chromatogram of the batch sample collected from an outdoor smog chamber toluene/ $\mathrm{NO}_{x}$ daytime experiment. . . . . . 183

6.5 Detailed Reconstructed $\mathrm{m} / \mathrm{z} 181$ ion chromatogram of the batch sample collected from an outdoor smog chamber toluene/ $\mathrm{NO}_{\mathrm{x}}$ daytime experiment.

6.6 Reconstructed $\mathrm{m} / \mathrm{z} 181$ ion chromatogram of the batch sample collected from an outdoor smog chamber p-xylene/ $\mathrm{NO}_{\mathrm{x}}$ daytime experiment. . . . 187

6.7 Reconstructed $\mathrm{m} / \mathrm{z} 181$ ion chromatogram of the batch sample collected from an outdoor smog chamber m-xylene/NO ${ }_{x}$ daytime experiment. . . . . 190 
6.8 Detailed Reconstructed $\mathrm{m} / \mathrm{z} 181$ ion chromatogram of the batch sample collected from an outdoor smog chamber m-xylene/NO $\mathrm{Nay}_{\mathrm{x}}$ dame experiment. 191

6.9 Reconstructed $\mathrm{m} / \mathrm{z} 181$ ion chromatogram of the batch sample collected from an outdoor smog chamber o-xylene/ $\mathrm{NO}_{\mathrm{x}}$ daytime experiment. . . . . 196

6.10 Reconstructed $\mathrm{m} / \mathrm{z} 181$ ion chromatogram of 1,3,5-trimethylbenzene/ $/ \mathrm{NO}_{\mathrm{x}}$ in an outdoor smog chamber daytime experiment. . . . . . . . . . . . 200

6.11 Detailed Reconstructed $\mathrm{m} / \mathrm{z} 181$ ion chromatogram of the batch sample collected from an outdoor smog chamber 1,3,5-trimethylbenzene/ $\mathrm{NO}_{\mathrm{x}}$ daytime experiment. . . . . . . . . . . . . . 201

6.12 Reconstructed $\mathrm{m} / \mathrm{z} 181$ ion chromatogram of the batch sample collected from an outdoor smog chamber 1,2,4-trimethylbenzene/ $\mathrm{NO}_{\mathrm{x}}$ daytime experiment. . . . . . . . . . . . . . . . . . 204

6.13 The MS of normal acetone and ${ }^{13} \mathrm{C}_{3}$-acetone. . . . . . . . . . . . . 208

6.14 Run Arom-1 Toluene:NO versus Xylene:NO ${ }_{x}$; VOC series. . . . . . . . . . 210

6.15 Run Arom-1 Toluene:NO $\mathrm{NO}_{\mathrm{x}}$ versus Xylene:NO ${ }_{\mathrm{x}}$; VOC profiles. . . . . . . . . 211

6.16 Ion trap methane CI spectra of the PFBHA derivatives of butendial (bottom), 4-oxo-2-pentenal (middle), and 3-hexene-2,5-dione (top). . . . . . . . . 215

6.17 Run Arom-1 Toluene:NO $\mathrm{NO}_{\mathrm{x}}$ versus Xylene:NO ${ }_{\mathrm{x}}$; VOC series. . . . . . . . . . 217

6.18 Run Arom-2 1,3,5-Trimethyl-benzene:O $\mathrm{O}_{\mathrm{x}}$ versus Xylene:NO $\mathrm{N}_{\mathrm{x}}$, VOC series. . 218

6.19 Run Arom-1 Toluene:NO ${ }_{x}$ versus Xylene:NO ${ }_{x}$; VOC series. . . . . . . . . . 219

6.20 Run Arom-3 1,2,4-Trimethyl-benzene: $\mathrm{O}_{x}$ versus o-Xylene: $\mathrm{NO}_{\mathrm{x}}$; VOC series. 220

6.21 Run Arom-3 1,2,4-Trimethyl-benzene:O $\mathrm{O}_{x}$ versus o-Xylene: $\mathrm{NO}_{\mathrm{x}}$; VOC series. 221

6.22 Run Arom-2 1,3,5-Trimethyl-benzene: $\mathrm{O}_{\mathrm{x}}$ versus Xylene: $\mathrm{NO}_{\mathrm{x}}$; VOC series. . 222

6.23 Ion trap CI mass of the PFBHA derivatives of two $\mathrm{C}_{5}$ hydroxy dicarbonyls. Top: peak at $27.82 \mathrm{~min}$, Bottom: peak at $28.78 \mathrm{~min}$. . . . . . . . . . . . . . 226

6.24 Time series of $\mathrm{C}_{5}$ hydroxy dicarbonyl peaks in the 1,3,5 TMB and 1,2,4 TMB experiments. . . . . . . . . . . . . . . 227

6.25 Time series of a $C_{4}$ hydroxy dicarbonyl, $C_{5}$ triones (or $C_{5}$ epoxy carbonyls), and $\mathrm{a}_{4}$ trione (or $\mathrm{a}_{4}$ epoxy dicarbonyl) peaks in the 1,3,5 TMB and 1,2,4 TMB experiments . . . . . . . . . . . . . . . . . . . . 230

6.26 Run Arom-2 1,3,5-Trimethyl-benzene: $\mathrm{O}_{x}$ versus p-Xylene: $\mathrm{NO}_{\mathrm{x}}$; VOC series. 231

6.27 Ion trap methane CI mass of the PFBHA derivative of glycidaldehyde. . . . 236

6.28 Ion trap methane CI mass of the PFBHA derivative of Epoxide A. . . . . . 239

6.29 Run Arom-2 1,3,5-Trimethyl-benzene:O $\mathrm{O}_{x}$ versus Xylene: $\mathrm{NO}_{x}$; VOC series. . 240

6.30 The formation of Epoxide A and its subsequent reactions. . . . . . . . . . 242

7.1 Air quality models must be modified to include new code to output the process rates. . . . . . . . . . . . . . . . . . . . . 254

7.2 Schematic of a 'complete' process analysis for a model scenario run. . . . 255 
7.3 One frame from Day Two of a UAM movie of ozone mixing ratios for a 1988 Charlotte, North Carolina episode. The darkness is not proportional to $\mathrm{O}_{3}$ in this figure and deep blue (for no $\mathrm{O}_{3}$ ) and bright red (for $\mathrm{O}_{3}$ equal to or exceeding $120 \mathrm{ppb}$ ) both show as dark colors. . . . . . . . . . . . . 259

7.4 Process time series for Charlotte 1987 episode. . . . . . . . . . . . . . . 261

7.5 Model Predicted and Observed VOC composition in 6 by $65-\mathrm{km}$ cells near downtown Charlotte North Carolina in 1987 UAM scenario. . . . . . . . . 262

7.6 UAM predicted and observed $\mathrm{O}_{3}$ mixing ratio and the change caused by each process in the UAM. . . . . . . . . . . . . . . 263

7.7 Process composition times series for $\mathrm{O}_{3}$ in Episode 287FC Charlotte, North Carolina UAM simulation . . . . . . . . . . . . . . . . . . . 265

7.8 Process composition spatial contribution to a single cell's $\mathrm{O}_{3}$ in Episode 287FC Charlotte, NC UAM simulation. . . . . . . . . . . . . . . . 267

7.9 The $\mathrm{OH}$ and $\mathrm{NO}_{\mathrm{x}}$ Cycles from Integrated Reaction Rates for $6 x 6$ Cell Area in UAM Scenario 287FC for Charlotte, NC. . . . . . . . . . . . . . . . . 268

7.10 The NO Cycle and Mass Balance from Integrated Reaction Rates for $6 x 6$ Cell Area in UAM Scenario 287FC for Charlotte, NC. . . . . . . . . . . . 269

7.11 The distribution of reacted VOC and ozone produced in an Urban Airshed Model simulation of Charlotte, $\mathrm{NC}$ in 1987. . . . . . . . . . . . . . . 272 


\section{List of Tables}

2.1 The VOC Mixtures used in the CRC Methanol-85 Chamber Study-Part 1 . 33

2.2 The VOC Mixtures used in the CRC Methanol-85 Chamber Study-Part 2 . 34

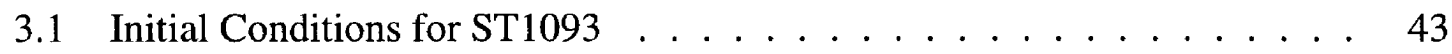

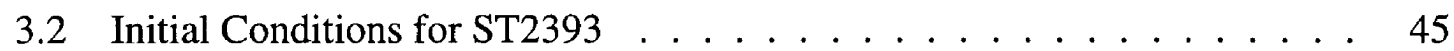

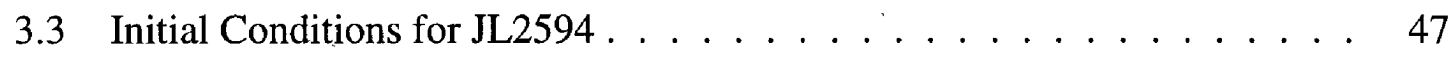

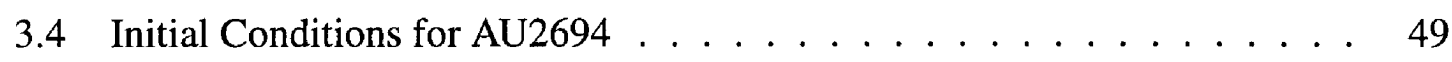

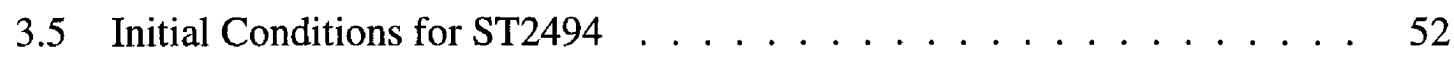

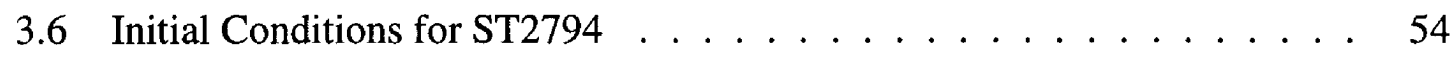

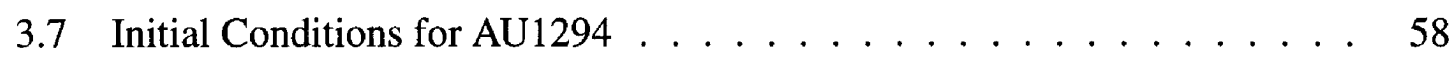

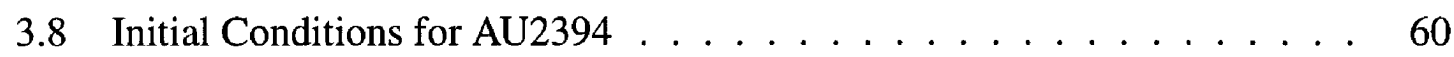

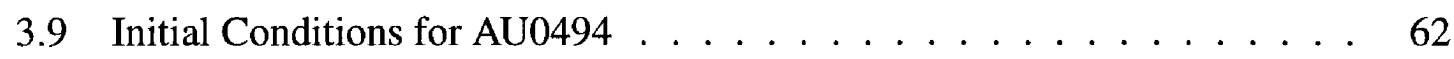

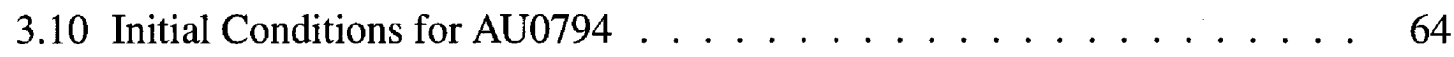

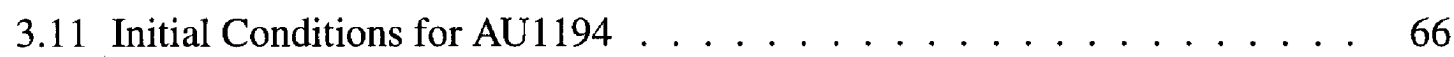

3.12 Initial Conditions for AU2494 . . . . . . . . . . . . . . . . 68

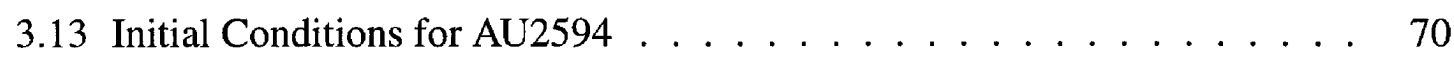

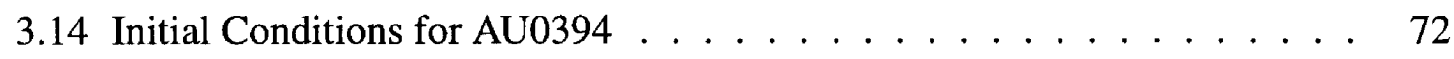

3.15 Initial Conditions for AU0894 . . . . . . . . . . . . . . . . . 74

3.16 Initial Conditions for $\mathrm{ST0994} \ldots \ldots \ldots \ldots$

3.17 Initial Conditions for $\mathbf{S T} 1194 \ldots \ldots \ldots$

3.18 Initial Conditions for $\mathrm{ST0894} \ldots \ldots \ldots \ldots$

3.19 Initial Conditions for $\mathrm{ST} 1494 \ldots \ldots \ldots \ldots$

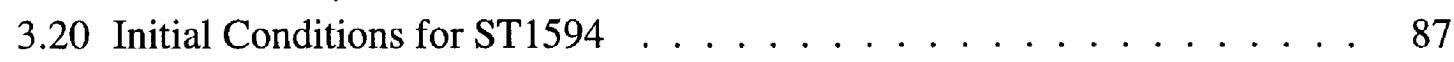

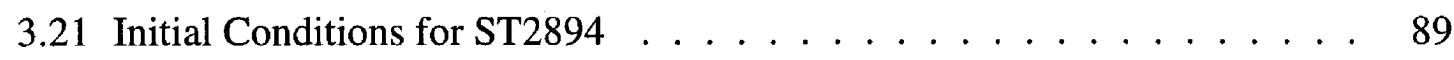

3.22 Initial Conditions for AU0994 . . . . . . . . . . . . . . 91

3.23 Initial Conditions for OC0994 . . . . . . . . . . . . . . . 93

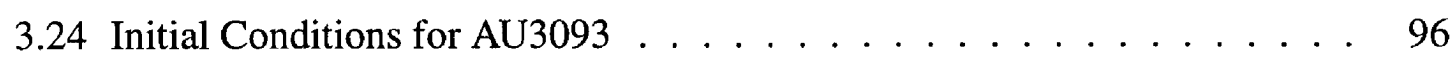

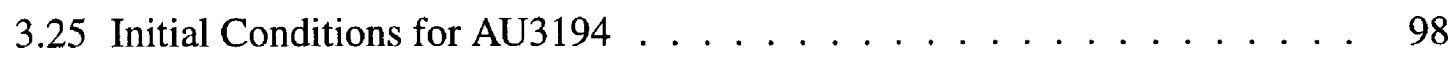

3.26 Initial Conditions for AU2994 . . . . . . . . . . . . . . . . . 100 
3.27 Initial Conditions for JL0194 _ . . . . . . . . . . . . . . . . . 102

3.28 Initial Conditions for ST1294 _ . . . . . . . . . . . . . . . . . . 104

3.29 Initial Conditions for JL1092 _ . . . . . . . . . . . . . . . . . . . . 107

3.30 Initial Conditions for JL1592 _ . . . . . . . . . . . . . . . . . . . . . 109

3.31 Initial Conditions for JL0992 . . . . . . . . . . . . . . . . 111

3.32 Initial Conditions for JL3092 . . . . . . . . . . . . . . . . . . . 113

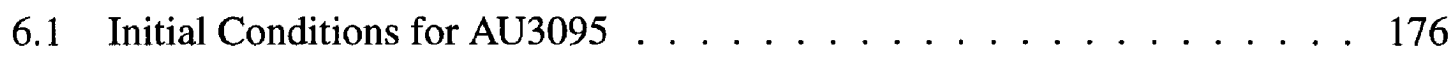

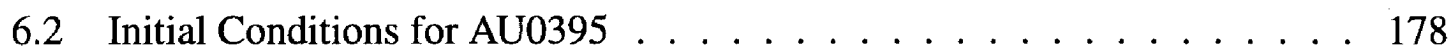

6.3 Initial Conditions for ST0195 _. . . . . . . . . . . . . . . 180

6.4 Carbonyl Products Detected in a Sample Collected from a Toluene/ $\mathrm{NO}_{\mathbf{x}}$ Daytime Smog Chamber Experiment . . . . . . . . . . . . . . . 185

6.5 (Continued) Carbonyl Products Detected in a Sample Collected from a Toluene/ $\mathrm{NO}_{x}$ Daytime Smog Chamber Experiment . . . . . . . . . . . . 186

6.6 Carbonyl Products Detected in a Sample Collected from a p-Xylene/ $\mathrm{NO}_{x}$ Daytime Smog Chamber Experiment . . . . . . . . . . . . . . 188

6.7 (Continued) Carbonyl Products Detected in a Sample Collected from a pXylene/ $\mathrm{NO}_{x}$ Daytime Smog Chamber Experiment . . . . . . . . . . . . . 189

6.8 Carbonyl Products Detected in a Sample Collected from a m-xylene/ $\mathrm{NO}_{x}$ Daytime Smog Chamber Experiment . . . . . . . . . . . . . . . . 192

6.9 (Continued) Carbonyl Products Detected in a Sample Collected from a mxylene/ $\mathrm{NO}_{\mathbf{x}}$ Daytime Smog Chamber Experiment . . . . . . . . . . .

6.10 (Continued) Carbonyl Products Detected in a Sample Collected from a mxylene/NO ${ }_{x}$ Daytime Smog Chamber Experiment . . . . . . . . . . . .

6.11 (Continued) Carbonyl Products Detected in a Sample Collected from a mxylene/NO ${ }_{x}$ Daytime Smog Chamber Experiment . . . . . . . . . . 195

6.12 Carbonyl Products Detected in a Sample Collected from a o-xylene/ $\mathrm{NO}_{\mathbf{x}}$ Daytime Smog Chamber Experiment . . . . . . . . . . . . . .

6.13 (Continued) Carbonyl Products Detected in a Sample Collected from a oxylene/NO x Daytime Smog Chamber Experiment . . . . . . . . . . 198

6.14 (Continued) Carbonyl Products Detected in a Sample Collected from a oxylene/NO Daytime Smog Chamber Experiment . . . . . . . . . . . . 199

6.15 Carbonyl Products Detected in a Sample Collected from a 1,3,5-trimethylbenzene/ $\mathrm{NO}_{x}$ Daytime Smog Chamber Experiment . . . . . . . . . . . . . . 202

6.16 (Continued) Carbonyl Products Detected in a Sample Collected from a 1,3,5-trimethylbenzene/ $\mathrm{NO}_{x}$ Daytime Smog Chamber Experiment . . . . . 203

6.17 Carbonyl Products Detected in a Sample Collected from a 1,2,4-trimethylbenzene/ $\mathrm{NO}_{x}$ Daytime Smog Chamber Experiment . . . . . . . . . . . . . 205

6.18 (Continued) Carbonyl Products Detected in a Sample Collected from a 1,2,4-trimethylbenzene $/ \mathrm{NO}_{x}$ Daytime Smog Chamber Experiment . . . . . 206 
6.19 List of Unknown Carbonyl Products . . . . . . . . . . . . . . 232

6.20 Structure II epoxy carbonyls formed from six alkyl benzenes. . . . . . . . 243

6.21 Structure III epoxy carbonyls formed from six alkyl benzenes. . . . . . . 244

6.22 Structure IV epoxy carbonyls formed from six alkyl benzenes. . . . . . 245

6.23 Structure $V$ epoxy carbonyls formed from six alkyl benzenes. . . . . . . 246

6.24 Structure VI epoxy carbonyls formed from six alkyl benzenes. . . . . . . 247

6.25 Structure VIII epoxy carbonyls formed from six alkyl benzenes. . . . . . 248

6.26 Structure X epoxy carbonyls formed from six alkyl benzenes. . . . . . . . 249

7.1 The First Part of the Integrated Process Rate Output . . . . . . . . . 256

7.2 The Second Part of the Integrated Process Rate Output . . . . . . . . 256

7.3 $\mathrm{NO}_{\mathrm{y}}$ Mass Balance from Integrated Reaction Rates for UAM Scenario 287FC for Charlotte . . . . . . . . . . . . . . . . . . . . 271 


\section{Chapter 1}

\section{Executive Summary}

\subsection{Purpose}

The goal of this project was to improve our ability to predict the changes in urban ozone resulting from the widespread use of alternative fuels in automobiles.

The purposes of the work reported here were:

- To conduct side-by-side outdoor chamber experiments using highly realistic, yet carefully formulated, synthetic mixtures of volatile organic compounds (VOCs) that represent the best estimate of the total emissions (tail pipe, evaporative, and running losses) from vehicles operated on alternative fuels. This work especially focused on compressed natural gas and ethanol blended with gasoline fuels. These experiments were designed to demonstrate directly the relative effectiveness of alternative fuels under realistic conditions and served to test photochemical reaction models in a stressful manner.

- To use mathematical models to simulate these experiments using the most commonly photochemical reaction mechanism now used in Eulerian airshed modeling and that are used in the U.S. Environmental Protection Agency's (EPA) regulatory models as a means of demonstrating the adequacy of these mechanisms to make accurate predictions of the large scale use of alternative fuels in an urban environment.

- To investigate the detailed reaction mechanisms for alkyl-substituted benzenes, which make up approximately $30 \%$ of urban atmospheric non-methane carbon. Despite significant effort in the last 15 years, atmospheric chemists have been unable to describe such reactions because of a lack of analytical ability to detect the form of the intermediate products subsequent to initial attack by hydroxyl radical, the major loss process for these type of compounds.

- To perform urban air shed model simulations and to investigate the role that alternative fuels may have on the ozone produced in these simulations. 


\subsection{Findings}

A summary of our findings and conclusions follows.

- Compressed natural gas (CNG) used as an alternative fuel produced significantly less ozone than did industry averaged gasoline (IAG), even at hydrocarbon to nitrogen oxides ratios $\left(\mathrm{HC}: \mathrm{NO}_{\mathbf{x}}\right)$ of 9:1. This difference was reproducible by the Carbon Bond Four photochemical reaction mechanism (CB4) used in a chamber mathematical simulation model, but there are caveats (see below) regarding the mechanism's formulation.

- When liquefied propane gas (LPG) was used as an alternative fuel, it also produced significantly less ozone than did IAG at a $\mathrm{HC}: \mathrm{NO}_{\mathrm{x}}$ of $6: 1$. This difference was also reproducible by the $C B 4$ mechanism in a chamber mathematical simulation model, but there are caveats.

- When ethanol blended with $15 \%$ gasoline (E85) was used as an alternative fuel, it produced significantly more ozone than did IAG at $\mathrm{HC}: \mathrm{NO}_{\times}$of 4.5:1 and 6:1, and produced the same ozone at 9:1. This difference was reproducible by the CB4 mechanism in a mathematical chamber simulation model, but there are caveats.

- The principal reason that blended ethanol mixtures produced more ozone than did IAG was demonstrated in experiments in which the ethanol mixture composition was varied. These showed that the significant fraction of acetaldehyde present in the exhaust was the cause. The reactions of acetaldehyde produce peroxyacyl nitrate (PAN) that, at the high concentrations of acetaldehyde in the ethanol blends, stores a significant amount of $\mathrm{NO}_{x}$. PAN, however, exhibits a strong temperature dependence on its decomposition rate, and as afternoon chamber temperatures increased, the PAN releases the $\mathrm{NO}_{x}$ back into the system where it can produce ozone. In the IAG systems, much less PAN is produced and the $\mathrm{NO}_{\mathrm{x}}$ is removed to more permanent stable products; thus, less is available in the afternoon to produce ozone. These effects were reproducible by the CB4 mechanism in a chamber mathematical simulation model, but there are caveats.

- Experiments conducted at two different air temperatures, but otherwise nearly identical conditions, show almost no temperature effect on ozone production and at best suggest a small negative effect. These experiments do suggest a negative effect on afternoon nitrogen-containing compounds that are measurable by the $\mathrm{NO}_{\mathrm{x}}$-channel of a standard analyzer (this includes nitrogen dioxide, PAN and PAN-like compounds, organic nitrates and nitrites, but little response to nitric acid). It appears that the strong positive temperature effect exhibited by alkane vocs such as n-butane are nearly perfectly offset by the temperature labile organic nitrogen products produced in our much more complex VOC mixtures that contain a variety of aromatic species.

- The CB4 mechanism does not reproduce the observed temperature effect. Instead, it is much too reactive at chamber temperatures above $90^{\circ} \mathrm{F}$, and at temperatures 
above $100^{\circ} \mathrm{F}$ it tends to over-predict observed ozone by as much as $200 \%$. At peak afternoon temperatures near $80^{\circ} \mathrm{F}$, the $\mathrm{CB} 4$ mechanism produces predictions that agree accurately with the chamber observations.

- The use of O-(2,3,4,5,6-pentafluorobenzyl)-hydroxylamine (PFBHA) to form oximes derivatives of polyfunctional carbonyls, which are then separated in a gas chromatograph and detected by ion trap mass spectrometry, is a very specific and sensitive new analytical method developed as part of this project. This method is capable of providing the molecular weight and some structural information about unknown carbonyls without the need for a standard.

- In indoor chamber and outdoor chamber experiments using single aromatic VOC species with $\mathrm{NO}_{x}$, the new PFBHA method identified more than 40 new carbonyl products formed in the photooxidation of these important urban atmospheric compounds. These included not only the previously detected aromatic aldheydes and the saturated dicarbonyls (e.g., glyoxal and methyl glyoxal), but also hydroxy carbonyls (e.g., glycolaldehyde, hydroxy acetone); hydroxy dicarbonyls; a variety of di-unsaturated 1,6-dicarbonyls; and unsaturated 1,4-dicarbonyls, quiones, and possiablly triones.

- The most significant products detected in these aromatic systems were a variety of epoxy carbonyls and epoxy dicarbonyls. These species, which may account for as much as as one-third of the aromatic oxidation pathway, are possibly mutagens. An epoxy carbonyl similar in structure to several of the intermediates we detected, 2,3epoxypropanal has been reported to be a potent mutagen in a variety of in vitro test systems and has been shown to induce sarcomas in rats by subcutaneous injection. As a bifuctional agent, 2,3-epoxy propanal is capable of forming cyclic adducts and crosslinks with DNA bases. Hence, the possible formation of epoxides from atmospheric oxidation of aromatic compounds is a significant public health concern, deserving additional laboratory and field work. If it becomes necessary to reduce or remove aromatic species from gasoline fuels because of their potential health effects, the use of alternative fuels such as $\mathrm{CNG}$ becomes even more compelling.

- Between this project and another UNC project, we developed a new method for improving the computed emissions from mobile sources in urban airshed models. This method combines the outputs of travel demand models and mobile source emissions models to more correctly temporally and spatially allocate the various components of mobile emissions in the gridded domain. These treatments resulted in up to $25 \%$ increases in the VOC emissions in downtown cells in the model, while reducing the concentrations along the Interstate highways.

- New methods for performing analysis of urban airshed model predictions were developed jointly between this project and yet another UNC project.

More details are given in the following sections. 


\subsection{Background}

In 1988, Congress passed the Alternative Motor Fuels Act (AMFA) with the intent to reduce: ambient air levels of ozone $\left(\mathrm{O}_{3}\right)$ and carbon monoxide $(\mathrm{CO})$; American dependence on foreign oil; and gases contributing to global warming.

The AMFA encourages the development and widespread use of alternative transportation fuels by providing for changes in corporate average fuel economy (CAFE) regulations and purchases of vehicles for the Federal fleet.

Although it is clear that alternative fuels can lead to less dependence on foreign oil and can perhaps result in reduced $\mathrm{CO}$ emissions, doubt remains about the first goal of AMFA. That is, methanol can be made from domestic coal and ethanol from corn, and both tail pipe and on-road measurements have shown about a $16 \%$ reduction in direct $\mathrm{CO}$ emissions when oxygenated fuels are used, but urban $\mathrm{O}_{3}$ is a secondary pollutant resulting from non-linear chemistry of dispersed reactants. This means that the case of large benefits arising from the use of alternative fuels is not so easily made.

Organic emissions from the exhaust of alternative fuel vehicles contain substantial fractions of the unburned fuel, as well as the aldehyde derivatives. For example, exhaust from vehicles running on methanol/gasoline blends contains large quantities of methanol and acetaldheyde( $\mathrm{CCHO}$ ), while exhust from vehicle running on ethanol fuels contains ethanol and formaldehyde $(\mathrm{HCHO})$. Although methanol or ethanol, by themselves, are generally thought of as less photochemically reactive than most of the hydrocarbons emitted from vehicles runing on gasoline, $\mathrm{HCHO}$ and $\mathrm{CCHO}$ are much more reactive. Furthermore, in $\mathrm{M} 85$ or E85 fuel for which gasoline comprises $15 \%$ of the fuel, gasoline-related emissions make up almost $40 \%$ of the exhaust carbon. In newer gasoline vehicles, exhaust is becoming a smaller fraction of the total vehicle emissions; other important sources are evaporative losses and running losses. The compositions of these emissions are dominated by the "light" species in the fuels, such as n-butane and isopentane. To estimate the benefits of alternative fuels to improve air quality, we first must understand how the fuel composition affects the vehicle exhaust, evaporative, and running loss compositions. Then we must make inferences about the effect of changes in these VOC compositions on subsequent $\mathrm{O}_{3}$ formation in an already complex urban chemical environment.

Figure 1.1 shows the many steps necessary to support the assertion that a particular alternative fuel might lead to the reduction of ozone in an urban area. We performed research in essentially all areas shown in this figure.

The mechanism whereby alternative fuels would have an impact on urban $\mathrm{O}_{3}$ is the supposedly lower "reactivity" of the alternative fuel exhausts and fuel losses. "Reactivity" refers to the extent to which a compound or a mixture of compounds contributes to atmospheric oxidation of $\mathrm{VOC}$, oxidation of $\mathrm{NO}$ to $\mathrm{NO}_{2}$, and subsequent $\mathrm{O}_{3}$ production in the ambient atmosphere. Unfortunately, reactivity is not an immutable characteristic of a VOC molecule, but rather it arises as a synergistic interaction among all reacting species. The reactivity of a VOC is as much a property of the chemical and physical environment in which it is reacting as it is of the VOC molecule itself. It has been shown both experimentally and 


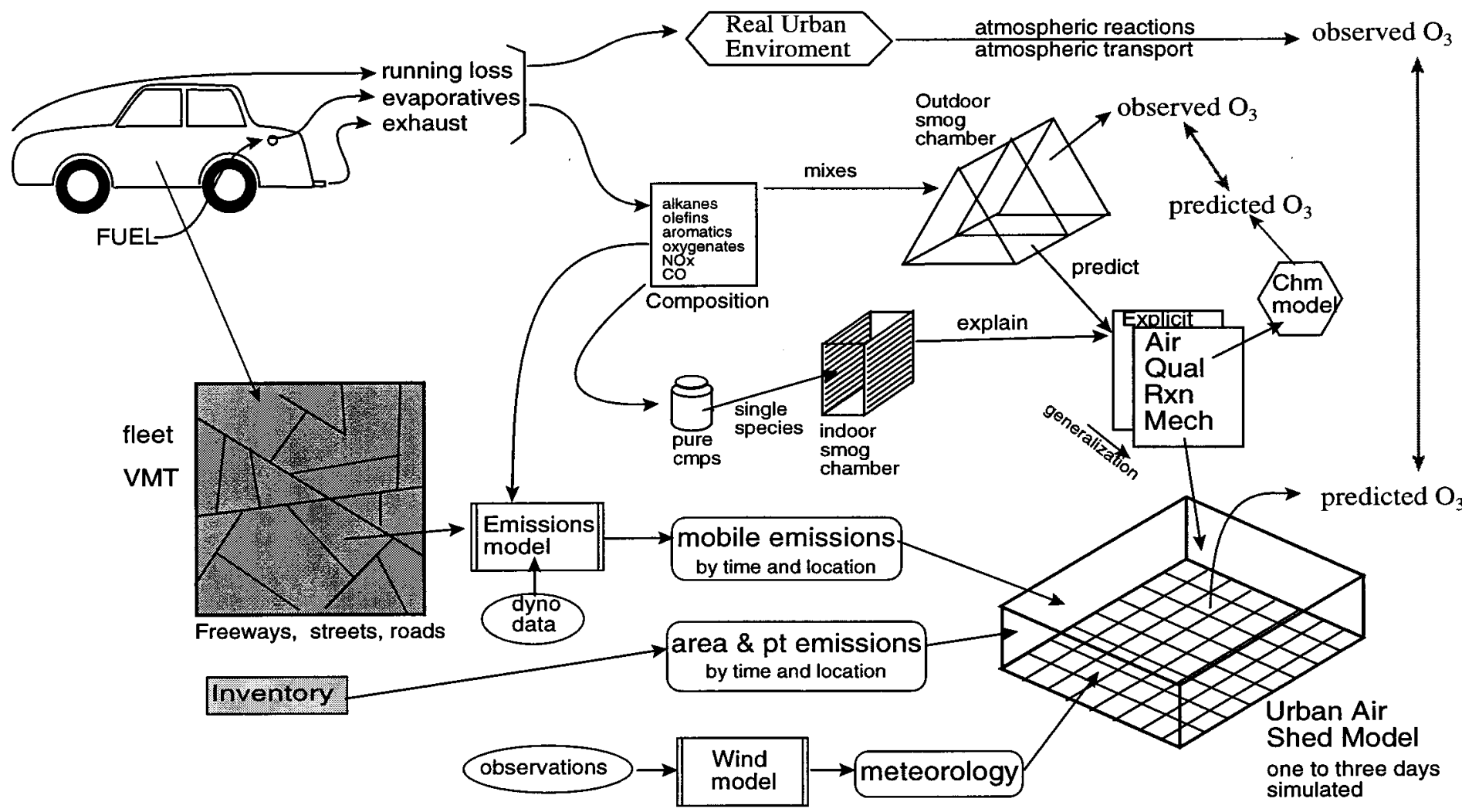

Figure 1.1: Schematic of the many steps needed to make inferences about the effect of alternative fuels on ozone production in urban areas. 
theoretically that if the conditions in which the reactions are occurring changes, then so do the reactivities of the compounds. Some compounds (e.g., toluene and longer paraffins) even change the direction of their reactivity effect, making a positive contribution under one set of conditions and a negative contribution under other conditions. Thus, one cannot talk about the reactivity of one compound relative to another without first making clear the specific environment in which the reactivity is occurring.

Thus, the most important information needed to understand how a compound will contribute to mixture reactivity is to know exactly which products are produced after hydroxyl radical $(\mathrm{OH})$ attack and to know the reactions subsequent to reaction of peroxy-radicals $\left(\mathrm{RO}_{2}\right)$ with NO. Current knowledge of the products formed after NO oxidation is quite incomplete, especially for aromatics, and the field is ripe for further investigation.

In urban atmospheric environments, aromatic hydrocarbons (e.g., toluene, the xylenes, and the trimethyl-benzenes) make up as much as $30 \%$ of the carbon [1]. It is understood that these species react with hydroxyl radicals, but the identity of many of the products formed and their subsequent reactions are unknown. These species have been the subject of many laboratory investigations over the last 20 years, but the products and mechanisms have yet to be completely explained [2,3]. The major reasons for this failure has been the lack of appropriate analytical methods to detect and identify the intermediates and the large number of potential reaction pathways.

Although it is necessary to have this detailed mechanistic understanding of how a particular VOC can react, it is the surrounding environment that determines the conditions under which it will react. Thus, results from smog chambers, which are useful for the former, do not tell us about the latter. For this, an urban airshed model is needed that can simulate the combined effects of emissions, meteorology, and chemistry. Such a model requires significant inputs including

- Spatial and temporally resolved emissions inventories of mobile, area, and point sources

- Spatial and temporally resolved wind fields, radiation fields, and temperature fields

- Temporally resolved boundary conditions at the sides and top of the model domain

- A relatively complete photochemical reaction mechanism. 


\subsubsection{Summary of Background}

- One of the reasons for switching to alternative fuels is to reduce urban $\mathrm{O}_{3}$.

- Urban $\mathrm{O}_{3}$ is a secondary pollutant resulting from non-linear chemistry of many dispersed reactants, and the case of benefits arising from the use of alternative fuels is not easily made.

- The industry-sponsored Auto/Oil vehicle emissions research program and programs sponsored by the U.S. Department of Energy (DOE) have made good progress in establishing the exhaust, evaporative, and running loss emissions compositions arising from the use of industry standard gasoline, various reformulated gasolines, methanol, and other alternative fuels in a fleet of new and older cars and light-duty trucks.

- Reactivity is the mechanism where by switching to alternative fuels is supposed to lead to changes in urban $\mathrm{O}_{3}$ formation.

- The reactivity of a given VOC is a not an immutable characteristic of a VOC molecule, but rather arises as a synergistic interaction among all reacting species, and can be positive in one set of circumstances and negative in others. In short, a VOC's reactivity is strongly dependent on the environment in which it is reacting.

- Most of the ozone in urban air is formed by the least reactive compounds in the VOC mix.

- The most reactive compounds in urban VOC mixtures are prolific sources of "new radicals." These new radicals arise for the most part via photolysis of the carbonyl products produced in the rapid oxidation of some of the vOCs.

- Current knowledge of these potentially photolyzable products is quite incomplete, especially for aromatics and biogenic-type VOCs, and the field is ripe for further investigation.

- Current air quality reaction mechanisms are only well-formulated for a few small VOCs (e.g., methane, formaldelhyde, ethene, acetaldehyde, and PAN); a few other VOC mechanisms are based on a limited set of chamber data. The vast majority of VOC species observed in urban air have never been tested in chambers or reactor systems, and the reactions mechanisms for these compounds are the result of speculation.

- The urban airshed model has limitations arising from its highly generalized chemical reaction mechanism, and from the quality of the input data used to drive simulations.

It is clear from these points that no single approach is adequate for determining the potential atmospheric benefits of alternative fuel use-both experimental and modeling studies are needed. Further, fundamental advances are needed to improve scientific understanding of reactivity. Practical studies are needed to bound modeling studies and to 
provide conclusive observational knowledge about reactivity effects likely to arise from fuel switching.

\subsection{Experimental Design}

\subsubsection{VOC Mixtures}

This study design was similar to that of our Coordinating Research Council (CRC) Methanol Fuels Reactivity Project [4] and relied on two of the mixtures produced in that study. These were:

- SynURB, a 54-component synthetic urban VOC mixture, and

- SynIAG, a 49-component synthetic mixture based on the weighted tail pipe, evaporative, and running loss emissions from a fleet of vehicles running on IAG.

These mixtures are the most complex yet high-precision composition mixtures ever used in smog chamber studies. About $65 \%$ of the carbon was explicitly represented in these mixtures (i.e., the exact compound is included in the mixture) and the rest of the carbon was represented by a similar species with similar structure. Some attention was paid to ease of analysis and to producing a precise composition from experiment to experiment.

For the current study we used new data supplied by EPA and by NIPER for the composition of emissions of CNG vehicles and for E85 vehicles to produce new synthetic mixtures. There were two sets of data for E85 vehicles with different exhaust composition and so we blended two slightly different E85 mixtures. These three mixtures were called SynCNG, SynE85E, and SynE85B.

The compositional details of all these mixtures is given in Chapter 2 .

\subsubsection{Experimental Design}

Figure 1.2 shows a legend and Figure 1.3 shows the originally planned experimental design. This too was similar to the plan used in the CRC project.

The two sides of the chamber were used to compare the effects of changing the vOC composition directly. Each comparison would take place at three $\mathrm{HC}: \mathrm{NO}_{x}$ ratios: 4.5:1, 6:1, and 9:1. Most urban centers are near 6:1 and for most mixtures reactivity effects disappear (because the ozone formation is $\mathrm{NO}_{\mathbf{x}}$-limited) under 9:1 conditions.

Because reactivity is as much a function of the chemical environment in which a VOC reacts as it is a function of the compound itself [1], to evaluate the relative reactivity of each alternative fuel we compared them in a background of SynURB, with $50 \%$ of the carbon being due to the alternative fuel. On the other side of the dual chamber we always used $50 \%$ SynURBwith 50\% SynIAG. That is, we compared 50:50 mixtures of SynURB:SynIAGwith 


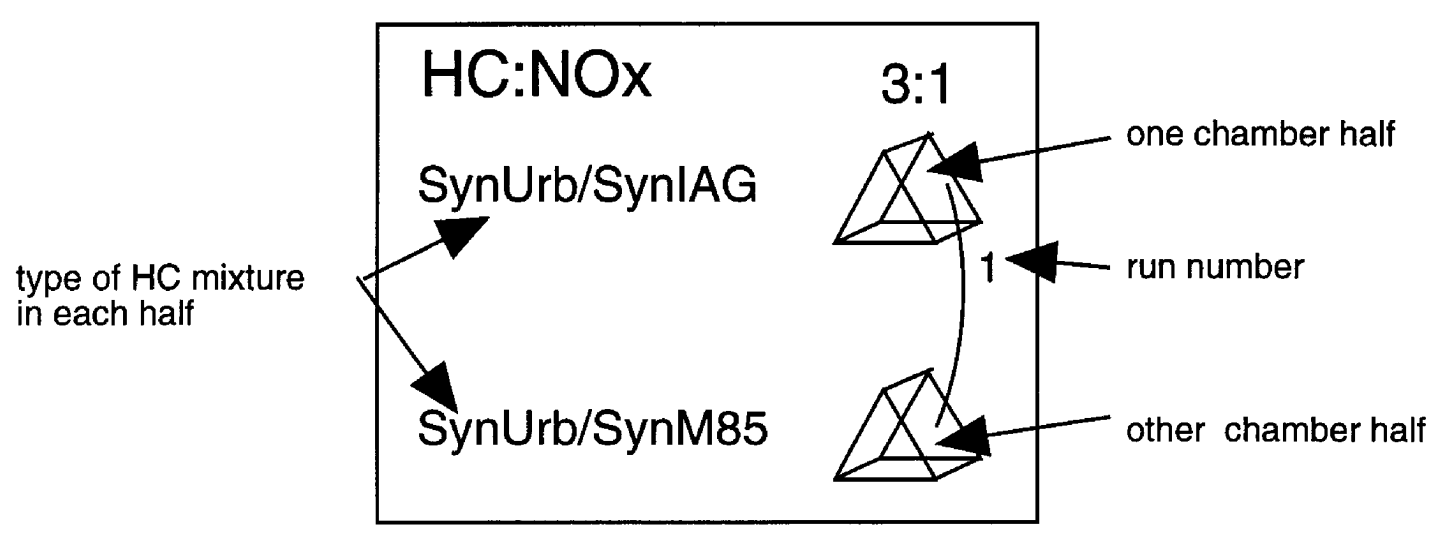

Figure 1.2: A legend for the experimental plan.

50:50 mixtures of SynURB:SynCNG, or with 50:50 mixtures of SynURB:SynE85E, or with 50:50 mixtures of SynURB:SynE85B.

The experiments were further organized into "Blocks" with a different objective for each block. As designed, the block objectives were:

1. The base CNG program which included a repeat of each of the three $\mathrm{HC}: \mathrm{NO}_{\mathrm{x}}$ conditions

2. The base E85 program which included all three $\mathrm{HC}: \mathrm{NO}_{\mathrm{x}}$ ratios

3. The single mixture comparison block at 6:1 $\mathrm{HC}: \mathrm{NO}_{\mathrm{x}}$ ratios

4. The special E-85 program block to demonstrate potential effects of various changes in the emissions

5. The liquefied propane gas (LPG) program block.

6. The chamber characterization run program block.

\subsection{Experimental Results}

The experimental results are described in a logical analysis order $(6,1,5,2,3,4)$ rather than the numerical order described above.

\subsubsection{Block 6: Chamber Characterization Tests}

As part of this project, we conducted five chamber characterization tests; other chamber characterization tests not shown here were conducted under other funding and are a part of the UNC Standard Chamber Database. 


SyC:

Figure 1.3: The original DOE experimental plan. 


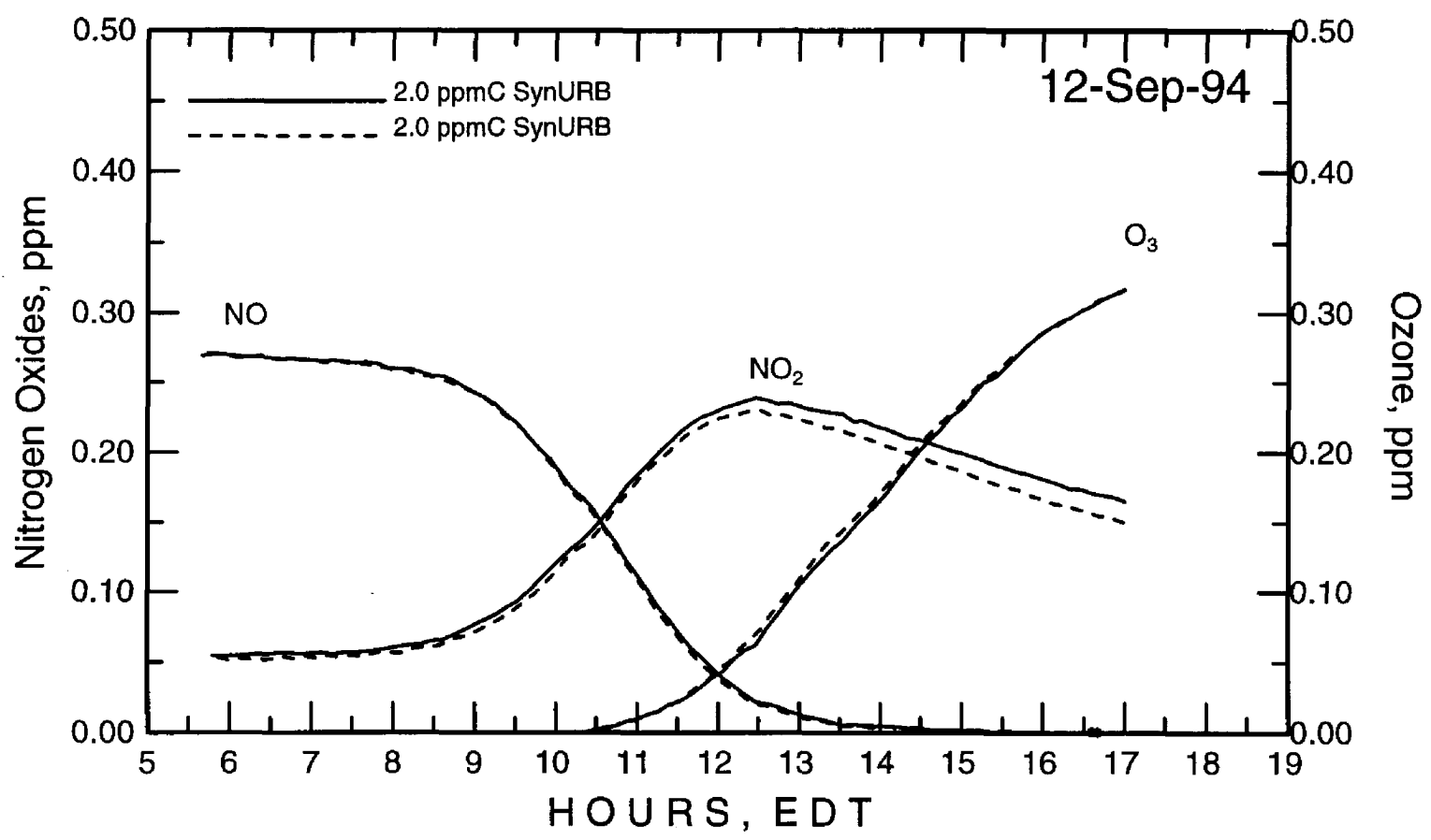

Figure 1.4: A chamber characterization test example showing matched chamber performance.

To demonstrate that the two sides of the dual chamber were well matched during the alternative fuel tests, we show one of the chamber characterization tests in Figure 1.4 in which both sides of the chamber had the same gases injected. Assuming that we achieved our target injection conditions in the other block runs, differences between the two sides can be taken to reflect differences in the chemistry of the two alternative fuels that were compared, and were not caused by different characteristics of the two sides of the chamber.

\subsubsection{Block 1: Base CNG Tests}

We conducted four experiments with a 50:50 mixture of SynURB/SynCNG compared to SynURB/SynIAG at two HC:NO ratios, 6:1 and 9:1. See the 23-Sep-93 experiment in Figure 1.5 for an example of these experiments.

CNG Finding: In all cases, SynCNG showed an ozone production of $40-60 \%$ that of SynIAG, even at 9:1 ratios.

\subsubsection{Block 5: Base LPG Tests}

We conducted only two experiments with a 50:50 mixture of SynURB/SynLPG compared to SynURB/SynIAG at a $\mathrm{HC}: \mathrm{NO}_{\mathrm{x}}$ ratio of $6: 1$; only one experiment took place under good 
meteorological conditions. See the 27-Sep-94 experiment in Figure 1.5 for an example of these experiments.

LPG Finding: In the one fall experiment, SynLPG showed an ozone production of $40-60 \%$ that of SynIAG, and was very similar to CNG.

\subsubsection{Block 2: Base E85 Tests}

Because there were two sources of of E85 composition (the EPA E85 composition had less acetaldehyde than the NIPER E85 composition), there were two different test mixtures used for conducting experiments.

We conducted seven experiments with a 50:50 mixture of SynURB/SynE85E compared to SynURB/SynIAG at three $\mathrm{HC}: \mathrm{NO}_{x}$ ratios, 4.5:1, 6:1, and 9:1. See the 7-Aug-94 experiment in Figure 1.6 for a good example of these experiments.

Two experiments were conducted with a 50:50 mixture of SynURB/SynE85B compared to SynURB/SynlAG at a $\mathrm{HC}: \mathrm{NO}_{\mathrm{x}}$ ratio of 6:1. See the 3-Aug-94 experiment in Figure 1.6 for an example of these experiments.

E85 Finding: At 4.5:1 and 6:1 $\mathrm{HC}: \mathrm{NO}_{\mathrm{x}}$ ratios, the SynE85E mixture produced $15 \%$ to $25 \%$ more ozone than did the SynIAG mixture when blended 50:50 with SynURB; the SynE85B mixture produced $60 \%$ more ozone than did the SynIAG mixture under the same conditions. At the 9:1 $\mathrm{HC}: \mathrm{NO}_{x}$ ratio, the SynE85E mixture produced exactly the same ozone as did the SynIAG mixture.

\subsubsection{Block 3: Single Mixture E85 Tests}

Three experiments were conducted with SynE85E. It was compared with SynURB at 6:1 and was compared with SynIAG also at 6:1. See Figure 1.7 for examples of these experiments.

E85 Finding: At a 6:1 $\mathrm{HC}: \mathrm{NO}_{\mathrm{x}}$ ratio, the SynE85E mixture produced $75 \%$ less ozone than the SynURB mixture, but produced nearly $100 \%$ more ozone than the SynIAG mixture.

\subsubsection{Block 4: E85 Mixture Variations Tests}

We conducted five experiments with a 50:50 mixture of SynURB/SynE85E. It was compared with only SynURB at 6:1 and was compared with $100 \%$ SynURB at $6: 1$, but without any ethanol in the SynE85E mixture. In another experiment, the amount of ethanol was increased. Finally, an "equal mass" experiment was conducted in which $0.84 \mathrm{ppmC}$ of SynE85E was compared to $1.0 \mathrm{ppmC}$ of SynIAG both in a blend with $1.0 \mathrm{ppmC}$ of SynURB. 

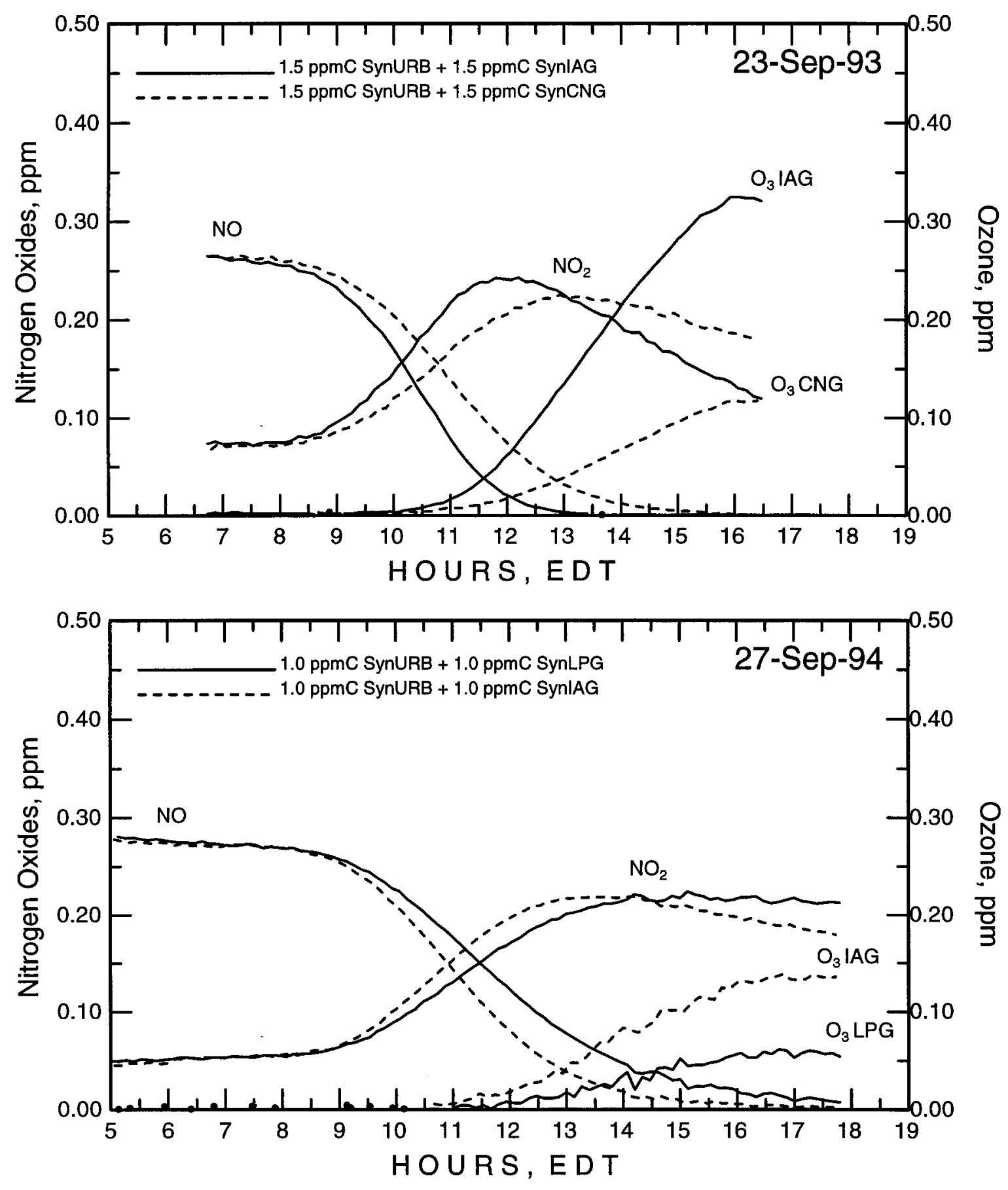

Figure 1.5: Base CNG and LPG test examples. 

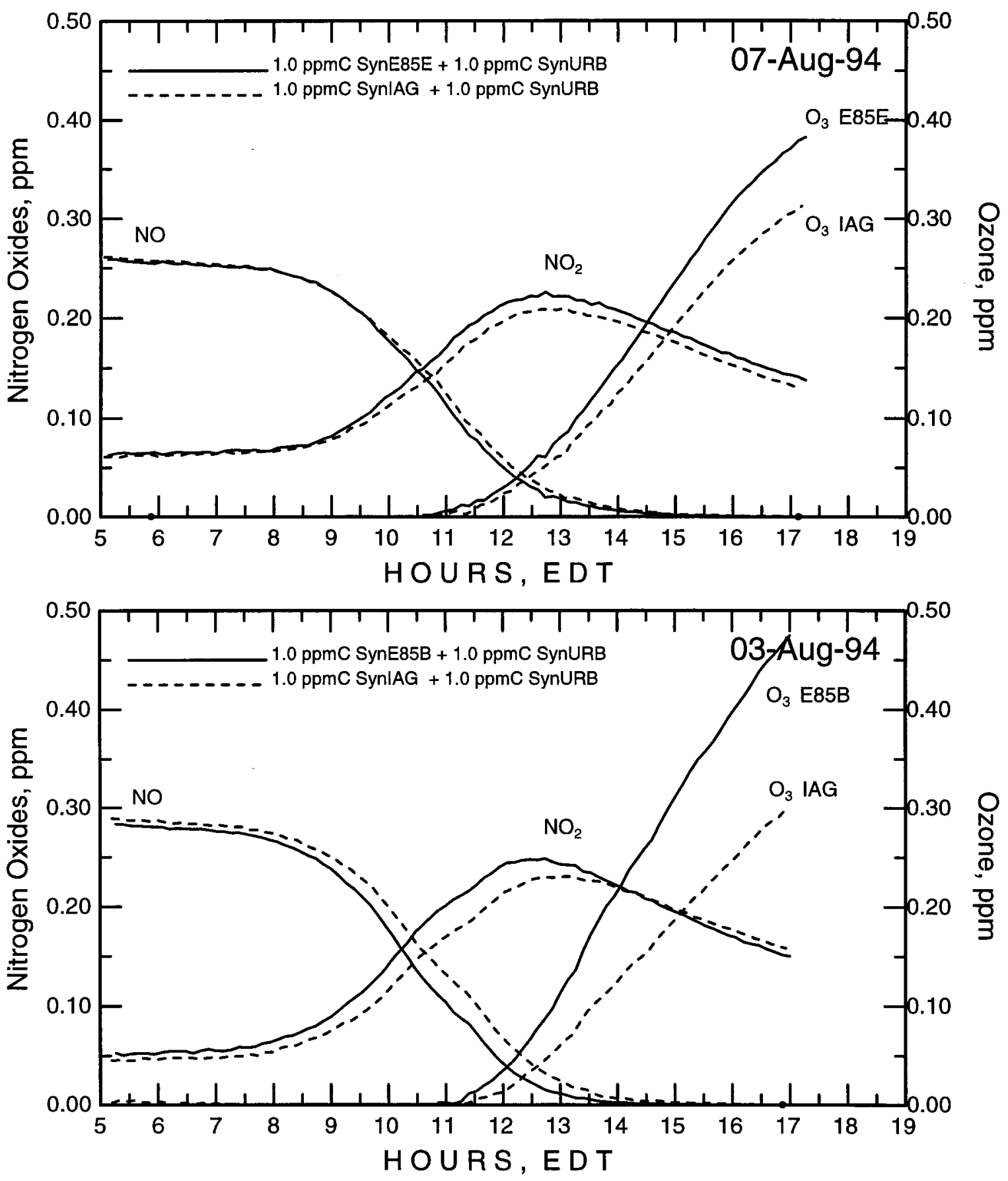

Figure 1.6: Base E85 test examples. 

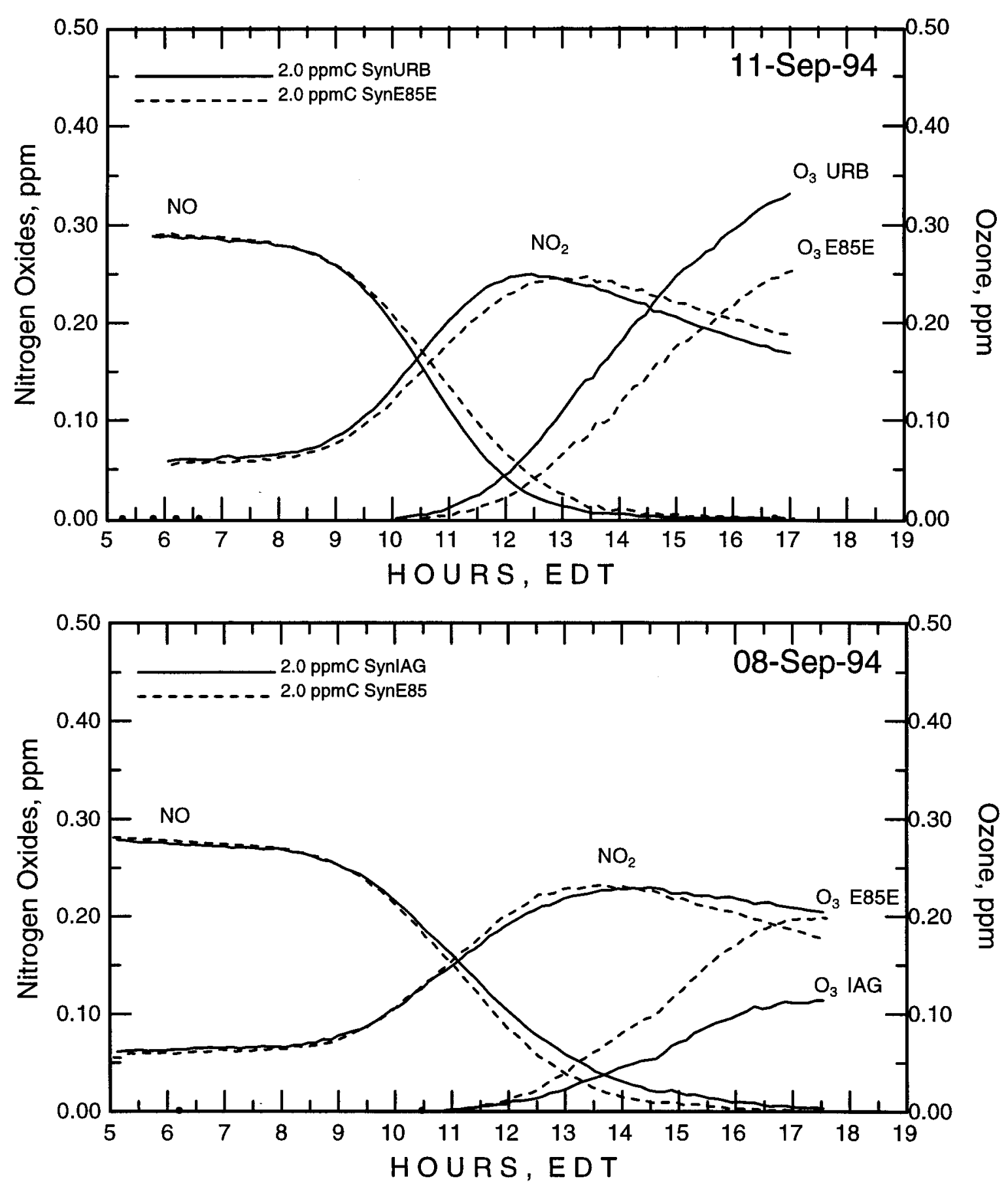

Figure 1.7: Single mixture E85 test examples. 
E85 Finding: At a 6:1 $\mathrm{HC}: \mathrm{NO}_{x}$ ratio, removing the ethanol from the SynE85E mixture decreased the ozone produced from $80 \%$ of the ozone produced by the SynURB-only mixture to $60 \%$ of the ozone produced by the SynURB-only mixture. Increasing the ethanol resulted in the SynURB/SynE85E mixture producing more ozone than did the SynURB-only mixture. Without the acetaldehyde the SynURBSynE85E mixture reactivity approached that of the SynURBSynIAG mixture. Because of the lower molar amount of carbon in the "equal mass" experiment, the SynE85E mixture and the the SynIAG mixture produced the same amount of ozone.

\subsection{Simulation Results}

\subsubsection{Temperature Effects}

Because new Teflon film was installed on the chamber in 1994, replacing the film that had been used for 15 years, there was a large decrease in in-chamber air temperature because the new film exhibits much less of a greenhouse effect than did the old film. The old film had become covered in fine layer of particulate matter that was absorbing infrared radiation. This resulted in two very interesting sets of experimental data:

- A 1991-92 SynURB and SynIAG data set from the CRC sponsored work with maximum chamber temperatures often exceeding $100^{\circ} \mathrm{F}$

- A 1994 SynURB and SynIAG data set from the current work with maximum chamber temperatures near $80^{\circ} \mathrm{F}$.

Several replicate experiments between these two data sets afforded the opportunity to examine how well CB4 could simulate temperature effects.

Comparisons of replicate runs with significantly different afternoon in-chamber temperatures showed that there was little effect of temperature on ozone formation (see, for example, Figure 1.8). This result appeared coorelated with an inverse relationship for afternoon response on the $\mathrm{NO}_{x}$ analyzer, that is, higher temperatures give lower afternoon $\mathrm{NO}_{x}$ analyzer readings. Our particular analyzer responds to $\mathrm{NO}_{2}$, PAN, organic nitrates, but does not respond to $\mathrm{HNO}_{3}$. Because PAN-like compounds would be expected to be temperaturelabile, they can store $\mathrm{NO}_{2}$ for subsequent release later in the run and thus maintain a higher $\mathrm{NO}_{x}$ concentration in the chamber during the afternoon. Because more PAN-like compounds would be available in cooler runs, more ozone could be produced, thus compensating for other processes that would be expected to lead to a decrease in ozone production. Because our VOC mixtures are so complex, we have a better chance to represent such complex internal compensation processes than have other chamber studies.

In the CRC report [4], we showed that the simulations of the complex VOC mixture experiments performed with the CB4 mechanism were very reactive relative to the chamber observations, on average predicting ozone about $150 \%$ too high. But in the simulations 

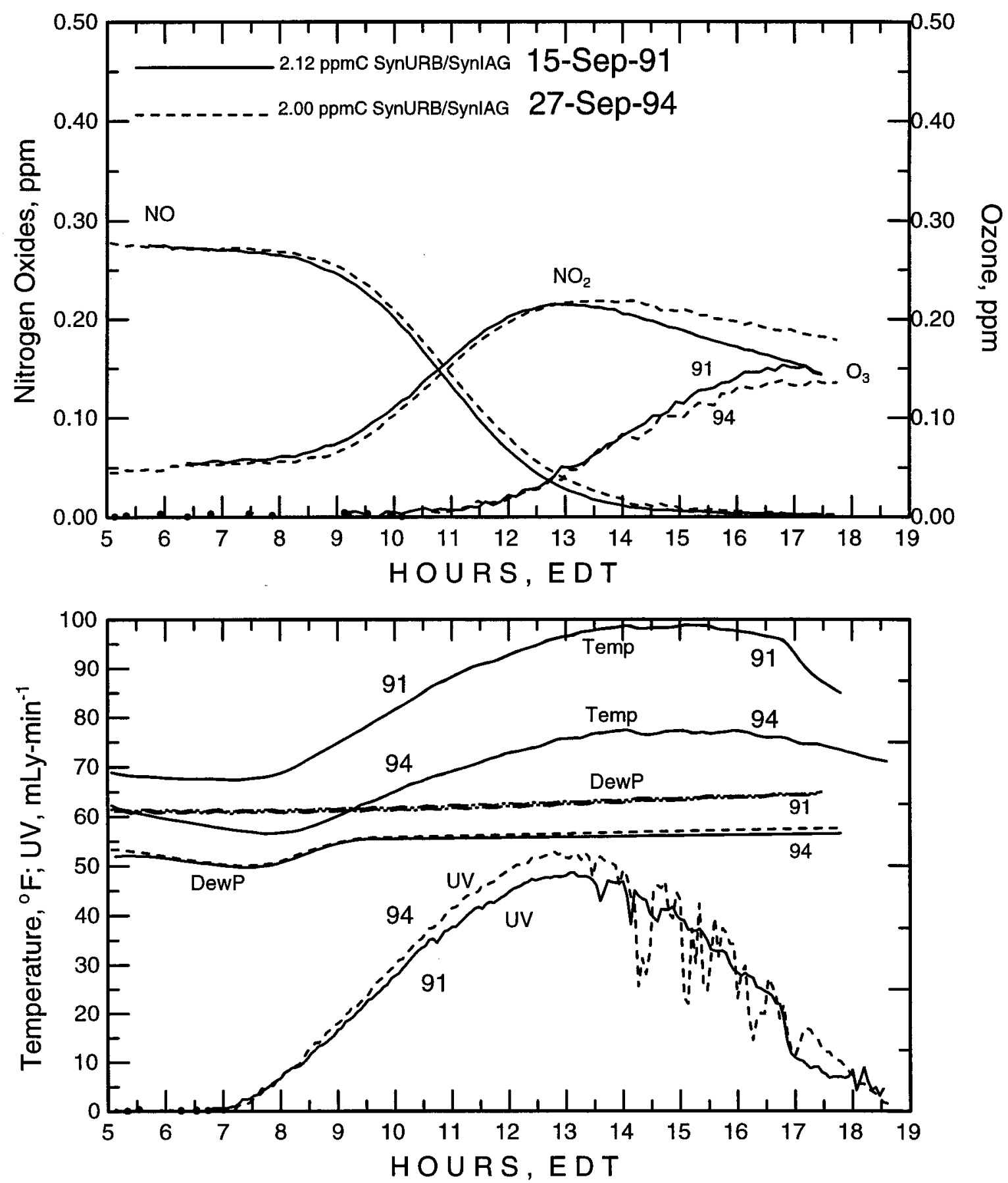

Figure 1.8: Comparison of SynURB/SynIAG experiments from 1991 and 1994. In the bottom plot, the lines marked Temp is the air temperature inside the chamber; the lines marked DewP is the dew point temperature of the chamber air; the lines marked UV are the ultraviolet irradiance on a tower adjacent to the chambers. 
performed here for the 1994 DOE experiments the CB4 simulations are in much better agreement with the chamber observations. This led us to conduct a series of comparisons of replicate experiments to investigate the observed temperature dependence of ozone production on temperature and to compare this with the CB4's temperature dependence. Further, we substituted the 1994 temperatures for the observed values in the 1992 simulations and showed that it was indeed the high in-chamber air temperatures that caused CB4's predictions to be so much higher than the chamber observations in the CRC study (see, for example, Figure 1.9).

Temperature Sensitivity Findings: Conclusions from these intercomparisons were:

- The outdoor smog chamber experimental results suggest that when using complex mixtures with the realistic compositions of synthetic urban and IAG, there is at best a small temperature effect. Also, some evidence exists to suggest that there may be a small inverse relationship between temperature and ozone production for these mixtures.

- The experimental evidence refutes the large positive effect of temperature on ozone production that is demonstrated by the CB4 mechanism.

These imply that while the CB4 simulations reported later in this document show excellent agreement with the chamber observations, this accuracy may be merely the result of having tuned the somewhat speculative organic chemistry portion of the CB4 using chamber experiments from the 1980's in which the chamber temperatures were more like those in the 1994 data set. In comparisons with hotter chamber data from 1992, the CB4 exhibits too much increase in ozone with increasing temperature and will not be a reliable predictor of ozone for conditions warmer than $80^{\circ} \mathrm{F}$.

\subsubsection{Block 6: Chamber Characterization Tests}

CB4 showed excellent agreement with all chamber characterization runs that did not involve complex mixtures; i.e., CB4 gave nearly perfect simulations for methane/ $\mathrm{NO}_{x}$ experiments and for $\mathrm{CO} / \mathrm{NO}_{x}$ experiments.

\subsubsection{Alternative Fuel Simulations}

The CB4 mechanism did reproduce the reactivity difference between 50:50 mixtures of SynURB/SynCNG and 50:50 mixtures of SynURB/SynlAGat 6:1 and at 9:1 HC:NO ratios (see, for example, Figure 1.10). CB4 performed similarly for the SynLPG experiments.

The CB4 mechanism did reproduce the reactivity difference between 50:50 mixtures of SynURB/SynE85E and 50:50 mixtures of SynURB/SynIAGat 6:1 and at 9:1 $\mathrm{HC}: \mathrm{NO}_{x}$ ratios 

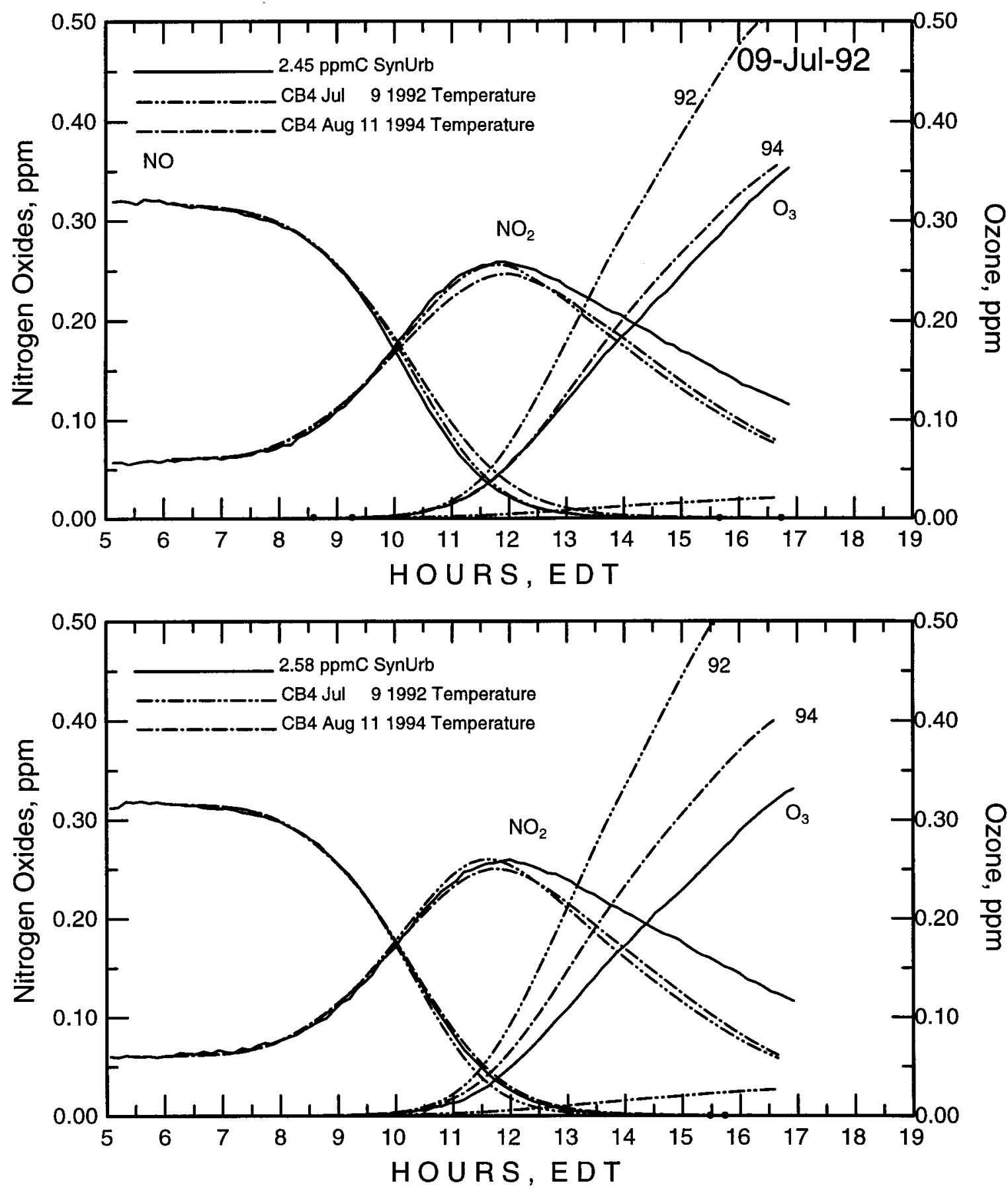

Figure 1.9:. Simulation results with the CB4 mechanism for JL0992 using different year's temperatures. 

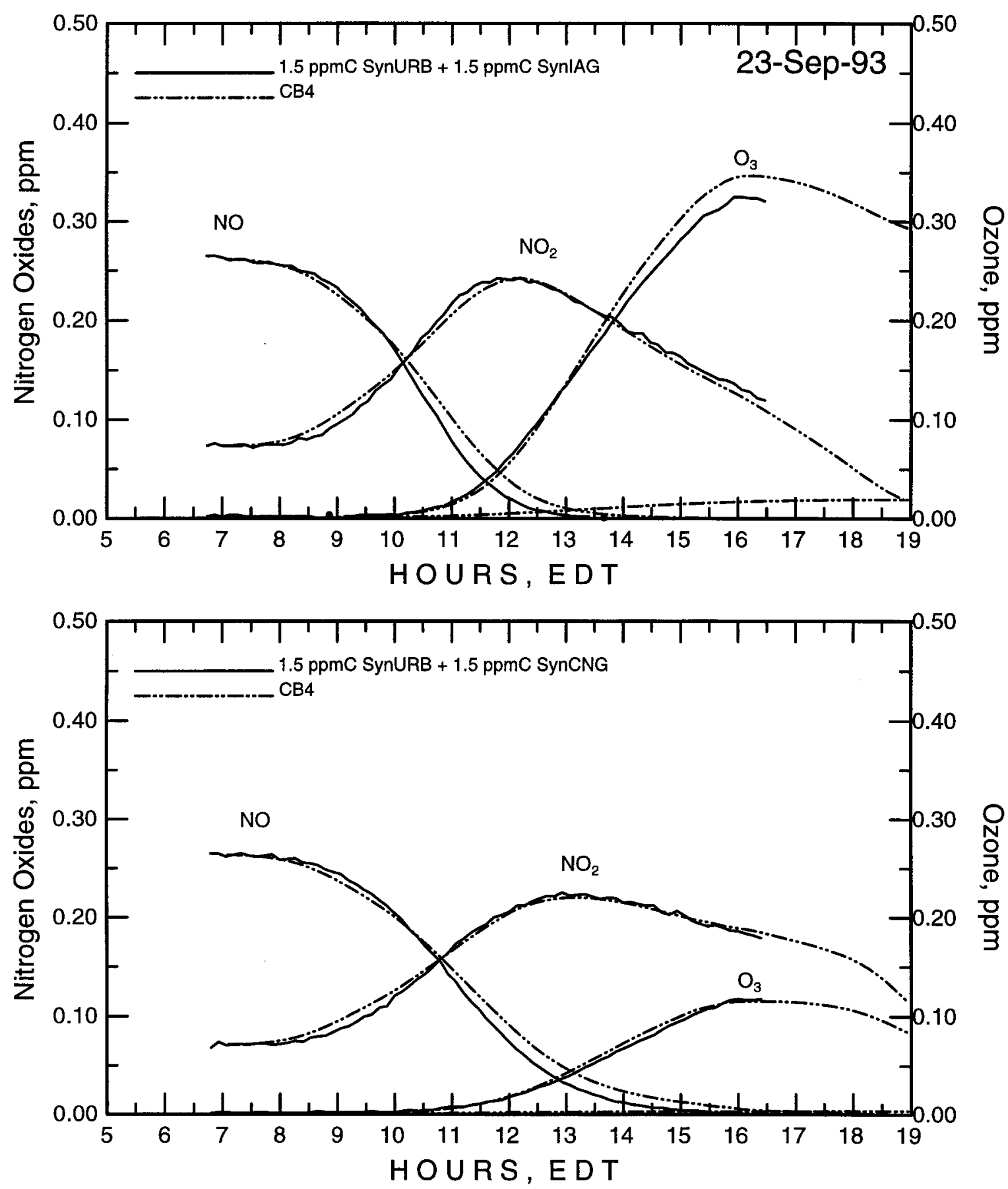

Figure 1.10:. Run 3-9:1 SynUrban/SynIAG:NOx versus 9:1 SynUrban/SynCNG:NO ; Carbon Bond Four Simulation. 

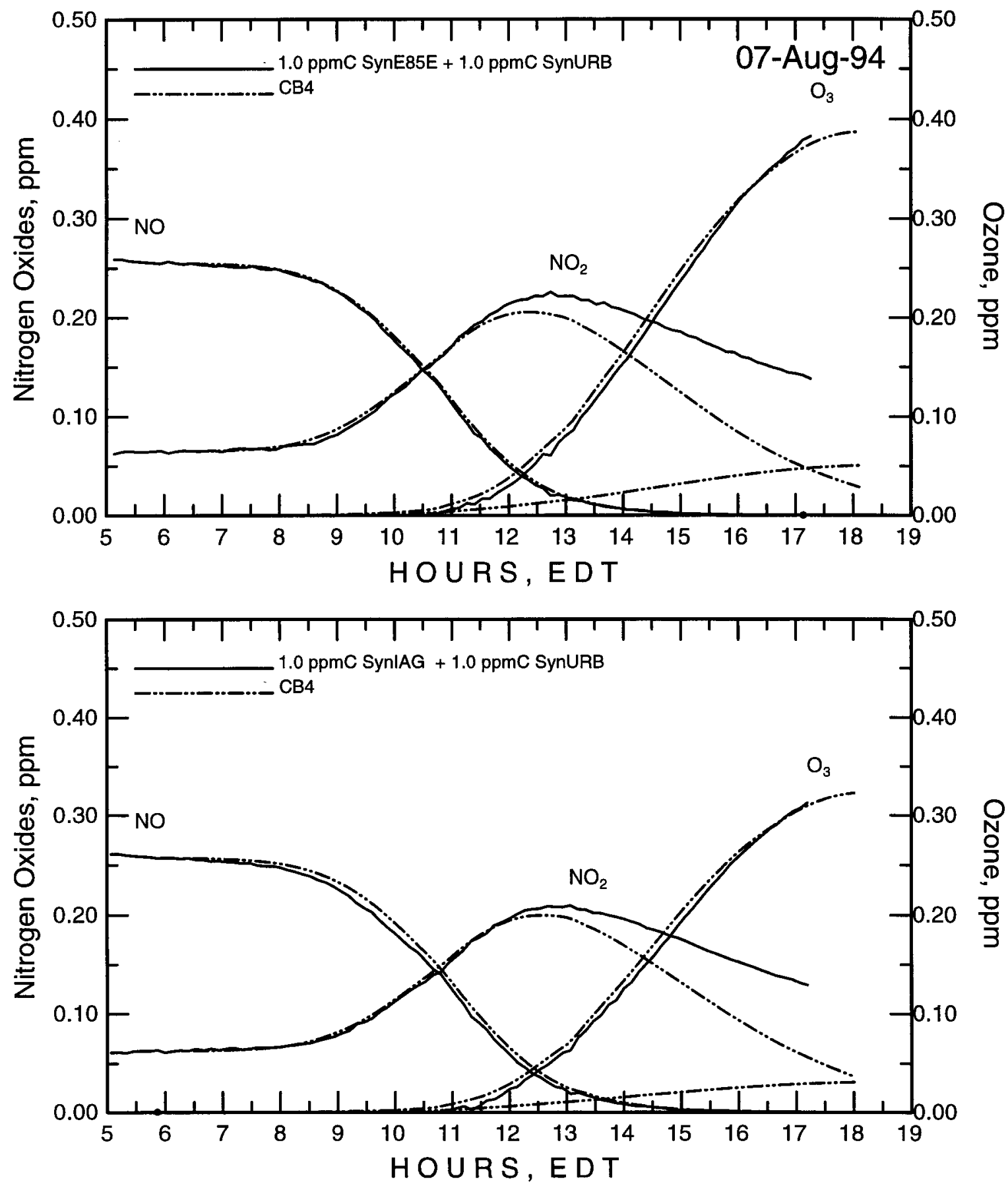

Figure 1.11:. Run 10-6:1 SynUrban/SynIAG:NO ${ }_{x}$ versus 6:1 SynUrban/SynE85E:NO ${ }_{x}$; Carbon Bond Four Simulation. 

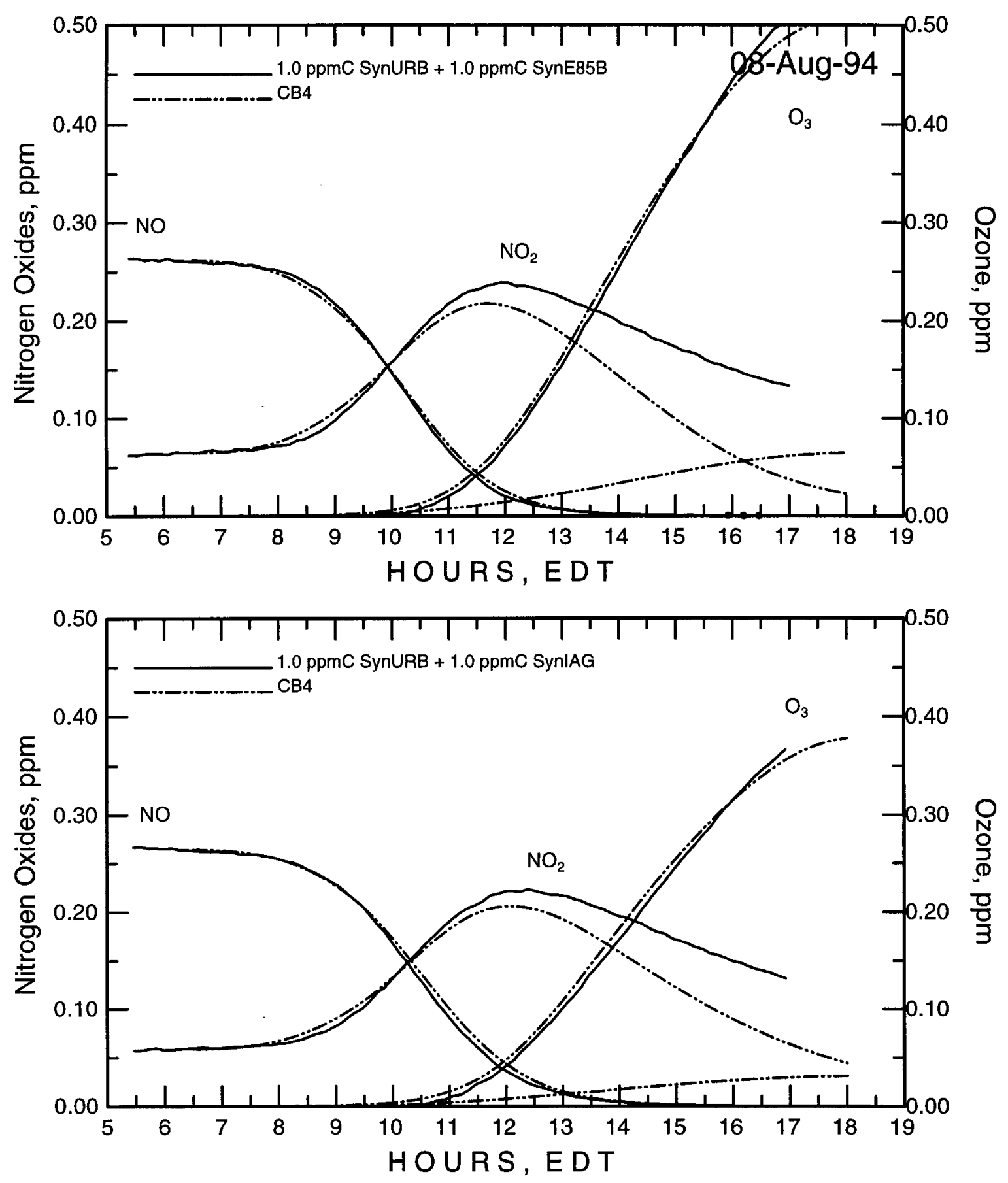

Figure 1.12:. Run 12-6:1 SynUrban/SynIAG:NO versus 6:1 SynUrban/SynE85B:NO ; Carbon Bond Four Simulation. 
(see, for example, Figure 1.11). CB4 also reproduced the reactivity difference between the EPA E85 composition and the NIPER E85 composition (see Figure 1.12).

On the other hand, CB4 did not perform as well when simulating just SynE85E (being somewhat over reactive), or simulating SynURB/SynE85E vs. just SynURB mixtures (again too over reactive for the E85 side).

\section{CB4 Tests Conclusion}

Given the various responses of CB4 reported above, it is clear that the processes in CB4 were carefully balanced under a particular set of chamber test conditions. Given that the temperature dependence for PAN formation and decay were changed in CB4 after it was formulated and there was no reevaluation of CB4 against a large set of chamber data, it is not surprising that CB4 exhibits an incorrect and overly sensitive temperature dependence for ozone formation.

CB4 Finding: On the other hand, when conditions are near those used to formulate CB4, which include air temperatures below $80^{\circ} \mathrm{F}$ and urban-like VOC mixtures, CB4 can accurately simulate the chamber results with a high level of accuracy. It must be remembered, however, that such accuracy is achieved by means of internal compensation among some correctly specified and some incorrectly specified processes in the model.

\subsection{New Analytical Methods}

One of the most successful parts of this project has been the development and refinement of a new analytical method for detecting and identifying unknown multi-functional carbonyls. Carbonyls are the ubiquitous intermediate products of all atmospheric organic oxidation reactions. Identifying and quantifying these carbonyls are critical to developing a scientific understanding of the reaction mechanisms and thus formulating an accurate reaction model for use in regulatory decision making.

This method has been published [6], and was in part responsible for Ms. Yu receiving the American Chemical Society's Best Graduate Student Paper Award in 1995.

The new method uses O-(2,3,4,5,6-pentafluorobenzyl)-hydroxylamine (PFBHA) to form oximes derivates of polyfunctional carbonyls. Figure 1.13 shows the PFBHA oxime of acetaldehyde. The presence of the five fluorine atoms not only raises the vapor pressure of the oxime permitting gas chromatography to be used for the separation, but also results in ready detection by electron capture (ECD). Furthermore, in a mass spectrometer, the stability of the $\mathrm{C}_{6} \mathrm{~F}_{5} \mathrm{CH}_{2}$ moieties produced by fragmentation gives a very strong $\mathrm{m} / \mathrm{z} 181$ peak that uniquely identifies the compound as being a carbonyl derivative.

The chemical ionization mass spectra using an ion trap produce direct molecular weight information and some of the fragmentation patterns assist in determining the structure of the 


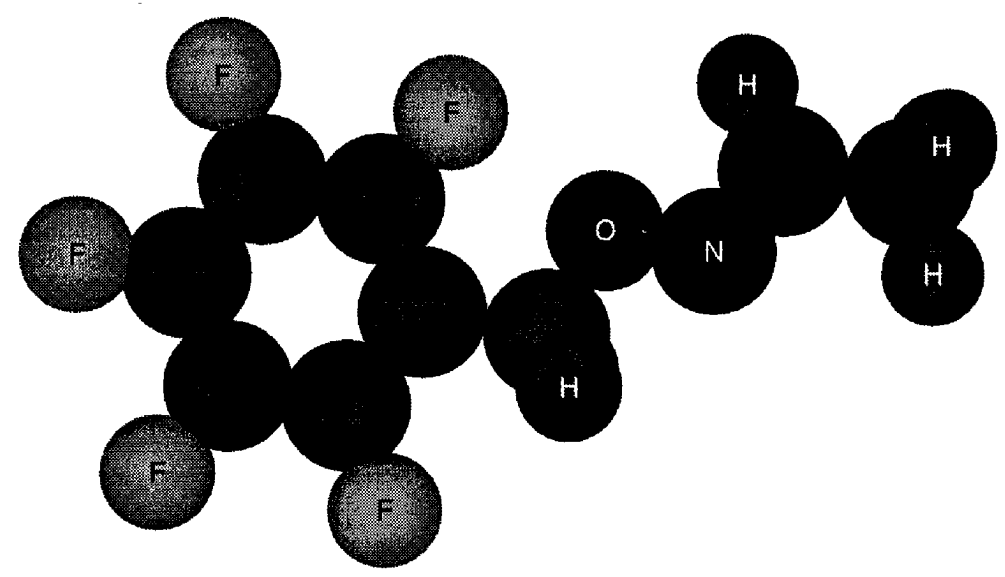

Figure 1.13: The O-(2,3,4,5,6)-pentafluorobenzyl oxime of of acetaldehyde.

carbonyls. An example mass spectrum will be shown in the following section. The ability to determine the molecular weight is the key to the power of this method, as many of the intermediate carbonyls are not available commercially for use as standards.

The method has been shown to work for all standard ketones and aldehydes and dicarbonyls from $\mathrm{HCHO}$ to $\mathrm{C}_{9}$ and for all hydroxy carbonyls for which we could obtain a standard. In addition, it has been show to work for epoxy carbonyls and dicarbonyls. It will not detect carboxylic acids (but a modification to use pentafluorobenzyl bromide (PFBBr) as the derivatizing agent has been shown in our laboratories to detect these acids).

\subsection{Aromatics Mechanism}

More than 20 indoor Teflon Bag Reactor (TBR) experiments with alkyl substituted benzene compounds and $\mathrm{NO}_{\mathrm{x}}$ were conducted as part of this work. These were used to develop the sampling and identification techniques. Although these are useful in determining the types of products that were produced in these systems, the photolytic spectrum for the TBR differs significantly from sunlight. Because many of the carbonyl products photolyze, outdoor experiments would more realistically represent the behavior of this complex system.

Three dual outdoor chamber experiments were performed for this project:

- toluene versus m-xylene

- -xylene versus 1,3,5-trimethyl benzene

- o-xylene versus 1,2,4-trimethyl benzene.

Batch and time-resolved samples were collected each side of each of these experiments and were analyzed using the new carbonyl method described above. The presence of carbonyl products in each batch sample was readily determined by examining the reconstructed 
ion chromatogram for the $\mathrm{m} / \mathrm{z}=181$ ion because the electron ionization spectra of PFBHA oximes always have a strong $\mathrm{m} / \mathrm{z}=181$ peak. The molecular weights of these carbonyl products are then determined from their methane chemical ionization mass spectra.

After methane, toluene is the most ubiquitous urban hydrocarbon, appearing among the top 25 most concentrated HC species in all the EPA canister samples. Aromatic species make up about $30 \%$ of all urban carbon. The reconstructed $\mathrm{m} / \mathrm{z} 181$ ion chromatogram from the toluene/ $\mathrm{NO}_{x}$ experiment is shown in Figure 1.14. Every peak in this figure is a carbonyl compound and it reveals the enormous diversity of the reaction products from toluene photooxidation. In the body of the report, we identify all the number peaks and present reaction paths leading to the formation of each carbonyl.

Although we have proposed a series of at least seven major reaction pathways leading to a large variety of intermediate and final products, the conversion of these reaction schemes into photochemical reaction mechanisms for use in models will require significant new work. Some of this work is currently underway in our laboratories under EPA funding and will require more than three years to complete. Other work, such as determining the absorption cross sections and quantum yields for the photolysis of the carbonyls, cannot be done in our laboratories and we need to establish collaborations and find funding for other investigators who can make these measurements. We have established a collaboration with organic synthesis chemists in the UNC Department of Chemistry in an effort to synthesize usable quantities of key intermediate multi-functional carbonyls and may be able to furnish samples to other investigators.

One of our most important discoveries in working with the aromatics photooxidation is that one of the important reaction pathways is via epoxy carbonyl production. This is consistent with a theoretical prediction of the formation of epoxide intermediates in the toluene oxidation that was made by Bartolotti and Edney [13] nearly simultaneously with our measurements. They used a series of density functional quantum mechanical calculations to identify potential intermediates following the $\mathrm{OH}$ addition to toluene. In working closely with Bartolotti, we produced the following reaction scheme:

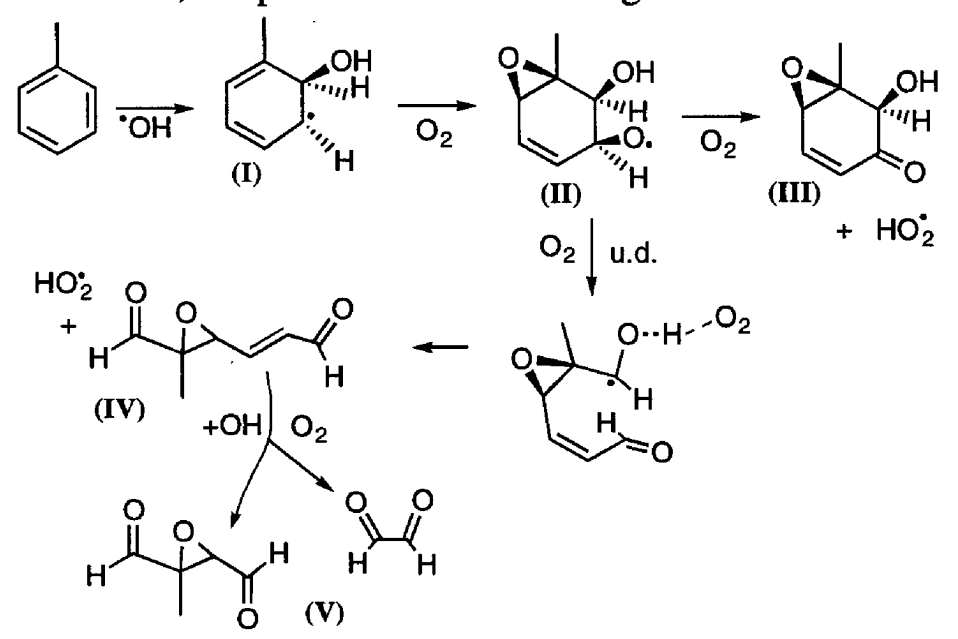

Bartolotti and Edney's calculations indicate that the epoxide structure II is the most stable 


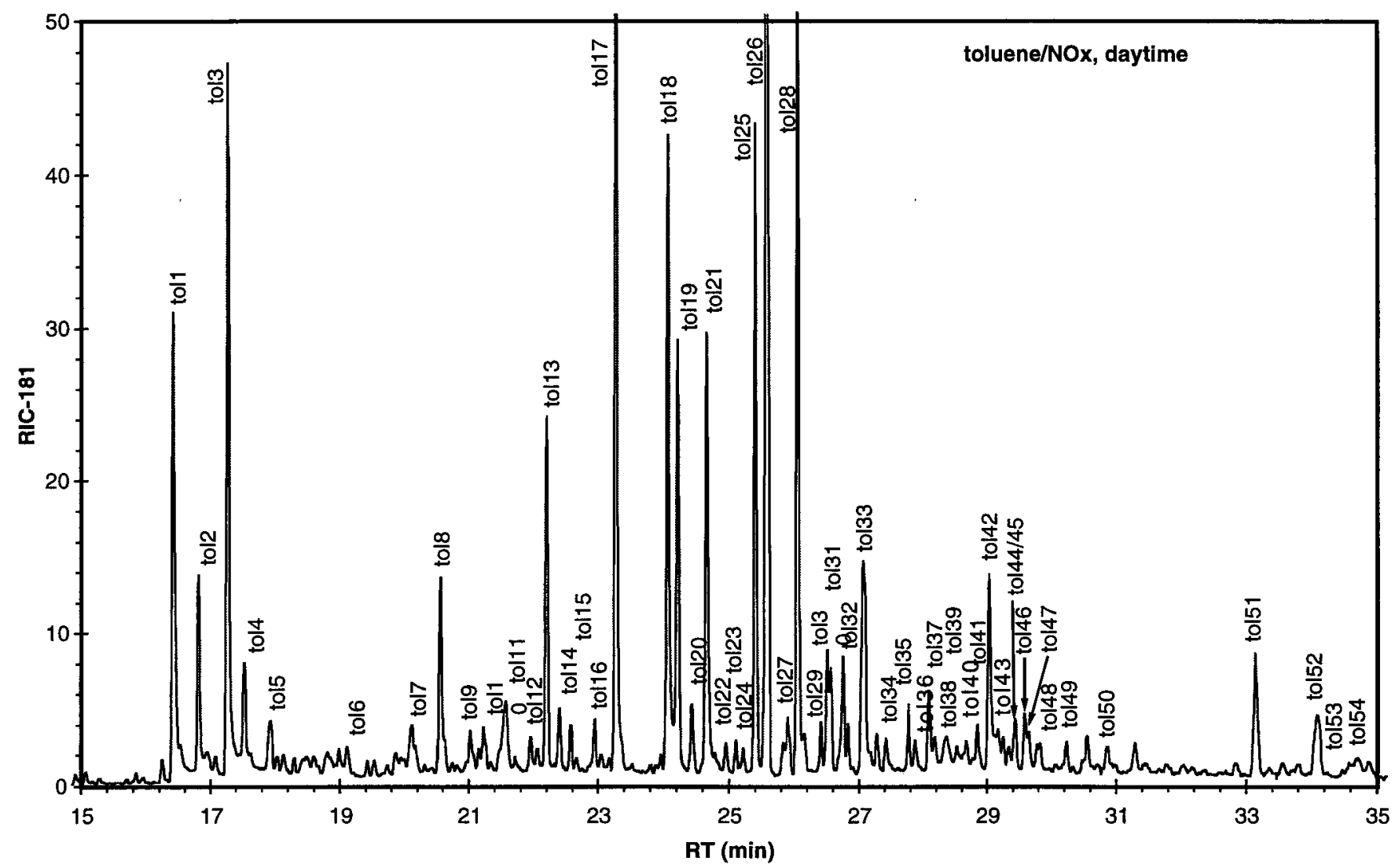

Figure 1.14:. Reconstructed $\mathrm{m} / \mathrm{z} 181$ ion chromatogram of the batch sample collected from an outdoor smog chamber toluene/ $\mathrm{NO}_{\mathrm{x}}$ daytime experiment. 
intermediate among the likely intermediates. We have detected the reaction products labeled III, IV, and V.

One of the most impressive mass spectra we have produced was from a 1,3,5-trimethyl benzene/ $/ \mathrm{NO}_{x}$ experiment in the outdoor chamber (see Figure 1.15). The existence of the first ring-opened epoxide, 2,3-epoxy-2,4-dimethyl-6-oxo-4-heptenal (called Epoxide A hereafter) is clearly revealed.

The detection of epoxide intermediates in the $\mathrm{OH}$ oxidation of aromatic compounds raises a concern over their possible toxicity. An epoxy carbonyl similar in structure to several intermediates we detected, 2,3-epoxypropanal (glycidaldehyde), has been reported to be a potent mutagen in a variety of in vitro test systems $[14,15]$ and has been shown to induce sarcomas in rats by subcutaneous injection [16]. As a bifunctional agent, 2,3-epoxy propanal is capable of forming cyclic adducts and crosslinks with DNA bases [17]. In general, the epoxy functional group can serve as electrophile to attack DNA, nucleosides [18-20]. It seems possible that the mutagenic activity of irradiated toluene/ $\mathrm{NO}_{x}$ mixtures reported by Shepson et al. [21] and by Shiraishi et al. [22] may have been due to the production of epoxide compounds. Hence, the possible formation of epoxides from atmospheric oxidation of aromatic compounds is a significant public health concern, deserving additional laboratory and field work.

\subsection{UAM Simulations and Analysis}

Chemistry is only one component in the predictions made by an airshed model. Meteorology and emissions are extremely important parameters in these models. Because of our involvement with the State of North Carolina in performing State Implementation Plan calculations for Charlotte, North Carolina, an ozone non-attainment area, we were able to investigate and improve a number of elements of the EPA's Urban Airshed Model (UAM) that would be used to investigate the effects of alternative fuels use.

The first of our efforts relates to improving the inventory, both area inventory and the mobile source inventory. Other efforts were directed at explaining the role of meteorology and its interaction with emissions and chemistry using a new technique developed in part on this project.

\subsubsection{Emissions Inventory Improvements}

In our UAM studies, we developed relational database management techniques and applied them to emissions inventory and spatial surrogate files for the North Carolina Modeling Domain [23]. Application of this code to quality assurance work on emissions inventory files resulted in many refinements of the spatial allocation of emissions.

The total gridded emissions may be correct, but may be in the wrong grid cells. The gridded emissions may be in the correct grid cells, but the total gridded emissions may be incorrect if the total fractional surrogates do not correctly sum to 1 . 


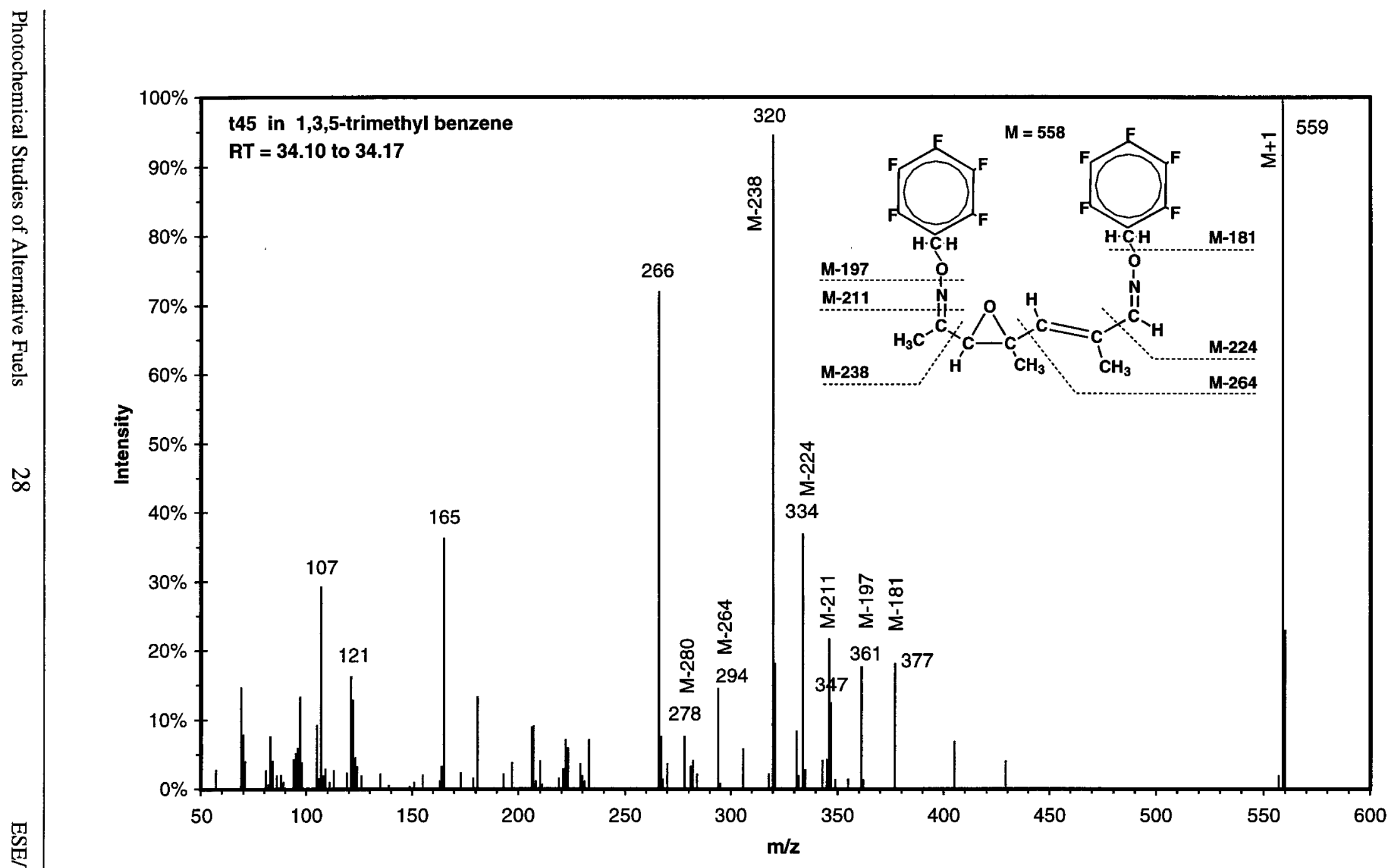

Figure 1.15: Ion trap methane CI mass of the PFBHA derivative of 2,3-epoxy-2,4-dimethyl-6-oxo-4-heptenal. 
Both types of errors were found in the North Carolina surrogate file. When allocating emissions using the original urban and water surrogates, the surrogates were zero for some counties, resulting in lost emissions during the gridding process of the Emisions Processing System codes (EPS). Also, the surrogates apparently originated from older land-use databases using a very coarse grid size, resulting in over-smoothing of the emissions field and the artificial spreading of emissions beyond the emissions sources.

To improve the mobile source inventory, we developed a new mobile inventory submodel [24] that:

- Implemented the current emission estimation methodology in a relational database management system to produce inventories in a more flexible and efficient manner

- Incorporated information from a UTPS-type transportation demand model to improve the spatial and temporal resolution of vehicle activity in the emission calculations.

\subsubsection{Process Analysis}

As part of this project (and others) we have developed a method called integrated process rate analysis, (IPRA), or just "process analysis" that allows us to fully explain a model's prediction. In process analysis, we focus on the change caused by each major physical and chemical process in the model, rather than just examining the concentration field for a pollutant. We thus output additional data for each cell in the model; these are data for the change over the model's time step caused by chemistry, horizontal transport, vertical transport, emissions, and deposition. In post processing programs, we subject these data to additional analysis by four major techniques:

1. Animation movies of major processes such as total odd oxygen production per cell per hour

2. Process time series plots

3. Process composition plots

4. Integrated reaction rate and mass balance system analysis.

These have proved to be invaluable in performing diagnostic analysis of particular episodes in which the simulation results were strange.

Some of the UAM simulation problems revealed by process analysis were:

- Because of the wind flow model's inability to treat correctly light and highly variable wind fields, there were major horizontal convergence problems that caused ozone to accumulate more than $50 \mathrm{ppb}$ above the observed peak and that resulted in the simulation having to be abandoned

- Incorrect shifts in vertical transport when there was a large change in horizontal wind direction 
- Incorrect ratio of toluene to xylene in the model's emissions inventory compared to ambient observations

- A $10 \% \mathrm{NO}_{x}$ mass balance error.

Examples of these are given in Chapter 7.

\subsubsection{UAM Simulation Results}

In this study we tested the sensitivity of the UAM's ozone predictions to three assumptions in the motor vehicle emission calculations: trip average emission factor, average daily temperature, and uniform speed by road classification. These were all preliminary to evaluating the effects of changing the mobile emissions composition to reflect the use of an alternative fuel.

The assumptions were tested by preparing a series of motor vehicle modeling inventories using conventional methodology (C), disaggregated emission factors (D), hourly temperature profiles (T), and speeds by road (S). These inventories were then merged with the area, point, biogenic, and nonroad mobile inventory for a 1988 ozone episode in Charlotte, North Carolina, and used in UAM simulations.

Spatially and temporally allocating the disaggregated emissions to trip starts, trip ends, and trip mileage increased both the maximum gridded hourly emissions and the variability of gridded hourly emissions. $\mathrm{VOC}$ and $\mathrm{CO}$ showed a larger change than $\mathrm{NO}_{\mathrm{x}}$. This reallocation moved emissions from major highway corridors to the central business district.

Varying the ambient temperature had little impact on either emission estimates or ozone predictions. Disaggregating the emission factor and varying speeds on individual links had significant impacts on the distribution and mass of emissions, particularly for $\mathrm{CO}$ and VOCs. The spatial redistribution caused by disaggregation was even more significant at higher grid resolutions. However, the impact of these changes on ozone predictions was small-peak ozone changed by less than $3 \mathrm{ppb}$ - but spatial ozone changes were more significant. This ozone result was primarily due to the $\mathrm{NO}_{\mathrm{x}}$-limited nature of the Charlotte area in 1988 . Effects on ozone concentrations in scenarios with different $\mathrm{VOC}$ to $\mathrm{NO}_{\mathrm{x}}$ ratios may be more significant. Furthermore, disaggregation may have a significant impact on predictions of CO concentrations.

Although the conventional methods for estimating motor vehicle emissions have been criticized as being unrealistic and unrepresentative, this work demonstrates that, under some conditions, major efforts to refine the emission estimates will have little impact on air quality predictions, at least at the current resolution of air quality models. Under different conditions, however, these changes in the emissions estimates may produce very significant air quality impacts.

The impact of a large scale use of natural gas vehicles in the Charlotte UAM scenario is currently being investigated under separate funding. 


\section{Chapter 2}

\section{Experimental Design}

\subsection{VOC Mixture Composition}

This study design was similar to that of our CRC Methanol Fuels Reactivity Project and relied on the SynURB and SynIAG complex VOC mixtures produced in that study. Detailed description of the analysis and creation of these mixtures is given in the CRC report [4].

These mixtures, each comprising about 55 compounds, are the most complex, yet high-precision, composition mixtures ever used in smog chamber studies (see Table 2.1, 2.2 , and Figure 2.1). They were based on extensive gas chromatographic analysis of early morning cannister samples collected by the EPA in 30 cites over a period of about 6 years. We did statistical analysis on more than 2000 samples with up to $300 \mathrm{HC}$ species to produce an average mixture of about 120 species. From these we selected the 55 species for inclusion in the synthetic mixtures based on the prevalence and concentration fraction of the species. About $65 \%$ of the carbon was explicitly represented in these mixtures (i.e., the exact compound is included in the mixture) and the rest of the carbon is represented by a similar species with similar structure. Some attention was given to ease of analysis and to producing a precise composition from experiment to experiment.

To meet the latter requirement, we divided the synthethic mixtures into two classes: those compounds that could be purchased in a high-pressure gas cylinder in nitrogen (14 components) and those species that were liquids ( 38 components). We purchased the former from a gas supply manufactor at $2 \%$ component accuracy and a total carbon concentration of about $10,000 \mathrm{ppmC}$. Injections of 15 liters of this tank into the chamber would produce $1.0 \mathrm{ppmC}$ of the mixture. We blended the liquid mixture using pure liquids and careful measurement of individual $\mathrm{HC}$ volumes to create a single blend liquid with the proper composition. Injection of a few 100 microliters of this liquid, along with evaporation with a hot air gun, was used to introduce these components into the chamber. Finally, the higher aldehydes were blended into another liquid separately (up to 5 aldehydes) and injected in a manner similar to the $\mathrm{HC}$ liquid. Formaldehyde $(\mathrm{HCHO}$ ) was injected using sublimation of a weighed amount (about $120 \mathrm{mg}$ ) of solid paraformaldehyde. 
For this study, we added to the SynURB and SynIAG mixtures a new mixture based on emissions from a compressed natural gas fueled vehicle called SynCNG (see Figure 2.2). We added two new mixtures mixtures based on composite emissions from ethanol fueled vehicles, one determined by EPA (called SynE85E) and one determined by NIPER (called SynE85B). The latter are described in Figure 2.3. 
Table 2.1: The VOC Mixtures used in the CRC Methanol-85 Chamber Study-Part 1

\begin{tabular}{|c|c|c|c|c|c|}
\hline \multirow{2}{*}{ Component } & \multicolumn{5}{|c|}{ ppbc in 1 ppmc injection } \\
\hline & \multirow{2}{*}{$\frac{\text { Urban }}{38.5}$} & \multirow{2}{*}{$\begin{array}{l}\mathbf{A G} \\
22.9\end{array}$} & \multirow{2}{*}{$\frac{\text { M85 }}{5.1}$} & \multicolumn{2}{|c|}{ Urb//AG Urb/M85 } \\
\hline 1 ethane & & & & 30.7 & 21.8 \\
\hline 2 propane & 46.1 & & & 23.0 & 23.0 \\
\hline 3 n-butane & 73.5 & 329.0 & 97.5 & 201.2 & 85.5 \\
\hline 4 isobutane & 32.9 & 10.0 & 6,5 & 21.4 & 19.7 \\
\hline $5 \mathrm{n}$-pentane & 31.1 & 41.0 & 53.3 & 36.0 & 42.2 \\
\hline 6 isopentane & 86.4 & 77.7 & 136.0 & 82.0 & 111.2 \\
\hline 72,2 -dimethylbutane & & 8.0 & & 4.0 & 0.0 \\
\hline 82,3 -dimethylbutane & 6.0 & 10.0 & 10.9 & 8.0 & 8.5 \\
\hline 9 2-methylpentane & 22.0 & 36.0 & 53.9 & 29.0 & 37.9 \\
\hline 103 -methylpentane & 16.0 & 17.0 & & 16.5 & 8.0 \\
\hline $11 \mathrm{n}$-hexane & 14.0 & 16.0 & 10.3 & 15.0 & 12.2 \\
\hline 122,3 -dimethylpentane & 17.0 & 13.0 & 4.8 & 15.0 & 10.9 \\
\hline 13 2-methylhexane & & 20.0 & 6.3 & 10.0 & 3.2 \\
\hline 14 3-methylhexane & 35.0 & & & 17.5 & 17.5 \\
\hline $15 \mathrm{n}$-heptane & 16.0 & 9.0 & & 12.5 & 8.0 \\
\hline $162,2,4$-trimethylpentane & 13.0 & 28.0 & 12.7 & 20.5 & 12.9 \\
\hline 172,5 -dimethylhexane & 11.0 & 5.0 & & 8.0 & 5.5 \\
\hline $182,3,4$-trimethylpentane & 10.0 & 9.0 & & 9.5 & 5.0 \\
\hline $19 \mathrm{n}$-octane & 12.0 & & & 6.0 & 6.0 \\
\hline 20 nonane & 16.0 & & & 8.0 & 8.0 \\
\hline $21 \mathrm{n}$-decane & 20.0 & & & 10.0 & 10.0 \\
\hline 22 4-methylnonane & 23.0 & & & 11.5 & 11.5 \\
\hline $23 \mathrm{n}$-dodecane & & & 2.9 & 0.0 & 1.5 \\
\hline 24 methylcyclopentane & 11.0 & 8.0 & 9.5 & 9.5 & 10.2 \\
\hline 25 cyclohexane & 10.0 & & & 5.0 & 5.0 \\
\hline 26 methylcyclohexane & 6.0 & & & 3.0 & 3.0 \\
\hline 27 ethene & 26.7 & 36.0 & 7.2 & 31.3 & 17.0 \\
\hline 28 propene & 8.4 & 14.2 & 2.5 & 11.3 & 5.4 \\
\hline 292 -methylpropene & 4.5 & 5.4 & & 4.9 & 2.2 \\
\hline 301 -butene & & 4.3 & & 2.1 & 0.0 \\
\hline $31 \mathrm{c}-2$-butene & & 4.0 & & 2.0 & 0.0 \\
\hline $32 t-2$-butene & 11.6 & & & 5.8 & 5.8 \\
\hline 331,3 -butadiene & 2.1 & 1.9 & & 2.0 & 1.1 \\
\hline 341 -pentene & 8.0 & & 2.3 & 4.0 & 5,2 \\
\hline $35 \mathrm{t}$-2-pentene & & 3.7 & 5.8 & 1.9 & 2.9 \\
\hline
\end{tabular}


Table 2.2: The VOC Mixtures used in the CRC Methanol-85 Chamber Study-Part 2

\begin{tabular}{|c|c|c|c|c|c|}
\hline \multirow{2}{*}{ Component } & \multicolumn{5}{|c|}{ ppbC in 1 ppmC injection } \\
\hline & \multirow{2}{*}{$\frac{\text { Urban }}{13.5}$} & \multirow{2}{*}{$\frac{\text { IAG }}{3.0}$} & \multirow{2}{*}{$\frac{\text { M85 }}{4.3}$} & \multicolumn{2}{|c|}{ Urb//AG Urb/M85 } \\
\hline $36 \mathrm{c}-2-$ pentene & & & & 8.2 & 8.9 \\
\hline 372 -methyl-1-butene & 4.2 & 3.2 & 5.7 & 3.7 & 5.0 \\
\hline 38 2-methyl-2-butene & & 8.6 & 9.8 & 4.3 & 4.9 \\
\hline 39 2-methy1-1-pentene & 8.0 & & & 4.0 & 4.0 \\
\hline 402 -methy $1-2$-pentene & 3.0 & & & 1.5 & 1.5 \\
\hline $412,3,3$-trimethy1-1-butene & 16.0 & & & 8.0 & 8.0 \\
\hline 421 -nonene & 7.0 & & & 3.5 & 3.5 \\
\hline 43 1-octene & 8.0 & & & 4.0 & 4.0 \\
\hline 44 cyclohexene & 1.0 & & 3.0 & 0.5 & 2.0 \\
\hline 45 isoprene & 3.0 & 1.0 & & 2.0 & 1.5 \\
\hline 46 cyclopentene & & 3.0 & & 1.5 & 0.0 \\
\hline 61 a-pinene & 6.0 & & & 3.0 & 3.0 \\
\hline 47 benzene & 22.0 & 52.0 & 22.8 & 37.0 & 22.4 \\
\hline 48 toluene & 69.0 & 72.0 & 35.9 & 70.5 & 52.5 \\
\hline 49 ethylbenzene & 11.0 & 20.0 & 8.0 & 15.5 & 9.5 \\
\hline $50 \mathrm{n}$-propylbenzene & 11.0 & & 2.9 & 5.5 & 7.0 \\
\hline $51 \mathrm{~m}$-xylene & 37.0 & 41.0 & 17.9 & 39.0 & 27.5 \\
\hline $52 \circ-x y l e n e$ & 16.0 & 13.0 & 6.5 & 14.5 & 11.3 \\
\hline $531,2,4$-trimethylbenzene & 56.0 & 10.0 & 6.8 & 33.0 & 31.4 \\
\hline $541,3,5$-trimethylbenzene & & 4.0 & & 2.0 & 0.0 \\
\hline 55 m-ethyltoluene & 10.0 & 14.0 & 7.5 & 12.0 & 8.7 \\
\hline 56 p-ethyltoluene & 15.0 & & & 7.5 & 7.5 \\
\hline 57 sec-butylbenzene & 7.0 & & & 3.5 & 3.5 \\
\hline 581,3 -diethylbenzene & 18.0 & 2.0 & & 10.0 & 9.0 \\
\hline $591,2,3,5$-tetramethylbenze & 6.0 & & & 3.0 & 3.0 \\
\hline 60 a-methylstyrene & 3.0 & & & 1.5 & 1.5 \\
\hline 62 styrene & & 2.0 & & 1.0 & 0.0 \\
\hline 63 naphthalene & & 1.0 & 1.0 & 0.5 & 0.5 \\
\hline 64 formaldehyde & 10.4 & 4.0 & 22.7 & 7.2 & 16.6 \\
\hline 65 acetaldehyde & 11.2 & 6.0 & 1.8 & 8.6 & 6.5 \\
\hline 66 propionaldehyde & & 1.0 & & 0.5 & 0.0 \\
\hline 67 p-tolualdehyde & & 3.0 & & 1.5 & 0.0 \\
\hline 68 crotonaldehyde & & 1.0 & & 0.5 & 0.0 \\
\hline 69 acetone & & 6.0 & 1.5 & 3.0 & 0.8 \\
\hline 70 methanol & & & 414.3 & & 207.1 \\
\hline Total & 1,000 & 1,000 & 1,000 & 1,000 & 1,000 \\
\hline
\end{tabular}




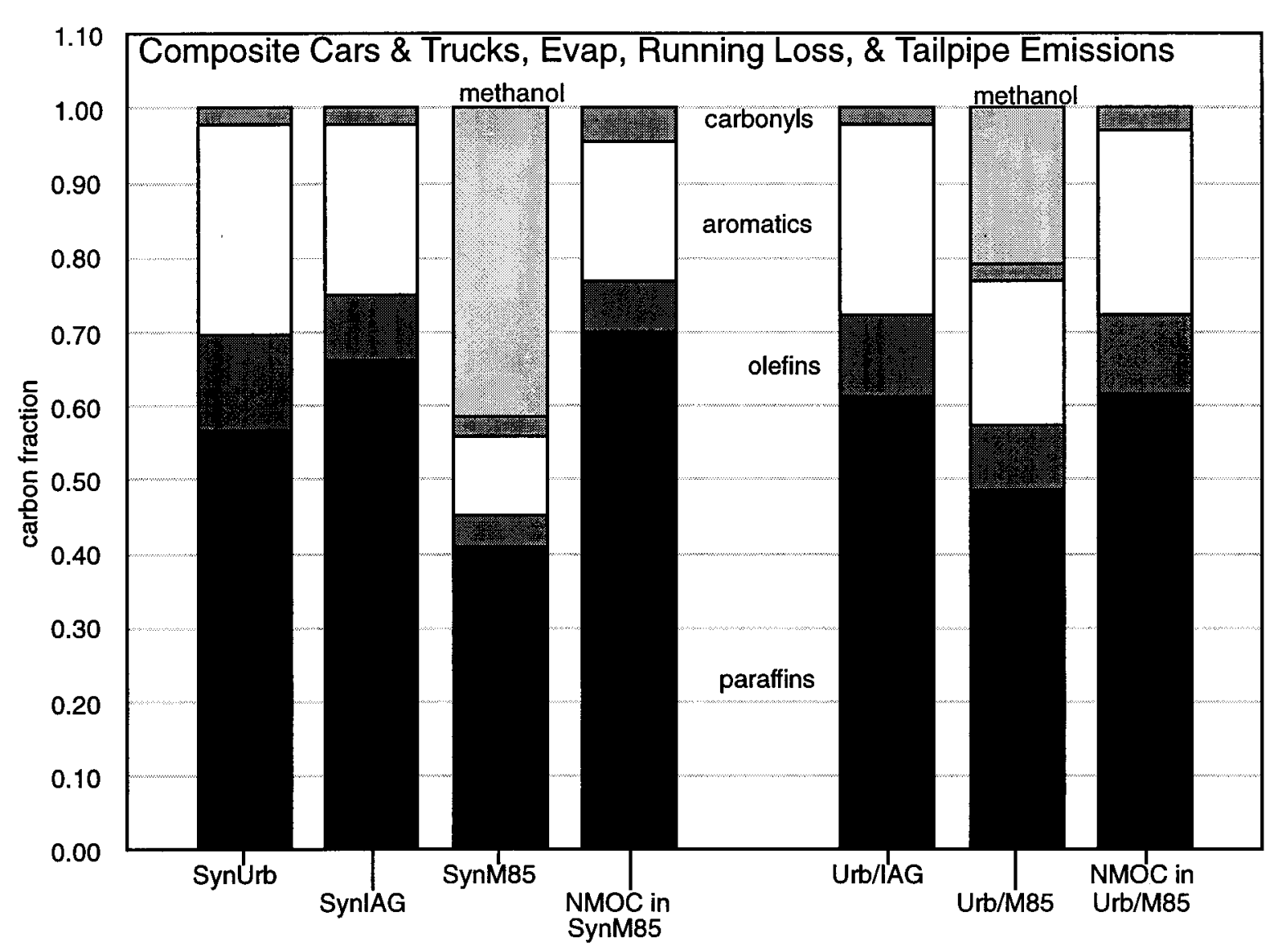

Figure 2.1: Summary composition of the CRC VOC mixtures. The NMOC bars are the distribution of the non-methanol fraction of the mixtures. 


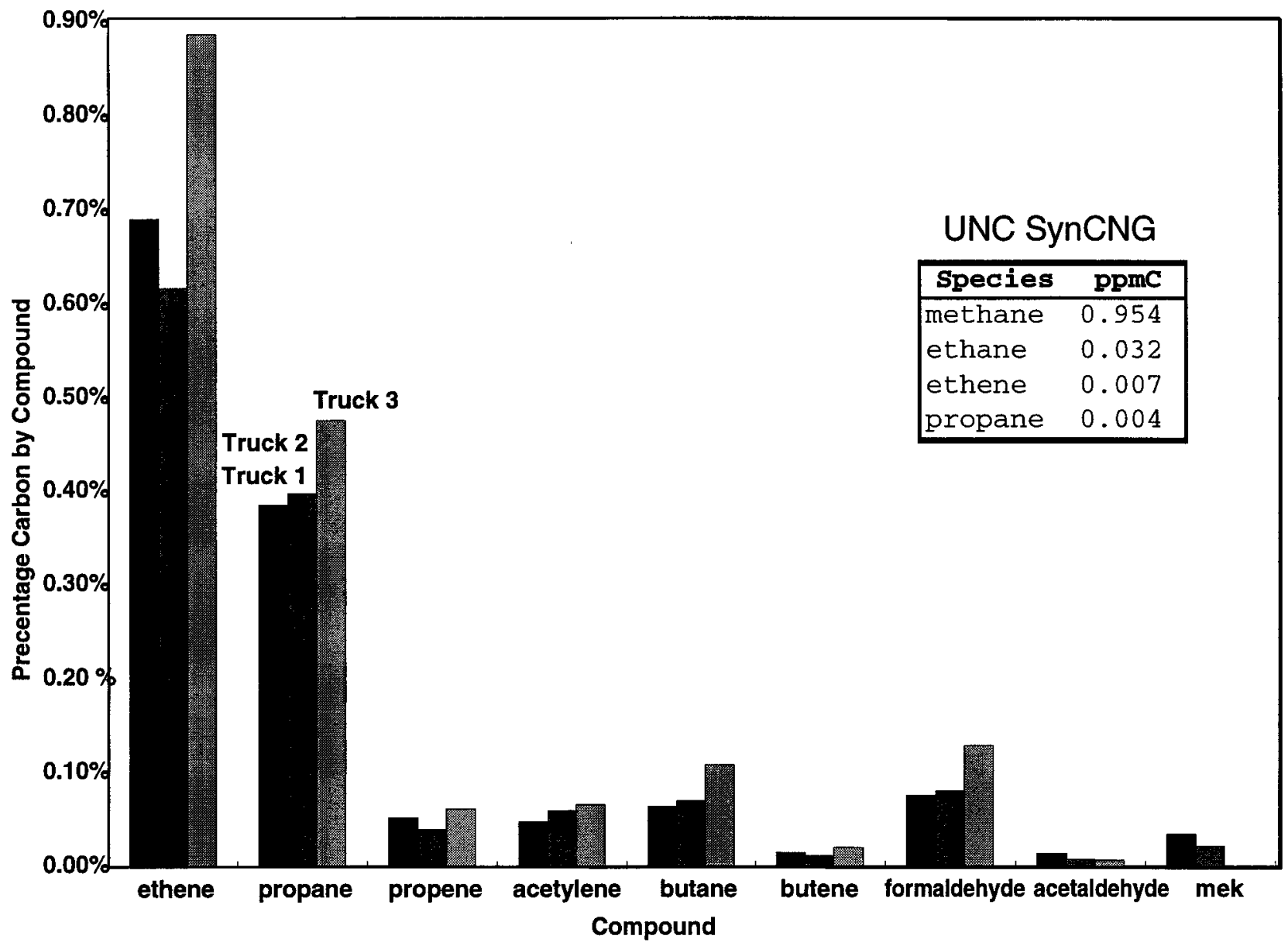

Figure 2.2: Summary composition of the compressed natural gas VOC mixtures. 


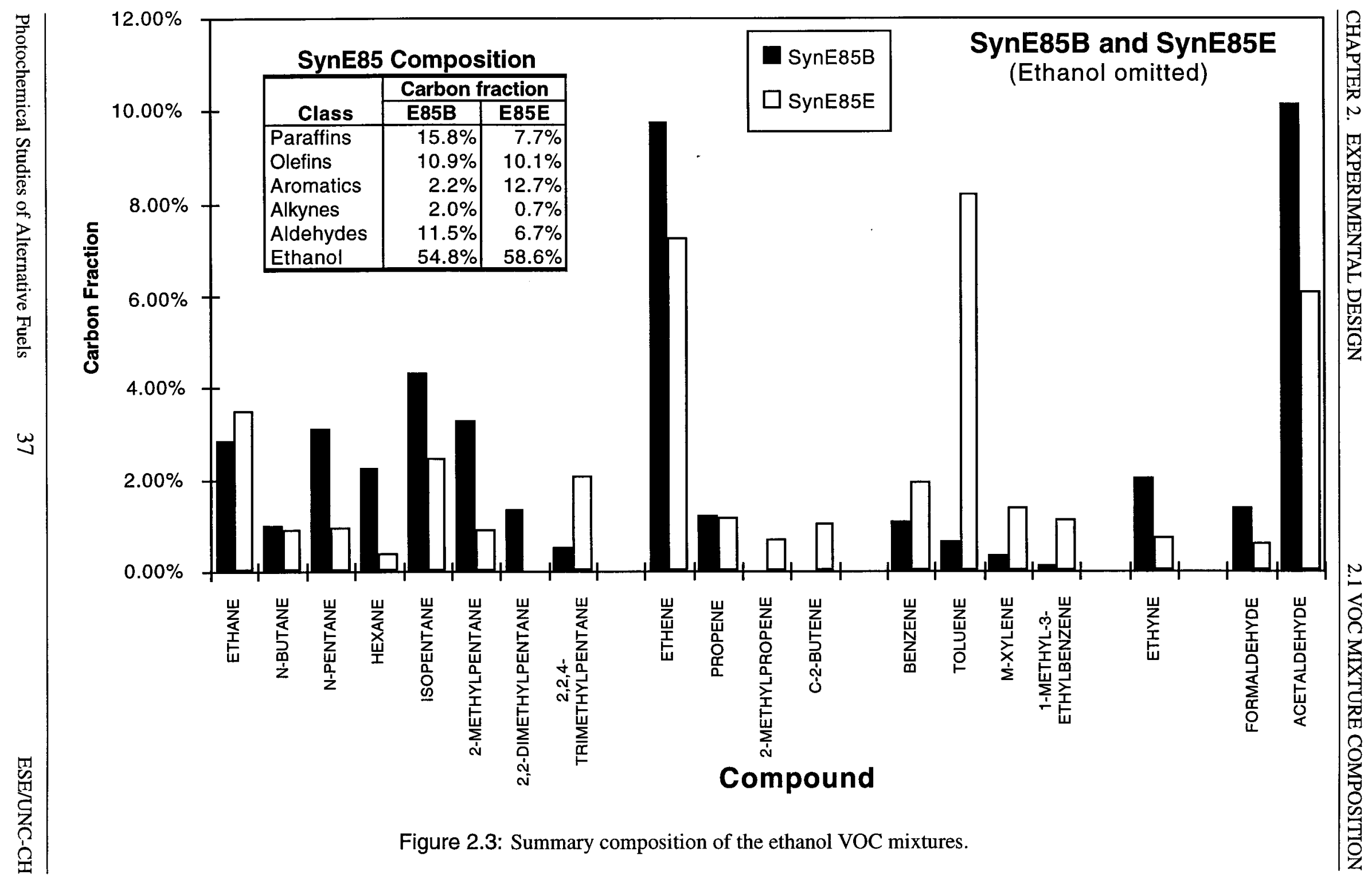




\subsection{Chamber Experimental Conditions}

Figure 2.4 shows the revised planned experimental design (the legend is the same as that shown in Figure 1.2). Revisions to the original plan shown in Figure 1.3 were made to accommodate further investigation of unexpected results obtained in early experiments. For example, we found that blends of SynE85E were more reactive than was SynIAG. Because this was an unexpected result, we devoted more chamber time to repeating this experiment and to creating new exprimental conditions to support these results.

The two sides of the chamber were used to compare the effects of changing the VOC composition directly. Each comparison would take place at three $\mathrm{HC}: \mathrm{NO}_{\mathrm{x}}$ ratios: 4.5:1, 6:1, and 9:1. Most urban centers are near 6:1 and for most mixtures, reactivity effects disappear (because the ozone formation is $\mathrm{NO}_{\mathrm{x}}$-limited) under 9:1 conditions. 


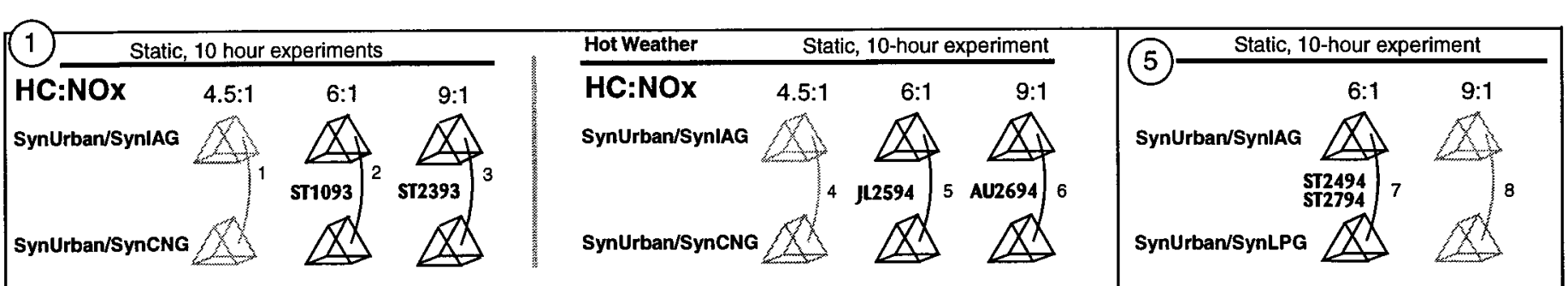

4 Static, 10 hour experiments
HC:NOX
SynUrban
SC:NOX
SynUrban
SynUrban/SynIAG

Figure 2.4: Revised DOE experimental plan. 


\section{Chapter 3}

\section{Experimental Results}

\subsection{Outdoor Smog Chamber Results}

A total of 29 alternative fuel outdoor smog chamber dual experiments were performed for this program. As indicated in Chapter 2, during the course of the work, we changed the experimental plan to explore more fully some of the surprising results obtained in our early experiments. This resulted in a greater number of replicate experiments for some conditions and a lower priority for other experimental conditions. Ultimately we did not perform four of the originally planned conditions. This is not a significant loss to the overall program, however, as the probable outcome of these conditions can be fairly well guessed, and it was more important to confirm the results for other conditions.

As usual, some experiments took place under unpredicted poor meteorological conditions and thus are not very useful for model testing. (See, for example, our first attempt at Run 7. It was conducted on September 24, 1994 and was nearly totally devoid of sunlight because of heavy overcast all day.) These had to be repeated under better weather conditions.

In other situations, significant modifications to the alternative-fuel-derived Voc compositions were used to demonstrate an hypothesis and to provide a full range of sensitive conditions. These modifications allowed us to challenge the photochemical models' ability to make accurate predictions for these unusual and stressful conditions.

The experimental results will be presented in the order of the experimental plan and block by block. Within each block, the relevant portion of the experimental plan plot will be shown, followed by a brief discussion of the experiments. Each experiment's results are shown here as an initial condition table and a SegFile standard plot. In the initial condition table, there are two main columns, one for the RED side of the chamber and one for the BLUE side of the chamber. The table has six sections,

- The total nitrogen oxides concentration (in bold), followed by the NO concentration and the $\mathrm{NO}_{2}$ concentration in each side at sunrise 
- The total HC mixture concentraton (in bold), followed by the total mixture component concentrations, and below a line, the total VOC mixture concentration (in bold) followed by the $\mathrm{CO}$ concentration (in bold) in each side at sunrise

- The total of the other measured organic or background vocs in each side at sunrise

- The estimated concentration of species known to be present, but not measured in each side at sunrise

- The estimated values of the chamber wall model parameters needed to simulate the experiment

- The three previous chamber events, i.e., the time and type of "runs" that proceeded the current experiment.

The SegFile standard plot shows in the top plot the concentration time series of $\mathrm{NO}, \mathrm{NO}_{2}$, and $\mathrm{O}_{3}$ on the two chamber sides and in the bottom plot shows time series for the meteorological data for the experiment (total solar radiation [TSR], ultraviolet radiation [UV], and the chamber air temperature and chamber dew point temperature [a form of absolute humidity]). In these plots,

- RED chamber data are always shown in SOLID lines.

- BLUE chamber data are always shown in DASHED lines.

- A legend identifies the contents of the RED and BLUE chamber sides.

- $\mathrm{NO}_{2}$ is $\left(\mathrm{NO}_{\mathrm{x}}-\mathrm{NO}\right)$ and includes PAN and organic nitrates $\left(\mathrm{RONO}_{2}\right)$, but not the $\mathrm{HNO}_{3}$.

- TSR (broadband 295-2800 $\mathrm{nm}$ radiation measured outside the chamber on a $10 \mathrm{~m}$ tower) is always shown as a SOLID line.

- UV (broadband 300-385 $\mathrm{nm}$ radiation measured outside the chamber on a $10 \mathrm{~m}$ tower) is always shown as a DASHED line.

- Air temperature inside one of the chamber-halves is always shown as a SOLID line.

- Dew point temperature inside each chamber is shown as a SOLID line for the RED side and a DASHED line for the BLUE side of the chamber.

The initial conditions shown here are within $\pm 5 \%$ of the actual values. The ozone, nitrogen oxides, temperature, dew point, and solar radiation have been processed with final calibration factors and are accurate within $\pm 2 \%$. 


\subsubsection{Block 1: Urb/IAG versus Urb/CNG}

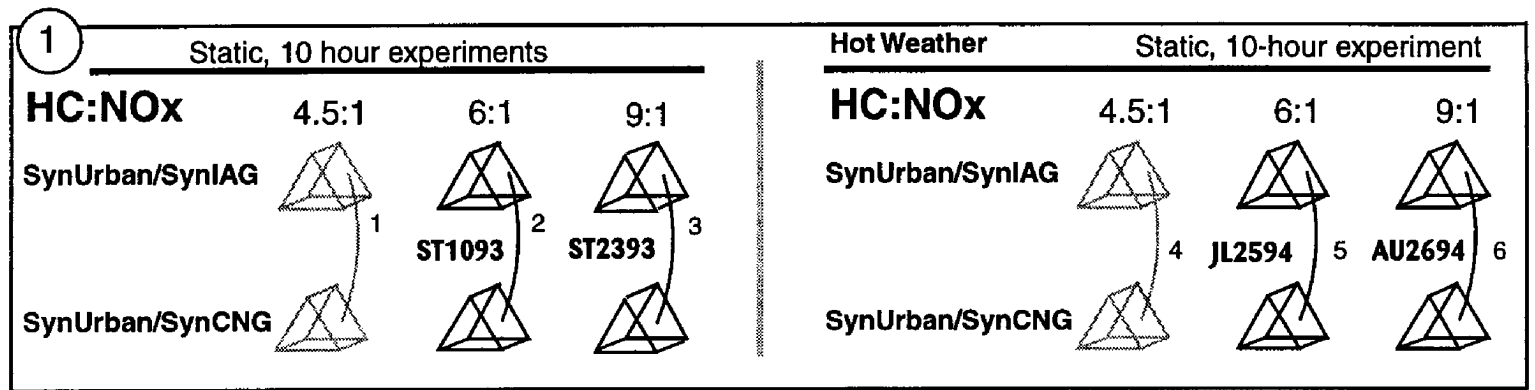

Block 1 experiments were designed to compare the reactivity of SynCNG with SynIAG with each mixture blended 50:50 with SynUrb. In an earlier chapter, we discussed the reasons for the experimental design of blending the different alternative fuel emissions test mixtures with SynUrb. In brief, this provides reasonably realistic test scenarios for direct observational comparisons to be made of substitution effects of these mixtures into urban atmospheres. In addition, these experiments provide good tests for the chemical mechanisms that will be used to model different urban control scenarios.

\section{Run 2, 6:1 Urb/IAG versus Urb/CNG}

ST1093 This run (Table 3.1 and Figure 3.1), shows that SynCNG has a significant benefit for reducing ozone compared to SynIAG even under cooler (fall) conditions.

\section{Run 3, 9:1 Urb/IAG versus Urb/CNG}

ST2393 Usually at 9:1 VOC:NO ${ }_{x}$, reactivity makes almost no difference. This run (Table 3.2 and Figure 3.2), however, shows that for CNG, the benefits extend to well beyond 9:1.

\section{Run 5, 6:1 Urb/IAG versus Urb/CNG}

JL2594 This run (Table 3.3 and Figure 3.3), is a repeat of ST1093, conducted in warm weather. This run shows that the benefits of CNG are sustained under warm, high intensity (summer) sun conditions.

\section{Run 6, 9:1 Urb/IAG versus Urb/CNG}

AU2694 This is a repeat of Run 3 (ST2393) under warm, high sun (summer) conditions. This run (Table 3.4 and Figure 3.4), shows that the benefits of CNG are sustained under warm, high sun conditions even for high $\mathrm{HC}: \mathrm{NO}_{\mathrm{x}}$ ratios, unlike $\mathrm{M} 85$ and E85 results. 
Table 3.1: Initial Conditions for ST1093

(Units are ppm or ppmC)

\begin{tabular}{|c|c|c|c|}
\hline \multicolumn{2}{|c|}{ RED SIDE } & \multicolumn{2}{|c|}{ BLUE SIDE } \\
\hline Compound & Amount & Compound & Amount \\
\hline \multicolumn{4}{|c|}{ Initial Nitrogen Oxides } \\
\hline $\mathrm{NO}_{\mathrm{x}}$ & 0.3387 & $\mathrm{NO}_{\mathrm{x}}$ & 0.3405 \\
\hline NO & 0.2940 & NO & 0.2899 \\
\hline $\mathrm{NO}_{2}$ & 0.0447 & $\mathrm{NO}_{2}$ & 0.0506 \\
\hline \multicolumn{4}{|c|}{ Initial Volatile Organic Compounds } \\
\hline SynUrban & 0.9997 & SynUrban & 0.9997 \\
\hline SYNURBGAS91 & 0.3953 & SYNURBGAS91 & 0.3952 \\
\hline SYNURBLIQ91 & 0.5909 & SYNURBLIQ91 & 0.5909 \\
\hline $\mathrm{CCHO}$ & 0.0070 & $\mathrm{CCHO}$ & 0.0070 \\
\hline $\mathrm{HCHO}$ & 0.0065 & $\mathrm{HCHO}$ & 0.0065 \\
\hline SynCNG & 0.9977 & SynIAG & 0.8575 \\
\hline $\mathrm{CH} 4$ & 0.9536 & $\mathrm{CH} 4$ & 0.1410 \\
\hline $\mathrm{CC}$ & 0.0316 & SYNIAGGAS91 & 0.4852 \\
\hline $\mathrm{C}: \mathrm{C}$ & 0.0073 & SYNIAGLPO92 & 0.1563 \\
\hline \multirow[t]{2}{*}{$\operatorname{ccc}$} & 0.0042 & SYNIAGARO92 & 0.1974 \\
\hline & & SYNIAGALD91 & 0.0146 \\
\hline $\mathrm{HCHO}$ & 0.0010 & $\mathrm{HCHO}$ & 0.0040 \\
\hline Total VOCs & 1.9974 & Total VOCs & 1.8572 \\
\hline $\mathrm{CO}$ & 1.3000 & $\mathrm{CO}$ & 1.3000 \\
\hline \multicolumn{4}{|c|}{ Other Measured Initial Compounds } \\
\hline BVOC9109 & 0.1440 & BVOC9109 & 0.1440 \\
\hline \multicolumn{4}{|c|}{ Estimated Initial Compounds } \\
\hline HONO & 0.007 & HONO & 0.007 \\
\hline $\mathrm{CH}_{4}$ & 1.790 & $\mathrm{CH}_{4}$ & 1.790 \\
\hline $\mathrm{H}_{2}$ & 0.580 & $\mathrm{H}_{2}$ & 0.580 \\
\hline \multicolumn{4}{|c|}{ Chamber Model Parameters } \\
\hline $\begin{array}{l}\mathrm{NO}_{2} \longrightarrow \mathrm{HONO} \\
\text { wall. } \mathrm{HNO}_{3} \longrightarrow \mathrm{NO}_{2}\end{array}$ & $\begin{array}{l}1.8 \times 10^{-3} \\
2.0 \times 10^{-3}\end{array}$ & $\begin{array}{l}\mathrm{NO}_{2} \longrightarrow \mathrm{HONO} \\
\text { wall. } \mathrm{HNO}_{3} \longrightarrow \mathrm{NO}_{2}\end{array}$ & $\begin{array}{l}1.8 \times 10^{-3} \\
2.0 \times 10^{-3}\end{array}$ \\
\hline
\end{tabular}

Three Previous Chamber Events

930830.SEG Delta CO run

930824.SEG Propylbenzene vs Ethylbenzene run

930823.SEG Delta Ethene run 

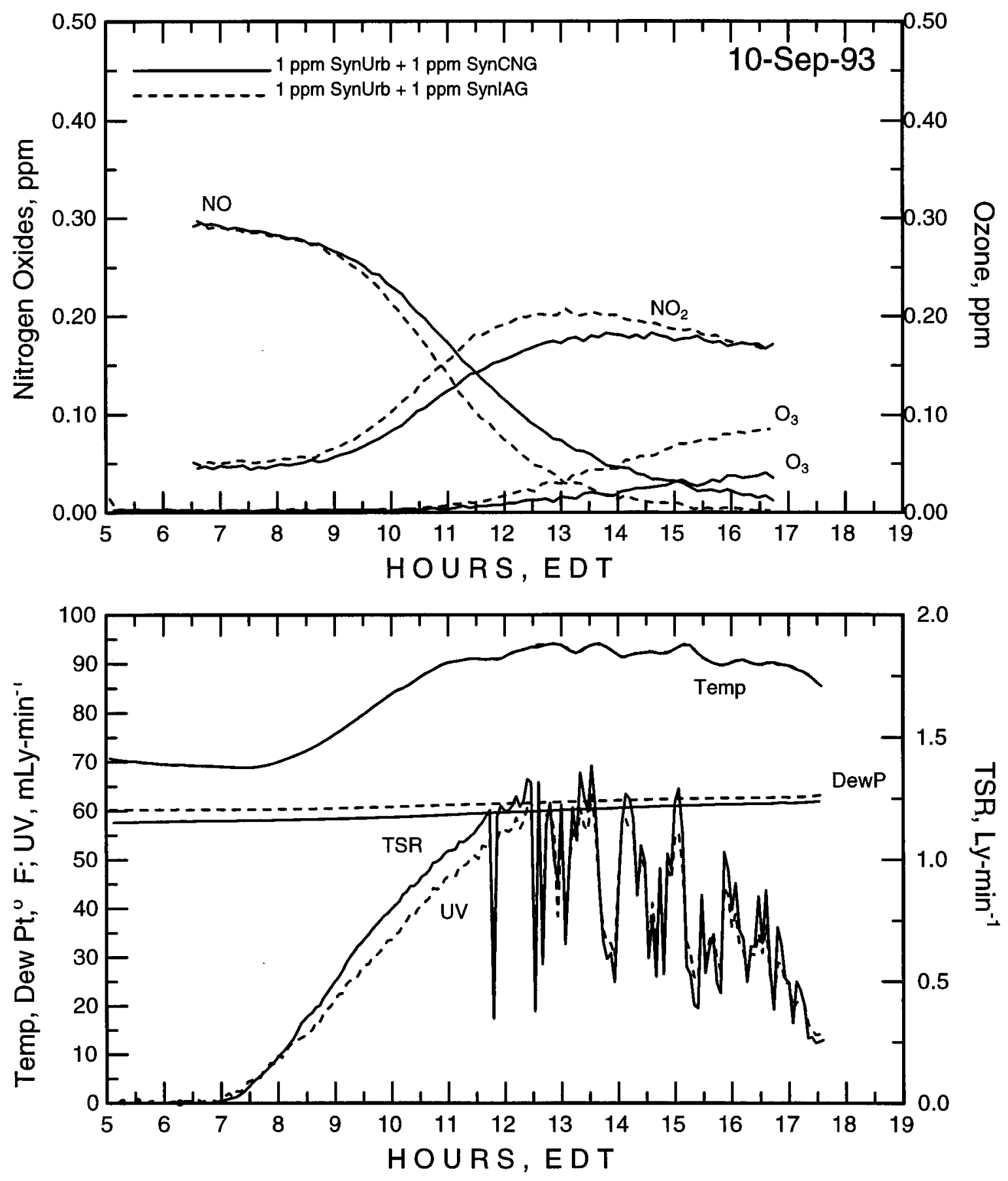

Figure 3.1: Run 2-6:1 SynUrban/SynIAG:NOx versus 6:1 SynUrban/SynCNG:NO . 
Table 3.2: Initial Conditions for ST2393

(Units are ppm or ppmC)

\begin{tabular}{|c|c|c|c|}
\hline \multicolumn{2}{|c|}{ RED SIDE } & \multicolumn{2}{|c|}{ BLUE SIDE } \\
\hline Compound & Amount & Compound & Amount \\
\hline \multicolumn{4}{|c|}{ Initial Nitrogen Oxides } \\
\hline $\mathrm{NO}_{\mathrm{x}}$ & 0.3342 & $\mathrm{NO}_{x}$ & 0.3349 \\
\hline NO & 0.2612 & NO & 0.2636 \\
\hline $\mathrm{NO}_{2}$ & 0.0730 & $\mathrm{NO}_{2}$ & 0.0713 \\
\hline \multicolumn{4}{|c|}{ Initial Volatile Organic Compounds } \\
\hline SynUrban & 1.4999 & SynUrban & 1.4999 \\
\hline SYNURBGAS91 & 0.5871 & SYNURBGAS91 & 0.5871 \\
\hline SYNURBLIQ91 & 0.8865 & SYNURBLIQ91 & 0.8865 \\
\hline $\mathrm{CCHO}$ & 0.0137 & $\mathrm{CCHO}$ & 0.0137 \\
\hline $\mathrm{HCHO}$ & 0.0126 & $\mathrm{HCHO}$ & 0.0126 \\
\hline SynIAG & 1.2894 & SynCNG & 1.4965 \\
\hline $\mathrm{CH} 4$ & 0.2115 & $\mathrm{CH} 4$ & 1.4304 \\
\hline SYNIAGGAS91 & 0.7281 & $\mathrm{CC}$ & 0.0474 \\
\hline SYNIAGLPO92 & 0.2362 & $C: C$ & 0.0110 \\
\hline SYNIAGARO92 & 0.2981 & $\mathrm{CCC}$ & 0.0063 \\
\hline SYNIAGALD91 & 0.0219 & & \\
\hline $\mathrm{HCHO}$ & 0.0051 & $\mathrm{HCHO}$ & 0.0014 \\
\hline Total VOCs & 2.7893 & Total VOCs & 2.9964 \\
\hline $\mathrm{CO}$ & 1.3000 & CO & 1.3000 \\
\hline \multicolumn{4}{|c|}{ Other Measured Initial Compounds } \\
\hline BVOC9109 & 0.1440 & BVOC9109 & 0.1440 \\
\hline \multicolumn{4}{|c|}{ Estimated Initial Compounds } \\
\hline HONO & 0.008 & HONO & 0.008 \\
\hline $\mathrm{CH}_{4}$ & 1.790 & $\mathrm{CH}_{4}$ & 1.790 \\
\hline $\mathrm{H}_{2}$ & 0.580 & $\mathrm{H}_{2}$ & 0.580 \\
\hline \multicolumn{4}{|c|}{ Chamber Model Parameters } \\
\hline $\begin{array}{l}\mathrm{NO}_{2} \longrightarrow \mathrm{HONO} \\
\text { wall. } \mathrm{HNO}_{3} \longrightarrow \mathrm{NO}_{2}\end{array}$ & $\begin{array}{l}1.8 \times 10^{-3} \\
2.0 \times 10^{-3}\end{array}$ & $\begin{array}{l}\mathrm{NO}_{2} \longrightarrow \mathrm{HONO} \\
\text { wall. } \mathrm{HNO}_{3} \longrightarrow \mathrm{NO}_{2}\end{array}$ & $\begin{array}{l}1.8 \times 10^{-3} \\
2.0 \times 10^{-3}\end{array}$ \\
\hline $\begin{array}{l}\text { Three Previous Cha } \\
\text { 930920.SEG Delta m } \\
\text { 930916.SEG Night Is } \\
\text { 930915.SEG Night Is }\end{array}$ & $\begin{array}{l}\text { nber Events } \\
\text { thane run } \\
\text { oprene run } \\
\text { oprene run }\end{array}$ & & \\
\hline
\end{tabular}



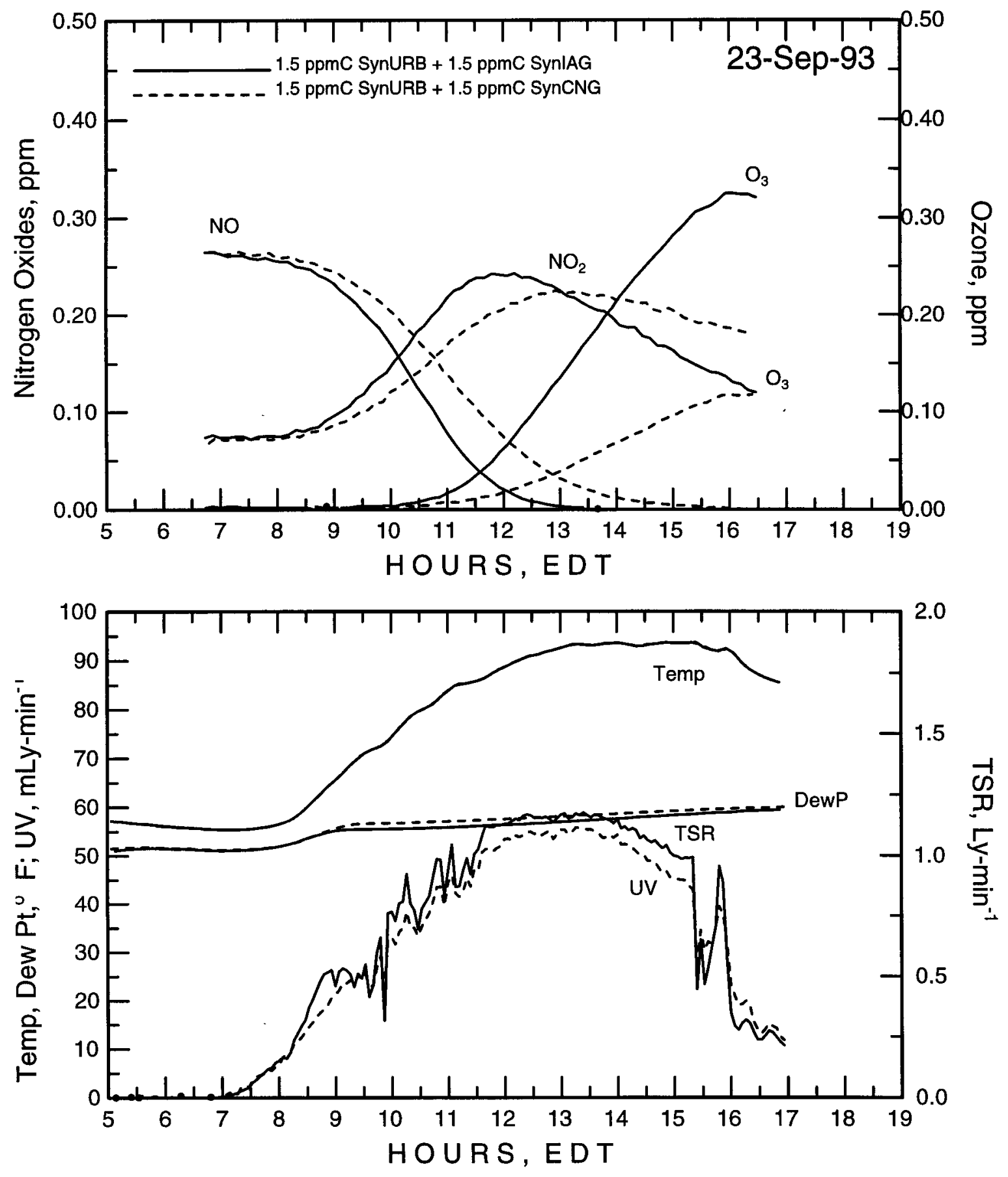

Figure 3.2: Run 3-9:1 SynUrban/SynIAG:NO versus 9:1 SynUrban/SynCNG:NO . 
Table 3.3: Initial Conditions for JL2594

(Units are ppm or ppmC)

\begin{tabular}{|c|c|c|c|}
\hline \multicolumn{2}{|c|}{ RED SIDE } & \multicolumn{2}{|c|}{ BLUE SIDE } \\
\hline Compound & Amount & Compound & Amount \\
\hline \multicolumn{4}{|c|}{ Initial Nitrogen Oxides } \\
\hline $\mathrm{NO}_{\mathrm{x}}$ & 0.3360 & $\mathrm{NO}_{\mathrm{x}}$ & 0.3347 \\
\hline NO & 0.2851 & NO & 0.2874 \\
\hline $\mathrm{NO}_{2}$ & 0.0509 & $\mathrm{NO}_{2}$ & 0.0473 \\
\hline \multicolumn{4}{|c|}{ Initial Volatile Organic Compounds } \\
\hline SynUrban & 0.9997 & SynUrban & 0.9997 \\
\hline SYNURBGAS91 & 0.3953 & SYNURBGAS91 & 0.3952 \\
\hline SYNURBLIQ91 & 0.5909 & SYNURBLIQ91 & 0.5909 \\
\hline $\mathrm{CCHO}$ & 0.0070 & $\mathrm{CCHO}$ & 0.0070 \\
\hline $\mathrm{HCHO}$ & 0.0065 & $\mathrm{HCHO}$ & 0.0065 \\
\hline SynCNG & 0.9977 & SynIAG & 0.8575 \\
\hline $\mathrm{CH} 4$ & 0.9536 & $\mathrm{CH} 4$ & 0.1410 \\
\hline $\mathrm{CC}$ & 0.0316 & SYNIAGGAS91 & 0.4852 \\
\hline$C: C$ & 0.0073 & SYNIAGLPO92 & 0.1563 \\
\hline \multirow[t]{2}{*}{$\operatorname{ccc}$} & 0.0042 & SYNIAGARO92 & 0.1974 \\
\hline & & SYNIAGALD91 & 0.0146 \\
\hline $\mathrm{HCHO}$ & 0.0010 & $\mathrm{HCHO}$ & 0.0040 \\
\hline Total VOCs & 1.9974 & Total VOCs & 1.8572 \\
\hline $\mathrm{CO}$ & 1.3000 & $\mathrm{CO}$ & 1.3000 \\
\hline \multicolumn{4}{|c|}{ Other Measured Initial Compounds } \\
\hline BVOC9109 & 0.1440 & BVOC9109 & 0.1440 \\
\hline \multicolumn{4}{|c|}{ Estimated Initial Compounds } \\
\hline HONO & 0.003 & HONO & 0.003 \\
\hline $\mathrm{CH}_{4}$ & 1.790 & $\mathrm{CH}_{4}$ & 1.790 \\
\hline $\mathrm{H}_{2}$ & 0.580 & $\mathrm{H}_{2}$ & 0.580 \\
\hline \multicolumn{4}{|c|}{ Chamber Model Parameters } \\
\hline $\begin{array}{l}\mathrm{NO}_{2} \longrightarrow \mathrm{HONO} \\
\text { wall. } \mathrm{HNO}_{3} \longrightarrow \mathrm{NO}_{2}\end{array}$ & $\begin{array}{l}2.5 \times 10^{-3} \\
2.0 \times 10^{-3}\end{array}$ & $\begin{array}{l}\mathrm{NO}_{2} \longrightarrow \mathrm{HONO} \\
\text { wall. } \mathrm{HNO}_{3} \longrightarrow \mathrm{NO}_{2}\end{array}$ & $\begin{array}{l}2.5 \times 10^{-3} \\
2.0 \times 10^{-3}\end{array}$ \\
\hline
\end{tabular}

Three Previous Chamber Events

940720.SEG Delta Toluene run

940715.SEG Delta Toluene run

940708.SEG m-Xylene vs Toluene run 

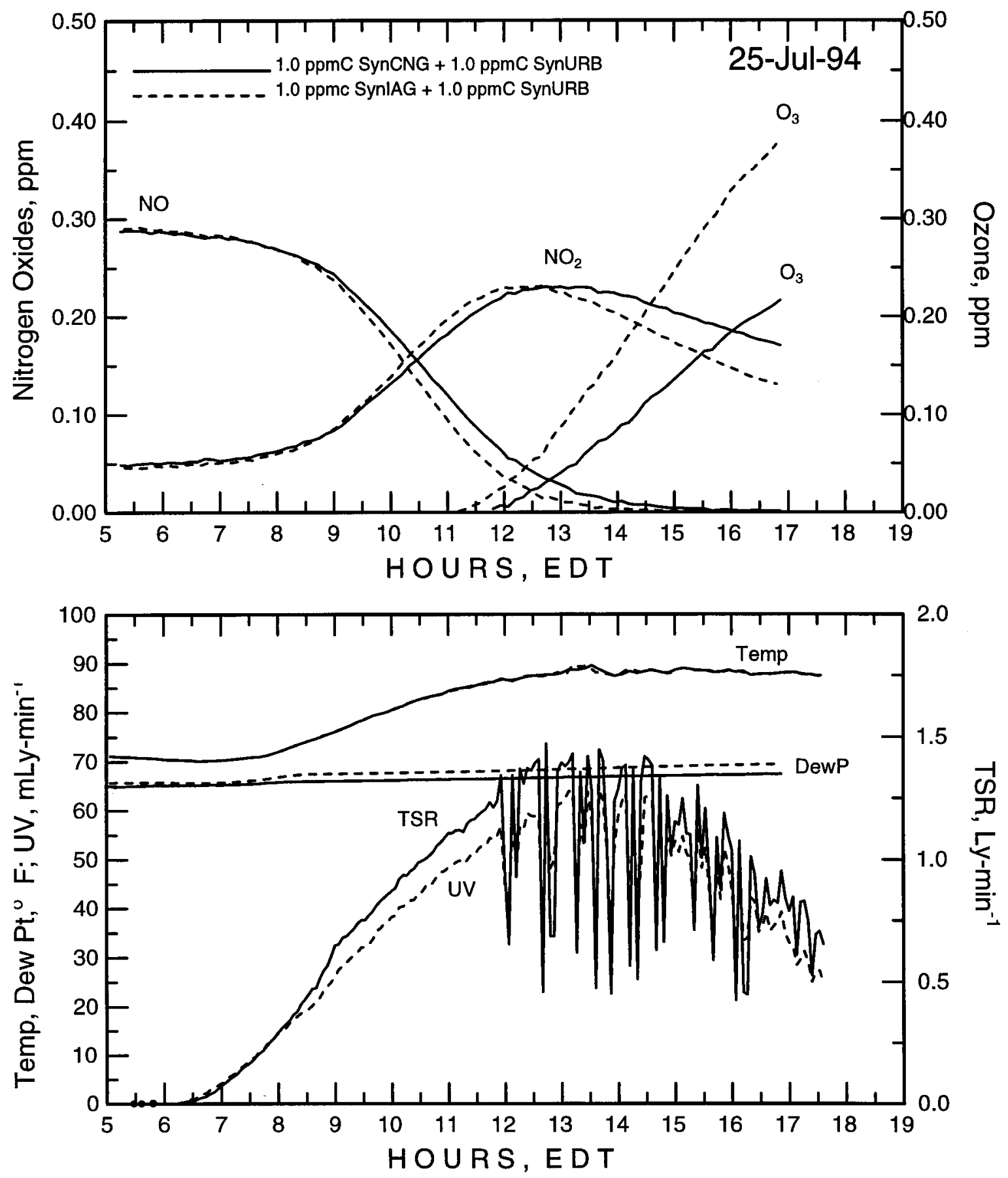

Figure 3.3: Run 5-6:1 SynUrban/SynIAG:NOx versus 6:1 SynUrban/SynCNG:NO . 
Table 3.4: Initial Conditions for AU2694

(Units are ppm or ppmC)

\begin{tabular}{|c|c|c|c|}
\hline \multicolumn{2}{|c|}{ RED SIDE } & \multicolumn{2}{|c|}{ BLUE SIDE } \\
\hline Compound & Amount & Compound & Amount \\
\hline \multicolumn{4}{|c|}{ Initial Nitrogen Oxides } \\
\hline $\mathrm{NO}_{\mathrm{x}}$ & 0.3208 & $\mathrm{NO}_{\mathrm{x}}$ & 0.3213 \\
\hline NO & 0.2619 & NO & 0.2635 \\
\hline $\mathrm{NO}_{2}$ & 0.0589 & $\mathrm{NO}_{2}$ & 0.0578 \\
\hline \multicolumn{4}{|c|}{ Initial Volatile Organic Compounds } \\
\hline SynUrban & 1.4999 & SynUrban & 1.4999 \\
\hline SYNURBGAS91 & 0.5871 & SYNURBGAS91 & 0.5871 \\
\hline SYNURBLIQ91 & 0.8865 & SYNURBLIQ91 & 0.8865 \\
\hline $\mathrm{CCHO}$ & 0.0137 & $\mathrm{CCHO}$ & 0.0137 \\
\hline $\mathrm{HCHO}$ & 0.0126 & $\mathrm{HCHO}$ & 0.0126 \\
\hline SynCNG & 1.4965 & SynIAG & 1.2894 \\
\hline $\mathrm{CH} 4$ & 1.4304 & $\mathrm{CH} 4$ & 0.2115 \\
\hline $\mathrm{CC}$ & 0.0474 & SYNIAGGAS91 & 0.7281 \\
\hline$C: C$ & 0.0110 & SYNIAGLPO92 & 0.2362 \\
\hline \multirow[t]{2}{*}{$\operatorname{ccc}$} & 0.0063 & SYNIAGARO92 & 0.2981 \\
\hline & & SYNIAGALD91 & 0.0219 \\
\hline $\mathrm{HCHO}$ & 0.0014 & $\mathrm{HCHO}$ & 0.0051 \\
\hline Total VOCs & 2.9964 & Total VOCs & 2.7893 \\
\hline $\mathrm{CO}$ & 1.3000 & $\mathrm{CO}$ & 1.3000 \\
\hline \multicolumn{4}{|c|}{ Other Measured Initial Compounds } \\
\hline BVOC9109 & 0.1440 & BVOC9109 & 0.1440 \\
\hline \multicolumn{4}{|c|}{ Estimated Initial Compounds } \\
\hline HONO & 0.005 & HONO & 0.005 \\
\hline $\mathrm{CH}_{4}$ & 1.790 & $\mathrm{CH}_{4}$ & 1.790 \\
\hline $\mathrm{H}_{2}$ & 0.580 & $\mathrm{H}_{2}$ & 0.580 \\
\hline \multicolumn{4}{|c|}{ Chamber Model Parameters } \\
\hline $\mathrm{NO}_{2} \longrightarrow \mathrm{HONO}$ & $3.0 \times 10^{-3}$ & $\mathrm{NO}_{2} \longrightarrow \mathrm{HONO}$ & $3.0 \times 10^{-3}$ \\
\hline wall. $\mathrm{HNO}_{3} \longrightarrow \mathrm{NO}_{2}$ & $2.0 \times 10^{-3}$ & wall. $\mathrm{HNO}_{3} \longrightarrow \mathrm{NO}_{2}$ & $2.0 \times 10^{-3}$ \\
\hline
\end{tabular}

Three Previous Chamber Events

940825.SEG SynE85E vs SynIAG run

940824.SEG SynE85E vs SynIAG run

940823.SEG SynE85E vs SynIAG run 

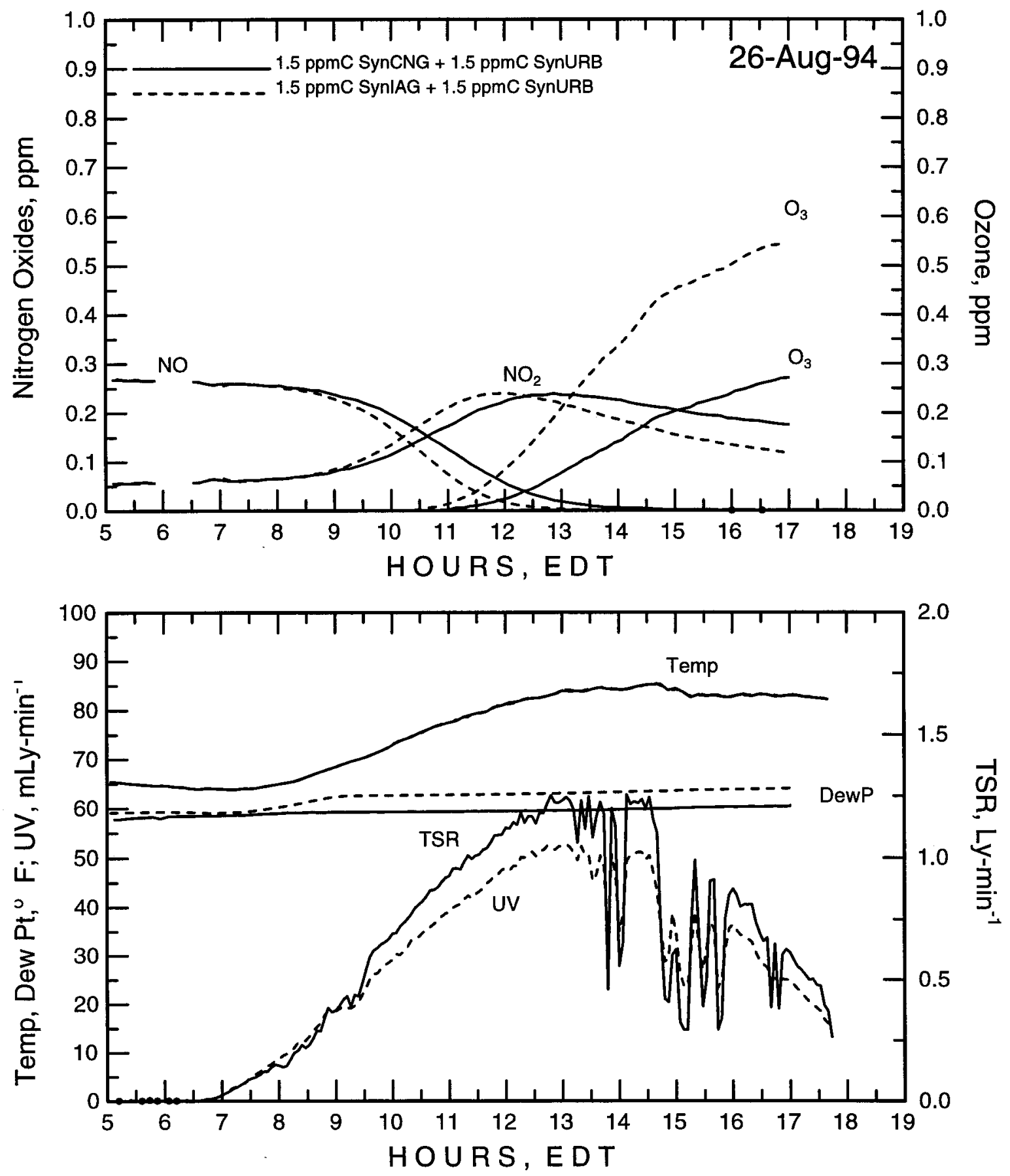

Figure 3.4: Run 6-9:1 SynUrban/SynIAG:NOx versus 9:1 SynUrban/SynCNG:NO . 


\subsubsection{Block 5: Urb/IAG versus Urb/LPG}

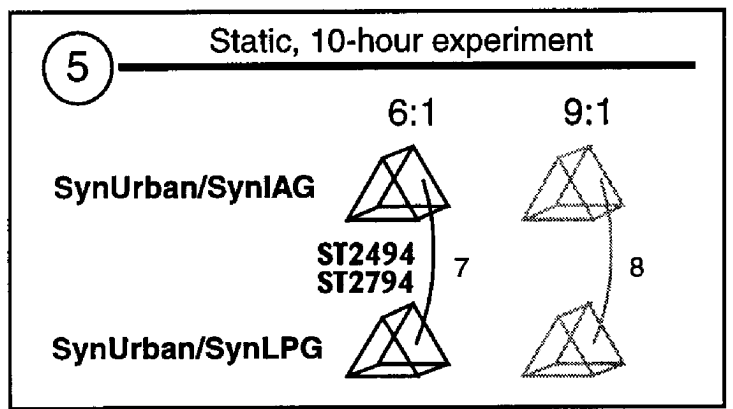

Block 5 experiments were designed to compare the reactivity of SynLPG with SynIAG, with each mixture blended 50:50 with SynUrb.

\section{Run 7, 6:1 Urb/IAG versus Urb/LPG}

ST2494 LPG shows a benefit very similar to CNG. This particular experiment, (Table 3.5 and Figure 3.5), had very cloudy conditions. The experiment was repeated.

ST2794 This experiment (Table 3.6 and Figure 3.6), is a repeat of the September 24 experiment with much better sun. 
Table 3.5: Initial Conditions for ST2494

(Units are ppm or ppmC)

\begin{tabular}{|c|c|c|c|}
\hline \multicolumn{2}{|c|}{ RED SIDE } & \multicolumn{2}{|c|}{ BLUE SIDE } \\
\hline Compound & Amount & Compound & Amount \\
\hline \multicolumn{4}{|c|}{ Initial Nitrogen Oxides } \\
\hline NO & 0.2792 & NO & 0.2761 \\
\hline $\mathrm{NO}_{2}$ & 0.0571 & $\mathrm{NO}_{2}$ & 0.0564 \\
\hline $\mathrm{NO}_{\mathrm{x}}$ & 0.3363 & $\mathrm{NO}_{\mathrm{x}}$ & 0.3325 \\
\hline \multicolumn{4}{|c|}{ Initial Volatile Organic Compounds } \\
\hline SynUrban & 0.9997 & SynUrban & 0.9997 \\
\hline SYNURBGAS91 & 0.3953 & SYNURBGAS91 & 0.3953 \\
\hline SYNURBLIQ91 & 0.5909 & SYNURBLIQ91 & 0.5909 \\
\hline $\mathrm{CCHO}$ & 0.0070 & $\mathrm{CCHO}$ & 0.0070 \\
\hline $\mathrm{HCHO}$ & 0.0065 & $\mathrm{HCHO}$ & 0.0065 \\
\hline SynLPG & 0.9512 & SynIAG & 1.0000 \\
\hline $\operatorname{CCC}$ & 0.7360 & SYNIAGGAS91 & 0.5650 \\
\hline $\mathrm{CH}_{4}$ & 0.1285 & SYNIAGLPO92 & 0.1830 \\
\hline $\mathrm{CC}$ & 0.0593 & SYNIAGARO92 & 0.2310 \\
\hline $\mathrm{CC}: \mathrm{C}$ & 0.0200 & & \\
\hline \multirow[t]{2}{*}{$\mathrm{C}: \mathrm{C}$} & 0.0054 & & \\
\hline & & SYNIAGALD91 & 0.0170 \\
\hline $\mathrm{HCHO}$ & 0.0020 & $\mathrm{HCHO}$ & 0.0040 \\
\hline Total VOCs & 1.9509 & Total VOCs & 1.9997 \\
\hline co & 1.3000 & $\mathrm{co}$ & 1.3000 \\
\hline \multicolumn{4}{|c|}{ Other Measured Initial Compounds } \\
\hline BVOC9109 & 0.1440 & BVOC9109 & 0.1440 \\
\hline \multicolumn{4}{|c|}{ Estimated Initial Compounds } \\
\hline HONO & 0.005 & HONO & 0.005 \\
\hline $\mathrm{CH}_{4}$ & 1.790 & $\mathrm{CH}_{4}$ & 1.790 \\
\hline $\mathrm{H}_{2}$ & 0.580 & $\mathrm{H}_{2}$ & 0.580 \\
\hline \multicolumn{4}{|c|}{ Chamber Model Parameters } \\
\hline $\mathrm{NO}_{2} \longrightarrow \mathrm{HONO}$ & $2.5 \times 10^{-3}$ & $\mathrm{NO}_{2} \longrightarrow \mathrm{HONO}$ & $2.5 \times 10^{-3}$ \\
\hline wall. $\mathrm{HNO}_{3} \longrightarrow \mathrm{NO}_{2}$ & $2.0 \times 10^{-3}$ & wall. $\mathrm{HNO}_{3} \longrightarrow \mathrm{NO}_{2}$ & $2.0 \times 10^{-3}$ \\
\hline
\end{tabular}

\section{Three Previous Chamber Events}

940920.SEG Night Isoprene run 940919.SEG Night Isoprene run 940915 .SEG SynE85E-EtOH vs SynURB run 

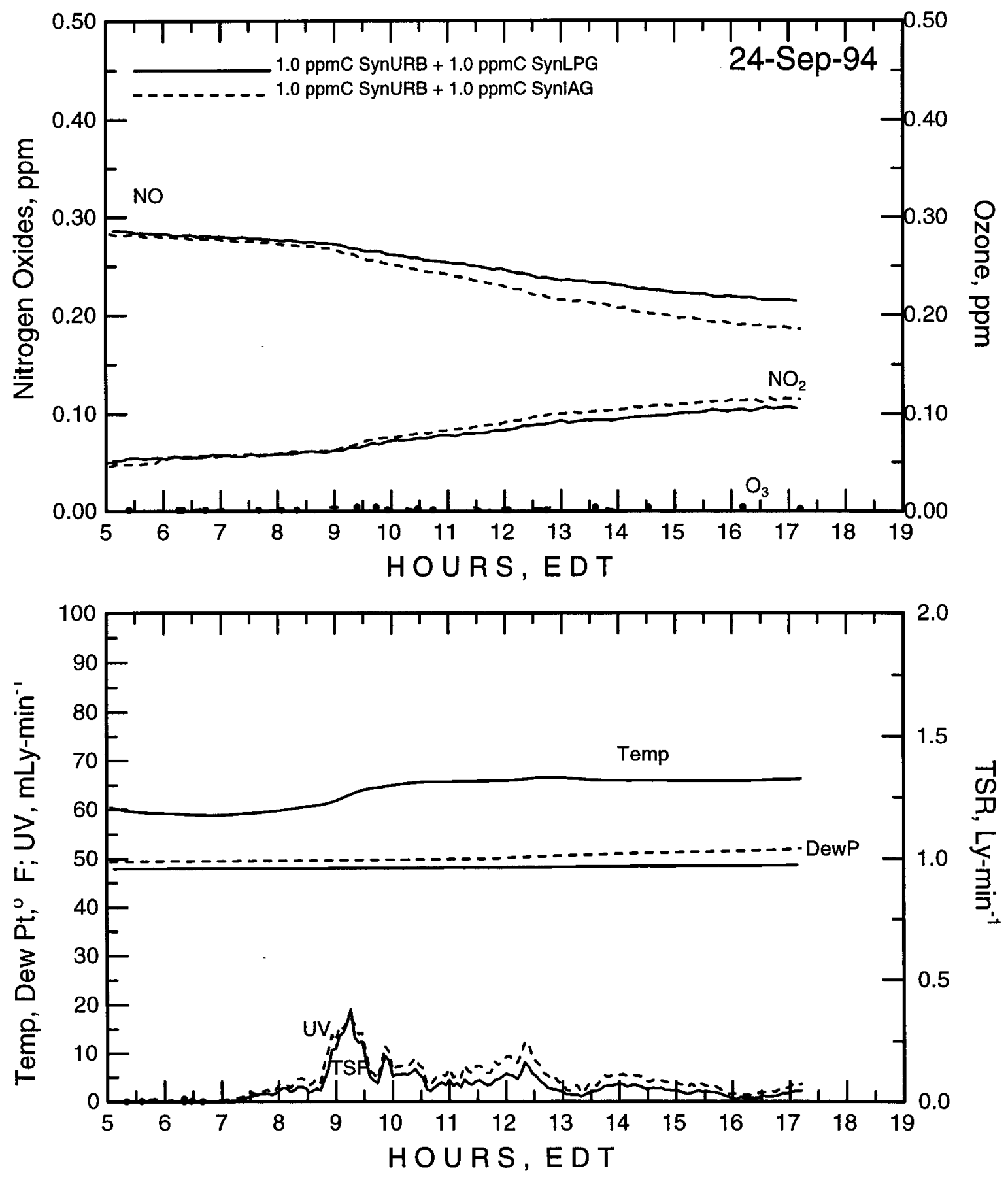

Figure 3.5: Run 7-6:1 SynUrban/SynIAG:NOx versus 6:1 SynUrban/SynLPG:NO . 
Table 3.6: Initial Conditions for ST2794

(Units are ppm or ppmC)

\begin{tabular}{|c|c|c|c|}
\hline \multicolumn{2}{|c|}{ RED SIDE } & \multicolumn{2}{|c|}{ BLUE SIDE } \\
\hline Compound & Amount & Compound & Amount \\
\hline \multicolumn{4}{|c|}{ Initial Nitrogen Oxides } \\
\hline $\mathrm{NO}_{\mathrm{x}}$ & 0.3254 & $\mathrm{NO}_{\mathrm{x}}$ & 0.3253 \\
\hline NO & 0.2718 & NO & 0.2721 \\
\hline $\mathrm{NO}_{2}$ & 0.0536 & $\mathrm{NO}_{2}$ & 0.0532 \\
\hline \multicolumn{4}{|c|}{ Initial Volatile Organic Compounds } \\
\hline SynUrban & 0.9997 & SynUrban & 0.9997 \\
\hline SYNURBGAS91 & 0.3953 & SYNURBGAS91 & 0.3953 \\
\hline SYNURBLIQ91 & 0.5909 & SYNURBLIQ91 & 0.5909 \\
\hline $\mathrm{CCHO}$ & 0.0070 & $\mathrm{CCHO}$ & 0.0070 \\
\hline $\mathrm{HCHO}$ & 0.0065 & $\mathrm{HCHO}$ & 0.0065 \\
\hline SynLPG & 0.9512 & SynlAG & 1.0000 \\
\hline $\operatorname{ccc}$ & 0.7360 & SYNIAGGAS91 & 0.5650 \\
\hline $\mathrm{CH} 4$ & 0.1285 & SYNIAGLPO92 & 0.1830 \\
\hline $\mathrm{CC}$ & 0.0593 & SYNIAGARO92 & 0.2310 \\
\hline $\mathrm{CC}: \mathrm{C}$ & 0.0200 & & \\
\hline \multirow{2}{*}{$C: C$} & 0.0054 & & \\
\hline & & SYNIAGALD91 & 0.0170 \\
\hline $\mathrm{HCHO}$ & 0.0020 & $\mathrm{HCHO}$ & 0.0040 \\
\hline Total VOCs & 1.9509 & Total VOCs & 1.9997 \\
\hline $\mathrm{CO}$ & 1.3000 & $\mathrm{CO}$ & 1.3000 \\
\hline \multicolumn{4}{|c|}{ Other Measured Initial Compounds } \\
\hline BVOC9109 & 0.1440 & BVOC9109 & 0.1440 \\
\hline \multicolumn{4}{|c|}{ Estimated Initial Compounds } \\
\hline HONO & 0.005 & HONO & 0.005 \\
\hline $\mathrm{CH}_{4}$ & 1.790 & $\mathrm{CH}_{4}$ & 1.790 \\
\hline $\mathrm{H}_{2}$ & 0.580 & $\mathrm{H}_{2}$ & 0.580 \\
\hline \multicolumn{4}{|c|}{ Chamber Model Parameters } \\
\hline $\mathrm{NO}_{2} \longrightarrow \mathrm{HONO}$ & $1.3 \times 10^{-3}$ & $\mathrm{NO}_{2} \longrightarrow \mathrm{HONO}$ & $1.3 \times 10^{-3}$ \\
\hline wall. $\mathrm{HNO}_{3} \longrightarrow \mathrm{NO}_{2}$ & $2.0 \times 10^{-3}$ & wall. $\mathrm{HNO}_{3} \longrightarrow \mathrm{NO}_{2}$ & $2.0 \times 10^{-3}$ \\
\hline
\end{tabular}

Three Previous Chamber Events

YYMM24.SEG SynLPG vs SynIAG run

YYMM20.SEG Night Isoprene run

YYMM19.SEG Night Isoprene run

Photochemical Studies of Alternative Fuels 

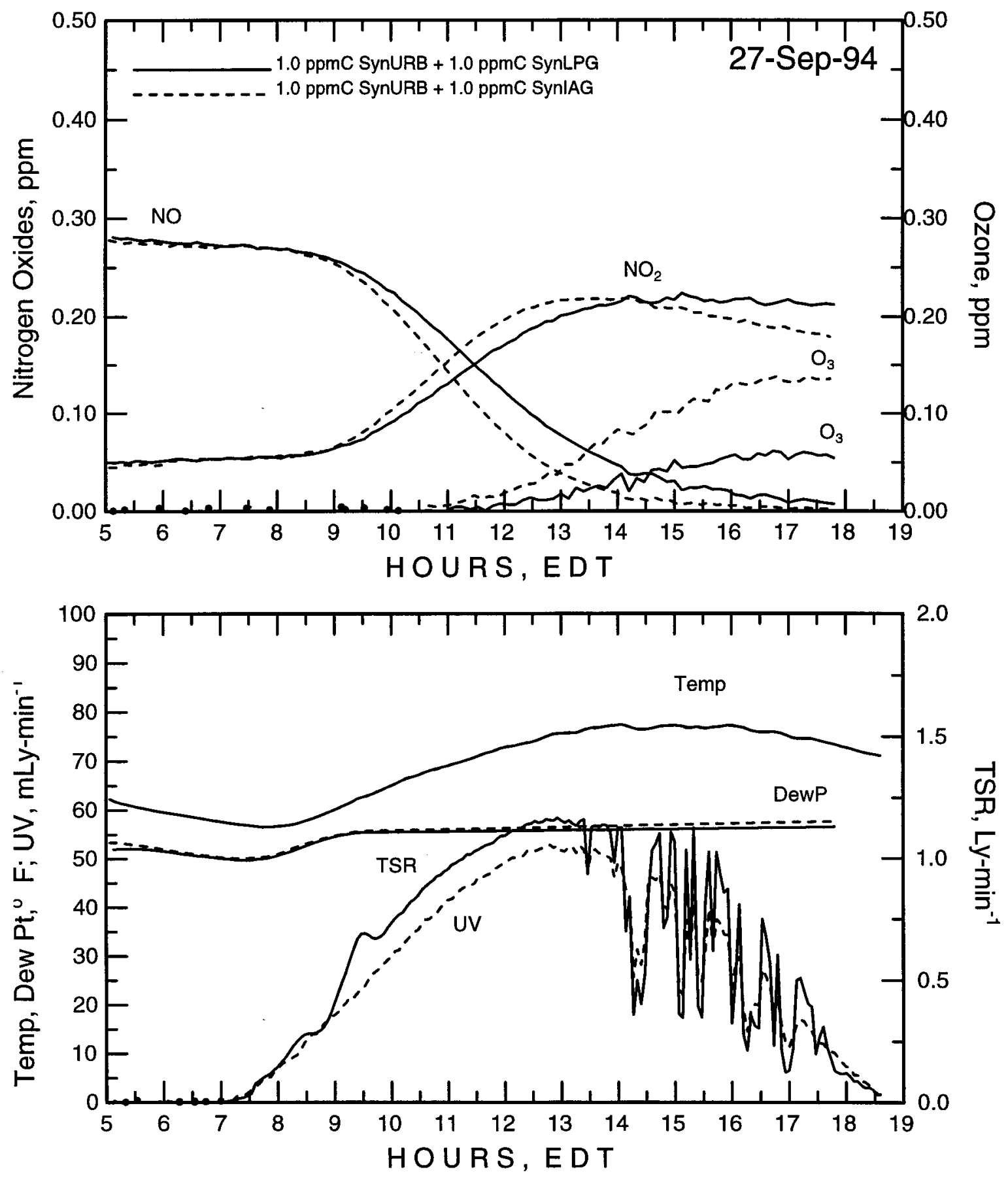

Figure 3.6: Run 7-6:1 SynUrban/SynIAG:NO versus 6:1 SynUrban/SynLPG:NO . 


\subsubsection{Block 2 - Urb/IAG versus Urb/E85}

(2) Static, 10 hour experiments $\quad$ Static, 10 hour experiments

Block 2 experiments qwere designed to compare the reactivity of SynE85E with SynIAG, with each mixture blended 50:50 with SynURB. Two sources of E85 composition data resulted in two different test mixtures being used to conduct experiments.

\section{Run 9, 4.5:1 Urb/IAG versus Urb/E85E}

AU1294 In this low HC concentration experiment (Table 3.7 and Figure 3.7), the EPA version of E85, which has less acetaldehyde ( $\mathrm{CCHO}$ ) than the NIPER version of the exhaust, is seen to be worse than IAG (is more reactive and produces more ozone) when blended 50:50 with the SynUrb mixture.

AU2394 This experiment, (Table 3.8 and Figure 3.8), is a repeat of AU1294 under clearer skies. The results, however, still indicate that E85E is worse than IAG when blended 50:50 with the SynUrb mixture.

\section{Run 10, 6:1 Urb/IAG versus Urb/E85E}

AU0494 In this intermediate HC concentration experiment (Table 3.9 and Figure 3.9) the sky was overcast all day. SynE85E is also worse than IAG under these conditions.

AU0794 This experiment was conducted with more sun than AU0494 (Table 3.10 and Figure 3.10). The results are the same as the previous experiments of this type.

AU1194 This experiment had more sun than either AU0494 or AU0794 (Table 3.11 and Figure 3.11). The results are the same as the previous experiments of this type.

\section{Run 11, 9:1 Urb/IAG versus Urb/E85E}

AU2494 Unlike the case with CNG, this 9:1 IAG versus E85 experiment (Table 3.12 and Figure 3.12) shows essentially no difference between the iwo sides. This is the normal experience for 9:1 runs, and helps show the unusually low reactivity of the CNG. 
AU2594 This experiment (Table 3.13 and Figure 3.13), duplicates the results of the AU2494 experiment.

\section{Run 12, 6:1 Urb/IAG versus Urb/E85B}

AU0394 This experiment used the NIPER E85 composition (Table 3.14 and Figure 3.14). It shows an even larger difference between IAG and E85 than does the EPA composition. This is because E85B has a larger fraction of acetaldehyde than does E85E.

AU0894 This experiment (Table 3.15 and Figure 3.15), duplicates the results of the AU0394 experiment. 
Table 3.7: Initial Conditions for AU1294

(Units are ppm or ppmC)

\begin{tabular}{|c|c|c|c|}
\hline \multicolumn{2}{|c|}{ RED SIDE } & \multicolumn{2}{|c|}{ BLUE SIDE } \\
\hline Compound & Amount & Compound & Amount \\
\hline \multicolumn{4}{|c|}{ Initial Nitrogen Oxides } \\
\hline $\mathrm{NO}_{\mathrm{x}}$ & 0.3230 & $\mathrm{NO}_{\mathrm{x}}$ & 0.3242 \\
\hline NO & 0.2630 & NO & 0.2629 \\
\hline $\mathrm{NO}_{2}$ & 0.0600 & $\mathrm{NO}_{2}$ & 0.0613 \\
\hline \multicolumn{4}{|c|}{ Initial Volatile Organic Compounds } \\
\hline SynUrban & 0.7498 & SynUrban & 0.7498 \\
\hline SYNURBGAS91 & 0.2980 & SYNURBGAS91 & 0.2980 \\
\hline SYNURBLIQ91 & 0.4432 & SYNURBLIQ91 & 0.4432 \\
\hline $\mathrm{CCHO}$ & 0.0045 & $\mathrm{CCHO}$ & 0.0045 \\
\hline $\mathrm{HCHO}$ & 0.0041 & $\mathrm{HCHO}$ & 0.0041 \\
\hline SynE85E & 0.7497 & SynlAG & 0.7494 \\
\hline SYNEPAE85GAS & 0.1401 & SYNIAGGAS91 & 0.4237 \\
\hline \multirow[t]{2}{*}{ SYNEPAE85LIQ } & 0.1201 & SYNIAGLPO92 & 0.1370 \\
\hline & & SYNIAGARO92 & 0.1730 \\
\hline $\mathrm{CCHO}$ & 0.0456 & SYNIAGALD91 & 0.0127 \\
\hline $\mathrm{HCHO}$ & 0.0045 & $\mathrm{HCHO}$ & 0.0030 \\
\hline ETOH & 0.4394 & & \\
\hline Total VOCs & 1.4995 & Total VOCs & 1.4992 \\
\hline $\mathrm{CO}$ & 1.3000 & $\mathrm{CO}$ & 1.3000 \\
\hline \multicolumn{4}{|c|}{ Other Measured Initial Compounds } \\
\hline BVOC9109 & 0.1440 & BVOC9109 & 0.1440 \\
\hline \multicolumn{4}{|c|}{ Estimated Initial Compounds } \\
\hline HONO & 0.003 & HONO & 0.003 \\
\hline $\mathrm{CH}_{4}$ & 1.790 & $\mathrm{CH}_{4}$ & 1.790 \\
\hline $\mathrm{H}_{2}$ & 0.580 & $\mathrm{H}_{2}$ & 0.580 \\
\hline \multicolumn{4}{|c|}{ Chamber Model Parameters } \\
\hline $\mathrm{NO}_{2} \longrightarrow \mathrm{HONO}$ & $\begin{array}{l}2.5 \times 10^{-3} \\
2.0 \times 10^{-3}\end{array}$ & $\mathrm{NO}_{2} \longrightarrow \mathrm{HONO}$ & $\begin{array}{l}2.5 \times 10^{-3} \\
20 \times 10^{-3}\end{array}$ \\
\hline
\end{tabular}

Three Previous Chamber Events

940811.SEG SynE85E vs SynIAG run

940809.SEG SynE85E-RCHO vs SynIAG run

940808.SEG SynE85B vs SynE85 run 

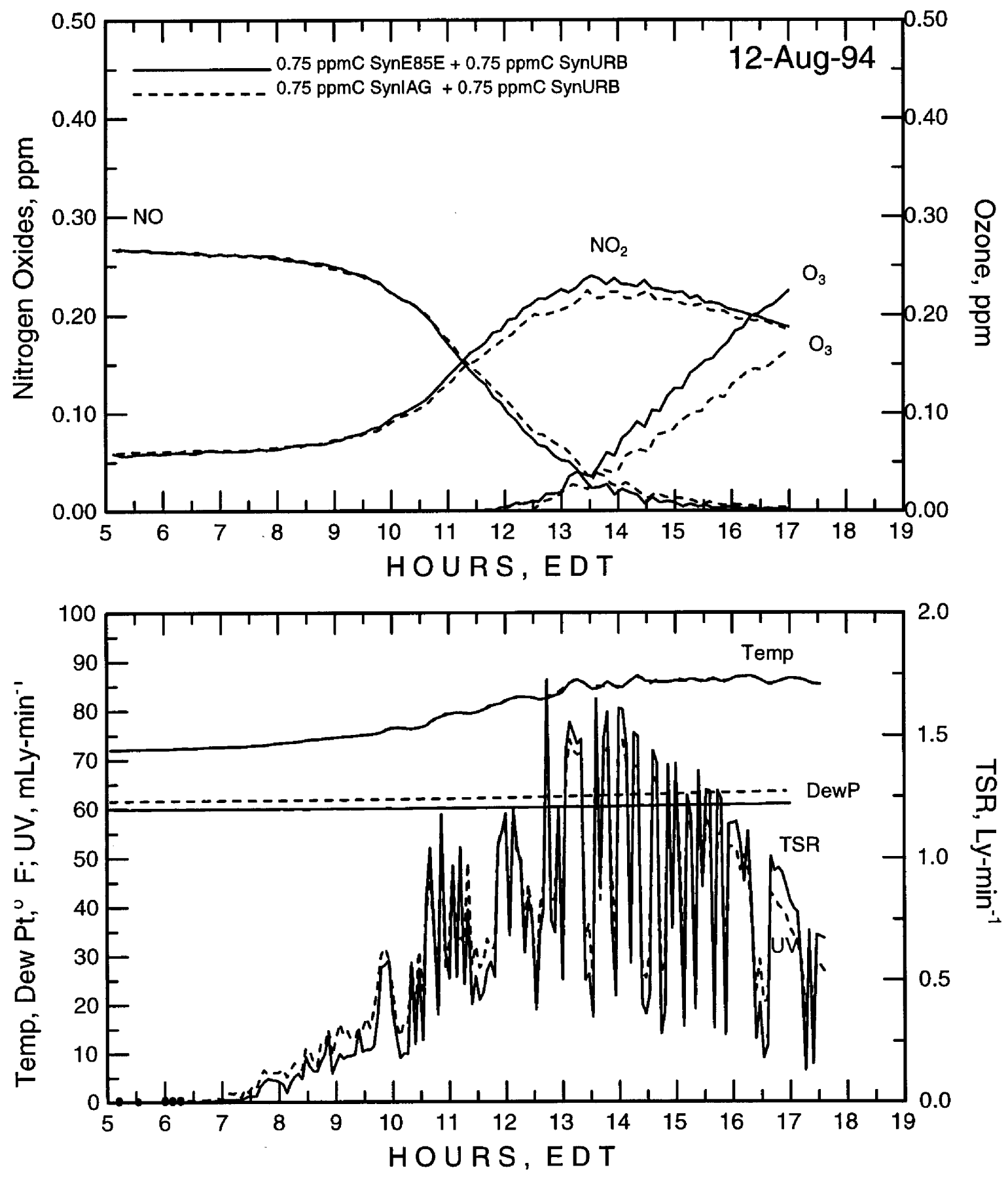

Figure 3.7: Run 9-4.5:1 SynUrban/SynIAG:NO versus 4.5:1 SynUrban/SynE85E:NO . 
Table 3.8: Initial Conditions for AU2394

(Units are ppm or ppmC)

\begin{tabular}{|c|c|c|c|}
\hline \multicolumn{2}{|c|}{ RED SIDE } & \multicolumn{2}{|c|}{ BLUE SIDE } \\
\hline Compound & Amount & Compound & Amount \\
\hline \multicolumn{4}{|c|}{ Initial Nitrogen Oxides } \\
\hline $\mathrm{NO}_{\mathrm{x}}$ & 0.3476 & $\mathrm{NO}_{\mathrm{x}}$ & 0.3453 \\
\hline NO & 0.2832 & NO & 0.2833 \\
\hline $\mathrm{NO}_{2}$ & 0.0644 & $\mathrm{NO}_{2}$ & 0.0620 \\
\hline \multicolumn{4}{|c|}{ Initial Volatile Organic Compounds } \\
\hline SynUrban & 0.7498 & SynUrban & 0.7498 \\
\hline SYNURBGAS91 & 0.2980 & SYNURBGAS91 & 0.2980 \\
\hline SYNURBLIQ91 & 0.4432 & SYNURBLIQ91 & 0.4432 \\
\hline $\mathrm{CCHO}$ & 0.0045 & $\mathrm{CCHO}$ & 0.0045 \\
\hline $\mathrm{HCHO}$ & 0.0041 & $\mathrm{HCHO}$ & 0.0041 \\
\hline SynlAG & 0.7494 & SynE85E & 0.7497 \\
\hline SYNIAGGAS91 & 0.4237 & SYNEPAE85GAS & 0.1401 \\
\hline SYNIAGLPO92 & 0.1370 & SYNEPAE85LIQ & 0.1201 \\
\hline SYNIAGARO92 & 0.1730 & & \\
\hline SYNIAGALD91 & 0.0127 & $\mathrm{CCHO}$ & 0.0456 \\
\hline \multirow[t]{2}{*}{$\mathrm{HCHO}$} & 0.0030 & $\mathrm{HCHO}$ & 0.0045 \\
\hline & & ETOH & 0.4394 \\
\hline Total VOCs & 1.4992 & Total VOCs & 1.4995 \\
\hline $\mathrm{CO}$ & 1.3000 & $\mathrm{CO}$ & 1.3000 \\
\hline \multicolumn{4}{|c|}{ Other Measured Initial Compounds } \\
\hline BVOC9109 & 0.1440 & BVOC9109 & 0.1440 \\
\hline \multicolumn{4}{|c|}{ Estimated Initial Compounds } \\
\hline HONO & 0.005 & HONO & 0.005 \\
\hline $\mathrm{CH}_{4}$ & 1.790 & $\mathrm{CH}_{4}$ & 1.790 \\
\hline $\mathrm{H}_{2}$ & 0.580 & $\mathrm{H}_{2}$ & 0.580 \\
\hline \multicolumn{4}{|c|}{ Chamber Model Parameters } \\
\hline $\mathrm{NO}_{2} \longrightarrow \mathrm{HONO}$ & $1.2 \times 10^{-3}$ & $\mathrm{NO}_{2} \longrightarrow \mathrm{HONO}$ & $1.2 \times 10^{-3}$ \\
\hline wall. $\mathrm{HNO}_{3} \longrightarrow \mathrm{NO}_{2}$ & $2.0 \times 10^{-3}$ & wall. $\mathrm{HNO}_{3} \longrightarrow \mathrm{NO}_{2}$ & $2.0 \times 10^{-3}$ \\
\hline $\begin{array}{l}\text { Three Previous Cha } \\
\text { 940812.SEG SynE85 } \\
\text { 940811.SEG SynE85 } \\
\text { 940809.SEG SynE85 }\end{array}$ & $\begin{array}{l}\text { aber Events } \\
\text { vs SynIAG } \\
\text { vs SynIAG } \\
\text {-RCHO vs S }\end{array}$ & G run & \\
\hline
\end{tabular}



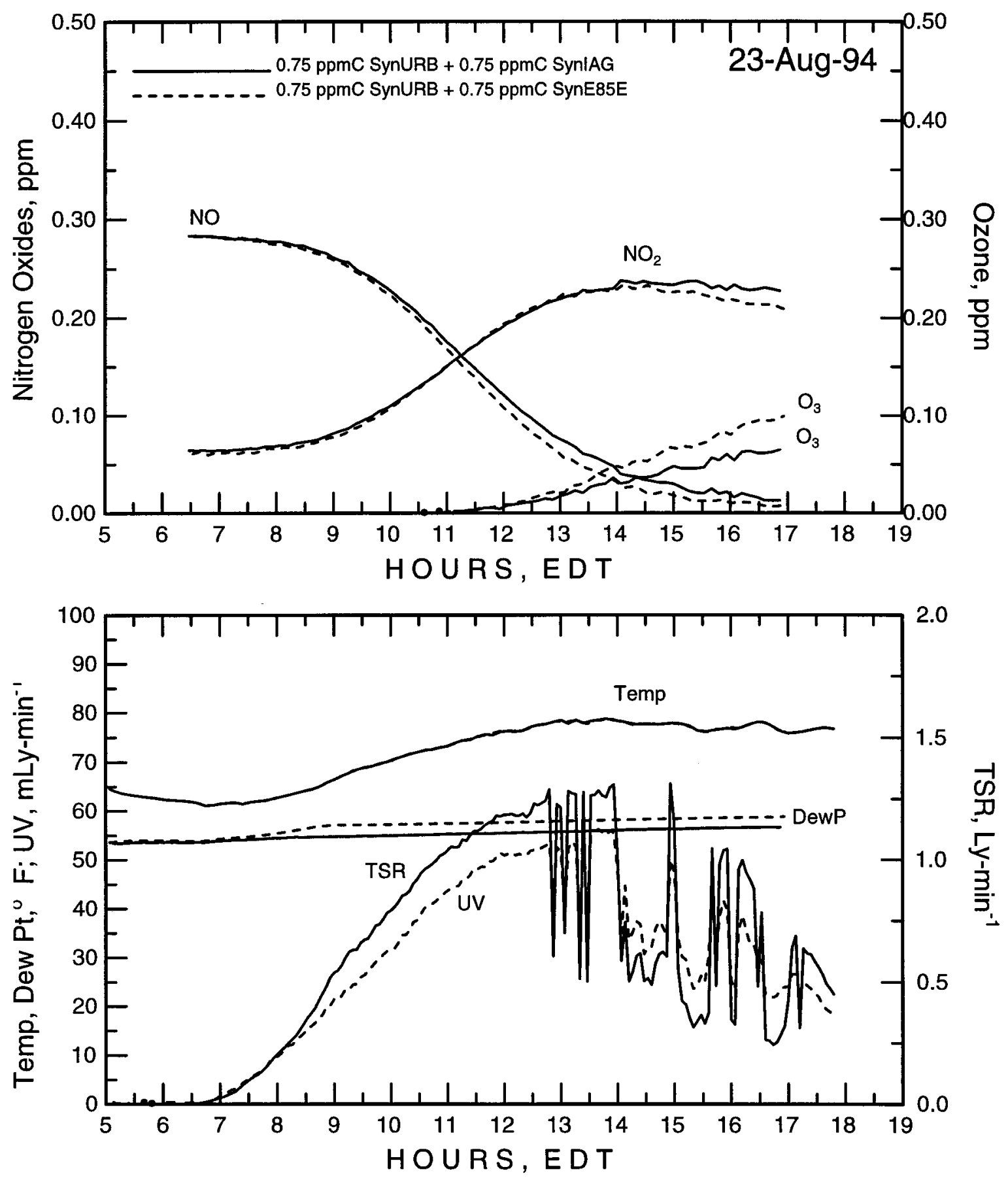

Figure 3.8: Run 9-4.5:1 SynUrban/SynIAG:NO versus 4.5:1 SynUrban/SynE85E:NOx. 
Table 3.9: Initial Conditions for AU0494

(Units are ppm or ppmC)

\begin{tabular}{|c|c|c|c|}
\hline \multicolumn{2}{|c|}{ RED SIDE } & \multicolumn{2}{|c|}{ BLUE SIDE } \\
\hline Compound & Amount & Compound & Amount \\
\hline \multicolumn{4}{|c|}{ Initial Nitrogen Oxides } \\
\hline $\mathrm{NO}_{x}$ & 0.3463 & $\mathrm{NO}_{\mathrm{x}}$ & $\mathbf{0 . 3 4 5 1}$ \\
\hline NO & 0.2806 & NO & 0.2790 \\
\hline $\mathrm{NO}_{2}$ & 0.0657 & $\mathrm{NO}_{2}$ & 0.0661 \\
\hline \multicolumn{4}{|c|}{ Initial Volatile Organic Compounds } \\
\hline SynUrban & 0.9997 & SynUrban & 0.9997 \\
\hline SYNURBGAS91 & 0.3953 & SYNURBGAS91 & 0.3953 \\
\hline SYNURBLIQ91 & 0.5909 & SYNURBLIQ91 & 0.5909 \\
\hline $\mathrm{CCHO}$ & 0.0070 & $\mathrm{CCHO}$ & 0.0070 \\
\hline $\mathrm{HCHO}$ & 0.0065 & $\mathrm{HCHO}$ & 0.0065 \\
\hline SynE85E & 0.9998 & SynIAG & 1.0000 \\
\hline SYNEPAE85GAS & 0.1869 & SYNIAGGAS91 & 0.5650 \\
\hline \multirow[t]{2}{*}{ SYNEPAE85LIQ } & 0.1601 & SYNIAGLPO92 & 0.1830 \\
\hline & & SYNIAGARO92 & 0.2310 \\
\hline $\mathrm{CCHO}$ & 0.0608 & SYNIAGALD91 & 0.0170 \\
\hline $\mathrm{HCHO}$ & 0.0061 & $\mathrm{HCHO}$ & 0.0040 \\
\hline ETOH & 0.5859 & & \\
\hline Total VOCs & 1.9995 & Total VOCs & 1.9997 \\
\hline $\mathrm{CO}$ & 1.3000 & $\mathrm{CO}$ & 1.3000 \\
\hline \multicolumn{4}{|c|}{ Other Measured Initial Compounds } \\
\hline BVOC9109 & 0.1440 & BVOC9109 & 0.1440 \\
\hline \multicolumn{4}{|c|}{ Estimated Initial Compounds } \\
\hline HONO & 0.002 & HONO & 0.002 \\
\hline $\mathrm{CH}_{4}$ & 1.790 & $\mathrm{CH}_{4}$ & 1.790 \\
\hline $\mathrm{H}_{2}$ & 0.580 & $\mathrm{H}_{2}$ & 0.580 \\
\hline \multicolumn{4}{|c|}{ Chamber Model Parameters } \\
\hline $\begin{array}{l}\mathrm{NO}_{2} \longrightarrow \mathrm{HONO} \\
\text { wall. } \mathrm{HNO}_{3} \longrightarrow \mathrm{NO}_{2}\end{array}$ & $\begin{array}{l}2.5 \times 10^{-3} \\
2.0 \times 10^{-3}\end{array}$ & $\begin{array}{l}\mathrm{NO}_{2} \longrightarrow \mathrm{HONO} \\
\text { wall. } \mathrm{HNO}_{3} \longrightarrow \mathrm{NO}_{2}\end{array}$ & $\begin{array}{l}2.5 \times 10^{-3} \\
2.0 \times 10^{-3}\end{array}$ \\
\hline
\end{tabular}

Three Previous Chamber Events

940803.SEG SynE85B vs SynIAG run

940801.SEG Delta Toluene run

940725.SEG SynCNG vs SynIAG run 

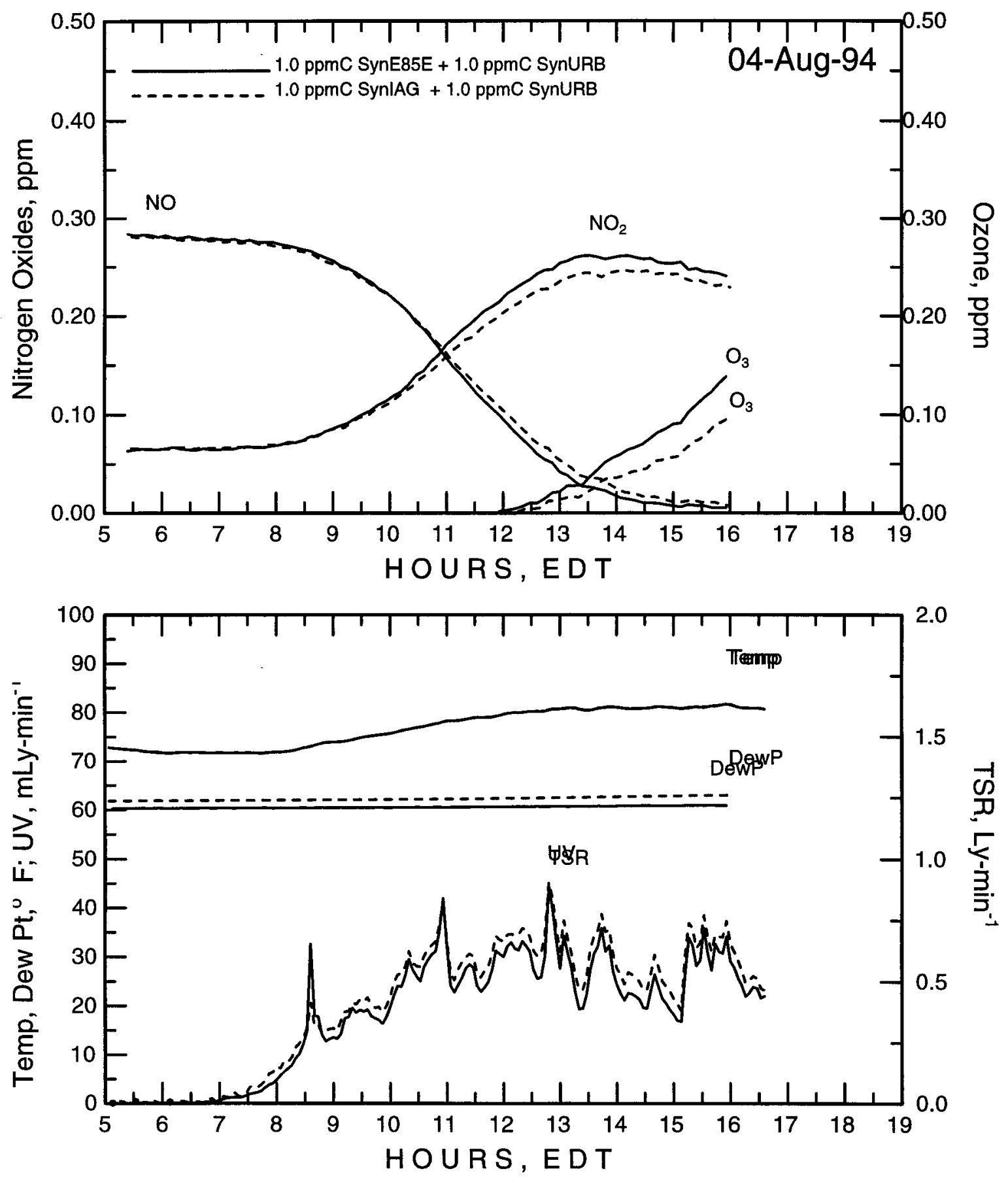

Figure 3.9: Run 10-6:1 SynUrban/SynIAG:NO versus 6:1 SynUrban/SynE85E:NO . 
Table 3.10: Initial Conditions for AU0794

(Units are ppm or ppmC)

\begin{tabular}{|c|c|c|c|}
\hline \multicolumn{2}{|c|}{ RED SIDE } & \multicolumn{2}{|c|}{ BLUE SIDE } \\
\hline Compound & Amount & Compound & Amount \\
\hline \multicolumn{4}{|c|}{ Initial Nitrogen Oxides } \\
\hline $\mathrm{NO}_{x}$ & 0.3200 & $\mathrm{NO}_{x}$ & 0.3195 \\
\hline NO & 0.2547 & NO & 0.2568 \\
\hline $\mathrm{NO}_{2}$ & 0.0653 & $\mathrm{NO}_{2}$ & 0.0627 \\
\hline \multicolumn{4}{|c|}{ Initial Volatile Organic Compounds } \\
\hline SynUrban & 0.9997 & SynUrban & 0.9997 \\
\hline SYNURBGAS91 & 0.3953 & SYNURBGAS91 & 0.3953 \\
\hline SYNURBLIQ91 & 0.5909 & SYNURBLIQ91 & 0.5909 \\
\hline $\mathrm{CCHO}$ & 0.0070 & $\mathrm{CCHO}$ & 0.0070 \\
\hline $\mathrm{HCHO}$ & 0.0065 & $\mathrm{HCHO}$ & 0.0065 \\
\hline SynE85E & 0.9998 & SynIAG & 1.0000 \\
\hline SYNEPAE85GAS & 0.1869 & SYNIAGGAS91 & 0.5650 \\
\hline \multirow[t]{2}{*}{ SYNEPAE85LIQ } & 0.1601 & SYNIAGLPO92 & 0.1830 \\
\hline & & SYNIAGARO92 & 0.2310 \\
\hline $\mathrm{CCHO}$ & 0.0608 & SYNIAGALD91 & 0.0170 \\
\hline $\mathrm{HCHO}$ & 0.0061 & $\mathrm{HCHO}$ & 0.0040 \\
\hline $\mathrm{ETOH}$ & 0.5859 & & \\
\hline Total VOCs & 1.9995 & Total VOCs & 1.9997 \\
\hline $\mathrm{CO}$ & 1.3000 & $\mathrm{CO}$ & 1.3000 \\
\hline \multicolumn{4}{|c|}{ Other Measured Initial Compounds } \\
\hline BVOC9109 & 0.1440 & BVOC9109 & 0.1440 \\
\hline \multicolumn{4}{|c|}{ Estimated Initial Compounds } \\
\hline HONO & 0.002 & HONO & 0.002 \\
\hline $\mathrm{CH}_{4}$ & 1.790 & $\mathrm{CH}_{4}$ & 1.790 \\
\hline $\mathrm{H}_{2}$ & 0.580 & $\mathrm{H}_{2}$ & 0.580 \\
\hline \multicolumn{4}{|c|}{ Chamber Model Parameters } \\
\hline $\begin{array}{l}\mathrm{NO}_{2} \longrightarrow \mathrm{HONO} \\
\text { wall. } \mathrm{HNO}_{3} \longrightarrow \mathrm{NO}_{2}\end{array}$ & $\begin{array}{l}1.2 \times 10^{-3} \\
2.0 \times 10^{-3}\end{array}$ & $\begin{array}{l}\mathrm{NO}_{2} \longrightarrow \mathrm{HONO} \\
\text { wall. } \mathrm{HNO}_{3} \longrightarrow \mathrm{NO}_{2}\end{array}$ & $\begin{array}{l}1.2 \times 10^{-3} \\
2.0 \times 10^{-3}\end{array}$ \\
\hline
\end{tabular}

Three Previous Chamber Events

940804.SEG SynE85E vs SynIAG run

940803.SEG SynE85B vs SynIAG run

940801.SEG Delta Toluene run 

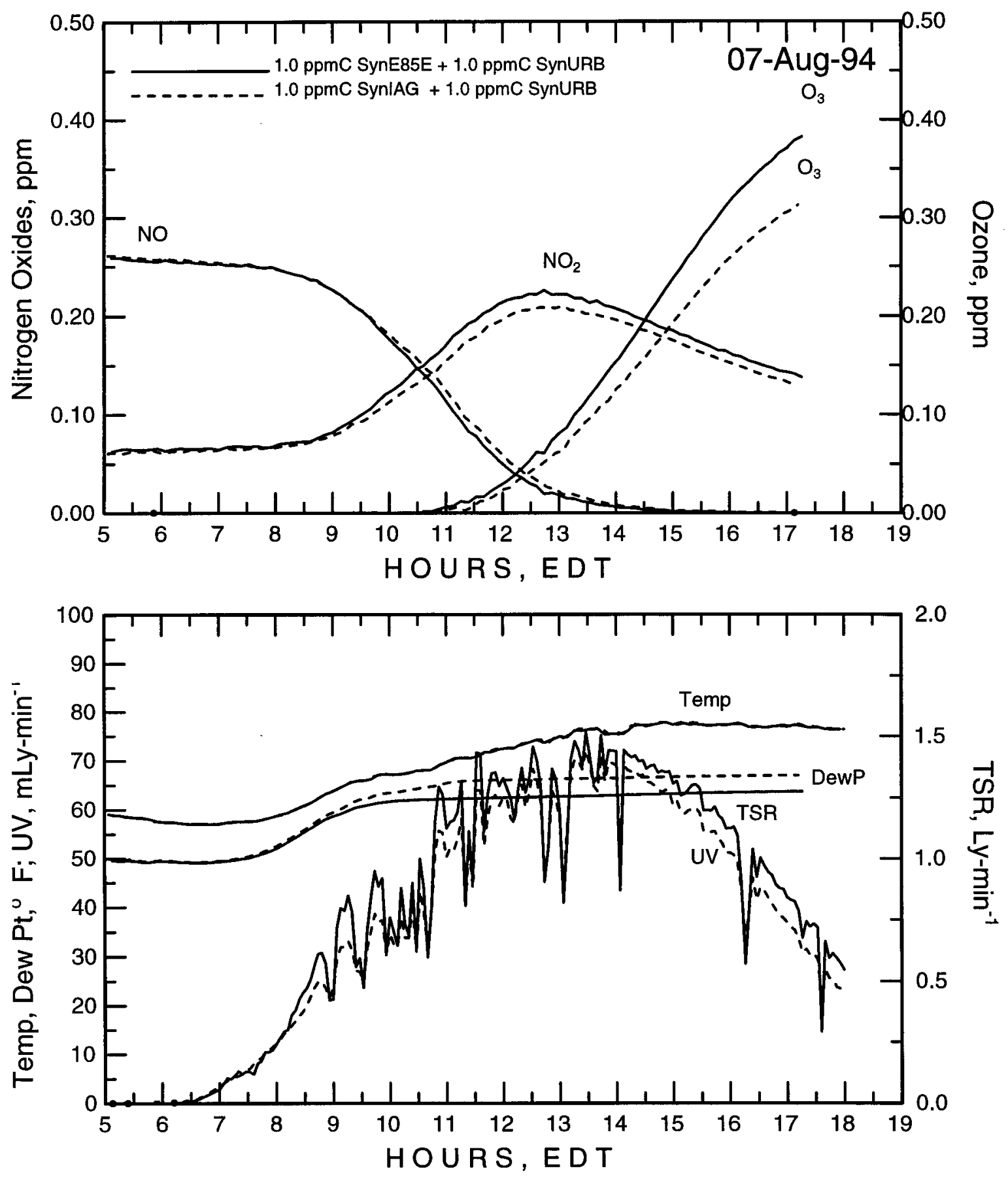

Figure 3.10: Run 10—6:1 SynUrban/SynIAG:NO versus 6:1 SynUrban/SynE85E: $\mathrm{NO}_{\mathrm{x}}$. 
Table 3.11: Initial Conditions for AU1194

(Units are ppm or ppmC)

\begin{tabular}{|c|c|c|c|}
\hline \multicolumn{2}{|c|}{ RED SIDE } & \multicolumn{2}{|c|}{ BLUE SIDE } \\
\hline Compound & Amount & Compound & Amount \\
\hline \multicolumn{4}{|c|}{ Initial Nitrogen Oxides } \\
\hline $\mathrm{NO}_{x}$ & 0.3286 & $\mathrm{NO}_{x}$ & 0.3287 \\
\hline NO & 0.2719 & NO & 0.2744 \\
\hline $\mathrm{NO}_{2}$ & 0.0567 & $\mathrm{NO}_{2}$ & 0.0543 \\
\hline \multicolumn{4}{|c|}{ Initial Volatile Organic Compounds } \\
\hline SynUrban & 0.9997 & SynUrban & 0.9997 \\
\hline SYNURBGAS91 & 0.3953 & SYNURBGAS91 & 0.3953 \\
\hline SYNURBLIQ91. & 0.5909 & SYNURBLIQ91 & 0.5909 \\
\hline $\mathrm{CCHO}$ & 0.0070 & $\mathrm{CCHO}$ & 0.0070 \\
\hline $\mathrm{HCHO}$ & 0.0065 & $\mathrm{HCHO}$ & 0.0065 \\
\hline SynE85E & 0.9998 & SynIAG & 1.0000 \\
\hline SYNEPAE85GAS & 0.1869 & SYNIAGGAS91 & 0.5650 \\
\hline \multirow[t]{2}{*}{ SYNEPAE85LIQ } & 0.1601 & SYNIAGLPO92 & 0.1830 \\
\hline & & SYNIAGARO92 & 0.2310 \\
\hline $\mathrm{CCHO}$ & 0.0608 & SYNIAGALD91 & 0.0170 \\
\hline $\mathrm{HCHO}$ & 0.0061 & $\mathrm{HCHO}$ & 0.0040 \\
\hline ETOH & 0.5859 & & \\
\hline Total VOCs & 1.9995 & Total VOCs & 1.9997 \\
\hline $\mathrm{CO}$ & 1.3000 & $\mathrm{CO}$ & 1.3000 \\
\hline \multicolumn{4}{|c|}{ Other Measured Initial Compounds } \\
\hline BVOC9109 & 0.1440 & BVOC9109 & 0.1440 \\
\hline \multicolumn{4}{|c|}{ Estimated Initial Compounds } \\
\hline HONO & 0.004 & HONO & 0.004 \\
\hline $\mathrm{CH}_{4}$ & 1.790 & $\mathrm{CH}_{4}$ & 1.790 \\
\hline $\mathrm{H}_{2}$ & 0.580 & $\mathrm{H}_{2}$ & 0.580 \\
\hline \multicolumn{4}{|c|}{ Chamber Model Parameters } \\
\hline $\begin{array}{l}\mathrm{NO}_{2} \longrightarrow \mathrm{HONO} \\
\text { wall. } \mathrm{HNO}_{3} \longrightarrow \mathrm{NO}_{2}\end{array}$ & $\begin{array}{l}2.1 \times 10^{-3} \\
2.0 \times 10^{-3}\end{array}$ & $\begin{array}{l}\mathrm{NO}_{2} \longrightarrow \mathrm{HONO} \\
\text { wall } . \mathrm{HNO}_{3} \longrightarrow \mathrm{NO}_{2}\end{array}$ & $\begin{array}{l}2.1 \times 10^{-3} \\
2.0 \times 10^{-3}\end{array}$ \\
\hline
\end{tabular}

Three Previous Chamber Events

940809.SEG SynE85E-RCHO vs SynIAG run

949808.SEG SynE85B vs SynIAG run

940807.SEG SynE85E vs SynIAG run 

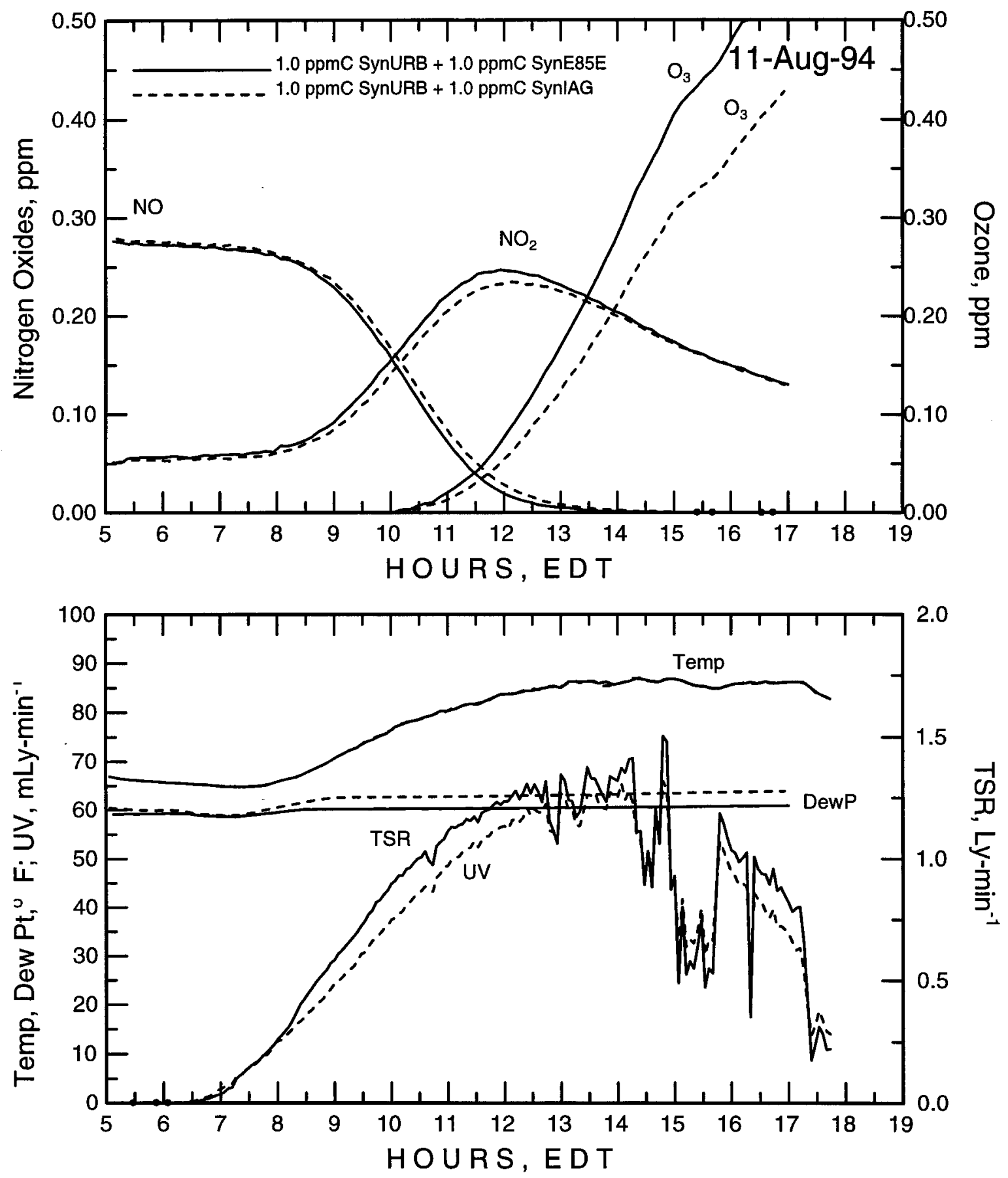

Figure 3.11: Run 10—6:1 SynUrban/SynIAG:NO versus 6:1 SynUrban/SynE85E:NO . 
Table 3.12: Initial Conditions for AU2494

(Units are ppm or ppmC)

\begin{tabular}{|c|c|c|c|}
\hline \multicolumn{2}{|c|}{ RED SIDE } & \multicolumn{2}{|c|}{ BLUE SIDE } \\
\hline Compound & Amount & Compound & Amount \\
\hline \multicolumn{4}{|c|}{ Initial Nitrogen Oxides } \\
\hline $\mathrm{NO}_{x}$ & 0.3227 & $\mathrm{NO}_{\mathrm{x}}$ & 0.3200 \\
\hline NO & 0.2633 & NO & 0.2659 \\
\hline $\mathrm{NO}_{2}$ & 0.0594 & $\mathrm{NO}_{2}$ & 0.0541 \\
\hline \multicolumn{4}{|c|}{ Initial Volatile Organic Compounds } \\
\hline SynUrban & 1.4999 & SynUrban & 1.4999 \\
\hline SYNURBGAS91 & 0.5871 & SYNURBGAS91 & 0.5871 \\
\hline SYNURBLIQ91 & 0.8865 & SYNURBLIQ91 & 0.8865 \\
\hline $\mathrm{CCHO}$ & 0.0137 & $\mathrm{CCHO}$ & 0.0137 \\
\hline $\mathrm{HCHO}$ & 0.0126 & $\mathrm{HCHO}$ & 0.0126 \\
\hline SynIAG & 1.5010 & SynE85E & 1.4995 \\
\hline SYNIAGGAS91 & 0.8475 & SYNEPAE85GAS & 0.2802 \\
\hline SYNIAGLPO92 & 0.2750 & SYNEPAE85LIQ & 0.2402 \\
\hline SYNIAGARO92 & 0.3470 & & \\
\hline SYNIAGALD91 & 0.0255 & $\mathrm{CCHO}$ & 0.0912 \\
\hline \multirow[t]{2}{*}{$\mathrm{HCHO}$} & 0.0060 & $\mathrm{HCHO}$ & 0.0090 \\
\hline & & ETOH & 0.8788 \\
\hline Total VOCs & 3.0009 & Total VOCs & 2.9994 \\
\hline $\mathrm{CO}$ & 1.3000 & $\mathrm{CO}$ & 1.3000 \\
\hline \multicolumn{4}{|c|}{ Other Measured Initial Compounds } \\
\hline BVOC9109 & 0.1440 & BVOC9109 & 0.1440 \\
\hline \multicolumn{4}{|c|}{ Estimated Initial Compounds } \\
\hline HONO & 0.005 & HONO & 0.005 \\
\hline $\mathrm{CH}_{4}$ & 1.790 & $\mathrm{CH}_{4}$ & 1.790 \\
\hline $\mathrm{H}_{2}$ & 0.580 & $\mathrm{H}_{2}$ & 0.580 \\
\hline \multicolumn{4}{|c|}{ Chamber Model Parameters } \\
\hline $\begin{array}{l}\mathrm{NO}_{2} \longrightarrow \mathrm{HONO} \\
\text { wall. } \mathrm{HNO}_{3} \longrightarrow \mathrm{NO}_{2}\end{array}$ & $\begin{array}{l}2.5 \times 10^{-3} \\
2.0 \times 10^{-3}\end{array}$ & $\begin{array}{l}\mathrm{NO}_{2} \longrightarrow \mathrm{HONO} \\
\text { wall. } \mathrm{HNO}_{3} \longrightarrow \mathrm{NO}_{2}\end{array}$ & $\begin{array}{l}2.5 \times 10^{-3} \\
2.0 \times 10^{-3}\end{array}$ \\
\hline
\end{tabular}

Three Previous Chamber Events

940823.SEG SynE85E vs SynIAG run 940812.SEG SynE85E vs SynIAG run 940811.SEG SynE85E vs SynIAG run 

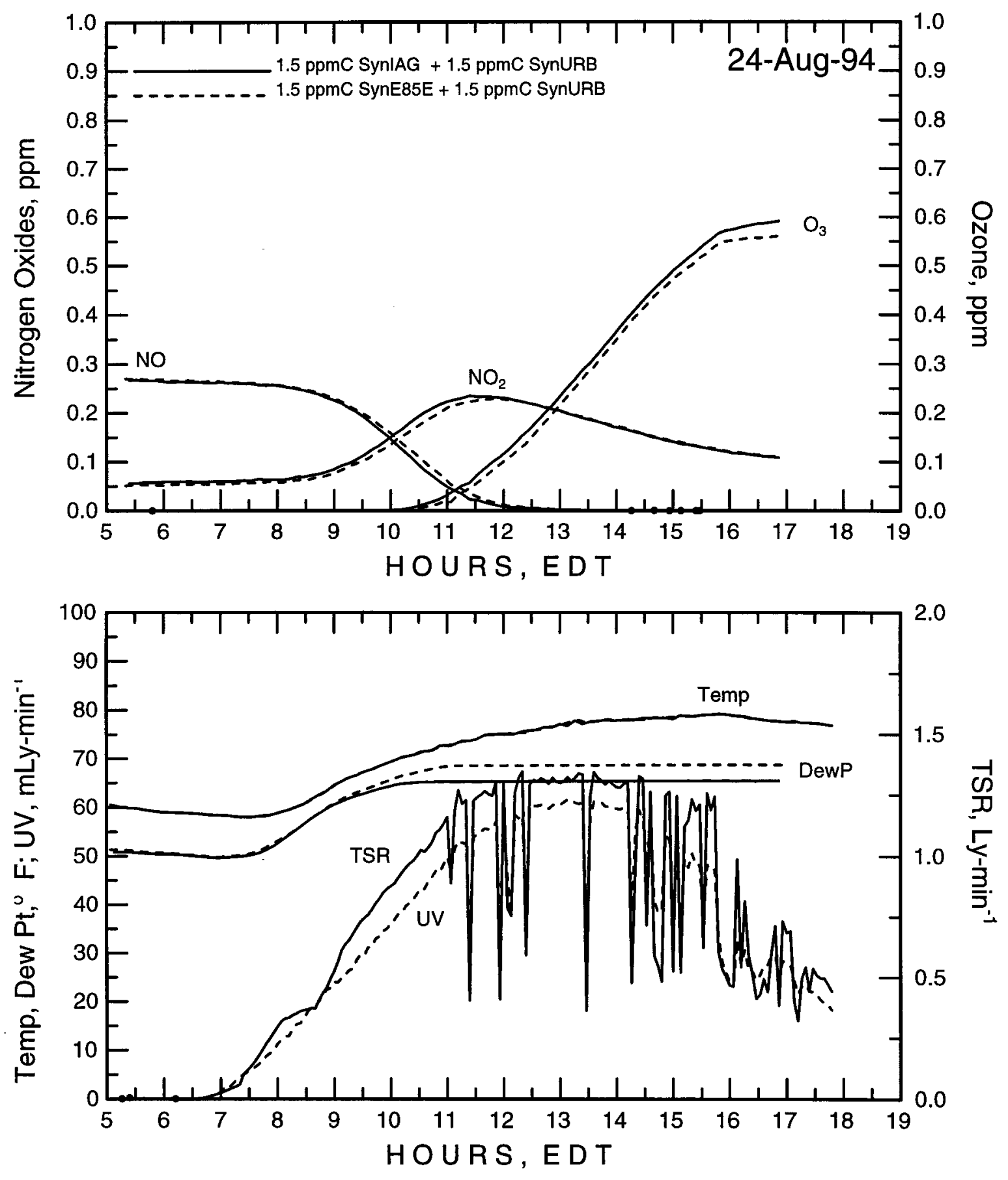

Figure 3.12: Run 11—9:1 SynUrban/SynIAG:NO versus 9:1 SynUrban/SynE85E:NOx. 
Table 3.13: Initial Conditions for AU2594

(Units are ppm or ppmC)

\begin{tabular}{|c|c|c|c|}
\hline \multicolumn{2}{|c|}{ RED SIDE } & \multicolumn{2}{|c|}{ BLUE SIDE } \\
\hline Compound & Amount & Compound & Amount \\
\hline \multicolumn{4}{|c|}{ Initial Nitrogen Oxides } \\
\hline $\mathrm{NO}_{x}$ & 0.3289 & $\mathrm{NO}_{\mathrm{x}}$ & 0.3267 \\
\hline NO & 0.2681 & NO & 0.2674 \\
\hline $\mathrm{NO}_{2}$ & 0.0608 & $\mathrm{NO}_{2}$ & 0.0593 \\
\hline \multicolumn{4}{|c|}{ Initial Volatile Organic Compounds } \\
\hline SynUrban & 1.4999 & SynUrban & 1.4999 \\
\hline SYNURBGAS91 & 0.5871 & SYNURBGAS91 & 0.5871 \\
\hline SYNURBLIQ91 & 0.8865 & SYNURBLIQ91 & 0.8865 \\
\hline $\mathrm{CCHO}$ & 0.0137 & $\mathrm{CCHO}$ & 0.0137 \\
\hline $\mathrm{HCHO}$ & 0.0126 & $\mathrm{HCHO}$ & 0.0126 \\
\hline SynlAG & 1.5010 & SynE85E & 1.4995 \\
\hline SYNIAGGAS91 & 0.8475 & SYNEPAE85GAS & 0.2802 \\
\hline SYNIAGLPO92 & 0.2750 & SYNEPAE85LIQ & 0.2402 \\
\hline SYNIAGARO92 & 0.3470 & & \\
\hline SYNIAGALD91 & 0.0255 & $\mathrm{CCHO}$ & 0.0912 \\
\hline \multirow[t]{2}{*}{$\mathrm{HCHO}$} & 0.0060 & $\mathrm{HCHO}$ & 0.0090 \\
\hline & & ETOH & 0.8789 \\
\hline Total VOCs & 3.0009 & Total VOCs & 2.9994 \\
\hline $\mathrm{CO}$ & 1.3000 & $\mathrm{CO}$ & 1.3000 \\
\hline \multicolumn{4}{|c|}{ Other Measured Initial Compounds } \\
\hline BVOC9109 & 0.1440 & BVOC9109 & 0.1440 \\
\hline \multicolumn{4}{|c|}{ Estimated Initial Compounds } \\
\hline HONO & 0.001 & HONO & 0.001 \\
\hline $\mathrm{CH}_{4}$ & 1.790 & $\mathrm{CH}_{4}$ & 1.790 \\
\hline $\mathrm{H}_{2}$ & 0.580 & $\mathrm{H}_{2}$ & 0.580 \\
\hline \multicolumn{4}{|c|}{ Chamber Model Parameters } \\
\hline $\mathrm{NO}_{2} \longrightarrow \mathrm{HONO}$ & $2.9 \times 10^{-3}$ & $\mathrm{NO}_{2} \longrightarrow \mathrm{HONO}$ & $2.9 \times 10^{-3}$ \\
\hline wall. $\mathrm{HNO}_{3} \longrightarrow \mathrm{NO}_{2}$ & $2.0 \times 10^{-3}$ & wall. $\mathrm{HNO}_{3} \longrightarrow \mathrm{NO}_{2}$ & $2.0 \times 10^{-3}$ \\
\hline
\end{tabular}

Three Previous Chamber Events

940824.SEG SynE85E vs SynIAG run

940823.SEG SynE85E vs SynIAG run

940812.SEG SynE85E vs SynIAG run 

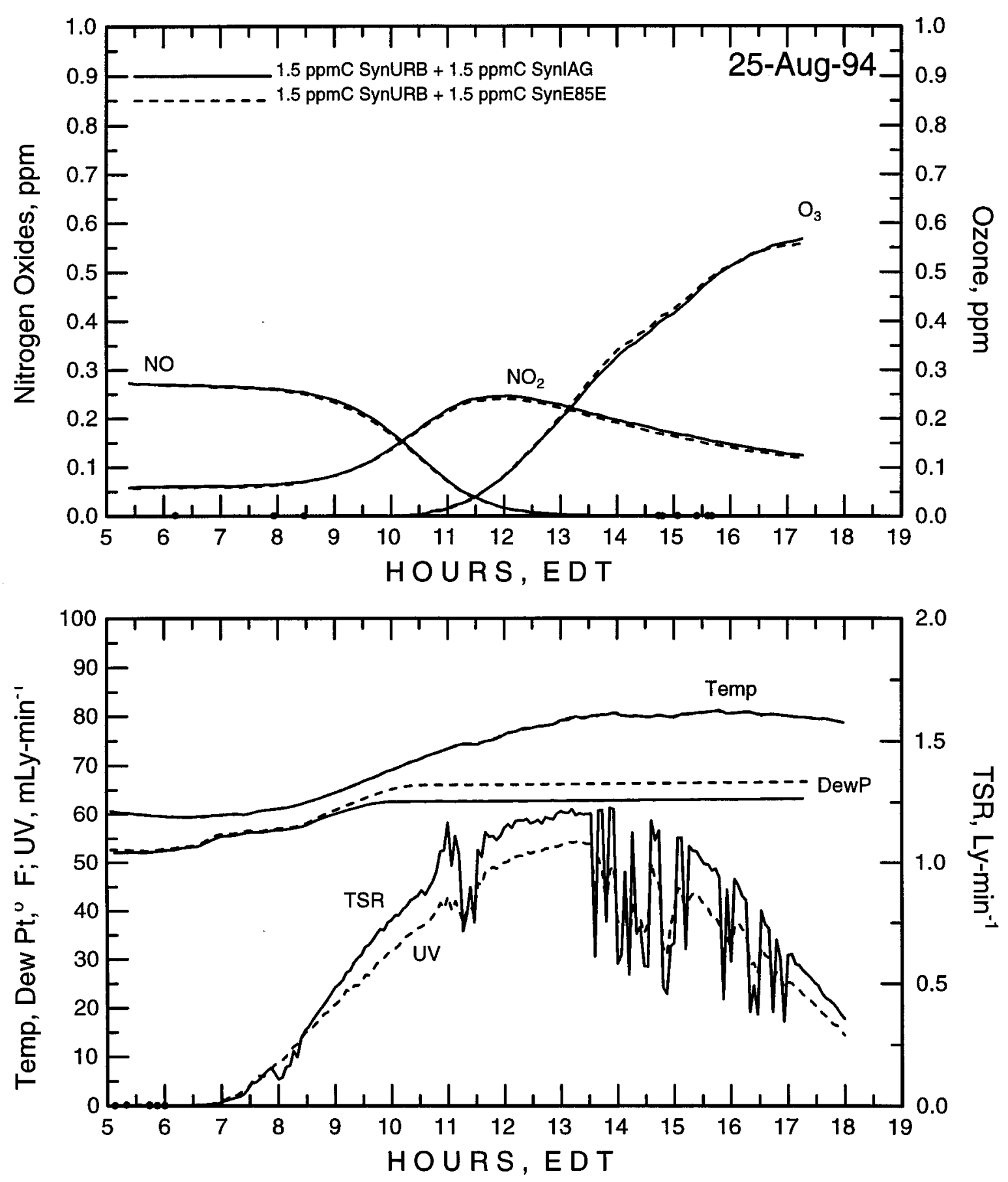

Figure 3.13: Run 11-9:1 SynUrban/SynIAG:NO versus 9:1 SynUrban/SynE85E:NOx. 
Table 3.14: Initial Conditions for AU0394

(Units are ppm or ppmC)

\begin{tabular}{|c|c|c|c|}
\hline \multicolumn{2}{|c|}{ RED SIDE } & \multicolumn{2}{|c|}{ BLUE SIDE } \\
\hline Compound & Amount & Compound & Amount \\
\hline \multicolumn{4}{|c|}{ Initial Nitrogen Oxides } \\
\hline $\mathrm{NO}_{\mathrm{x}}$ & 0.3332 & $\mathrm{NO}_{\mathrm{x}}$ & 0.3321 \\
\hline No & 0.2786 & NO & 0.2843 \\
\hline $\mathrm{NO}_{2}$ & 0.0546 & $\mathrm{NO}_{2}$ & 0.0478 \\
\hline
\end{tabular}

\section{Initial Volatile Organic Compounds}

\begin{tabular}{|c|c|c|c|}
\hline SynUrban & 0.9997 & SynUrban & 0.9997 \\
\hline SYNURBGAS91 & 0.3953 & SYNURBGAS91 & 0.3953 \\
\hline SYNURBLIQ91 & 0.5909 & SYNURBLIQ91 & 0.5909 \\
\hline $\mathrm{CCHO}$ & 0.0070 & $\mathrm{CCHO}$ & 0.0070 \\
\hline $\mathrm{HCHO}$ & 0.0065 & $\mathrm{HCHO}$ & 0.0065 \\
\hline SynE85E & 1.0134 & SynlAG & 1.0000 \\
\hline SYNE85BARTGAS & 0.2417 & SYNIAGGAS91 & 0.5650 \\
\hline \multirow[t]{2}{*}{ SYNE85BARTLIQ } & 0.0955 & SYNIAGLPO92 & 0.1830 \\
\hline & & SYNIAGARO92 & 0.2310 \\
\hline $\mathrm{CCHO}$ & 0.1148 & SYNIAGALD91 & 0.0170 \\
\hline $\mathrm{HCHO}$ & 0.0136 & $\mathrm{HCHO}$ & 0.0040 \\
\hline ETOH & 0.5478 & & \\
\hline Total VOCs & 2.0131 & Total VOCs & 1.9997 \\
\hline $\mathrm{CO}$ & 1.3000 & $\mathrm{CO}$ & 1.3000 \\
\hline \multicolumn{4}{|c|}{ Other Measured Initial Compounds } \\
\hline BVOC9109 & 0.1440 & BVOC9109 & 0.1440 \\
\hline \multicolumn{4}{|c|}{ Estimated Initial Compounds } \\
\hline HONO & 0.002 & HONO & 0.002 \\
\hline $\mathrm{CH}_{4}$ & 1.790 & $\mathrm{CH}_{4}$ & 1.790 \\
\hline $\mathrm{H}_{2}$ & 0.580 & $\mathrm{H}_{2}$ & 0.580 \\
\hline \multicolumn{4}{|c|}{ Chamber Model Parameters } \\
\hline $\begin{array}{l}\mathrm{NO}_{2} \longrightarrow \mathrm{HONO} \\
\text { wall. } \mathrm{HNO}_{3} \longrightarrow \mathrm{NO}_{2}\end{array}$ & $\begin{array}{l}2.5 \times 10^{-3} \\
2.0 \times 10^{-3}\end{array}$ & $\begin{array}{l}\mathrm{NO}_{2} \longrightarrow \mathrm{HONO} \\
\text { wall. } \mathrm{HNO}_{3} \longrightarrow \mathrm{NO}_{2}\end{array}$ & $\begin{array}{l}2.5 \times 10^{-3} \\
2.0 \times 10^{-3}\end{array}$ \\
\hline
\end{tabular}

Three Previous Chamber Events 940801.SEG Delta Toluene run 940725.SEG SynCNG vs SynIAG run 940720.SEG Delta Toluene run 

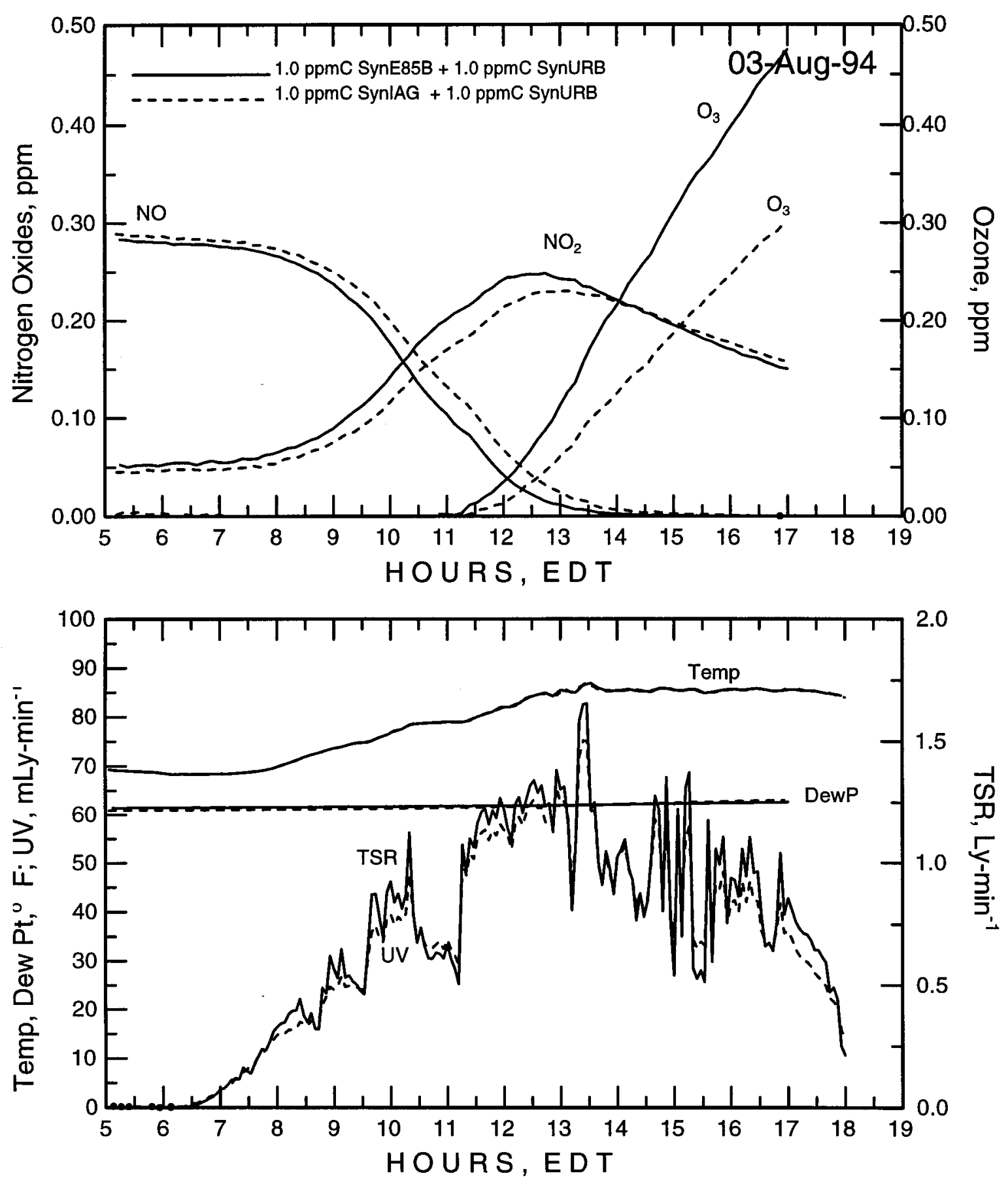

Figure 3.14: Run 12-6:1 SynUrban/SynIAG:NOx versus 6:1 SynUrban/SynE85B:NOx. 
Table 3.15: Initial Conditions for AU0894

(Units are ppm or ppmC)

\begin{tabular}{|c|c|c|c|}
\hline \multicolumn{2}{|c|}{ RED SIDE } & \multicolumn{2}{|c|}{ BLUE SIDE } \\
\hline Compound & Amount & Compound & Amount \\
\hline \multicolumn{4}{|c|}{ Initial Nitrogen Oxides } \\
\hline $\mathrm{NO}_{x}$ & 0.2613 & $\mathrm{NO}_{\mathrm{x}}$ & 0.3229 \\
\hline NO & 0.3262 & NO & 0.2642 \\
\hline $\mathrm{NO}_{2}$ & 0.0649 & $\mathrm{NO}_{2}$ & 0.0587 \\
\hline \multicolumn{4}{|c|}{ Initial Volatile Organic Compounds } \\
\hline SynUrban & 0.9997 & SynUrban & 0.9997 \\
\hline SYNURBGAS91 & 0.3953 & SYNURBGAS91 & 0.3953 \\
\hline SYNURBLIQ91 & 0.5909 & SYNURBLIQ91 & 0.5909 \\
\hline $\mathrm{CCHO}$ & 0.0070 & $\mathrm{CCHO}$ & 0.0070 \\
\hline $\mathrm{HCHO}$ & 0.0065 & $\mathrm{HCHO}$ & 0.0065 \\
\hline SynE85B & 1.0134 & SynIAG & 1.0000 \\
\hline SYNE85BARTGAS & 0.2417 & SYNIAGGAS91 & 0.5650 \\
\hline \multirow[t]{2}{*}{ SYNE85BARTLIQ } & 0.0955 & SYNIAGLPO92 & 0.1830 \\
\hline & & SYNIAGARO92 & 0.2310 \\
\hline $\mathrm{CCHO}$ & 0.1148 & SYNIAGALD91 & 0.0170 \\
\hline $\mathrm{HCHO}$ & 0.0136 & $\mathrm{HCHO}$ & 0.0040 \\
\hline ETOH & 0.5478 & & \\
\hline Total VOCs & 2.0131 & Total VOCs & 1.9997 \\
\hline $\mathrm{CO}$ & 1.3000 & $\mathrm{CO}$ & 1.3000 \\
\hline \multicolumn{4}{|c|}{ Other Measured Initial Compounds } \\
\hline BVOC9109 & 0.1440 & BVOC9109 & 0.1440 \\
\hline \multicolumn{4}{|c|}{ Estimated Initial Compounds } \\
\hline HONO & 0.004 & HONO & 0.004 \\
\hline $\mathrm{CH}_{4}$ & 1.790 & $\mathrm{CH}_{4}$ & 1.790 \\
\hline $\mathrm{H}_{2}$ & 0.580 & $\mathrm{H}_{2}$ & 0.580 \\
\hline \multicolumn{4}{|c|}{ Chamber Model Parameters } \\
\hline $\mathrm{NO}_{2} \rightarrow \mathrm{HONO}$ & $1.3 \times 10^{-3}$ & $\mathrm{NO}_{2} \longrightarrow \mathrm{HONO}$ & $1.3 \times 10^{-3}$ \\
\hline wall. $\mathrm{HNO}_{3} \longrightarrow \mathrm{NO}_{2}$ & $2.0 \times 10^{-3}$ & wall. $\mathrm{HNO}_{3} \longrightarrow \mathrm{NO}_{2}$ & $2.0 \times 10^{-3}$ \\
\hline
\end{tabular}

Three Previous Chamber Events

940807.SEG SynE85E vs SynIAG run

940804.SEG SynE85E vs SynIAG run

940803.SEG SynE85B vs SynIAG run 

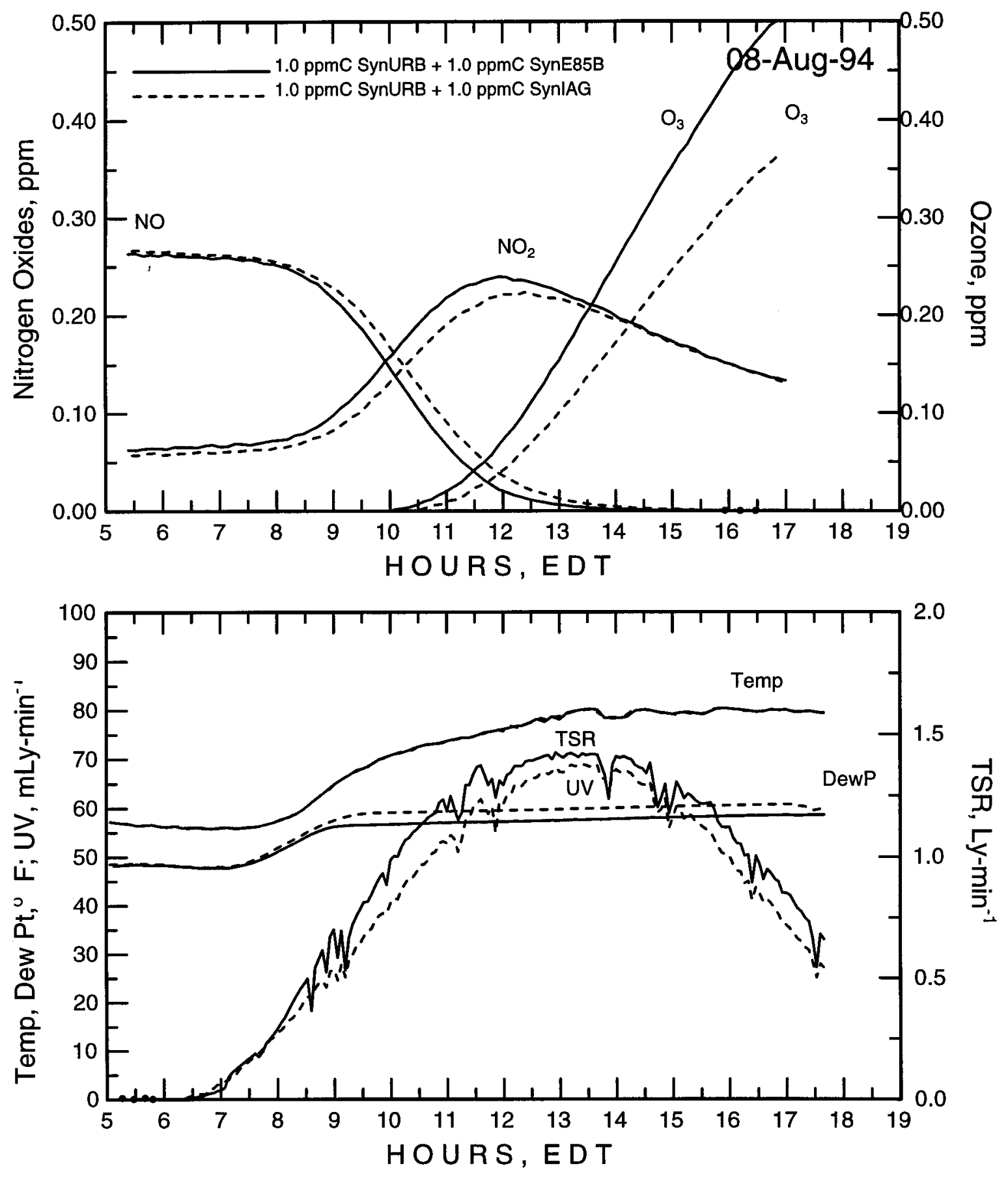

Figure 3.15: Run 12—6:1 SynUrban/SynIAG:NO versus 6:1 SynUrban/SynE85B:NO . 


\subsubsection{Block 3: Single Mixture Experiments}

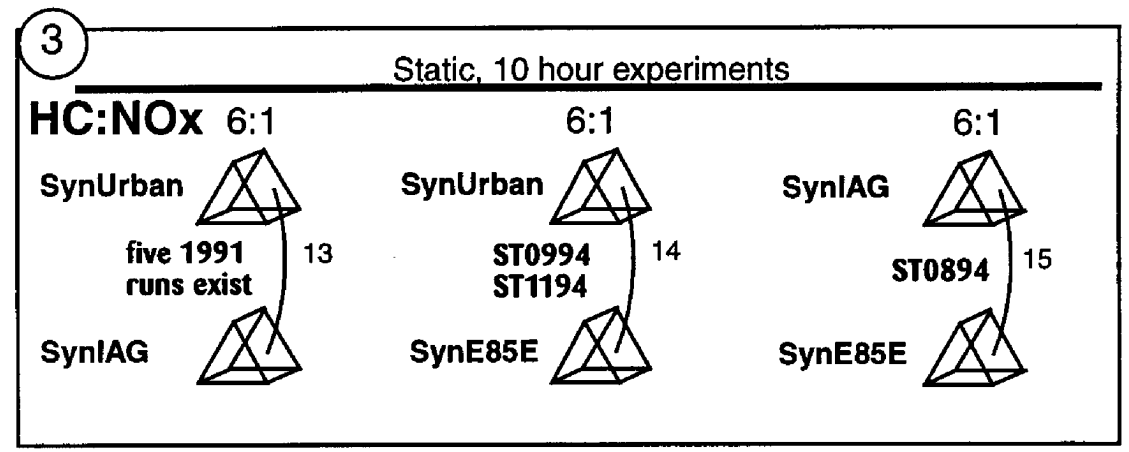

Block 3 experiments were conducted with different test mixtures, but without blending with SynUrb. These are part of the experimental design to provide experiments for modeling testing and to provide information to help understand the chemical processes in these pure mixtures.

\section{Run 14, 6:1 Urb vs.E85E}

ST0994 In this experiment E85 is compared directly against SynUrb (Table 3.16 and Figure 3.16). The E85 side resulted in an ozone maximum concentration $40 \%$ less than the SynUrb side. This experiment was repeated because of the cloudy conditions.

ST1194 This is the repeat of the September 9 experiment (Table 3.17 and Figure 3.17). The E85 side resulted in an ozone maximum concentration $25 \%$ less than the SynUrb side, under more sunny conditions.

\section{Run 15, 6:1 IAG vs. E85E}

ST0894 This dual experiment compares E85 against IAG (Table 3.18 and Figure 3.18). The E85 side produced an ozone maximum $80 \%$ higher than the IAG side by the end of the experiment. 
Table 3.16: Initial Conditions for ST0994

(Units are ppm or ppmC)

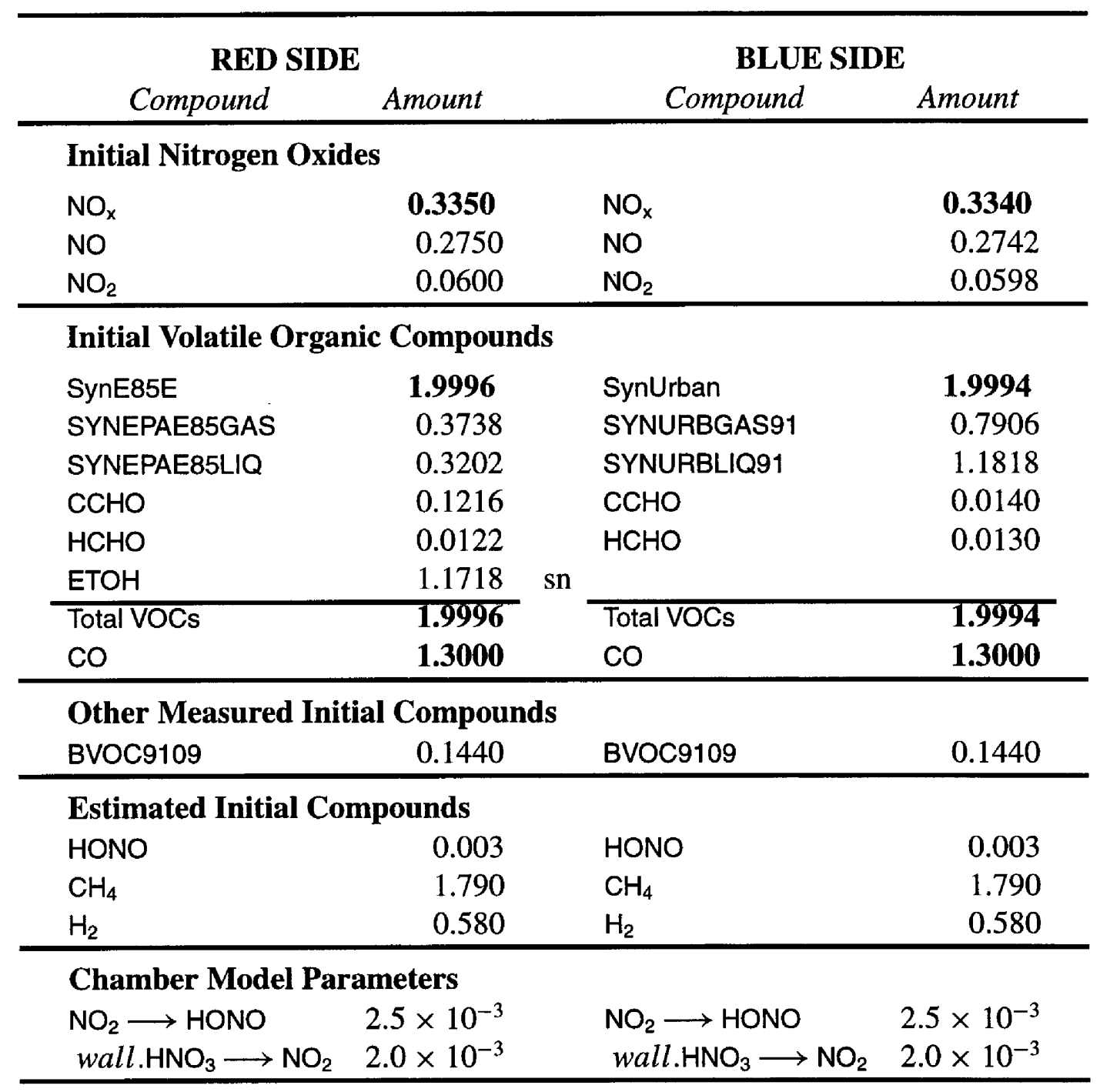

Three Previous Chamber Events

940908.SEG SynE85E vs SynIAG run

940831.SEG Delta CO run

940829.SEG Delta CO run 

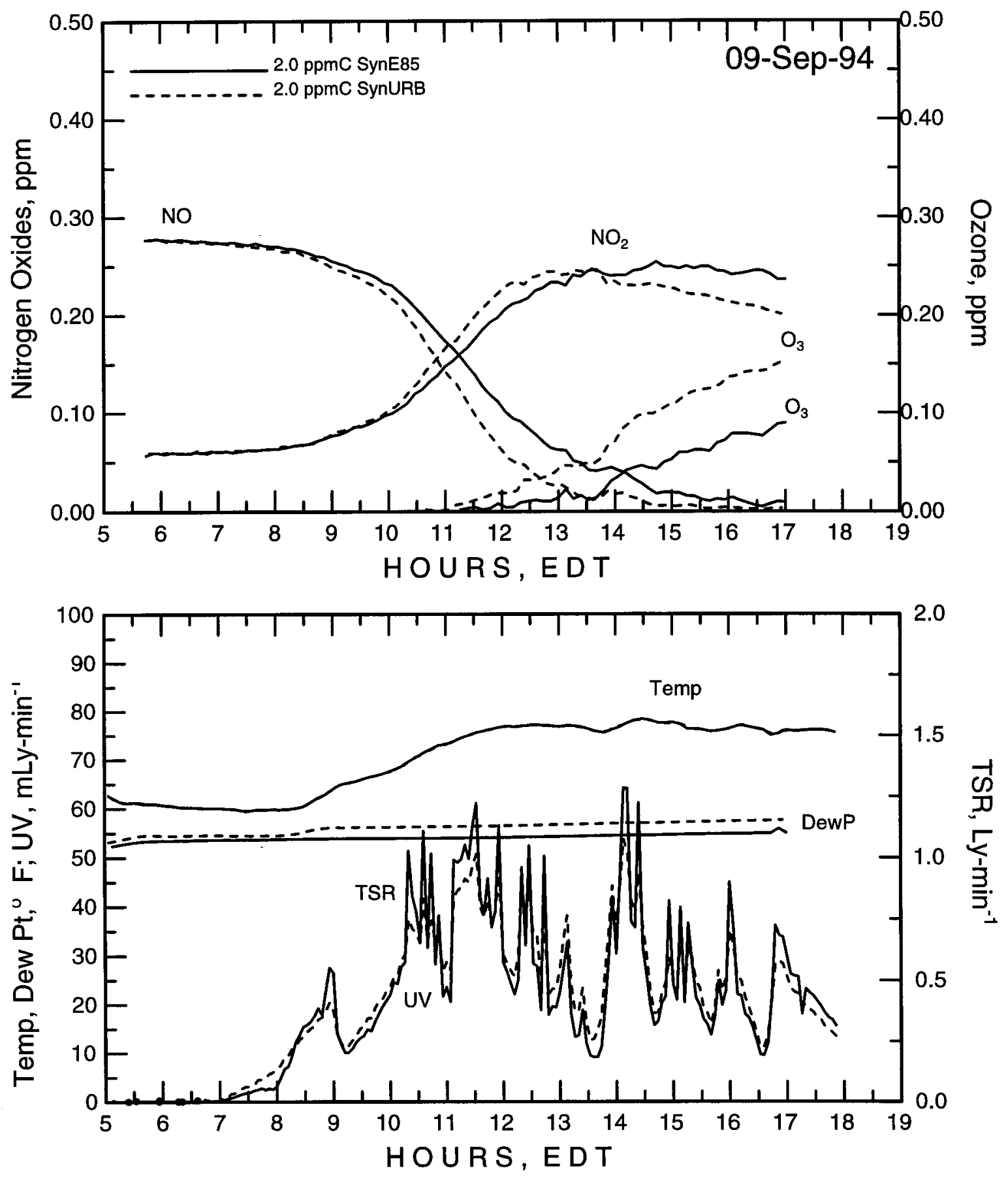

Figure 3.16: Run 14-6:1 SynUrban:NOx versus 6:1 SynE85E:NO . 
Table 3.17: Initial Conditions for ST1194

(Units are ppm or ppmC)

\begin{tabular}{|c|c|c|c|}
\hline \multicolumn{2}{|c|}{ RED SIDE } & \multicolumn{2}{|c|}{ BLUE SIDE } \\
\hline Compound & Amount & Compound & Amount \\
\hline \multicolumn{4}{|c|}{ Initial Nitrogen Oxides } \\
\hline $\mathrm{NO}_{x}$ & 0.3464 & $\mathrm{NO}_{\mathrm{x}}$ & 0.3448 \\
\hline NO & 0.2868 & NO & 0.2879 \\
\hline $\mathrm{NO}_{2}$ & 0.0596 & $\mathrm{NO}_{2}$ & 0.0569 \\
\hline \multicolumn{4}{|c|}{ Initial Volatile Organic Compounds } \\
\hline SynUrban & 1.9994 & SynE85E & 1.9996 \\
\hline SYNURBGAS91 & 0.7906 & SYNEPAE85GAS & 0.3738 \\
\hline SYNURBLIQ91 & 1.1818 & SYNEPAE85LIQ & 0.3202 \\
\hline $\mathrm{CCHO}$ & 0.0140 & $\mathrm{CCHO}$ & 0.1216 \\
\hline \multirow[t]{2}{*}{$\mathrm{HCHO}$} & 0.0130 & $\mathrm{HCHO}$ & 0.0122 \\
\hline & & ETOH & 1.1718 \\
\hline Total VOCs & 1.9994 & Total VOCs & 1.9996 \\
\hline $\mathrm{CO}$ & 1.3000 & $\mathrm{CO}$ & 1.3000 \\
\hline \multicolumn{4}{|c|}{ Other Measured Initial Compounds } \\
\hline BVOC9109 & 0.1440 & BVOC9109 & 0.1440 \\
\hline \multicolumn{4}{|c|}{ Estimated Initial Compounds } \\
\hline HONO & 0.005 & HONO & 0.005 \\
\hline $\mathrm{CH}_{4}$ & 1.790 & $\mathrm{CH}_{4}$ & 1.790 \\
\hline $\mathrm{H}_{2}$ & 0.580 & $\mathrm{H}_{2}$ & 0.580 \\
\hline \multicolumn{4}{|c|}{ Chamber Model Parameters } \\
\hline $\begin{array}{l}\mathrm{NO}_{2} \longrightarrow \mathrm{HONO} \\
\text { wall. } \mathrm{HNO}_{3} \longrightarrow \mathrm{NO}_{2}\end{array}$ & $\begin{array}{l}2.2 \times 10^{-3} \\
2.0 \times 10^{-3} \\
\end{array}$ & $\begin{array}{l}\mathrm{NO}_{2} \longrightarrow \mathrm{HONO} \\
\text { wall. } \mathrm{HNO}_{3} \longrightarrow \mathrm{NO}_{2}\end{array}$ & $\begin{array}{l}2.2 \times 10^{-3} \\
2.0 \times 10^{-3} \\
\end{array}$ \\
\hline \multicolumn{4}{|c|}{$\begin{array}{l}\text { Three Previous Chamber Events } \\
\text { 940909.SEG SynE85E vs SynURB run } \\
\text { 940908.SEG SynE85E vs SynIAG run } \\
\text { 940831.SEG Delta CO run }\end{array}$} \\
\hline
\end{tabular}



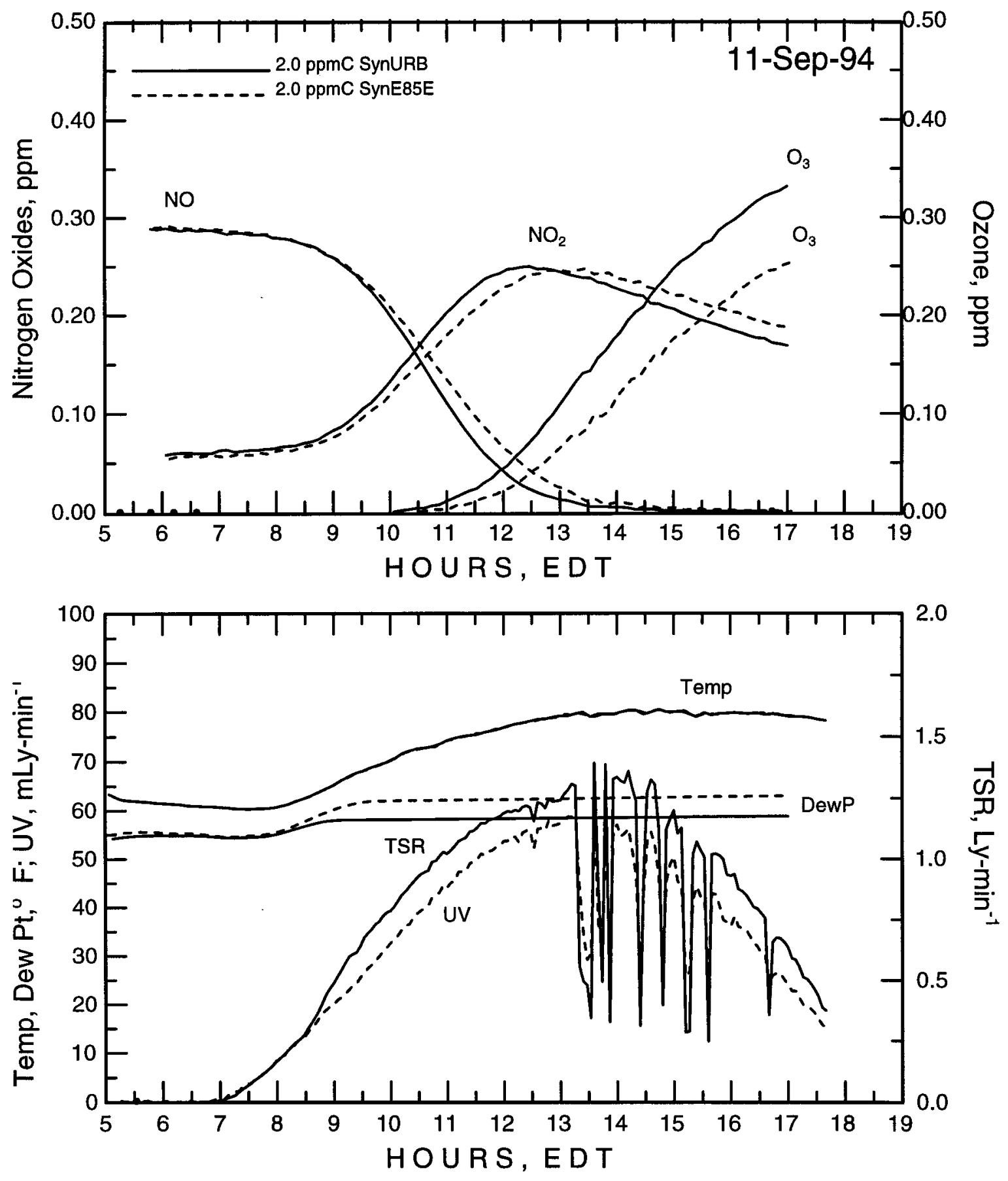

Figure 3.17: Run 14-6:1 SynUrban:NO versus 6:1 SynE85E:NO . 
Table 3.18: Initial Conditions for ST0894

(Units are ppm or ppmC)

\begin{tabular}{|c|c|c|c|}
\hline \multicolumn{2}{|c|}{ RED SIDE } & \multicolumn{2}{|c|}{ BLUE SIDE } \\
\hline Compound & Amount & Compound & Amount \\
\hline \multicolumn{4}{|c|}{ Initial Nitrogen Oxides } \\
\hline $\mathrm{NO}_{x}$ & 0.3380 & $\mathrm{NO}_{x}$ & 0.3374 \\
\hline NO & 0.2727 & NO & 0.2752 \\
\hline $\mathrm{NO}_{2}$ & 0.0653 & $\mathrm{NO}_{2}$ & 0.0622 \\
\hline \multicolumn{4}{|c|}{ Initial Volatile Organic Compounds } \\
\hline SynlAG & 2.0000 & SynE85E & 1.9996 \\
\hline SYNIAGGAS91 & 1.1300 & SYNEPAE85GAS & 0.3738 \\
\hline SYNIAGLPO92 & 0.3660 & SYNEPAE85LIQ & 0.3202 \\
\hline SYNIAGARO92 & 0.4620 & & \\
\hline SYNIAGALD91 & 0.0340 & $\mathrm{CCHO}$ & 0.1216 \\
\hline \multirow[t]{2}{*}{$\mathrm{HCHO}$} & 0.0080 & $\mathrm{HCHO}$ & 0.0122 \\
\hline & & ETOH & 1.1718 \\
\hline Total VOCs & 2.0000 & Total VOCs & 1.9996 \\
\hline $\mathrm{CO}$ & 1.3000 & $\mathrm{CO}$ & 1.3000 \\
\hline \multicolumn{4}{|c|}{ Other Measured Initial Compounds } \\
\hline BVOC9109 & 0.1440 & BVOC9109 & 0.1440 \\
\hline \multicolumn{4}{|c|}{ Estimated Initial Compounds } \\
\hline HONO & 0.003 & HONO & 0.010 \\
\hline $\mathrm{CH}_{4}$ & 1.790 & $\mathrm{CH}_{4}$ & 1.790 \\
\hline $\mathrm{H}_{2}$ & 0.580 & $\mathrm{H}_{2}$ & 0.580 \\
\hline \multicolumn{4}{|c|}{ Chamber Model Parameters } \\
\hline $\mathrm{NO}_{2} \longrightarrow \mathrm{HONO}$ & $2.5 \times 10^{-3}$ & $\mathrm{NO}_{2} \longrightarrow \mathrm{HONO}$ & $2.5 \times 10^{-3}$ \\
\hline wall. $\mathrm{HNO}_{3} \longrightarrow \mathrm{NO}_{2}$ & $2.0 \times 10^{-3}$ & wall. $\mathrm{HNO}_{3} \longrightarrow \mathrm{NO}_{2}$ & $2.0 \times 10^{-3}$ \\
\hline
\end{tabular}

Three Previous Chamber Events

940831.SEG Delta CO run 940829.SEG Delta CO run 940826.SEG SynCNG vs SynIAG run 

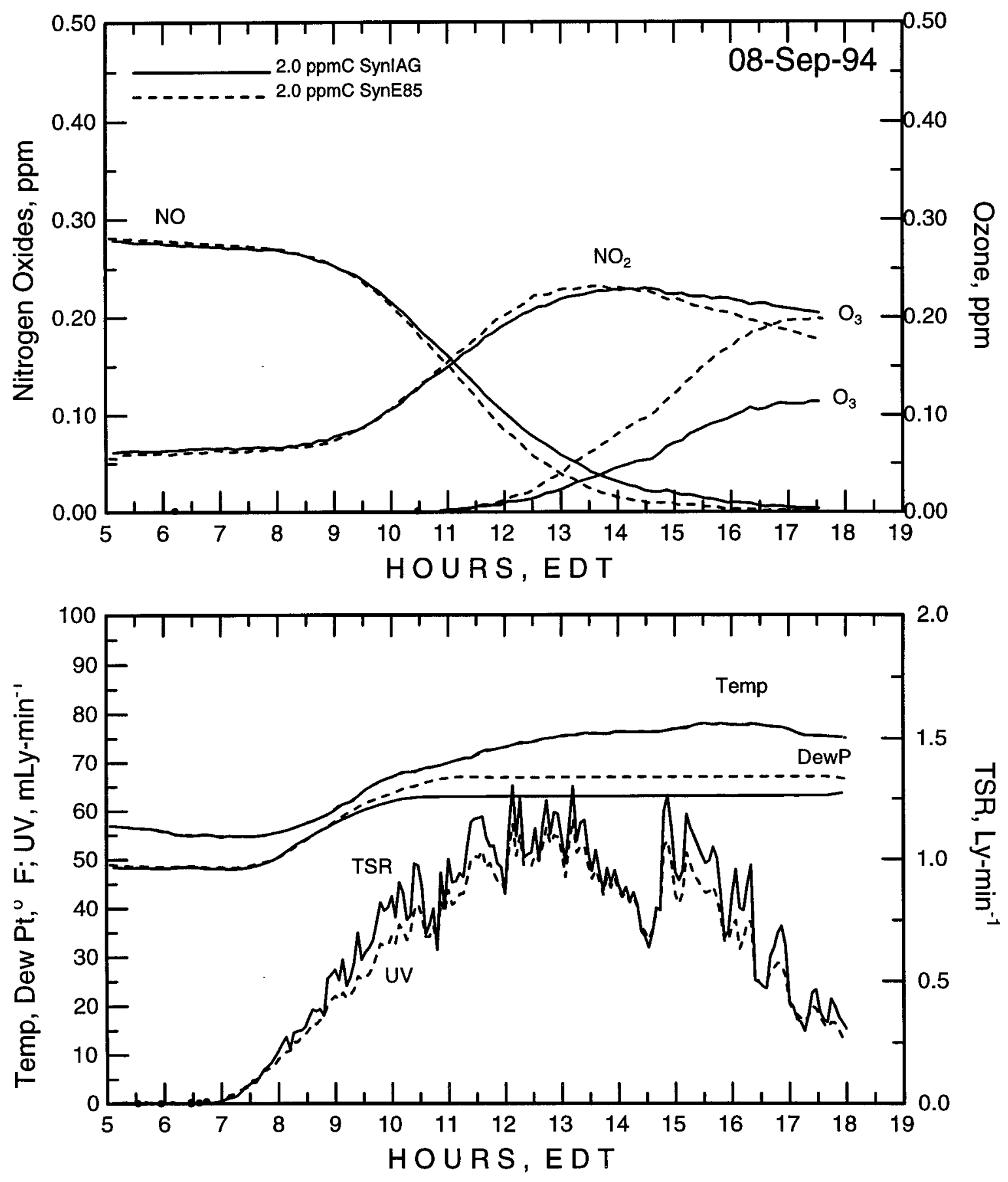

Figure 3.18: Run 15-6:1 SynIAG:NO versus 6:1 SynE85E:NO . 


\subsubsection{Block 4: Mixture Variations}

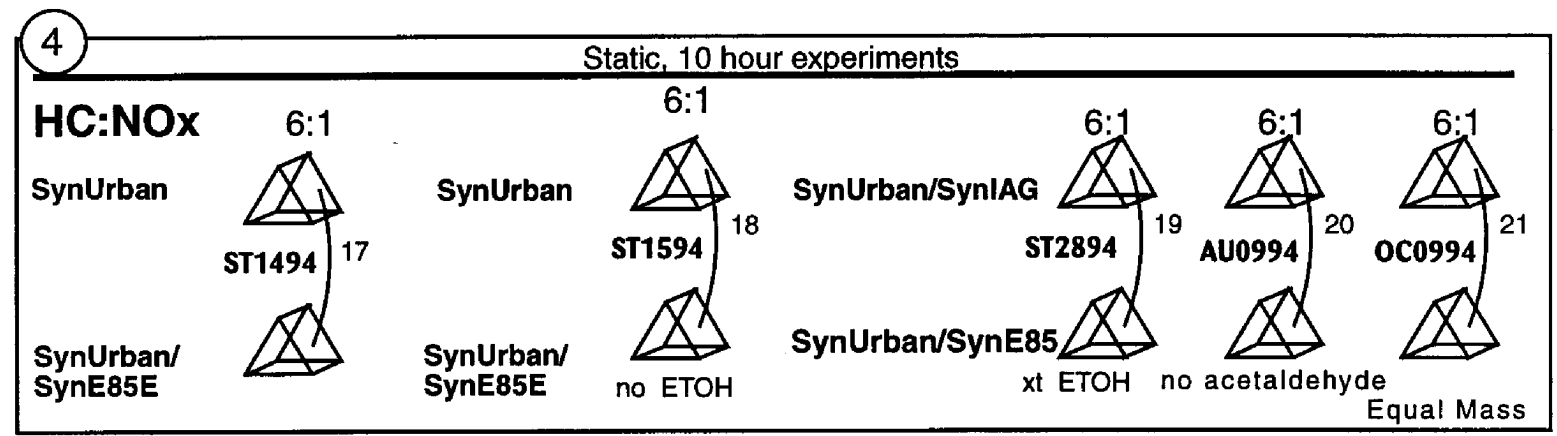

Block 4 experiments were conducted with test mixtures that were variations of the standard mixtures and our standard experiments. These were added as the results of other experiments were obtained and designed to help answer questions. Some of these were designed to demonstrate, by direct observation, the effects of modifying the composition, including injecting more or less of certain mixture components. One was designed to test the effect of comparing equal mass experiments rather than the standard matched carbon experiments. Although all of these are useful for chemical mechanism testing, a few were part of the experimental design more to provide experiments for modeling stress testing and to help understand the chemical processes in these pure, but modified, mixtures.

\section{Run 17, 6:1 Urb versus Urb/E85E}

ST1494 This experiment tests the effect of substituting 50\% of the SynUrb with the E85 mixture (Table 3.19 and Figure 3.19) by comparing directly against unsubstituted SynUrb. The E85 side resulted in a $20 \%$ reduction in ozone maximum concentration.

\section{Run 18, 6:1 Urb versus Urb/E85E (no ETOH)}

ST1594 This experiment tests the ozone contribution of the EtOH by removing the ethanol from the E85 mixture, in a experiment designed to compare with the September 14 substi. tution experiment (Table 3.20 and Figure 3.20).

\section{Run 19, 6:1 Urb/IAG versus Urb/E85 (xt EtOH)}

ST2894 This experiment was conducted with extra ethanol, to provide for a wider range of mechanism testing conditions (Table 3.21 and Figure 3.21).

\section{Run 20, 6:1 Urb/IAG versus Urb/E85 (no aldehyde)}

AU0994 This experiment was designed to show the effect of removing the acetaldehyde from the E85 mixture (Table 3.22 and Figure 3.22), to help understand the reactivity of the 
E85 mixture, and to provide a wider range of chemical mechanism tests. Even with no initial acetaldehyde, the E85 mixture was more reactive than IAG with aldehyde.

\section{Run 21, 6:1 Urb/IAG versus Urb/E85 (equal mass)}

OC0994 This experiment was designed to show the comparison of a matched-mass (including the mass of oxygen) version of E85 vs IAG when blended with the SynUrb (Table 3.23 and Figure 3.23). This experiment resulted in equal ozone production. 
Table 3.19: Initial Conditions for ST1494

(Units are ppm or ppmC)

\begin{tabular}{|c|c|c|c|}
\hline \multicolumn{2}{|c|}{ RED SIDE } & \multicolumn{2}{|c|}{ BLUE SIDE } \\
\hline Compound & Amount & Compound & Amount \\
\hline \multicolumn{4}{|c|}{ Initial Nitrogen Oxides } \\
\hline $\mathrm{NO}_{x}$ & 0.3469 & $\mathrm{NO}_{x}$ & 0.3440 \\
\hline NO & 0.2865 & NO & 0.2864 \\
\hline $\mathrm{NO}_{2}$ & 0.0604 & $\mathrm{NO}_{2}$ & 0.0576 \\
\hline \multicolumn{4}{|c|}{ Initial Volatile Organic Compounds } \\
\hline SynUrban & 0.9997 & SynUrban & 1.9994 \\
\hline SYNURBGAS91 & 0.3953 & SYNURBGAS91 & 0.7906 \\
\hline SYNURBLIQ91 & 0.5909 & SYNURBLIQ91 & 1.1818 \\
\hline $\mathrm{CCHO}$ & 0.0070 & $\mathrm{CCHO}$ & 0.0140 \\
\hline $\mathrm{HCHO}$ & 0.0065 & $\mathrm{HCHO}$ & 0.0130 \\
\hline SynE85E & 0.9998 & & \\
\hline SYNEPAE85GAS & 0.1869 & & \\
\hline SYNEPAE85LIQ & 0.1601 & & \\
\hline $\mathrm{CCHO}$ & 0.0608 & & \\
\hline $\mathrm{HCHO}$ & 0.0061 & & \\
\hline ETOH & 0.5859 & & \\
\hline Total VOCs & 1.9995 & Total VOCs & 1.9994 \\
\hline $\mathrm{CO}$ & 1.3000 & $\mathrm{CO}$ & 1.3000 \\
\hline \multicolumn{4}{|c|}{ Other Measured Initial Compounds } \\
\hline BVOC9109 & 0.1440 & BVOC9109 & 0.1440 \\
\hline \multicolumn{4}{|c|}{ Estimated Initial Compounds } \\
\hline HONO & 0.003 & HONO & 0.003 \\
\hline $\mathrm{CH}_{4}$ & 1.790 & $\mathrm{CH}_{4}$ & 1.790 \\
\hline $\mathrm{H}_{2}$ & 0.580 & $\mathrm{H}_{2}$ & 0.580 \\
\hline \multicolumn{4}{|c|}{ Chamber Model Parameters } \\
\hline $\mathrm{NO}_{2} \longrightarrow \mathrm{HONO}$ & $2.9 \times 10^{-3}$ & $\mathrm{NO}_{2} \longrightarrow \mathrm{HONO}$ & $2.9 \times 10^{-3}$ \\
\hline wall. $\mathrm{HNO}_{3} \longrightarrow \mathrm{NO}_{2}$ & $2.0 \times 10^{-3}$ & wall. $\mathrm{HNO}_{3} \longrightarrow \mathrm{NO}_{2}$ & $2.0 \times 10^{-3}$ \\
\hline $\begin{array}{l}\text { Three Previous Char } \\
\text { 940912.SEG Matched } \\
\text { 940911.SEG SynE841 } \\
\text { 940909.SEG SynE85 }\end{array}$ & $\begin{array}{l}\text { nber Events } \\
\text { SynURB run } \\
\text { vs SynURB } \\
\text { vs SynURB }\end{array}$ & & \\
\hline
\end{tabular}



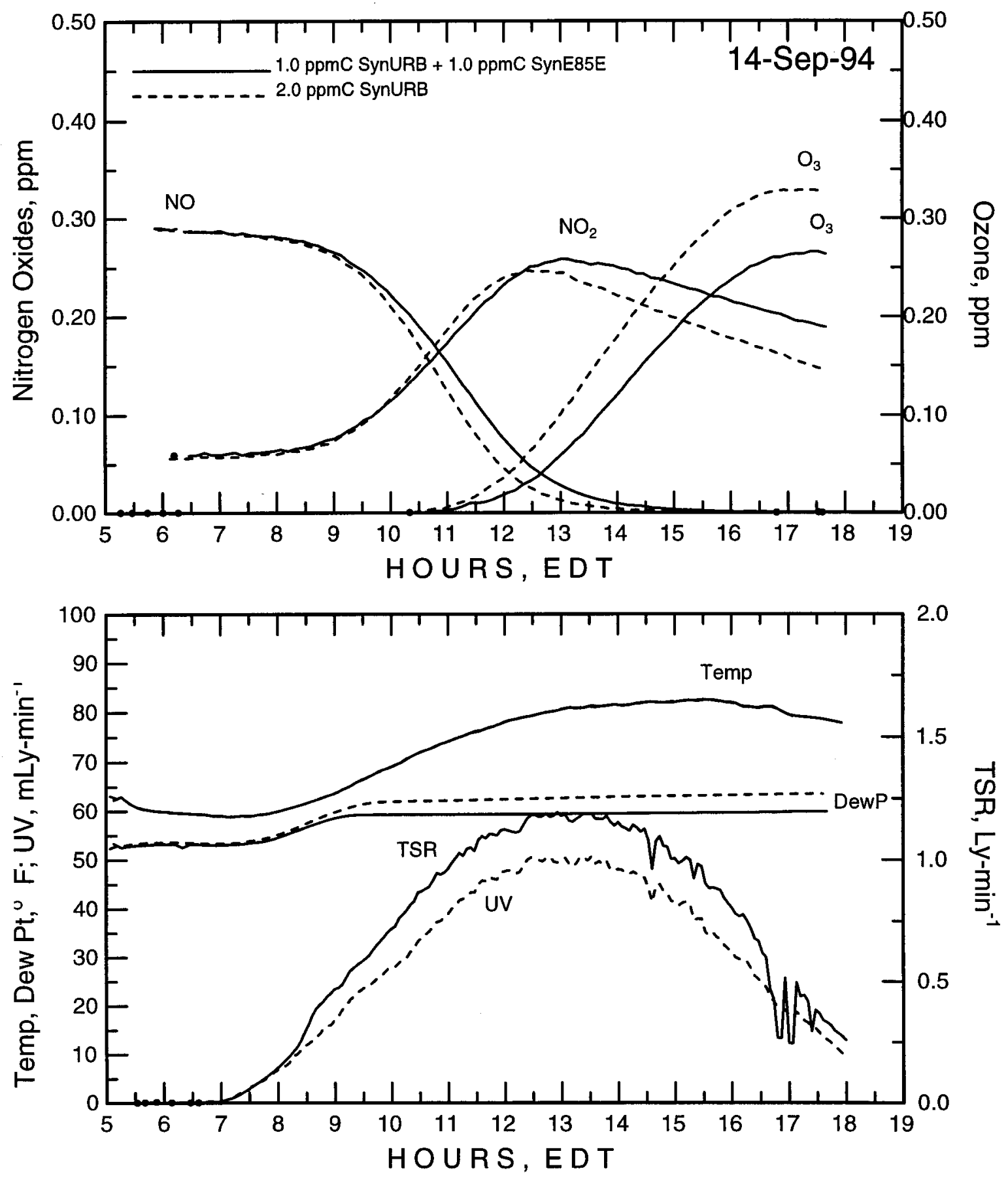

Figure 3.19: Run 17-6:1 SynUrban:NOx versus 6:1 SynUrban/SynE85E:NOx. 
Table 3.20: Initial Conditions for ST1594

(Units are ppm or ppmC)

\begin{tabular}{|c|c|c|c|}
\hline \multicolumn{2}{|c|}{ RED SIDE } & \multicolumn{2}{|c|}{ BLUE SIDE } \\
\hline Compound & Amount & Compound & Amount \\
\hline \multicolumn{4}{|c|}{ Initial Nitrogen Oxides } \\
\hline $\mathrm{NO}_{\mathrm{x}}$ & 0.3433 & $\mathrm{NO}_{x}$ & 0.3431 \\
\hline NO & 0.2794 & NO & 0.2799 \\
\hline $\mathrm{NO}_{2}$ & 0.0639 & $\mathrm{NO}_{2}$ & 0.0637 \\
\hline \multicolumn{4}{|c|}{ Initial Volatile Organic Compounds } \\
\hline SynUrban & 0.9997 & SynUrban & 1.9994 \\
\hline SYNURBGAS91 & 0.3953 & SYNURBGAS91 & 0.7906 \\
\hline SYNURBLIQ91 & 0.5909 & SYNURBLIQ91 & 1.1818 \\
\hline $\mathrm{CCHO}$ & 0.0070 & $\mathrm{CCHO}$ & 0.0140 \\
\hline $\mathrm{HCHO}$ & 0.0065 & $\mathrm{HCHO}$ & 0.0130 \\
\hline SynE85E & 0.4139 & & \\
\hline SYNEPAE85GAS & 0.1869 & & \\
\hline SYNEPAE85LIQ & 0.1601 & & \\
\hline $\mathrm{CCHO}$ & 0.0608 & & \\
\hline $\mathrm{HCHO}$ & 0.0061 & & \\
\hline Total VOCs & 1.4136 & Total VOCs & 1.9994 \\
\hline $\mathrm{CO}$ & 1.3000 & $\mathrm{CO}$ & 1.3000 \\
\hline \multicolumn{4}{|c|}{ Other Measured Initial Compounds } \\
\hline BVOC9109 & 0.1440 & BVOC9109 & 0.1440 \\
\hline \multicolumn{4}{|c|}{ Estimated Initial Compounds } \\
\hline HONO & 0.004 & HONO & 0.004 \\
\hline $\mathrm{CH}_{4}$ & 1.790 & $\mathrm{CH}_{4}$ & 1.790 \\
\hline $\mathrm{H}_{2}$ & 0.580 & $\mathrm{H}_{2}$ & 0.580 \\
\hline \multicolumn{4}{|c|}{ Chamber Model Parameters } \\
\hline $\begin{array}{l}\mathrm{NO}_{2} \longrightarrow \mathrm{HONO} \\
\text { wall. } \mathrm{HNO}_{3} \longrightarrow \mathrm{NO}_{2}\end{array}$ & $\begin{array}{l}2.9 \times 10^{-3} \\
2.0 \times 10^{-3}\end{array}$ & $\begin{array}{l}\mathrm{NO}_{2} \longrightarrow \mathrm{HONO} \\
\text { wall. } \mathrm{HNO}_{3} \longrightarrow \mathrm{NO}_{2}\end{array}$ & $\begin{array}{l}2.9 \times 10^{-3} \\
2.0 \times 10^{-3}\end{array}$ \\
\hline $\begin{array}{l}\text { Three Previous Cha } \\
\text { 940914.SEG SynE85 } \\
\text { 940912.SEG Matchec } \\
\text { 940911.SEG SynE85 }\end{array}$ & $\begin{array}{l}\text { nber Events } \\
\text { vs SynURB } \\
\text { SynURB rur } \\
\text { vs SynURB }\end{array}$ & & \\
\hline
\end{tabular}



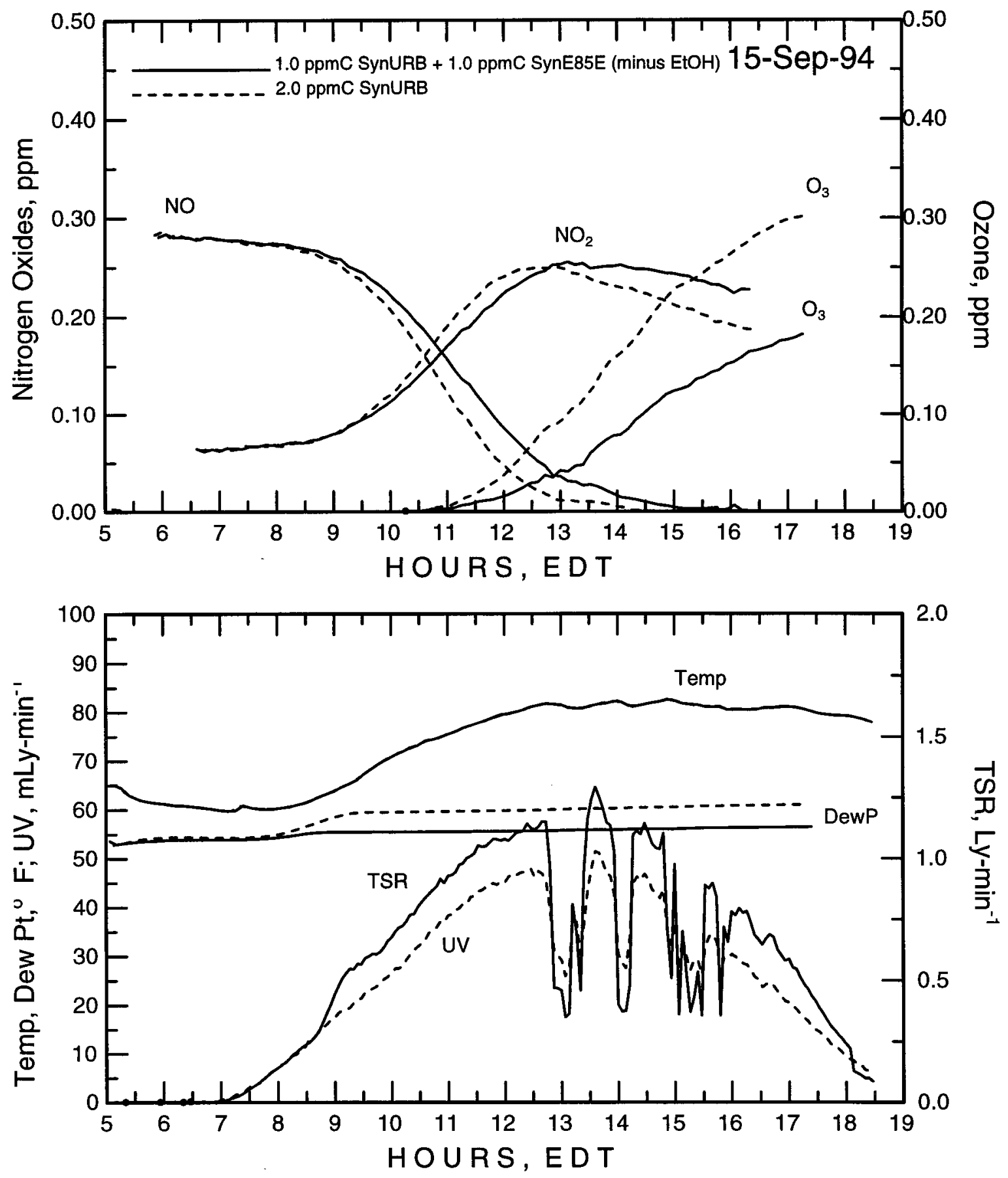

Figure 3.20: Run 18-6:1 SynUrban:NOx versus 6:1 SynUrban/SynE85E(no ETOH): $\mathrm{NO}_{\mathrm{x}}$. 
Table 3.21: Initial Conditions for ST2894

(Units are ppm or ppmC)

\begin{tabular}{|c|c|c|c|}
\hline \multicolumn{2}{|c|}{ RED SIDE } & \multicolumn{2}{|c|}{ BLUE SIDE } \\
\hline Compound & Amount & Compound & Amount \\
\hline \multicolumn{4}{|c|}{ Initial Nitrogen Oxides } \\
\hline $\mathrm{NO}_{x}$ & 0.3257 & $\mathrm{NO}_{x}$ & 0.3277 \\
\hline NO & 0.2600 & NO & 0.2641 \\
\hline $\mathrm{NO}_{2}$ & 0.0657 & $\mathrm{NO}_{2}$ & 0.0636 \\
\hline \multicolumn{4}{|c|}{ Initial Volatile Organic Compounds } \\
\hline SynUrban & 0.9997 & SynUrban & 0.9997 \\
\hline SYNURBGAS91 & 0.3953 & SYNURBGAS91 & 0.3953 \\
\hline SYNURBLIQ91 & 0.5909 & SYNURBLIQ91 & 0.5909 \\
\hline $\mathrm{CCHO}$ & 0.0070 & $\mathrm{CCHO}$ & 0.0070 \\
\hline $\mathrm{HCHO}$ & 0.0065 & $\mathrm{HCHO}$ & 0.0065 \\
\hline SynE85E & 0.9998 & SynIAG & 1.0000 \\
\hline SYNEPAE85GAS & 0.1869 & SYNIAGGAS91 & 0.5650 \\
\hline \multirow[t]{2}{*}{ SYNEPAE85LIQ } & 0.1601 & SYNIAGLPO92 & 0.1830 \\
\hline & & SYNIAGARO92 & 0.2310 \\
\hline $\mathrm{CCHO}$ & 0.0608 & SYNIAGALD91 & 0.0170 \\
\hline $\mathrm{HCHO}$ & 0.0061 & $\mathrm{HCHO}$ & 0.0040 \\
\hline $\mathrm{ETOH}$ & 0.7000 & & \\
\hline Total VOCs & 1.9995 & Total VOCs & 1.9997 \\
\hline $\mathrm{CO}$ & 1.3000 & $\mathrm{CO}$ & 1.3000 \\
\hline \multicolumn{4}{|c|}{ Other Measured Initial Compounds } \\
\hline BVOC9109 & 0.1440 & BVOC9109 & 0.1440 \\
\hline \multicolumn{4}{|c|}{ Estimated Initial Compounds } \\
\hline HONO & 0.006 & HONO & 0.006 \\
\hline $\mathrm{CH}_{4}$ & 1.790 & $\mathrm{CH}_{4}$ & 1.790 \\
\hline $\mathrm{H}_{2}$ & 0.580 & $\mathrm{H}_{2}$ & 0.580 \\
\hline \multicolumn{4}{|c|}{ Chamber Model Parameters } \\
\hline $\mathrm{NO}_{2} \longrightarrow \mathrm{HONO}$ & $1.6 \times 10^{-3}$ & $\mathrm{NO}_{2} \longrightarrow \mathrm{HONO}$ & $1.6 \times 10^{-3}$ \\
\hline wall. $\mathrm{HNO}_{3} \longrightarrow \mathrm{NO}_{2}$ & $2.0 \times 10^{-3}$ & wall. $\mathrm{HNO}_{3} \longrightarrow \mathrm{NO}_{2}$ & $2.0 \times 10^{-3}$ \\
\hline
\end{tabular}

Three Previous Chamber Events 940927.SEG SynLPG vs SynIAG run 940924.SEG SynLPG vs SynIAG run 940920.SEG Night Isoprene run 

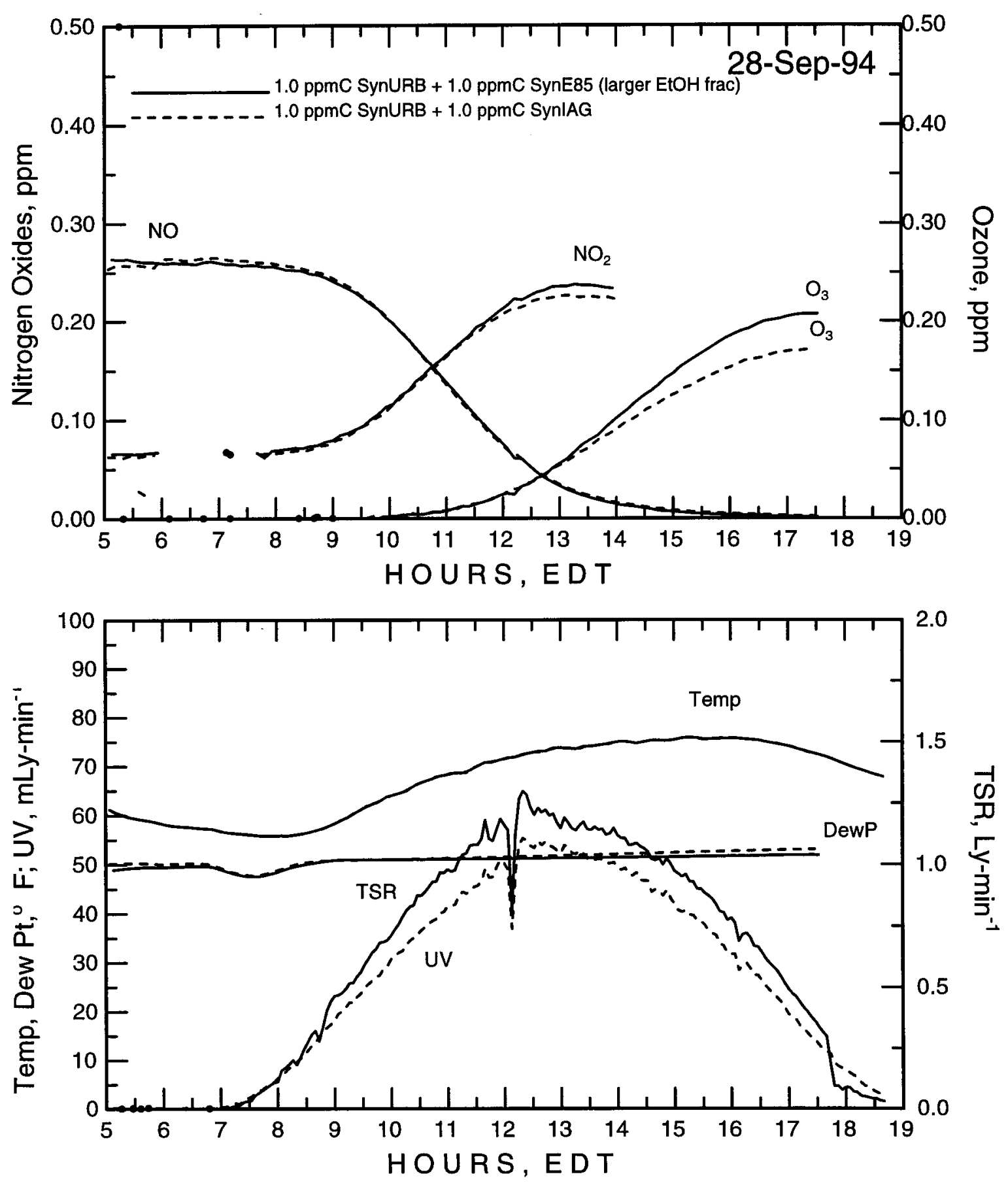

Figure 3.21: Run 19-6:1 SynUrban/SynIAG:NO versus 6:1 SynUrban/SynE85E (xt EtOH):NO . 
Table 3.22: Initial Conditions for AU0994

(Units are ppm or ppmC)

\begin{tabular}{|c|c|c|c|}
\hline \multicolumn{2}{|c|}{ RED SIDE } & \multicolumn{2}{|c|}{ BLUE SIDE } \\
\hline Compound & Amount & Compound & Amount \\
\hline \multicolumn{4}{|c|}{ Initial Nitrogen Oxides } \\
\hline $\mathrm{NO}_{x}$ & 0.3289 & $\mathrm{NO}_{x}$ & 0.3295 \\
\hline NO & 0.2605 & NO & 0.2626 \\
\hline $\mathrm{NO}_{2}$ & 0.0684 & $\mathrm{NO}_{2}$ & 0.0669 \\
\hline \multicolumn{4}{|c|}{ Initial Volatile Organic Compounds } \\
\hline SynUrban & 0.9997 & SynUrban & 0.9997 \\
\hline SYNURBGAS91 & 0.3953 & SYNURBGAS91 & 0.3953 \\
\hline SYNURBLIQ91 & 0.5909 & SYNURBLIQ91 & 0.5909 \\
\hline $\mathrm{CCHO}$ & 0.0070 & $\mathrm{CCHO}$ & 0.0070 \\
\hline $\mathrm{HCHO}$ & 0.0065 & $\mathrm{HCHO}$ & 0.0065 \\
\hline SynE85E & 0.9390 & SynIAG & 1.0000 \\
\hline SYNEPAE85GAS & 0.1869 & SYNIAGGAS91 & 0.5650 \\
\hline \multirow[t]{2}{*}{ SYNEPAE85LIQ } & 0.1601 & SYNIAGLPO92 & 0.1830 \\
\hline & & SYNIAGARO92 & 0.2310 \\
\hline $\mathrm{CCHO}$ & 0.0000 & SYNIAGALD91 & 0.0170 \\
\hline $\mathrm{HCHO}$ & 0.0061 & $\mathrm{HCHO}$ & 0.0040 \\
\hline ETOH & 0.5859 & & \\
\hline Total VOCs & 1.9387 & Total VOCs & 1.9997 \\
\hline $\mathrm{CO}$ & 1.3000 & $\mathrm{CO}$ & 1.3000 \\
\hline \multicolumn{4}{|c|}{ Other Measured Initial Compounds } \\
\hline BVOC9109 & 0.1440 & BVOC9109 & 0.1440 \\
\hline \multicolumn{4}{|c|}{ Estimated Initial Compounds } \\
\hline HONO & 0.005 & HONO & 0.005 \\
\hline $\mathrm{CH}_{4}$ & 1.790 & $\mathrm{CH}_{4}$ & 1.790 \\
\hline $\mathrm{H}_{2}$ & 0.580 & $\mathrm{H}_{2}$ & 0.580 \\
\hline \multicolumn{4}{|c|}{ Chamber Model Parameters } \\
\hline $\mathrm{NO}_{2} \longrightarrow \mathrm{HONO}$ & $2.3 \times 10^{-3}$ & $\mathrm{NO}_{2} \longrightarrow \mathrm{HONO}$ & $2.3 \times 10^{-3}$ \\
\hline wall. $\mathrm{HNO}_{3} \longrightarrow \mathrm{NO}_{2}$ & $2.0 \times 10^{-3}$ & wall. $\mathrm{HNO}_{3} \longrightarrow \mathrm{NO}_{2}$ & $2.0 \times 10^{-3}$ \\
\hline
\end{tabular}

Three Previous Chamber Events

940808.SEG SynE85B vs SynIAG run

940807.SEG SynE85E vs SynIAG run

940804.SEG SynE85E vs SynIAG run 

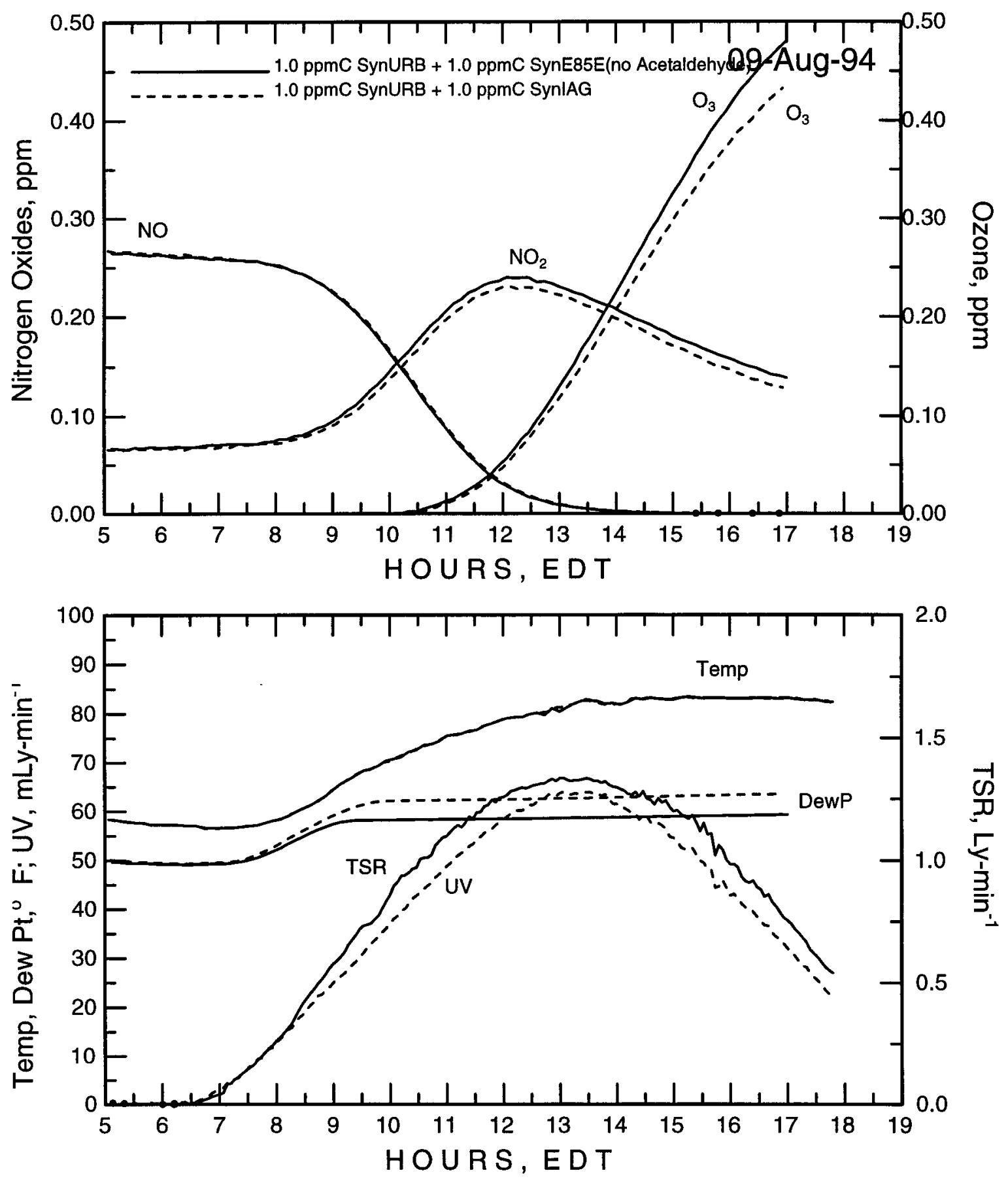

Figure 3.22:. Run 20-6:1 SynUrban/SynIAG:NO ${ }_{x}$ versus 6:1 SynUrban/SynE85E (no aldehyde): $\mathrm{NO}_{\mathrm{x}}$. 
Table 3.23: Initial Conditions for OC0994

(Units are ppm or ppmC)

\begin{tabular}{|c|c|c|c|}
\hline \multicolumn{2}{|c|}{ RED SIDE } & \multicolumn{2}{|c|}{ BLUE SIDE } \\
\hline Compound & Amount & Compound & Amount \\
\hline \multicolumn{4}{|c|}{ Initial Nitrogen Oxides } \\
\hline $\mathrm{NO}_{x}$ & 0.3191 & $\mathrm{NO}_{\mathrm{x}}$ & 0.3176 \\
\hline NO & 0.2683 & NO & 0.2650 \\
\hline $\mathrm{NO}_{2}$ & 0.0508 & $\mathrm{NO}_{2}$ & 0.0526 \\
\hline \multicolumn{4}{|c|}{ Initial Volatile Organic Compounds } \\
\hline SynUrban & 0.9997 & SynUrban & 0.9997 \\
\hline SYNURBGAS91 & 0.3953 & SYNURBGAS91 & 0.3953 \\
\hline SYNURBLIQ91 & 0.5909 & SYNURBLIQ91 & 0.5909 \\
\hline $\mathrm{CCHO}$ & 0.0070 & $\mathrm{CCHO}$ & 0.0070 \\
\hline $\mathrm{HCHO}$ & 0.0065 & $\mathrm{HCHO}$ & 0.0065 \\
\hline SynE85E & 0.8463 & SynIAG & 1.0000 \\
\hline SYNEPAE85GAS & 0.1743 & SYNIAGGAS91 & 0.5650 \\
\hline \multirow[t]{2}{*}{ SYNEPAE85LIQ } & 0.1494 & SYNIAGLPO92 & 0.1830 \\
\hline & & SYNIAGARO92 & 0.2310 \\
\hline $\mathrm{CCHO}$ & 0.0487 & SYNIAGALD91 & 0.0170 \\
\hline $\mathrm{HCHO}$ & 0.0049 & $\mathrm{HCHO}$ & 0.0040 \\
\hline ETOH & 0.4690 & & \\
\hline Total VOCs & 1.8460 & Total VOCs & 1.9997 \\
\hline $\mathrm{CO}$ & 1.3000 & $\mathrm{CO}$ & 1.3000 \\
\hline \multicolumn{4}{|c|}{ Other Measured Initial Compounds } \\
\hline BVOC9109 & 0.1440 & BVOC9109 & 0.1440 \\
\hline \multicolumn{4}{|c|}{ Estimated Initial Compounds } \\
\hline HONO & 0.003 & HONO & 0.003 \\
\hline $\mathrm{CH}_{4}$ & 1.790 & $\mathrm{CH}_{4}$ & 1.790 \\
\hline $\mathrm{H}_{2}$ & 0.580 & $\mathrm{H}_{2}$ & 0.580 \\
\hline \multicolumn{4}{|c|}{ Chamber Model Parameters } \\
\hline $\mathrm{NO}_{2} \longrightarrow \mathrm{HONO}$ & $2.5 \times 10^{-3}$ & $\mathrm{NO}_{2} \longrightarrow \mathrm{HONO}$ & $2.5 \times 10^{-3}$ \\
\hline wall. $\mathrm{HNO}_{3} \longrightarrow \mathrm{NO}_{2}$ & $2.0 \times 10^{-3}$ & wall. $\mathrm{HNO}_{3} \longrightarrow \mathrm{NO}_{2}$ & $2.0 \times 10^{-3}$ \\
\hline
\end{tabular}

Three Previous Chamber Events

940928.SEG SynE85E + EtOH vs SynIAG run

940927.SEG SynLPG vs SynIAG run

940924.SEG SynLPG vs SynIAG run 

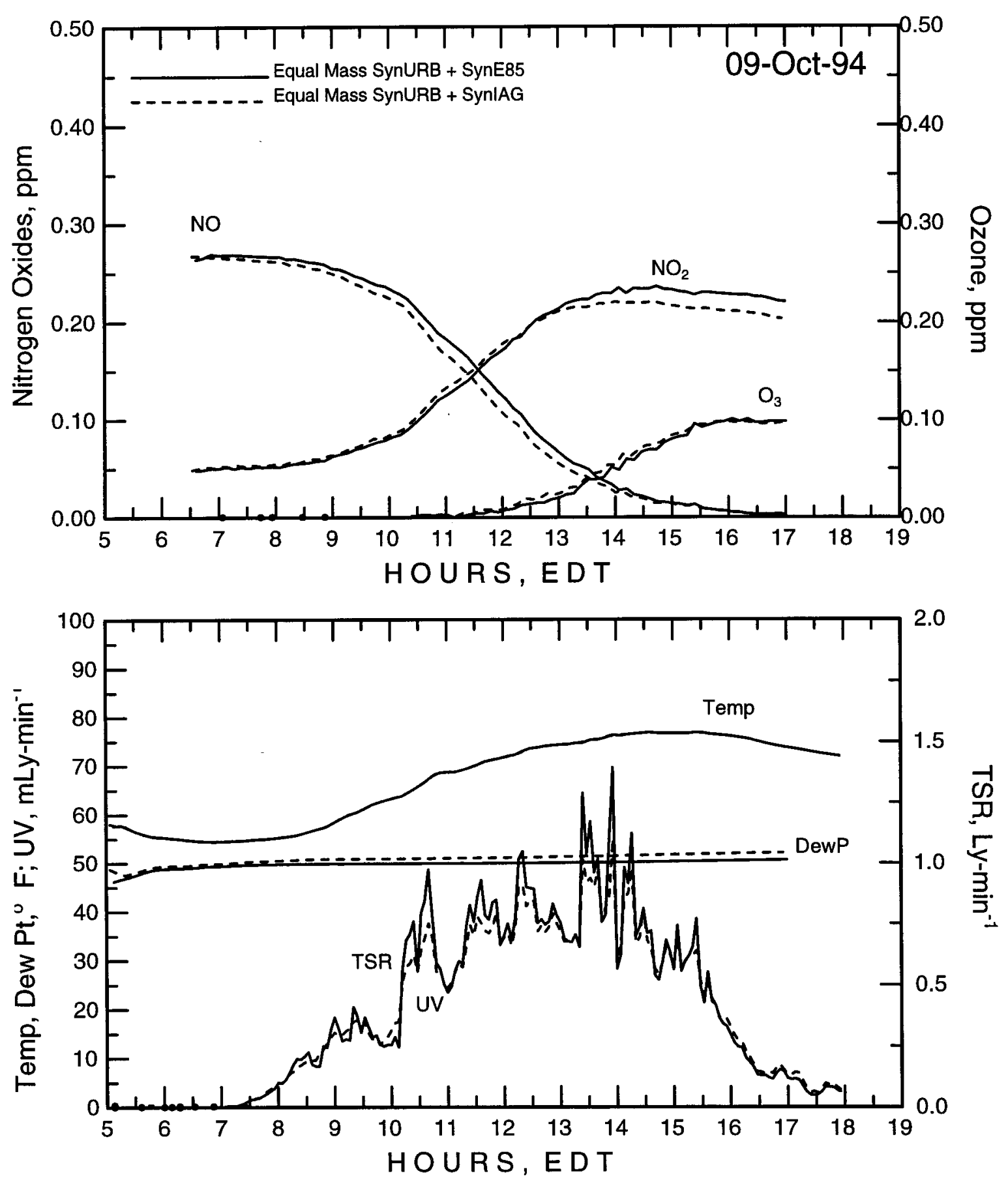

Figure 3.23: Run 21—6:1 SynUrban/SynIAG:NO ${ }_{x}$ versus SynUrban/SynE85E (equal mass): $\mathrm{NO}_{\mathrm{x}}$. 


\subsubsection{Block 6: Characterization}

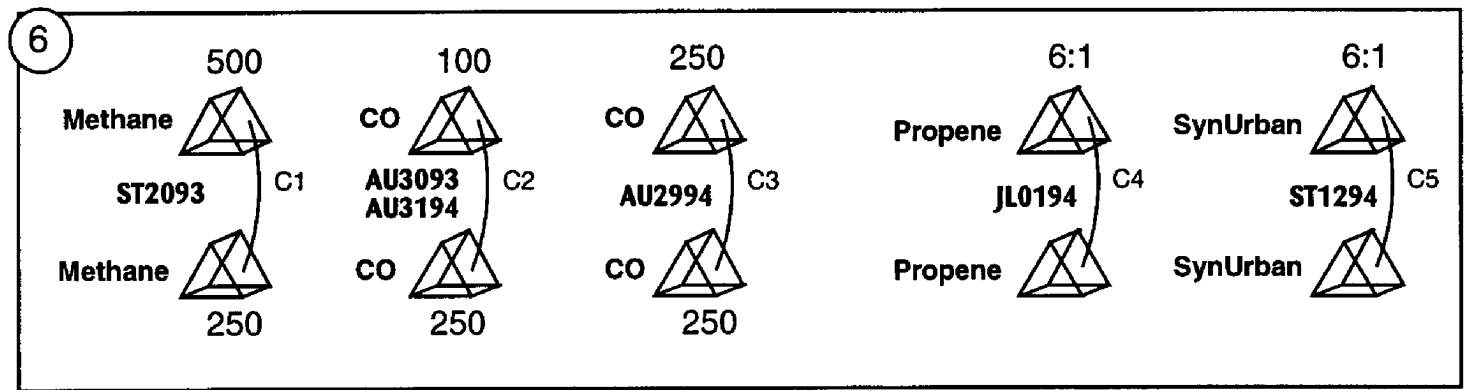

Block 6 experiments were used to determine the performance of the chamber and to identify its contribution to the results of the smog chamber experiments. Matched injection experiments demonstrated that the chambers were matched and there were no problems in injecting and replicating test mixtures into the chambers. Chamber matching assures that there is minimal dilution from sampling, no major leaks in chamber, no leaks in the sample lines, matched wall reactivity, and matched sun (no major shadows effecting one side of the chamber). Chamber characterization experiments are conducted with either simple compounds with better-understood chemistry or with a basic test mixture such as SynUrb.

\section{Run C2, 100 ppm CO versus 250 ppm CO}

AU3093 This experiment compares $100 \mathrm{ppm}$ of CO with $250 \mathrm{ppm}$ CO (Table 3.24 and Figure 3.24). It was designed to show the wall radical flux of the chambers.

AU3194 This experiment compares $100 \mathrm{ppm}$ of co with $250 \mathrm{ppm}$ CO (Table 3.25 and Figure 3.25). It was designed to show the wall radical flux of the chambers.

\section{Run C3, 250 ppm CO versus 250 ppm CO}

AU2994 This experiment compares $250 \mathrm{ppm}$ of $\mathrm{CO}$ with $250 \mathrm{ppm} \mathrm{CO}$ at different ratios of initial $\mathrm{NO}_{2}$ (Table 3.26 and Figure 3.26). It was designed to show the wall radical flux of the chambers.

\section{Run C4, Propene vs. Propene}

JL0194 This experiment compares $100 \%$ propene with $100 \%$ propene at 6:1 (Table 3.27 and Figure 3.27). It was designed to show that the two chamber halves are matched.

\section{Run C5, 6:1 Urb versus Urb}

ST1294 This experiment compares 100\% SynURB with 100\% SynURBat 6:1 (Table 3.28 and Figure 3.28). It was designed to show that the two chamber halves are matched and that we could replicate complex mixtures. 
Table 3.24: Initial Conditions for AU3093

(Units are ppm or ppmC)

\begin{tabular}{|c|c|c|c|}
\hline \multicolumn{2}{|c|}{ RED SIDE } & \multicolumn{2}{|c|}{ BLUE SIDE } \\
\hline Compound & Amount & Compound & Amount \\
\hline \multicolumn{4}{|c|}{ Initial Nitrogen Oxides } \\
\hline $\mathrm{NO}_{\mathrm{x}}$ & 0.3198 & $\mathrm{NO}_{x}$ & 0.3207 \\
\hline NO & 0.2757 & NO & 0.2748 \\
\hline $\mathrm{NO}_{2}$ & 0.0441 & $\mathrm{NO}_{2}$ & 0.0416 \\
\hline \multicolumn{4}{|c|}{ Initial Volatile Organic Compounds } \\
\hline Total VOCs & 0.0000 & Total VOCs & 0.0000 \\
\hline $\mathrm{CO}$ & 250.0000 & $\mathrm{CO}$ & 100.0000 \\
\hline \multicolumn{4}{|c|}{ Other Measured Initial Compounds } \\
\hline BVOC9109 & 0.1440 & BVOC9109 & 0.1440 \\
\hline \multicolumn{4}{|c|}{ Estimated Initial Compounds } \\
\hline HONO & 0.007 & HONO & 0.007 \\
\hline $\mathrm{CH}_{4}$ & 1.790 & $\mathrm{CH}_{4}$ & 1.790 \\
\hline $\mathrm{H}_{2}$ & 0.580 & $\mathrm{H}_{2}$ & 0.580 \\
\hline \multicolumn{4}{|c|}{ Chamber Model Parameters } \\
\hline $\begin{array}{l}\mathrm{NO}_{2} \longrightarrow \mathrm{HONO} \\
\text { wall. } \mathrm{HNO}_{3} \longrightarrow \mathrm{NO}_{2}\end{array}$ & $\begin{array}{r}0.37 \times 10^{-3} \\
2.0 \times 10^{-3}\end{array}$ & $\begin{array}{l}\mathrm{NO}_{2} \longrightarrow \mathrm{HONO} \\
\text { wall } \mathrm{HNO}_{3} \longrightarrow \mathrm{NO}_{2}\end{array}$ & $\begin{array}{l}0.7 \times 10^{-3} \\
2.0 \times 10^{-3}\end{array}$ \\
\hline
\end{tabular}

Three Previous Chamber Events 

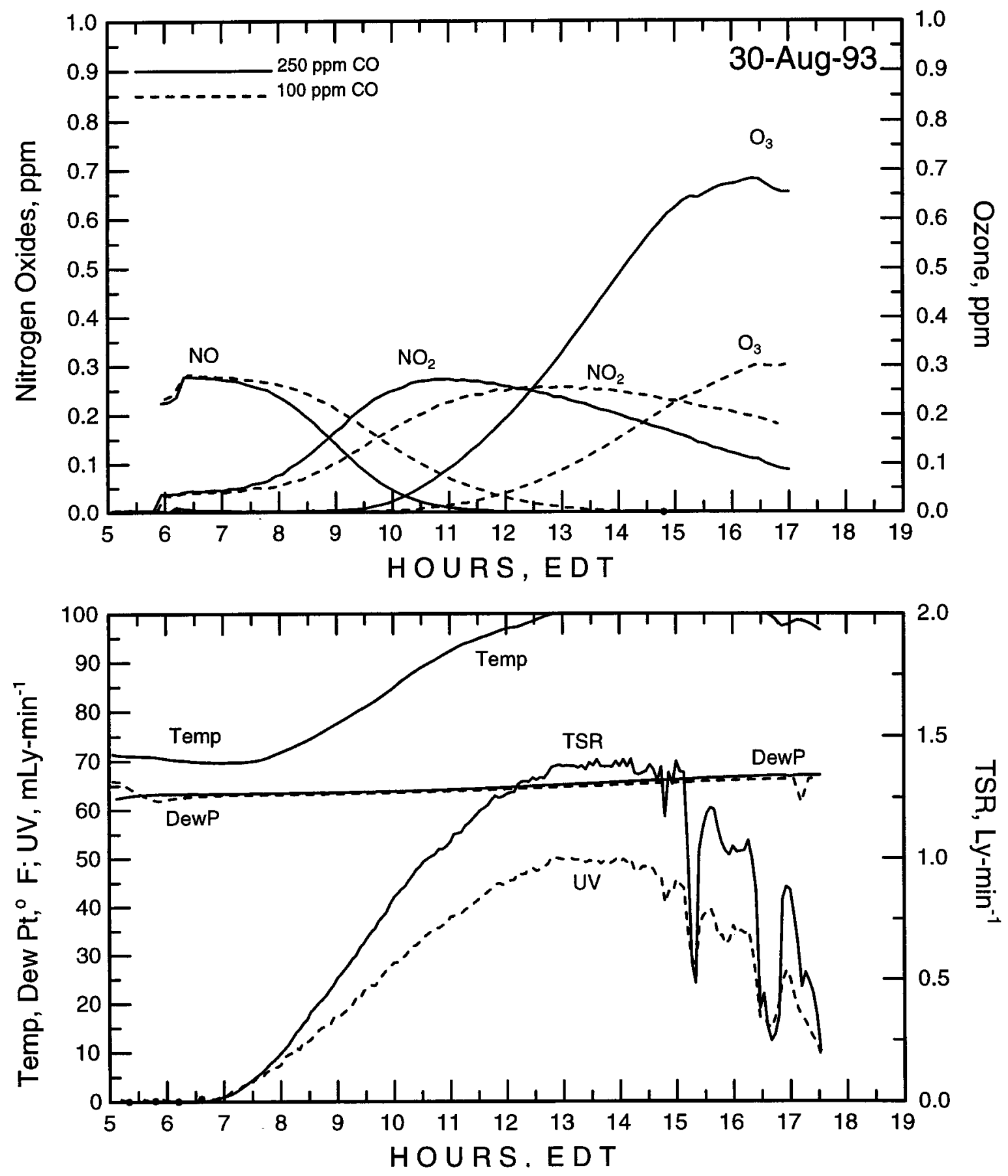

Figure 3.24: Run C2-100 ppm CO:NO versus 250 ppm CO:NO . 
Table 3.25: Initial Conditions for AU3194

(Units are ppm or ppmC)

\begin{tabular}{|c|c|c|c|}
\hline \multicolumn{2}{|c|}{ RED SIDE } & \multicolumn{2}{|c|}{ BLUE SIDE } \\
\hline Compound & Amount & Compound & Amount \\
\hline \multicolumn{4}{|c|}{ Initial Nitrogen Oxides } \\
\hline $\mathrm{NO}_{x}$ & 0.3594 & $\mathrm{NO}_{x}$ & 0.3614 \\
\hline NO & 0.2733 & NO & 0.2713 \\
\hline $\mathrm{NO}_{2}$ & 0.0861 & $\mathrm{NO}_{2}$ & 0.0901 \\
\hline \multicolumn{4}{|c|}{ Initial Volatile Organic Compounds } \\
\hline Total VOCs & 0.0000 & Total VOCs & 0.0000 \\
\hline $\mathrm{CO}$ & 250.0000 & $\mathrm{CO}$ & 100.0000 \\
\hline \multicolumn{4}{|c|}{ Other Measured Initial Compounds } \\
\hline BVOC9109 & 0.1440 & BVOC9109 & 0.1440 \\
\hline \multicolumn{4}{|c|}{ Estimated Initial Compounds } \\
\hline HONO & 0.007 & HONO & 0.007 \\
\hline $\mathrm{CH}_{4}$ & 1.790 & $\mathrm{CH}_{4}$ & 1.790 \\
\hline $\mathrm{H}_{2}$ & 0.580 & $\mathrm{H}_{2}$ & 0.580 \\
\hline \multicolumn{4}{|c|}{ Chamber Model Parameters } \\
\hline $\mathrm{NO}_{2} \longrightarrow \mathrm{HONO}$ & $\begin{array}{r}0.37 \times 10^{-3} \\
20 \times 10^{-3}\end{array}$ & $\begin{array}{l}\mathrm{NO}_{2} \longrightarrow \mathrm{HONO} \\
\text { wall } \mathrm{HNO}_{3} \longrightarrow \mathrm{NO}_{2}\end{array}$ & $\begin{array}{l}0.7 \times 10^{-3} \\
2.0 \times 10^{-3}\end{array}$ \\
\hline
\end{tabular}

Three Previous Chamber Events

940829.SEG Delta CO run 940826.SEG SynCNG vs SynIAG run

940825.SEG SynE85E vs SynIAG run 

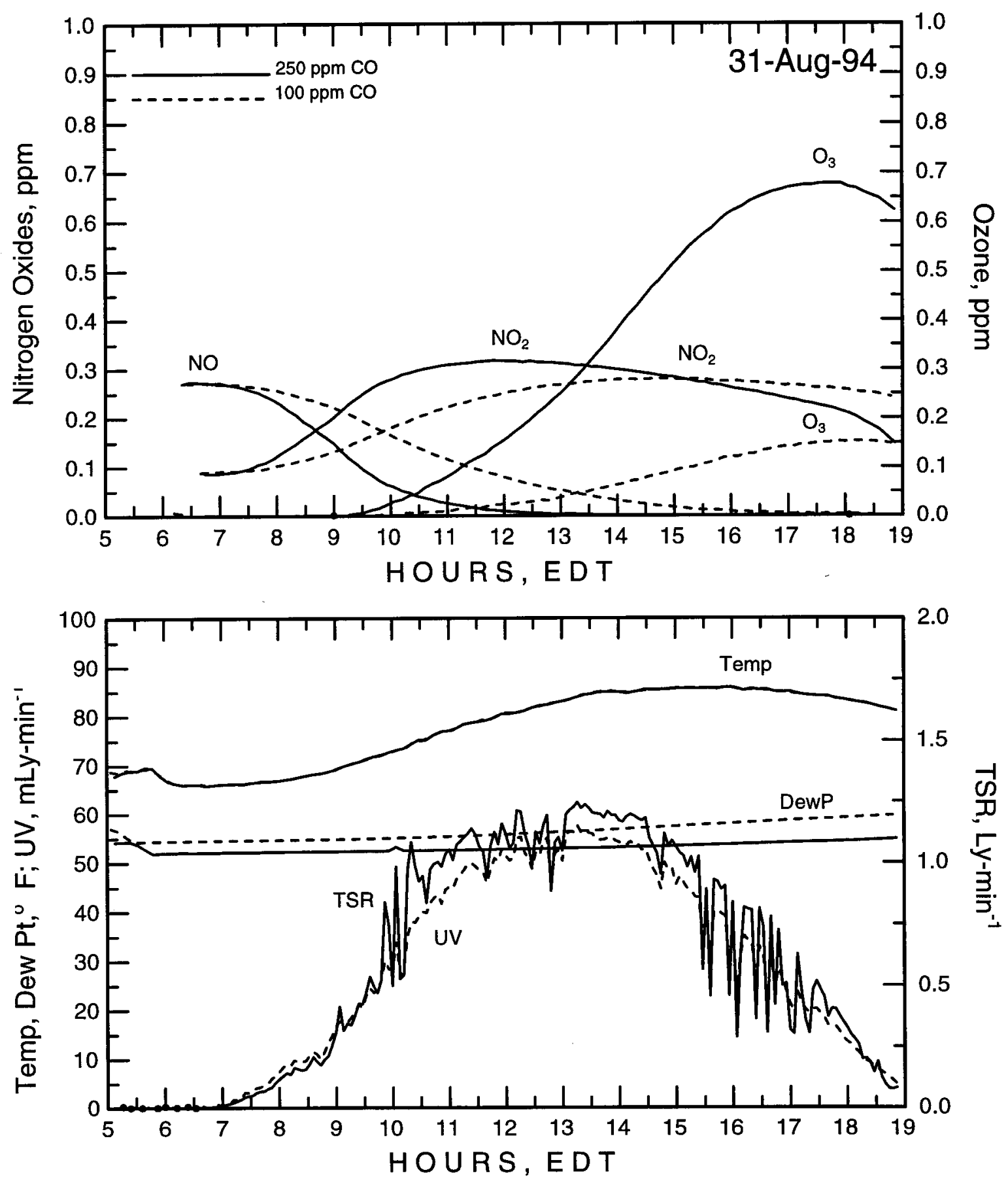

Figure 3.25: Run $\mathrm{C} 2-100 \mathrm{ppm} \mathrm{CO}: \mathrm{NO}_{\mathrm{x}}$ versus $250 \mathrm{ppm}$ CO: $\mathrm{NO}_{\mathrm{x}}$. 
Table 3.26: Initial Conditions for AU2994

(Units are ppm or ppmC)

\begin{tabular}{|c|c|c|c|}
\hline \multicolumn{2}{|c|}{ RED SIDE } & \multicolumn{2}{|c|}{ BLUE SIDE } \\
\hline Compound & Amount & Compound & Amount \\
\hline \multicolumn{4}{|c|}{ Initial Nitrogen Oxides } \\
\hline $\mathrm{NO}_{x}$ & 0.3413 & $\mathrm{NO}_{x}$ & 0.3415 \\
\hline NO & 0.2864 & NO & 0.2597 \\
\hline $\mathrm{NO}_{2}$ & 0.0549 & $\mathrm{NO}_{2}$ & 0.0818 \\
\hline \multicolumn{4}{|c|}{ Initial Volatile Organic Compounds } \\
\hline $\mathrm{CO}$ & 250.0000 & $\mathrm{CO}$ & 250.0000 \\
\hline \multicolumn{4}{|c|}{ Other Measured Initial Compounds } \\
\hline BVOC9109 & 0.1440 & BVOC9109 & 0.1440 \\
\hline \multicolumn{4}{|c|}{ Estimated Initial Compounds } \\
\hline HONO & 0.003 & HONO & 0.003 \\
\hline $\mathrm{CH}_{4}$ & 1.790 & $\mathrm{CH}_{4}$ & 1.790 \\
\hline $\mathrm{H}_{2}$ & 0.580 & $\mathrm{H}_{2}$ & 0.580 \\
\hline \multicolumn{4}{|c|}{ Chamber Model Parameters } \\
\hline $\begin{array}{l}\mathrm{NO}_{2} \longrightarrow \mathrm{HONO} \\
\text { wall. } \mathrm{HNO}_{3} \longrightarrow \mathrm{NO}_{2}\end{array}$ & $\begin{array}{l}2.5 \times 10^{-3} \\
2.0 \times 10^{-3}\end{array}$ & $\begin{array}{l}\mathrm{NO}_{2} \longrightarrow \mathrm{HONO} \\
\text { wall. } \mathrm{HNO}_{3} \longrightarrow \mathrm{NO}_{2}\end{array}$ & $\begin{array}{l}2.5 \times 10^{-3} \\
2.0 \times 10^{-3}\end{array}$ \\
\hline $\begin{array}{l}\text { Three Previous Cha } \\
\text { 940826.SEG SynCN } \\
\text { 940825.SEG SynE85 } \\
\text { 940824.SEG SynE85 }\end{array}$ & $\begin{array}{l}\text { nber Events } \\
\text { vs SynIAG } \\
\text { E vs SynIAG } \\
\text { E vs SynIAG }\end{array}$ & & \\
\hline
\end{tabular}



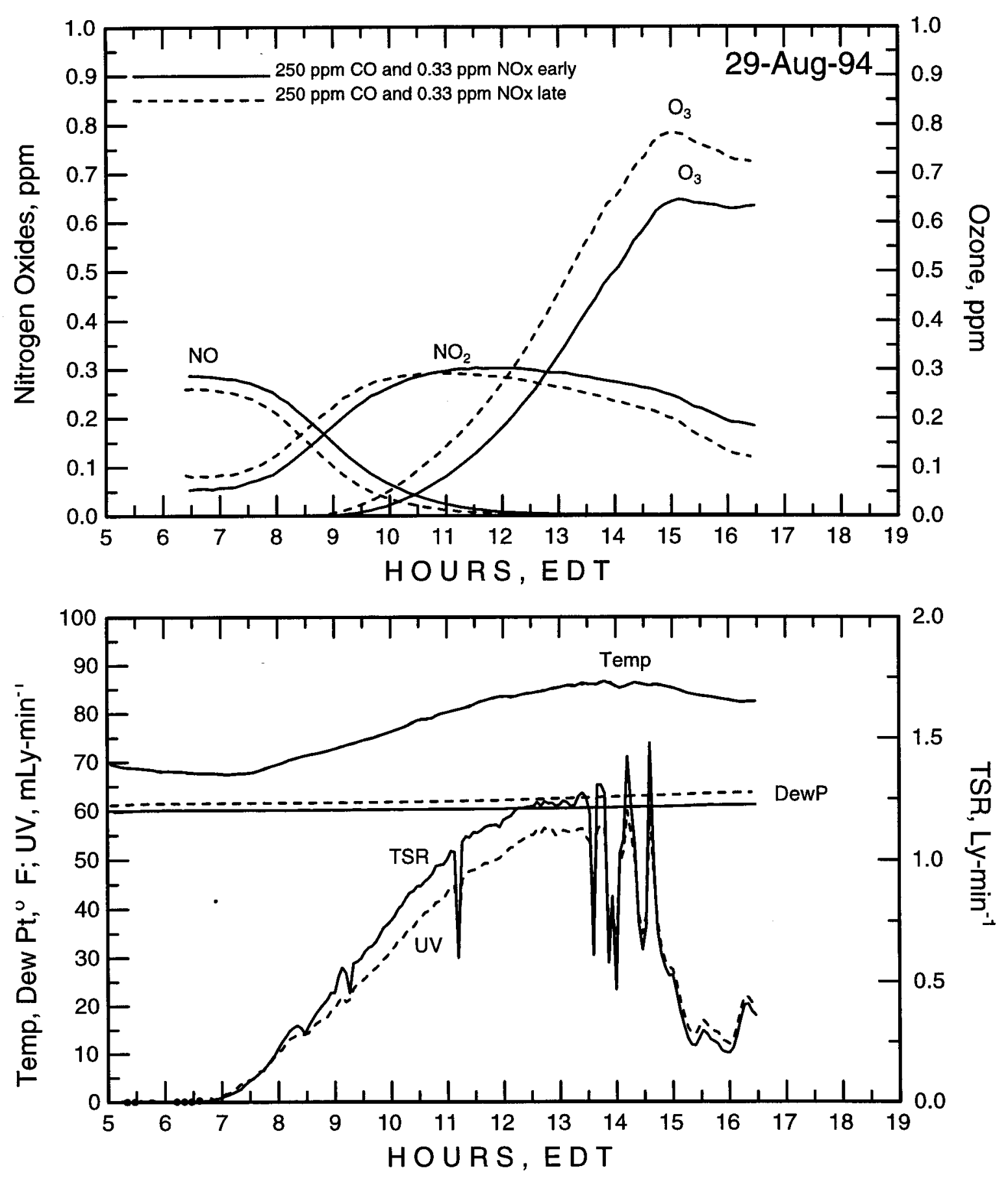

Figure 3.26: Run C3-250 ppm CO:NO versus $250 \mathrm{ppm}$ CO: $\mathrm{NO}_{\mathrm{x}}$. 
Table 3.27: Initial Conditions for JL0194

(Units are ppm or ppmC)

\begin{tabular}{|c|c|c|c|}
\hline \multicolumn{2}{|c|}{ RED SIDE } & \multicolumn{2}{|c|}{ BLUE SIDE } \\
\hline Compound & Amount & Compound & Amount \\
\hline \multicolumn{4}{|c|}{ Initial Nitrogen Oxides } \\
\hline $\mathrm{NO}_{\mathrm{x}}$ & 0.3021 & $\mathrm{NO}_{\mathrm{x}}$ & 0.3057 \\
\hline NO & 0.2047 & NO & 0.2095 \\
\hline $\mathrm{NO}_{2}$ & 0.0974 & $\mathrm{NO}_{2}$ & 0.0962 \\
\hline \multicolumn{4}{|c|}{ Initial Volatile Organic Compounds } \\
\hline $\mathrm{CC}: \mathrm{C}$ & 1.5000 & $\mathrm{CC}: \mathrm{C}$ & 1.5000 \\
\hline Total VOCs & 1.5000 & Total VOCs & $\mathbf{1 . 5 0 0 0}$ \\
\hline \multicolumn{4}{|c|}{ Other Measured Initial Compounds } \\
\hline BVOC9109 & 0.1440 & BVOC9109 & 0.1440 \\
\hline \multicolumn{4}{|c|}{ Estimated Initial Compounds } \\
\hline HONO & 0.003 & HONO & 0.003 \\
\hline $\mathrm{CH}_{4}$ & 1.790 & $\mathrm{CH}_{4}$ & 1.790 \\
\hline $\mathrm{H}_{2}$ & 0.580 & $\mathrm{H}_{2}$ & 0.580 \\
\hline \multicolumn{4}{|c|}{ Chamber Model Parameters } \\
\hline $\begin{array}{l}\mathrm{NO}_{2} \longrightarrow \mathrm{HONO} \\
\text { wall. } \mathrm{HNO}_{3} \longrightarrow \mathrm{NO}_{2}\end{array}$ & $\begin{array}{l}2.5 \times 10^{-3} \\
2.0 \times 10^{-3}\end{array}$ & $\begin{array}{l}\mathrm{NO}_{2} \longrightarrow \mathrm{HONO} \\
\text { wall. } \mathrm{HNO}_{3} \longrightarrow \mathrm{NO}_{2}\end{array}$ & $\begin{array}{l}2.5 \times 10^{-3} \\
2.0 \times 10^{-3}\end{array}$ \\
\hline
\end{tabular}

Three Previous Chamber Events

931006.SEG previous season run 930930.SEG previous season run 930929.SEG previous season run 

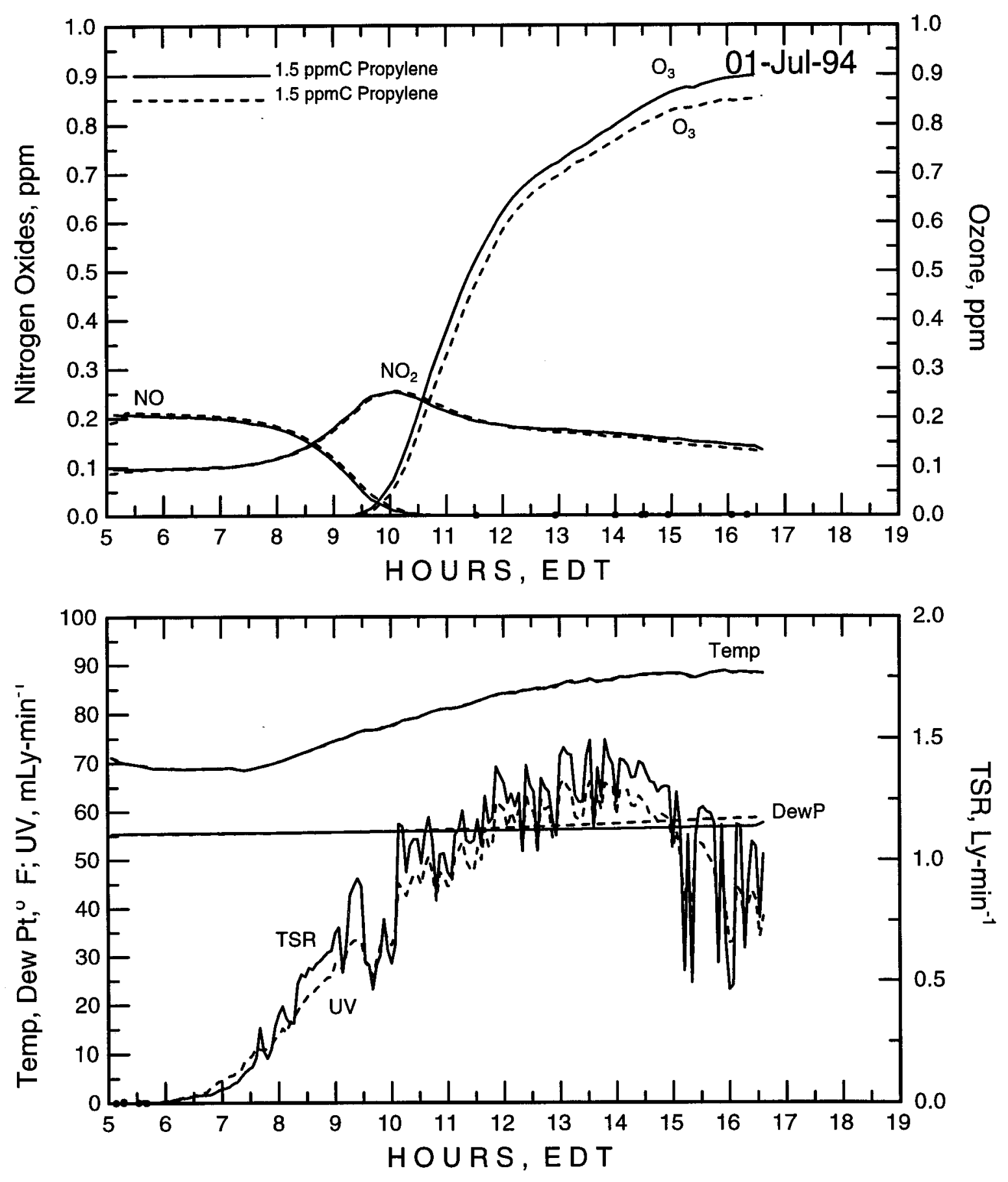

Figure 3.27: Run $\mathrm{C} 4-6: 1$ propene: $\mathrm{NO}_{\mathrm{x}}$ versus 6:1 propene:NO . 
Table 3.28: Initial Conditions for ST1294

(Units are ppm or ppmC)

\begin{tabular}{|c|c|c|c|}
\hline \multicolumn{2}{|c|}{ RED SIDE } & \multicolumn{2}{|c|}{ BLUE SIDE } \\
\hline Compound & Amount & Compound & Amount \\
\hline \multicolumn{4}{|c|}{ Initial Nitrogen Oxides } \\
\hline $\mathrm{NO}_{\mathrm{x}}$ & 0.3229 & $\mathrm{NO}_{\mathrm{x}}$ & 0.3196 \\
\hline NO & 0.2666 & NO & 0.2662 \\
\hline $\mathrm{NO}_{2}$ & 0.0563 & $\mathrm{NO}_{2}$ & 0.0534 \\
\hline \multicolumn{4}{|c|}{ Initial Volatile Organic Compounds } \\
\hline SynUrban & 1.9994 & SynUrban & 1.9994 \\
\hline SYNURBGAS91 & 0.7906 & SYNURBGAS91 & 0.7906 \\
\hline SYNURBLIQ91 & 1.1818 & SYNURBLIQ91 & 1.1818 \\
\hline $\mathrm{CCHO}$ & 0.0140 & $\mathrm{CCHO}$ & 0.0140 \\
\hline $\mathrm{HCHO}$ & 0.0130 & $\mathrm{HCHO}$ & 0.0130 \\
\hline Total VOCs & 1.9994 & Total VOCs & 1.9994 \\
\hline $\mathrm{CO}$ & 1.3000 & $\mathrm{CO}$ & 1.3000 \\
\hline \multicolumn{4}{|c|}{ Other Measured Initial Compounds } \\
\hline BVOC9109 & 0.1440 & BVOC9109 & 0.1440 \\
\hline \multicolumn{4}{|c|}{ Estimated Initial Compounds } \\
\hline HONO & 0.003 & HONO & 0.003 \\
\hline $\mathrm{CH}_{4}$ & 1.790 & $\mathrm{CH}_{4}$ & 1.790 \\
\hline $\mathrm{H}_{2}$ & 0.580 & $\mathrm{H}_{2}$ & 0.580 \\
\hline \multicolumn{4}{|c|}{ Chamber Model Parameters } \\
\hline $\begin{array}{l}\mathrm{NO}_{2} \longrightarrow \mathrm{HONO} \\
\text { wall. } \mathrm{HNO}_{3} \longrightarrow \mathrm{NO}_{2}\end{array}$ & $\begin{array}{l}2.1 \times 10^{-3} \\
2.0 \times 10^{-3}\end{array}$ & $\begin{array}{l}\mathrm{NO}_{2} \longrightarrow \mathrm{HONO} \\
\text { wall } . \mathrm{HNO}_{3} \longrightarrow \mathrm{NO}_{2}\end{array}$ & $\begin{array}{l}2.1 \times 10^{-3} \\
2.0 \times 10^{-3}\end{array}$ \\
\hline
\end{tabular}

Three Previous Chamber Events

940911.SEG SynE85E vs SynURB run 940909.SEG SynE85E vs SynURB run 940908.SEG SynE85E vs SynIAG run 

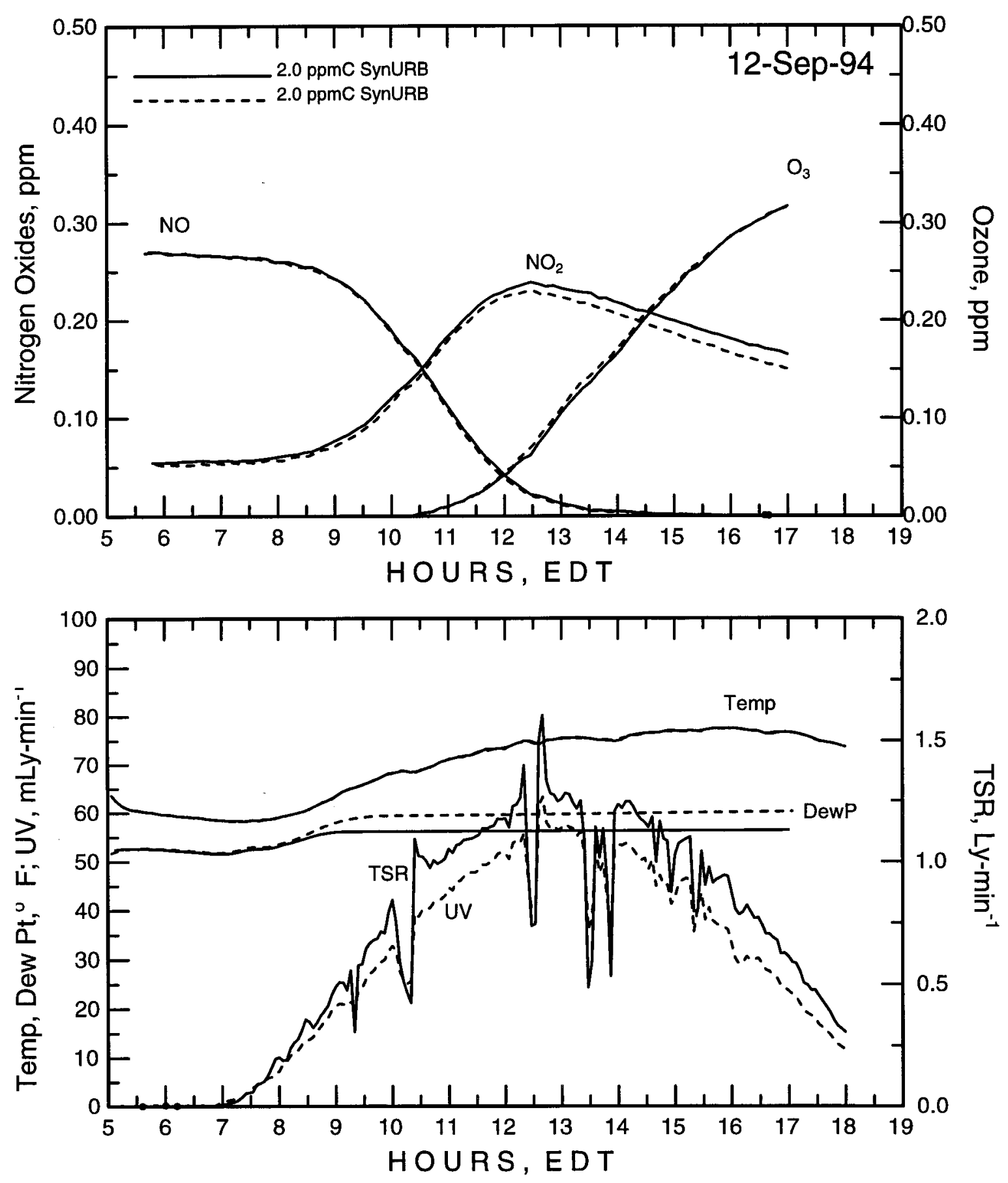

Figure 3.28: Run C5-6:1 SynUrban: $\mathrm{NO}_{\mathrm{x}}$ versus 6:1 SynUrban: $\mathrm{NO}_{\mathrm{x}}$. 


\subsection{Relevant CRC Methanol Study Results}

Because the chamber was re-built and the Teflon film was replaced with new film in early 1994, we found it useful to compare the results from the 1994 experiments with those for the same SynURB and SynIAG mixtures from the CRC study performed in 1991 and 1992. These older CRC experiments will be re-simulated in the next chapter in comparison to the new DOE experiments. To introduce these results in the same way as the DOE runs the initial conditions tables and standard plots are shown here.

Table 3.29 and Figure 3.29 show, on one side, a 6:1 HC-toNO $\mathrm{H}_{\mathrm{x}}$ experiment using 50:50 SynURB/SynIAG; we are not interested in the SynM85 parts of this experiment. Similarly, Table 3.30 and Figure 3.30 show, on one side, a 6:1 $\mathrm{HC}$-toNO $\mathrm{x}_{\mathrm{x}}$ experiment using 50:50 SynURB/SynIAG; we are also not interested in the SynM85 parts of this experiment. Table 3.31 and Figure 3.31 show a dual 6:1 $\mathrm{HC}$-toNO $\mathrm{N}_{\mathrm{x}}$ experiment using only SynURB. Finally, Table 3.32 and Figure 3.32 show a chamber characterization experiment performed with $500 \mathrm{ppm}$ and $250 \mathrm{ppm}$ methane. In addition to these, we will use an older experiment performed with just the paraffin/olefin portion of SynURB and an experiment performed with just the aromatic portion of SynURB (these will be shown in the next chapter). 
Table 3.29: Initial Conditions for JL1092

(Units are ppm or ppmC)

\begin{tabular}{|c|c|c|c|}
\hline \multicolumn{2}{|c|}{ RED SIDE } & \multicolumn{2}{|c|}{ BLUE SIDE } \\
\hline Compound & Amount & Compound & Amount \\
\hline \multicolumn{4}{|c|}{ Initial Nitrogen Oxides } \\
\hline $\mathrm{NO}_{x}$ & $\mathbf{0 . 3 5 1 7}$ & $\mathrm{NO}_{\mathrm{x}}$ & 0.3553 \\
\hline NO & 0.2676 & NO & 0.2691 \\
\hline $\mathrm{NO}_{2}$ & 0.0841 & $\mathrm{NO}_{2}$ & 0.0862 \\
\hline \multicolumn{4}{|c|}{ Initial Volatile Organic Compounds } \\
\hline SynUrban & $\mathbf{1 . 0 3 4 1}$ & SynUrban & 1.0457 \\
\hline SYNURBGAS91 & 0.4029 & SYNURBGAS91 & 0.4143 \\
\hline SYNURBLIQ91 & 0.6177 & SYNURBLIQ91 & 0.6179 \\
\hline $\mathrm{CCHO}$ & 0.0070 & $\mathrm{CCHO}$ & 0.0070 \\
\hline $\mathrm{HCHO}$ & 0.0065 & $\mathrm{HCHO}$ & 0.0065 \\
\hline SynlAG & 1.0413 & SynM85 & 1.0107 \\
\hline SYNIAGGAS91 & 0.5876 & SYNM85GAS91 & 0.3592 \\
\hline SYNIAGLIQ91 & 0.4327 & SYNM85LIQ91 & 0.2339 \\
\hline SYNIAGALD91 & 0.0170 & SYNM85ALD91 & 0.0033 \\
\hline \multirow[t]{2}{*}{$\mathrm{HCHO}$} & 0.0040 & $\mathrm{HCHO}$ & 0.0227 \\
\hline & & $\mathrm{MEOH}$ & 0.4143 \\
\hline Total VOCs & 2.0754 & Total VOCs & 2.0564 \\
\hline $\mathrm{CO}$ & 1.3200 & $\mathrm{CO}$ & 1.3000 \\
\hline \multicolumn{4}{|c|}{ Other Measured Initial Compounds } \\
\hline BVOC9109 & 0.150 & BVOC9109 & 0.150 \\
\hline \multicolumn{4}{|c|}{ Estimated Initial Compounds } \\
\hline HONO & 0.008 & HONO & 0.008 \\
\hline $\mathrm{CH}_{4}$ & 1.790 & $\mathrm{CH}_{4}$ & 1.790 \\
\hline $\mathrm{H}_{2}$ & 0.580 & $\mathrm{H}_{2}$ & 0.580 \\
\hline \multicolumn{4}{|c|}{ Chamber Model Parameters } \\
\hline $\mathrm{NO}_{2} \longrightarrow \mathrm{HONO}$ & $0.1 \times 10^{-3}$ & $\mathrm{NO}_{2} \longrightarrow \mathrm{HONO}$ & $0.1 \times 10^{-3}$ \\
\hline wall. $\mathrm{HNO}_{3} \longrightarrow \mathrm{NO}_{2}$ & $2.0 \times 10^{-3}$ & wall. $\mathrm{HNO}_{3} \longrightarrow \mathrm{NO}_{2}$ & $2.0 \times 10^{-3}$ \\
\hline
\end{tabular}

Three Previous Chamber Events

JL0992.SEG SynUrban vs. SynUrban

JL0892.SEG SynUrban vs. SynUrban

JL0792.SEG CO vs. CO 

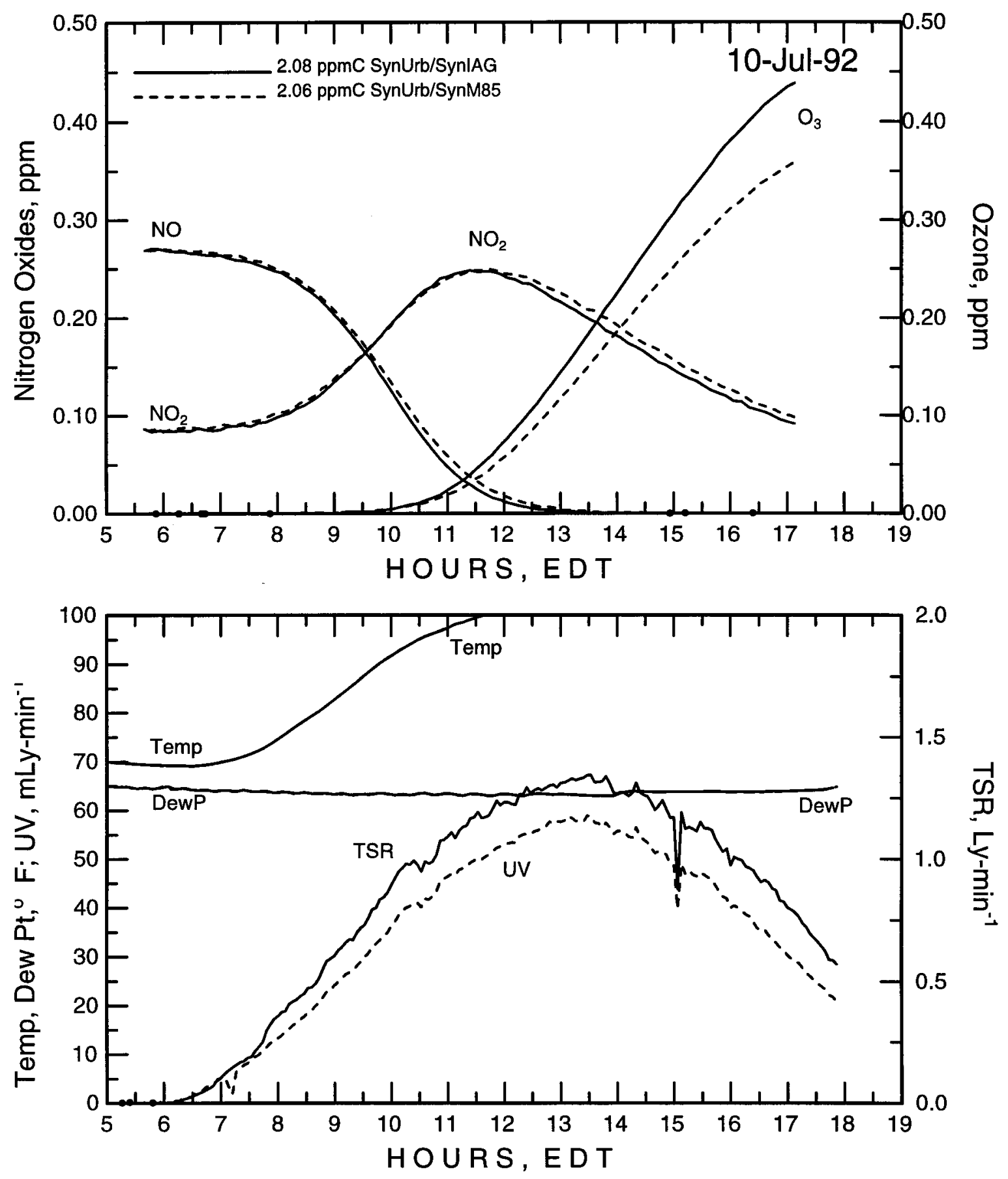

Figure 3.29: Run CRC-2—6:1 SynUrban/SynIAG:NO versus 6:1 SynUrban/SynM85:NOx. 
Table 3.30: Initial Conditions for JL1592

(Units are ppm or ppmC)

\begin{tabular}{|c|c|c|c|}
\hline \multicolumn{2}{|c|}{ RED SIDE } & \multicolumn{2}{|c|}{ BLUE SIDE } \\
\hline Compound & Amount & Compound & Amount \\
\hline \multicolumn{4}{|c|}{ Initial Nitrogen Oxides } \\
\hline $\mathrm{NO}_{x}$ & 0.3393 & $\mathrm{NO}_{x}$ & 0.3365 \\
\hline NO & 0.2760 & NO & 0.2750 \\
\hline $\mathrm{NO}_{2}$ & 0.0633 & $\mathrm{NO}_{2}$ & 0.0614 \\
\hline \multicolumn{4}{|c|}{ Initial Volatile Organic Compounds } \\
\hline SynUrban & 1.1223 & SynUrban & 1.0561 \\
\hline SYNURBGAS91 & 0.4276 & SYNURBGAS91 & 0.4164 \\
\hline SYNURBLIQ91 & 0.6819 & SYNURBLIQ91 & 0.6235 \\
\hline $\mathrm{CCHO}$ & 0.0070 & $\mathrm{CCHO}$ & 0.0070 \\
\hline $\mathrm{HCHO}$ & 0.0058 & $\mathrm{HCHO}$ & 0.0093 \\
\hline SynM85 & 1.1210 & SynlAG & 1.0668 \\
\hline SYNM85GAS91 & 0.3708 & SYNIAGGAS91 & 0.6072 \\
\hline SYNM85LIQ91 & 0.2582 & SYNIAGLIQ91 & 0.4368 \\
\hline SYNM85ALD91 & 0.0033 & SYNIAGALD91 & 0.0170 \\
\hline $\mathrm{HCHO}$ & 0.0612 & $\mathrm{HCHO}$ & 0.0058 \\
\hline MEOH & 0.4275 & & \\
\hline Total VOCs & 2.2433 & Total VOCs & 2.1229 \\
\hline $\mathrm{CO}$ & 1.3200 & $\mathrm{CO}$ & 1.2900 \\
\hline \multicolumn{4}{|c|}{ Other Measured Initial Compounds } \\
\hline BVOC9109 & 0.136 & BVOC9109 & 0.136 \\
\hline \multicolumn{4}{|c|}{ Estimated Initial Compounds } \\
\hline HONO & 0.009 & HONO & 0.009 \\
\hline $\mathrm{CH}_{4}$ & 1.790 & $\mathrm{CH}_{4}$ & 1.790 \\
\hline $\mathrm{H}_{2}$ & 0.580 & $\mathrm{H}_{2}$ & 0.580 \\
\hline \multicolumn{4}{|c|}{ Chamber Model Parameters } \\
\hline $\begin{array}{l}\mathrm{NO}_{2} \longrightarrow \mathrm{HONO} \\
\text { wall. } \mathrm{HNO}_{3} \longrightarrow \mathrm{NO}_{2}\end{array}$ & $\begin{array}{l}1.8 \times 10^{-3} \\
2.0 \times 10^{-3}\end{array}$ & $\begin{array}{l}\mathrm{NO}_{2} \longrightarrow \mathrm{HONO} \\
\text { wall. } \mathrm{HNO}_{3} \longrightarrow \mathrm{NO}_{2}\end{array}$ & $\begin{array}{l}1.4 \times 10^{-3} \\
2.0 \times 10^{-3}\end{array}$ \\
\hline
\end{tabular}

Three Previous Chamber Events

JL1492.SEG SynUrban/IAG vs. SynUrban/M85

JL1392.SEG CarterMix/SynUrban vs. SynUrban

JL1092.SEG SynUrban/IAG vs. SynUrban/M85 

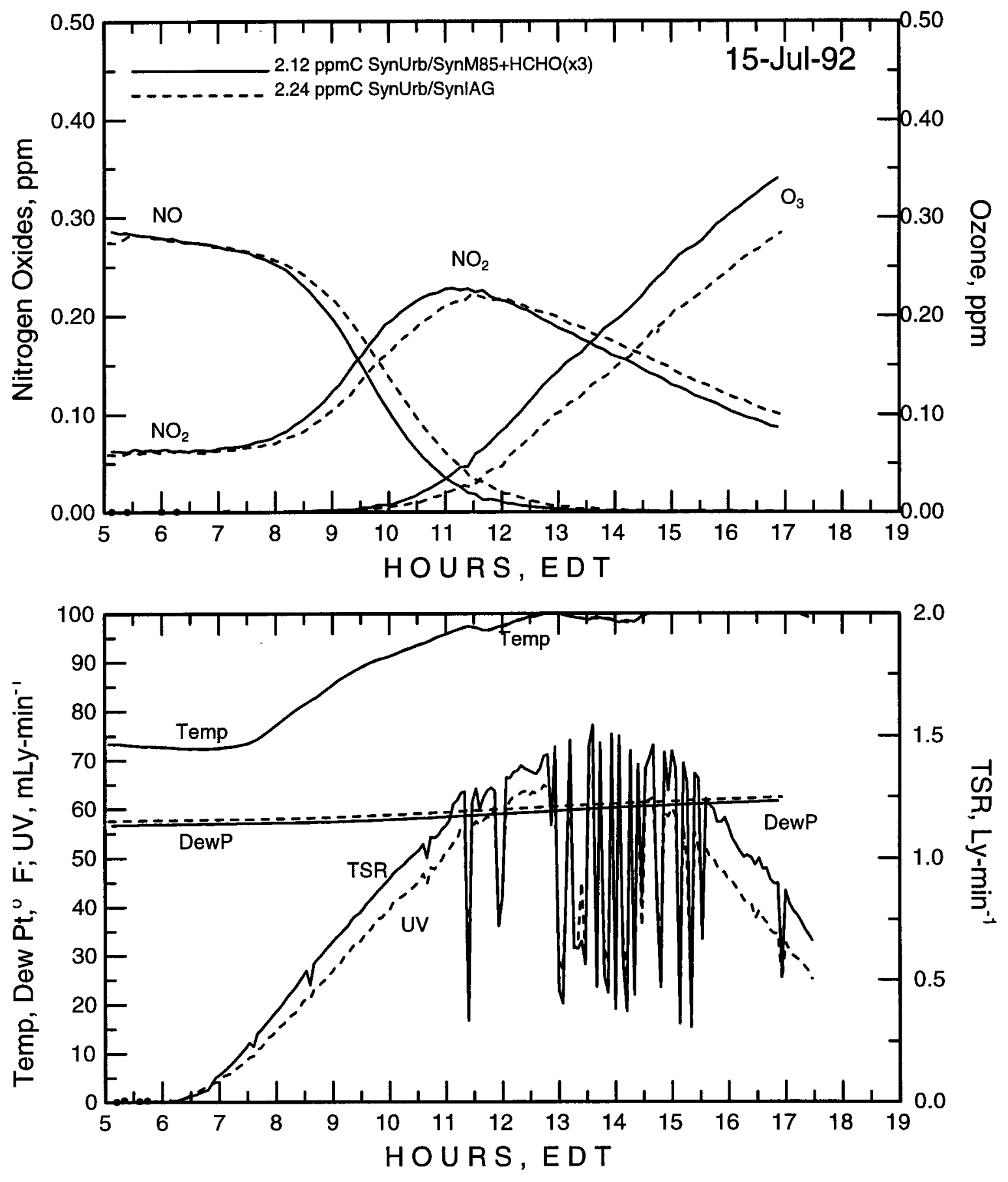

Figure 3.30: Run CRC-11-6:1 SynUrban/SynIAG:NOx versus 6:1 SynUrban/SynM85/HCHO:NOx. 
Table 3.31: Initial Conditions for JL0992

(Units are ppm or ppmC)

\begin{tabular}{|c|c|c|c|}
\hline \multicolumn{2}{|c|}{ RED SIDE } & \multicolumn{2}{|c|}{ BLUE SIDE } \\
\hline Compound & Amount & Compound & Amount \\
\hline \multicolumn{4}{|c|}{ Initial Nitrogen Oxides } \\
\hline $\mathrm{NO}_{x}$ & 0.3800 & $\mathrm{NO}_{x}$ & 0.3770 \\
\hline NO & 0.3170 & NO & 0.3160 \\
\hline $\mathrm{NO}_{2}$ & 0.0640 & $\mathrm{NO}_{2}$ & 0.0610 \\
\hline \multicolumn{4}{|c|}{ Initial Volatile Organic Compounds } \\
\hline SynUrban & 2.4494 & SynUrban & 2.5804 \\
\hline SYNURBGAS91 & 0.8811 & SYNURBGAS91 & 0.8606 \\
\hline SYNURBLIQ91 & 1.5459 & SYNURBLIQ91 & 1.6974 \\
\hline $\mathrm{CCHO}$ & 0.0224 & $\mathrm{CCHO}$ & 0.0224 \\
\hline Total VOCs & 2.4494 & Total VOCs & 2.5804 \\
\hline $\mathrm{CO}$ & 1.450 & $\mathrm{CO}$ & $\mathbf{1 . 4 5 0}$ \\
\hline \multicolumn{4}{|c|}{ Other Measured Initial Compounds } \\
\hline BVOC9109 & 0.150 & BVOC9109 & 0.150 \\
\hline \multicolumn{4}{|c|}{ Estimated Initial Compounds } \\
\hline HONO & 0.008 & HONO & 0.008 \\
\hline $\mathrm{CH}_{4}$ & 1.790 & $\mathrm{CH}_{4}$ & 1.790 \\
\hline $\mathrm{H}_{2}$ & 0.580 & $\mathrm{H}_{2}$ & 0.580 \\
\hline \multicolumn{4}{|c|}{ Chamber Model Parameters } \\
\hline $\begin{array}{l}\mathrm{NO}_{2} \longrightarrow \mathrm{HONO} \\
\text { wall. } \mathrm{HNO}_{3} \longrightarrow \mathrm{NO}_{2}\end{array}$ & $\begin{array}{l}2.5 \times 10^{-3} \\
2.0 \times 10^{-3} \\
\end{array}$ & $\begin{array}{l}\mathrm{NO}_{2} \longrightarrow \mathrm{HONO} \\
\text { wall. } \mathrm{HNO}_{3} \longrightarrow \mathrm{NO}_{2}\end{array}$ & $\begin{array}{l}2.5 \times 10^{-3} \\
2.0 \times 10^{-3} \\
\end{array}$ \\
\hline
\end{tabular}

Three Previous Chamber Events

JL0892.SEG SynUrban vs. SynUrban

JL0792.SEG CO run

JN2892.SEG methacrolein run 

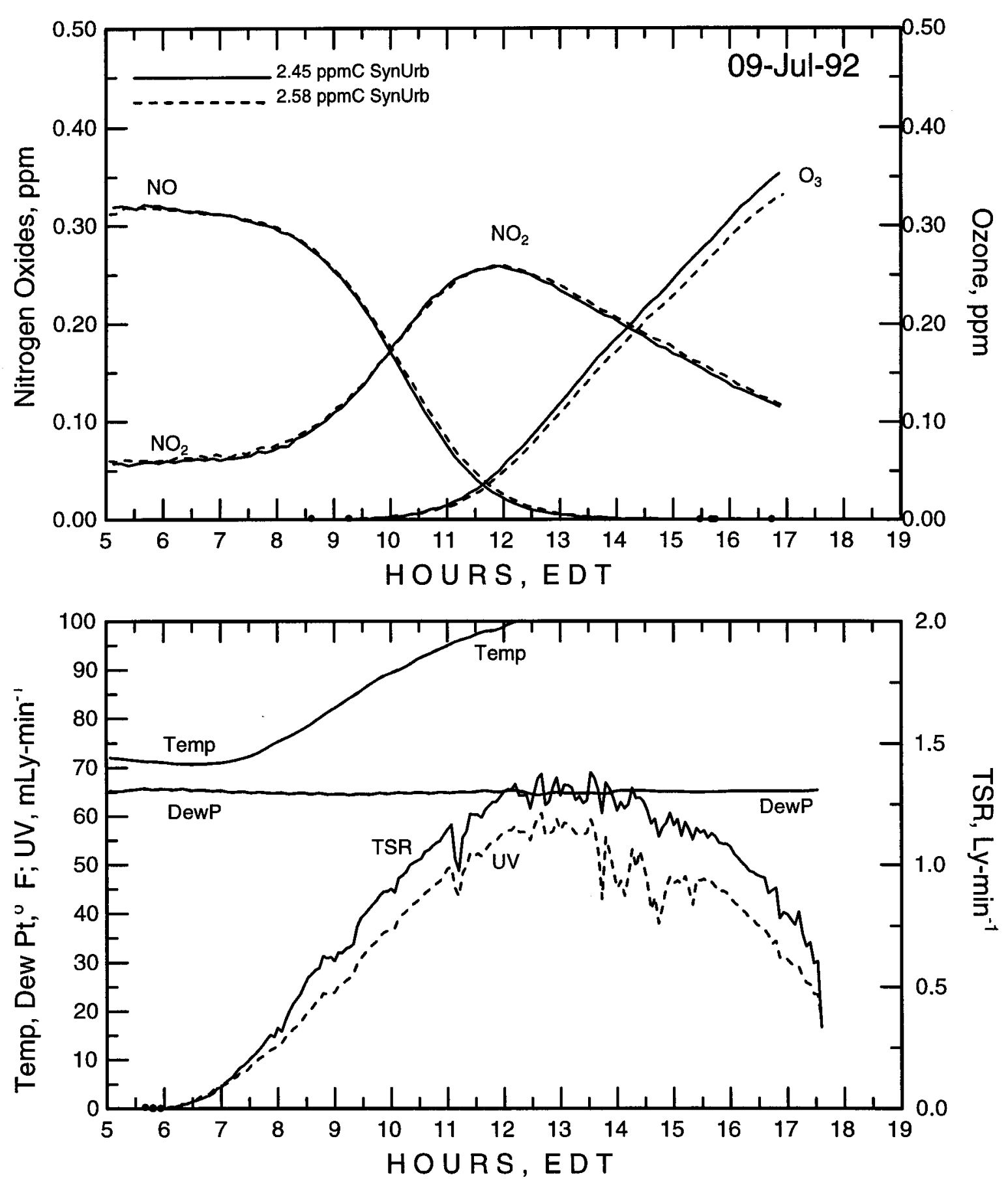

Figure 3.31: Run C5-6:1 SynUrban:NOx versus 6:1 SynUrban:NO . 
Table 3.32: Initial Conditions for JL3092

(Units are ppm or ppmC)

\begin{tabular}{|c|c|c|c|}
\hline \multicolumn{2}{|c|}{ RED SIDE } & \multicolumn{2}{|c|}{ BLUE SIDE } \\
\hline Compound & Amount & Compound & Amount \\
\hline \multicolumn{4}{|c|}{ Initial Nitrogen Oxides } \\
\hline $\mathrm{NO}_{x}$ & 0.3544 & $\mathrm{NO}_{\mathrm{x}}$ & 0.3544 \\
\hline NO & 0.3100 & NO & 0.3109 \\
\hline $\mathrm{NO}_{2}$ & 0.0445 & $\mathrm{NO}_{2}$ & 0.0439 \\
\hline \multicolumn{4}{|c|}{ Initial Volatile Organic Compounds } \\
\hline $\mathrm{CH} 4$ & 500.0000 & $\mathrm{CH} 4$ & 250.0000 \\
\hline \multicolumn{4}{|c|}{ Other Measured Initial Compounds } \\
\hline $\mathrm{CO}$ & 0.310 & $\mathrm{CO}$ & 0.310 \\
\hline BVOC9109 & 0.150 & BVOC9109 & 0.150 \\
\hline \multicolumn{4}{|c|}{ Estimated Initial Compounds } \\
\hline HONO & 0.008 & HONO & 0.012 \\
\hline $\mathrm{CH}_{4}$ & 1.790 & $\mathrm{CH}_{4}$ & 1.790 \\
\hline $\mathrm{H}_{2}$ & 0.580 & $\mathrm{H}_{2}$ & 0.580 \\
\hline \multicolumn{4}{|c|}{ Chamber Model Parameters } \\
\hline $\begin{array}{l}\mathrm{NO}_{2} \longrightarrow \mathrm{HONO} \\
\text { wall. } \mathrm{HNO}_{3} \longrightarrow \mathrm{NO}_{2}\end{array}$ & $\begin{array}{l}1.0 \times 10^{-3} \\
2.0 \times 10^{-3}\end{array}$ & $\begin{array}{l}\mathrm{NO}_{2} \longrightarrow \mathrm{HONO} \\
\text { wall. } \mathrm{HNO}_{3} \longrightarrow \mathrm{NO}_{2}\end{array}$ & $\begin{array}{l}1.4 \times 10^{-3} \\
2.0 \times 10^{-3}\end{array}$ \\
\hline
\end{tabular}

Three Previous Chamber Events

JL2992 aborted run

JL1592.SEG Urb:IAG vs. Urb:M85

JL1492 aborted run 

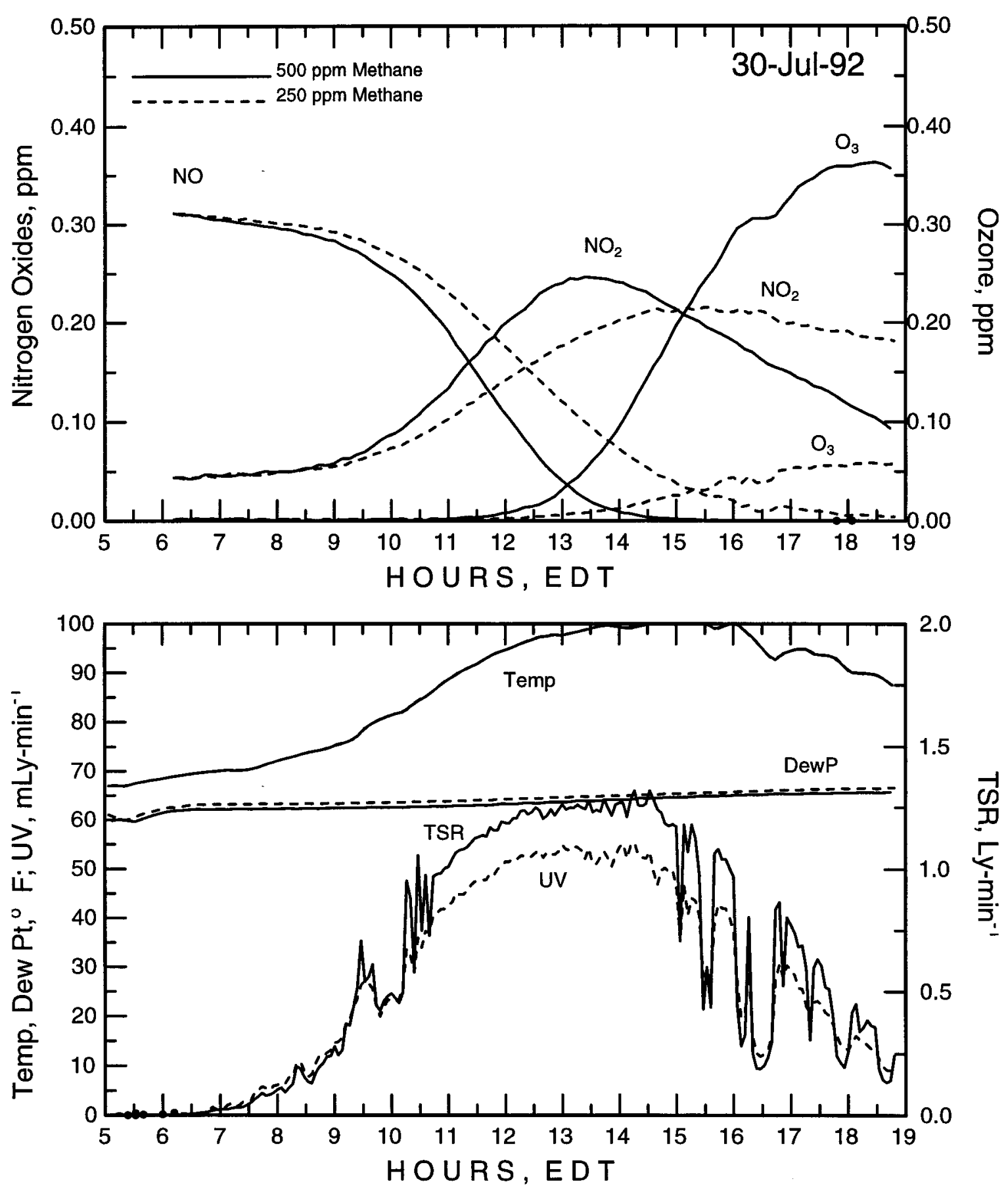

Figure 3.32: Run CRC-C1-250 ppm $\mathrm{CH}_{4}: \mathrm{NO}_{x}$ versus $500 \mathrm{ppm} \mathrm{CH}_{4}: \mathrm{NO}_{x}$. 


\section{Chapter 4}

\section{Modeling Results}

\subsection{The Simulation Process}

Figure 4.1, taken from the EPA's oxidant mechanism evaluation protocol guidance document [5], is a schematic representation of the chamber simulation process. In this figure it was assumed that in a full evaluation of a mechanism, chamber data from at least four different chambers would be used. In our evaluation below, we used only data produced in this DOE funded project and some data produced in the previous CRC methanol fuels project in the UNC dual chamber. Thus, we only have one Chamber Auxiliary Mechanism (i.e., UNCCham.rxn that contains chamber specific reactions for the UNC chamber) that was combined with only one Principal Reaction Mechanisms (i.e., the CB4) to produce one Combined Mechanism (CB4+UNCCham.rxn) that was the one actual tested.

For the UNC chamber, the vast majority of the operations in Figure 4.1 are automated, meaning that computer programs (with supporting input data bases) were used to perform the operations. Thus, documentation of the detail procedures exists in the form of the source codes for these programs. All of the processing programs (and documentation) needed for MS-DOS personal computers to carry out the operations shown in Figure 4.1, as well as all of the chamber data files, the simulation data files, and the summary measures files, are available from UNC. All can be obtained from the Internet in various sub-directories at URL ftp://airsite.unc.edu/pub/pgms/ese_unc/jeffries/.

Detail information on the simulation process, on the UNC Quality Assurance activities and descriptions of standard modeling procedures are available in UNC's CRC final report [4]. This report is also available on the World Wide Web at URL

http://airsite.unc.edu/atmchemunc/harveys_lib/harveyslib.html 


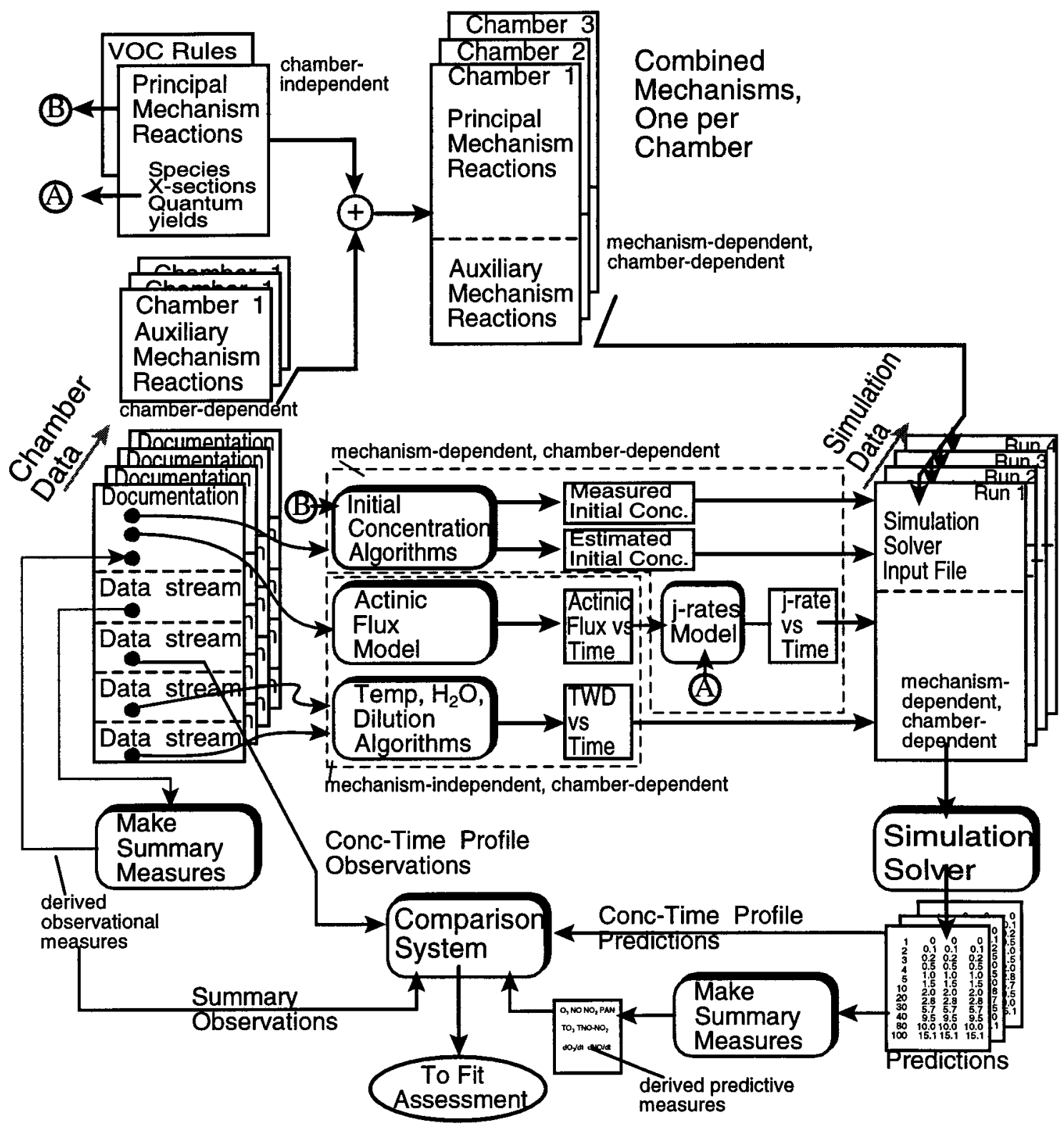

Figure 4.1: The Chamber simulation process. 


\subsection{Temperature Effects}

After the outdoor smog chamber was rebuilt in 1994, we observed much lower afternoon temperature maximums compared to previous recent seasons. In checking over the the entire 20 -year data set, we determined that in experiments conducted in the late 1970s and early 1980s the reported afternoon chamber temperatures were also lower than those measured in the early 1990's.

Fortunately, as part of our standard QA/QC efforts, we had installed a backup temperature measurement system to allow for frequent checking of the chamber's standard chamber temperature measurement system. This comparison record is extensive, spanning five years, and it includes frequent comparison of morning and afternoon temperature measurements by the two systems. In addition, on noticing the lower temperature measurements in 1994, we checked the temperature measurement system calibration with a reference mercury thermometer used in place with the other two systems. These efforts have assured us that the reported temperature data are accurate.

It is clear that changing the Teflon film after more than 15 years of use resulted in a large chamber temperature difference before and after the change. From direct analysis of the film surface, we know that the old Teflon film walls were coated with very fine particulate matter. We actually determined, by secondary ion emission time-of-flight mass spectrometry (SIMS/TOF) on samples of the aged film, that it contained significant sulfate on the surface. Because we have never injected sulfur-containing compounds into the chamber, we surmised that the sulfate came from atmospheric deposition of airborne sulfate particles introduced during chamber venting with ambient air. The charge on the Teflon walls attracts and holds such fine particulates. In addition, in the late 1980s and early 1990s we conducted inside and outside spectral radiation measurements in the chamber as part of the actinic flux mathematical model development. These spectral data show very elevated near-infrared radiation inside the chamber. Because Teflon film itself has only a few bands in the infrared, the increase in chamber heat is most likely due to an enhanced greenhouse effect caused by the particles absorbing outgoing radiation and reradiating it back into the chamber. The new Teflon film is much clearer than the old film, has not acquired much of a particle layer, and thus exhibits a significantly smaller greenhouse effect than did the older film.

\subsubsection{Effect of Temperature on Ozone}

We compared all replicate complex mixture smog chamber experiments conducted between 1990 and 1994 to determine the effect of the different temperature conditions on the experiments (we excluded low $\mathrm{HC}: \mathrm{NO}_{\mathrm{x}}$ ratio experiments and October experiments). Two types of replicate experiments of interest have been found: SynURB and the 50:50 blend of the IAG with the SynURB at two different levels of $H C$. Experiments used for comparison were similar in terms of initial conditions and sunlight quality. The following figures present the 
nitrogen oxides, ozone, temperature, dew point data, and the UV time series (TSR is omitted for clarity).

\section{SynURB-only experiments}

Figure 4.2 compares experiments conducted in the fall on three different days, September 12, and 15 of 1994 and September 12 1991. The initial conditions were similar. The 1991 day was hotter than the 1994 days. Little difference can be observed in the experimental results. The 1991 day did, however, produce less ozone even though it was the hotter day. Notice that the hottest day does have the lowest afternoon " $\mathrm{NO}_{2}$ ". (Remember this is not really $\mathrm{NO}_{2}$ but any nitrogen species that can be converted to NO by the instrument's converter. This includes PAN, organic nitrates, and some nitric acid.) The hotter conditions should result in less PAN, which is very temperature-labile; thus, more $\mathrm{NO}_{2}$ should be converted to organic nitrates and nitric acid. 

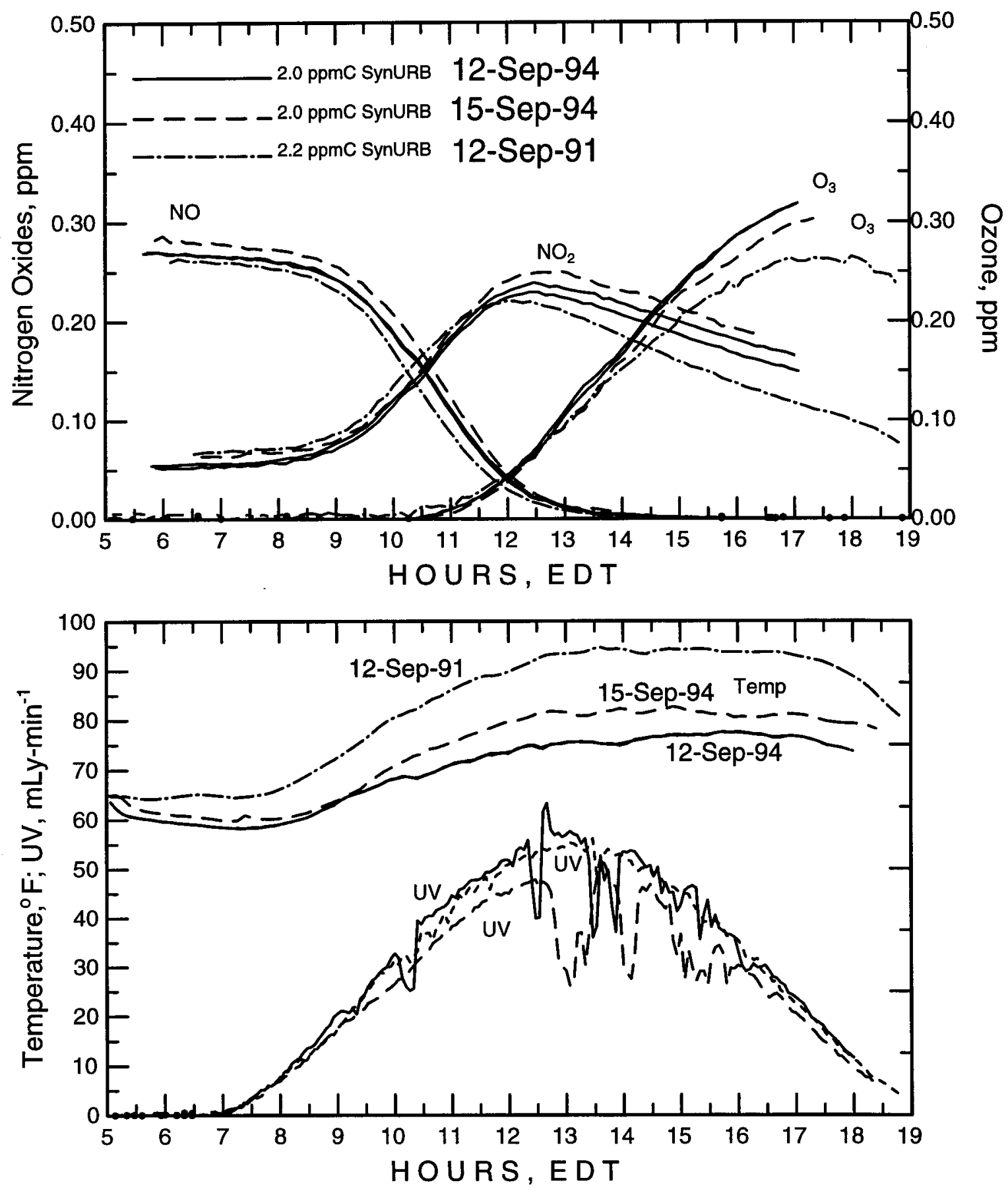

Figure 4.2: Comparison of SynURB-only experiments from 1991 and 1994. 


\section{SynURB/SynIAG blend experiments}

Figure 4.3 compares two blended IAG and SynURB experiments conducted in the fall. The initial conditions were within 5\%. The 1991 experiment was much hotter than the 1994 experiment, yet little difference is observed in the experimental results, and the differences that were observed might be explainable in terms of the "holes" in the afternoon solar radiation (caused by clouds) in the 1994 experiment. Notice again the difference in afternoon " $\mathrm{NO}_{2}$ ".

Figure 4.4 compares two blended IAG and SynURB experiments conducted in the summer. The initial conditions are similar except that the 1992 day contained approximately $15 \%$ more $\mathrm{NO}_{\mathrm{x}}$. The 1992 experiment was much hotter than the 1994 experiment. Again very little difference was observed between these two runs. We were quite surprised by this outcome. One normally assumes that additional $\mathrm{NO}_{\mathrm{x}}$ should have resulted in more ozone production, but the fact that the extra $\mathrm{NO}_{x}$ was mostly $\mathrm{NO}_{2}$ may have led to increased $\mathrm{OH}$ radical termination.

Figure 4.5 compares two blended IAG and SynURB experiments conducted in the summer. The initial conditions are similar except that the 1992 experiment contained approximately $10 \%$ more $\mathrm{NO}_{\mathrm{x}}$. The 1992 experiment was much hotter than the 1994 experiment. Again, little difference was observed in the ozone experimental results. 

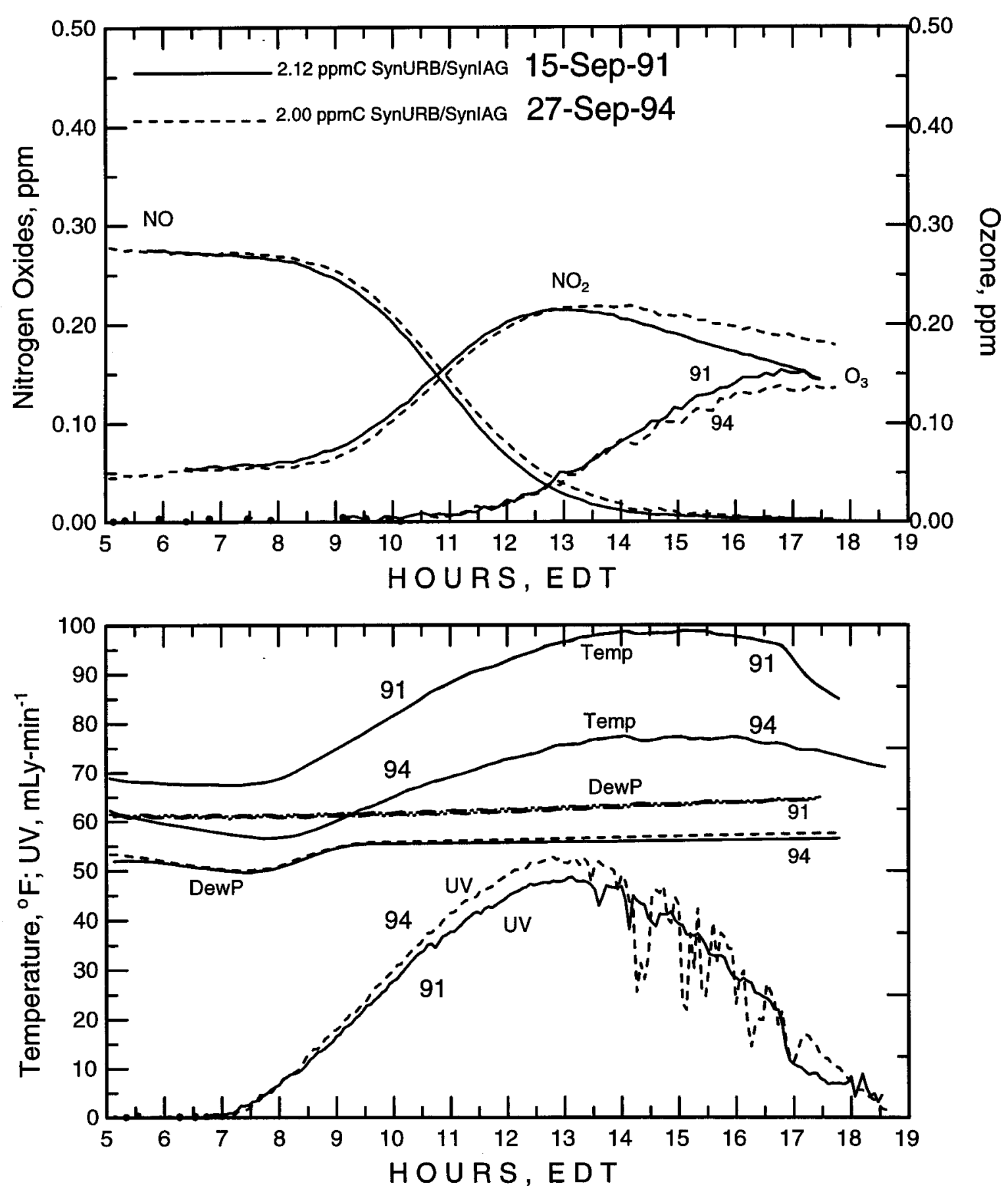

Figure 4.3: Comparison of SynURB/SynIAG experiments from 1991 and 1994. 

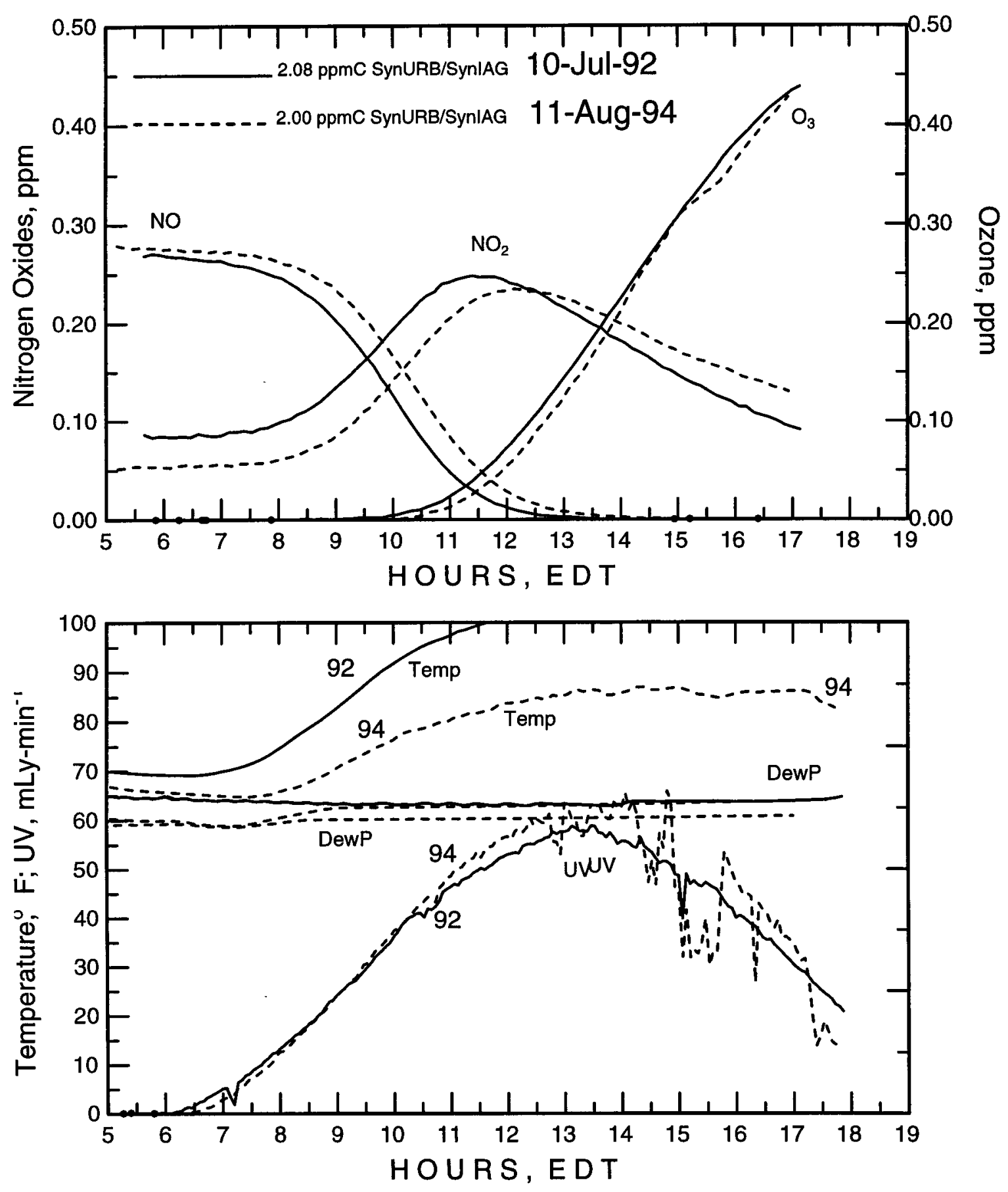

Figure 4.4: Comparison of SynURB/SynIAG experiments from 1992 and 1994. 

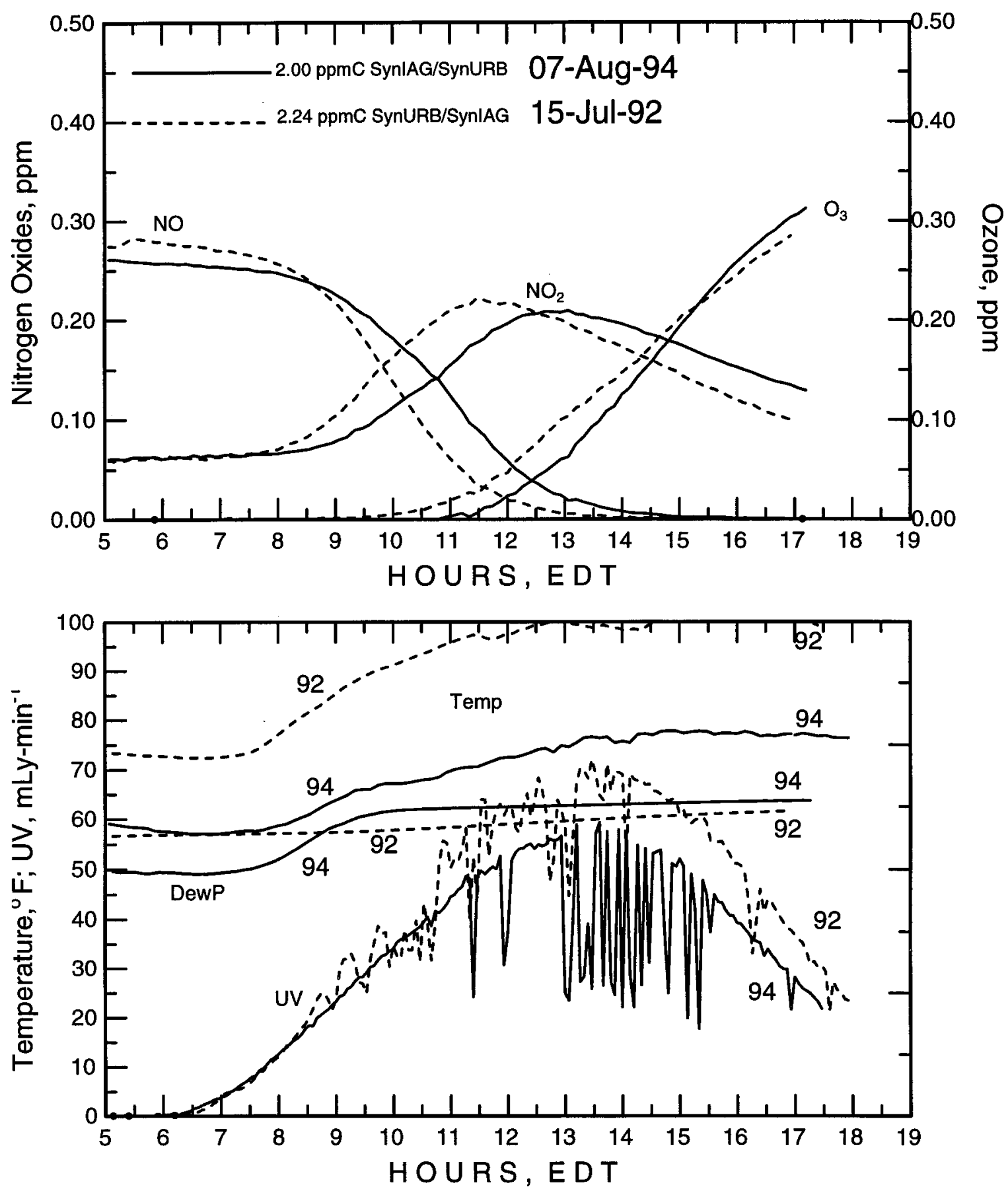

Figure 4.5: Comparison of SynURB/SynlAG experiments from 1992 and 1994. 
Two other experiments with similar initial conditions may be compared with the August 11, 1994 experiment. These were conducted on August 7, 1994, August 16, 1991 and August 25, 1992 (see Figure 4.6). Solar radiation was similar on these days (except that it was slightly more cloudy on August 25). The two pre-1994 days were not as hot as were the July 10 and 15,1992 days, being only 10 to $15^{\circ} \mathrm{F}$ warmer. This comparison shows much less ozone production in the 1991 and 1992 experiments even though these had slightly more initial $\mathrm{NO}_{\mathrm{x}}$. It appears that if the sun had been more clear on the August 25, 1992 day, it might have matched the ozone production better with the August 16, 1991 day.

Comparison can also be made at a higher $\mathrm{HC}: \mathrm{NO}_{x}$ ratio (see Figure 4.7). Again, the August 26, 1994 experiment produced more ozone than the closely matching replicate experiments conducted on August 21, 1991 and August 31, 1992. The two older experiments shown here were not as hot as the July 10 and 15,1992 experiments, being only 10 to $15^{\circ} \mathrm{F}$ hotter. 

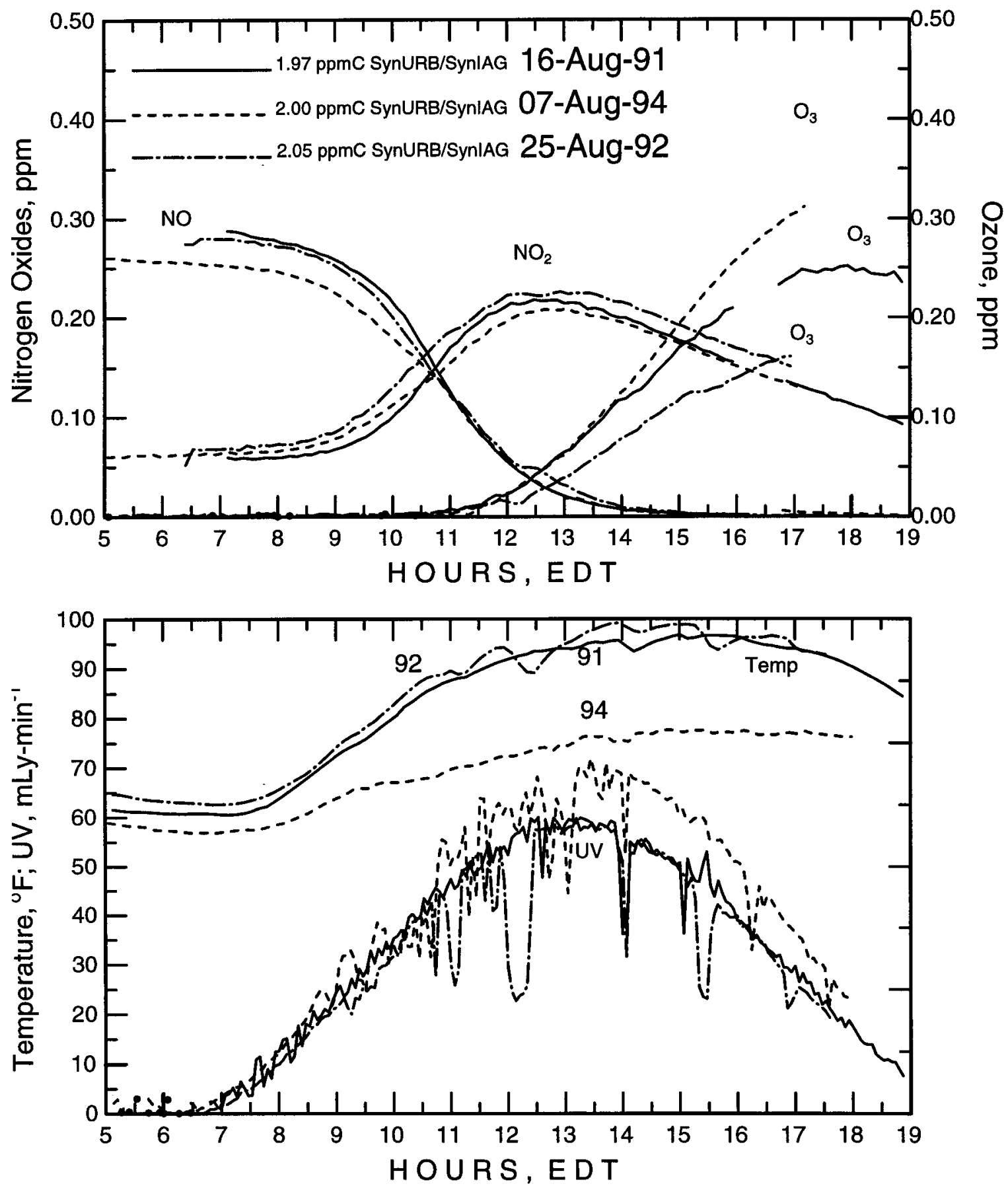

Figure 4.6: Comparison of SynURB/SynlAG experiments from 1992 and 1994. 

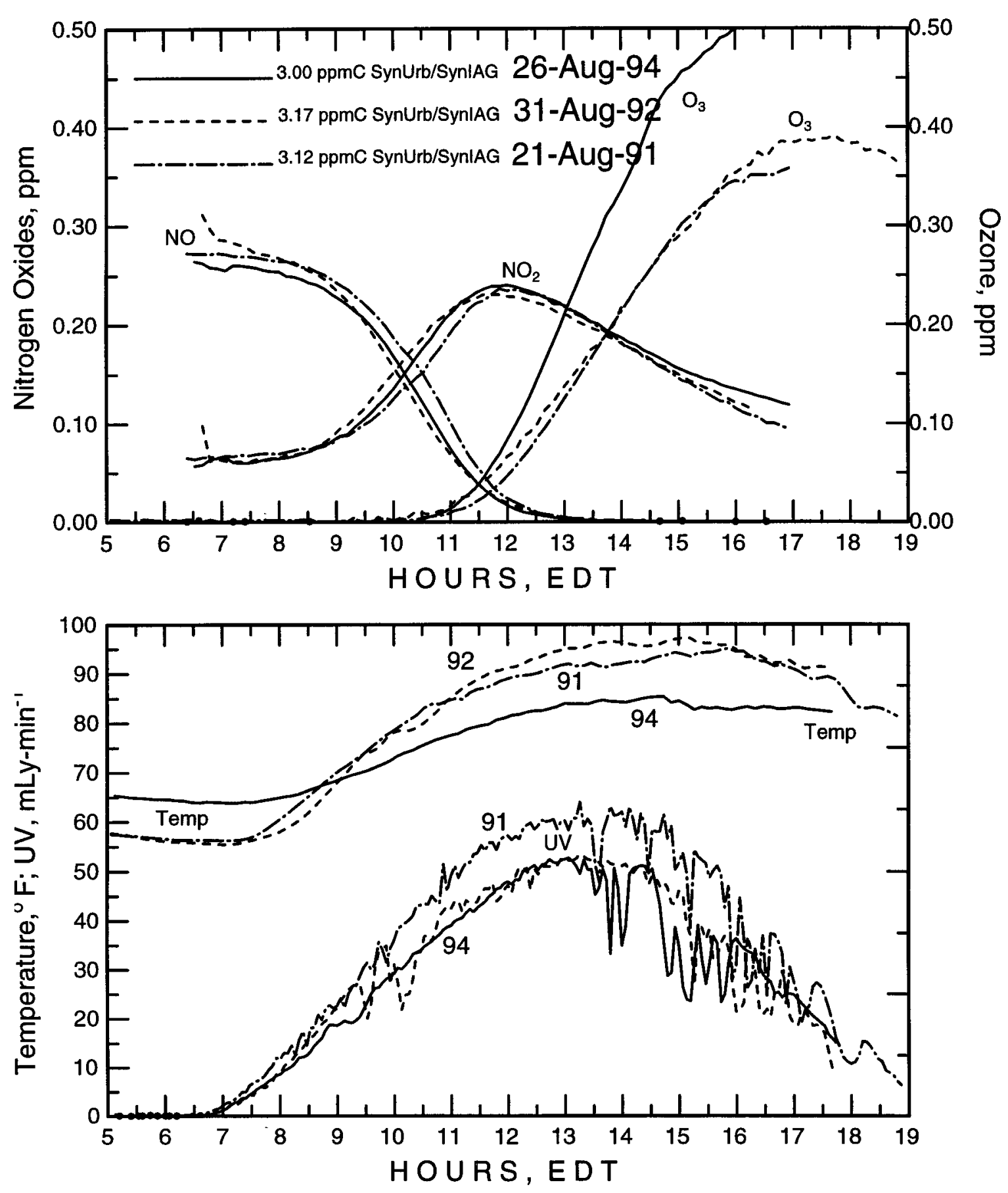

Figure 4.7: Comparison of SynURB/SynIAG experiments from 1992 and 1994. 


\subsubsection{Modeled Temperature Effect}

\section{Methane}

As a standard chamber characterization experiment, we performed high concentration methane/NO $\mathrm{x}$ experiments. Because the chemistries of methane and its products are believed to be fully understood, these methane experiments served to verify that we have the physical parameters of the chamber well characterized. This means that any difficulties in simulating the $\mathrm{CH}_{4} / \mathrm{NO}_{x}$ experiments implies that something is wrong with the specified chamber conditions, not that anything is wrong with the principal methane chemical reaction mechanisms. In the CRC study performed in 1991 and 1992 , we ran several $\mathrm{CH}_{4}$ experiments. One that is relevant to this discussion is shown in Figure 4.8 and Figure 4.9. The figures include the predictions of the CB4 mechanism using the photolytic and temperature conditions measured on this day. Clearly the CB4 mechanism (with an update to the $\mathrm{CH}_{4}+$ $\mathrm{OH}$ rate constant in the published $\mathrm{CB} 4$ mechanism) simulates methane/NO $\mathrm{N}_{\mathrm{x}}$ conditions well, and this supports the conclusion that the physical conditions specified for the chamber and the inorganic and basic organic radical chemistries in the CB4 mechanism are reasonably accurate representations of the real-world conditions.

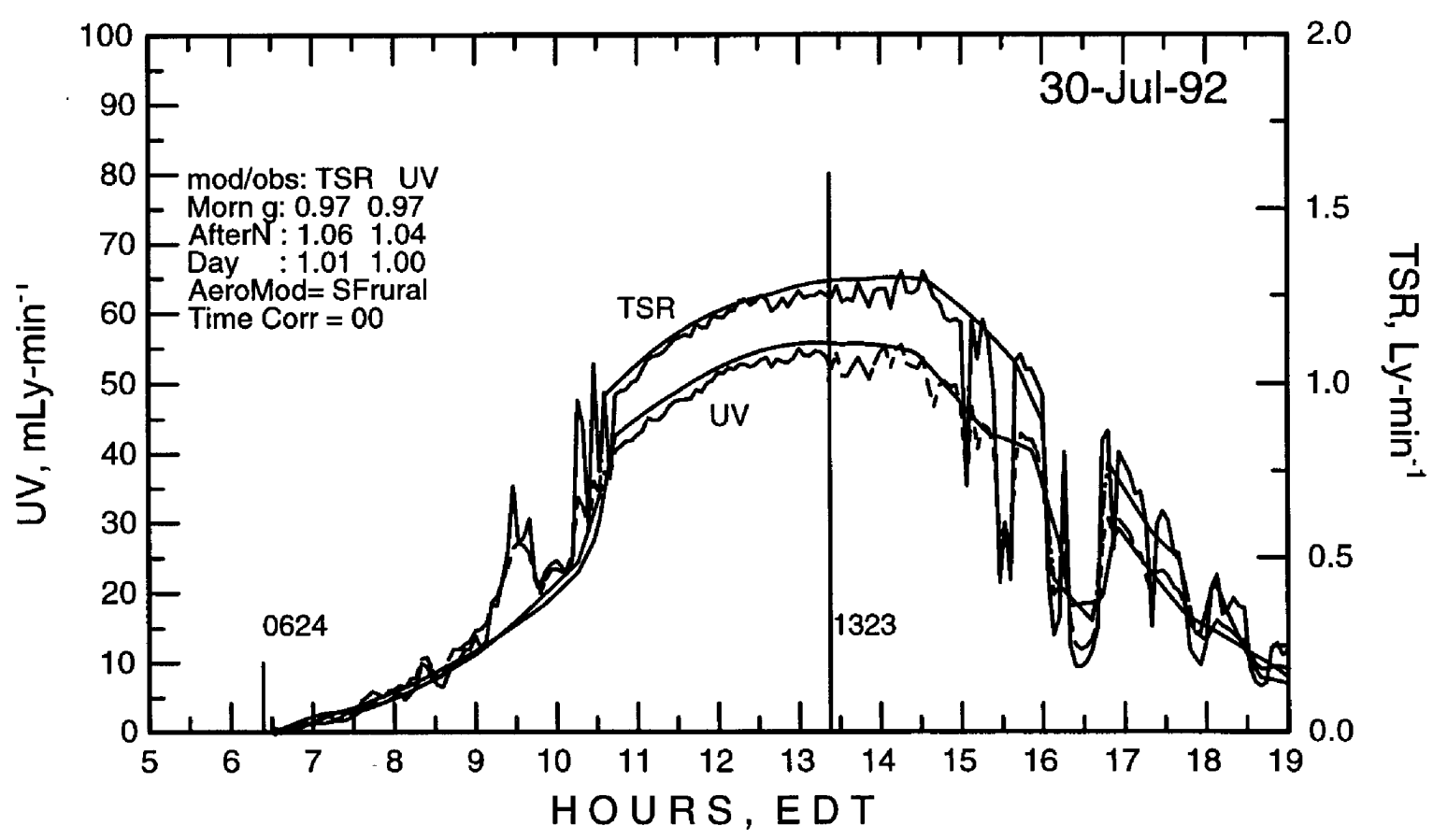

Figure 4.8: Solar radiation simulation for JL3092. 

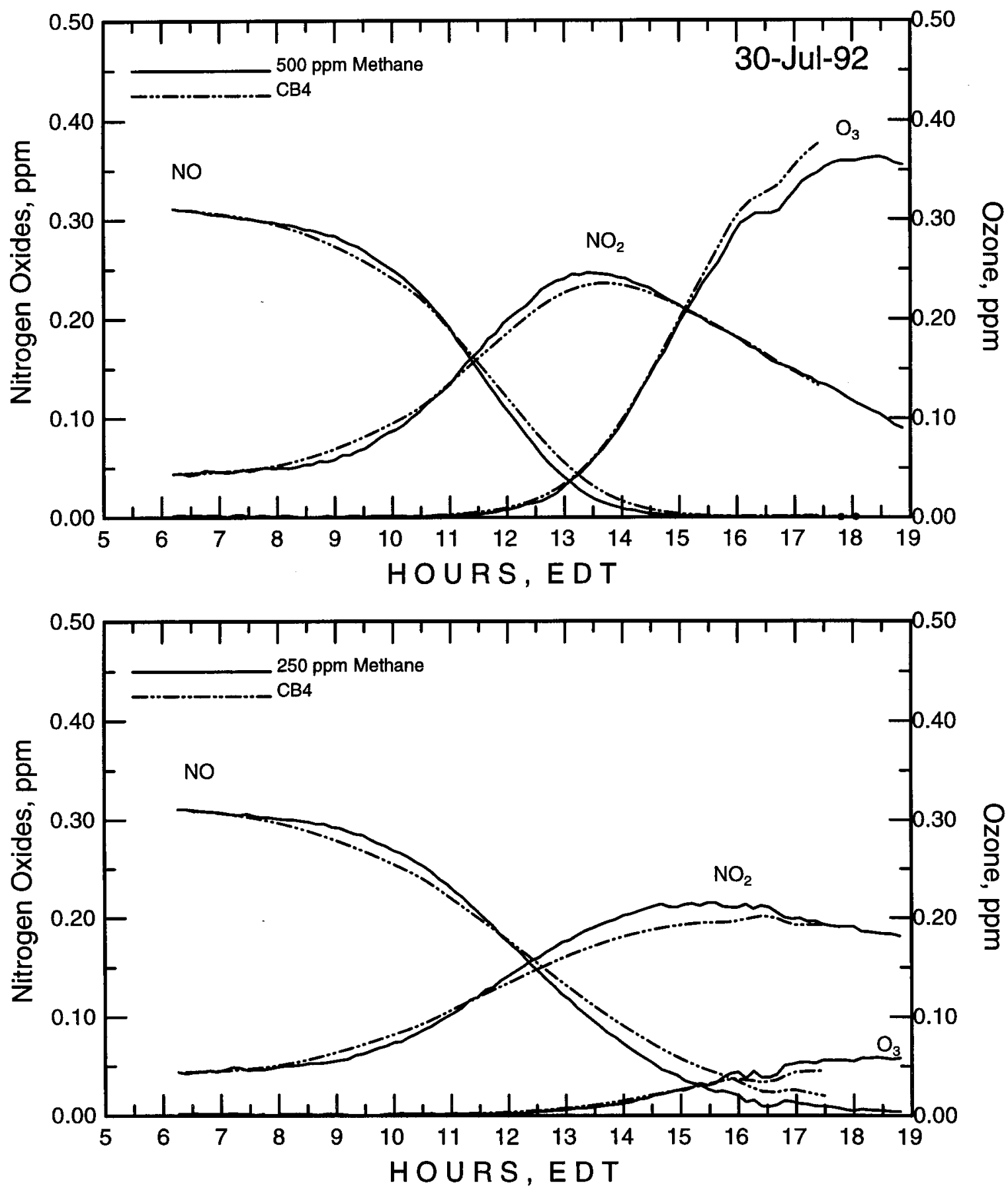

Figure 4.9: Carbon Bond Four simulation of methane/ $\mathrm{NO}_{\mathrm{x}}$ experiment. 


\section{SynURB Mixtures}

Figure 4.10 shows simulations of two special experiments we performed in the CRC project to investigate the difficulties simulations with the CB4 had in fitting the SynURB experiments. In the top plot, the only species included in the VOCs injected into the chamber were alkanes and alkenes. For this condition, the simulations agreed quite well with the observations. In the bottom plot of the figure, the only species included in the VOCs injected into the chamber were aromatic species. The models performed very poorly for this condition, grossly overpredicting the observations. These results are consistent with the methane simulation shown just above.

The CB4 mechanism exhibited poor performance in simulating the aromatic VOC components. The reactions of aromatic species that are used in CB4 were formulated without knowledge of the details of the true reaction mechanisms or even pathways for aromatic oxidation. The properties of the model species were adjusted to permit the gross features (i.e., $\mathrm{O}_{3}$ time-series and VOC decay) of a very limited number (ca. 6) of single aromatic compounds experiments to be simulated. As will be shown below, however, CB4 does fit the cooler chamber experiments for SynURB mixtures. This suggests that most of the temperature problem in the mechanism is located in the aromatics portion. Only new fundamental knowledge of aromatic oxidation (see Chapter 7) can solve these mechanism formulation problems.

In the CRC report, we showed that the simulations of the complex VOC mixture experiments performed with the CB4 mechanism were very reactive relative to the chamber observations, on average predicting ozone about $150 \%$ too high. As will be shown later in this chapter, however, the CB4 simulations of the 1994 experiments are in much better agreement with the chamber observations. This led us to conduct a series of modeling experiments in which we substituted the 1994 temperatures for the 1992 temperatures in the 1992 simulations (see Figure 4.11) to see if this was the cause of the model's large over-prediction in the CRC study. These results are shown in Figure 4.10 to 4.17. 

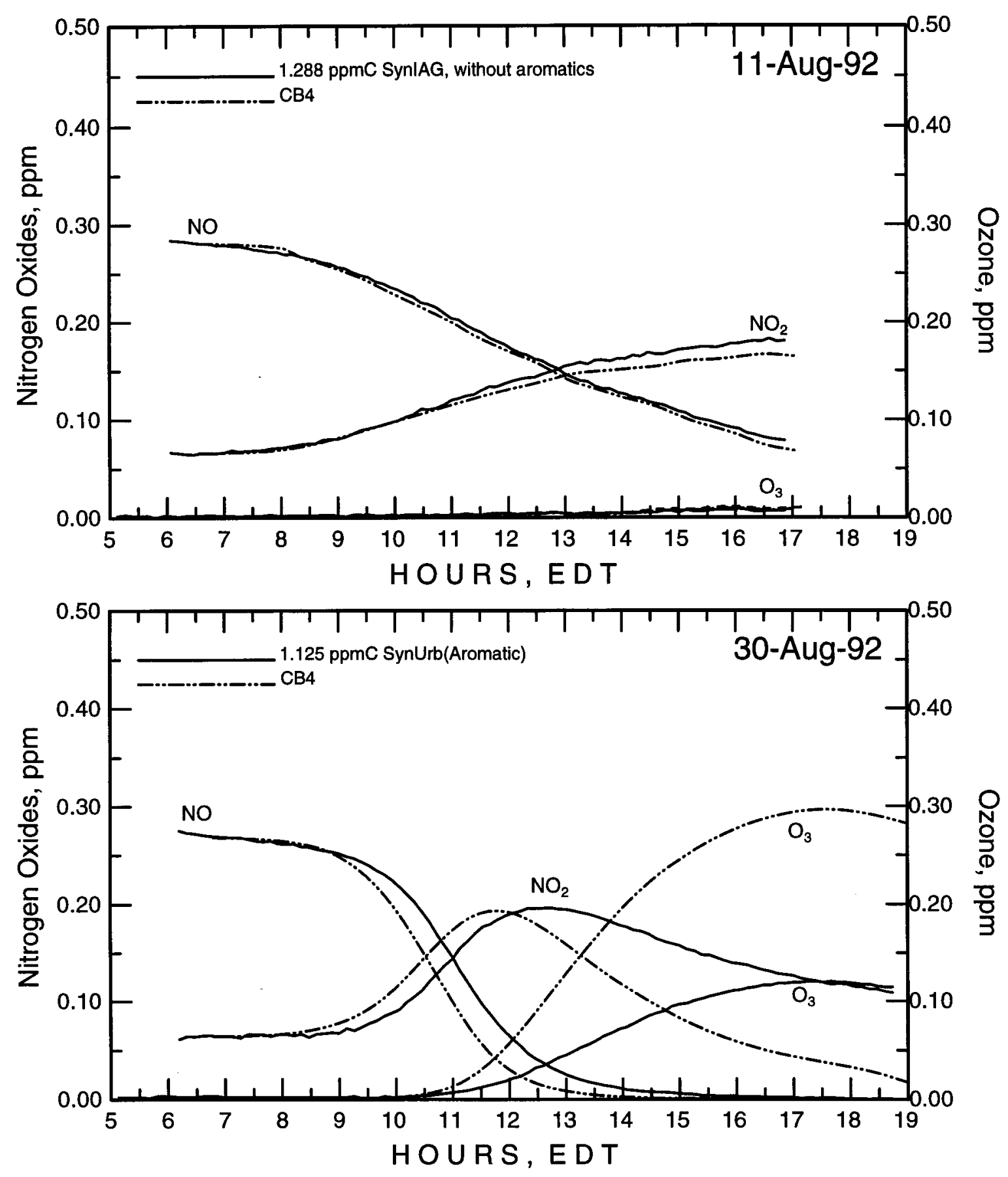

Figure 4.10:. EPA's Carbon Bond Four photochemical reaction mechanisms simulation of: TOP: Urban alkane/alkene-only urban voc mixture (39 species); BOTTOM: Urban aromatics-only urban voc mixture (13 species) in UNC's Outdoor Smog Chamber. 


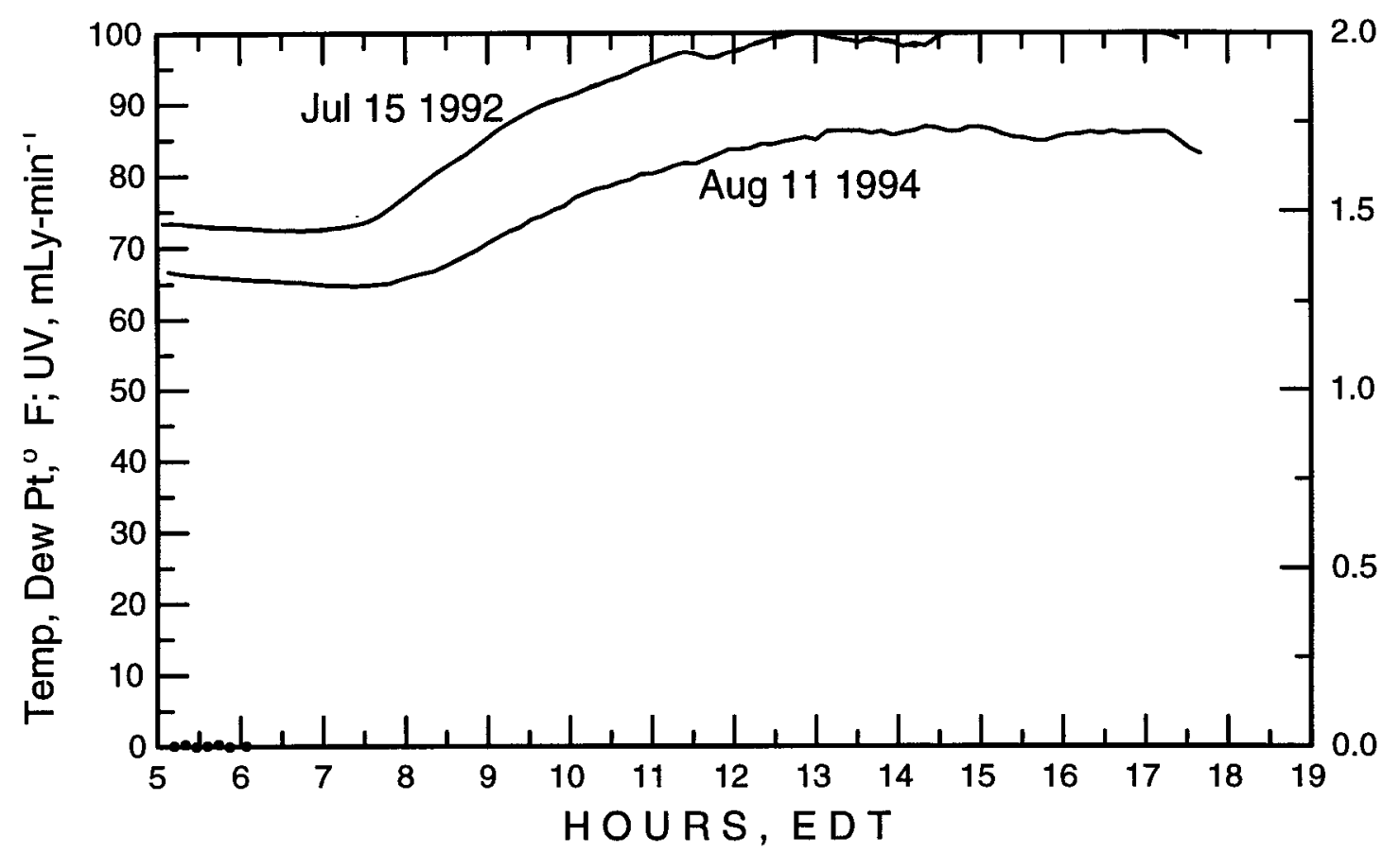

Figure 4.11: Comparison of in chamber temperature in 1992 and 1994.

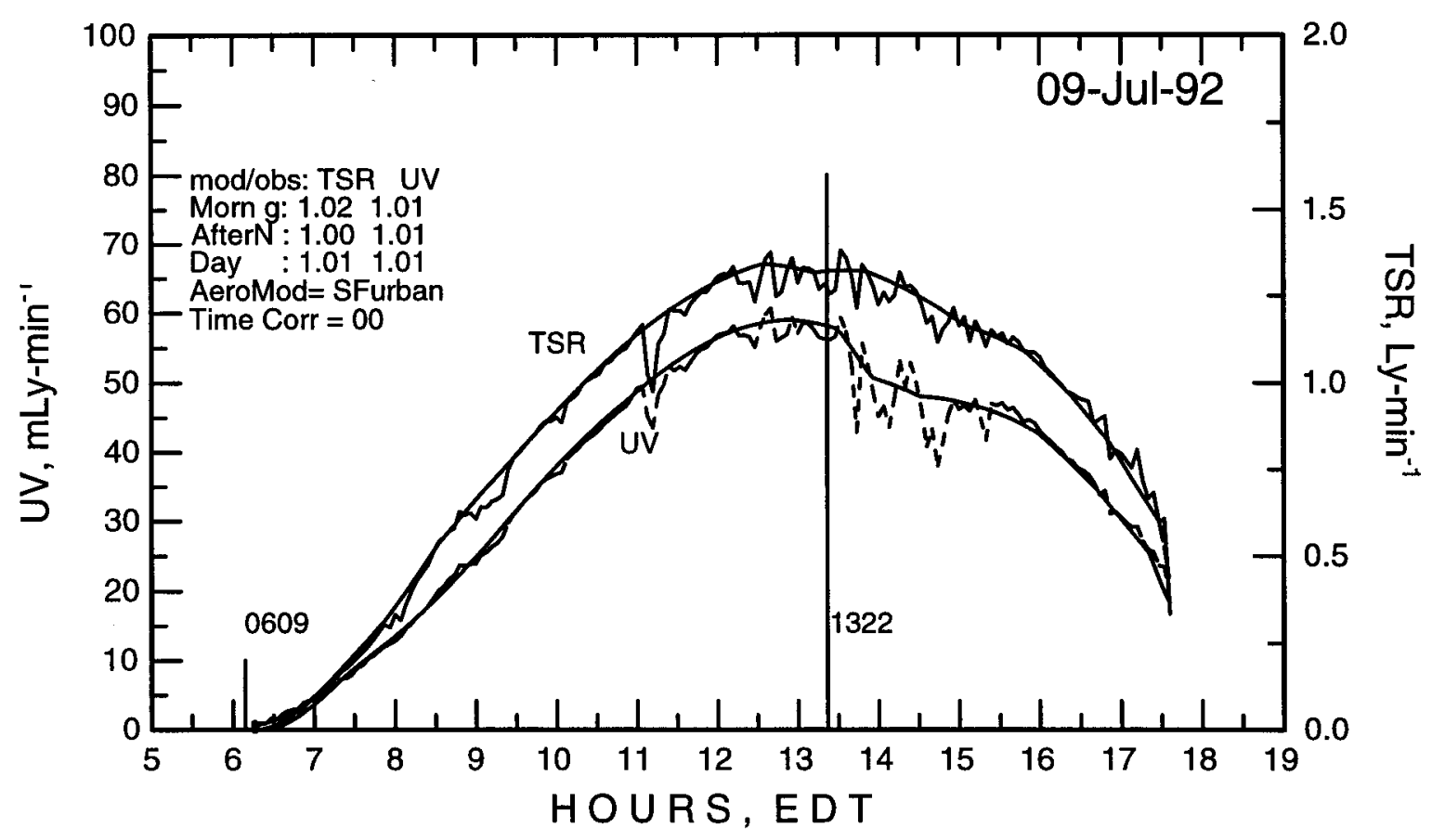

Figure 4.12: Solar radiation simulation for JL0992. 

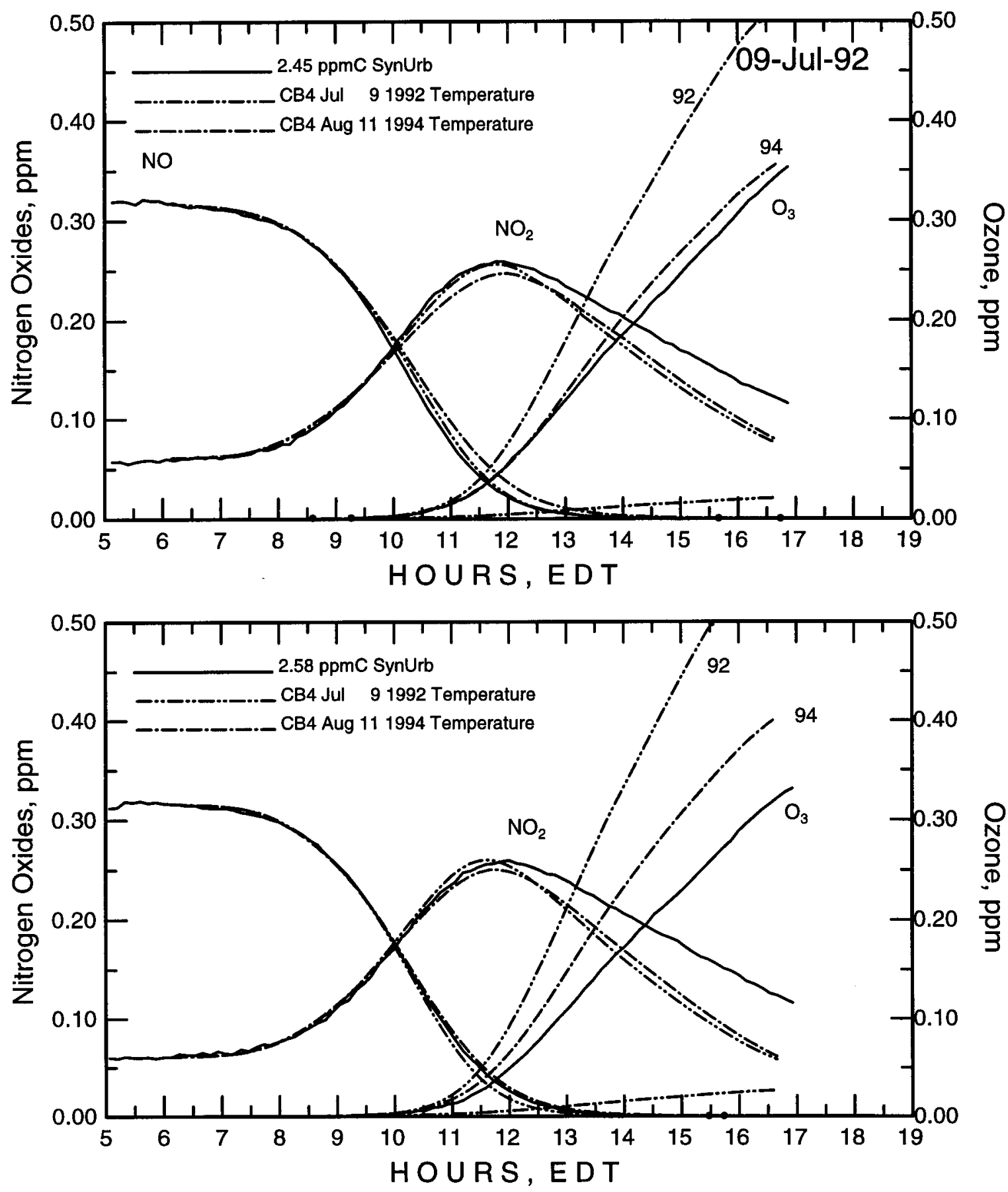

Figure 4.13: Carbon Bond Four simulation of JL0992 at different temperatures. 


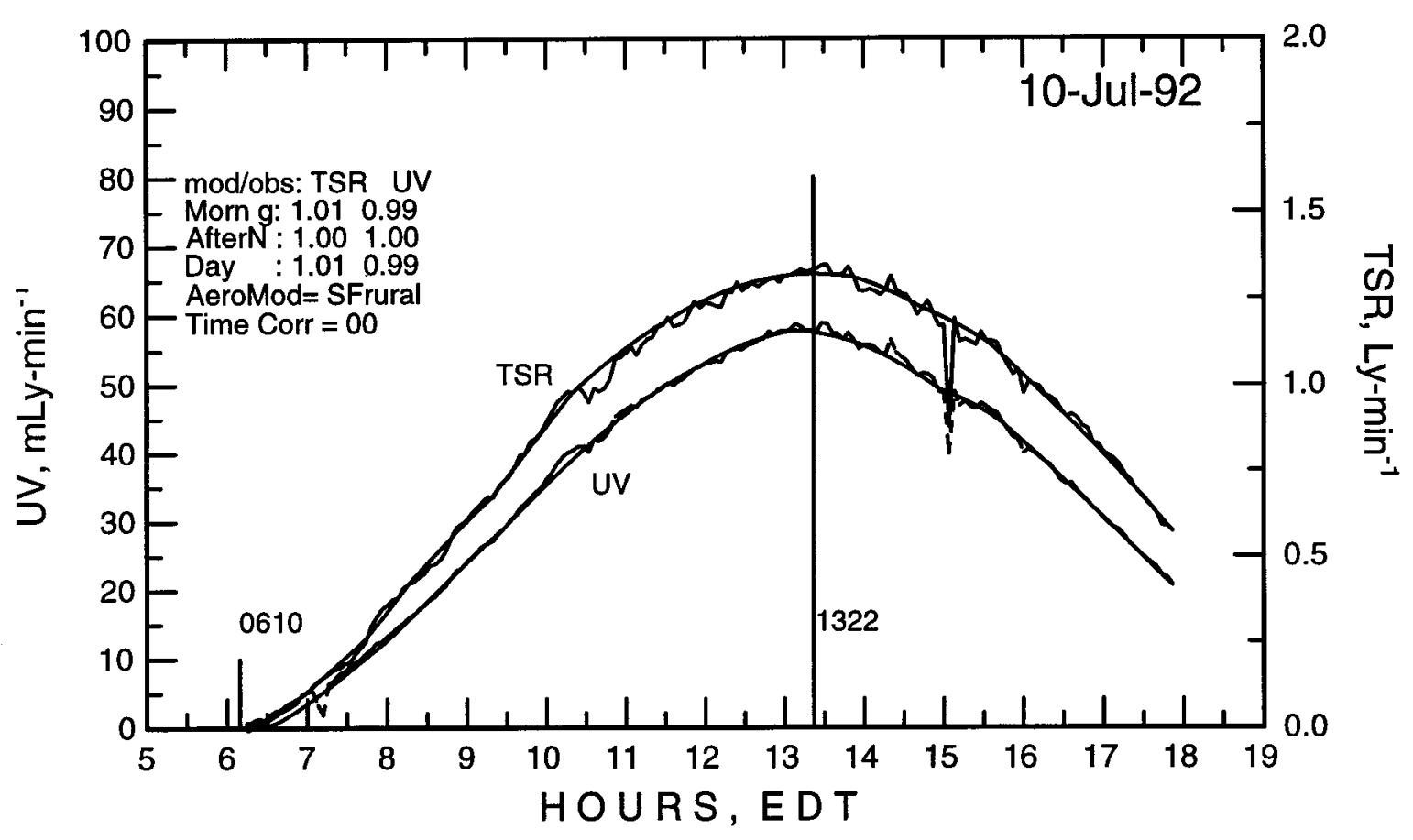

Figure 4.14: Solar radiation simulation for JL1092.

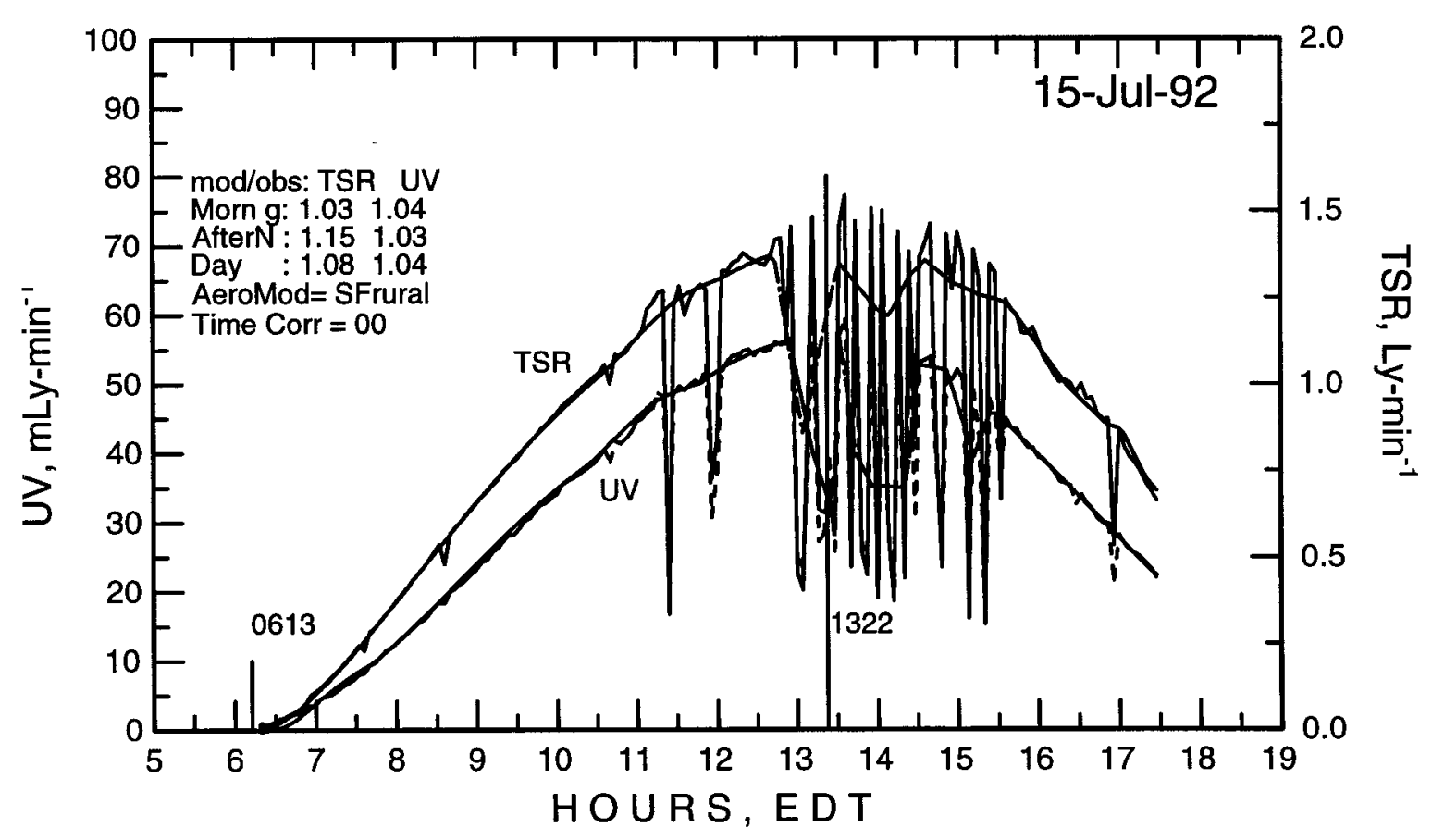

Figure 4.15: Solar radiation simulation for JL1592. 

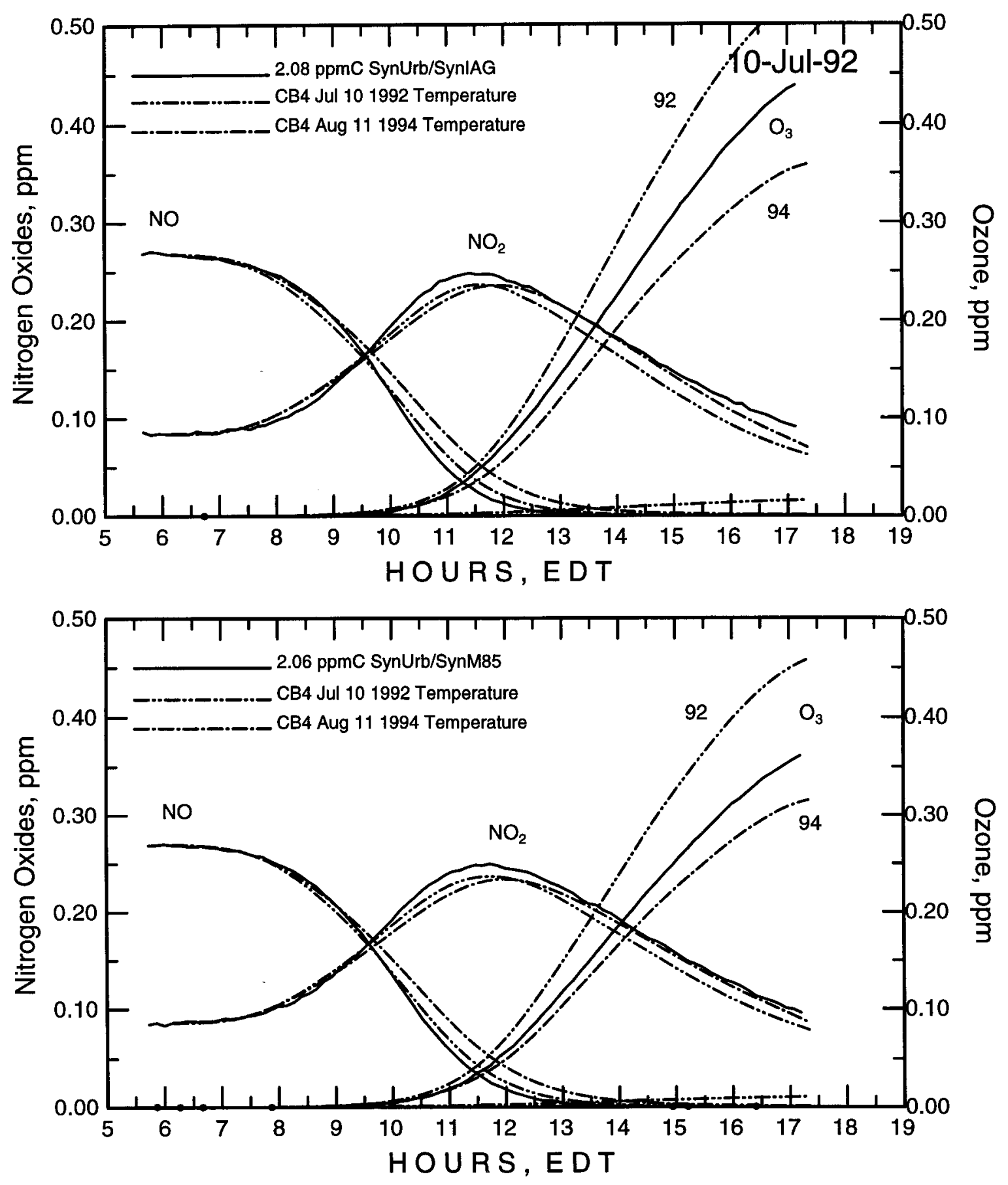

Figure 4.16: Carbon Bond Four simulation of JL1092 at different temperatures. 

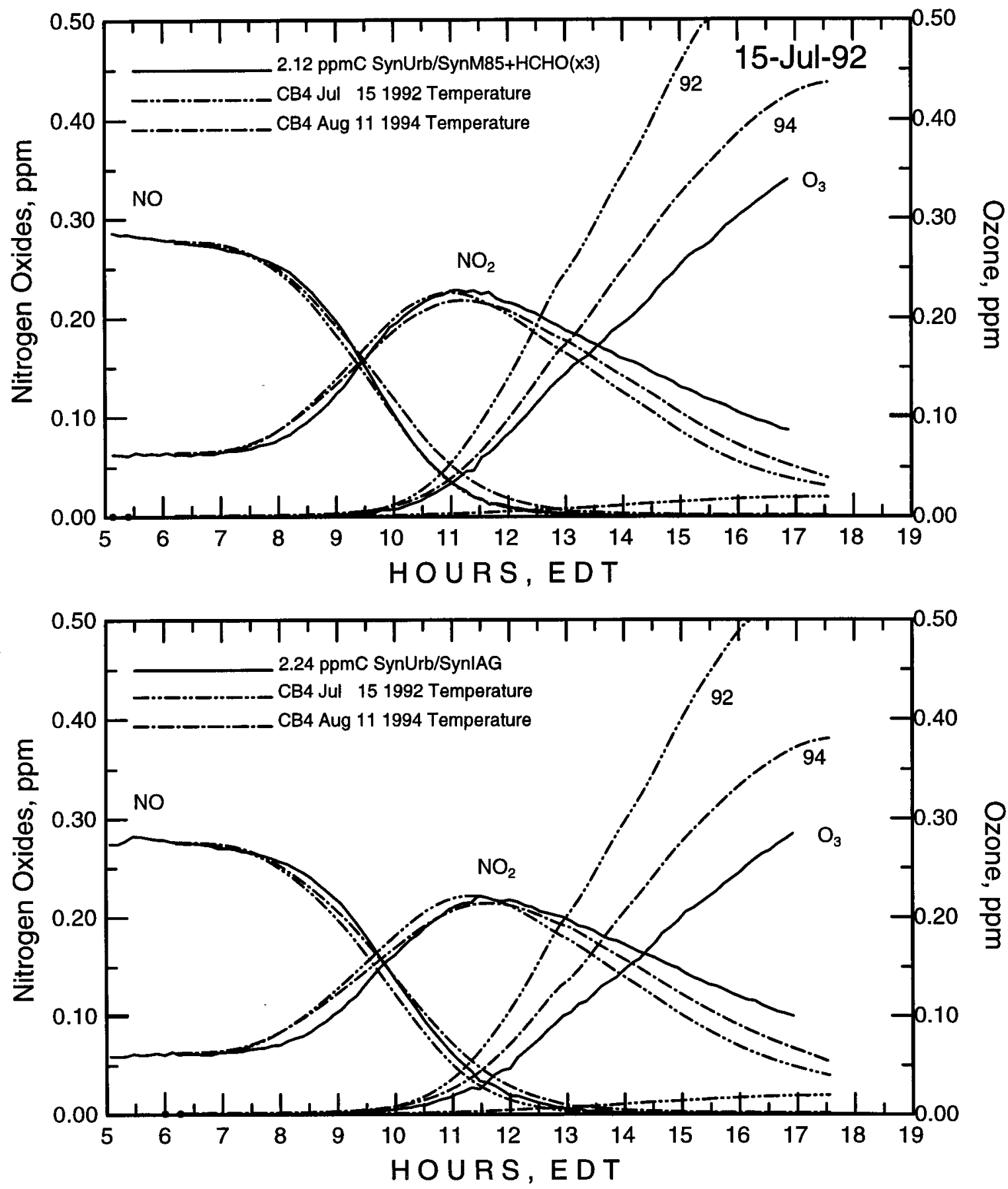

Figure 4.17: Carbon Bond Four simulation of JL1592 at different temperatures. 
These simulation results show that the CB4 mechanism exhibits a much larger temperature sensitivity for complex mixtures than is seen in the chamber observations. This explains why the CB4 performed so poorly for the CRC experiments, which were all "hot" relative to the DOE experiments reported here.

\subsubsection{Temperature Effect Conclusions}

The conclusions from these intercomparisons follow.

- The outdoor smog chamber experimental results suggest that when using complex mixtures with the realistic compositions of synthetic urban and IAG, there is at best a small temperature effect. In addition, there is some evidence to suggest that an inverse relationship may exist between temperature and ozone production for these mixtures. There does appear to be a difference in the afternoon $\mathrm{NO}_{y}$, such that higher temperatures give lower $\mathrm{NO}_{y}$ measurements suggesting that there may be a compensating effect between the amount of PAN-like compounds produced and the ozone produced.

- The experimental evidence refutes the large positive effect of temperature on ozone production that is demonstrated by the CB4 mechanism.

These imply that although the CB4 simulations reported later in this report show excellent agreement with the chamber observations, this accuracy may merely result from having tuned the more complex organic chemistry in the CB4 using chamber experiments from the 1980s in which the chamber temperatures were more like those in the 1994 data set. In comparisons with hotter chamber data from 1992, the CB4 exhibits too much increase of ozone with increasing temperature.

Fortunately, the main application region for CB4 in the Urban Airshed Model is more like the temperatures observed in the outdoor chamber in the 1994 experiments. Nevertheless, the results of such simulations must be taken with a "grain of salt." 


\subsection{Characterization Run Simulations}

\subsubsection{Block 6}

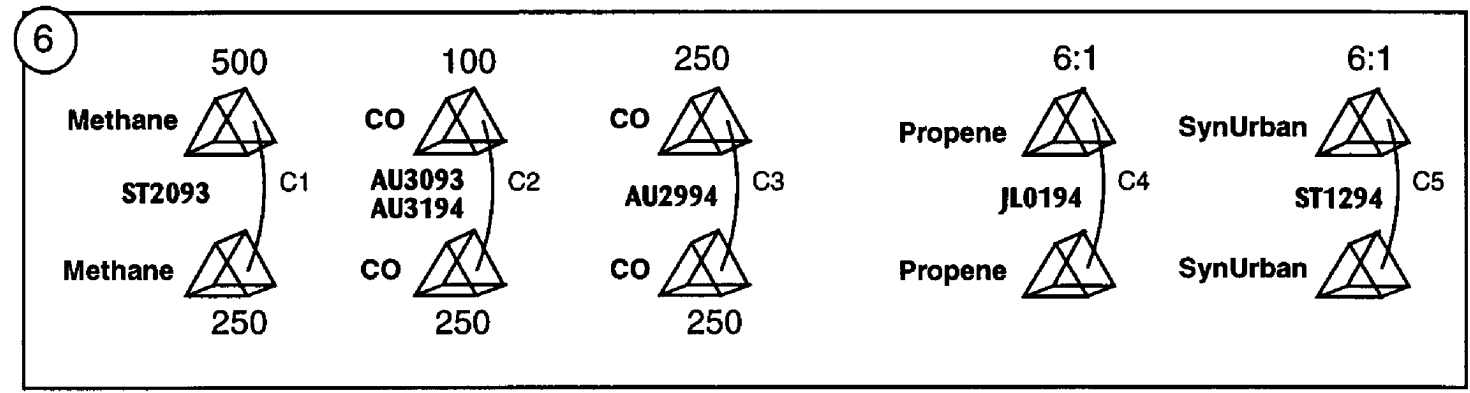

\section{Run C2, 100 ppm CO versus 250 ppm CO}

Earlier chapters discussed the importance of the smog chamber characterization experiments. Briefly, they

- Test and demonstrate that the chambers and associated sampling apparatus are operating properly

- Demonstrate the reproducability of establishing test conditions and experimental results

- Help determine the chamber background reactivity

- Allow demonstration that our measurement of light and calculation of basic photolytic rates is sufficient for modeling experiments with compounds with well understood chemistry.

AU3194 This experiment compares $100 \mathrm{ppm}$ of $\mathrm{CO}$ with $250 \mathrm{ppm}$ CO (see Figures 4.18 and 4.19). It is designed to show, and estimate by modeling, the wall radical flux of the chambers.

\section{Run C5, 6:1 Urb versus Urb}

ST1294 This experiment compares 100\% SynURB with 100\% SynURBat 6:1 (see Figures 4.20 and 4.21). It is designed to show that the two chamber halves and the sample systems are matched, as well as the ability to replicate complex mixtures. 

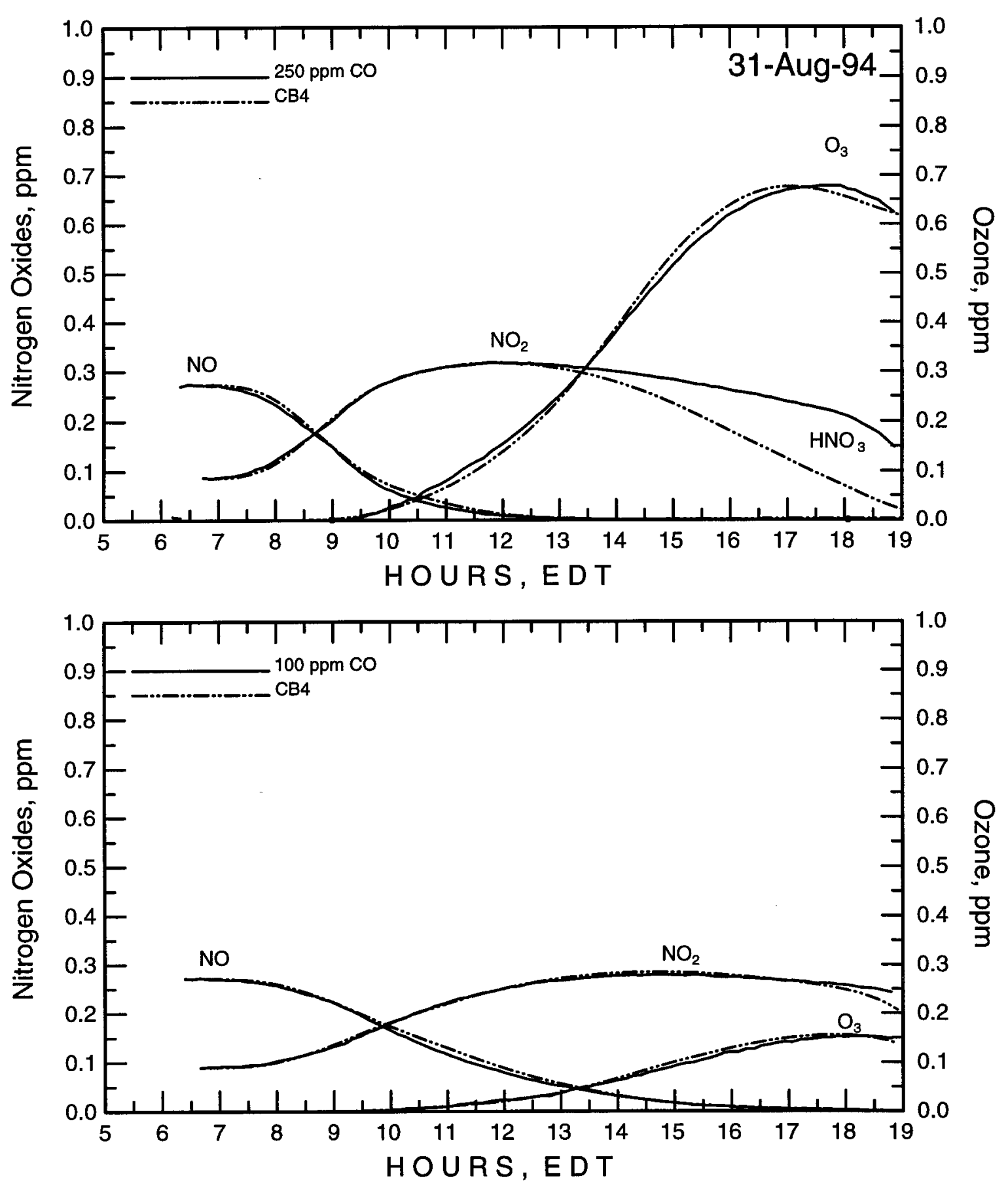

Figure 4.18: Run C2-100 ppm CO:NO verses 250 ppm CO:NO ; Carbon Bond Four simulation. 


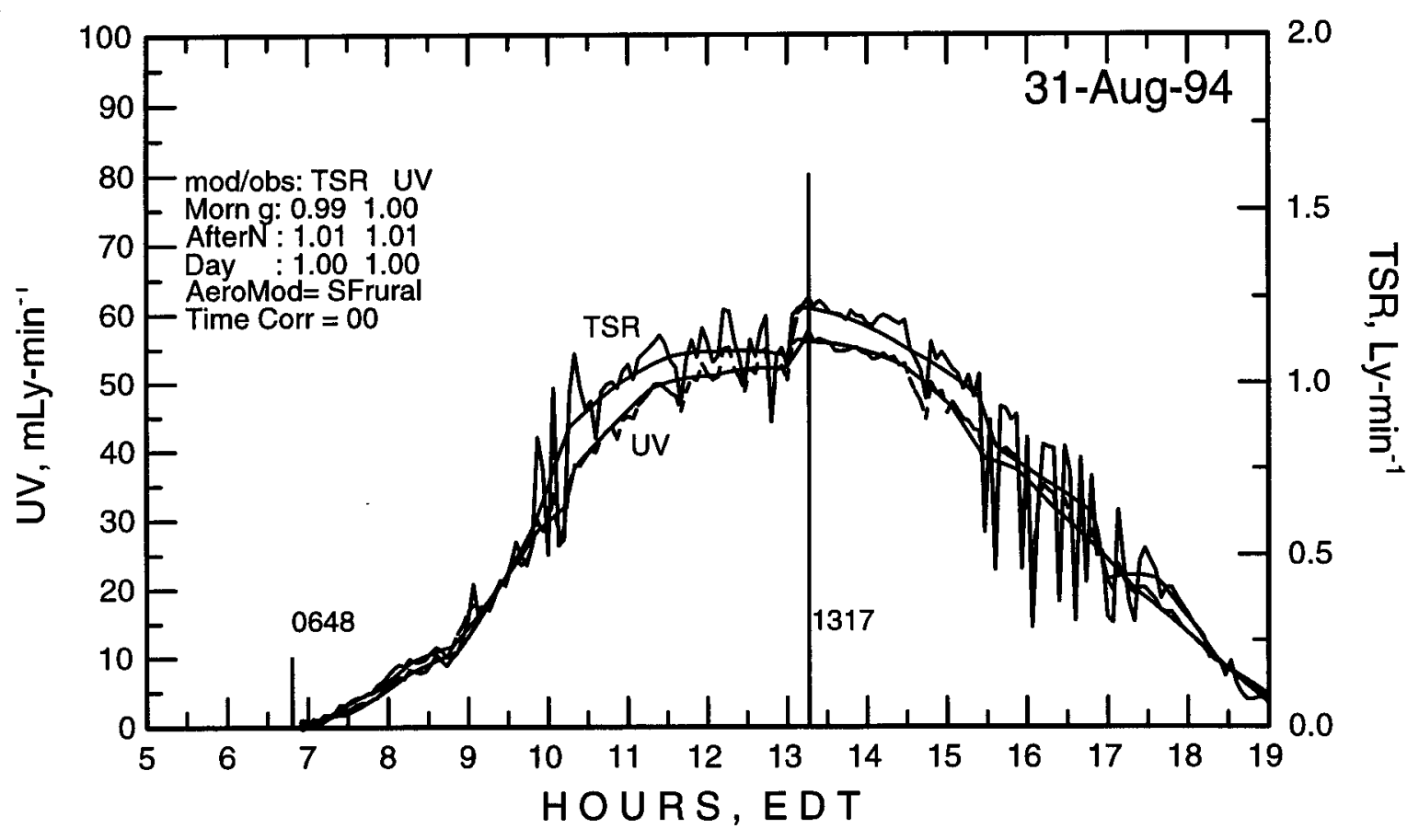

Figure 4.19: Run C2-Solar radiation simulation.

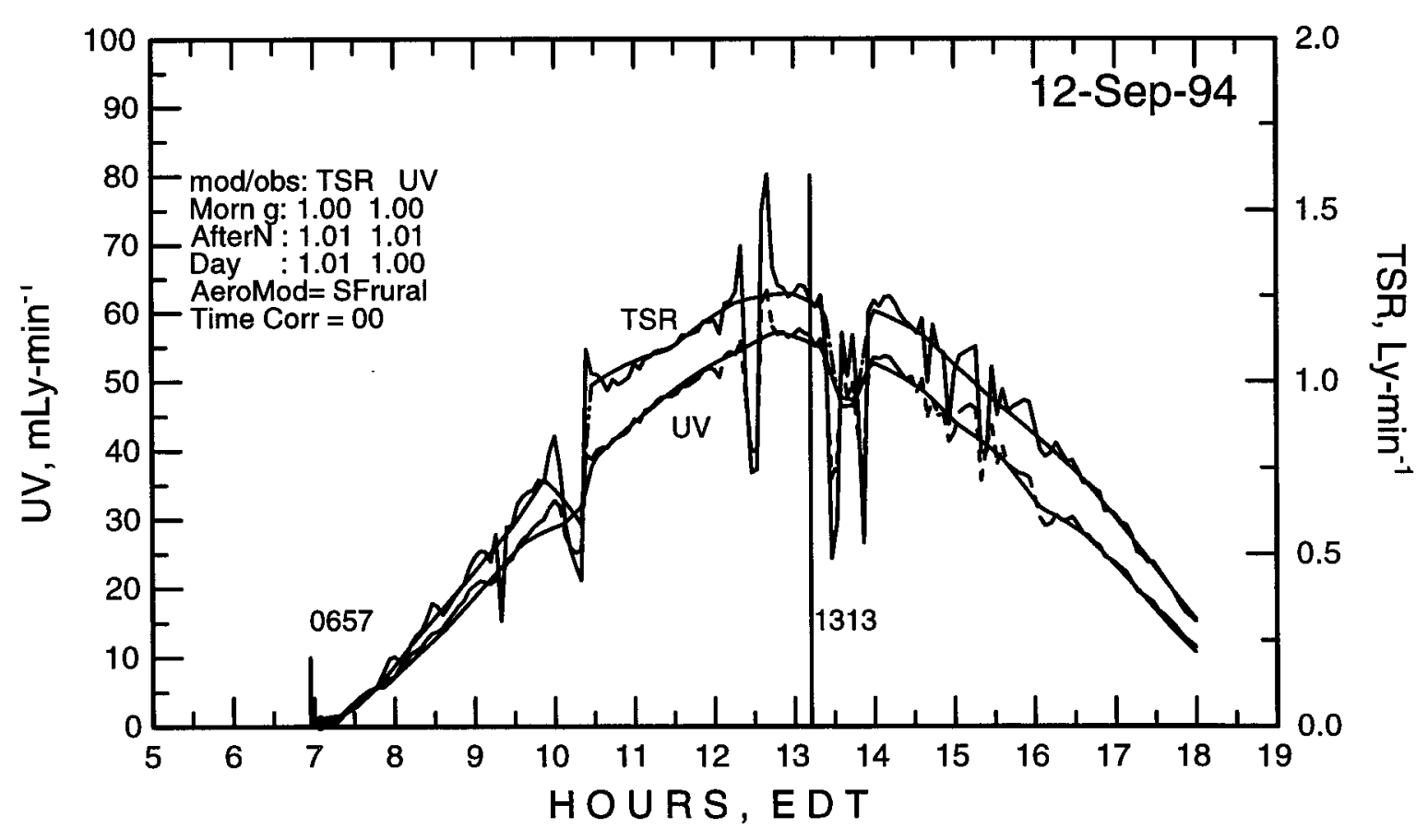

Figure 4.20: Run C5-Solar radiation simulation. 

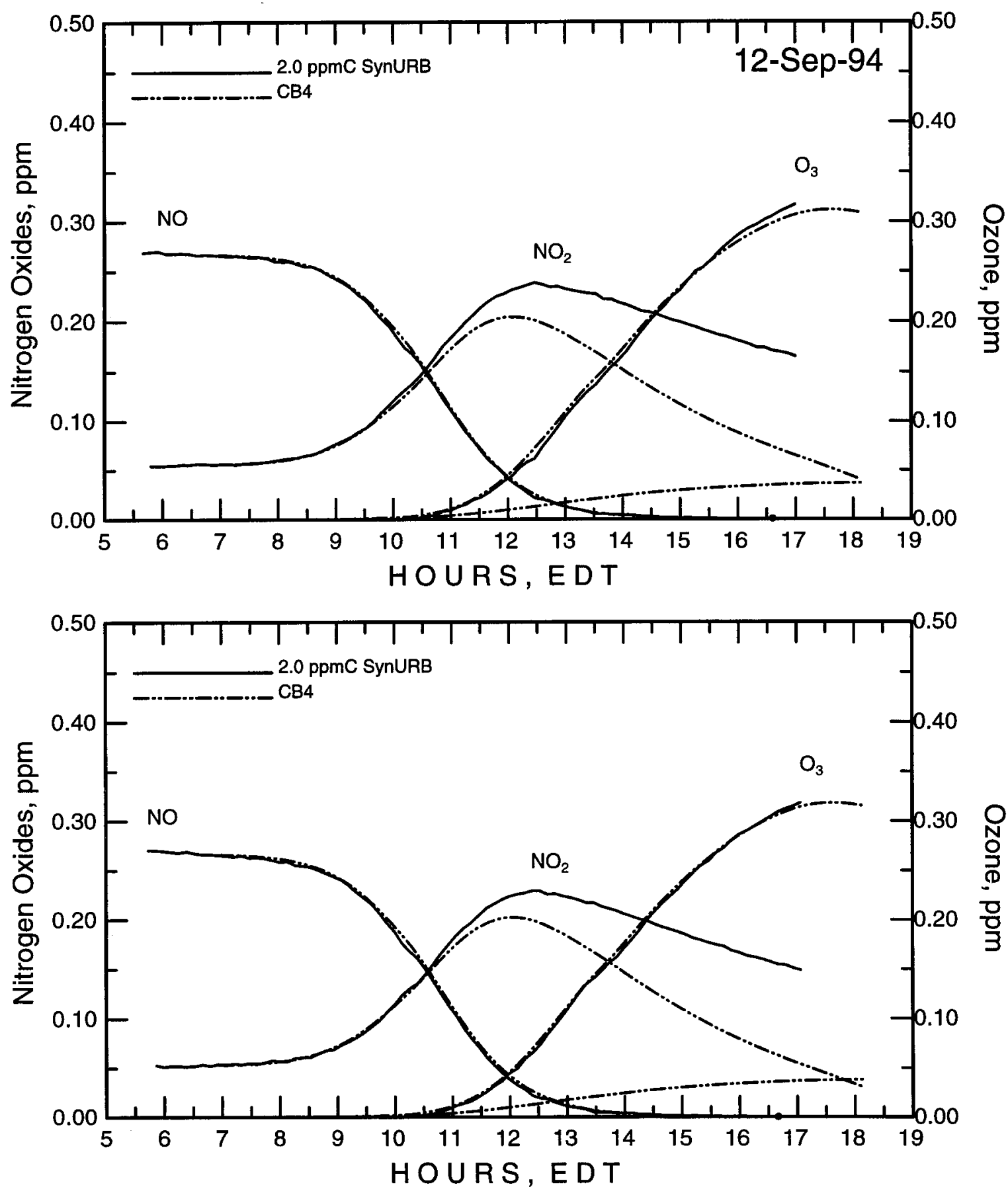

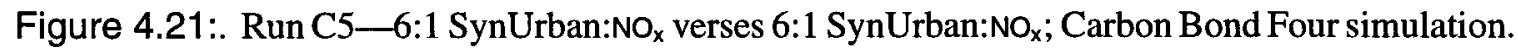




\subsection{Alternative Fuels Runs Simulations}

The initial conditions tables in the experimental results chapter show the estimated initial conditions of HONO and the chamber model parameters used to model these experiments. These values were estimated by repeated simulations after adjusting the values to obtain the best simulation agreements with the experimental results, while keeping within the bounds for these values (as determined from simulations of the chamber characterization experiments, consideration of the chamber history before each experiment, and from measurements of HONO made in other experiments). The same or similar estimates were usually used for each chamber side for each experiment unless recent chamber history of experiments with widely different reactivity justified different chamber background estimates.

\subsubsection{Block 1}

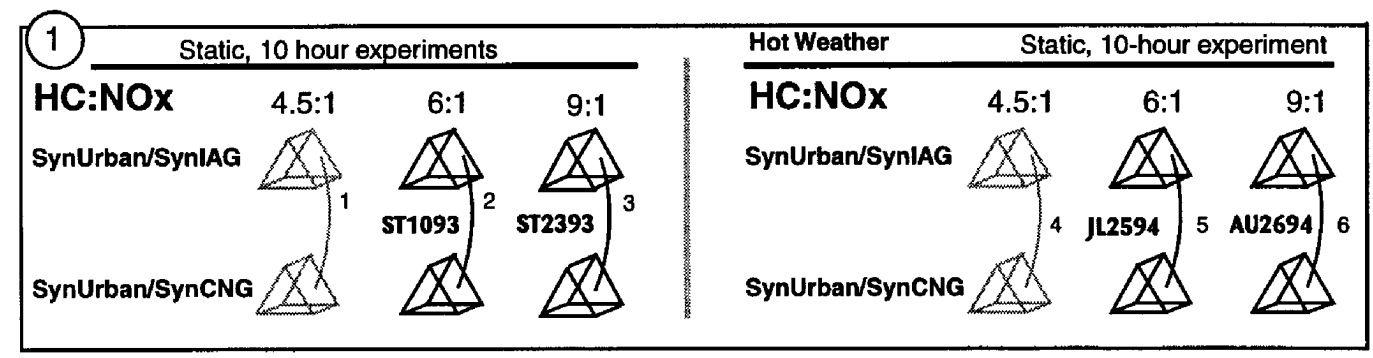

\section{Run 2, 6:1 Urb/IAG versus Urb/CNG}

ST1093 This run shows that SynCNG has a significant benefit for reducing ozone compared to SynIAG even under cooler, shorter-day, fall conditions (see Figures 4.22 and 4.23). The CB4 mechanism was successfully in simulating this experiment. Given that 10 days of chamber venting preceded this experiment (the chamber was not used), the same chamber background estimates were used to model each side. The IAG side was over-predicted somewhat compared to the relatively good simulation of the SynCNG side.

\section{Run 3, 9:1 Urb/IAG versus Urb/CNG}

ST2393 Usually at 9:1 VOC: $\mathrm{NO}_{x}$, reactivity makes almost no difference. This run (Figure 4.25), however, shows that for CNG, the benefits extend to well beyond 9:1. The solar radiation simulation is shown in Figure 4.24. The CB4 mechanism was used to successfully simulate this experiment. The IAG side was slightly over-predicted compared to the relatively good simulation of the SynCNG side. Given that two days of chamber venting preceded this experiment (the chamber was not used), and that the only previous experiment for six days prior was a methane run, the same chamber background estimates were used to model each side. It is gratifying that given that both this experiment and the September 10 experiment were preceded by minimal chamber use, the same chamber background 
estimates were used to successfully model these two experiments, which were of widely different chemical reactivity. Also, while the temperature maximum was the same for these two days $\left(94^{\circ} \mathrm{F}\right)$, the morning temperatures were significantly different $\left(69^{\circ} \mathrm{F}\right.$ on September 10 and $56^{\circ} \mathrm{F}$ for September 23).

\section{Run 5, 6:1 Urb/IAG versus Urb/CNG}

JL2594 This run (Figures 4.26 and 4.23 was a repeat of ST1093 in warm weather. This run shows that the benefits of CNG are sustained under warm, high sun conditions. The solar radiation simulation is shown in Figure 4.27. The CB4 mechanism was used to successfully simulate the IAG side (slightly early in ozone production). The SynCNG side was not simulated well. Standard chamber background estimates were used in the simulations. This chamber-side experiment is the only non-ethanol experiment that was poorly modeled (not even extreme chamber background esimates yielded a successful simulation). Given that the model simulation of this SynCNG experiment is consistent with other experimental results, we suspect that there is some undiscovered (at this time) problem with this particular experiment. The IAG side, however, is an excellent clear-sun, midsummer, dry experiment.

\section{Run 6, 9:1 Urb/IAG versus Urb/CNG}

AU2694 This was a repeat of Run 3 (ST2393) under warm, high-sun conditions. This run showed that the benefits of CNG are sustained under warm, high-sun conditions even for high $\mathrm{HC}: \mathrm{NO}_{x}$ ratios. The solar radiation simulation is shown in Figure 4.28. The CB4 mechanism was used to successfully simulate (very well) both sides of this experiment (Figure 4.29). Equal and relatively higher chamber background estimates were used to simulate both sides of this experiment, consistent with the chamber history. 

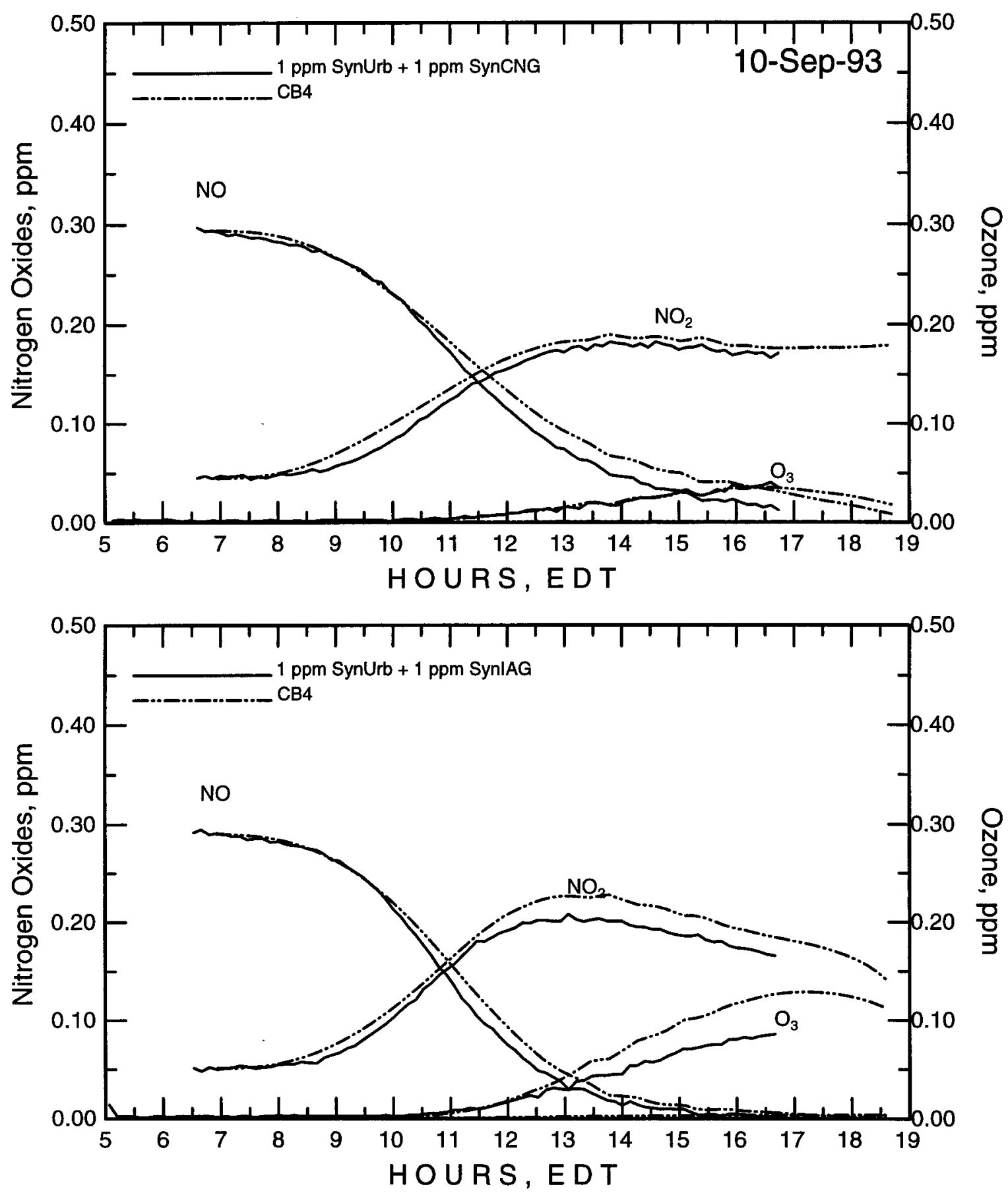

Figure 4.22:. Run 2-6:1 SynUrban/SynIAG:NO ${ }_{x}$ verses 6:1 SynUrban/SynCNG:NO ; Carbon Bond Four simulation. 


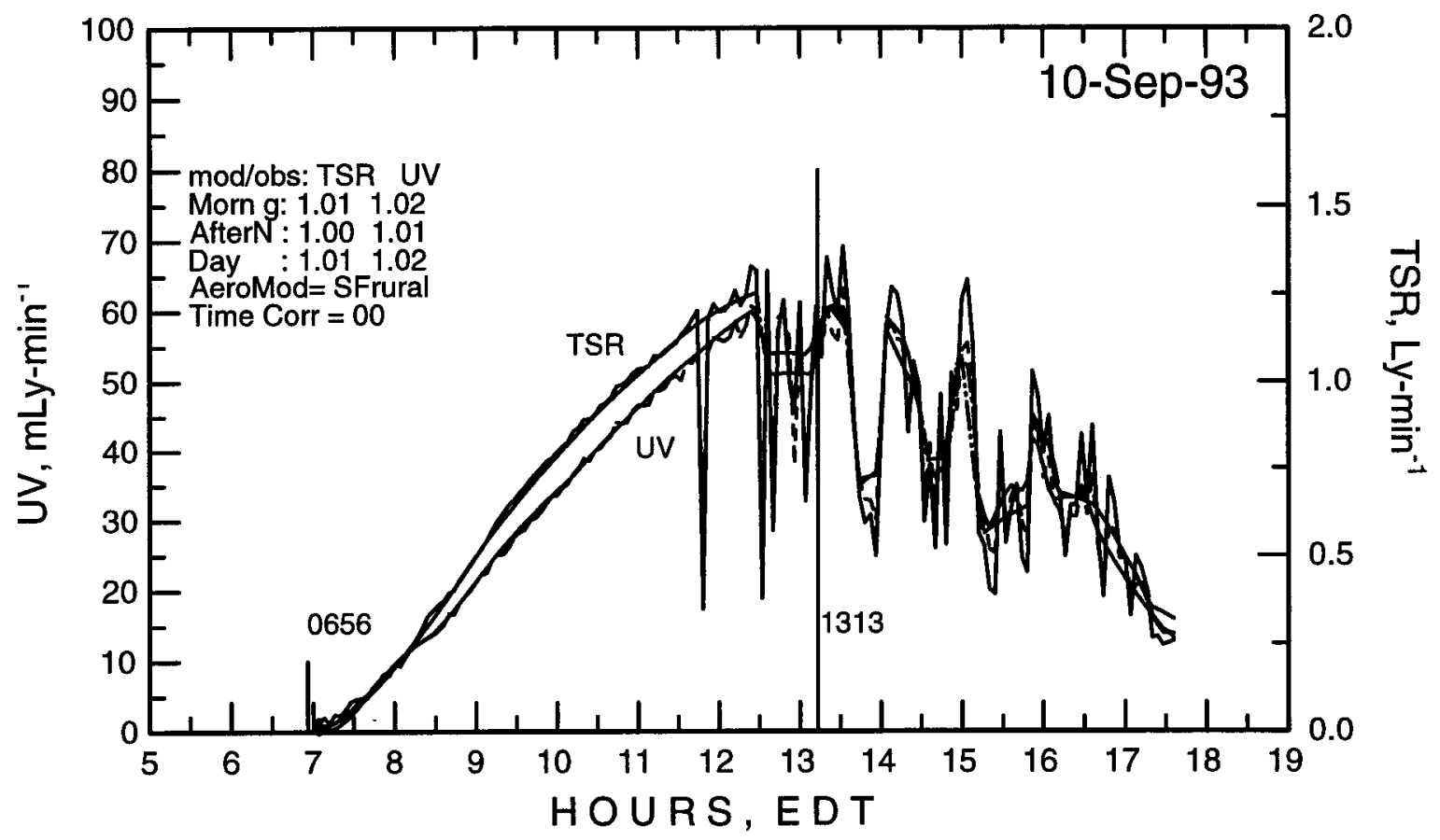

Figure 4.23: Run 2-Solar radiation simulation.

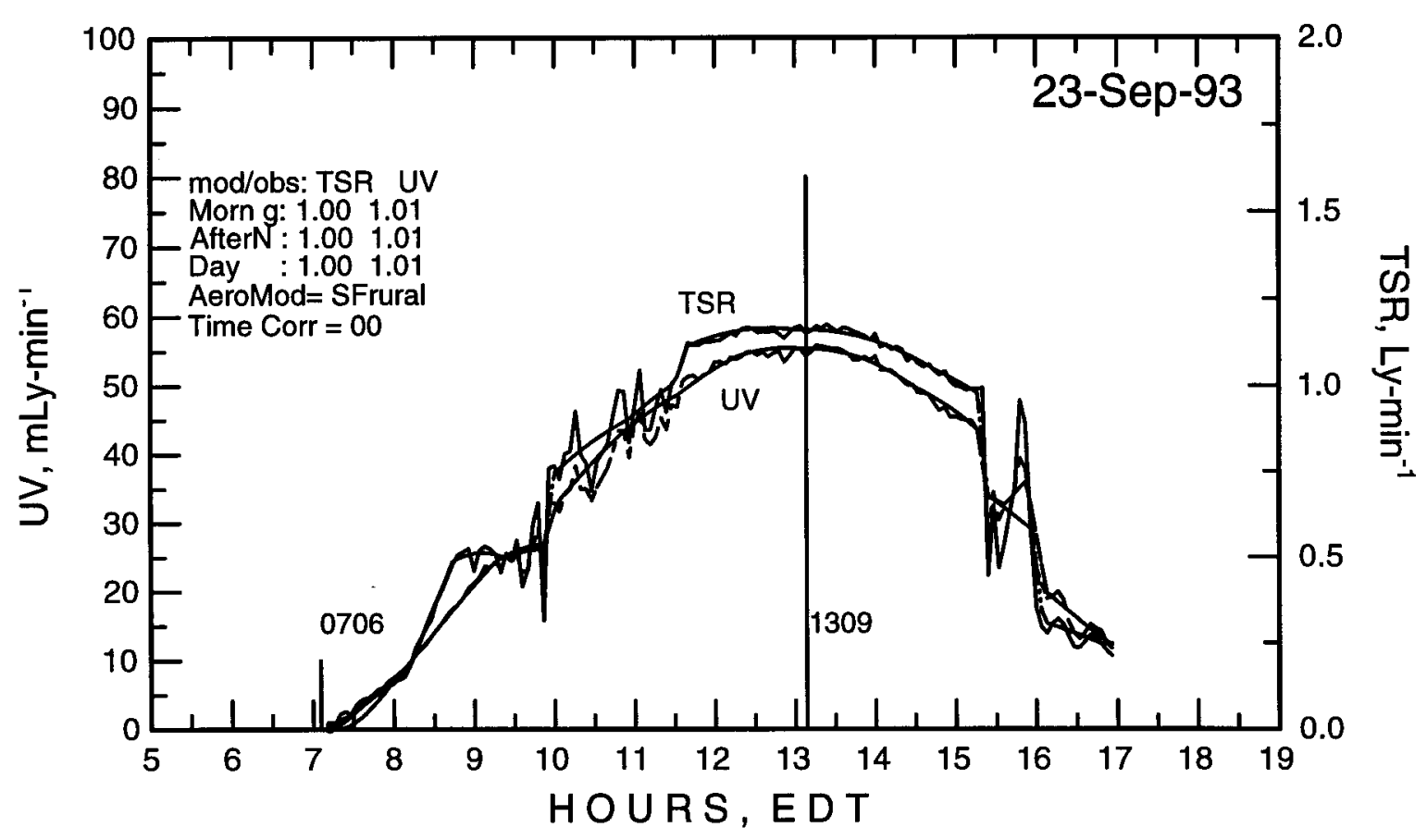

Figure 4.24: Run 3-Solar radiation simulation. 

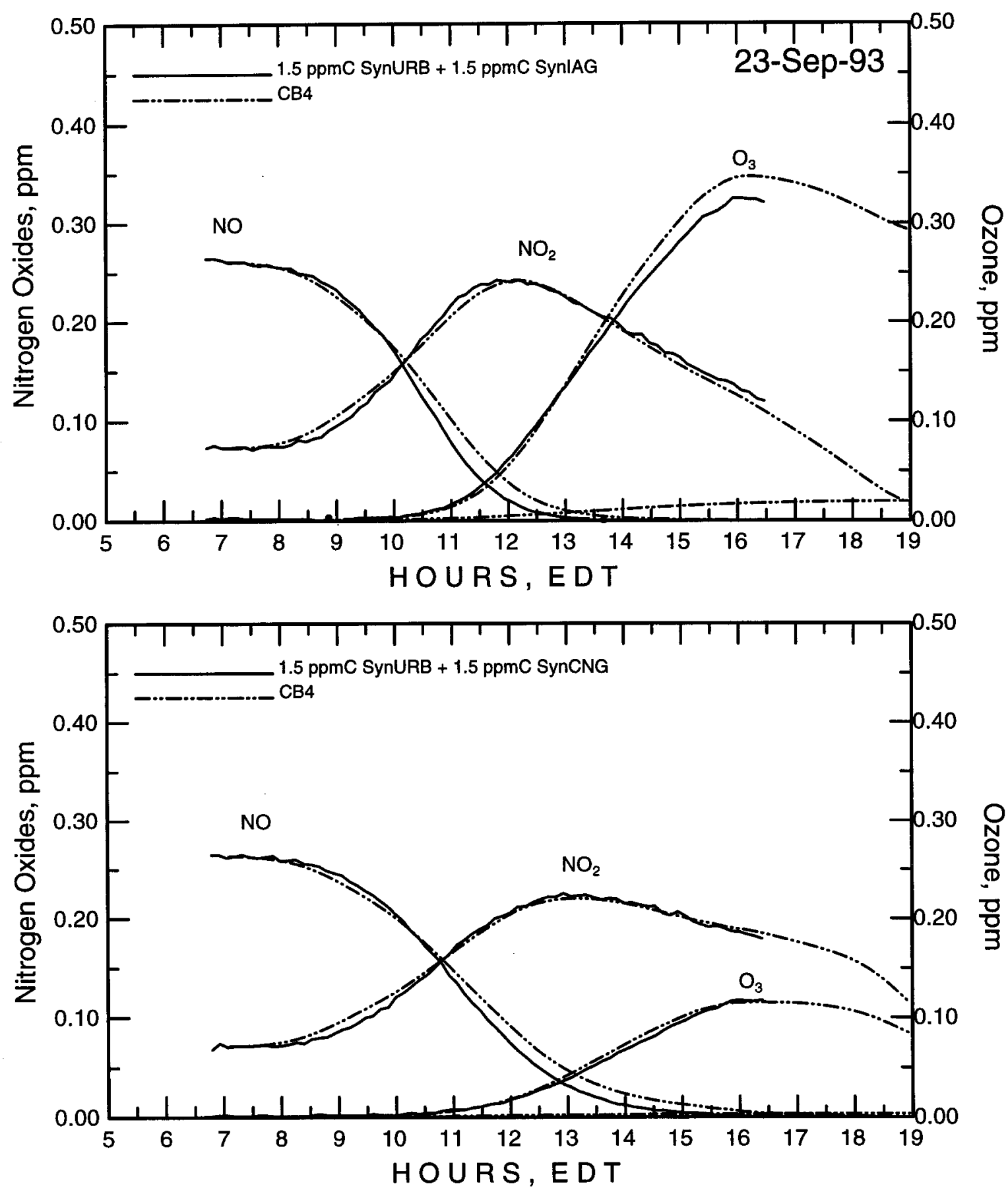

Figure 4.25:. Run 3-9:1 SynUrban/SynIAG:NOx verses 9:1 SynUrban/SynCNG:NO ; Carbon Bond Four simulation. 

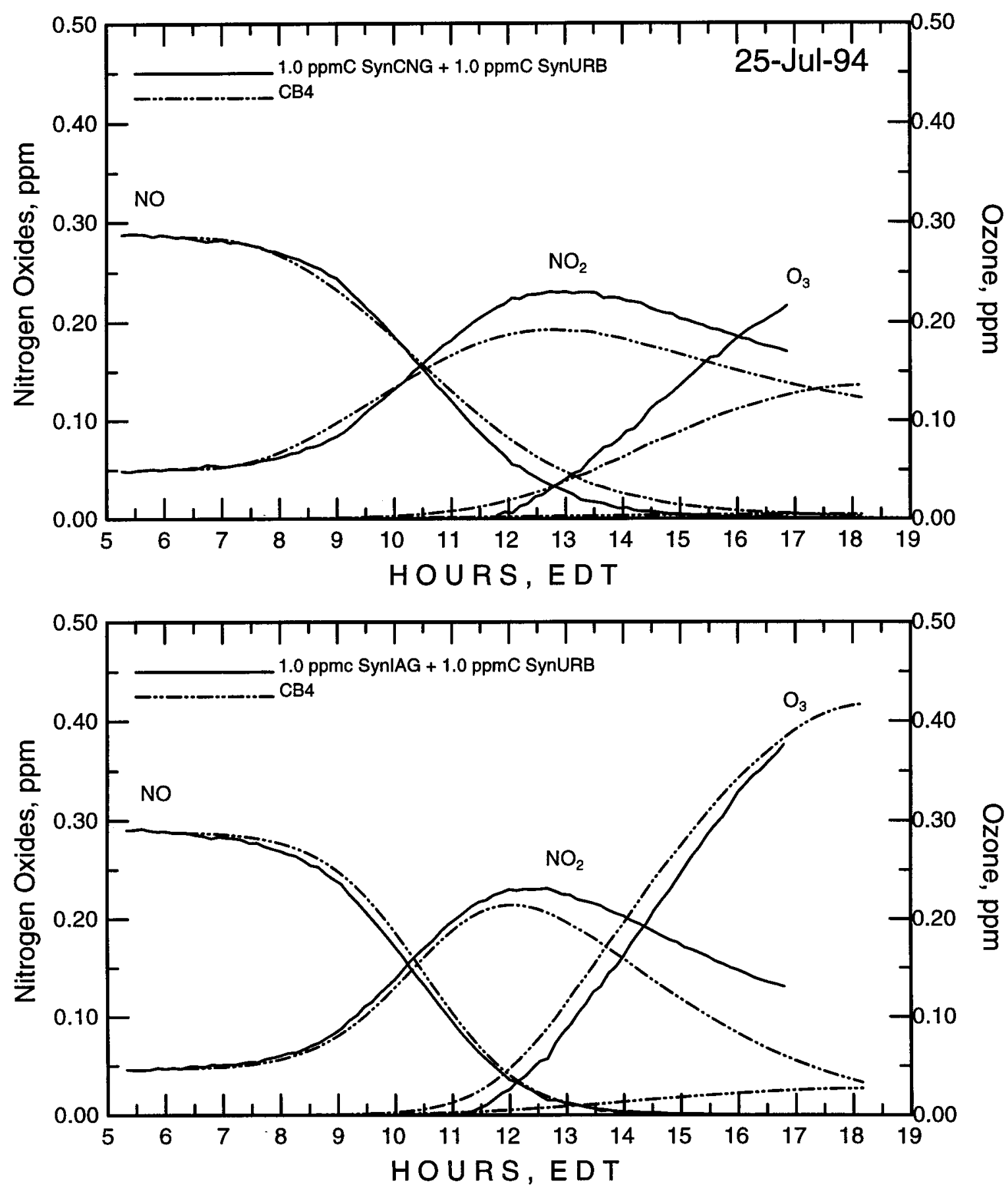

Figure 4.26:. Run 5-6:1 SynUrban/SynIAG:NO ${ }_{x}$ verses 6:1 SynUrban/SynCNG:NO ; Carbon Bond Four simulation. 


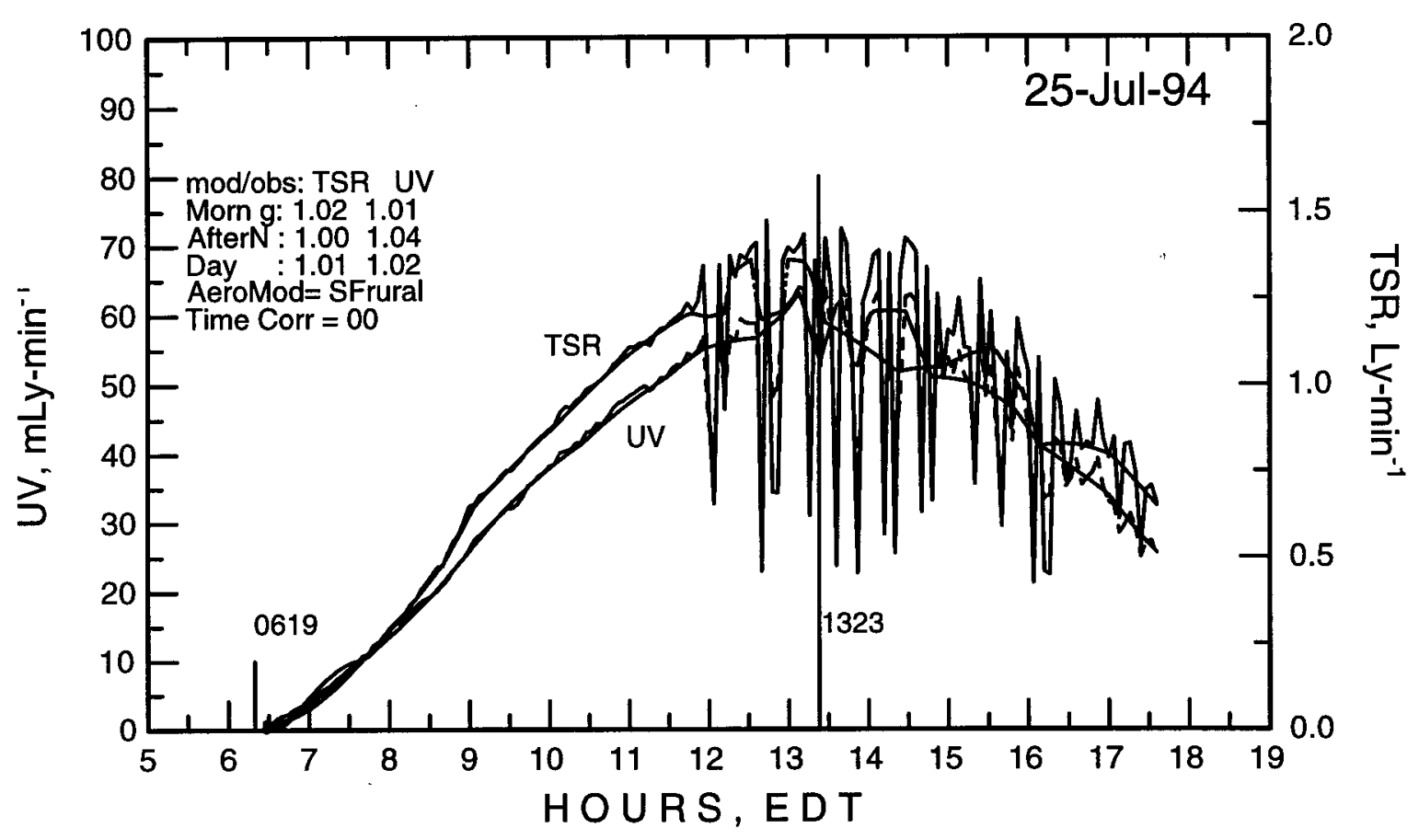

Figure 4.27: Run 5-Solar radiation simulation.

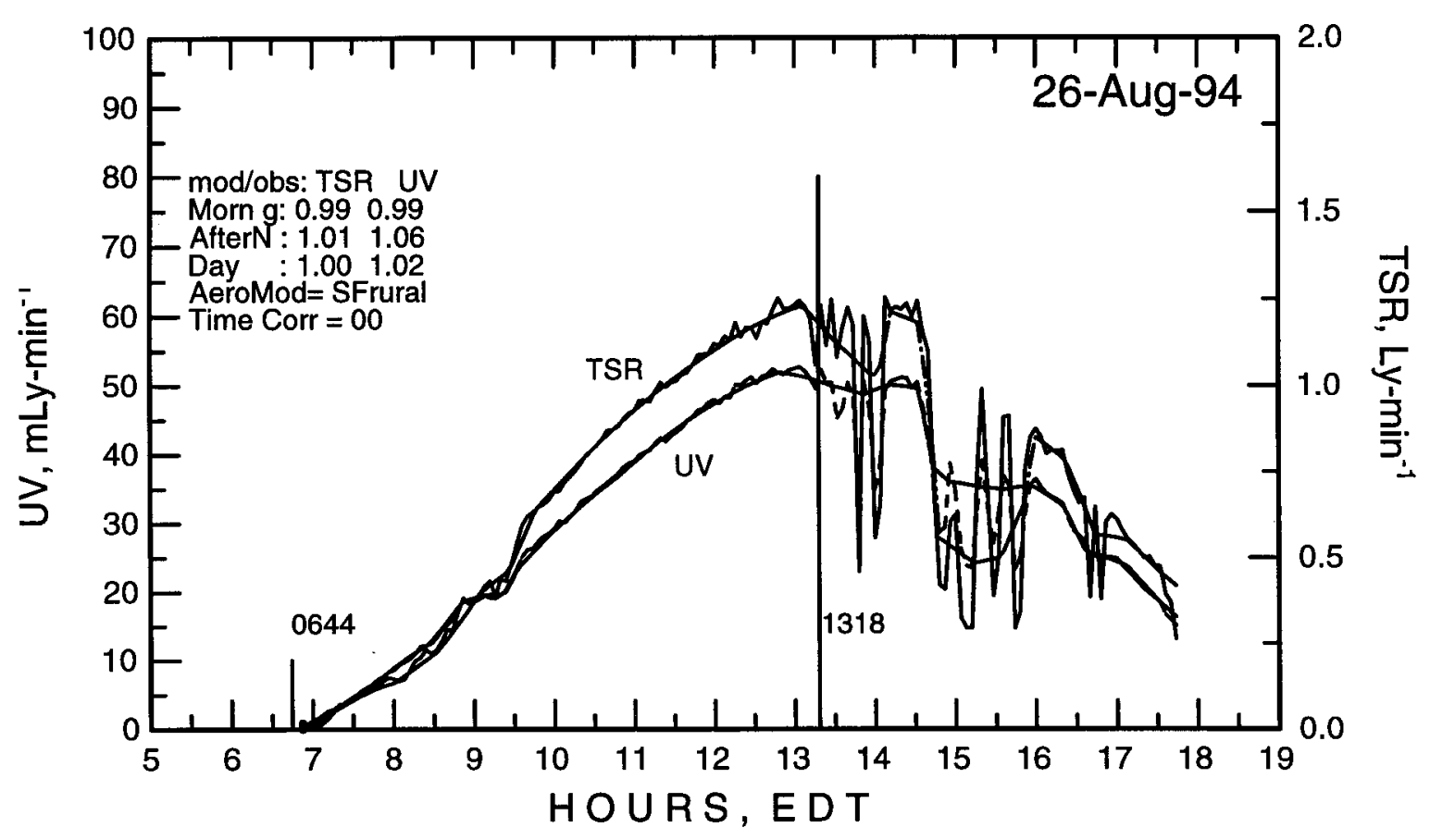

Figure 4.28: Run 6-Solar radiation simulation. 

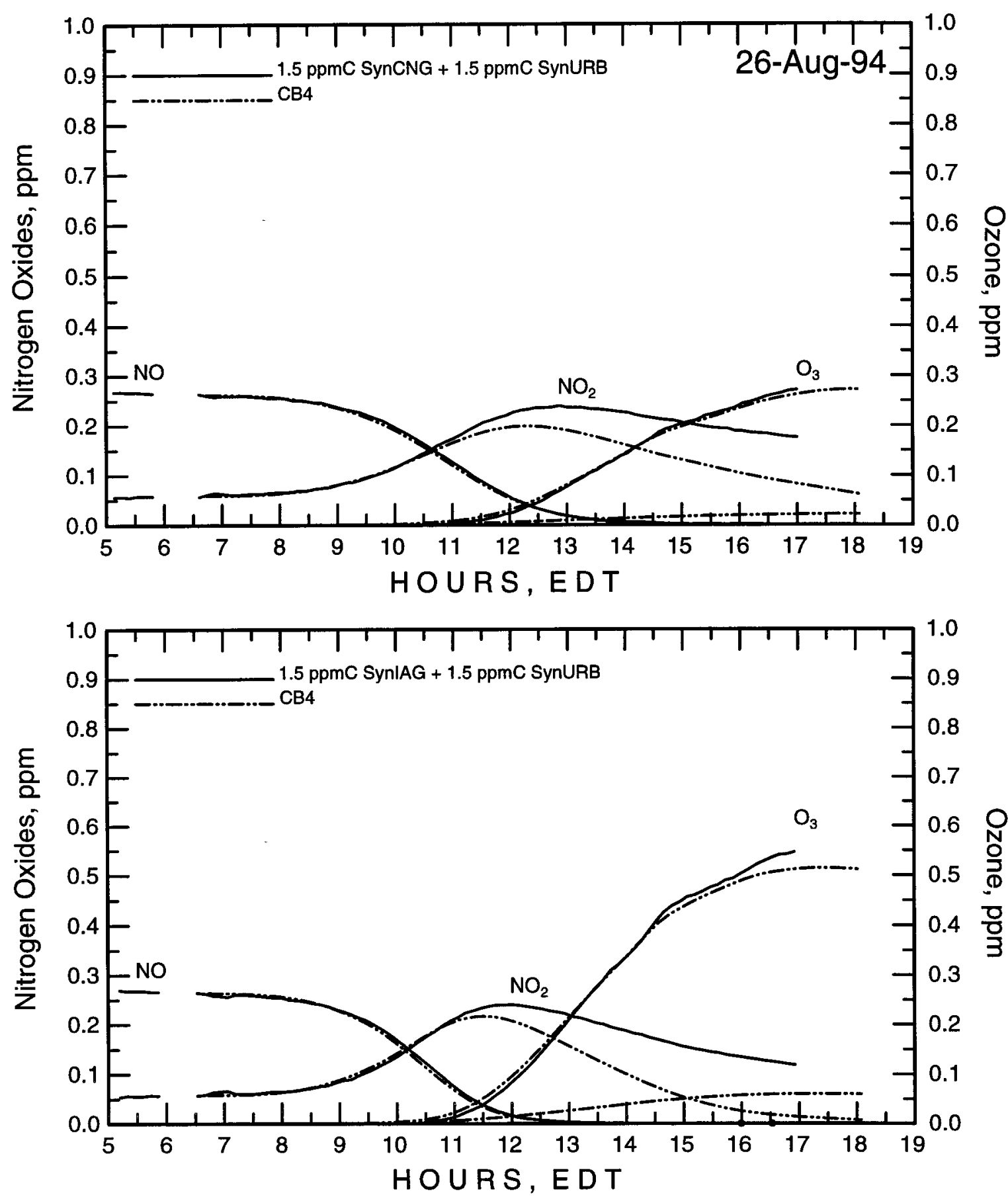

Figure 4.29:. Run 6-9:1 SynUrban/SynIAG:NOx verses 9:1 SynUrban/SynCNG:NO ; Carbon Bond Four simulation. 


\subsubsection{Block 5}

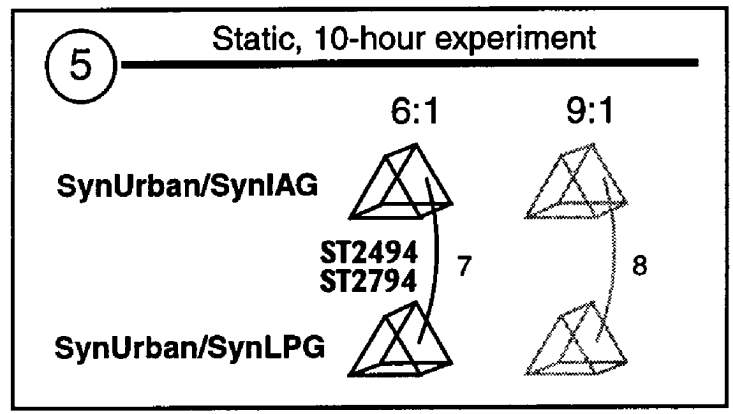

\section{Run 7, 6:1 Urb/IAG versus Urb/LPG}

ST2794 The LPG experiments show a benefit in ozone production similar to CNG. The solar radiation simulation is shown in Figure 4.30. The CB4 mechanism was used to successfully simulate (very well) both sides of this experiment (Figure 4.31). Equal and low chamber background estimates were used to simulate both sides of this experiment consistent with the chamber history.

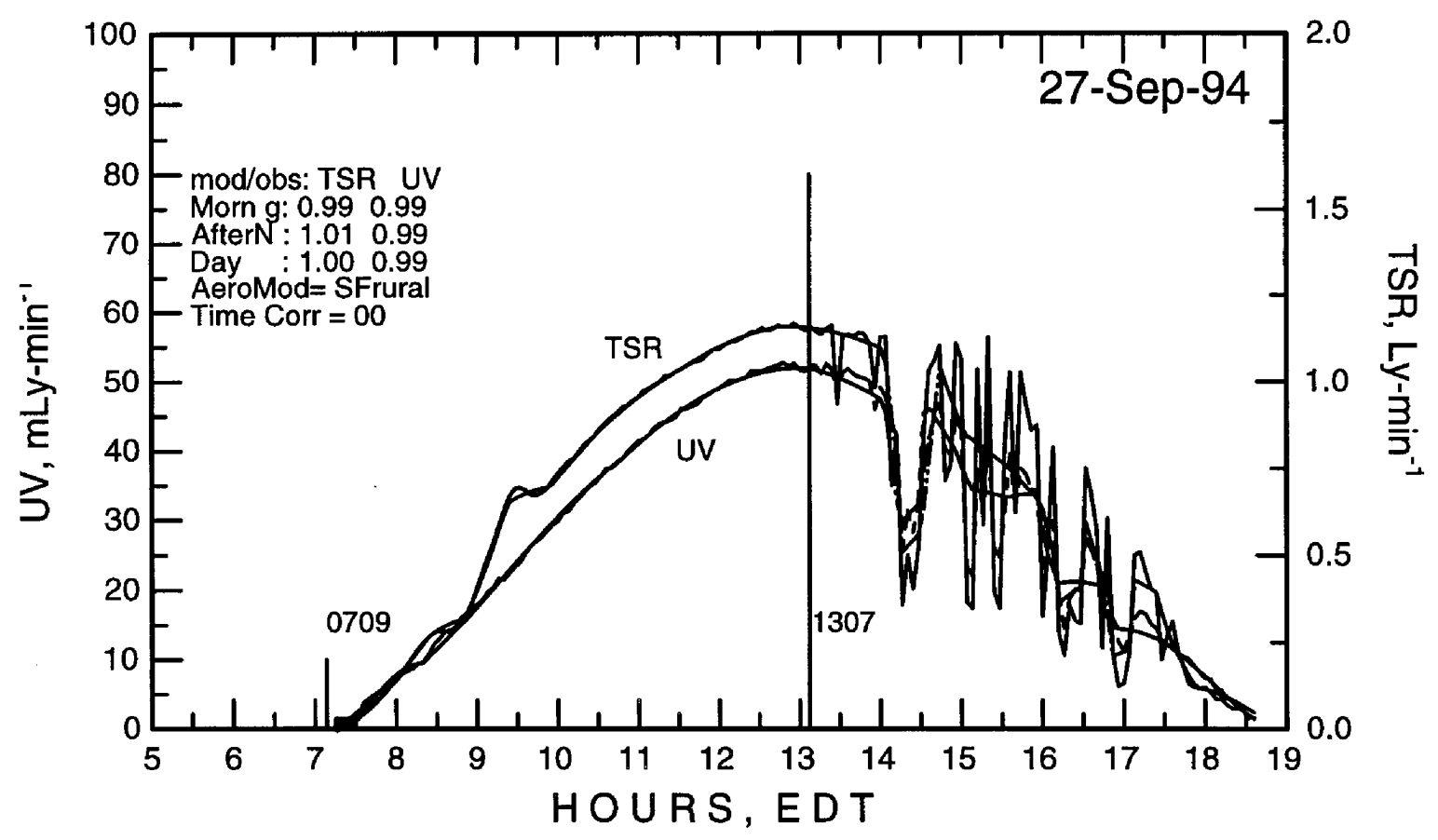

Figure 4.30: Run 7-Solar radiation simulation. 

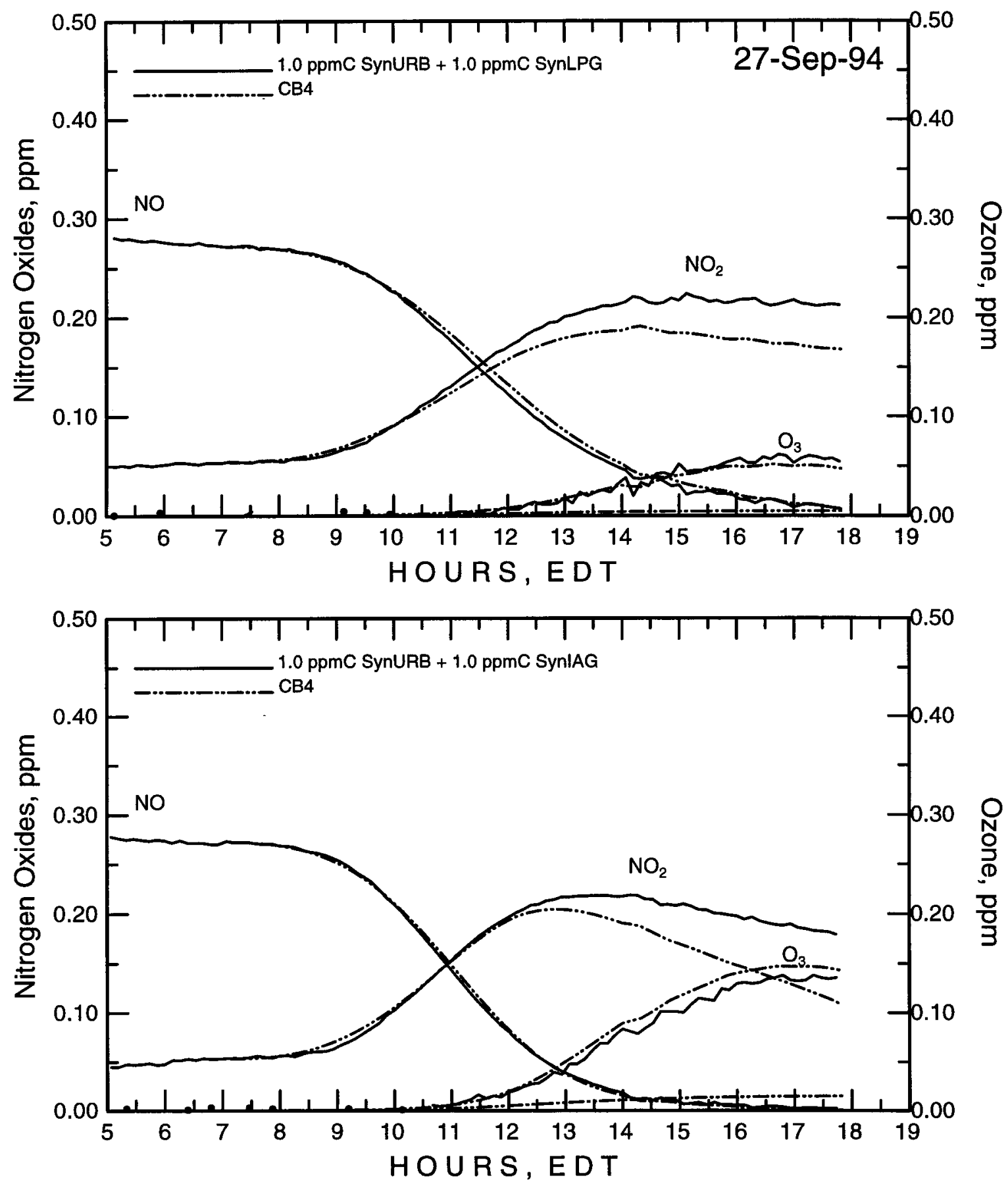

Figure 4.31:. Run 7-9:1 SynUrban/SynIAG:NOx verses 9:1 SynUrban/SynLPG:NO ; Carbon Bond Four simulation. 


\subsubsection{Block 2}

(2) $\frac{\text { Static, } 10 \text { hour experiments }}{\text { SC:NOX }}$

\section{Run 10, 6:1 Urb/IAG versus Urb/E85E}

AU0794 This experiment compareed the reactivity of the EPA E85 and IAG compositions with blending 50:50 with SynURB. The E85 side yielded an ozone maximum concentration approximately $15 \%$ higher than the IAG side. The CB4 mechanism was used to successfully simulate (very well) both sides of this experiment (Figures 4.32 and 4.33). Equal and low chamber background estimates were used to simulate both sides of this experiment, consistent with the chamber history.

AU1194 This experiment was a repeat of AU0794 with better sun. The results are consistent. The solar radiation simulation is shown in Figure 4.34. The CB4 mechanism was used to successfully simulate (very well) both sides of this experiment (Figure 4.35). Equal and medium chamber background estimates were used to simulate both sides of this experiment, consistent with the chamber history.

\section{Run 11, 9:1 Urb/IAG versus Urb/E85E}

AU2594 This experiment duplicated the results of the AU2494 experiment, showing matched reactivity at higher $\mathrm{HC}: \mathrm{NO}_{\mathrm{x}}$ ratios. The $\mathrm{CB} 4$ mechanism was used to successfully simulate both sides of this experiment (Figure 4.36 and 4.37). Both sides were slightly under-predicted after a cloudy period which occured before the separation between the data and model results. Equal and relatively higher chamber background estimates were used to simulate both sides of this experiment, consistent with the chamber history.

\section{Run 12, 6:1 Urb/IAG versus Urb/E85B}

AU0894 This experiment compared the reactivity of the NIPER E85 and IAG compositions with blending 50:50 with SynUrb. The E85 side yielded an ozone maximum concentration approximately 35 percent higher than the IAG side. The solar radiation simulation is shown in Figure 4.38. The CB4 mechanism was used to successfully simulate (very well) both 
sides of this experiment (Figure 4.39). Equal and medium chamber background estimates were used to simulate both sides of this experiment consistent with the chamber history. 

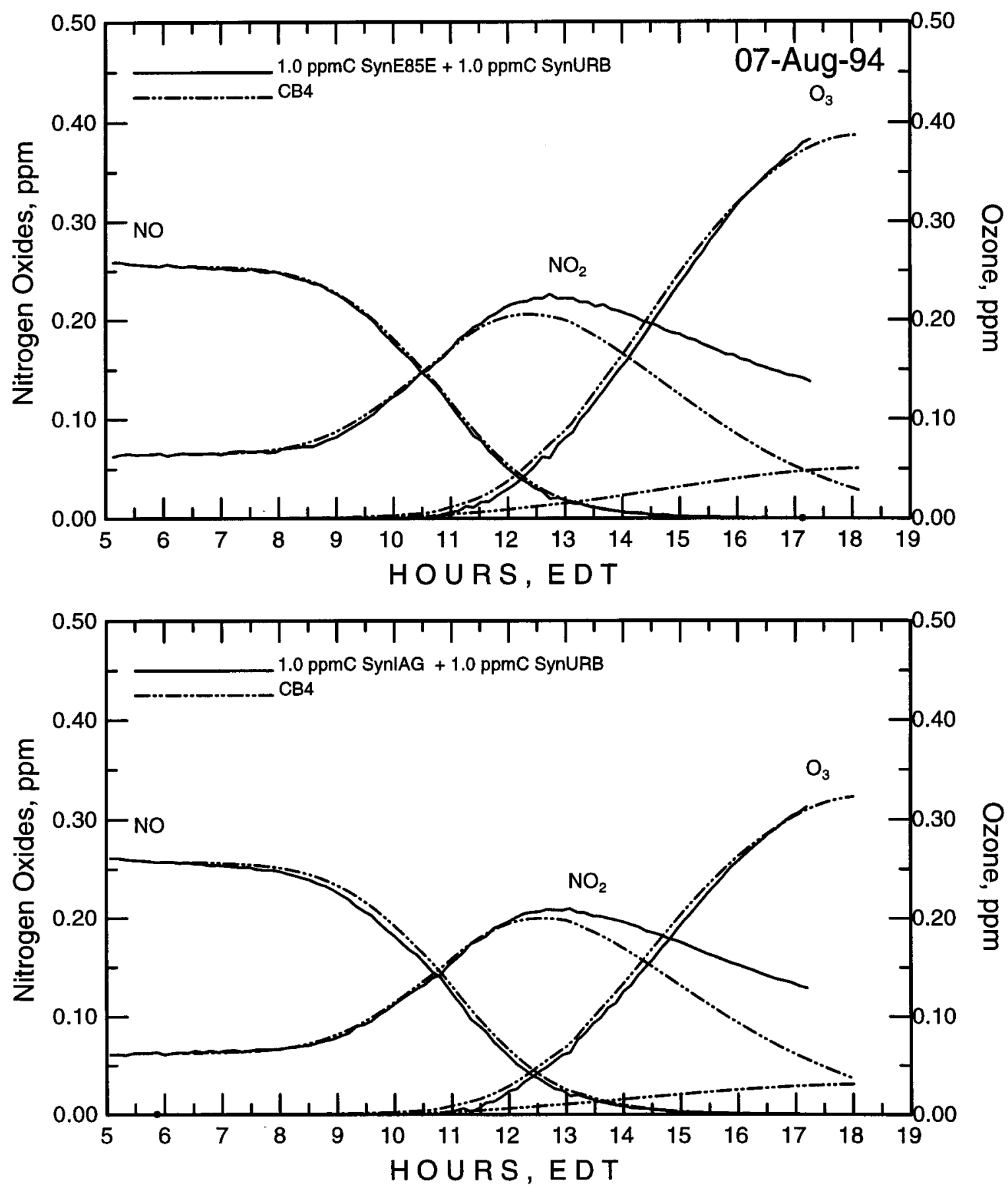

Figure 4.32:. Run 10—6:1 SynUrban/SynIAG:NO verses 6:1 SynUrban/SynEevE:NO ; Carbon Bond Four simulation. 


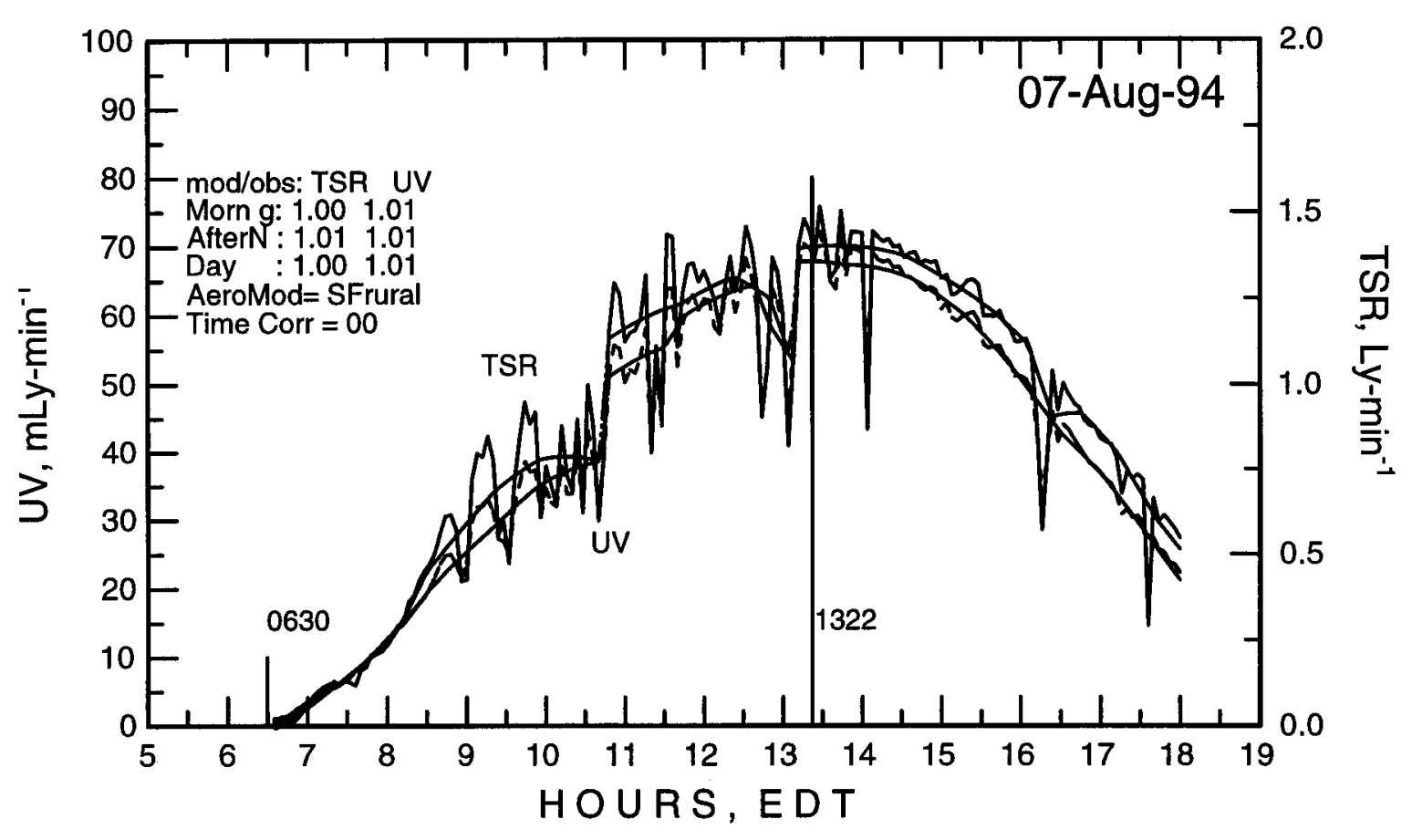

Figure 4.33: Run 10-Solar radiation simulation.

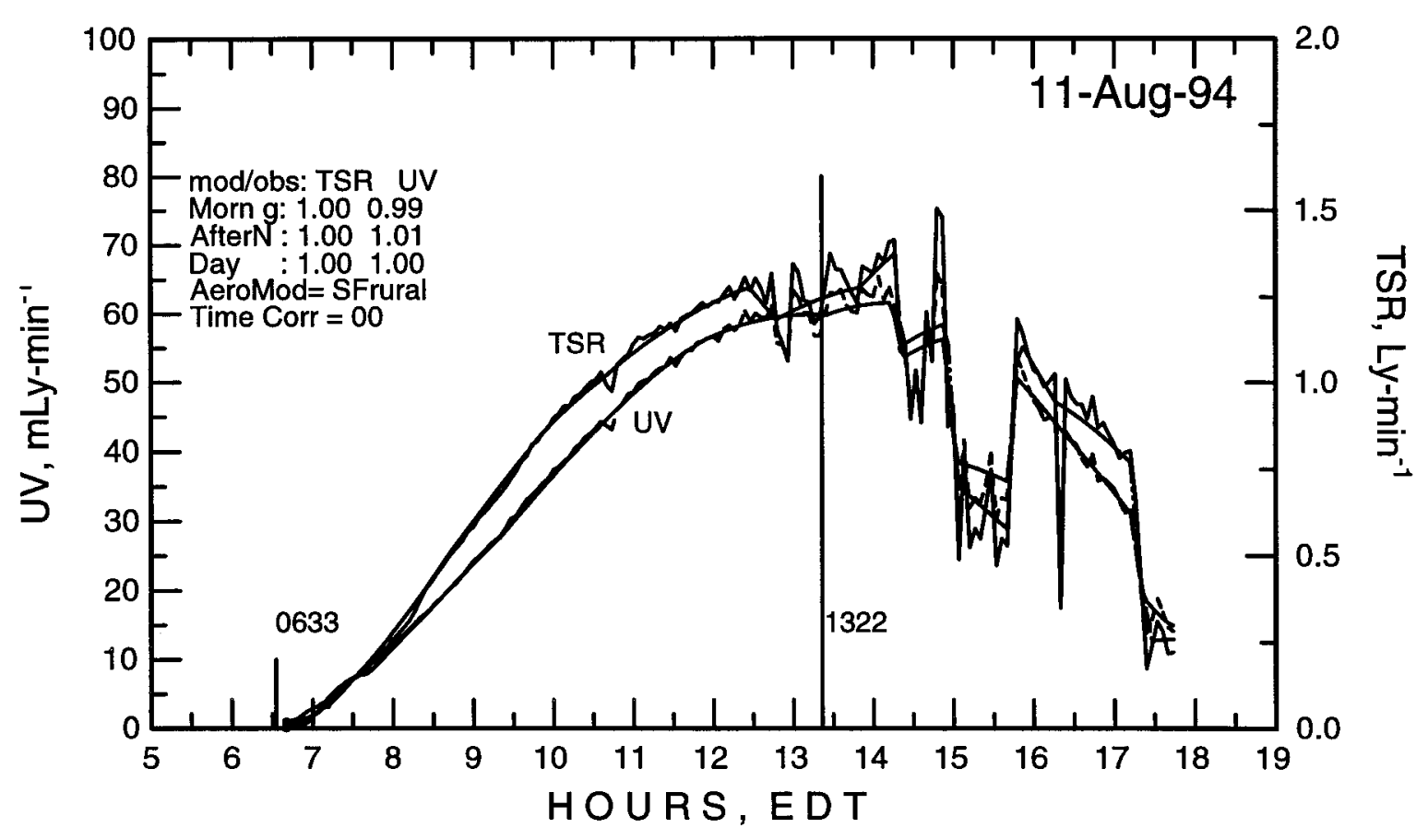

Figure 4.34: Run 10—Solar radiation simulation. 

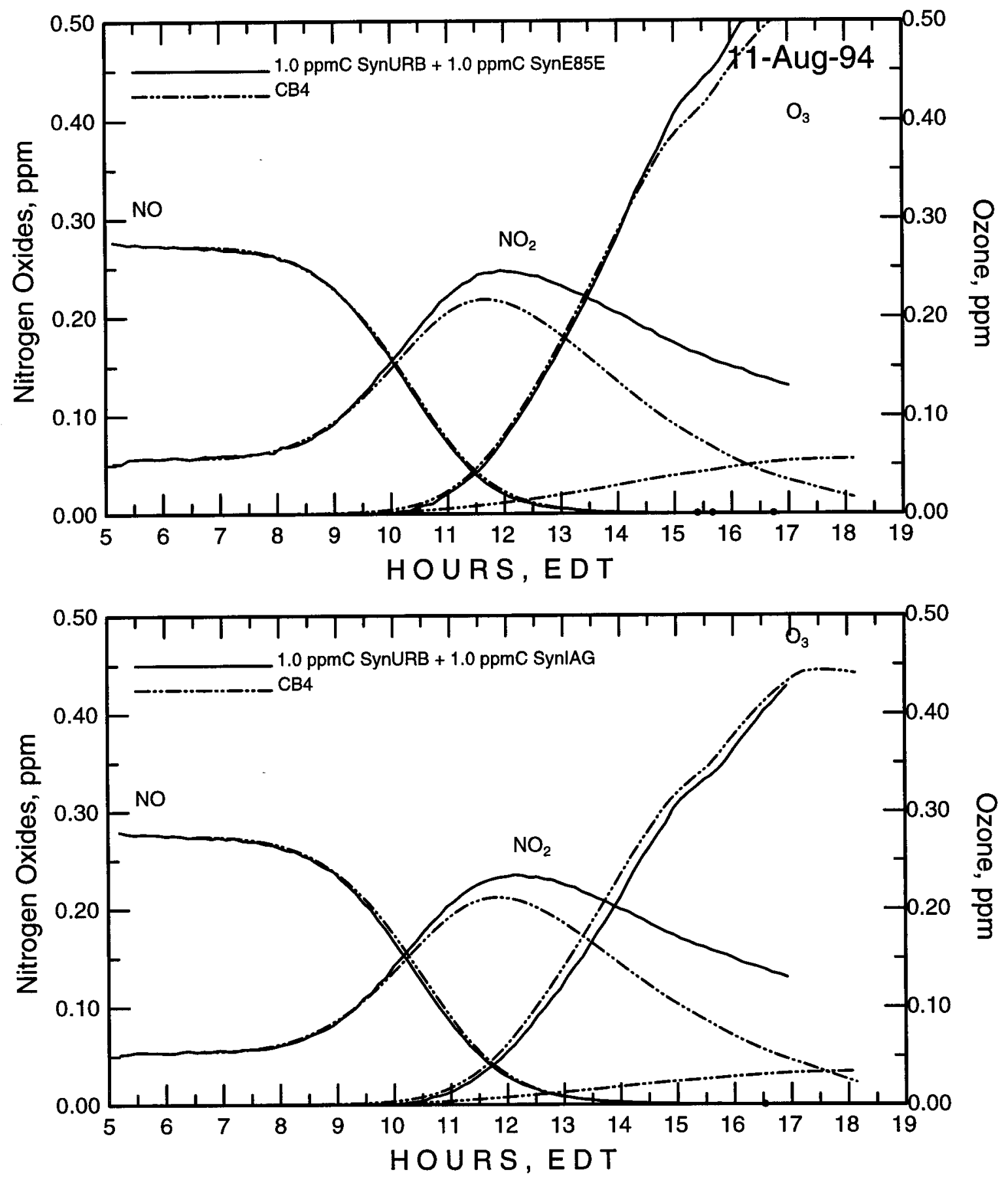

Figure 4.35:. Run 10-6:1 SynUrban/SynIAG:NOx verses 6:1 SynUrban/SynEevE:NOx; Camsrbon Bond Four simulation. 

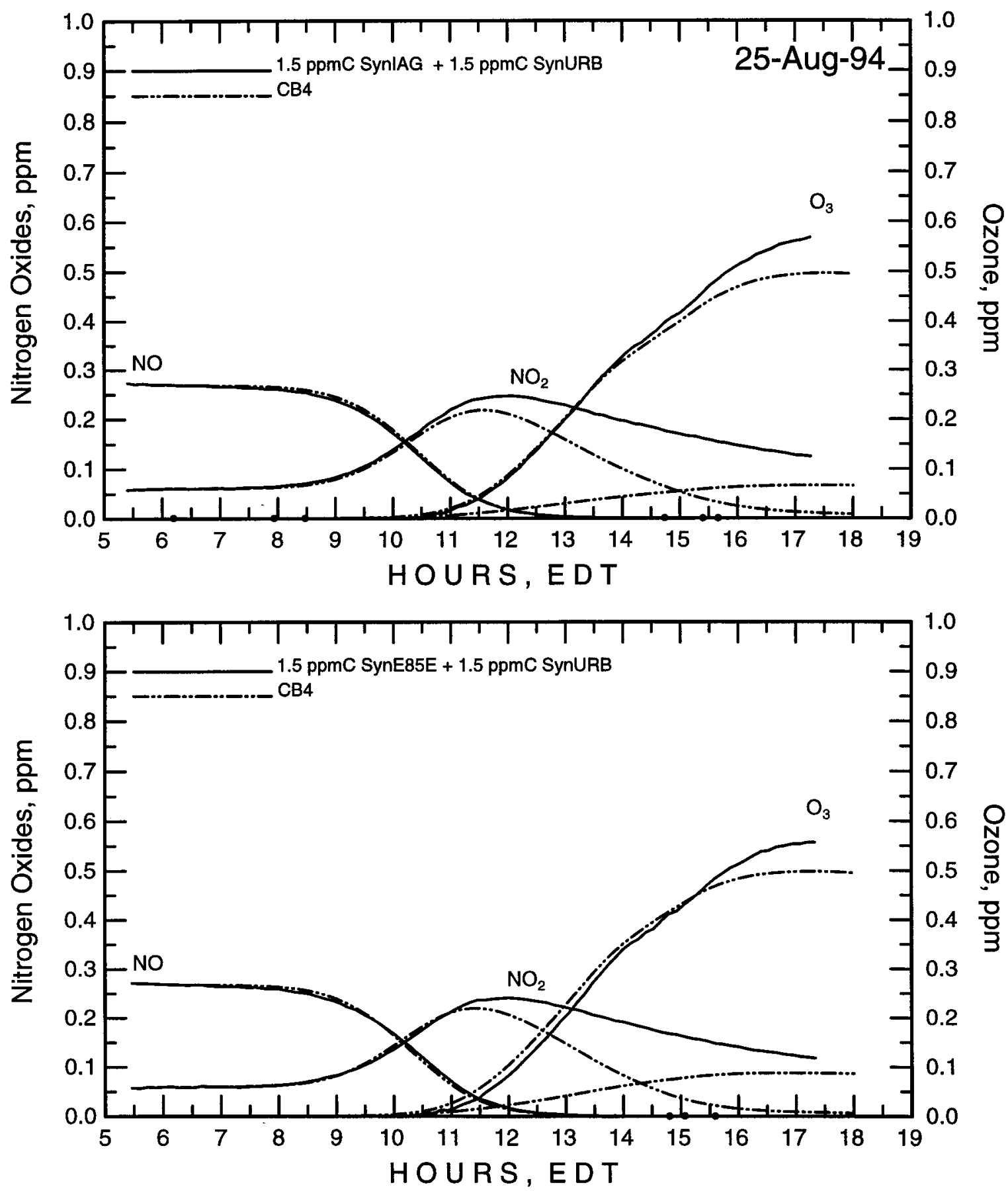

Figure 4.36:. Run 11-9:1 SynUrban/SynIAG:NOx verses 9:1 SynUrban/SynE85E:NO ; Carbon Bond Four simulation. 


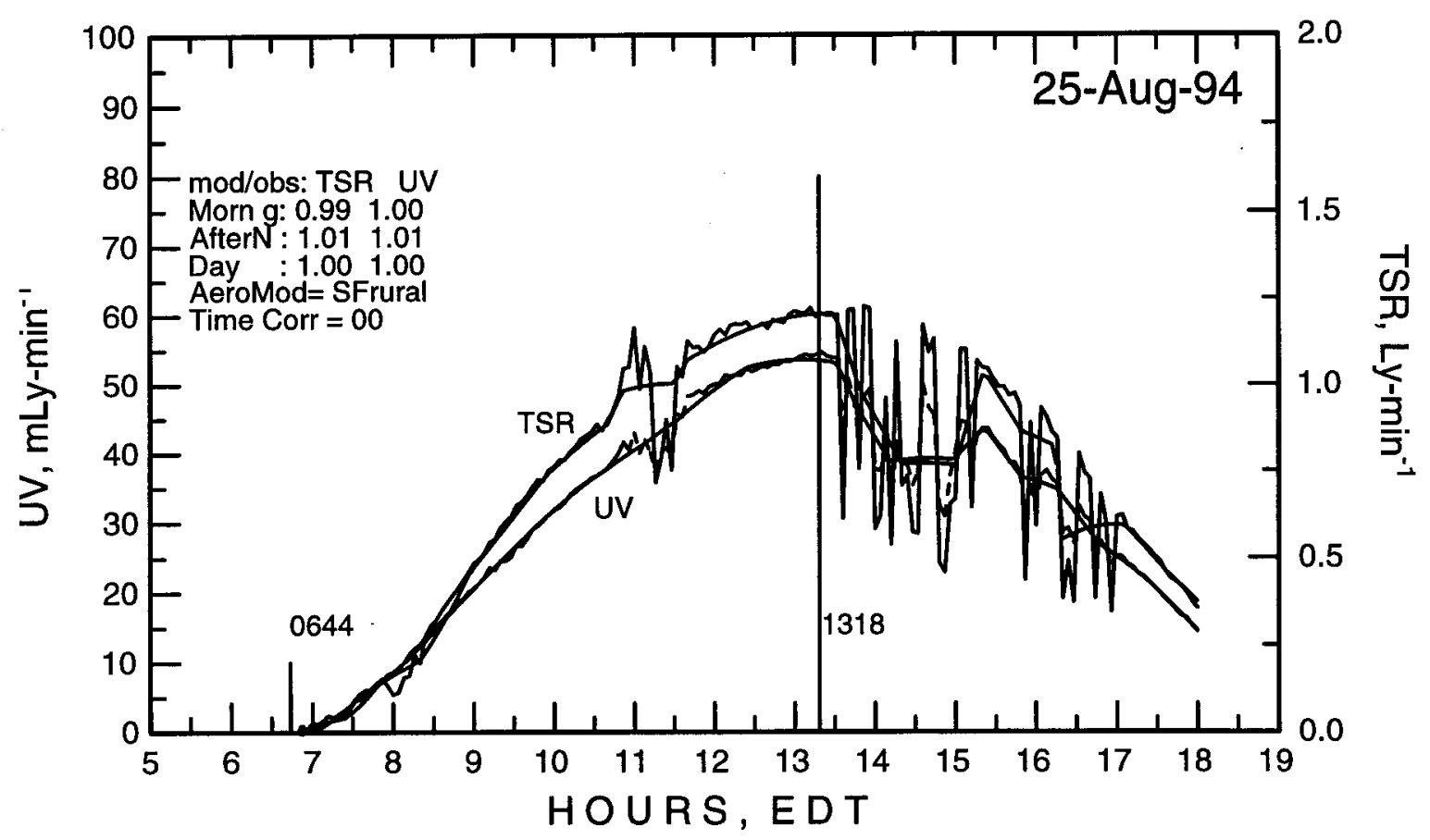

Figure 4.37: Run 11-Solar radiation simulation.

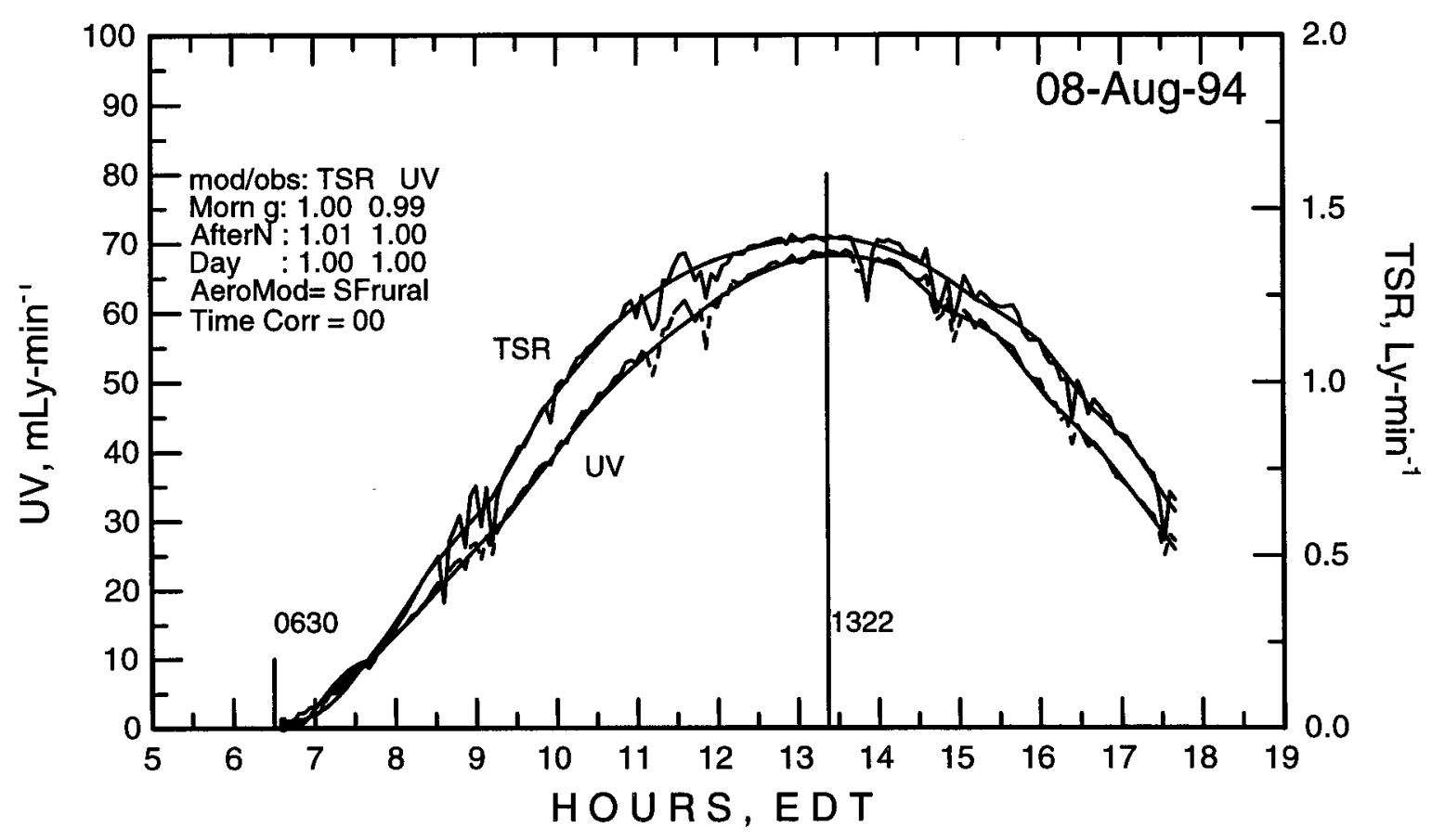

Figure 4.38: Run 12-Solar radiation simulation. 

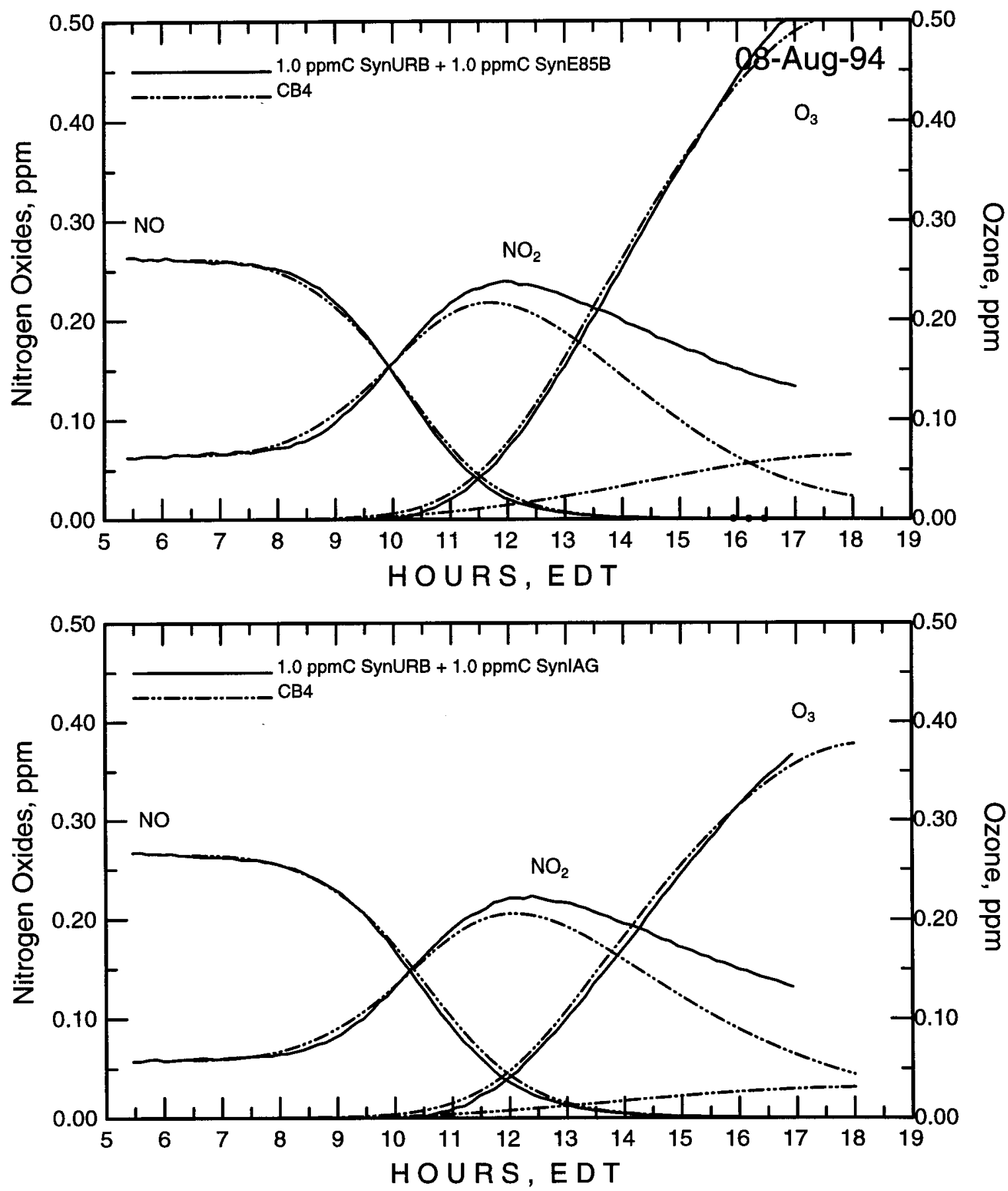

Figure 4.39:. Run 12-6:1 SynUrban/SynIAG:NOx verses 6:1 SynUrban/SynE85B:NO ; Carbon Bond Four simulation. 


\subsubsection{Block 3}

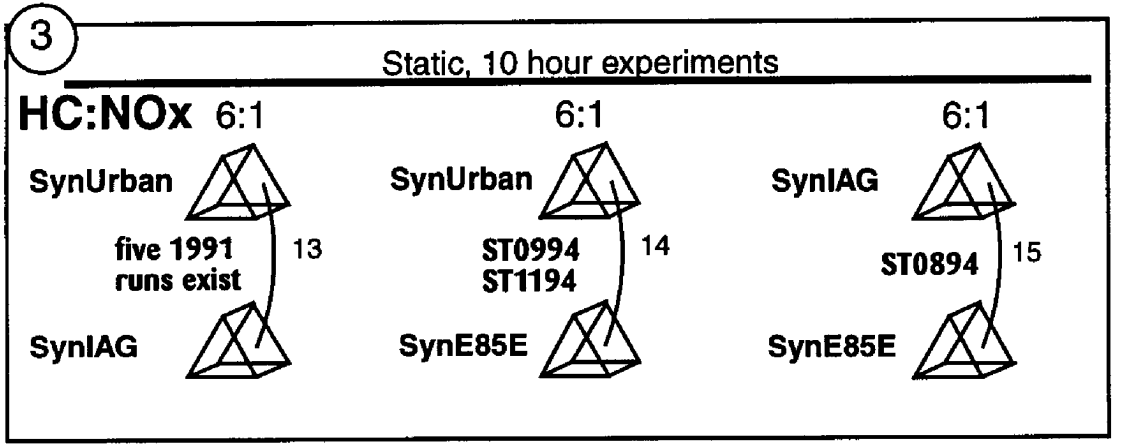

\section{Run 14, 6:1 Urb versus E85E}

ST1194 This experiment compared the reactivity of the EPA E85 and SynURB compositions directly without blending with other mixtures. The E85 side yielded an ozone maximum concentration approximately $23 \%$ lower than did the SynURB side. The solar radiation simulation is shown in Figure 4.40. The CB4 mechanism successfully simulated the SynURB side of this experiment (Figure 4.41), but over-predicted the E85 side. Equal and medium chamber background estimates were used to simulate both sides of this experiment, consistent with the chamber history.

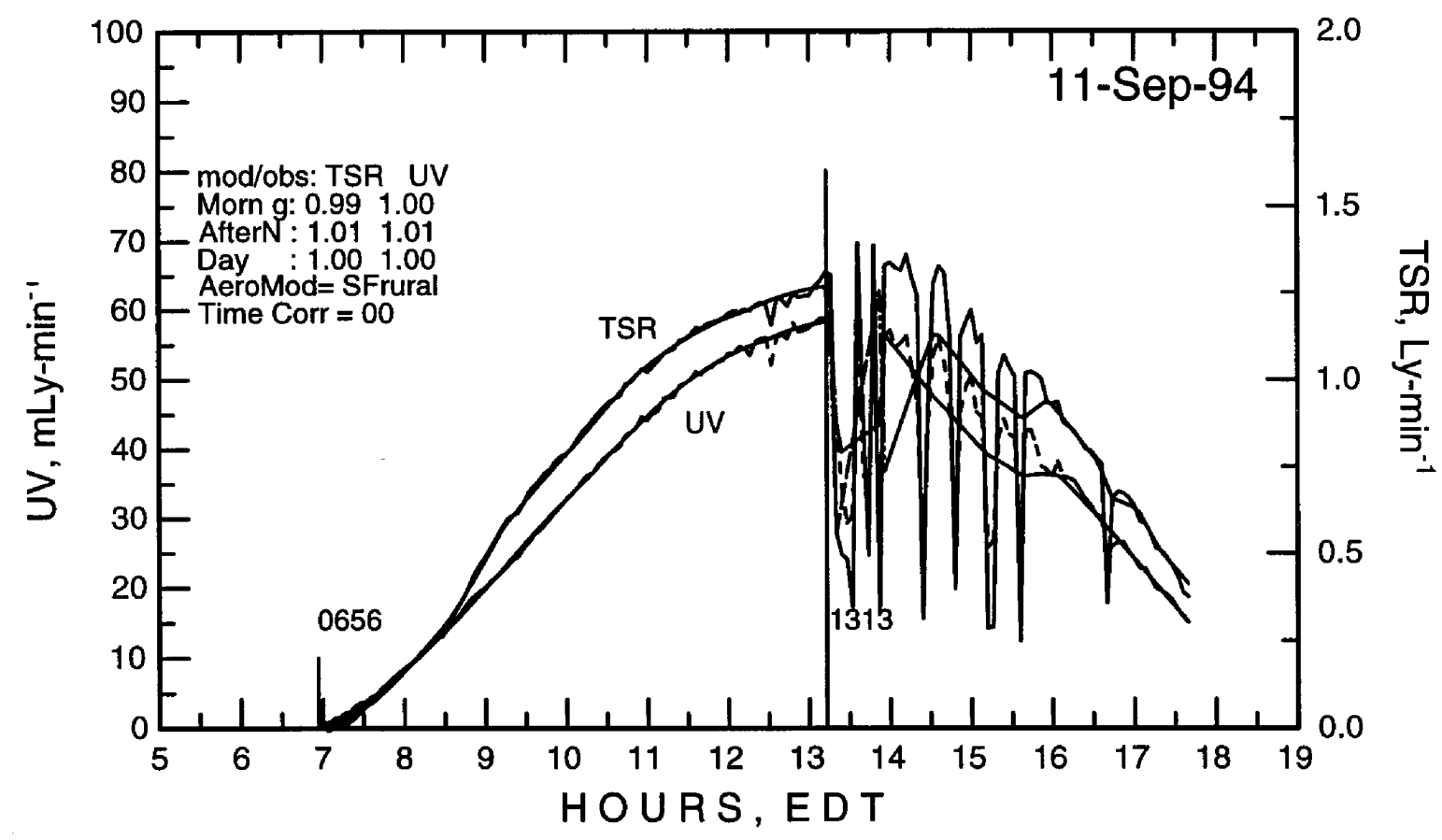

Figure 4.40: Run 14-Solar radiation simulation. 

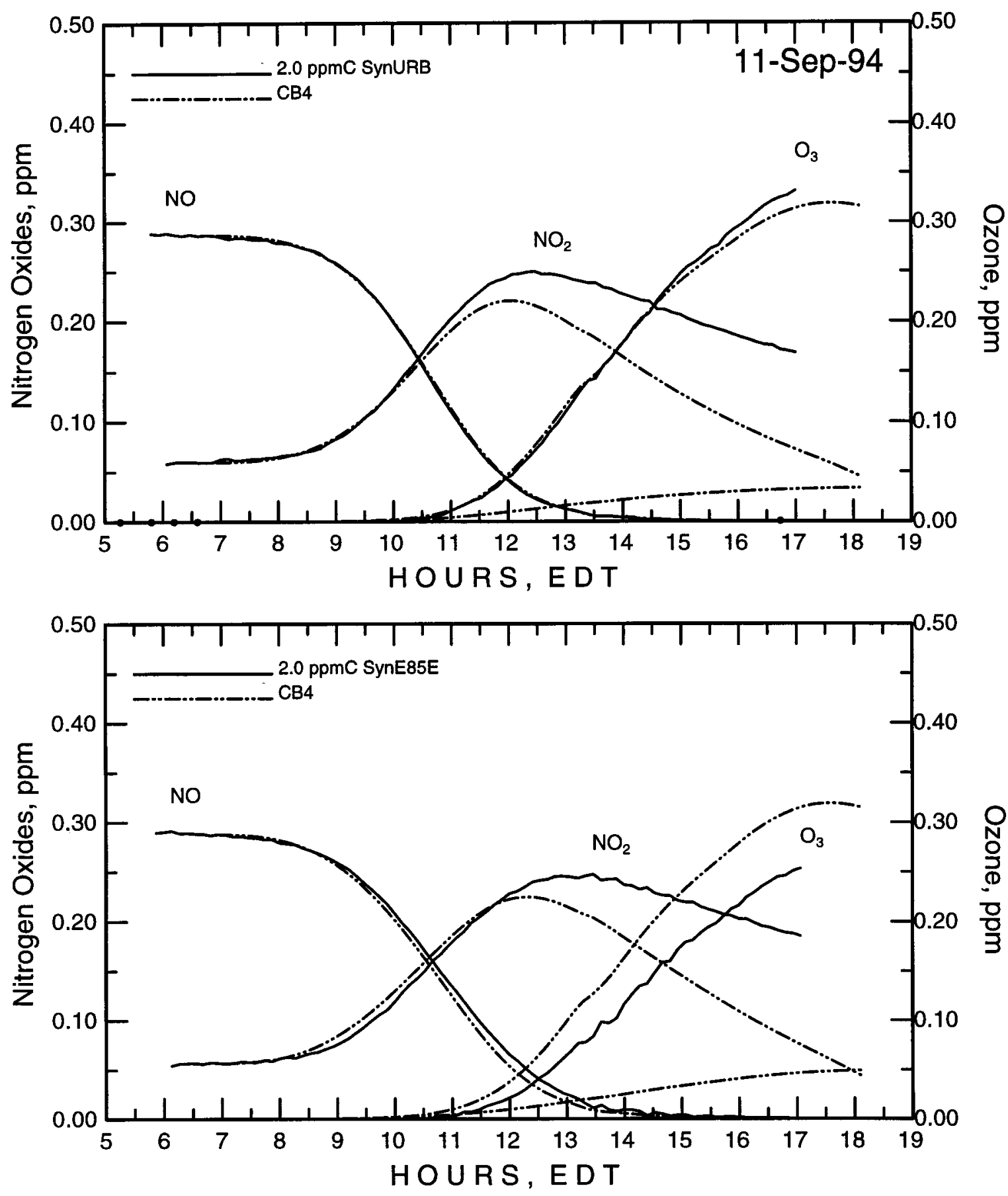

Figure 4.41:. Run 14-6:1 SynUrban:NOx verses 6:1 SynE85E:NO ; Carbon Bond Four simulation. 


\subsubsection{Block 4}

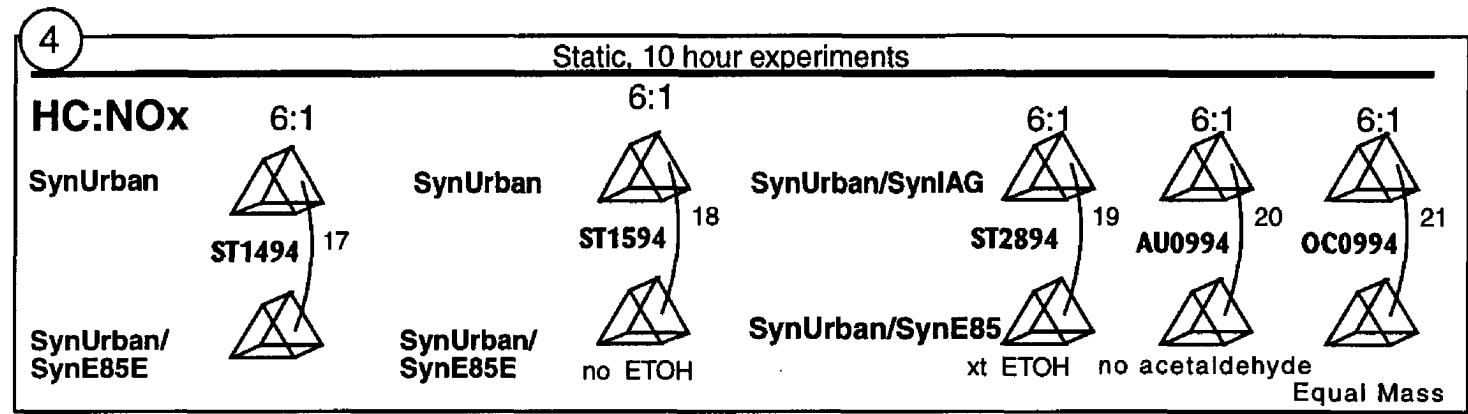

\section{Run 17, 6:1 Urb versus Urb/E85E}

ST1494 This experiment compared the reactivity of the EPA E85 blended 50:50 with SynURB (50\% substitution) against pure SynURB compositions. The E85 side yielded an ozone maximum concentration approximately $20 \%$ lower than did the SynURB-only side. The CB4 mechanism successfully simulated the SynURBside of this experiment, but over-predicted the E85 side (Figures 4.42 and 4.43). Equal and medium-high chamber background estimates were used to simulate both sides of this experiment consistent with the chamber history.

\section{Run 18, 6:1 Urb versus Urb/E85E (no ETOH)}

ST1594 This experiment repeated the September 14 experiment minus the ethanol (EtOH). The E85-minus-EtOH side yielded an ozone maximum concentration approximately $40 \%$ lower than did the SynURB-only side, indicating the significant role in reactivity when compared to the September 14 experiment. The solar radiation simulation is shown in Figure 4.44. The CB4 mechanism successfully simulated the SynURB side of this experiment, but over-predicted the E85 side by approximately $15 \%$ (Figure 4.45). Equal and mediumhigh chamber background estimates were used to simulate both sides of this experiment, consistent with the chamber history. Some lesser amounts of the chamber background estimates used in the modeling may have resulted in a slight under-prediction of the SynURB side and a lesser over-prediction of the E85 side. The values used were selected on the basis of chamber history.

\section{Run 19, 6:1 Urb/IAG versus Urb/E85 (extra EtOH)}

ST2894 This experiment repeated experiment 10 with additional EtOH. Experiment 10 compareed the reactivity of the EPA E85 and IAG compositions with blending 50:50 with SynURB. This experiment was, however, conducted in September. The E85 side yielded an ozone maximum concentration approximately $20 \%$ higher than the IAG side. The CB4 mechanism was used to successfully simulate (very well) the IAG side of this experiment, 
over-predicting the E85 side (Figures 4.46 and 4.47). Equal and medium chamber background estimates were used to simulate both sides of this experiment consistent with the chamber history.

\section{Run 20, 6:1 Urb/IAG versus Urb/E85 (no aldehyde)}

AU0994 This experiment was designed to show the effect of removing the acetaldehyde from the E85 mixture. It repeated experiment 10, the comparison of the reactivity of the EPA E85 (but without the acetaldehyde) and IAG compositions with blending each 50:50 with SynURB. This experiment was conducted in September, however. Even with no initial acetaldehyde, the E85 mixture was more reactive than IAG with aldehyde. The E85 side yielded an ozone maximum concentration approximately $20 \%$ higher than did the IAG side. The solar radiation simulation is shown in Figure 4.48. The CB4 mechanism was used to successfully simulate both sides of this experiment with some small underprediction of the IAG side near the end of this experiment (Figure 4.49). Equal and medium-high chamber background estimates were used to simulate both sides of this experiment, consistent with the chamber history. 

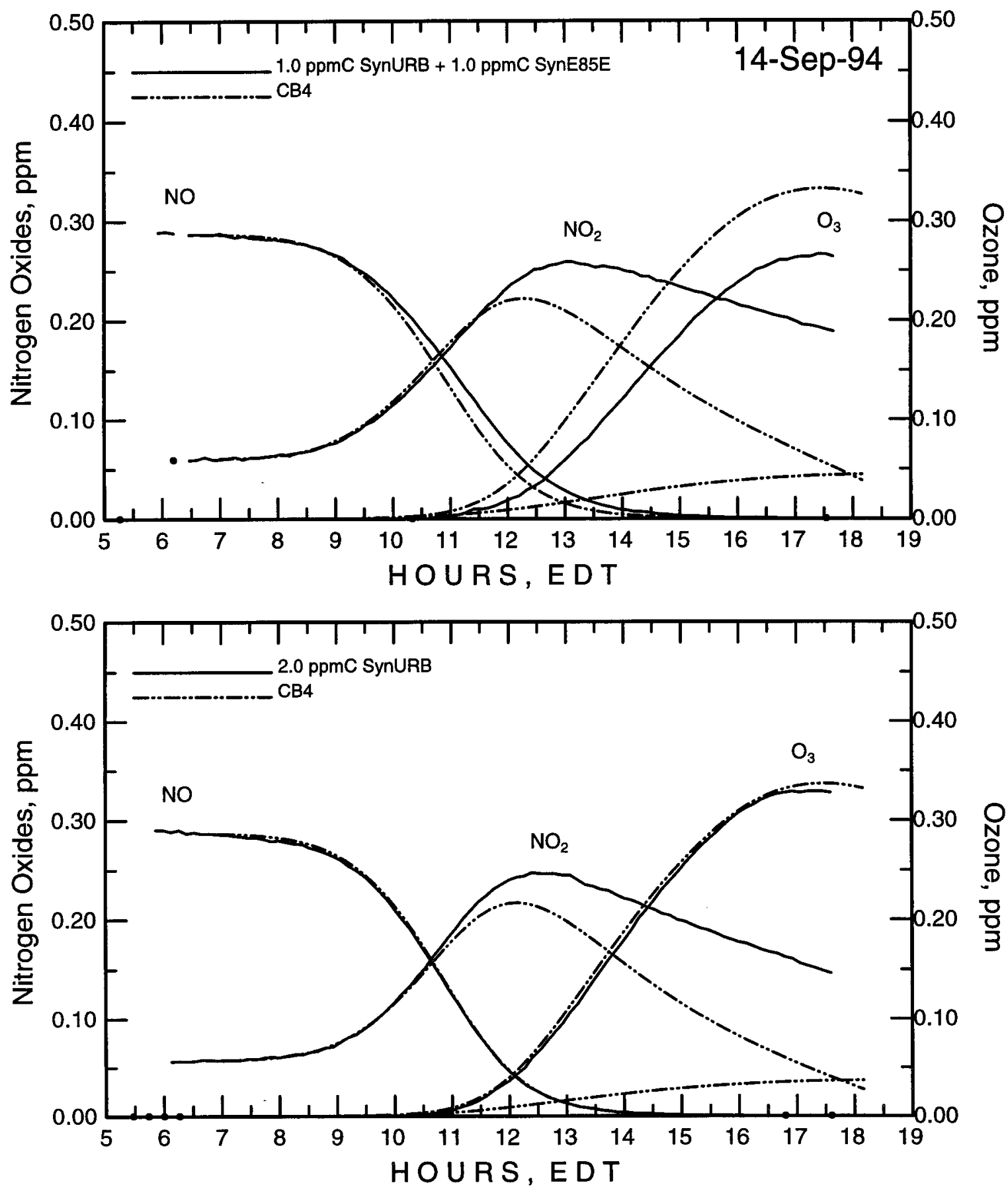

Figure 4.42:. Run 17-6:1 SynUrban:NO ${ }_{x}$ verses 6:1 SynUrban/SynE85E:NOx; Carbon Bond Four simulation. 


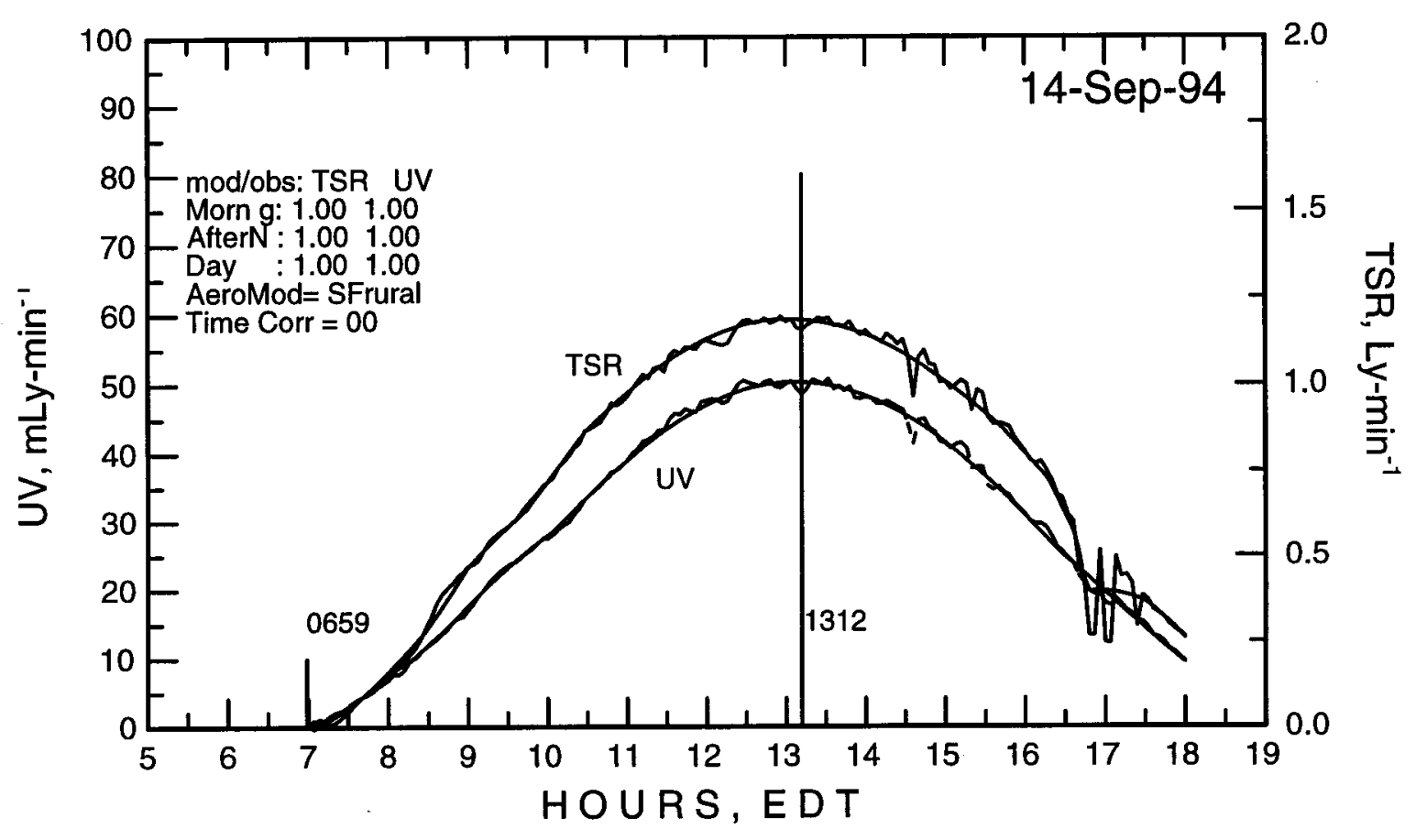

Figure 4.43: Run 17-Solar radiation simulation.

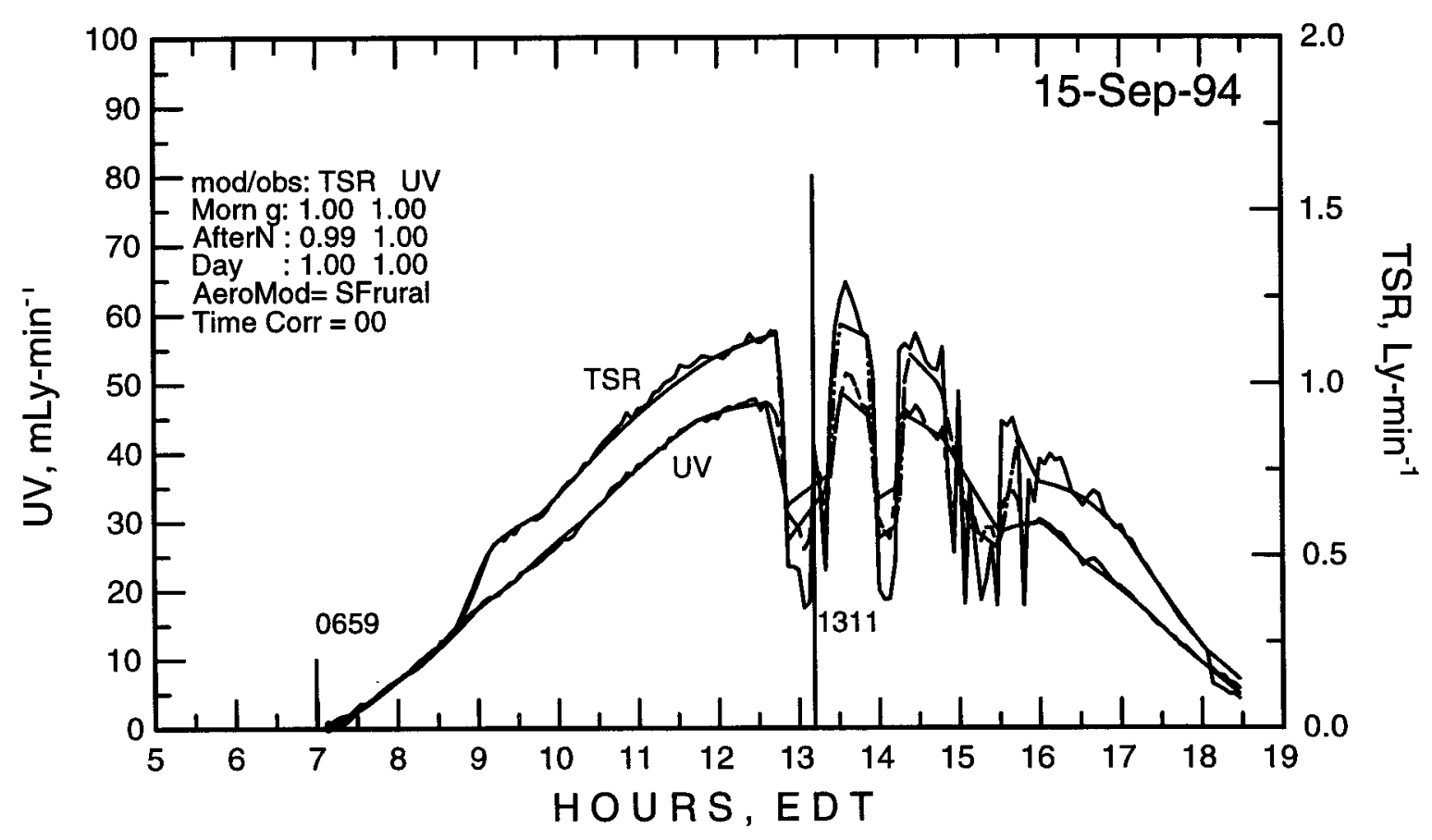

Figure 4.44: Run 18-Solar radiation simulation. 

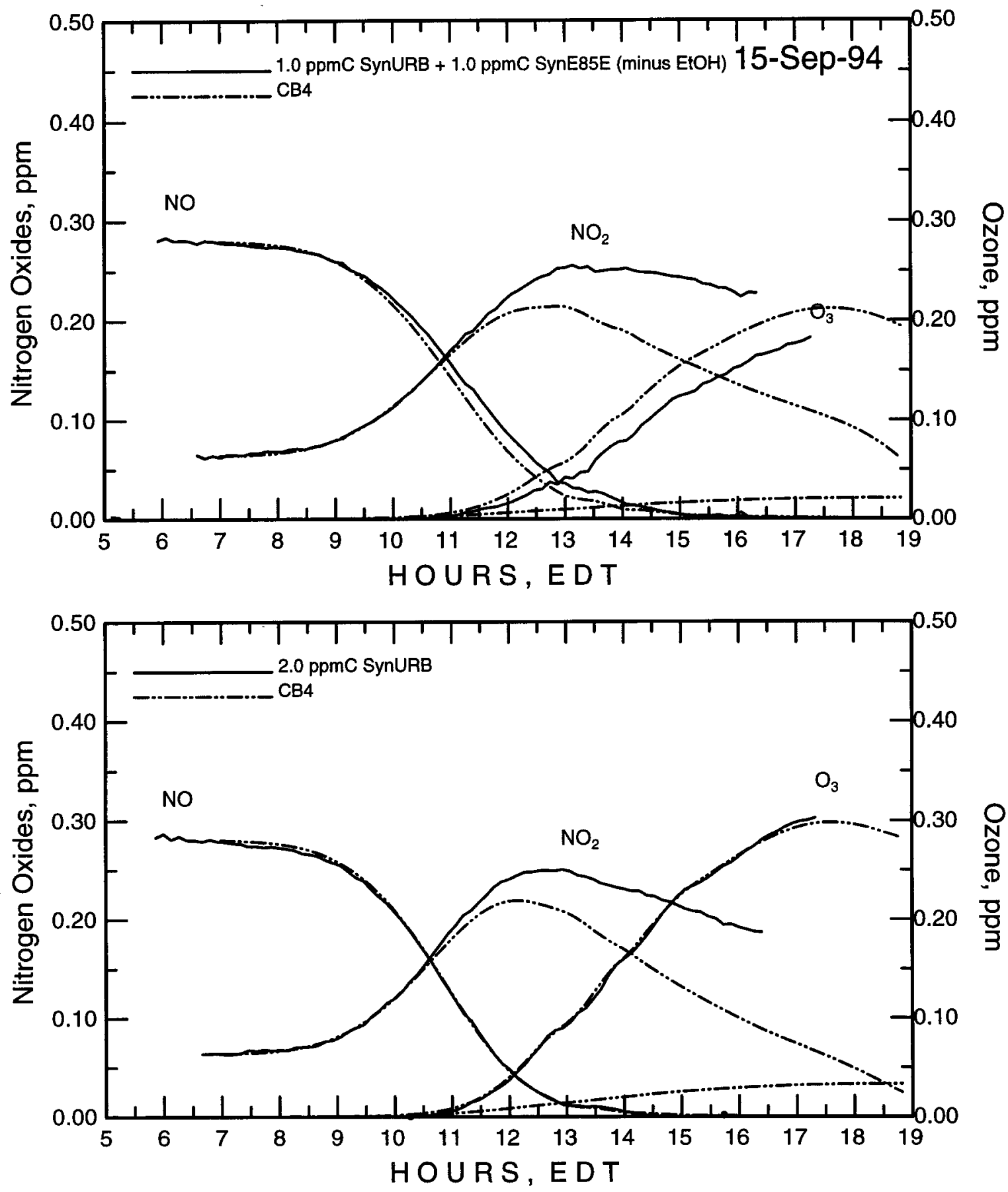

Figure 4.45:. Run 18-6:1 SynUrban:NO verses 6:1 SynUrban/SynE85E(no ETOH):NOx; Carbon Bond Four simulation. 

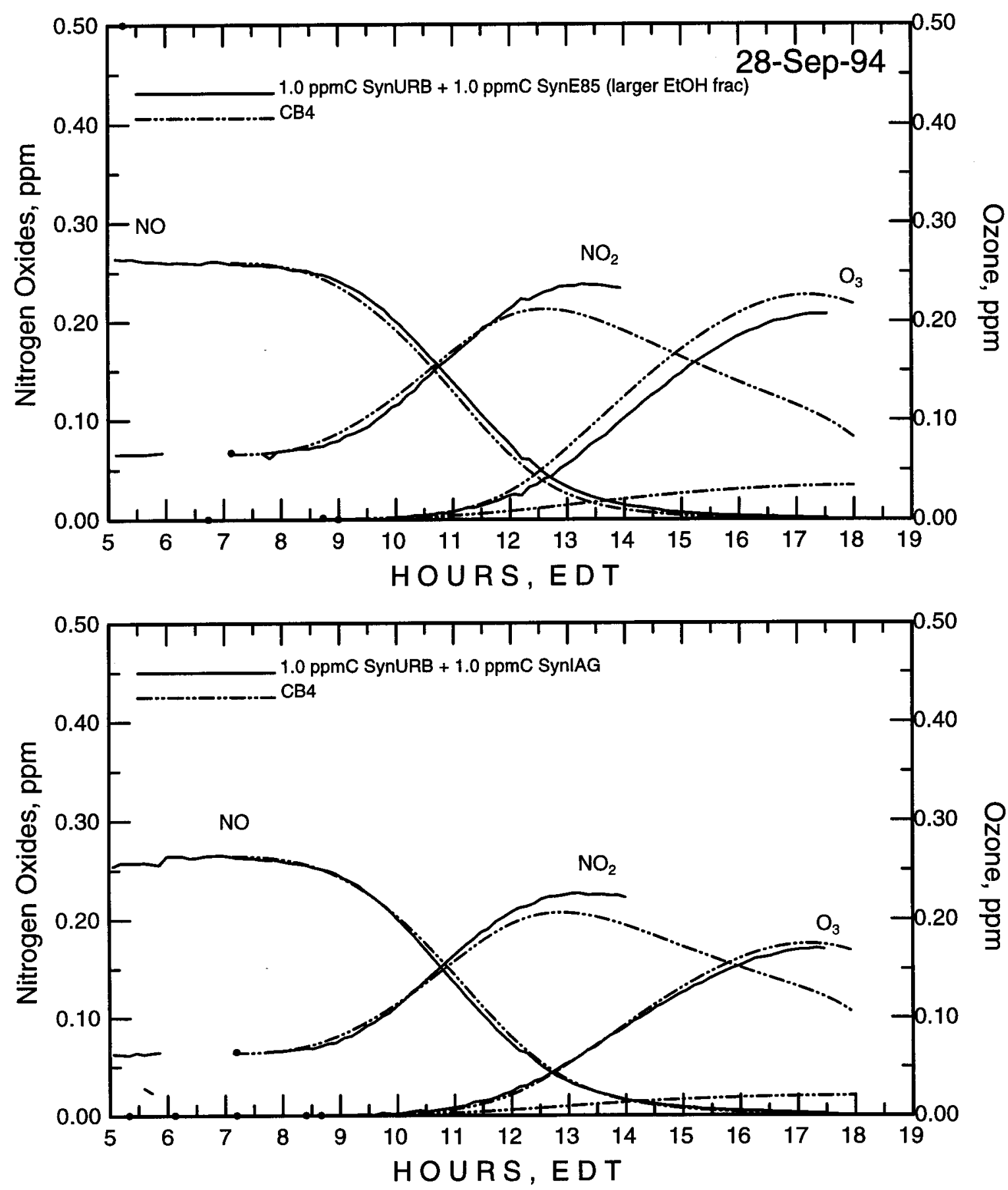

Figure 4.46:. Run 19-6:1 SynUrban/SynIAG:NOx verses 6:1 SynUrban/SynE85E (xt EtOH):NO Carbon Bond Four simulation. 


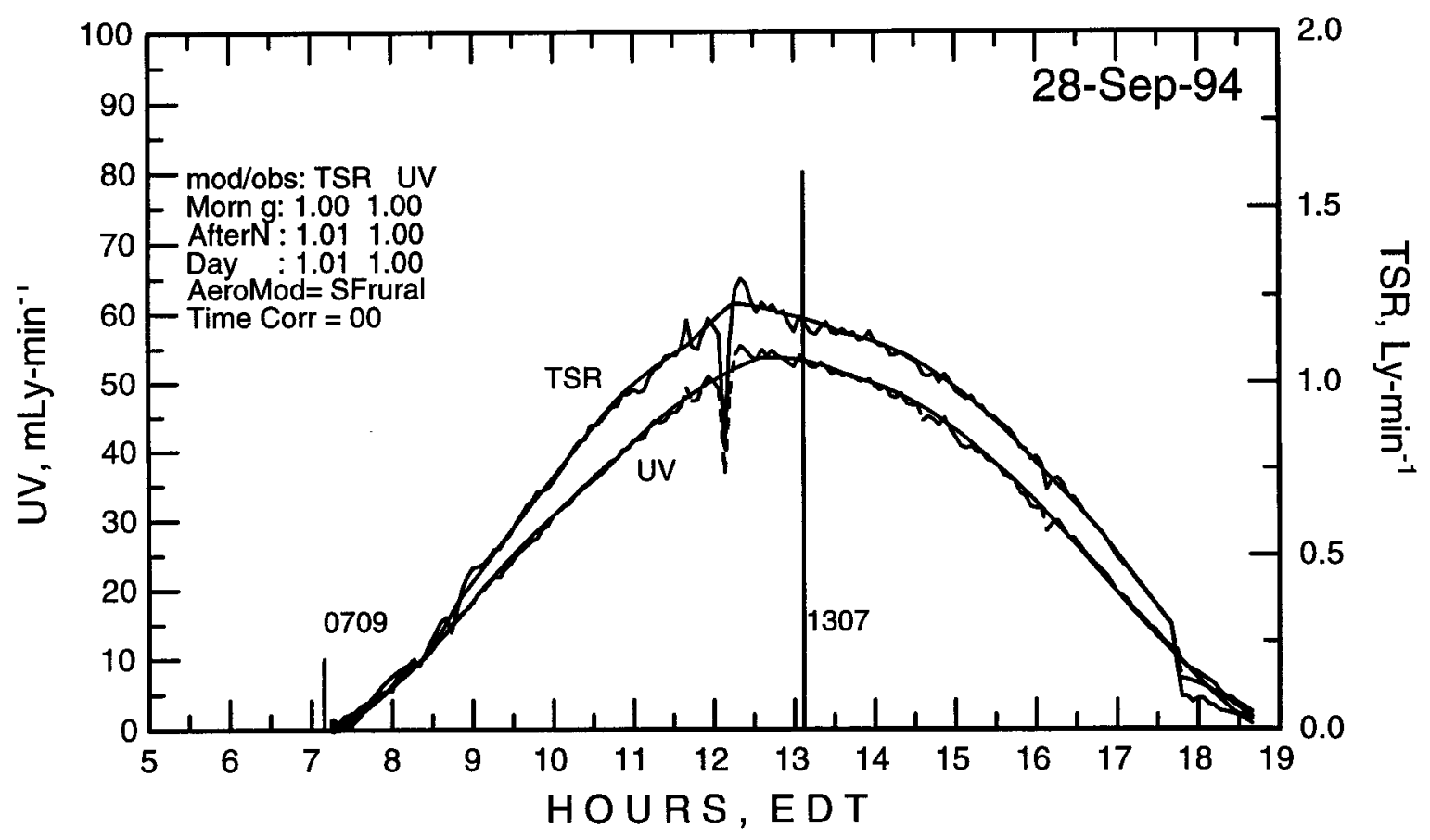

Figure 4.47: Run 19-Solar radiation simulation.

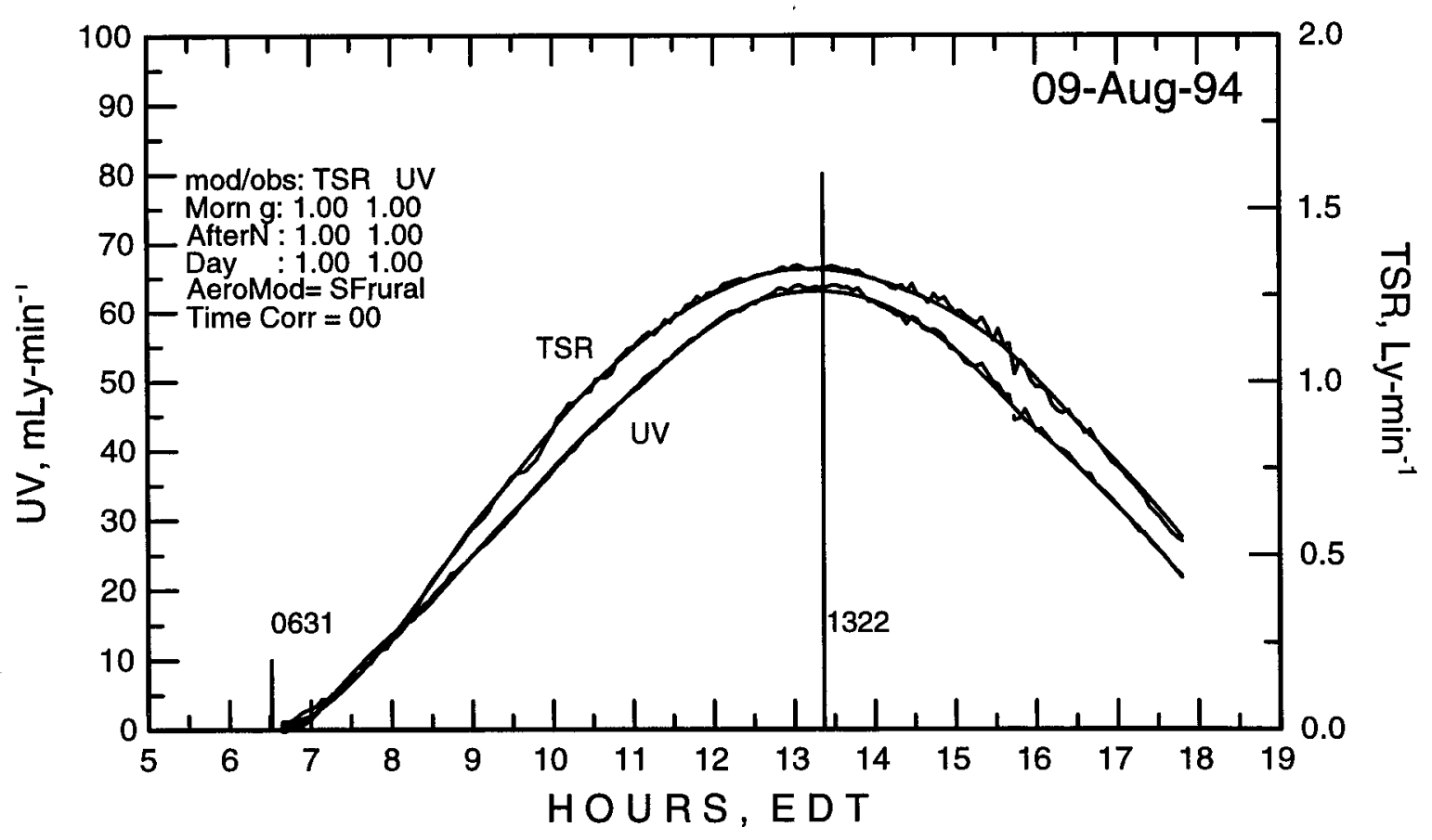

Figure 4.48: Run 20—Solar radiation simulation. 

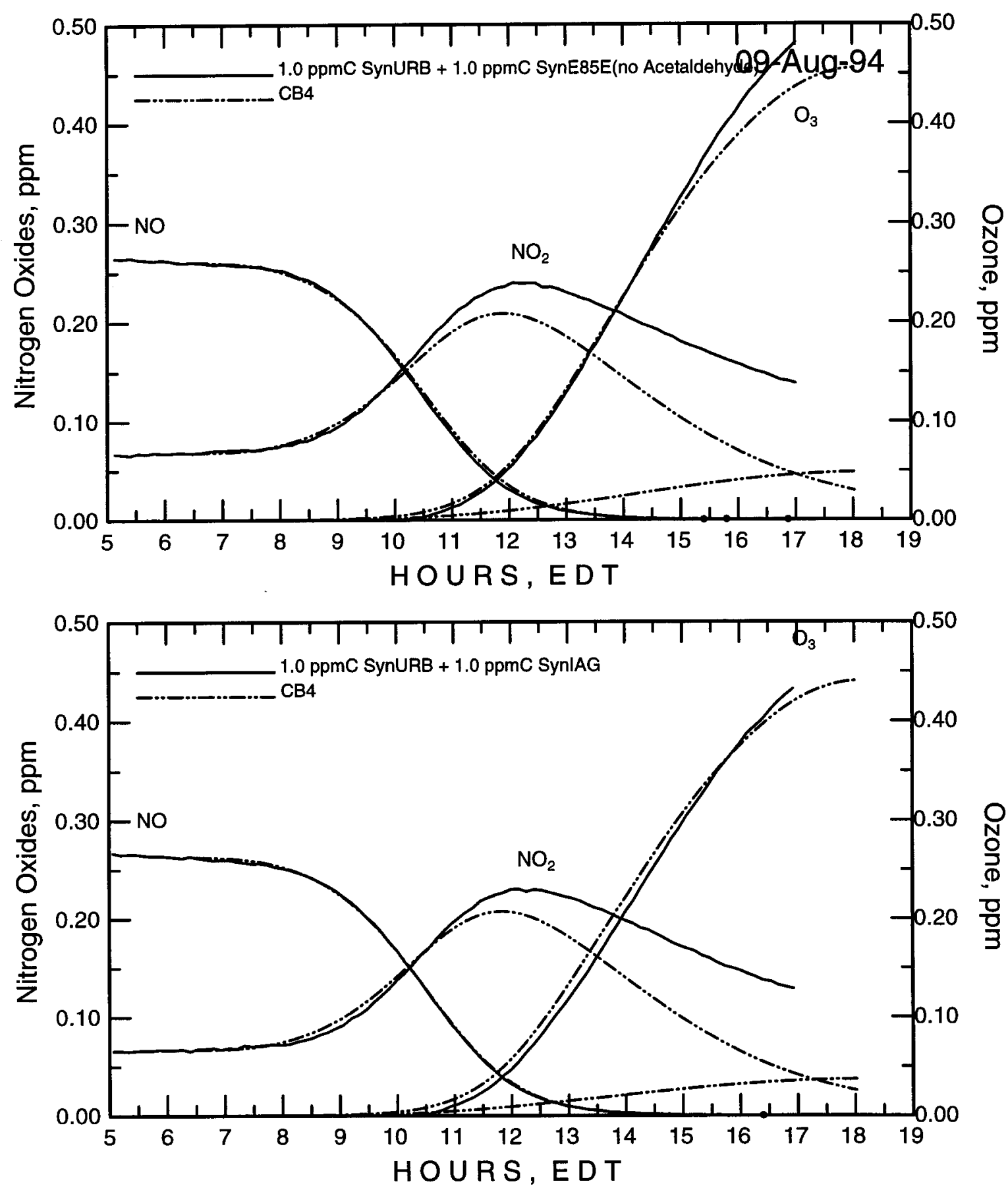

Figure 4.49:. Run 20-6:1 SynUrban/ SynIAG:NO ${ }_{x}$ verses 6:1 SynUrban/ SynE85E (no aldehyde): $\mathrm{NO}_{\mathbf{x}}$; Carbon Bond Four simulation. 


\section{Chapter 5}

\section{New Analytical Methods}

\subsection{Detection and Identification of Carbonyls}

O-(2,3,4,5,6-pentafluorobenzyl)-hydroxylamine or PFBHA has been shown to be an excellent derivatizing agent for detecting polyfunctional carbonyls [6-10]. The corresponding aldehyde or ketone oximes are easily resolved by gas chromatography (GC) and, because of the five fluorine atoms in the molecule, are amenable to detection by electron capture detection (ECD). GC coupled to mass spectrometery (MS) offers a powerful tool to identify unknown polyfunctional carbonyls as well as simple carbonyls.

PFBHA reacts with a carbonyl to produce both the $\mathrm{E}$ and $\mathrm{Z}$ geometric isomers of the corresponding oxime (see Figure 5.1). The reaction is generally conducted in aqueous solution with subsequent extraction of the oximes into an organic phase.

\subsubsection{The Derivatization Procedure}

We used the following derivatization procedure. It applies to either standards made from pure carbonyl compounds or to gas-phase samples collected in water by use of impingers. To the sample, an excess amount of PFBHA aqueous solution $(0.5 \mathrm{ml}$ of $5 \mathrm{mg} / \mathrm{ml}$ solution) is added to $5 \mathrm{ml}$ of the carbonyl solution. The mixture is allowed to stand at room temperature for $24 \mathrm{~h}$. Two drops of $37 \% \mathrm{HCl}$ are added to acidify the mixture just before $2 \mathrm{ml}$ of hexane are added to extract the PFBHA derivatives. Most of the excess PFBHA remains in the acidic aqueous phase, thus avoiding the deterioration of the column and detector by excess PFBHA. About $50 \mathrm{mg}$ of anhydrous $\mathrm{Na}_{2} \mathrm{SO}_{4}$ is added to the hexane extract to adsorb trace amounts of water remaining after separation. One microliter of the hexane extract is injected into a Varian Saturn II Gas Chromatograph and Ion Trap Mass Spectrometer system operated with an MS-grade $60 \mathrm{~m} \times 0.325 \mathrm{~mm} \mathrm{~J} \& \mathrm{~W}$ DB-5 chemically bonded, fused-silica capillary column that is directly connected to the ion trap. The temperature program used is: initial temperature $60^{\circ} \mathrm{C}$, hold for $1 \mathrm{~min}, 60^{\circ} \mathrm{C}$ to $100^{\circ} \mathrm{C}$ at $5^{\circ} \mathrm{C} / \mathrm{min}, 100^{\circ} \mathrm{C}$ to $280^{\circ} \mathrm{C}$ at $10^{\circ} \mathrm{C}$ $/ \mathrm{min}$, hold at $280^{\circ} \mathrm{C}$ for $10 \mathrm{~min}$. The injector has an initial temperature of $60^{\circ} \mathrm{C}$ and is kept 


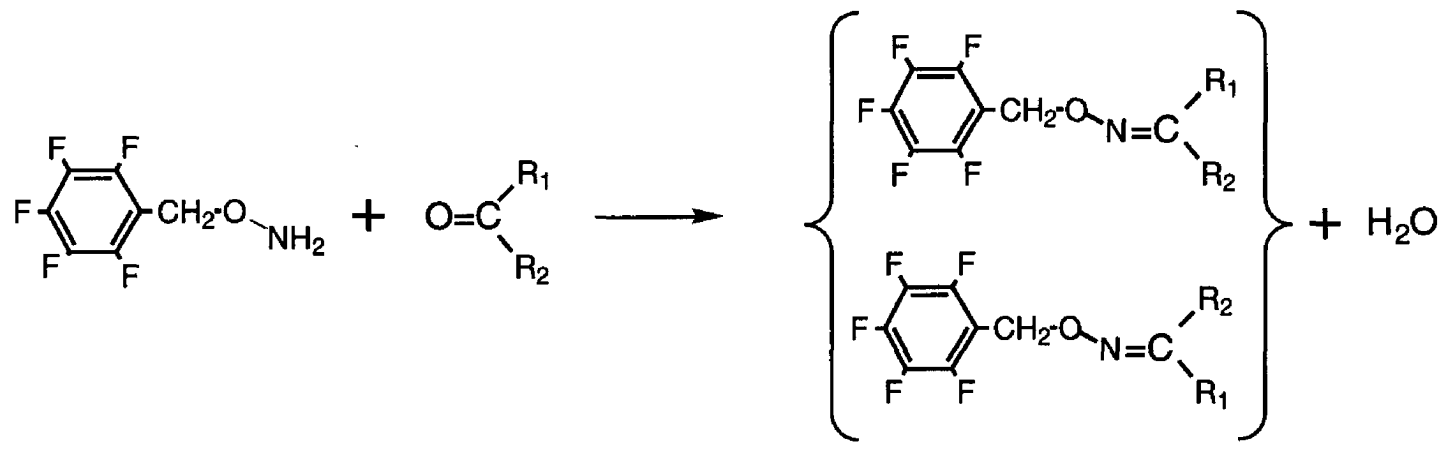

Figure 5.1: The reaction of O-(2,3,4,5,6)-pentafluorobenzyl) hydroxylamine with a carbonyl to produce two isomers for non-symmetrical carbonyls.

at this temperature for 0.80 minutes. Then the injector temperature is raised to $250^{\circ} \mathrm{C}$ at a rate of $180^{\circ} \mathrm{C} / \mathrm{min}$ and is held at this temperature until the analysis is finished. The transfer line between the column and the ion trap is kept at $280^{\circ} \mathrm{C}$. The ITMS can be readily operated in either EI (electron ionization) or CI (chemical ionization) mode, allowing back-to-back analysis of the same extract using different ionization modes. When CI mode was selected, the $\mathrm{CI}$ reagent gas used was methane. A mass scan range between 50 and 650 is usually chosen.

\subsubsection{Ion Trap Mass Spectra}

The Chromatography Systems Division of Varian Associates, Inc., Walnut Creek, California, has made a "permanent loan" of a Saturn II Gas Chromatography and Ion Trap Mass Spectrometer to our research group. This instrument is dedicated to our PFBHA work and is supported by a field service contract.

Figure 5.2 is a schematic diagram of the Varian Saturn gas chromatograph and ion trap mass spectrometer. Figure 5.3 is a detailled cross section of the ion trap itself. The GC uses a capillary column with helium carrier gas to separate species in the injected sample. The end of the column passes through a heated transfer line and exits into the cavity of an ion trap mass spectrometer (ITMS). The ion trap is small, only about 2 inches in diameter, and it sits atop a turbomolecular pump that is connected to an external roughing pump. The ion trap actually consists of a ring electrode and top and bottom end cap electrodes that form a particularly shaped cavity. Holes in the top and bottom end cap electrodes provide openings to the electron charging source above the top end cap and to the electron multiplier detector beneath the bottom end cap electrode. When sample flows into the ion trap cavity, the ion source is allowed to impart a charge to the sample molecules. The ITMS can be readily operated in either EI or CI modes (more on this below). After the sample molecules are charged, voltages on the electrodes cause these ions to be trapped in orbits inside the cavity. By manipulating the electrode voltages, ions of a given mass-to-charge ratio can be made to exit the cavity. The electron multiplier detects positive ions as they are ejected through the holes in the bottom end cap electrode. The electron multiplier has a gain of about $10^{5}$. 


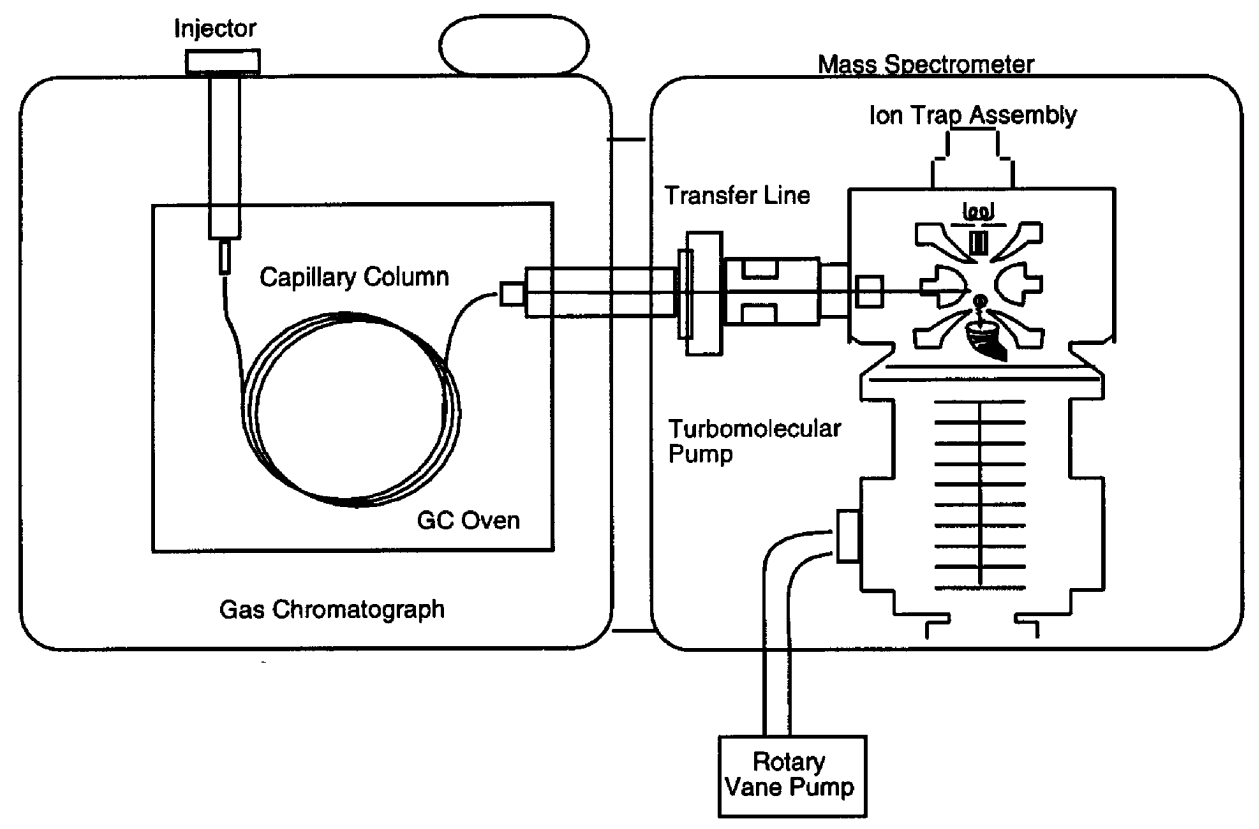

Figure 5.2:. A schematic of the Saturn II capillary column gas chromatograph with ion trap mass spectrometer detector.

A signal line connects the output of the electron multiplier to the processing electronics in the MS interface located in an MS-DOS 386 computer.

An AC voltage of constant fequency $(1.05 \mathrm{MHz}$ ) and variable amplitude (0 to 7500 volts zero to peak) is applied to the ring electrodes of the ion trap. Because of the frequency of this voltage, it is called the radio frequency or RF voltage. When the proper RF voltage is applied, the ion trap electrodes create a three-dimensional, hyperbolic electric field. In this field, ions can be trapped in stable, aperiodic orbits. Prior to ionization of sample molecules, the RF voltage applied to the ring electrode of the ion trap assembly is set to near 0 volts for $2 \mathrm{~ms}$. Above the ion trap is a heated filament that emits thermal electrons capapble of ionizing sample moleucles. These electons are allowed to enter the trap under selected conditions. During the ionization of sample molecules, the RF voltage is set to a specific voltage, called the storage voltage, which is appropiate to store or trap all ions of interest in the ion trap cavity. After the ionization period, the RF voltage is ramped quickly to the voltage apppropriate for the beginning of the mass analysis process (usually above $20 \mathrm{amu}$ to avoid $\mathrm{H}_{2} \mathrm{O}$ ). During mass analysis, the RF voltage is ramped from a low voltage to a higher voltage. As the RF voltage is increased, the trajectories of the ions become unstable in order of increasing mass per charge. Ions with unstable orbits are ejected from the ion trap and are detected by the electron multiplier located below the ion trap. The RF voltage is ramped at a constant rate approximately $5600 \mathrm{amu} / \mathrm{s}$. One complete scan at this fixed rate produces a microscan. Depending on the mass range and scan time, more than one microscan may be obtained during each scan. If two or more microscans are obtained, they are averaged by the data processing system, which improves the spectral quality. 


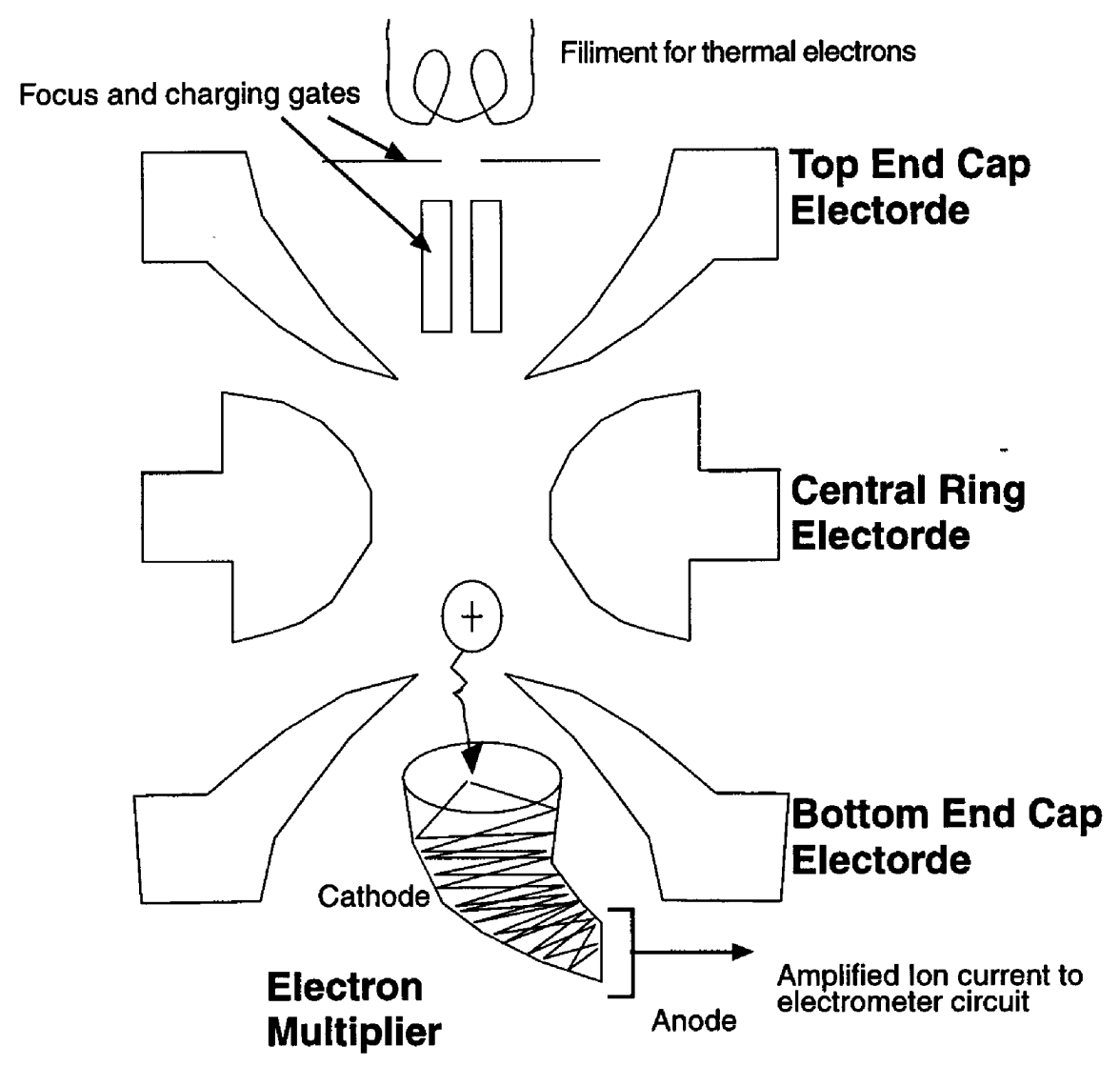

Figure 5.3: Cross section of the Saturn II ion trap mass spectrometer detector.

The flow of sample and helium carrier is controlled by the head pressure on the GC column and by the the Teflon ring spacers in the bottom of the ion trap exiting to the turbomolecular pump. The partial pressure is maintained at approximately $1 \times 10^{-3}$ torr $\left(1.33 \times 10^{-1} \mathrm{~Pa}\right)$ by the electronic flow controllers connected to the computer. Sample is present at about $5 \times 10^{-6}$ Torr. CI reagent gas is present at a partial pressure of $10^{-5}$ Torr if operating in the CI mode. The presence of helium in the ion trap cavity is important because it significantly enhances sensitivity and mass spectral resolution. Prior to their ejection from the ion trap cavity, sample ions collide with helium atoms. These collisions reduce the kinetic energy of the ions, thereby dampening the amplitude of their oscillations. As a result, the ions are focused into the center of the cavity rather than spread throughout the cavity.

The number of ions stored in the ion trap cavity is proportional to the ionization time and the filament emission current. Although the maximum ion-storage capacity of the ion trap is approximately $10^{6}$ to $10^{7}$ ions, space-charge repulsion occurs when the number of ions exceeds $10^{4}$ to $10^{5}$. Space-charge interactions causes a loss in unit mass resolution. To overcome this limitation, a small AC voltage, the axial modulation voltage, is applied to the end cap electrodes. This voltage reduces the effects of the space-charge and mass 
resolution is significantly improved.

$\mathrm{CI}$ is a "softer" ionization than is EI, meaning that CI imparts less energy to the sample molecule being ionized than does EI. Thus the ionized sample molecule undergoes less fragmentation, and an ion indicative of the molecular weight is more likely to be observed. In addition to molecular weight confirmation, CI mass spectra often provide other significant structural information that may not be available for EI mass spectra.

In CI, ionization of sample molecules is a two-step process. First, reagent gas ions are formed by interaction with electrons emitted by the filament to form reagent gas ions. In the second step, the reagent gas ions react with sample molecules in the ion trap to form sample ions. There are four principal types of reactions, described below, between reagent gas ions and sample molecules.

For the compounds examined here, the derivative molecules and the stable fragment ions $\mathrm{C}_{6} \mathrm{~F}_{5} \mathrm{CH}_{2}+$ are abundant in the ion trap chamber and they can form ion adducts. It is noted that the concentration used was high, so there were plenty of derivative molecules in the chamber. In a case where the analyte concentration is low, it is not surprising to find that the $\mathrm{M}+181$ ions are not always present. Actually this is confirmed by the isoprene run analysis, in which the mass spectra of weak unknown carbonyls didn't give the M+181 ion.

In the ion trap, a molecular ion in the EI mass spectra is present for all standard compounds examined. Because the presence of a benzene ring attributes to the stabilization of the molecular ion, aromatic aldehydes give strong molecular ions (RI>39\%). The protonated molecular ion $((\mathrm{MH})+)$ has a comparable intensity to the molecular ion. An adductive ion $(M+181)+$ is also present because of a special principle of the ITMS. The ITMS holds ions in orbits in a chamber and uses a radio frequency (RF) field to separate and analyze ions. As the RF voltage is scanned upwards, ions of increasing mass-to-charge ratio become unstable and are ejected out the chamber. Before ions are ejected, however, secondary ion reactions are possible. For the compounds examined here, the derivative molecules and the stable fragment ions $\mathrm{C}_{6} \mathrm{~F}_{5} \mathrm{CH}_{2}+$ are abundant in the ion trap and they have time to form ion adducts before the $\mathrm{m} / \mathrm{z} 181$ ions are ejected out the trap. We have observed, however, that when the analyte concentration is very low, the $(M+181)+$ ions are often below the detection limit. Other fragment ions characteristic of the molecular weight of the analytes also appear at $(M-17)+,(M-30)+,(M-181)+$ and $(M-197)+$, which are postulated to result from loss of $-\mathrm{OH}, \mathrm{NO}, \mathrm{C}_{6} \mathrm{~F}_{5} \mathrm{CH}_{2}$, and $\mathrm{C}_{6} \mathrm{~F}_{5} \mathrm{CH}_{2} \mathrm{O}$ moieties from the molecules. The intensity of the $\mathrm{M}-30$ ion for aromatic carbonyls is higher than that for aliphatic carbonyls. The $\mathrm{M}-17$ ion is considerably more stable for the hydroxyl carbonyls and ketones. Dicarbonyls show a strong ion at $\mathrm{m} / \mathrm{z} \mathrm{M}-197$ (RI $>9 \%)$ and the relative intensity increases with increasing molecular weight. All of these ions can be useful in confirming a proposed molecular weight. Based on its EI mass spectrum, the molecular weight can be determined for an aromatic carbonyl because ions containing molecular weight information are present with reasonably strong intensity.

The EI molecular ion is not always strong enough, however, for an unambiguous molecular weight determination for aliphatic carbonyls. The chemical ionization mass spectra (CIMS) of all PFBHA derivatives show a strong protonated molecular ion $\mathrm{MH}+$ 
with relative intensity ranging from $7 \%$ to $100 \%$. The intensity of $\mathrm{MH}+$ ion for hydroxy carbonyls are considerably lower than other carbonyls. The $\mathrm{MH}+$ ion is formed through proton transfer with the major ions formed from methane on electron impact such as $\mathrm{CH}_{5}+$, $\mathrm{C}_{2} \mathrm{H}_{5}+$, and $\mathrm{C}_{3} \mathrm{H}_{5}+$, e.g. An ion with $\mathrm{m} / \mathrm{z}=\mathrm{M}-197$, loss of $\mathrm{C}_{6} \mathrm{~F}_{5} \mathrm{CH}_{2} \mathrm{O}$ moiety from the neutral molecule, is consistently present with reasonably strong intensity (RI $>3 \%$, except for hydroxy carbonyls). Some of the isomers of hydroxyl carbonyl PFBHA derivatives have a relatively lower intensity ion at $m / z=M-197$. Ions with $m / z=M+29$ and $M+41$, arising from the reaction of neutral molecule with the major reagent ions $\mathrm{C}_{2} \mathrm{H}_{5}+$ and $\mathrm{C}_{3} \mathrm{H}_{5}+$, are observed for all carbonyls. A peak at $m / z=M+181$ is also present in CIMS to help confirm molecular weight diagnosis. These ions aid the determination of molecular weight of unknown carbonyls.

The PFBHA derivatives of hydroxy carbonyls in CIMS have a strong characteristic peak at $\mathrm{m} / \mathrm{z}=\mathrm{M}-17$ (RI $>23 \%$ ), corresponding to loss of $-\mathrm{OH}$ from the molecular ion or loss of $\mathrm{H}_{2} \mathrm{O}$ from $\mathrm{MH}+$ ions. Other carbonyl derivatives also show a peak at $\mathrm{M}-17$, but not so strong as hydroxy carbonyls $(\mathrm{RI}<12 \%)$. Therefore the presence of a strong ion of $M-17$ is a useful but not conclusive indicator of the presence of a hydroxy group in addition to the carbonyl functional group. Further confirmation of the presence of hydroxy group can be accomplished by a second derivatization step, e.g., derivatizing the hydroxyl group using silylation reagents or methylation reagents. The disappeance of the original PFBHA derivative peak and the emergence of a new peak serve as evidence of the presence of an hydroxyl group [10]. We are developing other confirmation methods to identify unsaturated carbonyls and carbonyls with adjacent hydroxy groups. These methods, which we have demonstrated to work on laboratory test samples, carry out additional reactions upon the extracted derivatives using selective oxidative reagents. In the first case, after performing the first injection into the GC/MS system, an aliquot of the extract is reacted with bromine in $\mathrm{CCl}_{4}$. Carbonyl compounds with unsaturated bonds will react with the bromine and be destroyed and new peaks of the brominated products will appear. In the second case, a similar process is carried out, but the reagent added to an aliquot of the extract is periodic acid $\left(\mathrm{HIO}_{4}\right)$, which will attack hydroxyl groups on adjacent carbons, cleave the carboncarbon bond, and produce aldehyde and ketone groups where there were hydroxyl groups. The latter method has been used for some time in carbohydrate chemistry to detect sugars. 


\section{Chapter 6}

\section{Aromatic Reaction Mechanisms}

\subsection{Experimental Results}

We conducted more than 20 indoor Teflon Bag Reactor (TBR) experiments with alkyl subsitututed benzene compounds and $\mathrm{NO}_{\mathrm{x}}$. These were used to develop the sampling and indentification techniques. Although these are useful in determining the types of products that were produced in these systems, the photolytic spectrum for the TBR differs significantly from sunlight. Because many of the carbonyl products produced also photolyze, outdoor experiments more realistically represent the behavior of this complex system.

Three dual outdoor chamber experiments were performed for this project:

- Toluene verses m-xylene

- $\mathrm{p}$-xylene verses 1,3,5-trimethyl benzene

- o-xylene verses 1,2,4-trimethyl benzene.

Detailed analysis of these experiments will be presented here.

The outdoor chamber initial conditions are given in Tables 6.1 to 6.3. Standard SegFile data plots of $\mathrm{NO}, \mathrm{NO}_{2}$ and $\mathrm{O}_{3}$ time series and the light conditions, temperature, dew point, TSR and UV intensity are given in Figures 6.1 to 6.3. For each of the aromatic compounds examined, a series of carbonyl products was detected using the PFBHA-GC/MS technique. These will be described in detail in subsequent figures. Analysis of the batch samples collected in the indoor TBR experiments showed similar carbonyl products to those detected in the outdoor smog chamber experiments. Therefore, only the results from the outdoor chamber experiments will be described in this report. 
Table 6.1: Initial Conditions for AU3095

(Units are ppm or ppmC)

\begin{tabular}{|c|c|c|c|}
\hline \multicolumn{2}{|c|}{ RED SIDE } & \multicolumn{2}{|c|}{ BLUE SIDE } \\
\hline Compound & Amount & Compound & Amount \\
\hline \multicolumn{4}{|c|}{ Initial Nitrogen Oxides } \\
\hline $\mathrm{NO}_{\mathrm{x}}$ & 0.6218 & $\mathrm{NO}_{\mathrm{x}}$ & 0.6171 \\
\hline NO & 0.5027 & NO & 0.5230 \\
\hline $\mathrm{NO}_{2}$ & 0.1191 & $\mathrm{NO}_{2}$ & 0.0941 \\
\hline \multicolumn{4}{|c|}{ Initial Volatile Organic Compounds } \\
\hline$m-x y l$ & 8.0200 & Toluene & 7.3100 \\
\hline \multicolumn{4}{|c|}{ Other Measured Initial Compounds } \\
\hline BVOC9109 & 0.1440 & BVOC9109 & 0.1440 \\
\hline $\mathrm{CO}$ & 0.250 & $\mathrm{CO}$ & 0.250 \\
\hline \multicolumn{4}{|c|}{ Estimated Initial Compounds } \\
\hline HONO & 0.003 & HONO & 0.003 \\
\hline $\mathrm{CH}_{4}$ & 1.790 & $\mathrm{CH}_{4}$ & 1.790 \\
\hline $\mathrm{H}_{2}$ & 0.580 & $\mathrm{H}_{2}$ & 0.580 \\
\hline \multicolumn{4}{|c|}{ Chamber Model Parameters } \\
\hline $\mathrm{NO}_{2} \longrightarrow \mathrm{HONO}$ & $2.5 \times 10^{-3}$ & $\mathrm{NO}_{2} \longrightarrow \mathrm{HONO}$ & $2.5 \times 10^{-3}$ \\
\hline wall. $\mathrm{HNO}_{3} \longrightarrow \mathrm{NO}_{2}$ & $2.0 \times 10^{-3}$ & wall. $\mathrm{HNO}_{3} \longrightarrow \mathrm{NO}_{2}$ & $2.0 \times 10^{-3}$ \\
\hline
\end{tabular}

Three Previous Chamber Events

950803.SEG Aromatics run

950801.SEG Delta Ethylene run

950726.SEG Matched Propylene run 

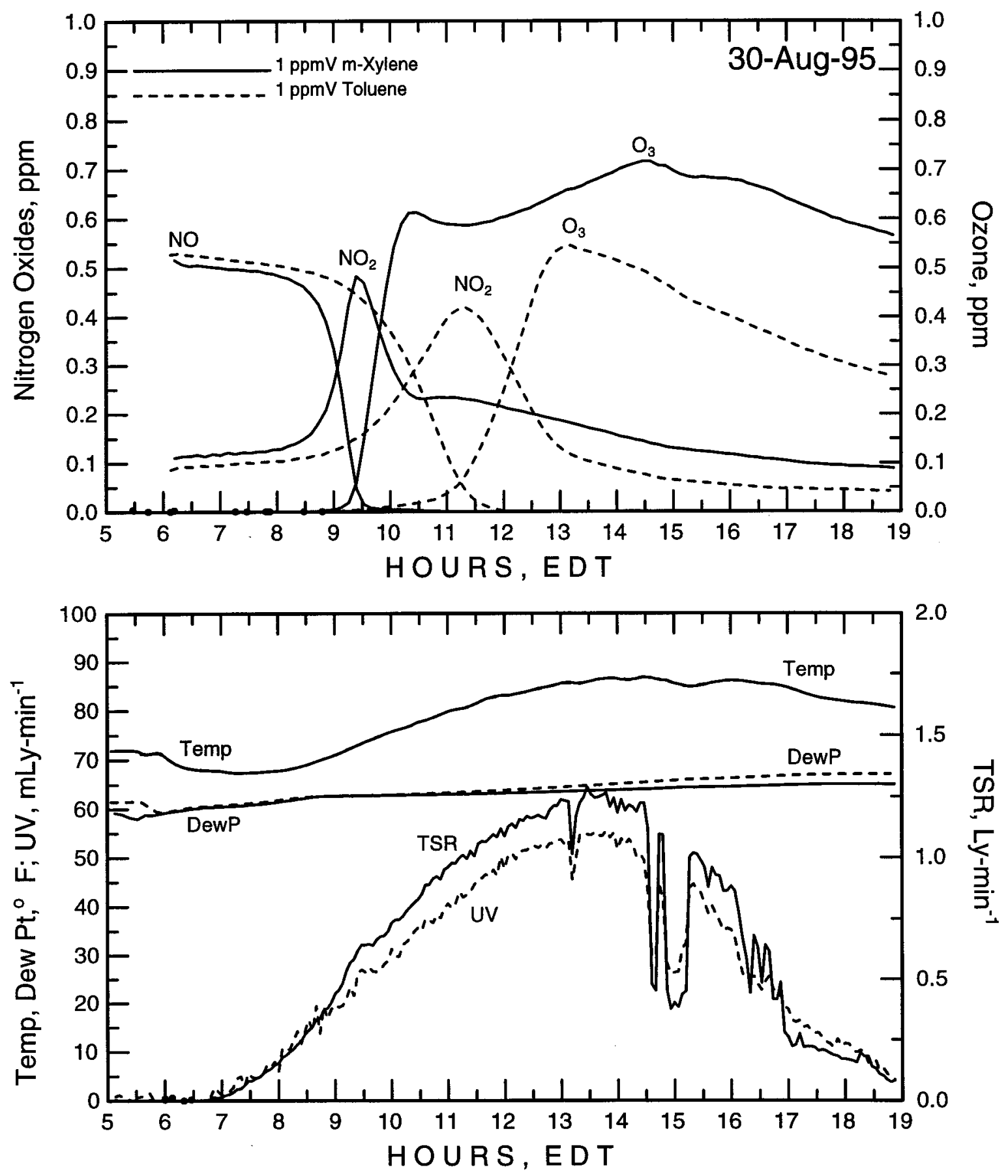

Figure 6.1: Run Arom-1 Toluene: $\mathrm{NO}_{\mathrm{x}}$ versus m-Xylene:NO 
Table 6.2: Initial Conditions for AU0395

(Units are ppm or ppmC)

\begin{tabular}{|c|c|c|c|}
\hline \multicolumn{2}{|c|}{ RED SIDE } & \multicolumn{2}{|c|}{ BLUE SIDE } \\
\hline Compound & Amount & Compound & Amount \\
\hline \multicolumn{4}{|c|}{ Initial Nitrogen Oxides } \\
\hline $\mathrm{NO}_{x}$ & 0.6407 & $\mathrm{NO}_{\mathrm{x}}$ & 0.6209 \\
\hline NO & 0.5323 & NO & 0.5368 \\
\hline $\mathrm{NO}_{2}$ & 0.0984 & $\mathrm{NO}_{2}$ & 0.0841 \\
\hline \multicolumn{4}{|c|}{ Initial Volatile Organic Compounds } \\
\hline$P-X y l$ & 8.0300 & $C-1(C-3) B z 5-C$ & 9.0000 \\
\hline \multicolumn{4}{|c|}{ Other Measured Initial Compounds } \\
\hline BVOC9109 & 0.1440 & BVOC9109 & 0.1440 \\
\hline $\mathrm{CO}$ & 0.250 & $\mathrm{CO}$ & 0.250 \\
\hline \multicolumn{4}{|c|}{ Estimated Initial Compounds } \\
\hline HONO & 0.003 & HONO & 0.003 \\
\hline $\mathrm{CH}_{4}$ & 1.790 & $\mathrm{CH}_{4}$ & 1.790 \\
\hline $\mathrm{H}_{2}$ & 0.580 & $\mathrm{H}_{2}$ & 0.580 \\
\hline \multicolumn{4}{|c|}{ Chamber Model Parameters } \\
\hline $\begin{array}{l}\mathrm{NO}_{2} \longrightarrow \mathrm{HONO} \\
\text { wall. } \mathrm{HNO}_{3} \longrightarrow \mathrm{NO}_{2}\end{array}$ & $\begin{array}{l}2.5 \times 10^{-3} \\
2.0 \times 10^{-3} \\
\end{array}$ & $\begin{array}{l}\mathrm{NO}_{2} \longrightarrow \mathrm{HONO} \\
\text { wall. } \mathrm{HNO}_{3} \longrightarrow \mathrm{NO}_{2}\end{array}$ & $\begin{array}{l}2.5 \times 10^{-3} \\
2.0 \times 10^{-3} \\
\end{array}$ \\
\hline $\begin{array}{l}\text { Three Previous Cha } \\
\text { 950801.SEG Delta E } \\
\text { 950726.SEG Matche } \\
\text { 950722.SEG CO run }\end{array}$ & $\begin{array}{l}\text { nber Events } \\
\text { hylene run } \\
\text { Propylene ru }\end{array}$ & & \\
\hline
\end{tabular}



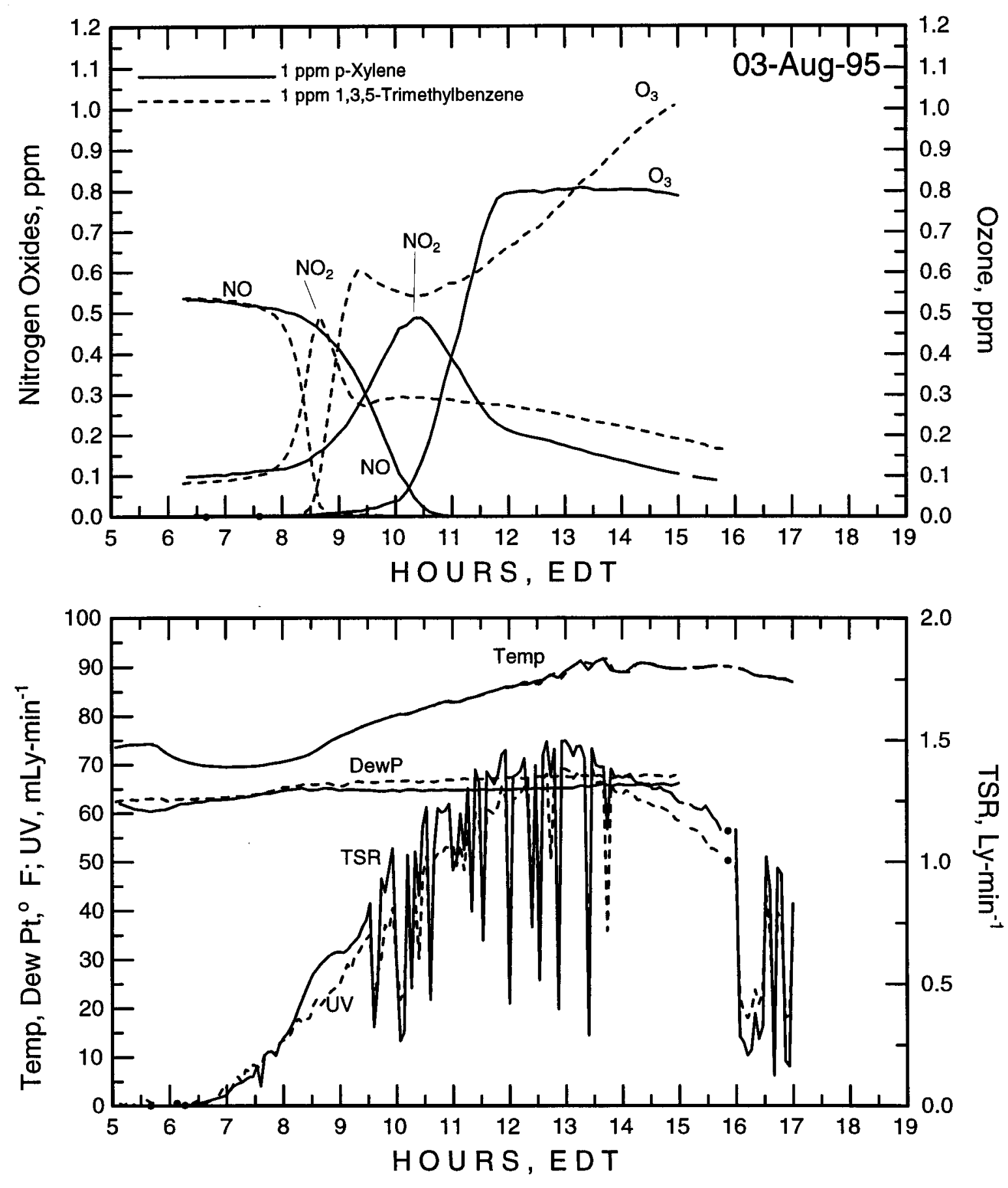

Figure 6.2: Run Arom-2 1,3,5-Trimethyl-benzene: $\mathrm{O}_{\mathrm{x}}$ versus p-Xylene: $\mathrm{NO}_{\mathrm{x}}$. 
Table 6.3: Initial Conditions for ST0195

(Units are ppm or ppmC)

\begin{tabular}{|c|c|c|c|}
\hline \multicolumn{2}{|c|}{ RED SIDE } & \multicolumn{2}{|c|}{ BLUE SIDE } \\
\hline Compound & Amount & Compound & Amount \\
\hline \multicolumn{4}{|c|}{ Initial Nitrogen Oxides } \\
\hline $\mathrm{NO}_{\mathrm{x}}$ & 0.6916 & $\mathrm{NO}_{\mathrm{x}}$ & 0.6502 \\
\hline NO & 0.5582 & NO & 0.5404 \\
\hline $\mathrm{NO}_{2}$ & 0.1334 & $\mathrm{NO}_{2}$ & 0.1098 \\
\hline \multicolumn{4}{|c|}{ Initial Volatile Organic Compounds } \\
\hline $\mathrm{C}-1(\mathrm{C}-2) \mathrm{Bz} 4-\mathrm{C}$ & 9.1300 & $0-X y l$ & 8.0800 \\
\hline \multicolumn{4}{|c|}{ Other Measured Initial Compounds } \\
\hline BVOC9109 & 0.1440 & BVOC9109 & 0.1440 \\
\hline $\mathrm{CO}$ & 0.250 & $\mathrm{CO}$ & 0.250 \\
\hline \multicolumn{4}{|c|}{ Estimated Initial Compounds } \\
\hline HONO & 0.003 & HONO & 0.003 \\
\hline $\mathrm{CH}_{4}$ & 1.790 & $\mathrm{CH}_{4}$ & 1.790 \\
\hline $\mathrm{H}_{2}$ & 0.580 & $\mathrm{H}_{2}$ & 0.580 \\
\hline \multicolumn{4}{|c|}{ Chamber Model Parameters } \\
\hline $\begin{array}{l}\mathrm{NO}_{2} \longrightarrow \mathrm{HONO} \\
\text { wall. } \mathrm{HNO}_{3} \longrightarrow \mathrm{NO}_{2}\end{array}$ & $\begin{array}{l}2.5 \times 10^{-3} \\
2.0 \times 10^{-3}\end{array}$ & $\begin{array}{l}\mathrm{NO}_{2} \longrightarrow \mathrm{HONO} \\
\text { wall. } \mathrm{HNO}_{3} \longrightarrow \mathrm{NO}_{2}\end{array}$ & $\begin{array}{l}2.5 \times 10^{-3} \\
2.0 \times 10^{-3}\end{array}$ \\
\hline $\begin{array}{l}\text { Three Previous Cha } \\
\text { 950830.SEG Aromat } \\
\text { 950803.SEG Aromat } \\
\text { 950801.SEG Delta E }\end{array}$ & $\begin{array}{l}\text { mber Events } \\
\text { cs run } \\
\text { cs run } \\
\text { hylene run }\end{array}$ & & \\
\hline
\end{tabular}



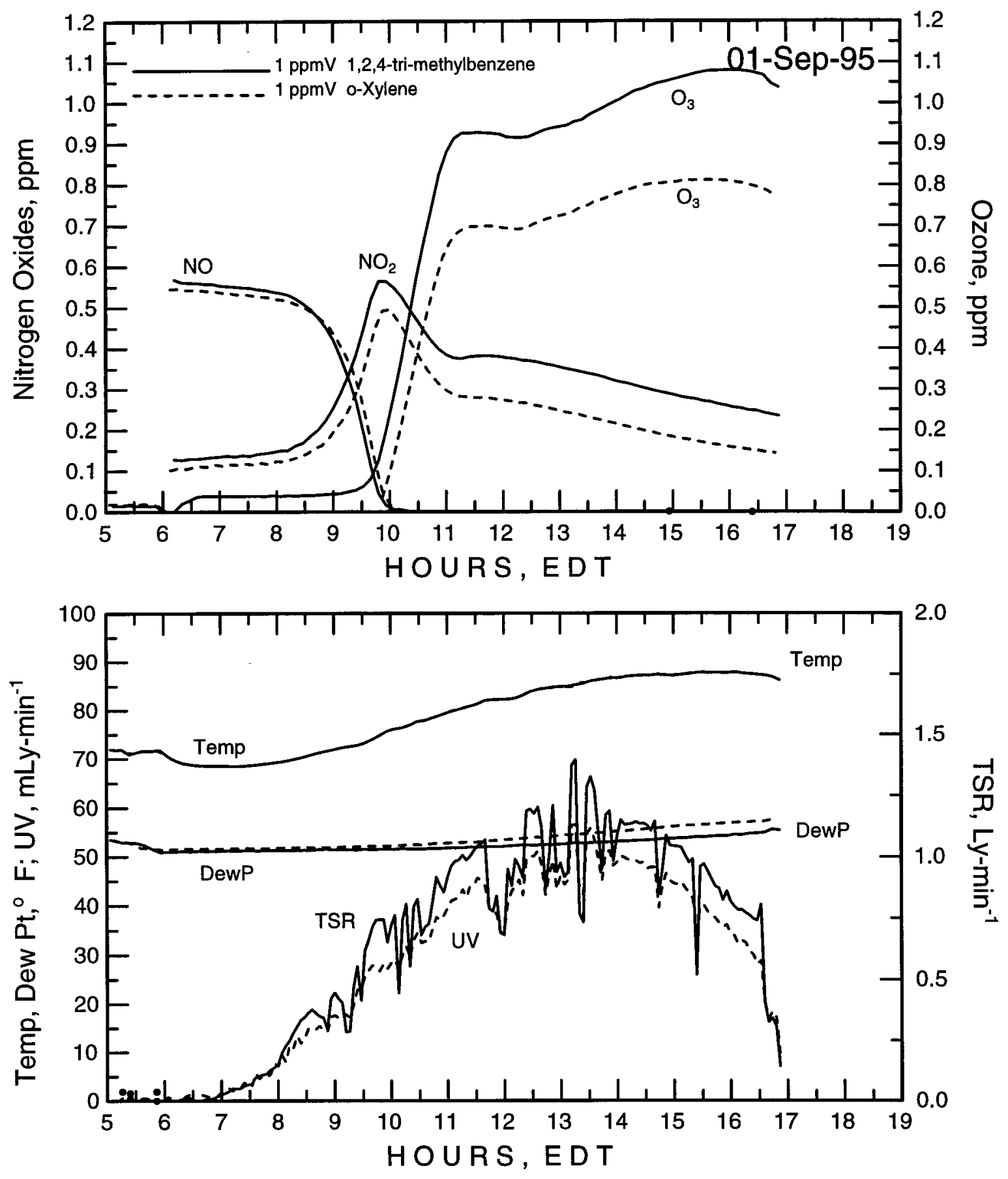

Figure 6.3: Run Arom-3 1,2,4-Trimethyl-benzene: $\mathrm{O}_{\mathrm{x}}$ versus o-Xylene: $\mathrm{NO}_{\mathbf{x}}$. 
The presence of carbonyl products in each batch sample was readily determined by examining the reconstructed ion chromatogram for the $\mathrm{m} / \mathrm{z}=181$ ion because the EI spectra of PFBHA oximes always have a strong $\mathrm{m} / \mathrm{z}=181$ peak. The molecular weights of these carbonyl products are then determined from their methane CI mass spectra.

A sample was taken from the background chamber air before every experiment and treated in the same way as the samples collected after the injection of the $\mathrm{HC}$ and $\mathrm{NO}_{\mathrm{x}}$. Carbonyl peaks detected in the background sample included small amounts of formaldehyde, acetaldehyde, acetone, pentafluorobenzaldehyde, glyoxal and methylglyoxal, as well as several very small unknown peaks. The possible sources for these background contaminants include the chamber air, laboratory air, distilled water, and the derivatizing reagent. Pentafluorobenzaldehyde was a by-product arising from the derivatization reaction.

The reconstructed $\mathrm{m} / \mathrm{z}=181$ ion chromatograms for the batch sample collected in the outdoor smog chamber experiments are shown in Figures 6.4 to 6.12 for the six aromatic compounds - toluene, p-xylene, m-xylene, o-xylene, 1, 3, 5TMB, and 1, 2, 4TMB. Tables 6.4 to 6.18 list the carbonyl products corresponding to the experiments, along with the molecular weigth information and their identities (when identification was possible). Carbonyl peaks eluting before glycolaldehyde-formaldehyde, acetaldehyde and acetone-are not shown here due to the limited information they provide. Trace amounts of these carbonyls are present in the laboratory air and/or the distilled water used in the derivatization process.

Although more than 45 carbonyl peaks were detected in each experiment, the number of carbonyl peaks detected does not reflect the actual number of carbonyl products. Except for symmetric carbonyls such as formaldehyde and acetone, PFBHA forms two or more geometric isomers depending on the structure of carbonyls. Non-symmetric mono-carbonyls, such as acetaldehyde, have two isomers for their PFBHA derivatives. Non-symmetric dicarbonyls, such as methylglyoxal, can form as many as four geometric isomers with PFBHA. When carbonyls have a $\mathrm{C}=\mathrm{C}$ in addition to two carbonyl function groups on each side of the $\mathrm{C}=\mathrm{C}$ bond, such as 4-oxo-2-pentenal, they can produce a maximum of eight geometric isomers because the carbonyls have cis- and trans- isomers and each of which could form four isomers of PFBHA derivatives. The GC conditions tested and used for this work were able to distinguish some, but not all, of these isomeric forms. 


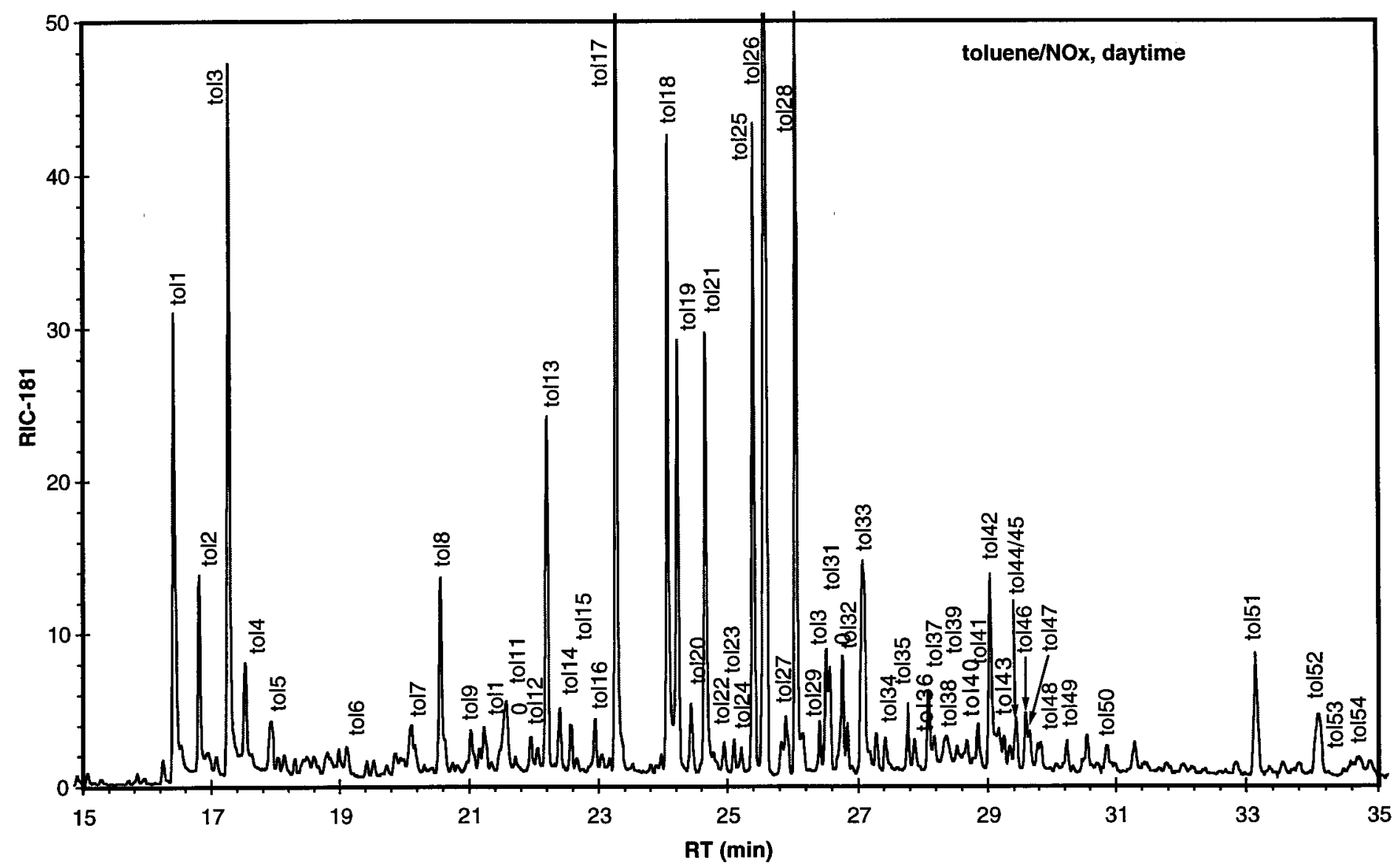

Figure 6.4: Reconstructed $\mathrm{m} / \mathrm{z} 181$ ion chromatogram of the batch sample collected from an outdoor smog chamber toluene/NO daytime experiment. 


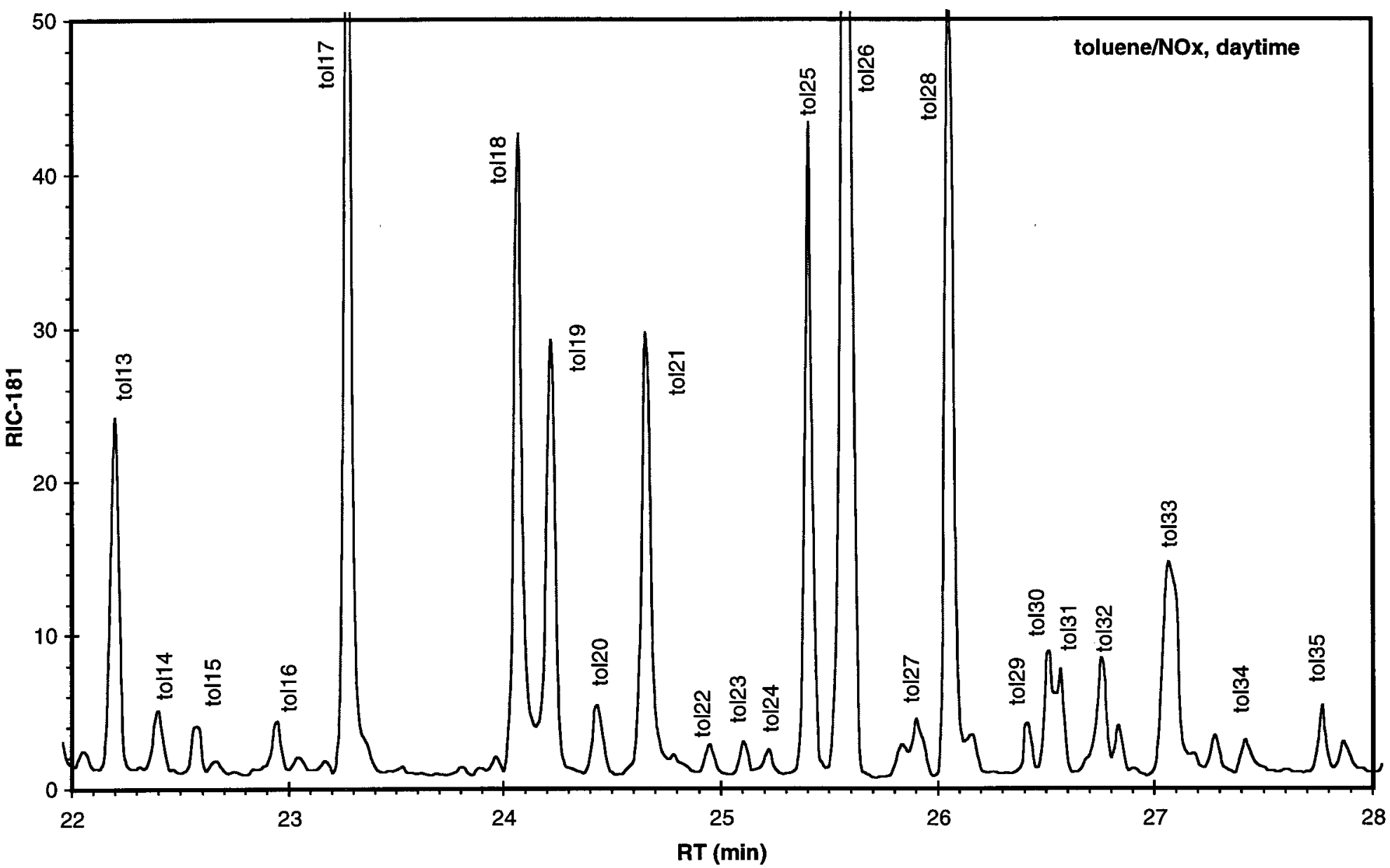

Figure 6.5:. Detailed Reconstructed $\mathrm{m} / \mathrm{z} 181$ ion chromatogram of the batch sample collected from an outdoor smog chamber toluene/NO daytime experiment. 
Table 6.4:

\section{Carbonyl Products Detected in a Sample Collected from a Toluene/NOx} Daytime Smog Chamber Experiment

\begin{tabular}{|c|c|c|c|c|}
\hline Peak \# & $\mathrm{RT}(\min )$ & Identity & $\mathbf{M W}^{2}$ & $\mathbf{M W}^{\mathrm{b}}$ \\
\hline tol1,tol2 & $16.42 / 16.81$ & glycolaldehyde & 255 & 60 \\
\hline tol3 & 17.28 & hydroxyacetone & 269 & 74 \\
\hline tol4 & 17.51 & hydroxyacetone or $\mathrm{C}_{4}$ hydroxy carbonyl & $269 / 283$ & $74 / 88$ \\
\hline tol5 & 17.93 & $\mathrm{C}_{5}$ hydroxy carbonyl & 297 & 102 \\
\hline tol6 & 19.13 & $\mathrm{C}_{6} \mathrm{~F}_{5} \mathrm{CHO}$ (by product of derivatizing reaction) & 391 & 196 \\
\hline tol7 & 19.75 & mono-deri. of $\mathrm{C}_{4}$ hydroxy dicarbonyl & 297 & 102 \\
\hline tol8 & 20.56 & $\mathrm{C}_{6} \mathrm{~F}_{5} \mathrm{CHO}$ (by product of derivatizing reaction) & 391 & 196 \\
\hline tol9 & 21.03 & mono-deri. of MW 504 carbonyl & 309 & 114 \\
\hline tol10 & 21.25 & $\begin{array}{l}\mathrm{C}_{6} \text { di-unsaturated carbonyl or furural/ methyl } \\
\text { furural or acetyl furan }\end{array}$ & $291 / 305$ & $96 / 110$ \\
\hline tol11 & 21.56 & mono-deri. of MW 488 carbonyl & 293 & 98 \\
\hline tol12,tol13 & $22.06 / 22.20$ & benzaldehyde & 301 & 106 \\
\hline tol14,tol15 & $22.40 / 22.58$ & methyl furural or acetyl furan & 305 & 110 \\
\hline tol16 & 22.94 & $\begin{array}{l}\mathrm{C}_{6} \text { unsaturated hydroxy epoxy cyclic carbonyl, see } \\
\text { Table } 6.6\end{array}$ & 321 & 126 \\
\hline toll7 & 23.27 & mono-deri. of benzoquinone & 303 & 108 \\
\hline tol18,tol19 & $24.06 / 24.23$ & mono-deri. of methyl benzoquinone & 317 & 122 \\
\hline tol20 & 24.43 & mono-deri. of MW 518 carbonyl & 323 & 128 \\
\hline tol21 & 24.66 & mono-deri. of methyl benzoquinone & 317 & 122 \\
\hline tol22 & 24.95 & unknown, containing -OH group & 337 & 142 \\
\hline tol23 & 25.10 & methylglyoxal & 462 & 72 \\
\hline tol24 & 25.18 & see table 6.1 & 335 & 140 \\
\hline tol25 & 25.40 & glyoxal & 448 & 58 \\
\hline tol26 & 25.58 & glyoxal/methylglyoxal & $448 / 462$ & $58 / 72$ \\
\hline
\end{tabular}

$\mathrm{MW}^{\mathrm{a}}$ : molecular weight of PFBHA-carbonyl derivative

$\mathrm{MW}^{\mathrm{b}}$ : molecular weight of carbonyl 
Table 6.5:

(Continued) Carbonyl Products Detected in a Sample Collected from a Toluene/NOx Daytime Smog Chamber Experiment

\begin{tabular}{|c|c|c|c|c|}
\hline Peak \# & RT (min) & Identity & $\mathrm{MW}^{\mathrm{a}}$ & $\mathbf{M W}^{\mathrm{b}}$ \\
\hline tol27 & 25.86 & $\mathrm{C}_{7}$ unsaturated epoxy dicarbonyl/methylglyoxal & $333 / 462$ & $128 / 72$ \\
\hline tol28 & 26.06 & methylglyoxal & 462 & 72 \\
\hline tol29 & 26.41 & propandial & 462 & 72 \\
\hline tol30 & 26.51 & $\mathrm{C}_{4}$ saturated dicarbonyl or $\mathrm{C}_{3}$ trione & 476 & 86 \\
\hline tol31,tol32 & $26.58 / 26.74$ & $\mathrm{C}_{7}$ unsaturated epoxy dicarbonyl & 333 & 128 \\
\hline tol33 & 27.08 & $\mathrm{C}_{4}$ saturated dicarbonyl or $\mathrm{C}_{3}$ trione & 476 & 86 \\
\hline tol34 & 27.43 & $\mathrm{C}_{4}$ hydroxy dicarbonyl & 492 & 102 \\
\hline tol35 & 27.76 & $\mathrm{C}_{4}$ epoxy dicarbonyl/ $\mathrm{C}_{4}$ trione & 490 & 100 \\
\hline tol36 & 28.09 & $\mathrm{C}_{4}$ hydroxy dicarbonyl & 492 & 102 \\
\hline tol37 & 28.19 & $\mathrm{C}_{4}$ epoxy dicarbonyl/ $\mathrm{C}_{4}$ trione & 490 & 100 \\
\hline tol38 & 28.34 & $\mathrm{C}_{5}$ epoxy dicarbonyl/ $\mathrm{C}_{5}$ trione & 504 & 114 \\
\hline tol39 & 28.53 & $\mathrm{C}_{4}$ hydroxy dicarbonyl & 492 & 102 \\
\hline tol40 & 28.66 & 4-oxo-2-pentenal & 488 & 98 \\
\hline tol41 & 28.84 & butendial & 474 & 84 \\
\hline tol42 & 29.04 & $\mathrm{C}_{4}$ hydroxy dicarbonyl & 492 & 102 \\
\hline tol43 & 29.18 & 4-oxo-2-pentenal & 488 & 98 \\
\hline tol44,tol45 & $29.24 / 29.33$ & butendial & 474 & 84 \\
\hline tol46 & 29.43 & $\mathrm{C}_{5}$ epoxy dicarbonyl/ $\mathrm{C}_{5}$ trione & 504 & 114 \\
\hline tol47 & 29.60 & 4-oxo-2-pentenal/ butendial & $488 / 474$ & $98 / 84$ \\
\hline tol48,tol49 & $29.78 / 30.23$ & 4-oxo-2-pentenal & 488 & 98 \\
\hline tol50 & 30.86 & $\mathrm{C}_{6}$ unsaturated hydroxy dicarbonyl & 518 & 128 \\
\hline tol51 & 33.14 & benzoquinone & 498 & 108 \\
\hline tol52 & 34.09 & methyl-p-benzoquinone & 512 & 122 \\
\hline tol53 & 34.24 & $\mathrm{C}_{7}$ di-unsaturated dicarbonyl & 514 & 124 \\
\hline tol54 & 34.67 & $\mathrm{C}_{7}$ unsaturated epoxy dicarbonyl & 528 & 138 \\
\hline
\end{tabular}




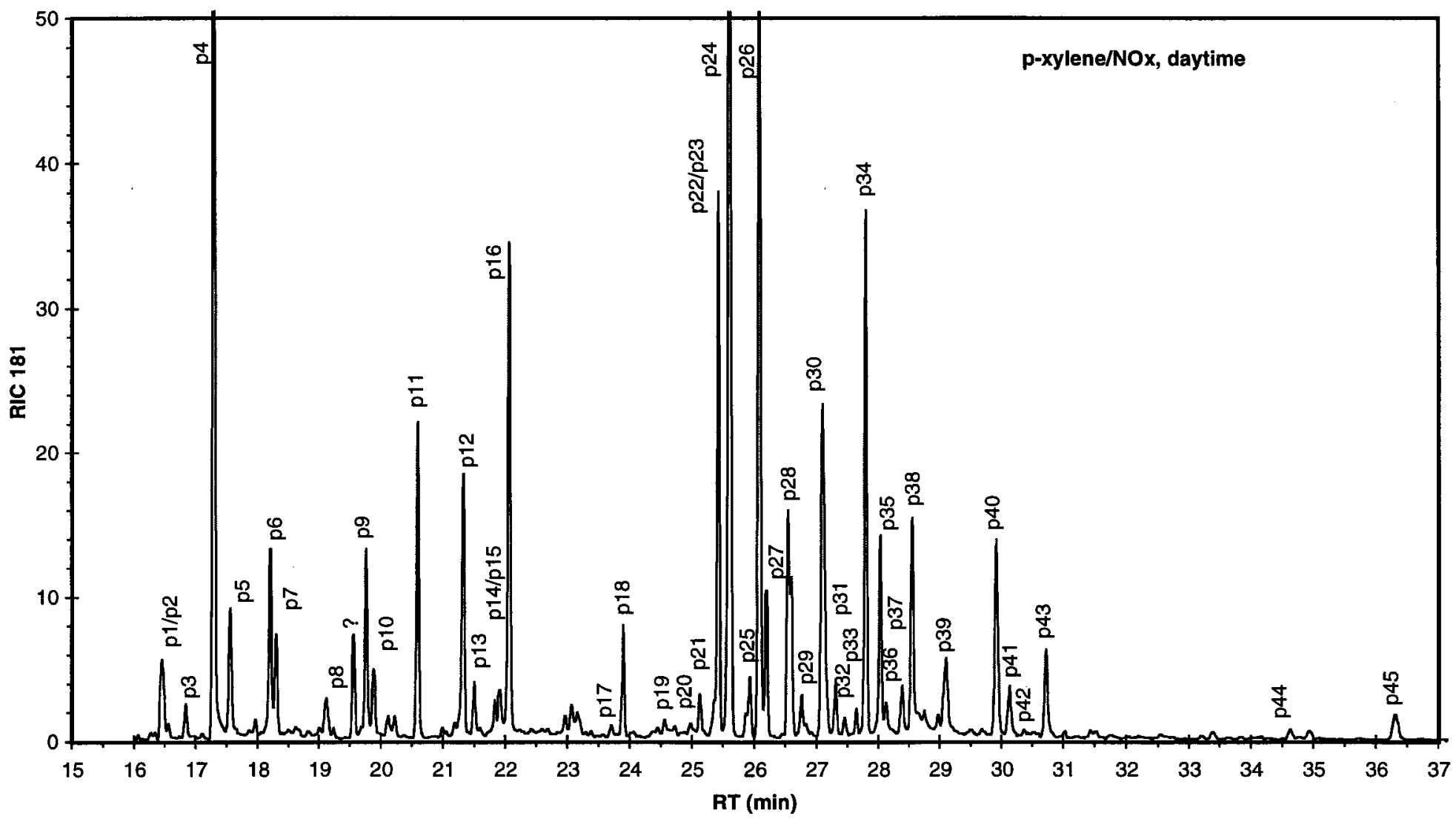

Figure 6.6:. Reconstructed $\mathrm{m} / \mathrm{z} 181$ ion chromatogram of the batch sample collected from an outdoor smog chamber $\mathrm{p}-\mathrm{xylene} / \mathrm{NO}_{\mathrm{x}}$ daytime experiment. 
Table 6.6:

\section{Carbonyl Products Detected in a Sample Collected from a p-Xylene/NOx} Daytime Smog Chamber Experiment

\begin{tabular}{|c|c|c|c|c|}
\hline Peak & RT (min) & Identity & $\mathrm{MW}^{\mathrm{a}}$ & $\mathbf{M W}^{\mathrm{b}}$ \\
\hline$\overline{\mathrm{pl}}$ & 16.45 & glycolaldehyde/2-hydroxy propanal & $255 / 269$ & $60 / 74$ \\
\hline$\overline{\mathrm{p} 2}$ & 16.55 & 2-hydroxy propanal & 269 & 74 \\
\hline $\mathrm{p3}$ & 16.83 & glycolaldehyde & 255 & 60 \\
\hline $\mathrm{p} 4, \mathrm{p} 5$ & $17.29 / 17.56$ & hydroxyacetone & 269 & 74 \\
\hline $\mathrm{p} 6, \mathrm{p} 7$ & $18.20 / 18.29$ & $\mathrm{C}_{4}$ epoxy/C $\mathrm{C}_{5}$ saturated carbonyl & 281 & 86 \\
\hline $\mathrm{p} 8$ & 19.08 & $\mathrm{C}_{6} \mathrm{~F}_{5} \mathrm{CHO}$ (derivatizing reaction byproduct) & 391 & 196 \\
\hline $\mathrm{p} 9$ & 19.76 & mono-deri. of $\mathrm{C}_{4}$ hydroxy dicarbonyl & 297 & 102 \\
\hline $\mathrm{p} 10$ & 19.88 & mono-deri. of 3-hexene-2,5-dione & 307 & 112 \\
\hline $\mathrm{p} 11$ & 20.58 & mono-deri. of 3-hexene-2,5-dione $/ \mathrm{C}_{6} \mathrm{~F}_{5} \mathrm{CHO}$ & $307 / 391$ & $112 / 196$ \\
\hline $\mathrm{p} 12$ & 21.31 & mono-deri. of 3-hexene-2,5-dione & 307 & 112 \\
\hline $\mathrm{p} 13$ & 21.49 & $\mathrm{C}_{6}$ unsaturated carbonyl & $293 / 325$ & $98 / 130$ \\
\hline $\mathrm{p} 14$ & 21.83 & $\mathrm{C}_{7}$ hydroxy carbonyl & 325 & 130 \\
\hline $\mathrm{p} 15$ & 21.91 & $\mathrm{C}_{4}$ epoxy/ $\mathrm{C}_{5}$ saturated carbonyl & 281 & 86 \\
\hline \multirow[t]{2}{*}{ p16 } & 22.06 & mono-deri. of 3-hexene-2,5-dione & 307 & 112 \\
\hline & 23.28 & benzoquinone & 303 & 108 \\
\hline $\mathrm{p} 17, \mathrm{p} 18$ & $23.70 / 23.89$ & p-tolualdehyde & 315 & 120 \\
\hline p19 & 24.56 & $\begin{array}{l}\text { epoxy carbonyl/ mono-deri. of } \mathrm{C}_{8} \text { di-unsaturated } \\
\text { dicarbonyl }\end{array}$ & $335 / 333$ & $140 / 138$ \\
\hline $\mathrm{p} 20$ & 24.73 & $\mathrm{C}_{7}$ unsaturated epoxy carbonyl & 321 & 126 \\
\hline $\mathrm{p} 21$ & 25.11 & methylglyoxal & 462 & 72 \\
\hline $\mathrm{p} 22$ & 25.32 & $\begin{array}{l}\text { mono-deri. of dimethyl-p-benzoquinone/ } \mathrm{C}_{8} \\
\text { hydroxy unsaturated epoxy cyclic carbonyl }\end{array}$ & $331 / 349$ & $136 / 154$ \\
\hline $\mathrm{p} 23$ & 25.41 & glyoxal & 448 & 58 \\
\hline p24 & 25.62 & glyoxal/methylglyoxal & $448 / 462$ & $58 / 72$ \\
\hline $\mathrm{p} 25$ & 25.86 & $\mathrm{C}_{7}$ unsaturated epoxy carbonyl/methylglyoxal & $321 / 462$ & $126 / 72$ \\
\hline p26 & 26.08 & methylglyoxal & 462 & 72 \\
\hline
\end{tabular}


Table 6.7:

Carbonyl Products Detected in a Sample Collected from a p-Xylene/NOx Daytime Smog Chamber Experiment (Continued)

\begin{tabular}{|c|c|c|c|c|}
\hline Peak & $\mathrm{RT}(\min )$ & Identity & $\mathrm{MW}^{\mathrm{a}}$ & $\mathrm{MW}^{\mathrm{b}}$ \\
\hline $\mathrm{p} 27$ & 26.18 & $\mathrm{C}_{5}$ epoxy carbonyl/ $\mathrm{C}_{4}$ epoxy carbonyl & $504 / 490$ & $114 / 100$ \\
\hline$\overline{\mathrm{p} 28}$ & 26.53 & $\mathrm{C}_{4}$ saturated dicarbonyl $/ \mathrm{C}_{3}$ trione & 476 & 86 \\
\hline p29 & 26.74 & $\begin{array}{l}\mathrm{C}_{4} \text { epoxy carbonyl or } \mathrm{C}_{4} \text { trione/ } \mathrm{C}_{4} \text { saturated } \\
\text { dicarbonyl or } \mathrm{C}_{3} \text { trione }\end{array}$ & $490 / 476$ & $100 / 86$ \\
\hline $\mathrm{p} 30$ & 27.08 & $\begin{array}{l}\mathrm{C}_{4} \text { saturated dicarbonyl or } \mathrm{C}_{3} \text { trione } / \mathrm{C}_{4} \text { hydroxy } \\
\text { dicarbonyl }\end{array}$ & $476 / 492$ & $86 / 102$ \\
\hline $\mathrm{p} 31, \mathrm{p} 32$ & $27.30,27.43$ & $\mathrm{C}_{4}$ hydroxy dicarbonyl & 492 & 102 \\
\hline $\mathrm{p} 33, \mathrm{p} 34$ & $27.63 / 27.80$ & $\mathrm{C}_{4}$ epoxy carbonyl or $\mathrm{C}_{4}$ trione & 490 & 100 \\
\hline p35 & 28.01 & 3-hexene-2,5-dione & 502 & 112 \\
\hline $\mathrm{p} 36, \mathrm{p} 37$ & $28.13 / 28.36$ & $\mathrm{C}_{4}$ hydroxy dicarbonyl & 492 & 102 \\
\hline p38 & 28.55 & 3-hexene-2,5-dione $/ \mathrm{C}_{4}$ hydroxy dicarbonyl & $502 / 492$ & $112 / 102$ \\
\hline \multirow[t]{2}{*}{ p39 } & 29.10 & 3-hexene-2,5-dione & 502 & 112 \\
\hline & 29.88 & $\mathrm{C}_{5}$ unsaturated dicarbonyl & 488 & 98 \\
\hline $\mathrm{p} 40, \mathrm{p} 41$ & $29.90 / 30.15$ & 3-hexene-2,5-dione & 502 & 112 \\
\hline $\mathrm{p} 42$ & 30.34 & $\mathrm{C}_{5}$ unsaturated dicarbonyl & 488 & 98 \\
\hline $\mathrm{p} 43$ & 30.71 & 3-hexene-2,5-dione/ $C_{5}$ unsaturated dicarbonyl & $502 / 488$ & $112 / 98$ \\
\hline$\overline{\mathrm{p} 44}$ & 34.63 & 2-methyl-butendial & 488 & 98 \\
\hline $\mathrm{p} 45$ & 36.30 & 2-methyl-butendial & 488 & 98 \\
\hline
\end{tabular}




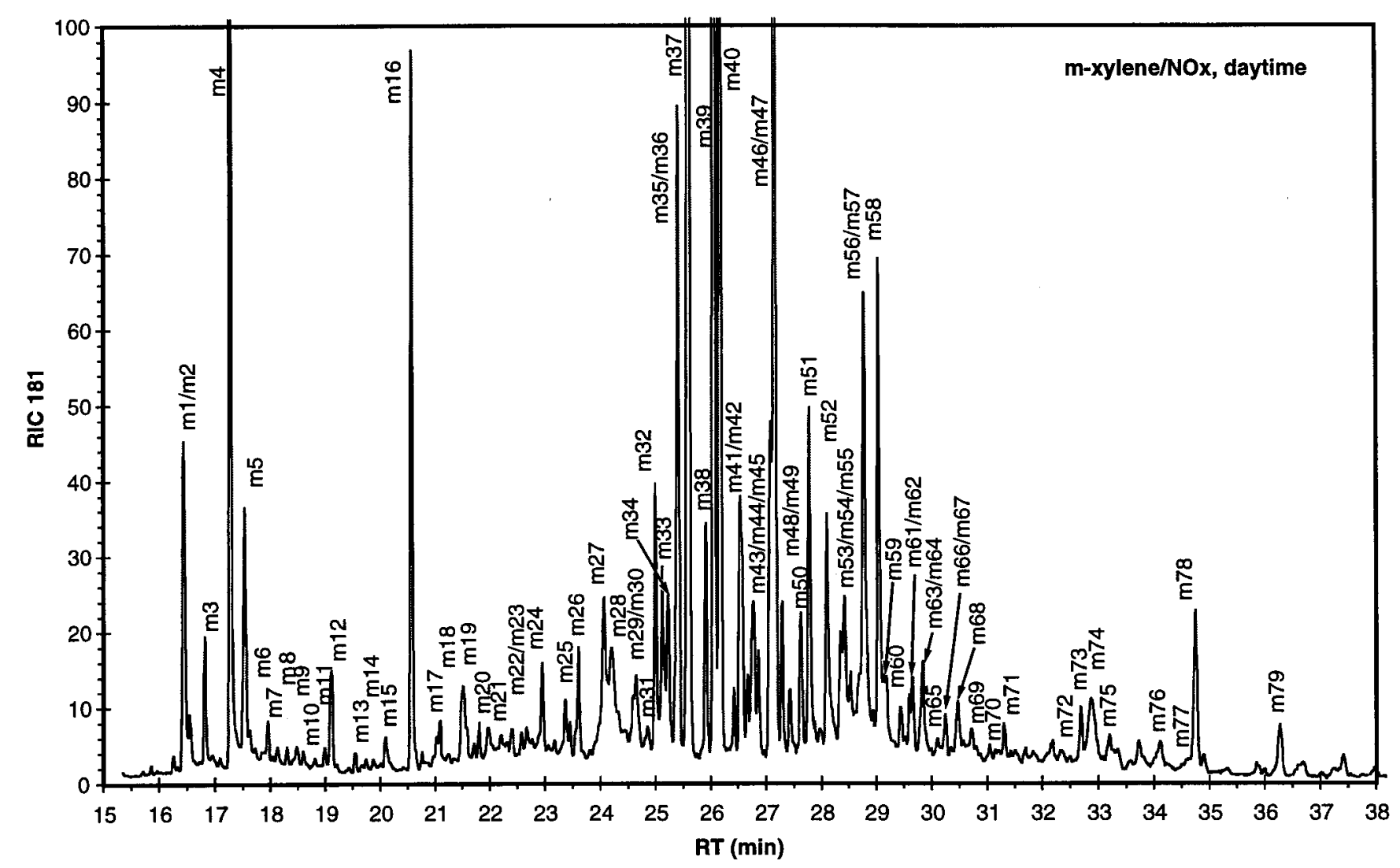

Figure 6.7: Reconstructed $\mathrm{m} / \mathrm{z} 181$ ion chromatogram of the batch sample collected from an outdoor smog chamber $\mathrm{m}$-xylene/NO daytime experiment. 


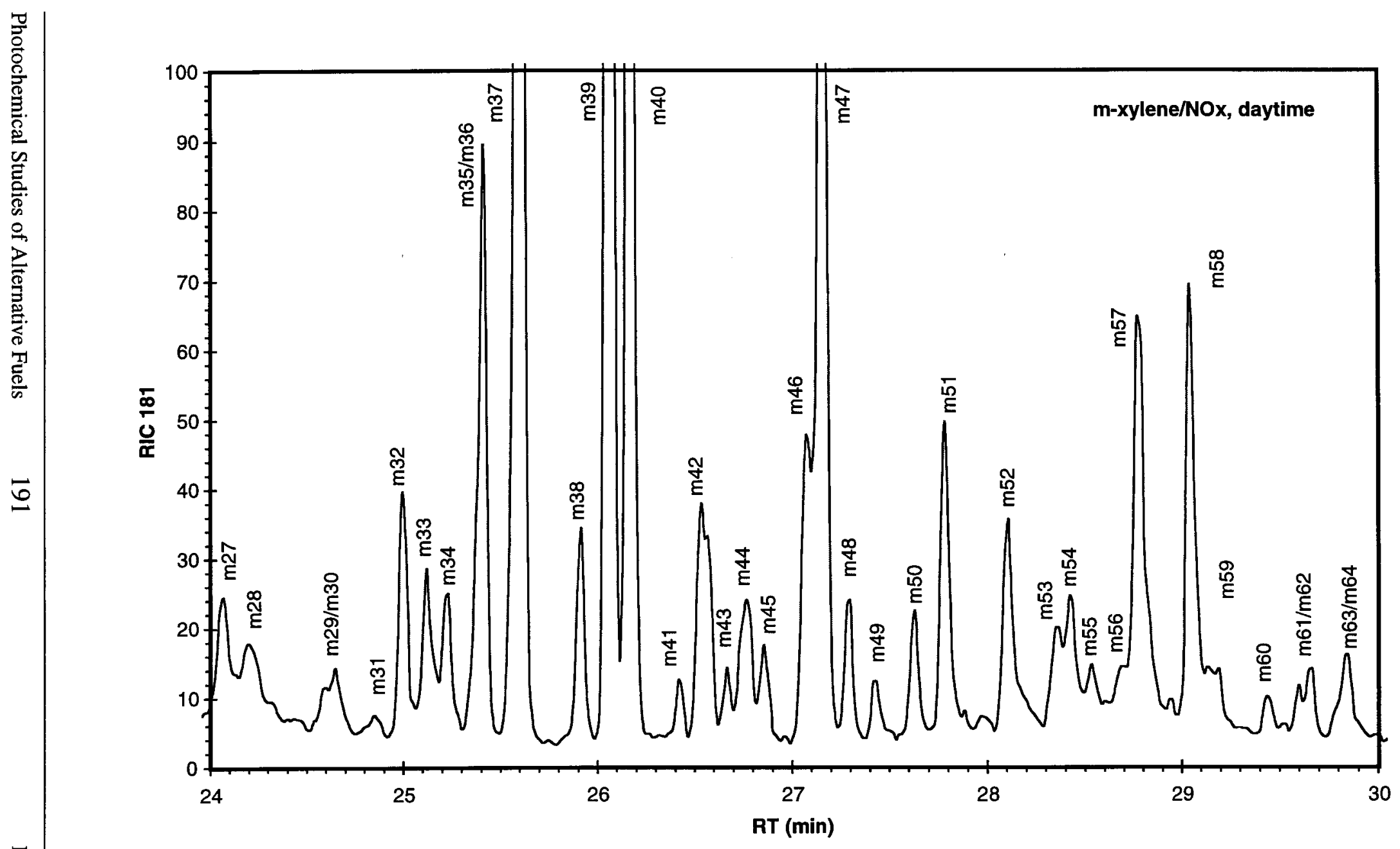

Figure 6.8:. Detailed Reconstructed $\mathrm{m} / \mathrm{z} 181$ ion chromatogram of the batch sample collected from an outdoor smog chamber $\mathrm{m}-\mathrm{xylene} / \mathrm{NO}_{\mathrm{x}}$ daytime experiment. 
Table 6.8:

Carbonyl Products Detected in a Sample Collected from an m-xylene/NOx Daytime Smog Chamber Experiment

\begin{tabular}{|c|c|c|c|c|}
\hline Peak & RT (min) & Identity & $\mathbf{M W}^{\mathrm{a}}$ & $\mathrm{MW}^{\mathrm{b}}$ \\
\hline$\overline{\mathrm{ml}}$ & $16.43 / 16.81$ & glycolaldehyde/2-hydroxy propanal & $255 / 269$ & $60 / 74$ \\
\hline $\mathrm{m} 2$ & 16.55 & 2-hydroxy propanal & 269 & 74 \\
\hline $\mathrm{m3}$ & 16.81 & glycolaldehyde & 255 & 60 \\
\hline $\mathrm{m4}$ & $17.28 / 17.53$ & hydroxy acetone & 269 & 74 \\
\hline$\overline{\mathrm{m} 5}$ & 17.53 & hydroxy acetone/ $\mathrm{C}_{4}$ hydroxy carbonyl & $269 / 283$ & $74 / 88$ \\
\hline $\mathrm{m6}$ & 17.63 & $\mathrm{C}_{4}$ hydroxy carbonyl & 283 & 88 \\
\hline$\overline{\mathrm{m} 7}$ & 17.94 & mono-deri. of $\mathrm{C}_{4}$ hydroxy dicarbonyl & 297 & 102 \\
\hline$\overline{\mathrm{m} 8}$ & 18.13 & $\mathrm{C}_{4}$ epoxy $/ \mathrm{C}_{5}$ saturated carbonyl & 281 & 86 \\
\hline$\overline{\mathrm{m} 9}$ & 18.59 & $\mathrm{C}_{5}$ epoxy $/ \mathrm{C}_{6}$ saturated carbonyl & 295 & 100 \\
\hline $\mathrm{m} 10$ & 18.81 & $\mathrm{C}_{4}$ hydroxy carbonyl & 283 & 88 \\
\hline$\overline{\mathrm{m} 11}$ & 18.98 & $\mathrm{C}_{5}$ di-unsaturated carbonyl & 279 & 84 \\
\hline $\mathrm{m} 12$ & 19.11 & $\mathrm{C}_{6} \mathrm{~F}_{5} \mathrm{CHO} / \mathrm{C}_{3}$ hydroxy carbonyl & $391 / 269$ & $196 / 74$ \\
\hline $\mathrm{m} 12$ & 19.11 & mono-deri. of $\mathrm{C}_{5}$ hydroxy carbonyl & 311 & 116 \\
\hline $\mathrm{m} 13$ & 19.74 & mono-deri. of $\mathrm{C}_{5}$ hydroxy carbonyl & 311 & 116 \\
\hline $\mathrm{m} 14$ & 19.86 & $\mathrm{C}_{6}$ unsaturated carbonyl & 293 & 98 \\
\hline $\mathrm{m} 15$ & 20.09 & $\mathrm{C}_{4}$ hydroxy carbonyl & 283 & 88 \\
\hline $\mathrm{m} 16$ & 20.56 & $\mathrm{C}_{6} \mathrm{~F}_{5} \mathrm{CHO}$ & 391 & 196 \\
\hline $\mathrm{ml7}$ & 21.08 & mono-deri. of $\mathrm{C}_{5}$ unsaturated dicarbonyl & 307 & 112 \\
\hline $\mathrm{m} 18$ & 21.25 & $\mathrm{C}_{7}$ di-unsaturated carbonyl & 305 & 110 \\
\hline $\mathrm{m} 19$ & 21.51 & $\begin{array}{l}\mathrm{C}_{6} \text { unsaturated carbonyl/ } \mathrm{C}_{7} \text { di-unsaturated } \\
\text { carbonyl }\end{array}$ & $293 / 305$ & $98 / 110$ \\
\hline $\mathrm{m} 20, \mathrm{~m} 21$ & $21.81,22.26$ & $\mathrm{C}_{8}$ di-unsaturated carbonyl & 319 & 124 \\
\hline $\mathrm{m} 22$ & 22.39 & $\mathrm{C}_{7} / \mathrm{C}_{8}$ di-unsaturated carbonyl & $319 / 305$ & $124 / 110$ \\
\hline $\mathrm{m} 23$ & 22.58 & $\mathrm{C}_{7} / \mathrm{C}_{8}$ di-unsaturated carbonyl & $319 / 305$ & $124 / 110$ \\
\hline $\mathrm{m} 24$ & 22.94 & $\mathrm{C}_{7}$ unsaturated epoxy carbonyl & 321 & 126 \\
\hline $\mathrm{m} 25$ & 23.44 & $\mathrm{C}_{7}$ hydroxy unsaturated epoxy cyclic carbonyl & 335 & 140 \\
\hline $\mathrm{m} 26$ & 23.59 & $\begin{array}{l}\mathrm{C}_{7} \text { hydroxy unsaturated epoxy cyclic carbonyl / } \\
\text { m-tolualdehyde }\end{array}$ & $335 / 315$ & $\overline{140 / 120}$ \\
\hline
\end{tabular}


Table 6.9:

Carbonyl Products Detected in a Sample Collected from an m-xylene/NOx Daytime Smog Chamber Experiment (Continued)

\begin{tabular}{|c|c|c|c|c|}
\hline Peak & $\mathrm{RT}$ (min) & Identity & $\mathrm{MW}^{\mathrm{a}}$ & $\mathbf{M W}^{\mathrm{b}}$ \\
\hline $\mathrm{m} 27$ & 24.06 & $\begin{array}{l}\mathrm{C}_{7} \text { unsaturated epoxy carbonyl/ mono-deri. of } \\
\text { methylbenzoquinone }\end{array}$ & $321 / 317$ & $126 / 122$ \\
\hline $\mathrm{m} 28$ & 24.19 & $\begin{array}{l}\mathrm{C}_{7} \text { unsaturated epoxy carbonyl/ mono-deri. of } \\
\text { methylbenzoquinone }\end{array}$ & $321 / 317$ & $126 / 122$ \\
\hline $\mathrm{m} 29$ & 24.59 & $\mathrm{C}_{7}$ hydroxy unsaturated epoxy cyclic carbonyl & 335 & 140 \\
\hline $\mathrm{m} 30$ & 24.64 & $\begin{array}{l}\text { mono-deri. of methylbenzoquinone/ } \mathrm{C}_{7} \\
\text { unsaturated epoxy carbonyl }\end{array}$ & $317 / 321$ & $122 / 126$ \\
\hline$\overline{\mathrm{m} 31}$ & 24.69 & $\mathrm{C}_{7}$ hydroxy unsaturated epoxy cyclic carbonyl & 335 & 140 \\
\hline $\mathrm{m} 32$ & 24.99 & mono-deri. of dimethylbenzoquinone & 331 & 136 \\
\hline $\mathrm{m} 33$ & 25.12 & $\begin{array}{l}\text { methylglyoxal/ } C_{7} \text { hydroxy unsaturated epoxy } \\
\text { cyclic carbonyl }\end{array}$ & $462 / 335$ & $72 / 140$ \\
\hline $\mathrm{m34}$ & 25.23 & $\mathrm{C}_{7}$ unsaturated epoxy carbonyl & 321 & 126 \\
\hline $\mathrm{m} 35$ & 25.35 & unknown & 365 & 170 \\
\hline $\mathrm{m} 36$ & 25.40 & glyoxal & 448 & 58 \\
\hline $\mathrm{m} 37$ & 25.60 & glyoxal/methylglyoxal & $448 / 462$ & $58 / 72$ \\
\hline $\mathrm{m} 38$ & 25.91 & methylglyoxal/unknown, containing -OH group & $462 / 351$ & $72 / 156$ \\
\hline $\mathrm{m} 39$ & 26.06 & methylglyoxal & 462 & 72 \\
\hline $\mathrm{m} 40$ & 26.16 & $\mathrm{C}_{5}$ epoxy dicarbonyl or $\mathrm{C}_{5}$ trione & 504 & 114 \\
\hline $\mathrm{m} 41$ & 26.41 & $\mathrm{C}_{8}$ hydroxy unsaturated epoxy cyclic carbonyl & 349 & 154 \\
\hline $\mathrm{m} 42$ & 26.53 & $\mathrm{C}_{4}$ saturated dicarbonyl or $\mathrm{C}_{3}$ trione/unknown & $476 / 365$ & $86 / 170$ \\
\hline $\mathrm{m} 43$ & 26.66 & unknown, containing -OH & 365 & 170 \\
\hline $\mathrm{m} 44$ & 26.73 & $\begin{array}{l}\mathrm{C}_{4} \text { saturated dicarbonyl or } \mathrm{C}_{3} \text { trione/ } \mathrm{C}_{3} \\
\text { dicarbonyl }\end{array}$ & $476 / 462$ & $86 / 72$ \\
\hline $\mathrm{m} 45$ & 26.85 & $\mathrm{C}_{3}$ dicarbonyl & 462 & 72 \\
\hline $\mathrm{m} 45$ & 26.85 & $\begin{array}{l}\mathrm{C}_{4} \text { epoxy dicarbonyl or } \mathrm{C}_{4} \text { trione/ } \mathrm{C}_{6} \text { saturated or } \\
\mathrm{C}_{5} \text { epoxy carbonyl }\end{array}$ & $490 / 347$ & $100 / 152$ \\
\hline
\end{tabular}


Table 6.10:

Carbonyl Products Detected in a Sample Collected from an m-xylene/NOx Daytime Smog Chamber Experiment (Continued)

\begin{tabular}{|c|c|c|c|c|}
\hline Peak & $\mathrm{RT}$ (min) & Identity & $\mathrm{MW}^{\mathrm{a}}$ & $\mathrm{MW}^{\mathrm{b}}$ \\
\hline $\mathrm{m} 46$ & 27.07 & $\begin{array}{l}\mathrm{C}_{4} \text { saturated dicarbonyl or } \mathrm{C}_{3} \text { trione/ } \mathrm{C}_{4} \text { hydroxy } \\
\text { dicarbonyl }\end{array}$ & $476 / 492$ & $86 / 102$ \\
\hline $\mathrm{m} 47$ & 27.15 & $\mathrm{C}_{5}$ epoxy dicarbonyl or $\mathrm{C}_{5}$ trione & 504 & 114 \\
\hline $\mathrm{m} 48, \mathrm{~m} 49$ & $27.28 / 27.43$ & $\mathrm{C}_{4}$ hydroxy dicarbonyl & 492 & 102 \\
\hline $\mathrm{m} 50$ & 27.63 & $\mathrm{C}_{5}$ hydroxy dicarbonyl & 506 & 116 \\
\hline m51 & 27.78 & $\begin{array}{l}\mathrm{C}_{4} \text { epoxy dicarbonyl or } \mathrm{C}_{4} \text { trione/ } \mathrm{C}_{6} \text { saturated or } \\
\mathrm{C}_{5} \text { epoxy carbonyl }\end{array}$ & $490 / 347$ & $100 / 152$ \\
\hline $\mathrm{m51}$ & 27.81 & $\mathrm{C}_{5}$ hydroxy dicarbonyl & 506 & 116 \\
\hline $\mathrm{m} 52$ & 28.10 & $\mathrm{C}_{4}$ hydroxy dicarbonyl/ $\mathrm{C}_{5}$ unsaturated dicarbonyl & $492 / 488$ & $102 / 98$ \\
\hline $\mathrm{m} 53$ & 28.10 & $\mathrm{C}_{5}$ epoxy carbonyl or $\mathrm{C}_{5}$ trione & 504 & 114 \\
\hline $\mathrm{m54}$ & 28.34 & $\begin{array}{l}\mathrm{C}_{5} \text { epoxy carbonyl or } \mathrm{C}_{5} \text { trione/ } \mathrm{C}_{4} \text { hydroxy } \\
\text { dicarbonyl }\end{array}$ & $504 / 492$ & $114 / 102$ \\
\hline $\mathrm{m55}$ & 28.42 & $\mathrm{C}_{5}$ hydroxy dicarbonyl & 506 & 116 \\
\hline $\mathrm{m} 55$ & 28.53 & $\mathrm{C}_{4}$ hydroxy dicarbonyl/ $\mathrm{C}_{6}$ unsaturated dicarbonyl & $492 / 502$ & $102 / 112$ \\
\hline $\mathrm{m56}$ & 28.53 & $\mathrm{C}_{6}$ unsaturated hydroxy dicarbonyl & 518 & 128 \\
\hline $\mathrm{m56}$ & 28.66 & 4-oxo-2-pentenal & 488 & 98 \\
\hline $\mathrm{m} 57$ & 28.76 & $\mathrm{C}_{5}$ hydroxy dicarbonyl & 506 & 116 \\
\hline m58 & 29.03 & $\mathrm{C}_{4}$ hydroxy dicarbonyl & $\overline{492}$ & 102 \\
\hline m59 & 29.19 & 4-oxo-2-pentenal & 488 & 98 \\
\hline $\mathrm{m60}$ & 29.30 & 2-methyl-4-oxo-2-pentenal & 502 & 112 \\
\hline m61 & 29.43 & $\mathrm{C}_{5}$ epoxy carbonyl or $\mathrm{C}_{5}$ trione & 504 & 114 \\
\hline $\mathrm{m} 62, \mathrm{~m} 63$ & 29.59 & 4-oxo-2-pentenal & 488 & 98 \\
\hline $\mathrm{m63}$ & 29.64 & 4-oxo-2-pentenal and 2-methyl butendial & 488 & 98 \\
\hline m64 & 29.83 & 4-oxo-2-pentenal & 488 & 98 \\
\hline $\mathrm{m} 65$ & 30.09 & 2-methyl-4-oxo-2-pentenal & 502 & 112 \\
\hline $\mathrm{m} 66, \mathrm{~m} 67$ & $30.24 / 30.35$ & 4-oxo-2-pentenal/2-methyl-butendial & 488 & 98 \\
\hline $\mathrm{m} 68$ & 30.46 & $\mathrm{C}_{7}$ unsaturated dicarbonyl/unknown & $516 / 500$ & $126 / 110$ \\
\hline m69 & 30.71 & 2-methyl-butendial /2-methyl-4-oxo-2-pentenal & $488 / 502$ & $98 / 112$ \\
\hline
\end{tabular}


Table 6.11:

Carbonyl Products Detected in a Sample Collected from an m-xylene/NOx Daytime Smog Chamber Experiment (Continued)

\begin{tabular}{|l|c|l|c|c|}
\hline Peak & RT (min) & \multicolumn{1}{|c|}{ Identity } & $\mathrm{MW}^{\mathrm{a}}$ & $\mathrm{MW}^{\mathrm{b}}$ \\
\hline $\mathrm{m} 70$ & 31.04 & $\mathrm{C}_{7}$ unsaturated dicarbonyl & 516 & 126 \\
\hline $\mathrm{m} 71, \mathrm{~m} 72$ & $31.29 / 32.33$ & unknown & 500 & 110 \\
\hline $\mathrm{m} 73$ & 32.68 & unknown & 558 & 168 \\
\hline $\mathrm{m} 74$ & 32.86 & unknown & 542 & 152 \\
\hline $\mathrm{m} 75$ & 33.18 & 2-methyl-4-oxo-2-pentenal & 502 & 112 \\
\hline $\mathrm{m} 76$ & 33.73 & $\mathrm{C}_{7}$ unsaturated dicarbonyl/unknown & $516 / 542$ & $126 / 152$ \\
\hline $\mathrm{m} 77$ & 34.58 & 2-methyl-butendial & 488 & 98 \\
\hline $\mathrm{m} 78$ & 34.75 & 2-methyl-4-oxo-2-pentenal & 502 & 112 \\
\hline $\mathrm{m} 79$ & 36.27 & 2-methyl-butendial & 488 & 98 \\
\hline $\mathrm{m} 80$ & 36.63 & $\mathrm{C}_{8}$ di-saturated dicarbonyl & 528 & 138 \\
\hline $\mathrm{m} 81$ & 37.96 & $\mathrm{C}_{5}$ unsaturated carbonyl & 488 & 98 \\
\hline
\end{tabular}




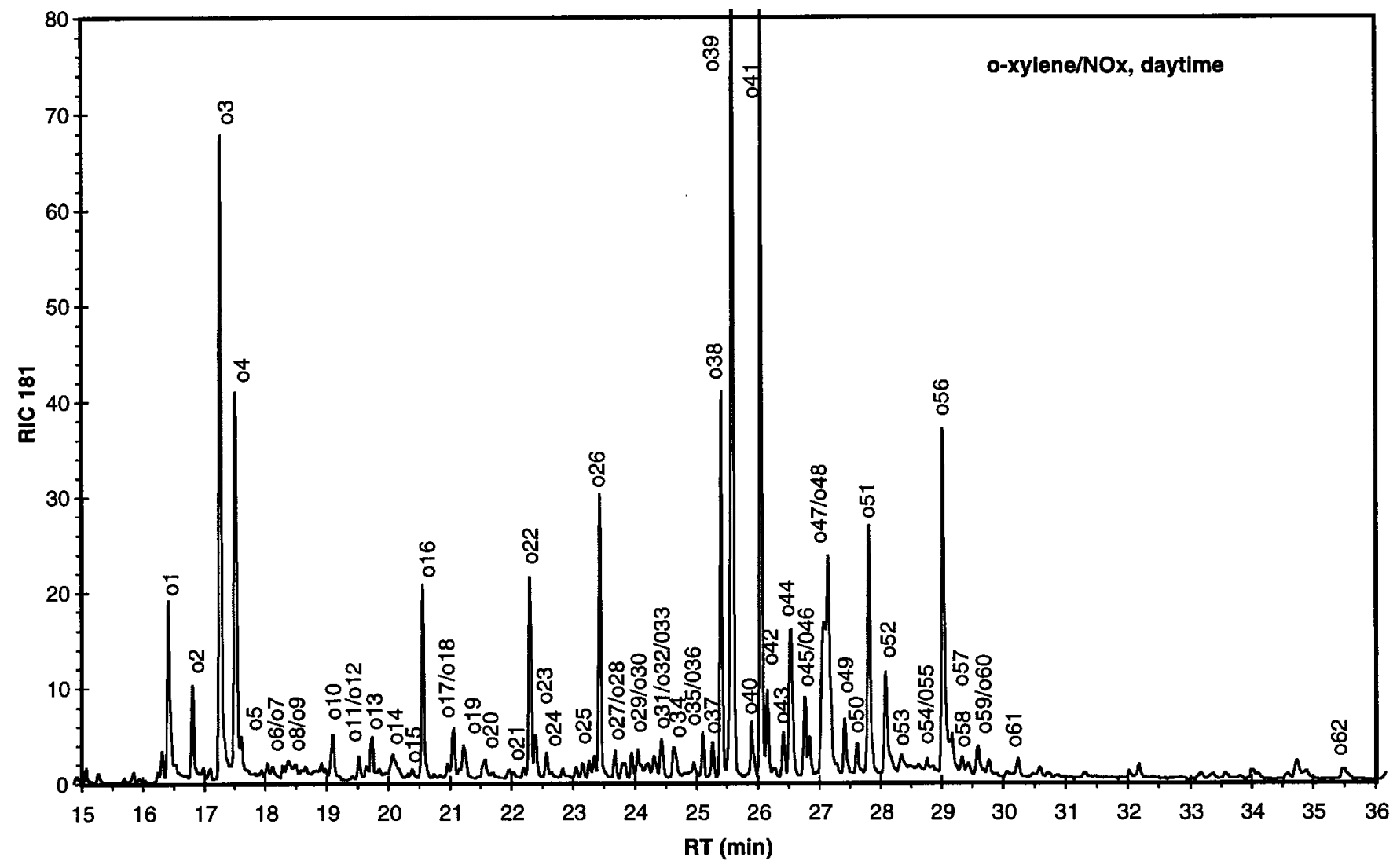

Figure 6.9:. Reconstructed $\mathrm{m} / \mathrm{z} 181$ ion chromatogram of the batch sample collected from an outdoor smog chamber o-xylene/NO ${ }_{x}$ daytime experiment. 
Table 6.12:

Carbonyl Products Detected in a Sample Collected from an o-Xylene/NOx Daytime Smog Chamber Experiment

\begin{tabular}{|c|c|c|c|c|}
\hline Peak & $\mathrm{RT}(\min )$ & Identity & $\mathrm{MW}^{\mathrm{a}}$ & $\mathrm{MW}^{\mathrm{b}}$ \\
\hline 01,02 & $16.41 / 16.79$ & glycolaldehyde & 255 & 60 \\
\hline 03 & 17.25 & hydroxyacetone & 269 & 74 \\
\hline 04 & 17.51 & hydroxyacetone/ $\mathrm{C}_{4}$ hydroxy carbonyl & 283 & 88 \\
\hline 05 & 17.61 & $\begin{array}{l}\mathrm{C}_{4} \text { hydroxy carbonyl or mono-deri. of } \mathrm{C}_{3} \text { hydroxy } \\
\text { dicarbonyl }\end{array}$ & 283 & 88 \\
\hline$\infty 6$ & 18.03 & $\mathrm{C}_{4}$ unsaturated carbonyl & 265 & 70 \\
\hline o7 & 18.11 & $\mathrm{C}_{4}$ epoxy/ $\mathrm{C}_{5}$ saturated carbonyl & 281 & 86 \\
\hline 08 & 18.38 & $\mathrm{C}_{4}$ hydroxy carbonyl/C $\mathrm{C}_{5}$ epoxy carbonyl & $283 / 295$ & $88 / 100$ \\
\hline$\infty$ & 18.54 & $\mathrm{C}_{5}$ epoxy/ $\mathrm{C}_{6}$ saturated carbonyl & 295 & 100 \\
\hline 010 & 19.10 & $\mathrm{C}_{6} \mathrm{~F}_{5} \mathrm{CHO} / \mathrm{C}_{3}$ hydroxy carbonyl & $391 / 269$ & $196 / 74$ \\
\hline 011 & 19.41 & furural or $\mathrm{C}_{6}$ di-unsaturated carbonyl & 291 & $\overline{\mathbf{9}}$ \\
\hline o12 & 19.63 & $\mathrm{C}_{5}$ unsaturated carbonyl & 293 & 98 \\
\hline ol3 & 19.73 & mono-deri. of $\mathrm{C}_{4}$ hydroxy dicarbonyl & 297 & 102 \\
\hline o14 & 20.06 & $\mathrm{C}_{6}$ epoxy carbonyl/ $\mathrm{C}_{4}$ hydroxy dicarbonyl & $309 / 283$ & $114 / 88$ \\
\hline 015 & 20.30 & $\mathrm{C}_{7}$ di-unsaturated carbonyl & 305 & 110 \\
\hline 016 & 20.55 & $\mathrm{C}_{6} \mathrm{~F}_{5} \mathrm{CHO}$ & 391 & 196 \\
\hline 017,018 & $20.95 / 21.05$ & $\mathrm{C}_{7}$ di-unsaturated carbonyl & 305 & 110 \\
\hline 019 & 21.21 & $\mathrm{C}_{7}$ di-unsaturated carbonyl & 305 & 110 \\
\hline o20 & 21.57 & $\mathrm{C}_{5}$ unsaturated carbonyl & 293 & 98 \\
\hline 021 & 22.18 & benzaldehyde & 301 & 106 \\
\hline 022 & 22.29 & $\mathrm{C}_{7}$ unsaturated epoxy carbonyl & 321 & 126 \\
\hline 023,024 & $22.38 / 22.56$ & $\mathrm{C}_{7}$ di-unsaturated carbonyl & 305 & 110 \\
\hline o25 & 23.26 & benzoquinone & 303 & 108 \\
\hline
\end{tabular}


Table 6.13:

Carbonyl Products Detected in a Sample Collected from an o-Xylene/NOx Daytime Smog Chamber Experiment (Continued)

\begin{tabular}{|c|c|c|c|c|}
\hline Peak & RT (min) & Identity & $\mathrm{MW}^{\mathrm{a}}$ & $\mathrm{MW}^{\mathrm{b}}$ \\
\hline 026 & 23.43 & $\mathrm{C}_{7}$ hydroxy unsaturated epoxy cyclic carbonyl & 335 & 140 \\
\hline 027 & 23.68 & o-tolualdehyde & 315 & 120 \\
\hline 028 & 23.83 & $\mathrm{C}_{8}$ di-unsaturated carbonyl & 319 & 124 \\
\hline o29 & 23.95 & mono-deri. of $\mathrm{C}_{8}$ di-unsaturated dicarbonyl & 333 & 138 \\
\hline 030 & 24.04 & $\begin{array}{l}\text { mono-deri. of methyl benzoquinone } / \mathrm{C}_{7} \\
\text { unsaturated epoxy carbonyl }\end{array}$ & $317 / 321$ & $122 / 126$ \\
\hline 031 & 24.16 & $\begin{array}{l}\text { mono-deri. of methyl benzoquinone } / \mathrm{C}_{7} \\
\text { unsaturated epoxy carbonyl }\end{array}$ & $317 / 321$ & $122 / 126$ \\
\hline 032 & 24.30 & 2,3-butanedione & 476 & 86 \\
\hline 033 & 24.43 & mono-deri. of 2-methyl-4-oxo-2-pentenal & 307 & 112 \\
\hline 034 & 24.63 & mono-deri. of methyl benzoquinone & 317 & 122 \\
\hline 035 & 24.95 & $\mathrm{C}_{8}$ hydroxy unsaturated epoxy cyclic carbonyl & 349 & 154 \\
\hline 036 & 25.09 & methylglyoxal & 462 & 72 \\
\hline 037 & 25.25 & 2,3-butanedione & 476 & 86 \\
\hline 038 & 25.39 & glyoxal & 448 & 58 \\
\hline 039 & 25.58 & glyoxal/methylglyoxal & $448 / 462$ & $58 / 72$ \\
\hline 040 & 25.89 & methylglyoxal & 462 & 72 \\
\hline 040 & 25.89 & mono-deri. of dimethylbenzoquinone & $331 / 347$ & $136 / 152$ \\
\hline 041 & 26.04 & methylglyoxal & 462 & 72 \\
\hline 042 & 26.15 & $\mathrm{C}_{5}$ epoxy dicarbonyl or $\mathrm{C}_{5}$ trione & 504 & 114 \\
\hline 043 & 26.41 & $\mathrm{C}_{3}$ dicarbonyl & 462 & $\overline{72}$ \\
\hline 044 & 26.53 & 2,3-butandione & 476 & 86 \\
\hline 045 & 26.74 & $\mathrm{C}_{3}$ dicarbonyl & 462 & 72 \\
\hline 046 & 26.81 & $\mathrm{C}_{3}$ dicarbonyl & $462 / 347$ & $72 / 152$ \\
\hline 047 & 27.06 & $\mathrm{C}_{4}$ dicarbonyl or $\mathrm{C}_{3}$ trione/ $\mathrm{C}_{4}$ hydroxy dicarbonyl & $476 / 492$ & $86 / 102$ \\
\hline 048 & 27.13 & unknown & 343 & 148 \\
\hline
\end{tabular}


Table 6.14:

Carbonyl Products Detected in a Sample Collected from an o-Xylene/NOx Daytime Smog Chamber Experiment (Continued)

\begin{tabular}{|l|c|l|c|c|}
\hline Peak & RT (min) & \multicolumn{1}{|c|}{ Identity } & $M^{\mathrm{a}}$ & $\mathrm{MW}^{\mathrm{b}}$ \\
\hline 049 & 27.41 & $\mathrm{C}_{4}$ hydroxy dicarbonyl & 492 & 102 \\
\hline 050 & 27.61 & $\mathrm{C}_{5}$ hydroxy dicarbonyl & 506 & 116 \\
\hline 051 & 27.81 & $\mathrm{C}_{5}$ hydroxy dicarbonyl & 506 & 116 \\
\hline 052 & 28.08 & $\mathrm{C}_{4}$ hydroxy dicarbonyl & 492 & 102 \\
\hline 053 & 28.33 & $\mathrm{C}_{3}$ hydroxy dicarbonyl & 478 & 88 \\
\hline 054 & 28.76 & 4-oxo-2-pentenal & 488 & 98 \\
\hline 055 & 28.86 & butendial & 474 & 84 \\
\hline 056 & 29.01 & C 4 hydroxy dicarbonyl & 492 & 102 \\
\hline 057 & 29.16 & 4-oxo-2-pentenal /C 4 hydroxy dicarbonyl & $488 / 492$ & $98 / 102$ \\
\hline 057 & 29.24 & butendial & 474 & 84 \\
\hline 058 & 29.31 & butendial & 474 & 84 \\
\hline 059 & 29.58 & butendial/4-oxo-2-pentenal & $474 / 488$ & $84 / 98$ \\
\hline 060 & 29.76 & 4-oxo-2-pentenal & 488 & 98 \\
\hline 061 & 30.23 & 4-oxo-2-pentenal & 488 & 98 \\
\hline 062 & 35.48 & o-phathalicdicarboxyaldehyde & 524 & 134 \\
\hline
\end{tabular}




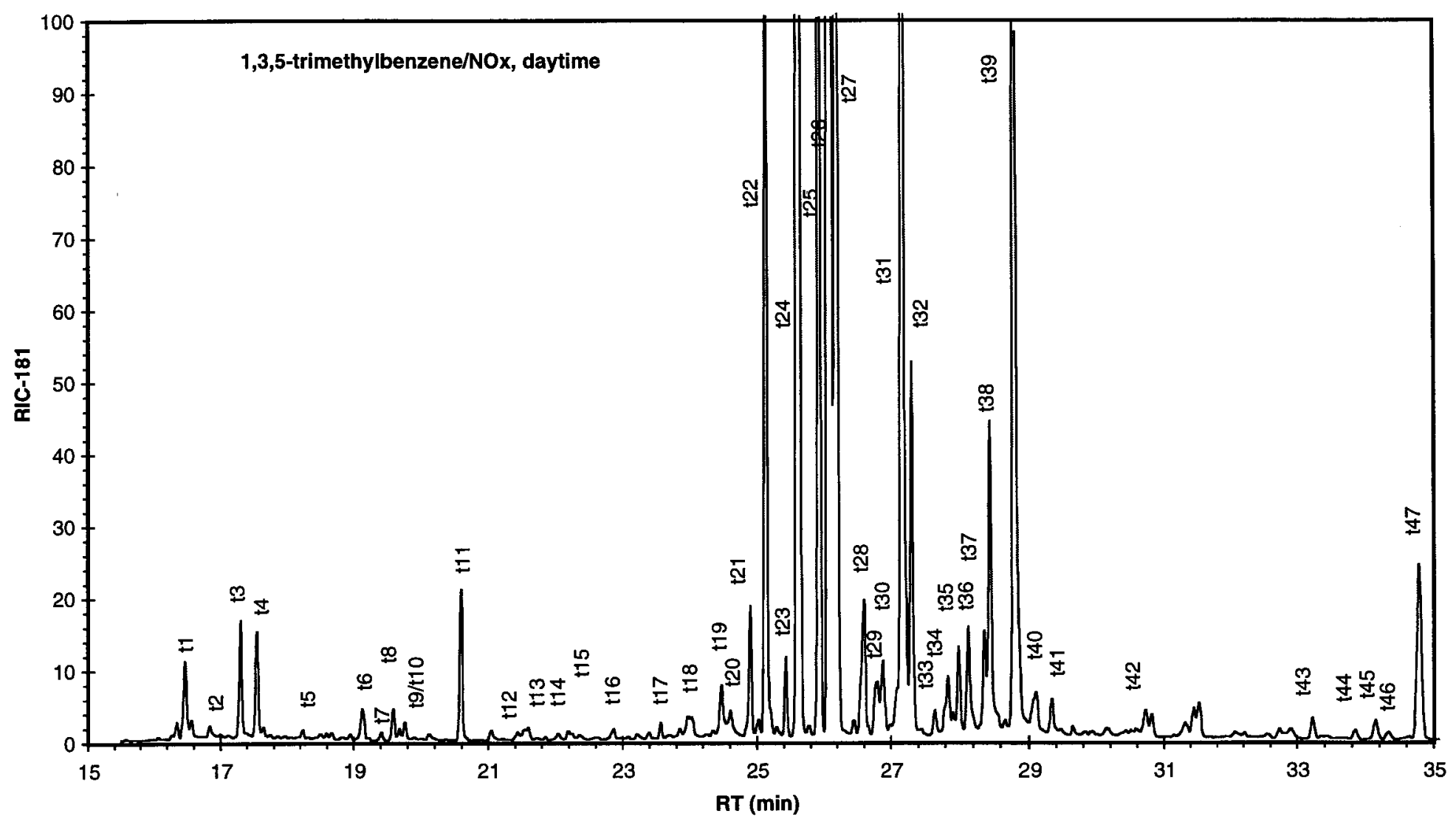

Figure 6.10: Reconstructed m/z 181 ion chromatogram of 1,3,5-trimethylbenzene/ $\mathrm{NO}_{\mathrm{x}}$ in an outdoor smog chamber daytime experiment. 


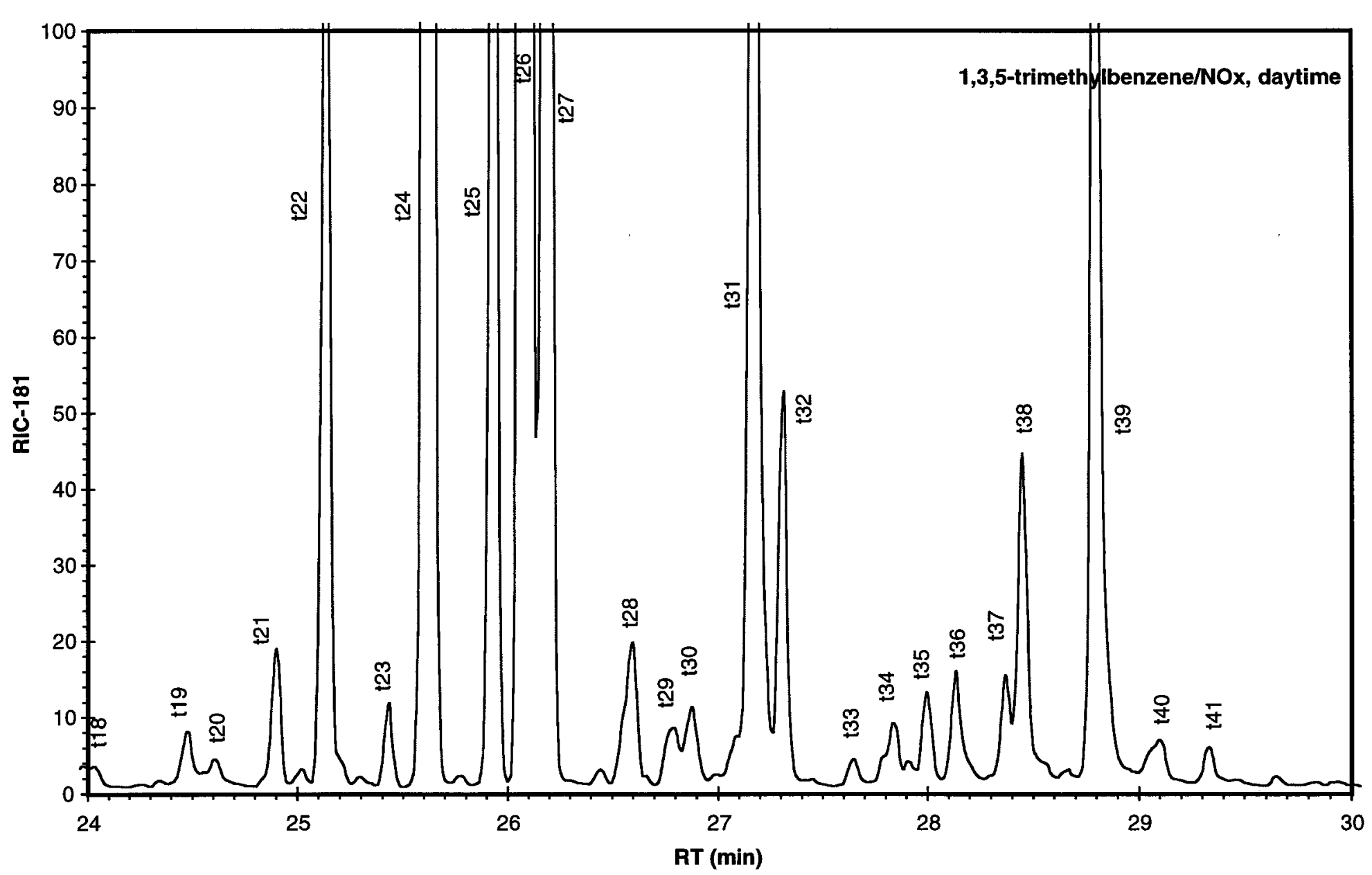

Figure 6.11:. Detailed Reconstructed $\mathrm{m} / \mathrm{z} 181$ ion chromatogram of the batch sample collected from an outdoor smog chamber 1,3,5trimethylbenzene/ $\mathrm{NO}_{x}$ daytime experiment. 
Table 6.15:

\section{Carbonyl Products Detected in a Sample Collected from an} 1,3,5-Trimethylbenzene/NOx Daytime Smog Chamber Experiment

\begin{tabular}{|c|c|c|c|c|}
\hline Peak & $\mathrm{RT}(\mathrm{min})$ & Identity & $\mathbf{M W}^{\mathrm{a}}$ & $\mathrm{MW}^{\mathrm{b}}$ \\
\hline $\mathrm{t} 1, \mathrm{t} 2$ & $16.46 / 16.79$ & glycolaldehyde & 255 & 60 \\
\hline $\mathrm{t} 3, \mathrm{t} 4$ & $17.30 / 17.54$ & hydroxyacetone & 269 & 74 \\
\hline$\overline{\mathrm{t} 5}$ & 18.64 & mono deri. of m504 carbonyl & 309 & 114 \\
\hline$\overline{\mathrm{t} 6}$ & 19.09 & mono-deri. of $\mathrm{C}_{5}$ hydroxy carbonyl $/ \mathrm{C}_{6} \mathrm{~F}_{5} \mathrm{CHO}$ & 311 & 116 \\
\hline $\mathrm{t} 7, \mathrm{t} 8$ & $19.40 / 19.58$ & mono-deri. of $\mathrm{C}_{5}$ hydroxy carbonyl & 311 & 116 \\
\hline $\mathrm{t} 9, \mathrm{t} 10$ & $19.66,19.75$ & mono-deri. of $\mathrm{C}_{5}$ hydroxy carbonyl & 311 & 116 \\
\hline t11 & 20.56 & $\mathrm{C}_{6} \mathrm{~F}_{5} \mathrm{CHO}$ & 391 & 196 \\
\hline $\mathrm{t} 12$ & 21.44 & mono-deri. of $\mathrm{C}_{6}$ unsaturated dicarbonyl & 307 & 112 \\
\hline $\mathrm{t} 13$ & 21.53 & $\mathrm{C}_{7}$ di-unsaturated carbonyl & 305 & 110 \\
\hline $\mathrm{t} 14$ & 21.84 & mono-deri. of $\mathrm{C}_{6}$ unsaturated dicarbonyl & 307 & 112 \\
\hline $\mathrm{t} 15$ & 22.34 & $\mathrm{C}_{5}$ epoxy $/ \mathrm{C}_{6}$ saturated carbonyl & 295 & 100 \\
\hline$\overline{\mathrm{t16}}$ & 22.83 & mono-deri. of $\mathrm{C}_{8}$ di-unsaturated dicarbonyl & 333 & 138 \\
\hline$\overline{t 17}$ & 23.56 & $\mathrm{C}_{8}$ unsaturated epoxy carbonyl & 335 & 140 \\
\hline $\mathrm{t} 18$ & 23.98 & $\mathrm{C}_{8}$ hydroxy unsaturated epoxy cyclic carbonyl & 349 & 154 \\
\hline t19 & 24.46 & 3,5-dimethyl benzaldehyde & 329 & 134 \\
\hline $\mathrm{t} 20$ & 24.60 & $\begin{array}{l}\mathrm{C}_{8} \text { unsaturated epoxy carbonyl/ } \mathrm{C}_{8} \text { hydroxy } \\
\text { unsaturated epoxy cyclic carbonyl }\end{array}$ & $335 / 349$ & $140 / 154$ \\
\hline \multirow[t]{2}{*}{$\mathrm{t} 21$} & 24.90 & 3,5-dimethyl benzaldehyde & 329 & 134 \\
\hline & 25.01 & dimethylbenzoquinone & 331 & 136 \\
\hline $\mathrm{t} 22$ & 25.13 & methylglyoxal & 462 & 72 \\
\hline t23 & 25.43 & glyoxal & 448 & 58 \\
\hline 124 & 25.61 & glyoxal/methylglyoxal & $448 / 462$ & $58 / 72$ \\
\hline 25 & 25.91 & methylglyoxal & 462 & $\overline{72}$ \\
\hline 126 & 26.08 & methylglyoxal/ $\mathrm{C}_{5}$ epoxy dicarbonyl/ $\mathrm{C}_{4}$ trione & $462 / 504$ & $72 / 114$ \\
\hline
\end{tabular}


Table 6.16:

Carbonyl Products Detected in a Sample Collected from an 1,3,5-Trimethylbenzene/NOx Daytime Smog Chamber Experiment (Continued)

\begin{tabular}{|c|c|c|c|c|}
\hline Peak & RT (min) & Identity & $\mathrm{MW}^{\mathrm{a}}$ & $\mathrm{MW}^{\mathrm{b}}$ \\
\hline $\mathrm{t} 27$ & 26.15 & $\mathrm{C}_{5}$ epoxy dicarbonyl or $\mathrm{C}_{5}$ trione & 504 & 114 \\
\hline $\mathrm{t} 28, \mathrm{t} 29$ & $26.59 / 26.76$ & $\mathrm{C}_{4}$ saturated dicarbonyl or $\mathrm{C}_{3}$ trione & 476 & 86 \\
\hline \multirow[t]{2}{*}{130} & 26.86 & $\mathrm{C}_{4}$ epoxy carbonyl or $\mathrm{C}_{4}$ trione & 490 & 100 \\
\hline & 27.06 & trimethyl-benzoquinone & 345 & 150 \\
\hline $\mathrm{t} 31$ & 27.16 & $\begin{array}{l}\mathrm{C}_{5} \text { epoxy carbonyl or } \mathrm{C}_{5} \text { trione/ } \mathrm{C}_{4} \text { epoxy carbonyl } \\
\text { or } \mathrm{C}_{4} \text { trione }\end{array}$ & $504 / 490$ & $114 / 100$ \\
\hline t32 & 27.29 & $\mathrm{C}_{4}$ hydroxy dicarbonyl & 492 & 102 \\
\hline $\mathrm{t} 33$ & 27.61 & $\mathrm{C}_{5}$ hydroxy dicarbonyl & 506 & 116 \\
\hline t34 & 27.82 & $\mathrm{C}_{5}$ hydroxy dicarbonyl/ $\mathrm{C}_{4}$ saturated carbonyl & $506 / 476$ & $116 / 86$ \\
\hline $\mathrm{t} 35$ & 27.98 & $\begin{array}{l}\mathrm{C}_{7} \text { unsaturated dicarbonyl/ } \mathrm{C}_{6} \text { unsaturated } \\
\text { dicarbonyl }\end{array}$ & $516 / 502$ & $126 / 112$ \\
\hline t36 & 28.13 & $\begin{array}{l}\mathrm{C}_{4} \text { unsaturated dicarbonyl/ } \mathrm{C}_{5} \text { epoxy dicarbonyl or } \\
\mathrm{C}_{5} \text { trione }\end{array}$ & $474 / 504$ & $86 / 114$ \\
\hline t37 & 28.35 & $\mathrm{C}_{4}$ hydroxy dicarbonyl & 492 & 102 \\
\hline $\mathrm{t} 38, \mathrm{t} 39$ & $28.49,28.78$ & $\mathrm{C}_{5}$ hydroxy dicarbonyl & 506 & 116 \\
\hline $\mathrm{t} 40$ & 29.04 & $\begin{array}{l}\text { 2-methyl-4-oxo-2-pentenal } / \mathrm{C}_{5} \text { unsaturated } \\
\text { dicarbonyl }\end{array}$ & $502 / 488$ & $112 / 98$ \\
\hline $\mathrm{t} 41, \mathrm{t} 42$ & $29.33 / 30.73$ & 2-methyl-4-oxo-2-pentenal & 502 & 112 \\
\hline t43 & 33.23 & 2-methyl-4-oxo-2-pentenal & 502 & 112 \\
\hline $\mathrm{t} 44, \mathrm{t} 45$ & $33.83 / 34.13$ & $C_{9}$ unsaturated epoxy dicarbonyl & 558 & 168 \\
\hline t46 & 34.33 & & 572 & 182 \\
\hline$\$ 47$ & 34.76 & 2-methyl-4-oxo-2-pentenal & 502 & 112 \\
\hline
\end{tabular}




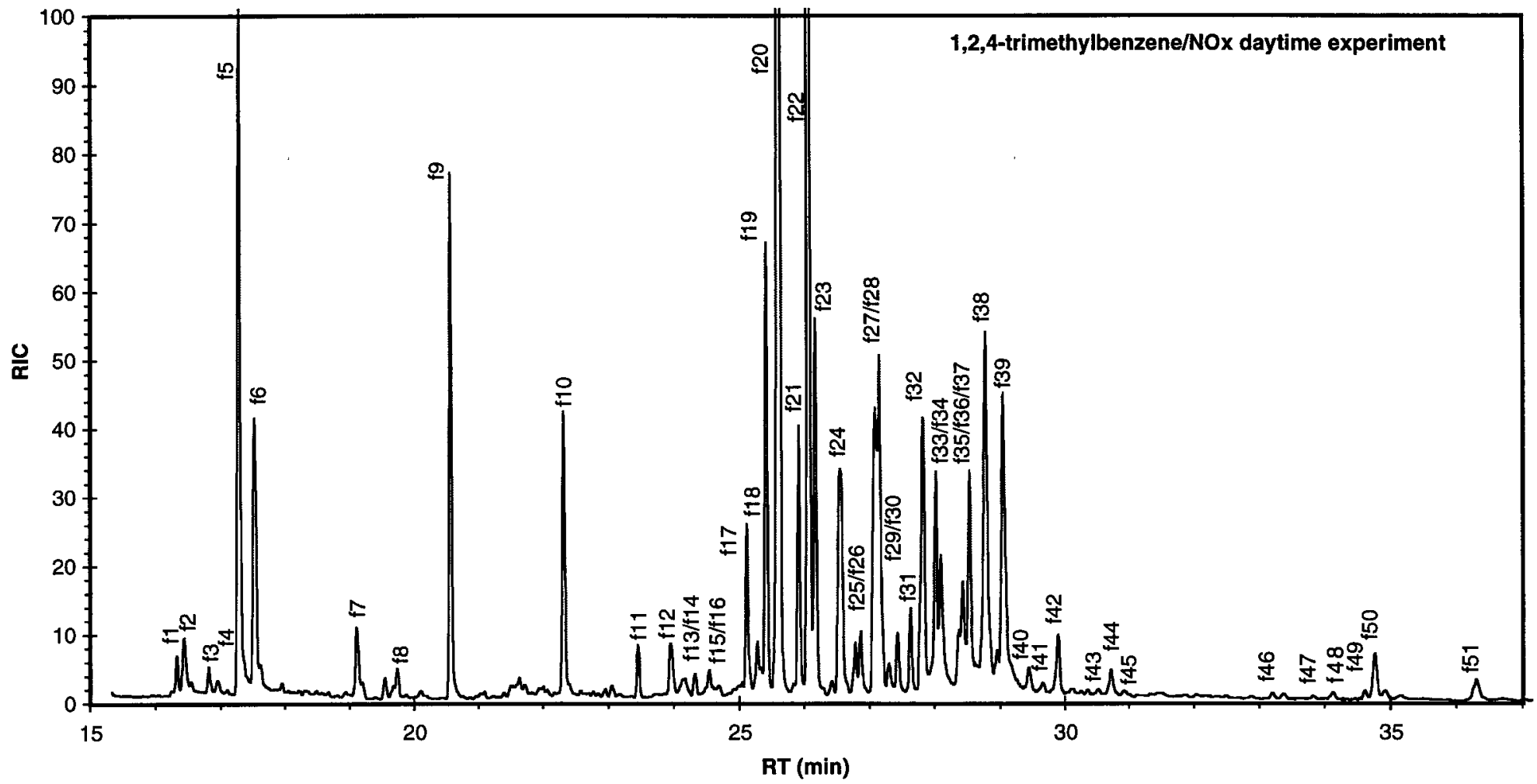

Figure 6.12:. Reconstructed $\mathrm{m} / \mathrm{z} 181$ ion chromatogram of the batch sample collected from an outdoor smog chamber 1,2,4trimethylbenzene $/ \mathrm{NO}_{x}$ daytime experiment. 
Table 6.17:

\section{Carbonyl Products Detected in a Sample Collected from an} 1,2,4-Trimethylbenzene/NOx Daytime Smog Experiment

\begin{tabular}{|c|c|c|c|c|}
\hline Peak & $\mathrm{RT}(\min )$ & Identity & $\mathrm{MW}^{\mathrm{a}}$ & $\mathrm{MW}^{\mathrm{b}}$ \\
\hline fl & 16.33 & $\mathrm{C}_{5}$ saturated $/ \mathrm{C}_{4}$ epoxy carbonyl & 281 & 86 \\
\hline $\mathrm{f} 2$ & 16.44 & glycolaldehyde/2-hydroxy propanal & $255 / 269$ & $60 / 74$ \\
\hline $\mathrm{f3}$ & 16.56 & 2-hydroxy propanal & 269 & 74 \\
\hline $\mathrm{f4}$ & 16.81 & glycolaldehyde & 255 & 60 \\
\hline f5 & 17.28 & hydroxyacetone & 269 & 74 \\
\hline f6 & 17.53 & hydroxyacetone/ $\mathrm{C}_{4}$ hydroxy carbonyl & $269 / 283$ & $74 / 88$ \\
\hline f7 & 19.11 & $\mathrm{C}_{3}$ hydroxy carbonyl/ $\mathrm{C}_{6} \mathrm{~F}_{5} \mathrm{CHO}$ & $269 / 391$ & $74 / 196$ \\
\hline 88 & 19.73 & mono deri. of $\mathrm{C}_{5}$ hydroxy dicarbonyl & 311 & 116 \\
\hline 19 & 20.56 & $\mathrm{C}_{6} \mathrm{~F}_{5} \mathrm{CHO}$ & 391 & 196 \\
\hline $\mathrm{flo}$ & 22.31 & mono-deri. of $\mathrm{C}_{7}$ unsaturated dicarbonyl & 321 & 126 \\
\hline f11 & 23.44 & $\mathrm{C}_{8}$ unsaturated epoxy carbonyl & 335 & 140 \\
\hline $\mathrm{f} 12$ & 23.94 & $\begin{array}{l}\mathrm{C}_{\mathrm{g}} \text { hydroxy unsaturated epoxy cyclic carbonyl / } \\
\text { unknown }\end{array}$ & $349 / 365$ & $154 / 170$ \\
\hline $\mathrm{fl3}$ & 24.15 & $\mathrm{C}_{\mathrm{g}}$ hydroxy unsaturated epoxy cyclic carbonyl & 349 & 154 \\
\hline$\overline{\mathrm{fl} 4}$ & 24.33 & 2,3-butandione & 476 & 86 \\
\hline f15 & 24.54 & $\mathrm{C}_{8}$ unsaturated epoxy carbonyl & 335 & 140 \\
\hline fl6 & 24.69 & $\mathrm{C}_{8}$ unsaturated epoxy carbonyl & 335 & 140 \\
\hline $\mathrm{f} 17$ & 25.11 & methylglyoxal/ $\mathrm{C}_{8}$ unsaturated epoxy carbonyl & $462 / 335$ & $72 / 140$ \\
\hline f18 & 25.25 & 2,3-butandione/ dimethyl benzaldehyde & $476 / 329$ & $86 / 134$ \\
\hline f19 & $25.36 / 25.41$ & dimethylbenzoquinone/ glyoxal & $331 / 448$ & $136 / 58$ \\
\hline $\mathrm{f} 20$ & 25.59 & glyoxal/methylglyoxal & $448 / 462$ & $58 / 72$ \\
\hline 220 & 25.66 & dimethyl benzaldehyde & 329 & 134 \\
\hline$f 21, f 22$ & $25.91 / 26.06$ & methylglyoxal & 462 & 72 \\
\hline 623 & 26.16 & $\mathrm{C}_{5}$ epoxy dicarbonyl or $\mathrm{C}_{5}$ trione & 504 & 114 \\
\hline $\mathrm{f} 24$ & 26.54 & 2,3-butandione & 476 & 86 \\
\hline $\mathrm{f} 25$ & 26.78 & $\mathrm{C}_{5}$ hydroxy dicarbonyl & 506 & 116 \\
\hline
\end{tabular}


Table 6.18:

Carbonyl Products Detected in a Sample Collected from an 1,2,4-Trimethylbenzene/NOx Daytime Smog Experiment (Continued)

\begin{tabular}{|c|c|c|c|c|}
\hline Peak & RT (min) & Identity & $\mathrm{MW}^{\mathrm{a}}$ & $\mathrm{MW}^{\mathrm{b}}$ \\
\hline $\mathrm{f} 26$ & 26.86 & $\mathrm{C}_{4}$ epoxy dicarbonyl or $\mathrm{C}_{4}$ trione & 490 & 100 \\
\hline$\overline{\mathrm{f} 27}$ & 27.08 & $\mathrm{C}_{4}$ hydroxy dicarbonyl & 492 & 102 \\
\hline 128 & 27.14 & $\mathrm{C}_{5}$ epoxy dicarbonyl or $\mathrm{C}_{5}$ trione & 504 & 114 \\
\hline $\mathrm{f} 28$ & 27.19 & $\mathrm{C}_{4}$ epoxy dicarbonyl or $\mathrm{C}_{4}$ trione & 490 & 100 \\
\hline $\mathrm{f} 29, \mathrm{f} 30$ & $27.29 / 27.43$ & $\mathrm{C}_{4}$ hydroxy dicarbonyl & 492 & 102 \\
\hline $\mathrm{f} 31, \mathrm{f} 32$ & $27.63 / 27.81$ & $\mathrm{C}_{5}$ hydroxy dicarbonyl & 506 & 116 \\
\hline$\overline{\mathrm{f} 33}$ & 28.02 & 3-hexene-2,5-dione & 502 & 112 \\
\hline $\mathrm{f} 34, \mathrm{f35}$ & $28.09 / 28.36$ & $\mathrm{C}_{4}$ hydroxy dicarbonyl & 492 & 102 \\
\hline f36 & 28.43 & $\mathrm{C}_{5}$ hydroxy dicarbonyl & 506 & 116 \\
\hline f37 & 28.53 & 3-hexene-2,5-dione & 502 & 112 \\
\hline f38 & 28.78 & $\mathrm{C}_{5}$ hydroxy dicarbonyl & 506 & 116 \\
\hline \multirow[t]{2}{*}{ f39 } & $29.05 / 29.10$ & $\mathrm{C}_{4}$ hydroxy dicarbonyl/3-hexene-2,5-dione & $492 / 502$ & $102 / 112$ \\
\hline & 29.33 & 2-methyl-4-oxo-2-pentenal & 502 & 112 \\
\hline $\mathrm{f40}$ & 29.44 & $\mathrm{C}_{7}$ unsaturated dicarbonyl & 516 & 126 \\
\hline f41 & 29.66 & 2-methyl-butendial & 488 & 98 \\
\hline $\mathrm{f} 42$ & 29.91 & 3-hexene-2,5-dione & 502 & 112 \\
\hline $\mathrm{f} 43$ & 30.36 & 2-methyl-butendial & 488 & 98 \\
\hline f44 & 30.71 & 3-hexene-2,5-dione or 2-methyl-4-oxo-2-pentenal & 502 & 112 \\
\hline f45 & 30.91 & $\mathrm{C}_{7}$ unsaturated dicarbonyl & 516 & 126 \\
\hline $\mathrm{f} 46$ & 33.19 & 2-methyl-4-oxo-2-pentenal & 502 & 112 \\
\hline $\mathrm{f} 47, \mathrm{f} 48$ & $33.83 / 33.41$ & $\mathrm{C}_{9}$ unsaturated epoxy dicarbonyl & 558 & 168 \\
\hline f49 & 34.59 & 2-methyl-butendial & 488 & 98 \\
\hline $\mathrm{f50}$ & 34.76 & 2-methyl-4-oxo-2-pentenal & 502 & 112 \\
\hline f51 & 36.28 & 2-methyl-butendial & 488 & 98 \\
\hline
\end{tabular}


Some of the carbonyl products coeluted with other peaks because of the limited separation power of the GC column and the high number of carbonyl products present. For example, the second peak of glyoxal-PFBHA derivative (tol26 in Figure 6.5) coelutes with the second peak of methylglyoxal. The mass spectrum of this peak contains unique ions of each derivative, making it possible to determine the molecule weight of the individual component. This peak, however, can not be used to quantify either glyoxal or methylglyoxal when both are present, because the $\mathrm{m} / \mathrm{z} 181$ ion used for quantification comes from both derivatives. Fortunately, the first peak of the glyoxal derivative (tol25) and the fourth peak (tol28) of methylglyoxal are free from interference from other carbonyls present. Therefore, these two peaks are used for the quantification of glyoxal and methylglyoxal. To generalize: whenever some isomer peaks of a carbonyl coelute with another peak, the peaks without a coelution problem are used for quantification; when all the isomer peaks are free of coelution, the sum of area of all the isomer peaks are used.

The concentrations of glyoxal, methylglyoxal, 2,3-butandione, glycolaldehyde, hydroxyacetone, and methyl-p-benzoquinone (MBQ) were computed using the calibration factors established in the TBR. Because benzaldehyde did not have a calibration factor from the TBR, and because p-tolaldehyde showed similar calibration factors in the TBR and the outdoor chamber, the concentrations of benzaldehyde and p-tolualdehyde were computed using calibration factors established in the outdoor chamber. The calibration factor for p-tolualdehyde was also used to estimate the concentrations of m-tolualdehyde, o-tolualdehyde, and dimethylbenzaldehyde. The calibration factor for MBQ was used to compute the concentrations for benzoquinone and dimethylbenzoquinone.

No estimation of calibration factors was made for other carbonyl products, and the relative peak areas-defined as the peak area ratio between $\mathrm{m} / \mathrm{z}=181$ ion of a target carbonyl and $\mathrm{m} / \mathrm{z}=59$ ion of ${ }^{13} \mathrm{C}_{3}$-acetone - added as an internal standard - were used to represent the concentration of any particular carbonyl product in the chamber. The ability to detect the ${ }^{13} \mathrm{C}_{3}$-acetone relative to normal acetone is shown in Figure 6.13.

The estimation of the yields for carbonyl products is difficult for reaction systems in experimental setting such as the UNC outdoor smog chamber. As mentioned above, carbonyls can undergo photolysis and reaction with $\mathrm{OH}$ radicals in the atmosphere, so calculation of their yields must take into account the loss that results from both processes. The use of sunlight as the photolysis light source and a lack of wavelength-dependent quantum yields for the compounds of interest makes it difficult to calculate the loss of carbonyls resulting from photolysis. For these reasons, no carbonyl yields were estimated for the work reported here.

Each of the aromatic compounds tested here can have produced a number of different carbonyl forms. For ease of presentation, the results shown here have been organized by carbonyl type in the following discussion of possible reaction pathways. Each type is described in a separate section, with the chemical structures for the relevant carbonyl forms shown at the top of that section. 

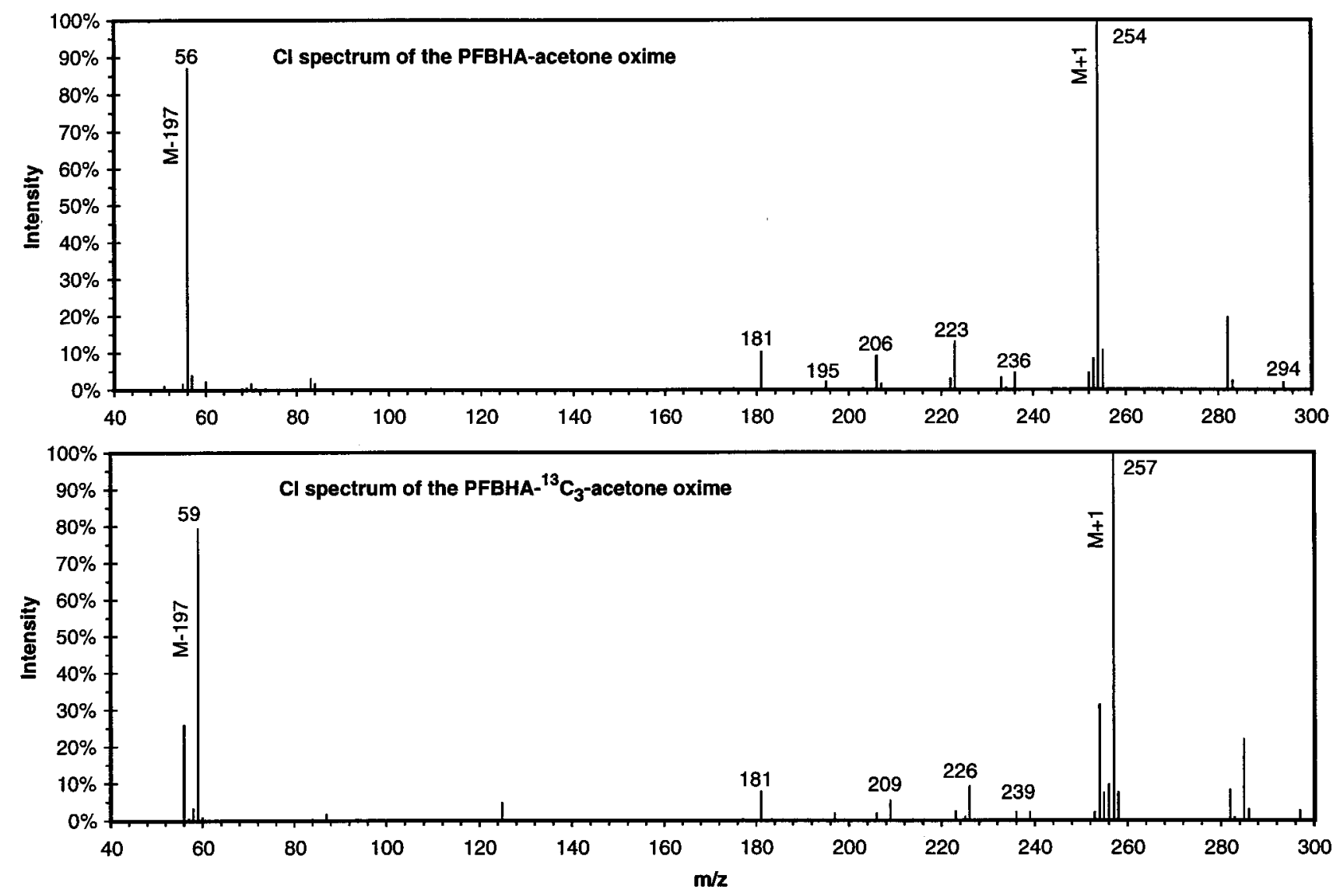

Figure 6.13: The MS of normal acetone and ${ }^{13} \mathrm{C}_{3}$-acetone. 


\subsubsection{Aromatic Aldehydes}

Benzaldehyde (tol12 and tol13), p-tolualdehyde (4-methyl-benzaldehyde) (p17 and p18), $\mathrm{m}$-tolualdehyde (m26) and o-tolualdehyde (027), are positively identified in the toluene, p-xylene, $\mathrm{m}$-xylene, and o-xylene experiments by matching the retention times and mass spectra of the carbonyls with those of the appropriate standards.<smiles>O=Cc1ccccc1</smiles>

benzaldehyde

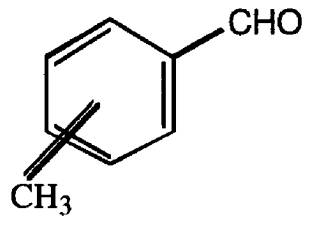

tolualdehyde<smiles>Cc1ccc(C=O)cc1</smiles>

dimethylbenzaldehyde

The molecular weight of the benzaldehyde-PFBHA derivative, 301, is clearly indicated by five strong ions characteristic of molecular weight: ions at m/z 104 (M-197), resulting from loss of $\mathrm{C}_{6} \mathrm{~F}_{5} \mathrm{CH}_{2} \mathrm{O}$ moiety; $\mathrm{m} / \mathrm{z} 271$ (M-30), resulting from loss of $\mathrm{NO} ; \mathrm{m} / \mathrm{z} 302(\mathrm{M}+1)$, resulting from protonation of neutral molecule; $\mathrm{m} / \mathrm{z} 330(\mathrm{M}+29)$, resulting from addition of the reagent ion $\mathrm{C}_{2} \mathrm{H}_{5}+$; and $\mathrm{m} / \mathrm{z} 482(\mathrm{M}+181)$, resulting from addition of $\mathrm{C}_{6} \mathrm{~F}_{5} \mathrm{CH}_{2}+$ ion to the neutral molecule.

Peaks t19 and t21 (Figure 6.10) from the 1, 3, 5TMB experiment were tentatively identified as 3,5-dimethylbenzaldehyde and peaks f18 and f20 (Figure 6.12) in the 1,2,4-TMB experiment were tentatively identified as a dimethylbenzaldehyde because the molecular weight data obtained from the CI mass spectra match that of a dimethylbenzaldehyde. In the 1, 3, 5TMB system, 3,5-dimethylbenzaldehyde is the only dimethylbenzaldehyde expected because of the symmetric structure of $1,3,5 \mathrm{TMB}$. 1,2,4-TMB, however, could produce three possible dimethylbenzaldehydes: 2,4-; 2,5-; and 3,4-dimethylbenzaldehyde. Additional differentiation among the three possible isomers cannot made based on the CI spectrum.

Benzaldehyde reacts with $\mathrm{OH}$ radicals at a rate two times faster than its parent compound toluene; tolualdehydes react with $\mathrm{OH}$ at a rate comparable to their parent compounds xylenes. The time profiles of benzaldehyde and m-tolualdehyde are shown in Figure 6.15, along with their parent compounds-toluene and m-xylene. $\mathrm{p}$-Tolualdehyde, o-tolualdehyde, dimethylbenzaldehydes have similar time series to those of benzaldehyde and $\mathrm{m}$-tolualdehyde. Degradation of the aromatic aldehydes (as shown in Figure 6.15) is because of reaction with $\mathrm{OH}$ and photolysis.

\subsubsection{Quinones}

Methyl-p-benzoquinone (MBQ) was positively identified in the samples from toluene (tol18, tol19, tol21, and tol52), $\mathrm{m}$-xylene (m27, $\mathrm{m} 28$, and $\mathrm{m} 30$ ), and o-xylene (o30, o31, and $\mathrm{o} 33$ ) photooxidation, but no MBQ was observed in the p-xylene experiment. 
<smiles>O=C1C=CC(=O)C=C1</smiles>

benzoquinone<smiles>CC1=CC(=O)C=CC1=O</smiles>

methyl-p-benzoquinone<smiles>CC1=CC(=O)C(C)=CC1=O</smiles>

dimethyl-p-benzoquinone

The PFBHA derivatives of MBQ could result in three peaks from the mono-derivative and one peak from the di-derivative, however, the di-derivative is formed with a lower yield than the mono-derivative and could be below the detection limit when the concentration is low. This possibility may explain why the di-derivative was not detected in the m-xylene and o-xylene experiments.

Benzoquinone (BQ) was identified as a product from photooxidation of toluene (tol17 and tol51), p-xylene, and o-xylene (o25). Additional outdoor smog chamber experiments conducted using benzene and ethylbenzene as the parent hydrocarbons showed benzoquinone is also a product of their photooxidation. Like MBQ, BQ could form both monoand di-derivatives with PFBHA and the di-derivative formed again at a lower yield than the mono-derivative.

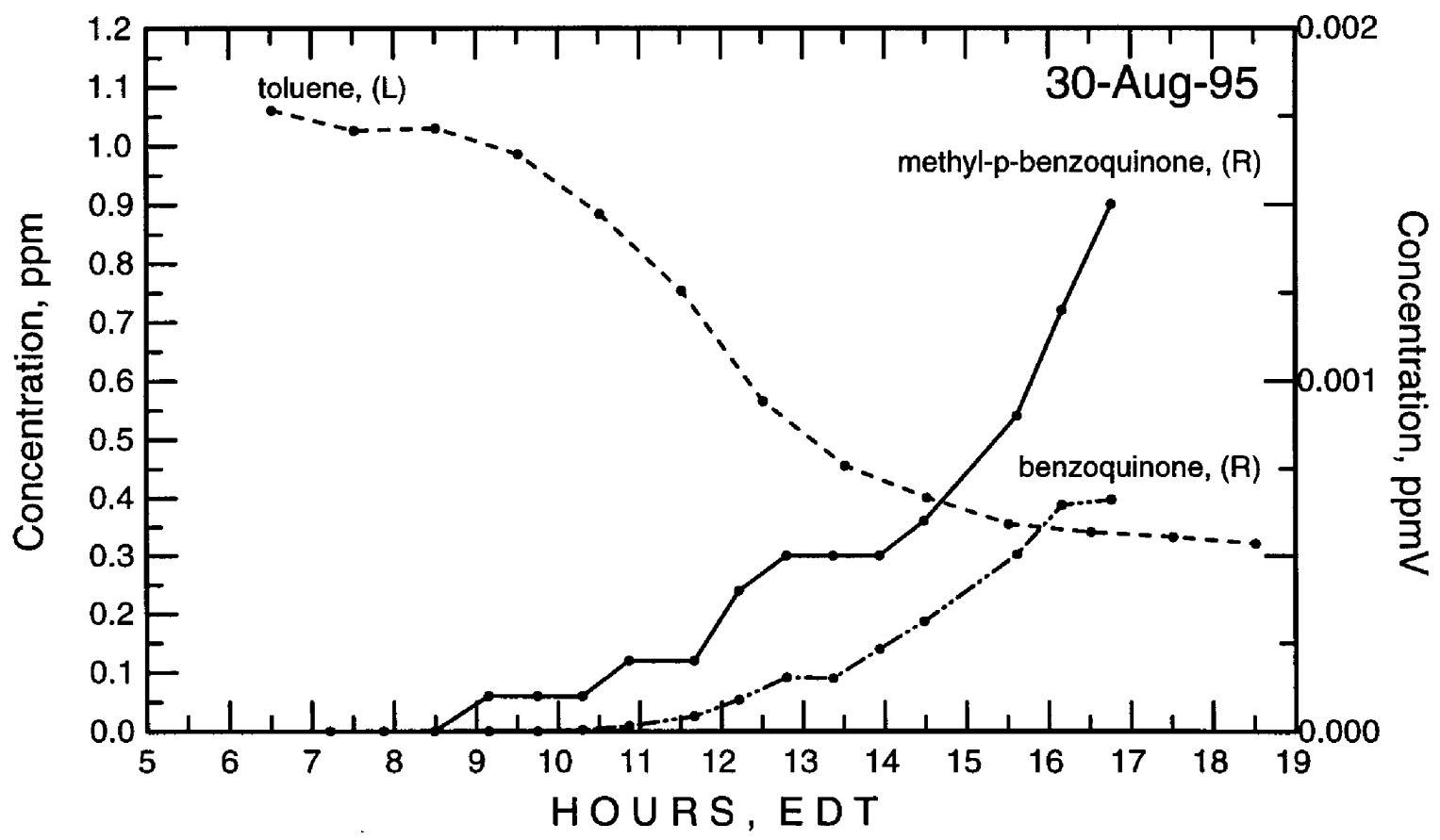

Figure 6.14:. Time series of benzoquinone and methyl-p-benzoquinone in the toluene outdoor chamber experiment. 

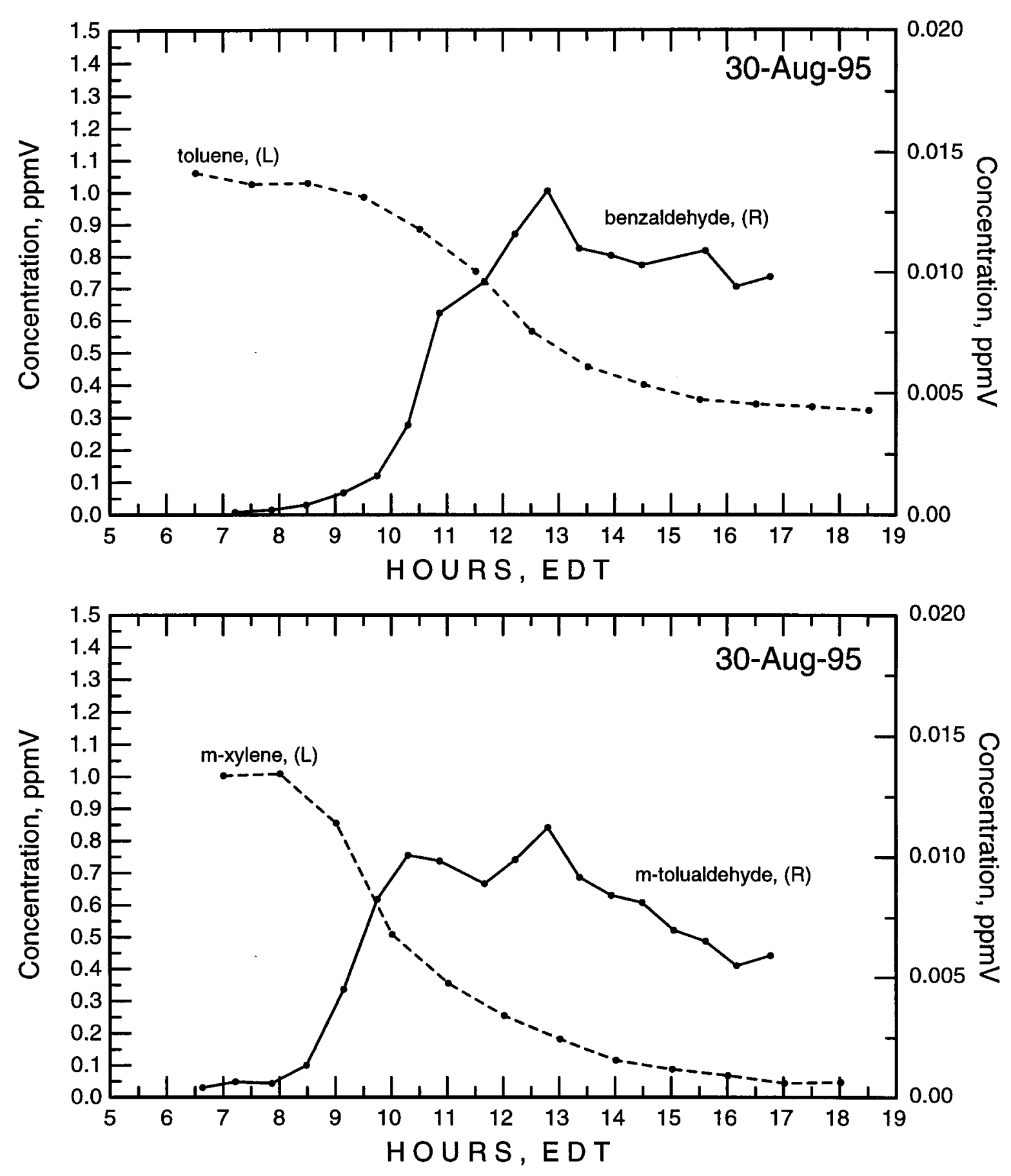

Figure 6.15:. Time series of benzaldehyde, m-tolualdehyde, and their parent compounds in the toluene and $\mathrm{m}$-xylene outdoor chamber experiments. 
Dimethylbenzoquinones (DMBQ) were present in the three xylenes (p22, m32 and o40), 1, 2, 4TMB (f19) and 1, 3, 5TMB systems. The retention times showed that the DMBQ formed from 1, 2, 4TMB is the same as the one from p-xylene and that the DMBQ from 1, 3, 5TMB the same as the one from $m$-xylene. Trace amounts of trimethylbenzoquinones were observed in 1, 3, 5TMB and 1, 2, 4TMB experiments.

The time series of benzoquinone and methyl-p-benzoquinone in the toluene outdoor chamber experiment are shown in Figure 6.14. They are typical of the quinone products. From the time series, it appears that benzoquinone, methylbenzoquinone and dimethylbenzoquinone are stable once they are formed, and likely are secondary products. Gery also detected trace amount of p-benzoquinone from photooxidation of benzaldehyde and methyl-p-benzoquinone from o-tolualdehyde. In addition, they showed that 2,6-dimethylp-benzoquinone was a product of 2,6-dimethylphenol photooxidation. Thus, two possible precursors of quinone products are aromatic aldehydes and phenols.

Using the calibration factors determined for MBQ in the TBR, maximum concentrations of quinone up to $2 \mathrm{ppbV}$ in the toluene, $\mathrm{m}$-xylene and $\mathrm{o}$-xylene systems and $8 \mathrm{ppbV}$ in the p-xylene system, and even lower in the two TMB systems were detected. Considering the low concentrations detected, quinone formation probably does not constitute an important reaction pathway for degradation of aromatic compounds.

\subsubsection{Di-unsaturated 1,6-Dicarbonyls}

Trace amounts of $\mathrm{C}_{7}$ and $\mathrm{C}_{8}$ di-unsaturated 1,6-dicarbonyls were tentatively identified in both the indoor TBR and the outdoor chamber experiments of toluene and xylenes.

$\mathrm{C}_{7}$ di-unsaturated-1,6-dicarbonyl<smiles>CC(C=O)=CC=CC=O</smiles>

$\mathrm{C}_{8}$ di-unsaturated-1,6-dicarbonyl<smiles>CC(=O)C=CC(C)=CC=O</smiles>

$\mathrm{C}_{9}$ di-unsaturated-1,6-dicarbonyl<smiles>CC(=O)C=C(C)C=CC=O</smiles>

Although $C_{9}$ di-unsaturated 1,6-dicarbonyls were not detected in the outdoor experiments of TBRs, they were observed in the TBR experiments. This discrepancy is most likely explained by the fact that much higher initial concentrations of the TBRs used in the TBR, producing the intermediates in a quantity sufficient to be observed.

Klotz et al [11] studied the photooxidation chemistry of a $C_{6}$ di-unsaturated 1,6- 
dicarbonyls, 2,4-hexadienedial, and a $\mathrm{C}_{7}$ 2-methyl-2,4-hexadienedial. Their results indicated that the di-unsaturated dicarbonyls are extremely reactive with $\mathrm{OH}$ radicals, having a reaction rate of about $1 \times 10^{-10} \mathrm{~cm}^{3}$-molecules ${ }^{-1}-\mathrm{s}^{-1}$, a rate similar to that of isoprene with $\mathrm{OH}$. Thus, the low concentrations of di-unsaturated dicarbonyls likely are the results of their strong reactivities.

\subsubsection{Unsaturated 1,4-Dicarbonyls}

Three 1,4-unsaturated dicarbonyls-butendial, 4-oxo-2-pentenal, and 3-hexene-2,5-dionewere synthesized by oxidizing furan, 2-methyl furan, and 2,5-dimethyl furan through reaction with $\mathrm{OH}$ radicals in the TBR [3]. The formation of butendial from the reaction of $\mathrm{OH}$ radicals with furan is proposed as follows:

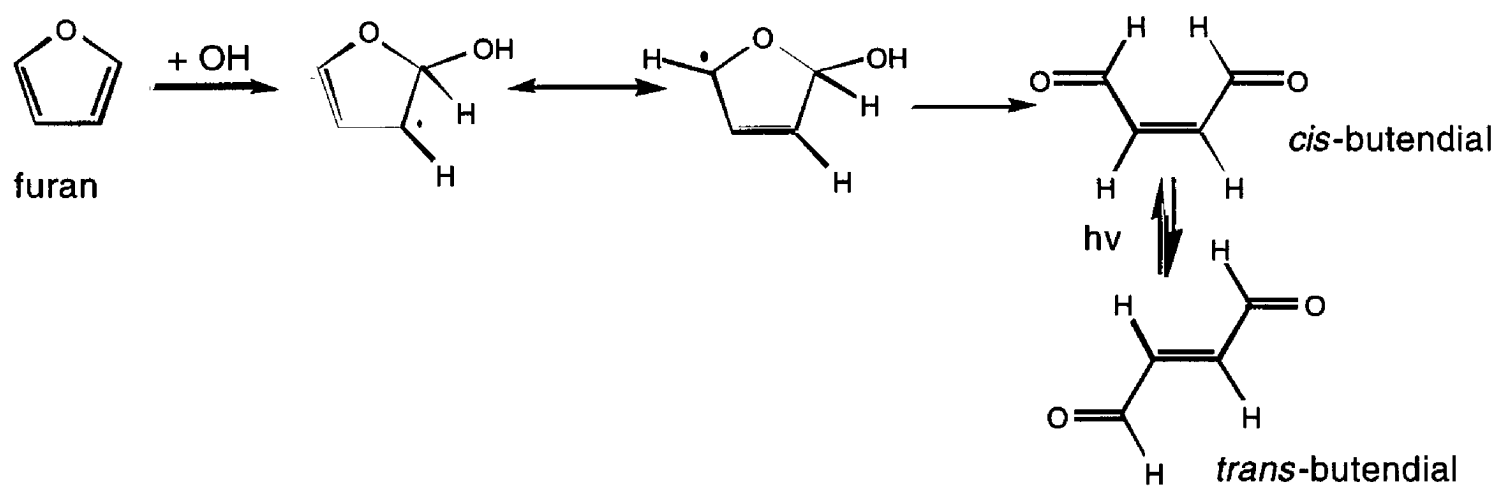

In a similar way, 4-oxo-2-pentenal and 3-hexene-2,5-dione are formed from 2-methyl furan and 2,5-dimethylfuran:

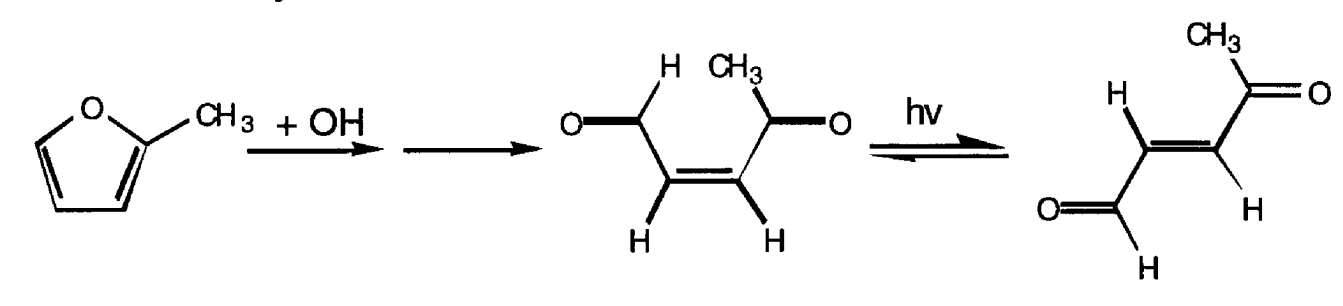

2-methyl furan

cis-4-oxo-2-pentenal

trans-4-oxo-2-pentenal

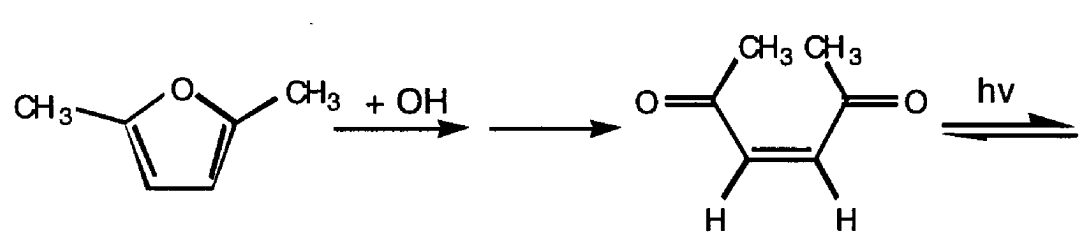

2,5-dimethyl furan

cis-3-hexen-2,5-dione<smiles>CC(=O)/C=C/C(C)=O</smiles>

trans-3-hexene-2,5-dione

In the TBR experiments, UV light was present to provide $\mathrm{OH}$ radicals from photolysis of $\mathrm{H}_{2} \mathrm{O}_{2}$. Studies by Bierbach et al. [3] and Tuazon et al. [12] showed that unsaturated 1,4-dicarbonyls undergo photoisomerization between cis- and trans-isomers; therefore, the 
resulting products are a mixture of cis- and trans-isomers. In this research, no attempt was made to differentiate among these. The PFBHA derivatives of butendial isomers show four peaks with retention times of $28.86,29.24,29.33$, and $29.61 \mathrm{~min}$. The PFBHA oximes of 4-oxo-2-pentenal isomers show five peaks at retention times $28.68,29.18,29.61,29.80$, and 30.23 min. Five peaks were observed to represent the PFBHA derivatives of 3-hexene-2,5dione isomers under the analytical conditions used for this work. The mass spectra of the isomers for each compound are similar, and hence only one spectrum for each carbonyl is shown in Figure 6.16.

\subsubsection{Unsaturated 1,4-dicarbonyls}

By matching retention times and mass spectra with the synthesized standard, butendial was positively identified as a product in the toluene and $o$-xylene photooxidation experiments, as was 4-oxo-2-pentenal in the toluene, o-xylene and m-xylene experiments, and as was 3hexene-2,5-dione in the p-xylene and 1, 2, 4TMB experiment (see Figure 6.16). The common product butendial from toluene and o-xylene reflects the common structure moiety among the three compounds, i.e., four adjacent unsubstituted sites on the benzene ring. Similarly, one methyl group and three adjacent unsubstituted positions common to toluene, m-xylene, and o-xylene, leads to the formation of 4-oxo-2-pentenal in those experiments. The formation of 3-hexene-2,5-dione in p-xylene and 1,2, 4TMB experiments results from another common moiety: two methyl groups in para-position with two unsubstituted positions between.

The time series of butendial, 4-oxo-2-pentenal and 3-hexene-2,5-dione indicate that they quickly react after their formation and likely are primary products since their concentration maxima were early in the morning (Figures 6.17 to 6.22). The reaction with $\mathrm{OH}$ radicals of these unsaturated dicarbonyls are about one half the rate of $\mathrm{OH}$-isoprene reaction [3], which explains their rapid loss.

In the 1, 2, 4TMB photooxidation, two peaks (f40 and f45), with a MW of 516 matching the PFBHA derivatives of $\mathrm{C}_{7}$ unsaturated dicarbonyls, were detected. These are most probably 3-methyl-3-hexene-2,5-dione.

Six $\mathrm{C}_{6}$ unsaturated dicarbonyl peaks at retention times of 27.98, 29.05, 29.33, 30.73, 33.23 , and $34.76 \mathrm{~min}(\mathrm{t} 35, \mathrm{t} 40, \mathrm{t} 41, \mathrm{t} 42, \mathrm{t} 43$, and $\mathrm{t} 47)$ were observed in the $1,3,5 \mathrm{TMB}$ experiments. The six carbonyl peaks exhibit three types of time series pattern (Figure 6.22). Peaks at $27.98,29.33$, and $30.73 \mathrm{~min}(\mathrm{t} 35, \mathrm{t} 41$, and $\mathrm{t} 42)$ have an early maximum near 0830 EDT, with the peak at $30.73 \mathrm{~min}$ being the strongest and the peak at $27.98 \mathrm{~min}$ the weakest among the three. Carbonyl peaks eluting at 33.23 and $34.78 \mathrm{~min}$ ( $\mathrm{t} 43$ and $\mathrm{t} 47$ ) have a later maximum near 1130 EDT and do not drop as precipitously as the previous three. The peak at $29.05 \mathrm{~min}$ (t40) continues to accumulate over time and no decline is seen during the entire experimental duration. Of these, the peak at $34.78 \mathrm{~min}$ is more than five times stronger than the others.

Four of the above six $\mathrm{C}_{6}$ unsaturated carbonyls (excluding the two weak peaks at 27.98 and $29.05 \mathrm{~min}$ ) were also observed in the $\mathrm{m}$-xylene $(\mathrm{m} 60, \mathrm{~m} 69, \mathrm{~m} 75$, and $\mathrm{m} 78)$ and 

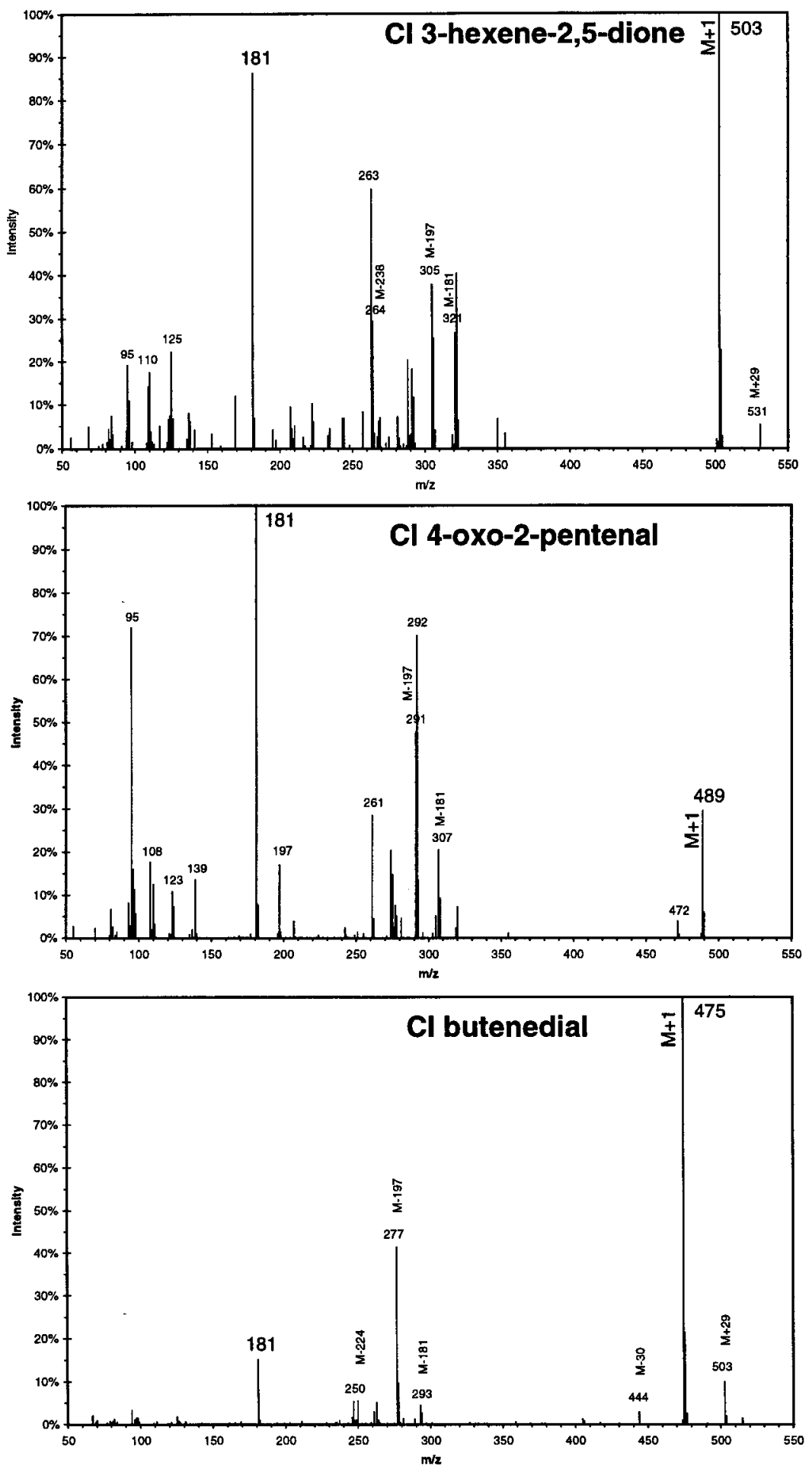

Figure 6.16:. Ion trap methane CI spectra of the PFBHA derivatives of butendial (bottom), 4-oxo-2pentenal (middle), and 3-hexene-2,5-dione (top). 
$1,2,4$ TMB (f44, f46, and f50) experiments. This observation is in agreement with the fact that these three aromatics species possess a common structure, two methyl groups in the meta-position. In the m-xylene and 1,2, 4TMB experiments, the absence of the two peaks is likely caused by lower concentrations resulting from the lower yields of this carbonyl. The similar time series patterns were observed (see Figures 6.19 and 6.21). The time series of two peaks at 29.33 and $30.73 \mathrm{~min}$ (f44) in the 1, 2, 4TMB experiment are not shown because the 30.73 min peak coeluted with 3-hexene-2,5-dione, a major product from the 1,2, 4TMB system and the 29.33 min peak is very small.

Considering the common moiety in m-xylene, 1, 2, 4TMB, and 1, 3, 5TMB, the six $\mathrm{C}_{6}$ unsaturated dicarbonyl peaks most likely represent 2-methyl-4-oxo-2-pentenal.

A common set of five $C_{5}$ unsaturated dicarbonyl peaks at retention times of 29.66 , $30.36,30.71,34.59$, and 36.30 min were observed in the p-xylene (p42, p43, p44 and p45), $\mathrm{m}$-xylene (m63, m67, m69, m77, and $\mathrm{m} 79$ ), and 1, 2, 4TMB (f41, f43, f44, f49, and f51) experiments. These peaks probably represent 2-methyl-butendial, formed as a result of the common structure: a methyl group with three adjacent unsubstituted sites on the benzene ring, two on one side of methyl group and one on another side. Two types of time series were observed, peaks at 29.66, 30.36 and 30.71 min peaking in the morning and peaks at 34.58 and $36.36 \mathrm{~min}$ peaking in the afternoon (see Figures 6.18 and 6.21, labeled as $\mathrm{m} 488$ u1). The $29.66 \mathrm{~min}$ peak coeluted with one of the 4-oxo-2-pentenal peaks in the m-xylene experiment; the $30.71 \mathrm{~min}$ peak coeluted with one of the 3-hexene-2,5-dione peaks. 

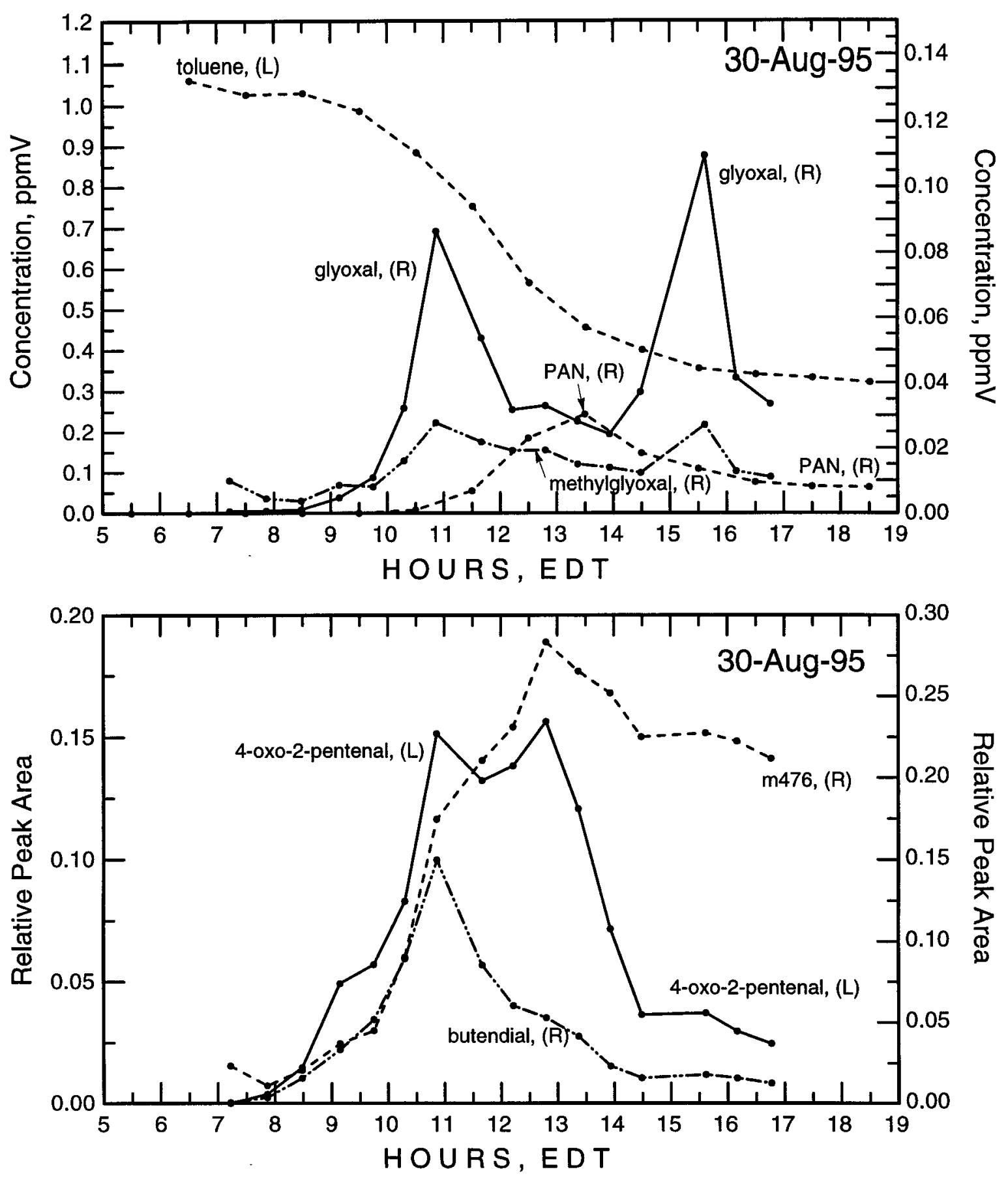

Figure 6.17:. Time series of unsaturated 1,4-dicarbonyls, saturated dicarbonyls, $\mathrm{CH}_{3} \mathrm{C}(\mathrm{O}) \mathrm{O}_{2} \mathrm{NO}_{2}$ and $\mathrm{a} \mathrm{C}_{3}$ trione (or $\mathrm{C}_{4}$ saturated dicarbonyl, labeled $\mathrm{m} 476$ ) in the toluene outdoor chamber experiment. 

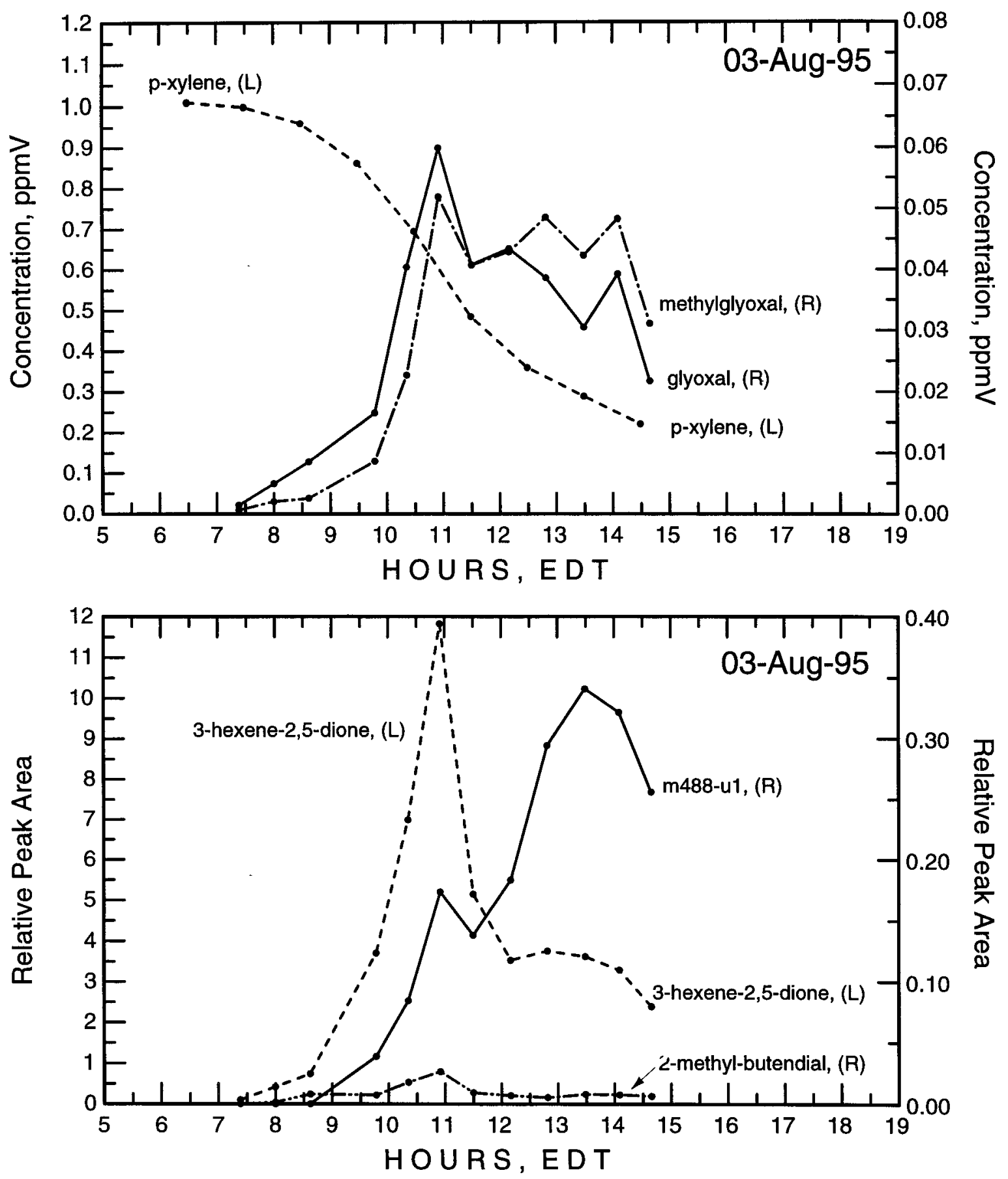

Figure 6.18:. Time series of unsaturated dicarbonyls and saturated dicarbonyls in the p-xylene outdoor chamber experiment. 

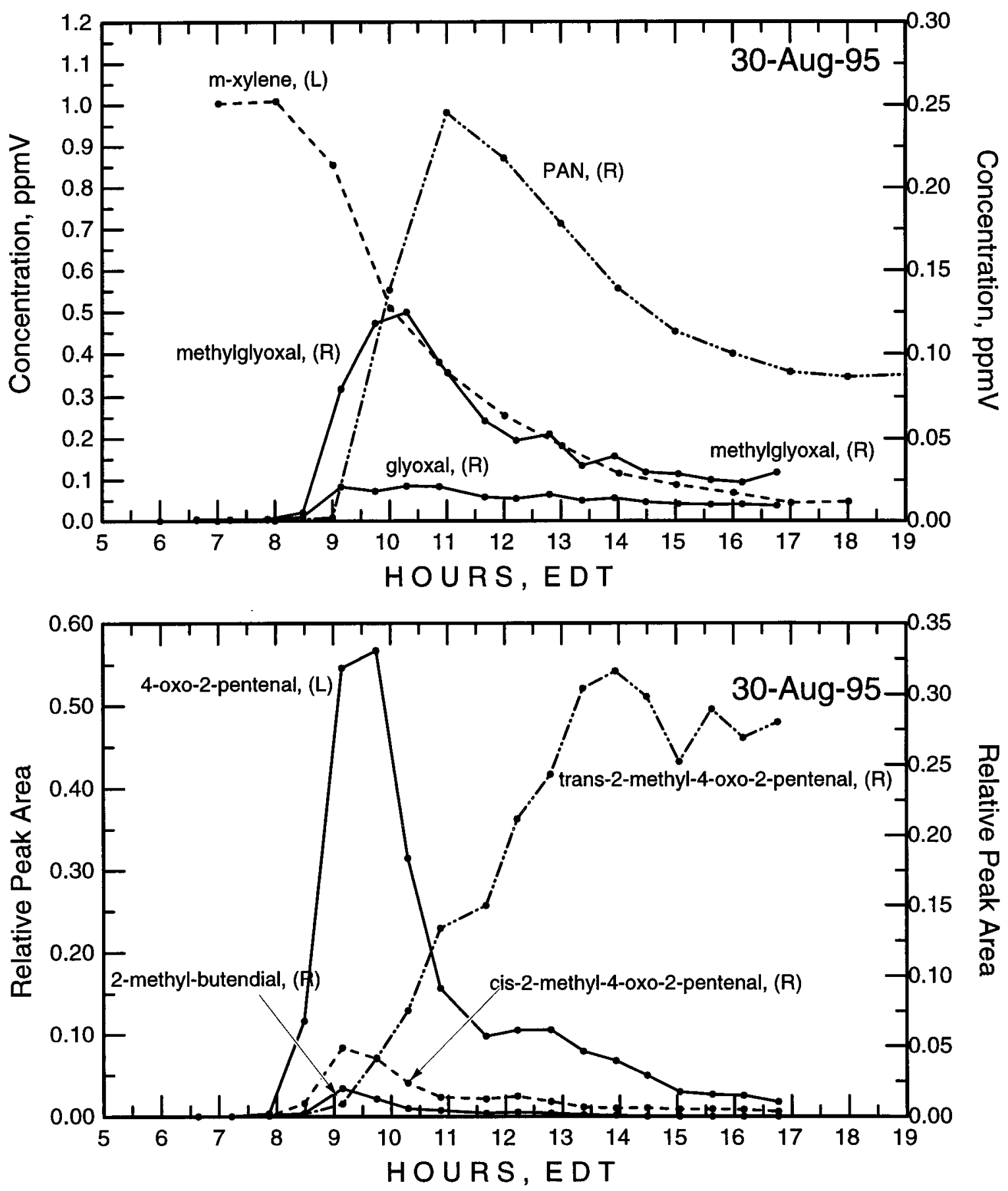

Figure 6.19:. Time series of unsaturated dicarbonyls, saturated dicarbonyls, and $\mathrm{CH}_{3} \mathrm{C}(\mathrm{O}) \mathrm{O}_{2} \mathrm{NO}_{2}$ in the m-xylene outdoor chamber experiment. 

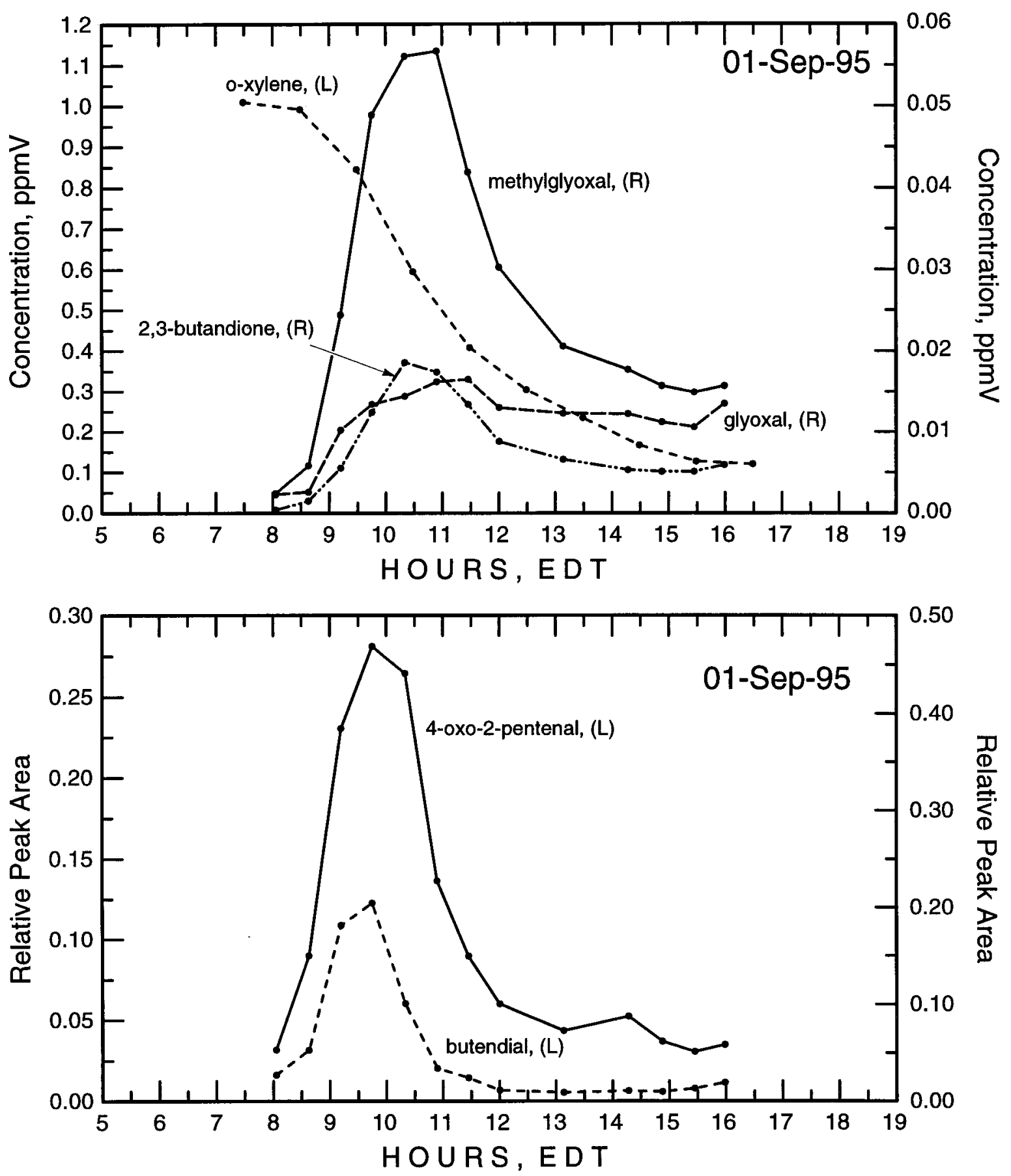

Figure 6.20:. Time series of unsaturated dicarbonyls and saturated dicarbonyls in the o-xylene outdoor chamber experiment. 

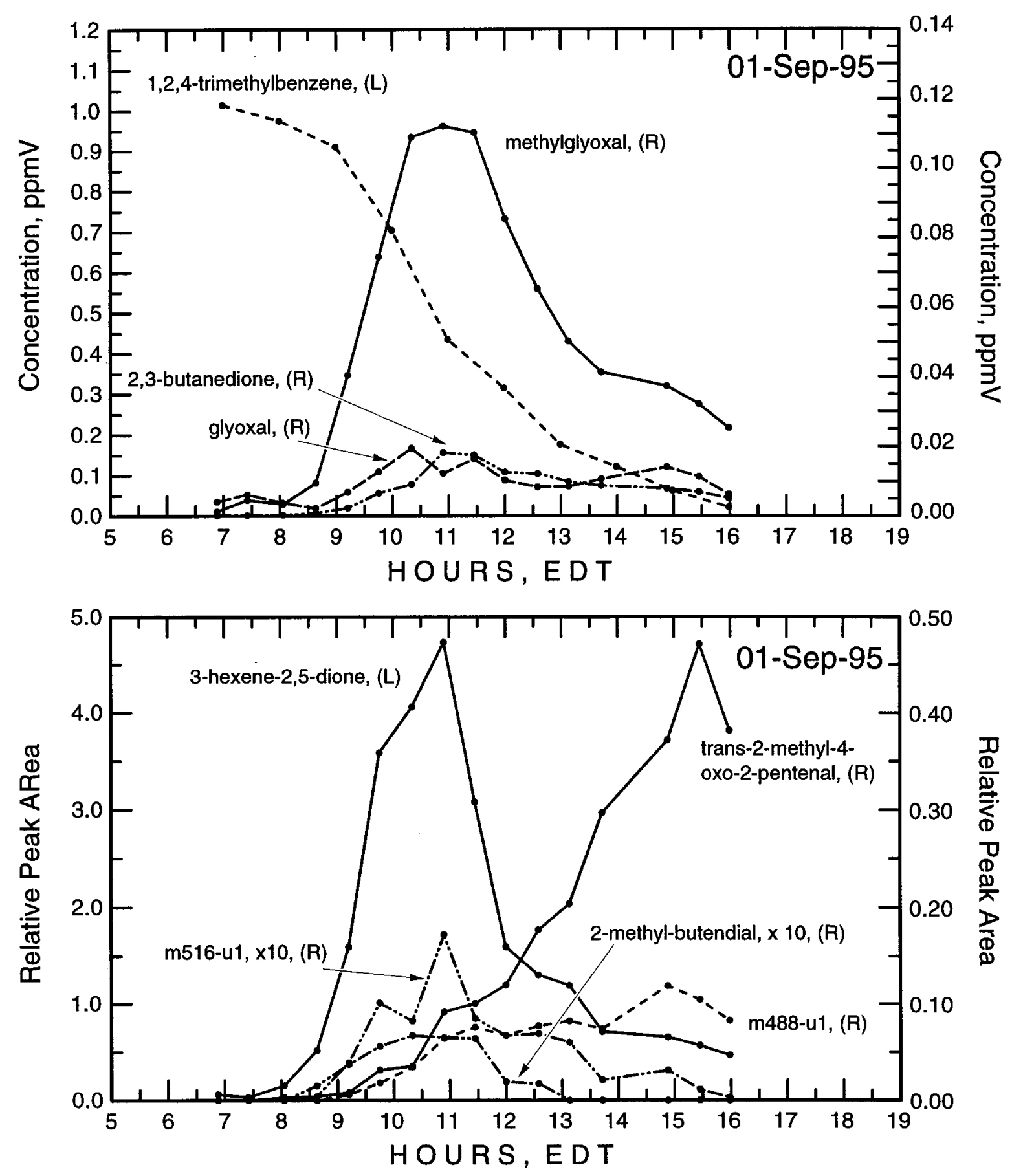

Figure 6.21:. Time series of unsaturated dicarbonyls and saturated dicarbonyls in the 1,2,4 TMB outdoor chamber experiment. 

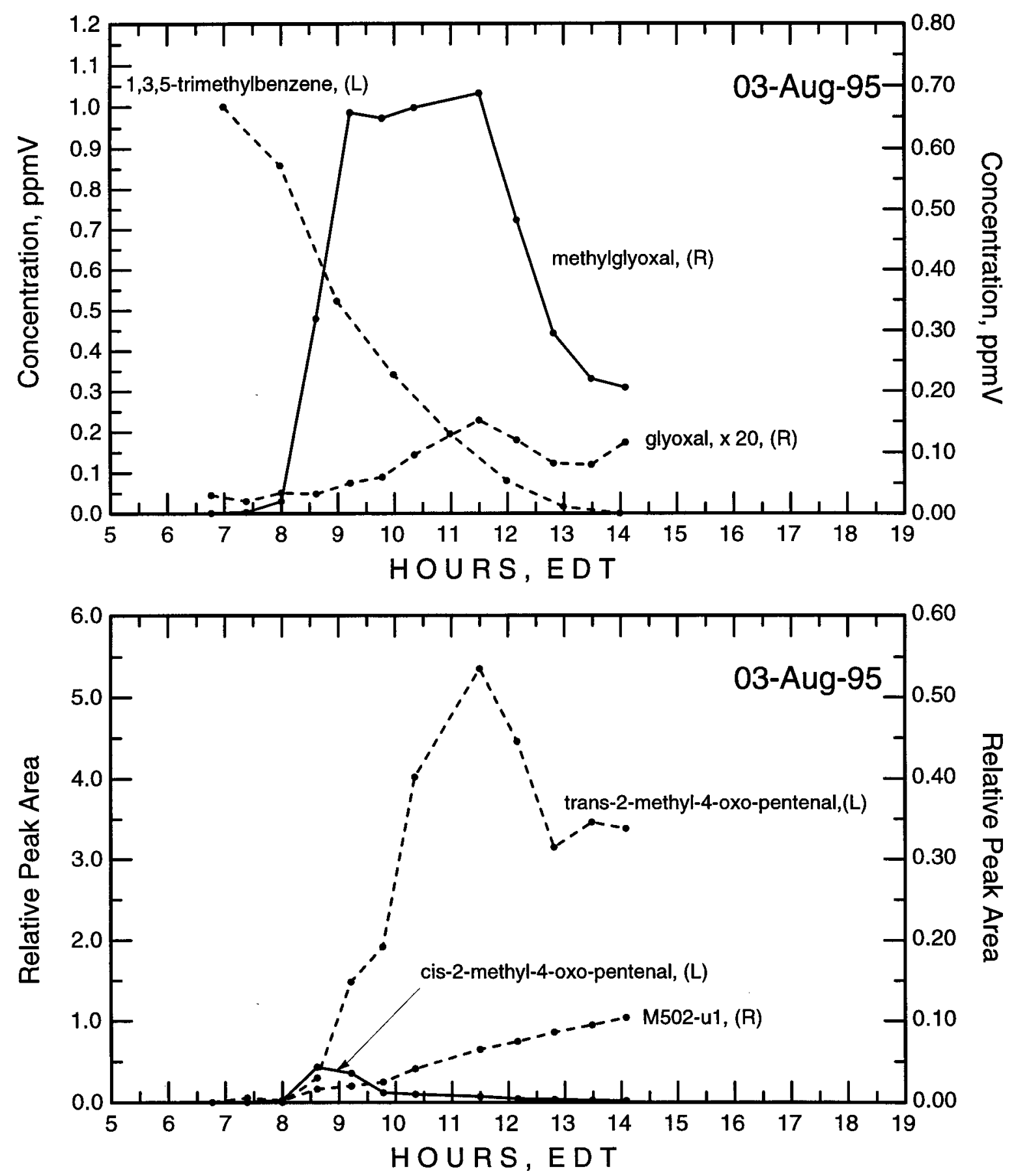

Figure 6.22:. Time series of unsaturated dicarbonyls and saturated dicarbonyls in the 1,3,5 TMB outdoor chamber experiment. 


\subsubsection{Saturated Dicarbonyls}

Glyoxal and methylglyoxal were observed as products in every aromatic system.

glyoxal<smiles>O=CC=O</smiles>

methylglyoxal<smiles>CC(=O)C=O</smiles>

2,3-butanedione<smiles>CC(=O)C(C)=O</smiles>

$1,3,5 T M B$ produced the largest concentration of methylglyoxal $(678 \mathrm{ppbV})$ and toluene produced the least $(27.3 \mathrm{ppbV})$. The largest concentration of glyoxal (108 ppbV) was seen in the toluene system; the lowest $(7.5 \mathrm{ppbV})$ was observed in the $1,3,5 \mathrm{TMB}$ system. The time series of glyoxal and methylglyoxal are shown in Figures 6.17 to 6.22 . In the toluene experiment, double maxima were observed for glyoxal and methylglyoxal, indicating they are both primarily and secondary products or that they are secondary products for both fast and slow reaction intermediates.

Biacetyl or 2,3-butanedione was identified as a product from the o-xylene and 1, 2, 4TMB systems, as expected because of the presence of two adjacent methyl groups. A maximum concentration of $18 \mathrm{ppbV}$ was detected in both systems. The time series of 2,3-butandione showed similar pattern to those of methylglyoxal in the o-xylene and 1,2,4TMB systems (Figures 6.20 and 6.21).

\subsubsection{Hydroxy Dicarbonyls}

Three different hydroxy dicarbonyls were observed:

$\mathrm{C}_{3}$ hydroxy dicarbonyl<smiles>O=CC(O)C=O</smiles>

$\mathrm{C}_{4}$ hydroxy dicarbonyl<smiles>CC(=O)C(O)C=O</smiles>

$\mathrm{C}_{5}$ hydroxy dicarbonyl<smiles>CC(=O)C(O)C(C)=O</smiles>

A common set of four peaks at $27.61,27.82,28.44$, and 28.78 min with MW 506 
were present in the m-xylene (m50, m51, m55, and $\mathrm{m} 57) ; 1,3,5 \mathrm{TMB}(\mathrm{t} 33, \mathrm{t} 34, \mathrm{t} 38$, and $\mathrm{t} 39$ ); and 1, 2, 4TMB (f31, f32, f36, and f38) experiments. Two of the above MW 506 peaks at 27.61 and $27.82 \mathrm{~min}$ (o50 and 051 ) were detected in the o-xylene experiment. The two peaks at 28.44 and $28.78 \mathrm{~min}$ ( $\mathrm{t} 34$ and $\mathrm{t} 39$ ) showed similar CI mass spectra (see Figure 6.23). The presence of a strong $\mathrm{m} / \mathrm{z}=489$ ion, resulting from the loss of $-\mathrm{OH}$ from neutral molecule, signifies the presence of this group in the carbonyl. Coupled with the molecular weight information, these two peaks can be identified as $\mathrm{C}_{5}$ hydroxy dicarbonyls. The strong $\mathrm{m} / \mathrm{z}=268$ ion, resulting from the loss of the moiety $\mathrm{C}_{6} \mathrm{~F}_{5} \mathrm{CH}_{2} \mathrm{ON}=\mathrm{CCH}_{3}$ from the neutral molecule, indicates the presence of a methyl group adjacent to the carbonyl group. These two peaks likely belong to the PFBHA derivative of the hydroxy dicarbonyl 3-hydroxy-2,4-pentanedione.

The sum of the relative peak areas for the two peaks at 28.44 and 28.79 min was plotted in Figure 6.24 and labeled as m506-u1. These two peaks peaked early in the morning followed by a rapid decline, indicating either that they are highly reactive with $\mathrm{OH}$ radicals or that they undergo photolysis rapidly.

The peak at 27.61 min shows a CI spectrum similar to the peaks at 28.44 and 28.78 min and its time series showed an accumulating trend followed by slight decline or leveling off (labeled as m506-u2 in Figure 6.24).

The peak at 27.82 min shows a different CI mass spectrum from those at TBR28.44 and TBR28.78 $\mathrm{min}$ (see Figure 6.23). The strong ion at $\mathrm{m} / \mathrm{z}=282$, resulting from the loss of $\mathrm{C}_{6} \mathrm{~F}_{5} \mathrm{CH}_{2} \mathrm{ON}=\mathrm{CH}$ from the neutral molecules, indicates the presence of an aldehyde carbonyl functionality. A strong ion at $\mathrm{m} / \mathrm{z}=268$ is also present, indicating a methyl group adjacent to the second carbonyl group. This peak could possibly be one of the PFBHA derivatives of the following dicarbonyls:<smiles>CC(=O)C(C)(O)C=O</smiles><smiles>CC(=O)C(O)CC=O</smiles><smiles>CC(=O)CC(O)C=O</smiles>

Distinguishing further among the three possibilities is not possible based on the CI spectra. The time series of this peak (labeled m506-u3 in Figure 6.24) in o-xylene and $1,2,4 \mathrm{TMB}$ experiments showed the concentration accumulates and then levels off. A decline, however, was observed of this peak in the 1,3,5TMB experiment after reaching the maximum (Figure 6.24). This peak coeluted with two other peaks in the m-xylene experiment, therefore, its time series was not obtained.

$\mathrm{C}_{4}$ hydroxy dicarbonyls having a MW of 492 for the PFBHA derivatives were observed for every aromatic compound examined. A common set of 7 peaks at 27.06, 27.28, 27.43, $28.09,28.36,28.53$, and 29.04 min were present in the toluene, p-xylene, m-xylene and 1, 2, 4TMB photooxidation experiments. Four of these seven peaks-27.06, 27.41, 28.08 and $29.01 \mathrm{~min}$ - were also detected in the o-xylene photooxidation experiment. Five of the above peaks at $27.29,27.43,28.35,28.55$ and 29.04 min were observed in the $1,3,5$ TMB 
experiment. These $\mathrm{C}_{4}$ hydroxy dicarbonyls were often weak and some of them coelute with another carbonyl products. The typical time series for $\mathrm{C}_{4}$ hydroxy dicarbonyls showed a gradual increase followed by leveling off (Figure 6.24).

Two $C_{3}$ hydroxy dicarbonyl peaks at 28.33 (o53) and 28.61 min were observed in the o-xylene and toluene experiment. because of their low intensity, not all the above peaks were labeled. 

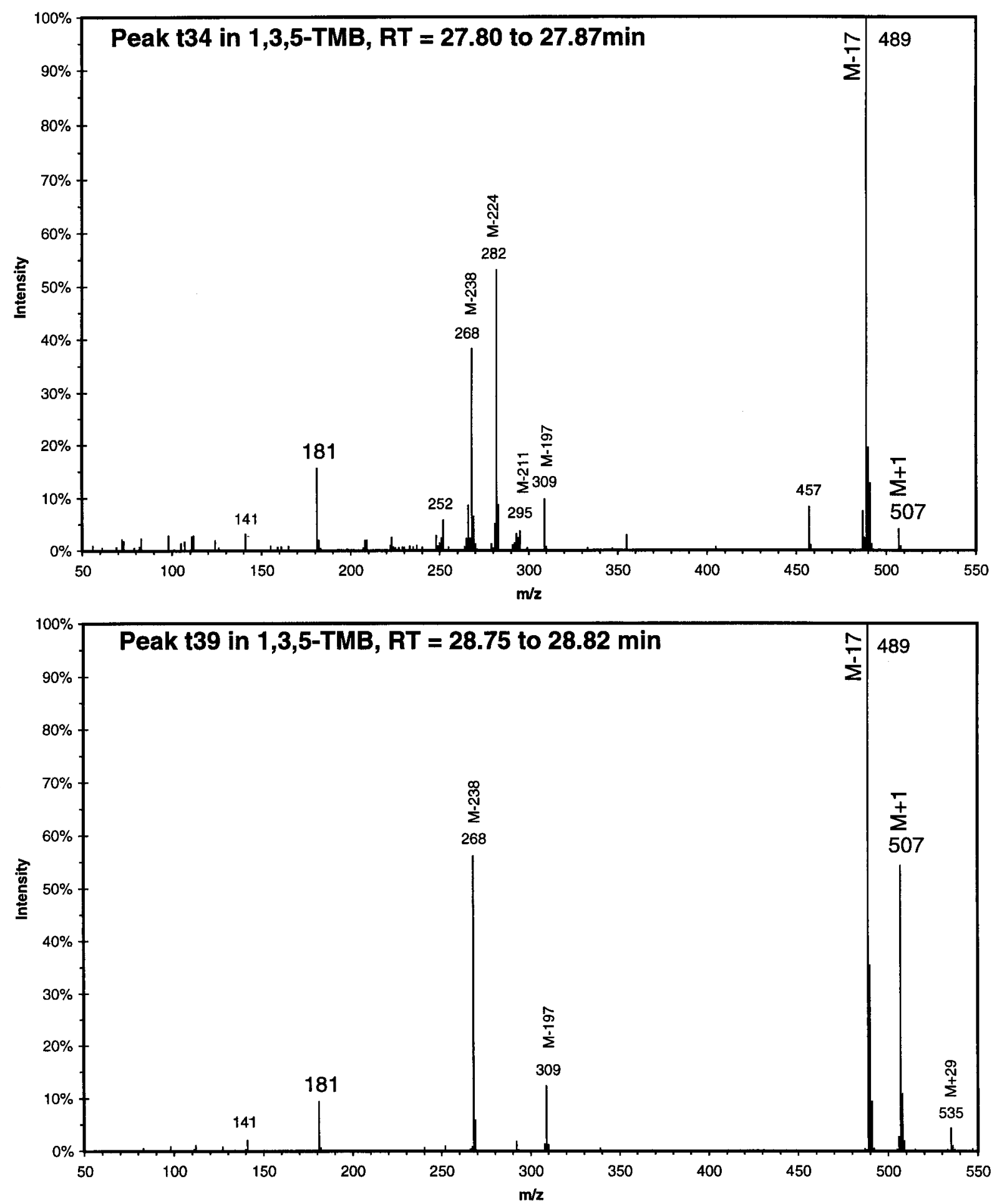

Figure 6.23:. Ion trap CI mass of the PFBHA derivatives of two $C_{5}$ hydroxy dicarbonyls.Top: peak at $27.82 \mathrm{~min}$, Bottom: peak at $28.78 \mathrm{~min}$. 

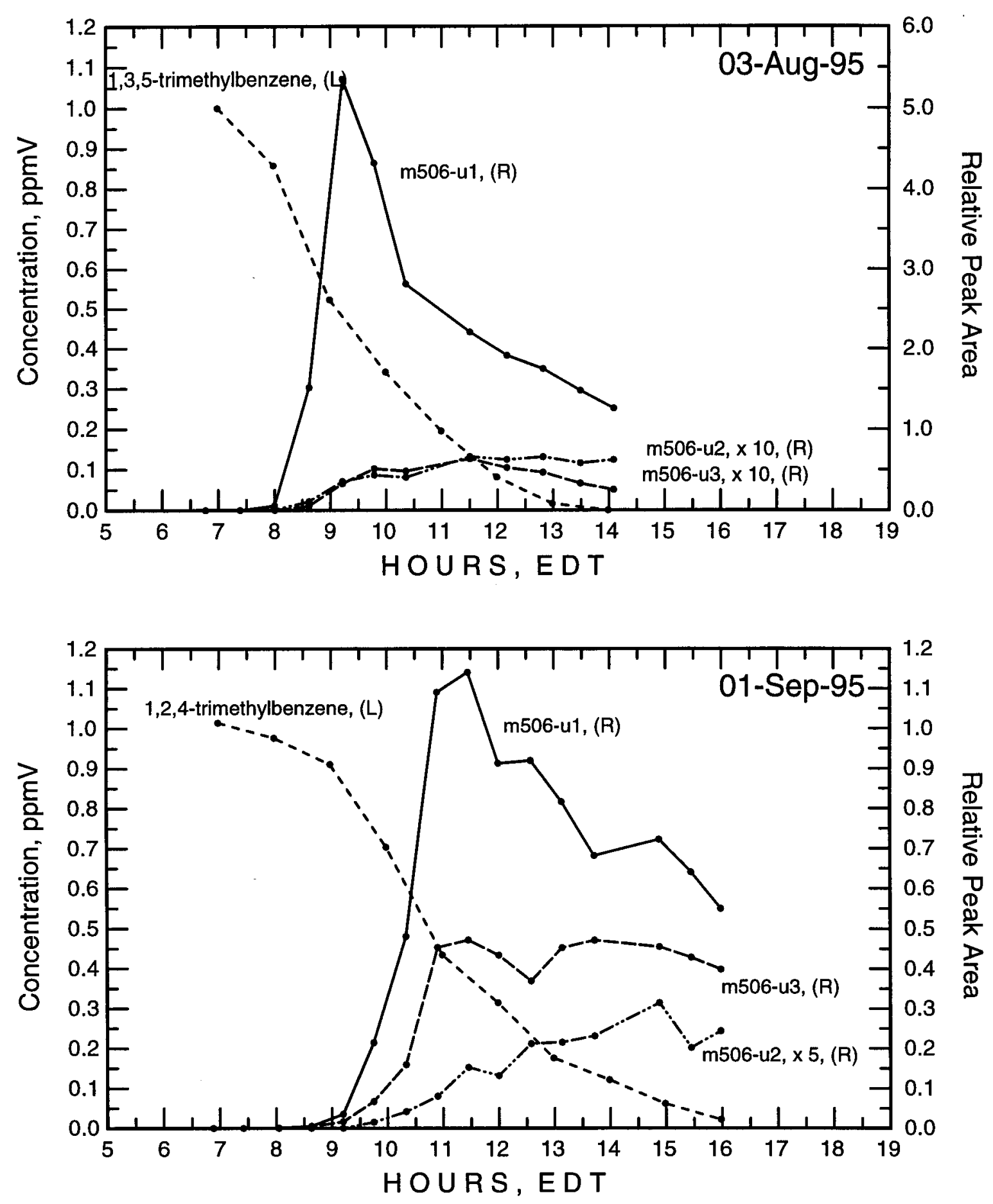

Figure 6.24:. Time series of $c_{5}$ hydroxy dicarbonyl peaks in the 1,3,5 TMB and 1,2,4 TMB experiments. m506-u1: sum of two peaks at 28.44 and $28.79 \mathrm{~min}$; m506-u2: the peak at $27.61 \mathrm{~min} ; \mathrm{m} 506-\mathrm{u} 3$ : the peak at $27.82 \mathrm{~min}$. 


\subsubsection{Triones}

There is evidence for the detection of a series of triones:

$\mathrm{C}_{3}$ trione<smiles>O=CC(=O)C=O</smiles>

$\mathrm{C}_{4}$ trione<smiles>CC(=O)C(=O)C=O</smiles>

$\mathrm{C}_{5}$ trione<smiles>CC(=O)C(=O)C(C)=O</smiles>

Three carbonyl peaks with a MW of 504 at 26.15, 27.16, and 28.13 min were detected in the m-xylene (m40, m47, and m53); 1, 3, 5TMB ( $\mathrm{t} 27, \mathrm{t} 31$ and $\mathrm{t} 36$ ); and 1, 2, 4TMB (f23, f28 and f34) photooxidation experiments. In these, the $26.15 \mathrm{~min}$ peak was the strongest, and the 28.13 min peak the weakest. This common set of carbonyl peaks for three aromatic compounds coincides with the occurrence of a common set of $\mathrm{C}_{5}$ hydroxy dicarbonyl peaks and $C_{5}$ unsaturated dicarbonyl peaks, which tends to suggest a link between the three types of carbonyls. The time series of these three peaks in the 1,3,5TMB and 1, 2, 4TMB experiments showed a pattern of accumulation throughout the entire experimental period, indicating that the MW 504 carbonyls are not reactive (Figure 6.25).

There are three types of carbonyl compounds matching the MW of 504: $\mathrm{C}_{6}$ saturated dicarbonyls, $\mathrm{C}_{5}$ epoxy dicarbonyls, and $\mathrm{C}_{5}$ triones.

Carbonyl peaks with a MW of 490 were also detected in every aromatic system. Two common MW 490 peaks at 26.86 and 27.19 min were observed in the m-xylene ( $\mathrm{m} 45$ and $\mathrm{m} 46$ ); 1, 3, 5TMB (t30 and $\mathrm{t} 31$ ); and 1, 2, 4TMB (f26 and f28) experiments. One common MW 490 peak at 27.76 min was present in toluene (tol35) and every xylene experiment. Again, there are three types of carbonyl compounds matching the MW of 490: $\mathrm{C}_{5}$ saturated dicarbonyls, $\mathrm{C}_{4}$ epoxy dicarbonyls, and $\mathrm{C}_{4}$ triones.

Two common peaks with a MW of 476 at 26.51 and 27.08 min were present in each aromatic experiment. The PFBHA derivative of $\mathrm{a}_{4}$ saturated dicarbonyl, or the di-derivative of a $\mathrm{C}_{3}$ trione, would each have a MW of 476 . The possibility of 2,3-butandione was ruled out because 2,3-butandione shows three peaks at 24.30,25.25, and $26.54 \mathrm{~min}$, and the first two peaks were not observed in the toluene, p-xylene, $\mathrm{m}$-xylene, and 1, 3, 5TMB experiments. In the o-xylene and the 1, 2, 4TMB experiments, 2,3-butandione was a product, and all three peaks were observed. Another $\mathrm{C}_{4}$ saturated dicarbonyl, 2-oxo-butanal which elutes at 26.04, 26.15 , and $26.28 \mathrm{~min}$, can also be ruled out based on a retention time mismatch. Therefore, the candidates for these two MW 476 peaks are reduced to the following:<smiles>O=CC(=O)C=O</smiles><smiles>CC(=O)CC=O</smiles><smiles>O=CCCCC=O</smiles> 
The time series of these two peaks in the toluene experiment are shown in Figure 6.17, indicating a slow reactivity in the smog chamber.

\subsubsection{Glycolaldehyde and Hydroxyacetone}

Glycolaldehyde and hydroxyacetone were observed in every aromatic system examined.

Glycolaldehyde

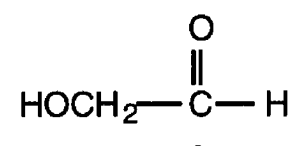

Hydroxyacetone<smiles>CC(=O)CO</smiles>

The 1, 3, 5TMB system produced the largest concentration of glycolaldehyde (up to 18 $\mathrm{ppbV}$ ) and $\mathrm{p}$-xylene system produced the most hydroxyacetone (up to $46 \mathrm{ppbV}$ ). The time series indicate that they are secondary products (see Figure 6.26). 

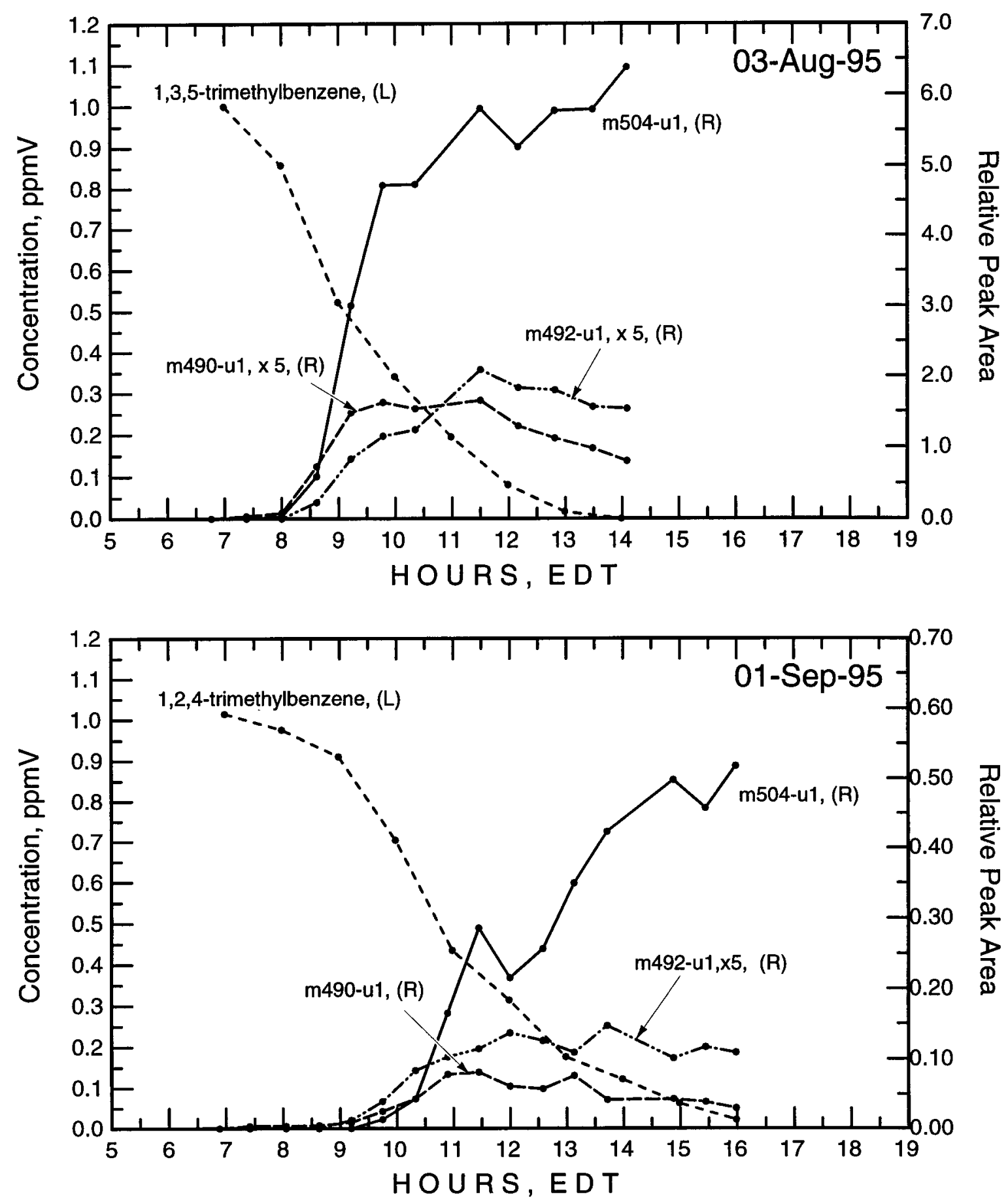

Figure 6.25:. Time series of a $C_{4}$ hydroxy dicarbonyl, $c_{5}$ triones (or $c_{5}$ epoxy carbonyls), and a $c_{4}$ trione (or a $\mathrm{C}_{4}$ epoxy dicarbonyl) peaks in the 1,3,5 TMB and 1,2,4 TMB experiments. m492-u1: a $\mathrm{C}_{4}$ hydroxy dicarbonyl at $27.31 \mathrm{~min}$; m504-u1: $\mathrm{C}_{5}$ triones or $\mathrm{C}_{5}$ epoxy dicarbonyls, sum of three peaks at 26.18, 27.17 and $28.09 \mathrm{~min}$; $\mathrm{m} 490-\mathrm{u} 1: \mathrm{a}_{4}$ trione or a $\mathrm{C}_{4}$ epoxy dicarbonyl at $26.86 \mathrm{~min}$. 

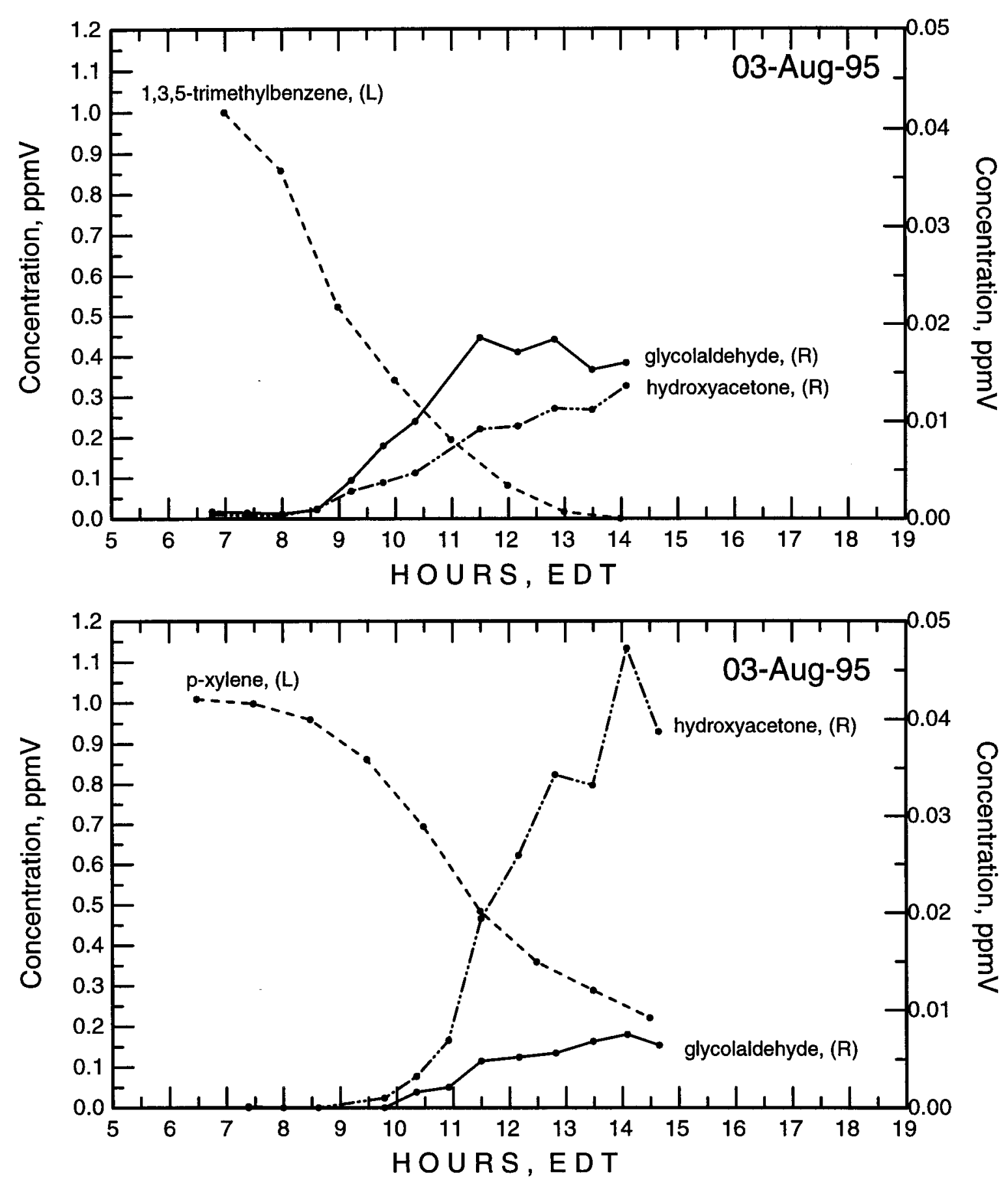

Figure 6.26:. Time series of glycolaldehyde and hydroxyacetone in the p-xylene and 1,3,5 TMB outdoor chamber experiments 


\subsubsection{Unknown Carbonyls}

Despite the long list of carbonyls described in this chapter, there are still some carbonyls that can not fit into the above categories. Table 6.19 lists several detected but unknown carbonyl products in the six alkylbenzene systems.

Table 6.19:

\section{List of Unknown Carbonyl Products}

\begin{tabular}{|l|l|l|l|l|}
\hline Aromatics & peak & RT (min) & $\mathrm{MW}^{\mathrm{a}}$ & $\mathrm{MW}^{\mathrm{b}}$ \\
\hline toluene & $\mathrm{o} 22$ & 24.95 & 337 & 142 \\
\hline m-xylene & $\mathrm{m} 35$ & 25.35 & 365 & 170 \\
\hline m-xylene & $\mathrm{m} 68^{\mathrm{c}}$ & 30.36 & 500 & 110 \\
\hline m-xylene & $\mathrm{m} 71$ & 31.29 & 500 & 110 \\
\hline m-xylene & $\mathrm{m} 72$ & 32.33 & 500 & 110 \\
\hline m-xylene & $\mathrm{m} 74$ & 32.86 & 542 & 152 \\
\hline m-xylene & $\mathrm{m} 76^{\mathrm{c}}$ & 33.73 & 542 & 152 \\
\hline o-xylene & $\mathrm{o} 48$ & 27.13 & 343 & 148 \\
\hline $1,3,5-T M B$ & $\mathrm{t} 46$ & 34.33 & 572 & 182 \\
\hline $1,2,4-$ TMB & $\mathrm{f} 12^{*}$ & 23.94 & 365 & 170 \\
\hline
\end{tabular}

${ }^{a}$ molecular weight of PFBHA-carbonyl derivative

"molecular weight of carbonyl

ccoelute with another peak 


\subsection{Epoxy Carbonyls}

Bartolotti and Edney [13] performed a series of density functional quantum mechanical calculations to identify potential intermediates following the $\mathrm{OH}$ addition to toluene. The calculations indicate that the epoxide structure $(\mathbf{I})$ is the most stable intermediate among the likely intermediates.<smiles>C[C@]12O[C@H]1C=C[C@H]([O])[C@H]2O</smiles>

The subsequent reactions of $I$ leads to the formation of three epoxy carbonyls (structures II, III, and IV); the abstraction of $\mathrm{H}$-atom by $\mathrm{O}_{2}$ yields a $\mathrm{C}_{7}$ unsaturated hydroxy epoxy cyclic carbonyl (II); the decomposition of $\mathbf{I}$ results in a $\mathrm{C}_{7}$ unsaturated epoxy dicarbonyl (IV); and, intermediate I may also undergo loss of $\mathrm{H}_{2}$ to form $\mathrm{a} \mathrm{C}_{7}$ unsaturated epoxy cyclic dicarbonyl (III).

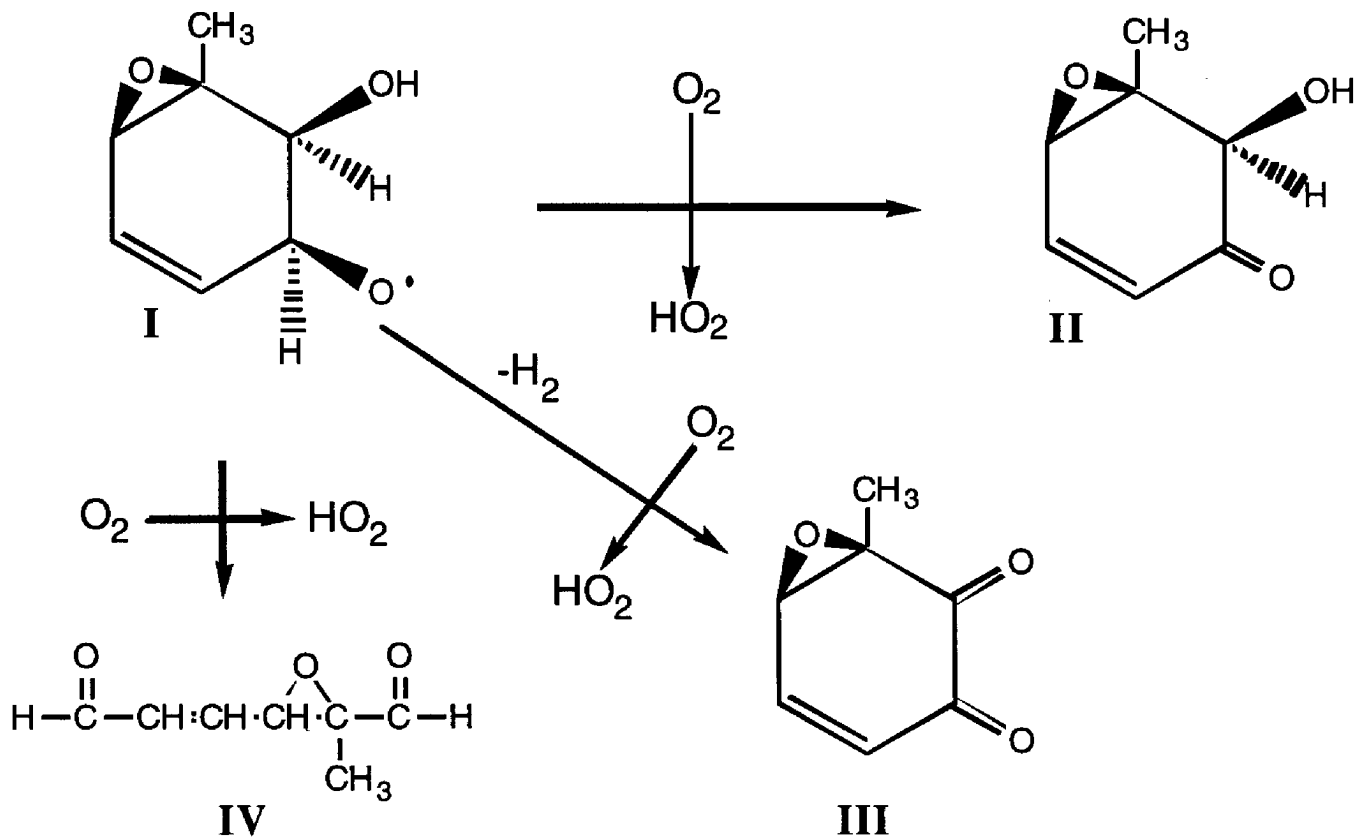

\subsubsection{Epoxide Toxicity}

If epoxide intermediates play a role in the $\mathrm{OH}$ oxidation of aromatic compounds, their possible toxicity will be a matter of some concern. An epoxy carbonyl similar in structure to structure IV, 2,3-epoxypropanal (glycidaldehyde) has been reported to be a potent mutagen 
in a variety of in vitro test system $[14,15]$ and has been shown to induce sarcomas in rats by subcutaneous injection. [16] As a bifunctional agent, 2,3-epoxy propanal is capable of forming cyclic adducts and crosslinks with DNA bases. [17] In general, the epoxy functional group can serve as electrophile to attack DNA and nucleosides [18-20]. It seems possible that the mutagenic activity of irradiated toluene/NOx mixtures reported by Shepson et al. [21] and by Shiraishi et al. [22] may have been because of the production of epoxide compounds. Hence, the possible formation of epoxides from atmospheric oxidation of aromatic compounds is a significant public health concern, deserving additional laboratory and field work.

\subsubsection{Theoretical Considerations}

Structure IV is expected to further react with $\mathrm{OH}$ radicals or ozone to form one epoxy dicarbonyl $(V)$ and one saturated dicarbonyl by the pathways shown in the reaction scheme above. In addition, IV can undergoes photolysis, one possible pathway being a Norrish Type I reaction, i.e., loss of CO to form an unsaturated epoxy carbonyl with one lower carbon number (Structure VI). The reactivity of the saturated epoxy dicarbonyl is at this point unknown.

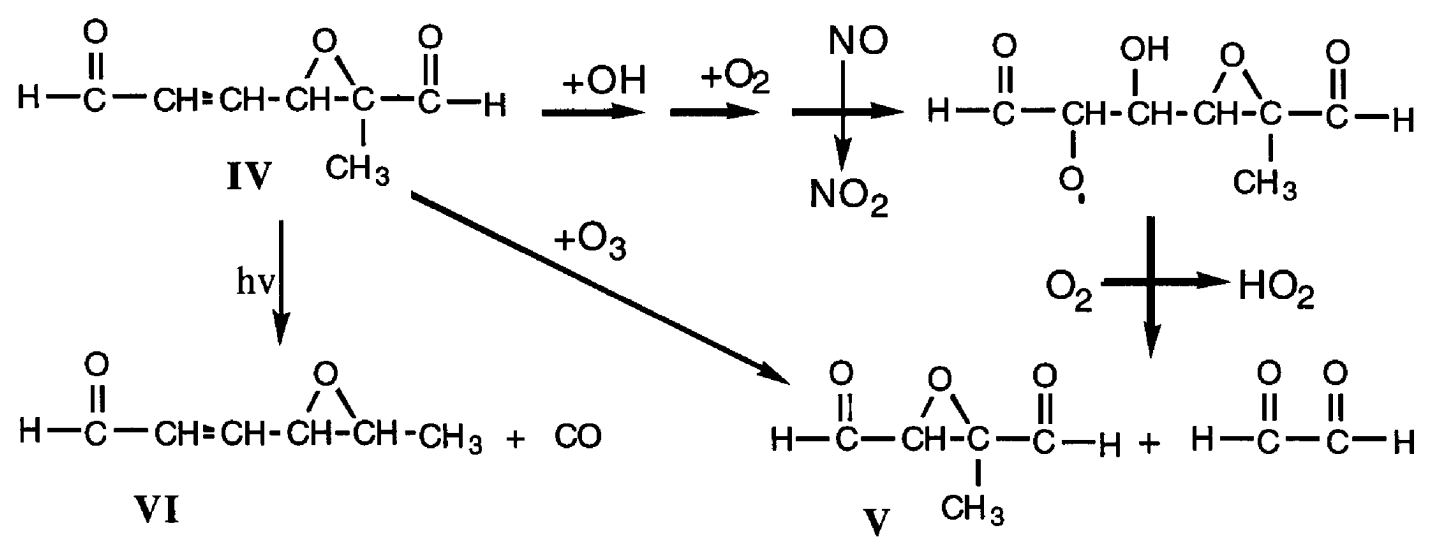

According to Bartolotti and Edney's calculations, structure VII has the second lowest energy of all the possible epoxide intermediates. When the energy barriers to the corresponding transition states are comparable, the formation of intermediate VII can be competitive with that of I. The subsequent reactions of structure VII will lead to carbonyl products (VIII, IX and X) different from those from Structure I. 


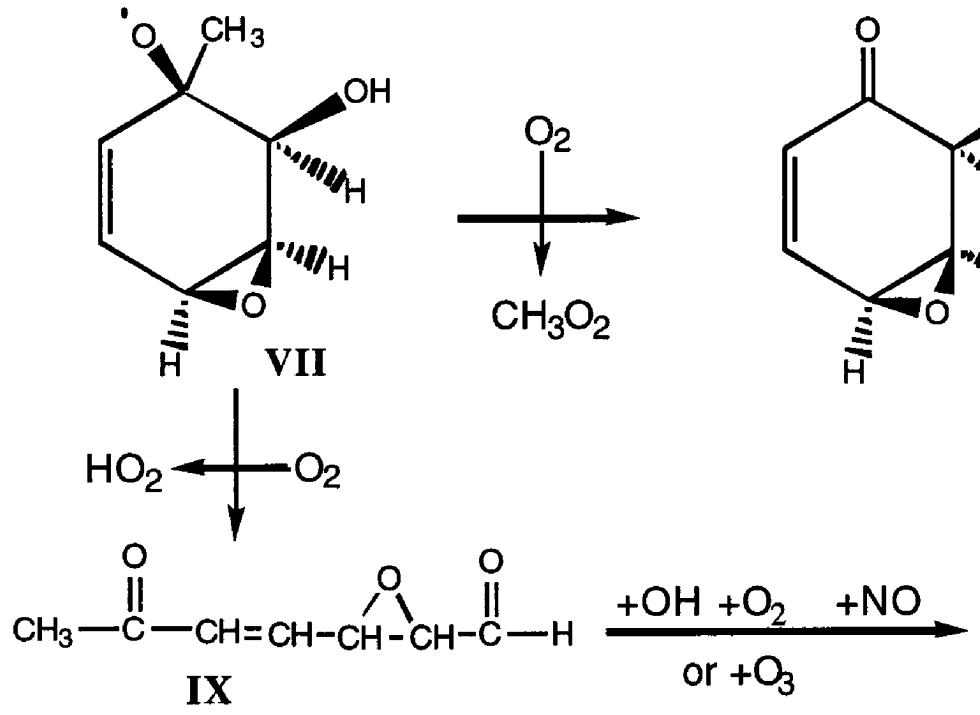

Similar epoxy carbonyls are also expected to form from $\mathrm{OH}$ oxidation of other aromatic compounds. Evidence supporting the formation of the intermediates I and VII will be discussed, based on the carbonyl products detected in the toluene, xylenes, and 1, 3, 5TMB experiments.

First, however, we must show that the PFBHA method is capable of detecting and identifying these epoxy carbonyls. Because none of the epoxy carbonyls described above are available commercially, we must conduct our demonstration using the few commercially available epoxy carbonyls and a few simple epoxy carbonyls that we can synthesize as model compounds.

\subsubsection{Model Epoxides}

Epoxides are known to hydrolyze slowly in water [20]. A commercially available epoxy carbonyl-1,2-epoxy-3-cyclohexanone-and three synthesized epoxy carbonyls-glycidaldehyde, 2-epoxy-3-butanone (methyl vinyl ketone epoxide), and 4,5-epoxy-pentanal-were employed to demonstrate that an epoxy carbonyl could survive the PFBHA derivatization procedure conducted in aqueous solution and be detected by GC/MS. The model epoxy carbonyls were successfully derivatized by PFBHA in water solution without loss of the epoxide functionality and all were detected by GC/MS. They generally elute later than the simple carbonyls, but earlier than the hydroxy carbonyls with the same carbon number. For example, glycidaldehyde eluted after the $\mathrm{C}_{3}$ simple carbonyls such as acetone, propanal and acrolein, but earlier than hydroxyacetone. Figure 6.27 illustrates the full spectrum of the PFBHA derivative of glycidaldehyde. The CI mass spectra patterns are similar to those simple carbonyls described in the paper by Yu et al. [6], showing strong $M+1$ and $M-197$ ions. The $M+181$ ion is often present to confirm the determination of molecular weight; however, there is not a particular ion characteristic of the epoxy functional group. 


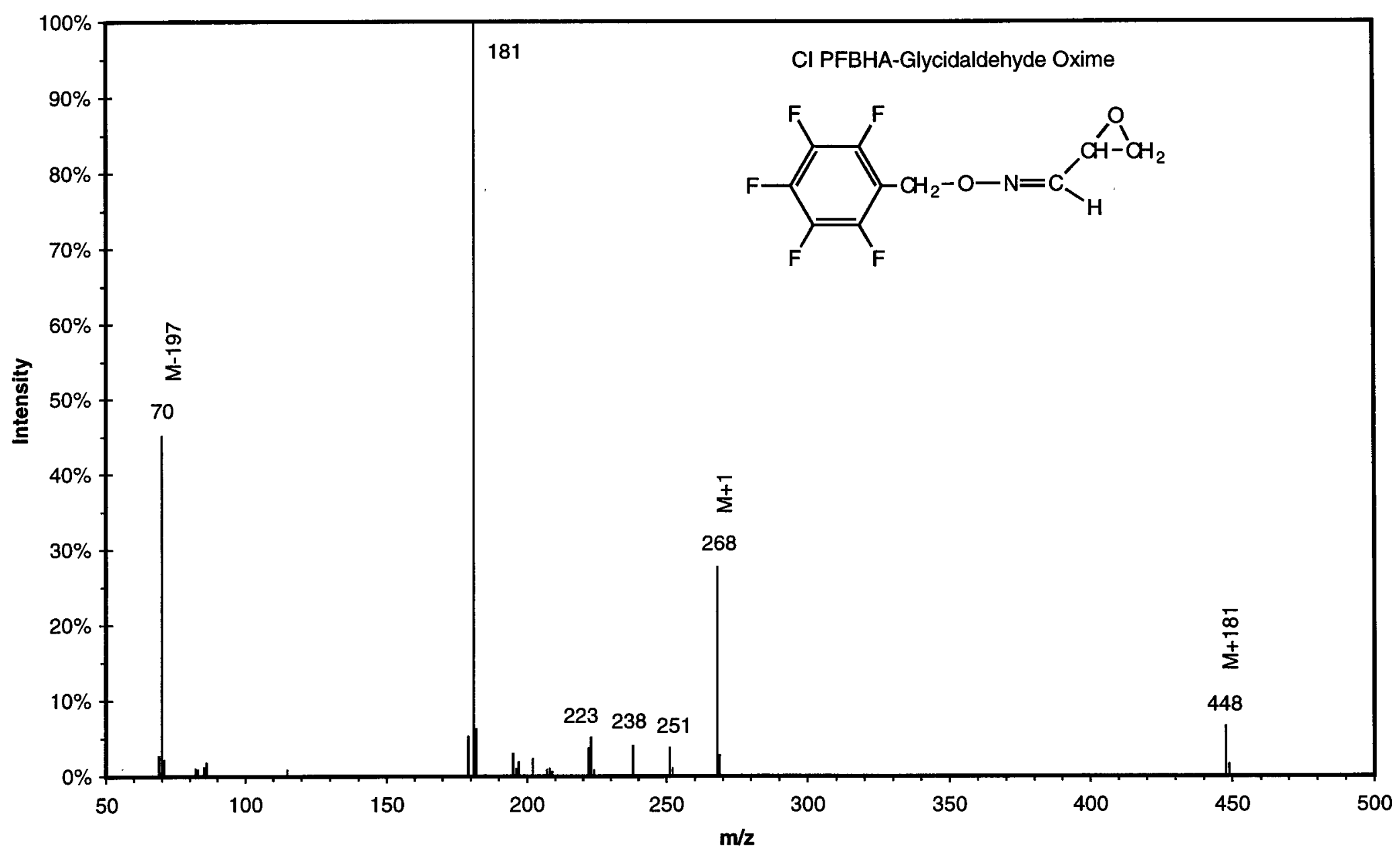

Figure 6.27: Ion trap methane CI mass of the PFBHA derivative of glycidaldehyde. 
A literature search confirmed that no study exists on the atmospheric chemistry of unsaturated epoxides having structures IV and VI. Hence, two simple unsaturated epoxides, 5,6-epoxide-1-hexene and 3,4-epoxide butene (butadiene monooxide) were chosen as model compounds for additional experimental investigation. A simple epoxide, propene oxide, was also examined. TBR experiments were conducted using $10 \mathrm{ppm}$ epoxide with $10 \mathrm{ppm}$ NO, and samples were collected and analyzed in the same manner as earlier TBR samples.

Carbonyl products resulting from the $\mathrm{OH}$ addition reactions with the carbon-carbon unsaturated bond were found to be the major products. A strong $\mathrm{C}_{4}$ hydroxy carbonyl is also observed among the carbonyl products from the $\mathrm{OH}$ reaction with butadiene monooxide, likely resulting from the following pathway:<smiles>C=CC1CO1</smiles>

In the $\mathrm{OH}$ oxidation of propene oxide, a trace amount of hydroxyacetone was positively identified as a product by comparison with an authentic standard. This observation indicates the $\mathrm{OH}$ attacking the $\mathrm{C}$-atom adjacent to the epoxide $\mathrm{O}$-atom pathway is operative. This pathway could be facilitated by either an adjacent $\mathrm{C}=\mathrm{C}$ or $\mathrm{C}=0$ group stabilizing the alkoxy radical.

\subsubsection{Epoxy Carbonyls in Experiments}

Tables 6.20 to 6.26 list the primary epoxy carbonyl products formed from the epoxide intermediates via the pathways depicted above. Carbonyl peaks with MW matching the predicted products having the form of structures II, III, IV, and VII types have been observed for all aromatic compounds examined. These peaks are all weak, indicating that these carbonyls are either very reactive, or are not formed with significant yields.

It is difficult to assign a definite structure to every observed carbonyl peak based on the MW information because often a given MW corresponds to a number of possible structures with different functionality. For example, the mono-derivative of structure IV type carbonyls have the same MW as structure II type carbonyls. A carbonyl peak with a MW of 140 in the toluene experiment, for example, can have either one of the structures:<smiles>CC12OC1C=CC(=O)C2O</smiles><smiles>CC1(C=O)OC1C=CC=O</smiles>

The limited information from mass spectra does not permit distinguishing between the two possibilities, but the presence of either of the carbonyls supports the supposition of an epoxide intermediate precursor. 
In other cases, however, only one particular structure can fit the observed molecular weight and structural information data obtained from the mass spectrum. For example, two peaks with a MW of 558 were observed in the sample collected from the 1, 3, 5TMB outdoor chamber experiment. The CI spectrum of one peak is shown in Figure 6.28, along with the fragmentation diagram. This compound is very likely 2,3-epoxy-2,4-dimethyl-6-oxo-4heptenal (Epoxide A hereafter) (see Table 6.22), a product logically expected to result from the decomposition pathway of the epoxide intermediate for 1,3,5TMB following the same pattern as was shown for toluene. Few other structures could match the combination of functionality identification, the observed molecular weight data, and the CI fragmentation pattern. Therefore, the 2,3-epoxy-2,4-dimethyl-6-oxo-4-heptenal assignment seems to be the only logical fit for this compound. In addition, two peaks with MW of 558 were also detected in the $1,2,4 \mathrm{TMB} / \mathrm{NO}_{x}$ experiment, which tends to confirm the proposed formation pathway for structure IV type epoxy carbonyls. The di-derivatives of structure IV type epoxy dicarbonyls were not detected in the toluene and xylenes experiments, but carbonyl peaks with MW matching those of the mono-derivatives were detected.

The time series of Epoxide A in Figure 6.29 shows a peak concentration near 1130 EDT, followed by decay to one-third of its peak concentration within $2.5 \mathrm{~h}$. Epoxide $\mathrm{A}$ is expected to have two possible loss pathways: $\mathrm{OH}$ attacking the unsaturated bond to form methylglyoxal and $\mathrm{a}_{6}$ epoxy dicarbonyl, 2,3-epoxy-2-methyl-2-one-pentanal; and loss of $\mathrm{CO}$ via photolysis to form a $\mathrm{C}_{8}$ unsaturated epoxy carbonyl, 5,6-epoxy-4-methyl-2-one-3heptene. Indeed, two carbonyl peaks with a MW matching $C_{8}$ unsaturated epoxy carbonyls (i.e., 335) were present. The time series of the sum of the two MW 335 peaks shown in Figure 6.29 indicates a concurrent increase in concentrations of MW 335 and Epoxide A peaks, which tends to support the proposed mechanism.

Products resulting from the photolysis pathway of structure IV type epoxy carbonyls for other aromatic compounds were also observed and are listed in Table 6.24. The expected $\mathrm{C}_{6}$ epoxy dicarbonyl from Epoxide A (MW 518) was not detected in the outdoor experiments, but was detected in the TBR experiments for p-xylene, and for 1,3,5TMB. A $\mathrm{C}_{5}$ epoxy dicarbonyl with a MW of 504 was also detected in the toluene TBR experiments. Because the radiation field in the TBR is signficantly weaker (and differs spectrally), these epoxy diacarbonyls may have a much longer lifetime in the TBR compared to that in the outdoor chamber under intense sunlight. Unfortunately, no available standards exist to test this compound further. In the toluene and xylenes systems, carbonyl peaks with MW matching the corresponding epoxy dicarbonyls were observed and are listed in Table 6.23. However, as discussed above, a trione-a likely product from hydroxy dicarbonyl-has the same MW as an epoxy dicarbonyl of the same carbon number. For example, the following two carbonyls have the same MW of 114 and their PFBHA derivatives have the same MW of 504.<smiles>CC(=O)C(=O)C(C)=O</smiles><smiles>CC1(C=O)OC1C=O</smiles> 


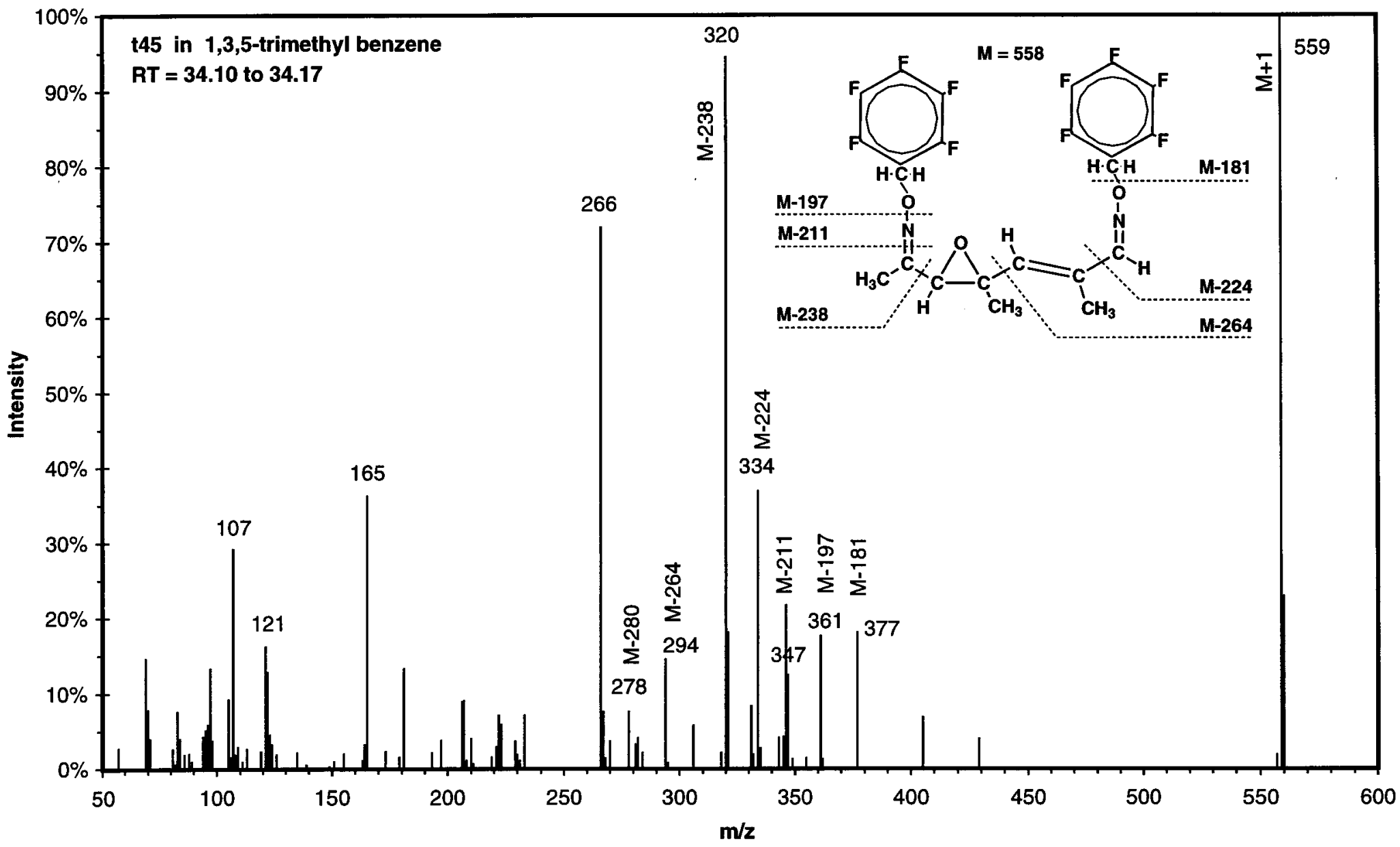

Figure 6.28: Ion trap methane CI mass of the PFBHA derivative of Epoxide A. 


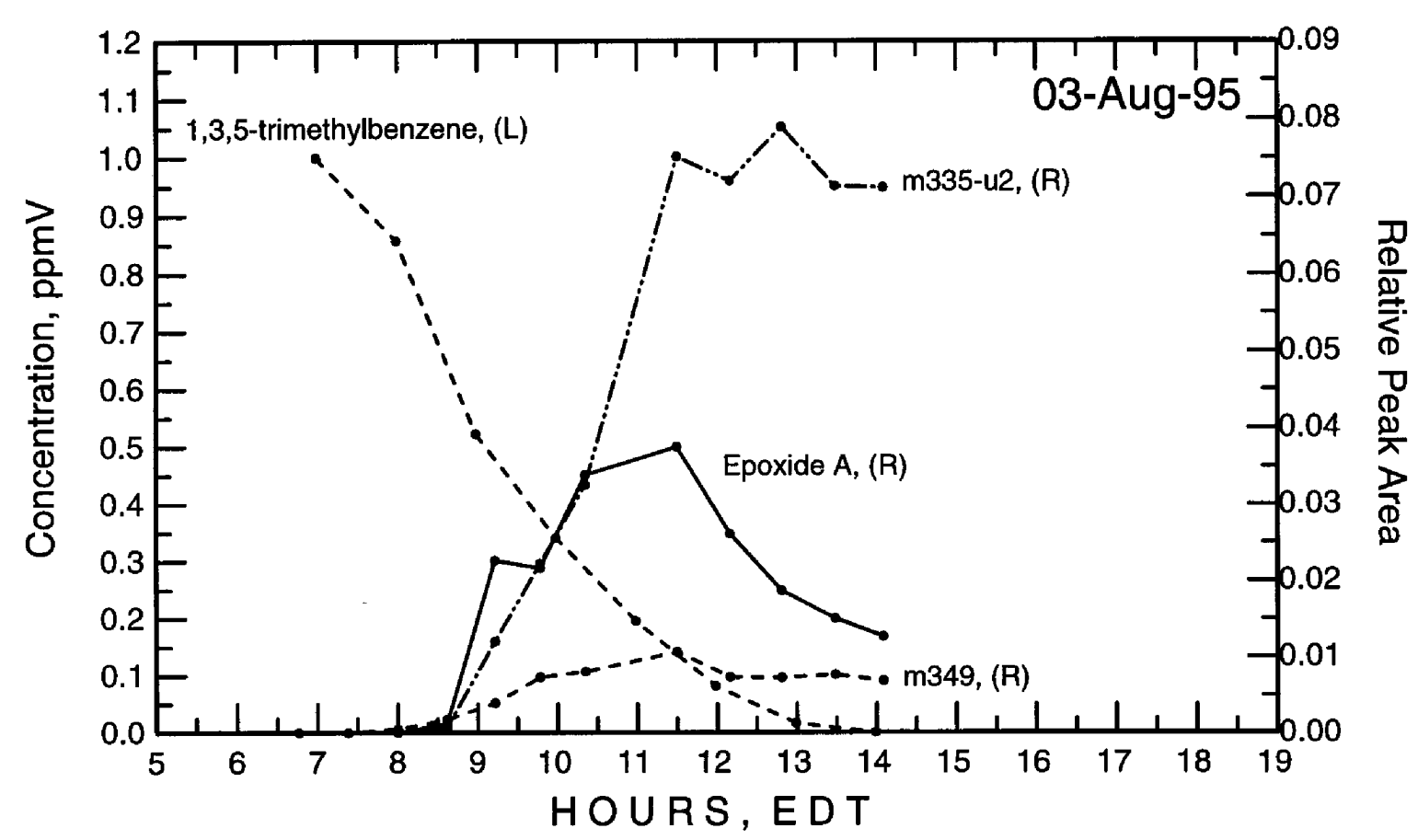

Figure 6.29:. Time series of unsaturated dicarbonyls and saturated dicarbonyls in the 1,3,5 TMB outdoor chamber experiment.

One interesting observation is that two strong $\mathrm{C}_{5}$ hydroxy carbonyls were present early in the outdoor smog chamber experiment (Figure 6.24). The $\mathrm{OH}$ addition reaction to a $\mathrm{C}_{6}$ epoxy dicarbonyl followed by unimolecular decomposition could lead to a $\mathrm{C}_{5}$ hydroxy carbonyl (Figure 6.30), a similar reaction pathway to the one in our model epoxide studies described above.

As discussed previously, however, unsaturated 1,4-dicarbonyls also serve as a possible source for hydroxy dicarbonyls; thus, the observation of hydroxy dicarbonyls is not proof of the reaction scheme shown in Figure 6.30.

\section{Other Epoxides}

Peaks with MW of 321,335 and 349 matching $C_{6}, C_{7}$, and $C_{8}$ hydroxy unsaturated epoxy cyclic carbonyl, were detected from the toluene, xylenes, and trimethylbenzenes experiments, respectively. They would be epoxide carbonyls similar in structure to that of Structure II, but having one less carbon (Table 6.25). This observation can be explained by invoking structure VII, a second-lowest intermediate. This results in such epoxy carbonyls by loss of a methyl group. As an example, Figure 6.29 shows the time series of MW 349 peaks in the 1,3,5TMB experiments: the pattern of gradually accumulating concentrations over time suggests that these types of carbonyls are not reactive in the outdoor chamber. 


\subsubsection{Conclusions}

The observation of a series of carbonyl peaks with molecular weights of PFBHA derivatives matching the proposed epoxy carbonyls strongly supports the supposition of the formation of epoxide intermediates as suggested by theoretical calculations. Confirmation of the observation of these in actual experimental conditions, however, still requires standards. These epoxide compounds may play a role in the $\mathrm{OH}$ oxidation of aromatic compounds, and their potential toxicity and mutagenicity raise public health concerns. For these reasons, the atmospheric chemistry of these epoxide compounds should be studied in greater detail with indoor and outdoor chamber and field experiments in order to assess their role in the urban atmosphere. 


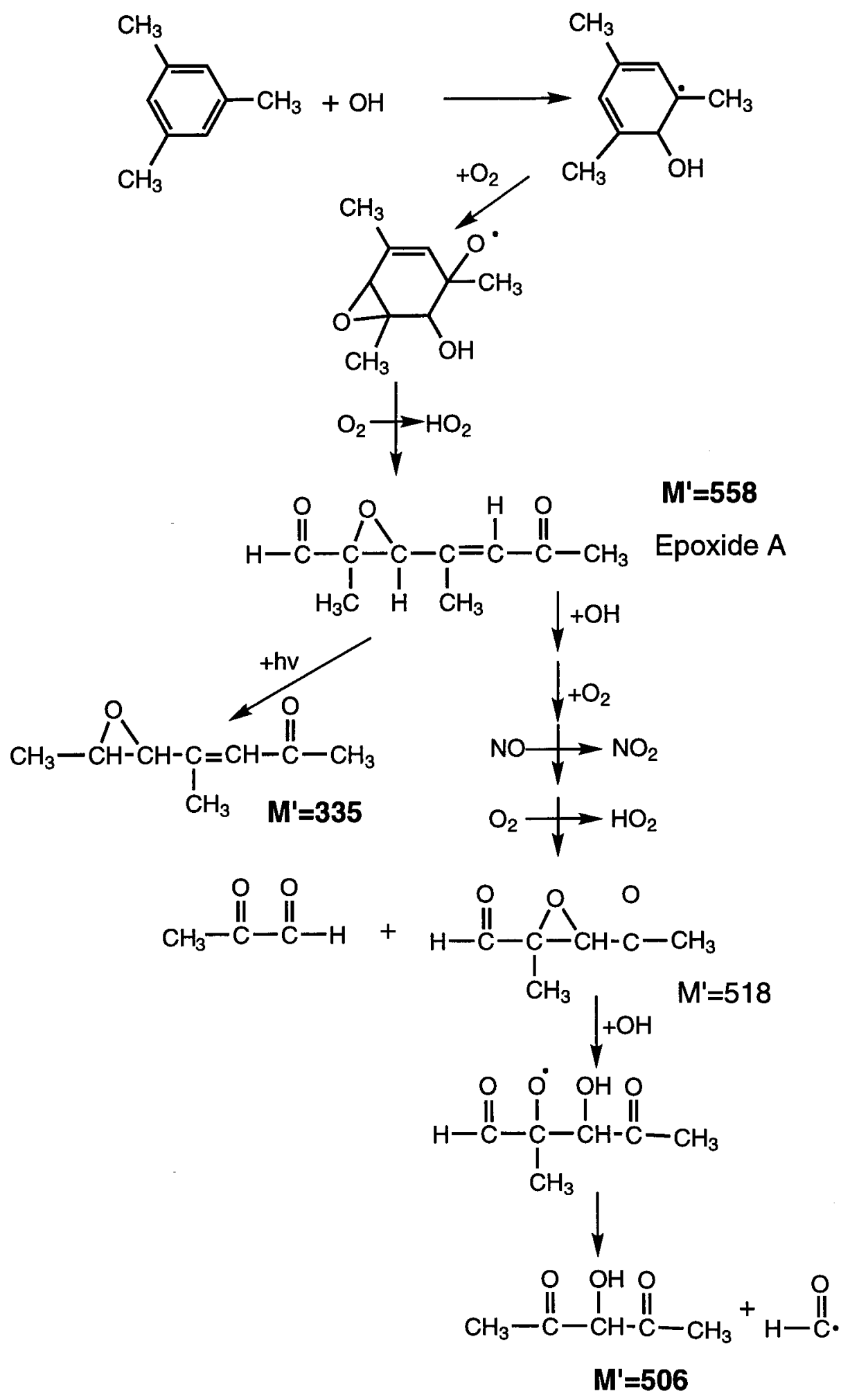

Figure 6.30: The formation of Epoxide $A$ and its subsequent reactions. 
Table 6.20:

Structure II Type Epoxy Carbonyls Formed from Six Alkylbenzenes

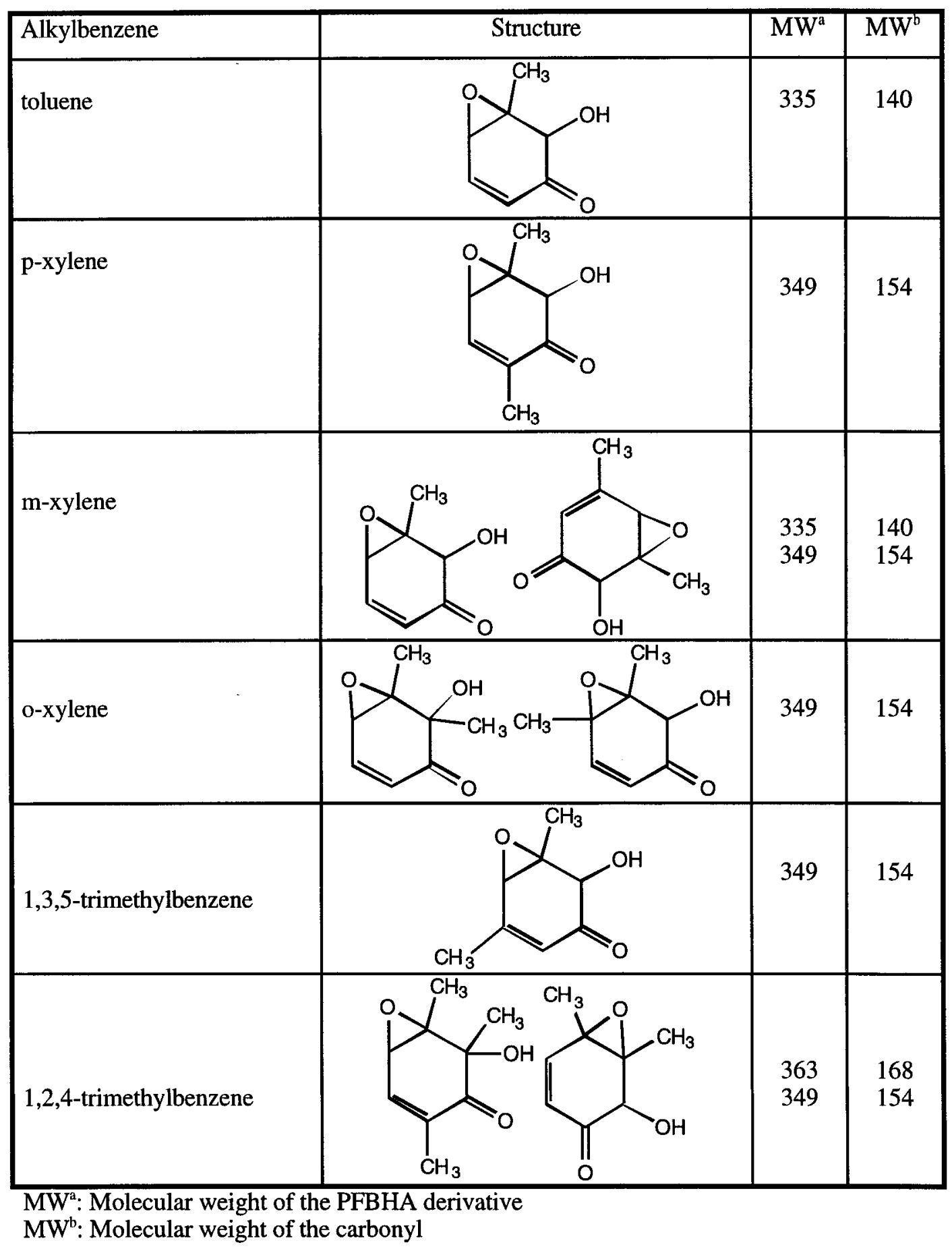


Table 6.21:

Structure III Type Epoxy Carbonyls Formed from Six Alkylbenzenes

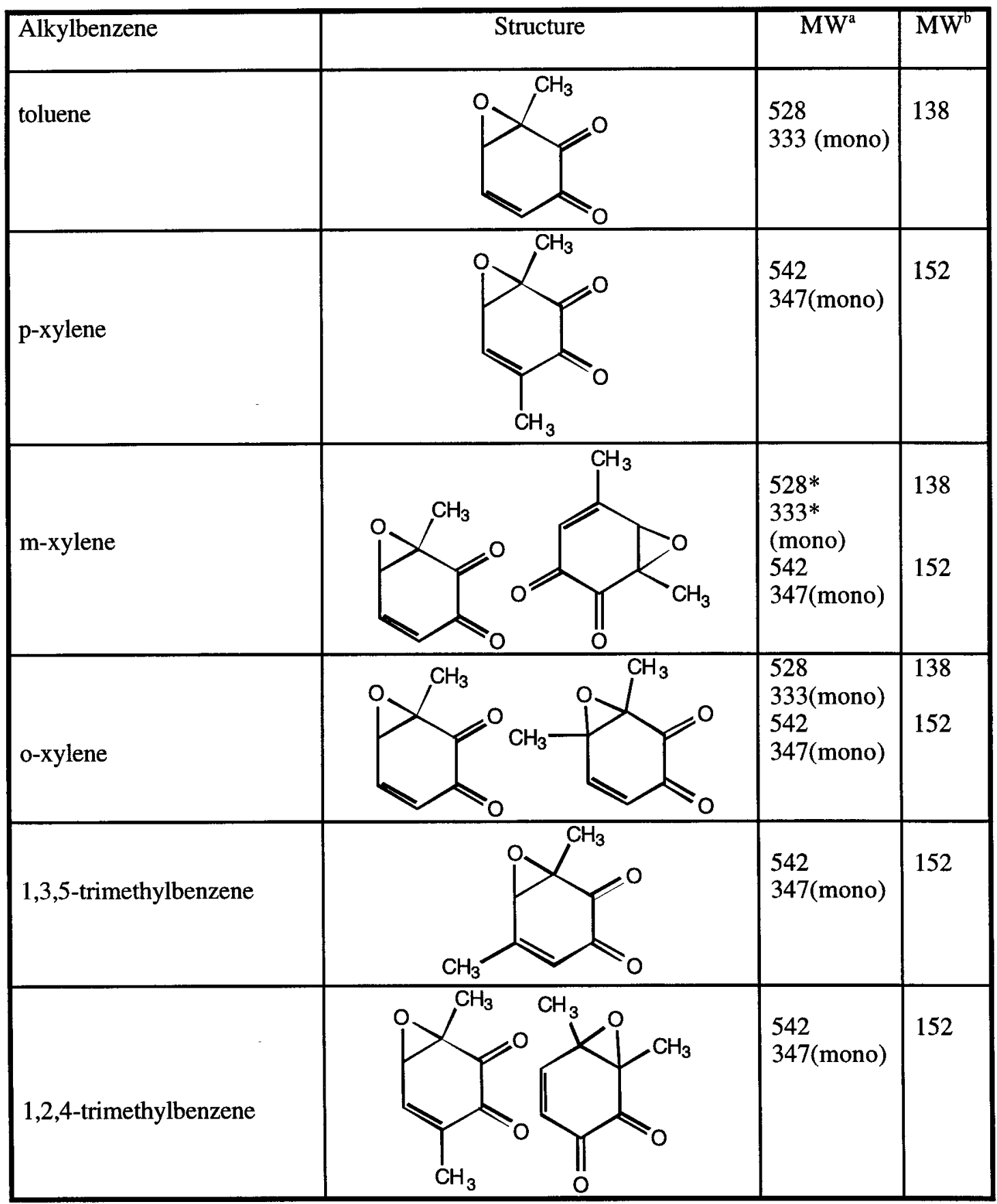

* not observed 
Table 6.22:

Structure IV Type Epoxy Carbonyls Formed from Six Alkylbenzenes

\begin{tabular}{|c|c|c|c|}
\hline Alkylbenzene & Structure & $\mathrm{MW}^{\mathrm{a}}$ & $\mathrm{MW}^{\mathrm{b}}$ \\
\hline toluene & $\mathrm{H}-\stackrel{\mathrm{O}}{\mathrm{C}}-\underset{\mathrm{CH}_{3}}{\stackrel{\mathrm{O}}{\mathrm{C}}} \mathrm{CH}-\mathrm{CH}=\mathrm{CH}-\stackrel{\mathrm{O}}{\|}-\mathrm{H}$ & $\begin{array}{l}530 \\
335 \text { (mono) }\end{array}$ & 140 \\
\hline p-xylene & 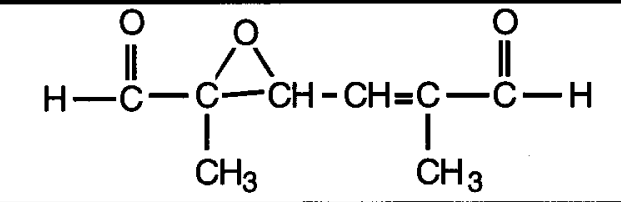 & $\begin{array}{l}544 \\
349 \text { (mono) }\end{array}$ & 154 \\
\hline m-xylene & $\stackrel{\stackrel{O}{\|}}{\|}-\mathrm{CH}=\mathrm{CH}-\mathrm{CH}$ & $\begin{array}{l}544 \\
349(\text { mono })\end{array}$ & 154 \\
\hline o-xylene & $\stackrel{\mathrm{O}}{\|} \underset{\mathrm{C}}{\mathrm{O}}-\stackrel{\mathrm{O}}{\mathrm{CH}}-\mathrm{CH}-\mathrm{CH}=\underset{\mathrm{C}}{\mathrm{C}}-\stackrel{\mathrm{O}}{\|}-\mathrm{CH}_{3}$ & $\begin{array}{l}544 \\
349(\text { mono })\end{array}$ & 154 \\
\hline 1,3,5-trimethylbenzene & $\mathrm{H}-\stackrel{\mathrm{O}}{\mathrm{C}}-\underset{\mathrm{CH}_{3}}{\stackrel{\mathrm{O}}{-}} \underset{\mathrm{C}}{\mathrm{C}}-\underset{\mathrm{CH}}{\mathrm{C}}=\mathrm{CH}-\stackrel{\mathrm{O}}{\mathrm{C}}-\mathrm{CH}_{3}$ & $\begin{array}{l}558 \\
363 \text { (mono) }\end{array}$ & 168 \\
\hline 1,2,4-trimethylbenzene & $\mathrm{CH}_{3}-\stackrel{\mathrm{O}}{\mathrm{C}}-\underset{\mathrm{CH}_{3}}{\mathrm{C}}-\underset{\mathrm{C}}{\mathrm{O}} \mathrm{C}-\mathrm{CH}=\underset{\mathrm{CH}}{\mathrm{C}}-\stackrel{\mathrm{O}}{\mathrm{C}}-\mathrm{H}$ & $\begin{array}{l}558 \\
363 \text { (mono) }\end{array}$ & 168 \\
\hline
\end{tabular}


Table 6.23:

Structure V Type Epoxy Carbonyls and Companying Saturated Dicarbonyls Formed from Six Alkylbenzenes

\begin{tabular}{|c|c|c|c|}
\hline Alkylbenzene & Structure & $\mathrm{MW}^{\mathrm{a}}$ & $\mathrm{MW}^{\mathrm{b}}$ \\
\hline toluene & 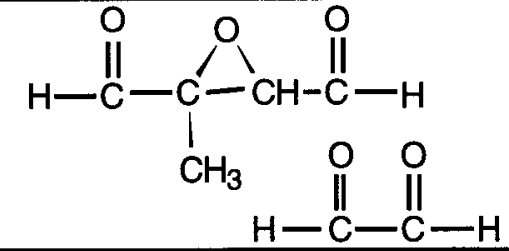 & $\begin{array}{l}504 \\
448\end{array}$ & $\begin{array}{l}114 \\
58\end{array}$ \\
\hline p-xylene & 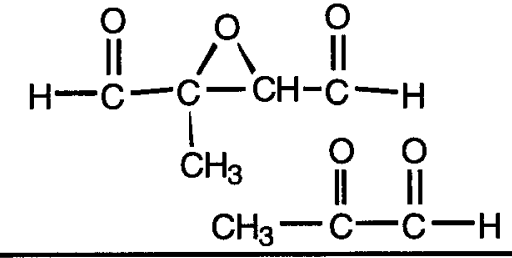 & $\begin{array}{l}504 \\
462\end{array}$ & $\begin{array}{l}114 \\
72\end{array}$ \\
\hline m-xylene & 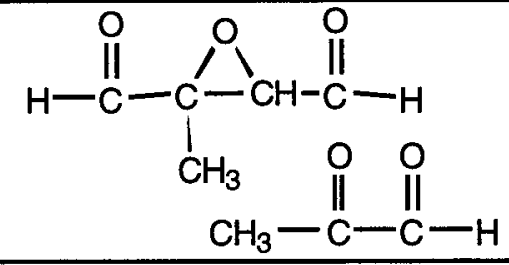 & $\begin{array}{l}504 \\
462\end{array}$ & $\begin{array}{l}114 \\
72\end{array}$ \\
\hline o-xylene & 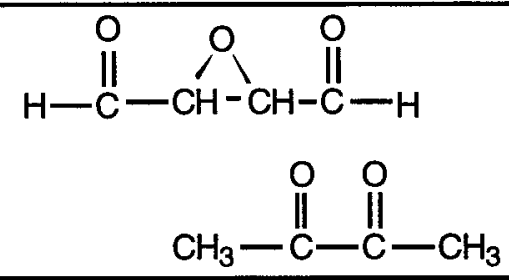 & $\begin{array}{l}490 \\
476\end{array}$ & $\begin{array}{l}100 \\
86\end{array}$ \\
\hline 1,3,5-trimethylbenzene & 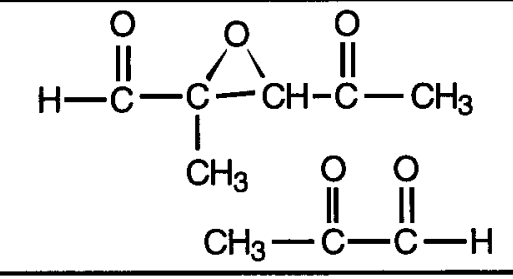 & $\begin{array}{l}518 * \\
462\end{array}$ & $\begin{array}{l}128 \\
72\end{array}$ \\
\hline 1,2,4-trimethylbenzene & 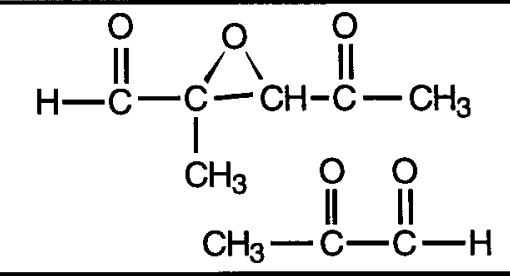 & $\begin{array}{l}518 * \\
462\end{array}$ & $\begin{array}{l}128 \\
72\end{array}$ \\
\hline
\end{tabular}

* not observed 
Table 6.24:

Structure VI Type Epoxy Carbonyls Formed from Six Alkylbenzenes

\begin{tabular}{|c|c|c|c|}
\hline Alkylbenzene & Structure & $\mathrm{MW}^{\mathrm{a}}$ & $\mathrm{MW}^{\mathrm{b}}$ \\
\hline toluene & $\mathrm{CH}-\mathrm{CH}=\mathrm{CH}_{2}$ & 307 & 112 \\
\hline p-xylene & $\mathrm{CH}=\mathrm{CH}-\mathrm{CH}_{3}$ & 321 & 126 \\
\hline m-xylene & $\mathrm{CH}_{3}-\stackrel{\mathrm{O}}{\mathrm{O}}-\mathrm{CH}-\mathrm{CH}=\mathrm{CH}-\stackrel{\mathrm{C}}{\|}-\mathrm{CH}_{3}$ & 321 & 126 \\
\hline o-xylene & $\mathrm{CH}_{2} \stackrel{\mathrm{O}}{\stackrel{\mathrm{C}}{-}} \mathrm{CH}-\mathrm{CH}=\underset{\mathrm{CH}_{3}}{\mathrm{C}}-\stackrel{\mathrm{O}}{\mathrm{C}}-\mathrm{CH}_{3}$ & 321 & 126 \\
\hline 1,3,5-trimethylbenzene & 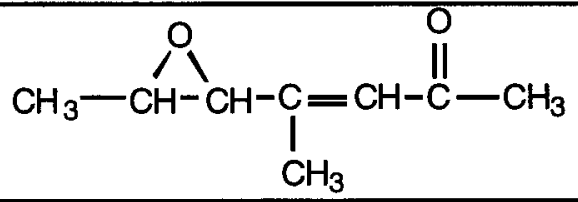 & 335 & 140 \\
\hline 1,2,4-trimethylbenzene & $\mathrm{CH}_{3}-\stackrel{\mathrm{O}}{\mathrm{C}}-\underset{\mathrm{CH}_{3}}{\mathrm{C}}-\stackrel{\mathrm{O}}{-} \mathrm{CH}-\mathrm{CH}=\mathrm{CH}-\mathrm{CH}_{3}$ & 335 & 140 \\
\hline
\end{tabular}


Table 6.25:

Structure VIII Type Epoxy Carbonyls Formed from Six Alkylbenzenes

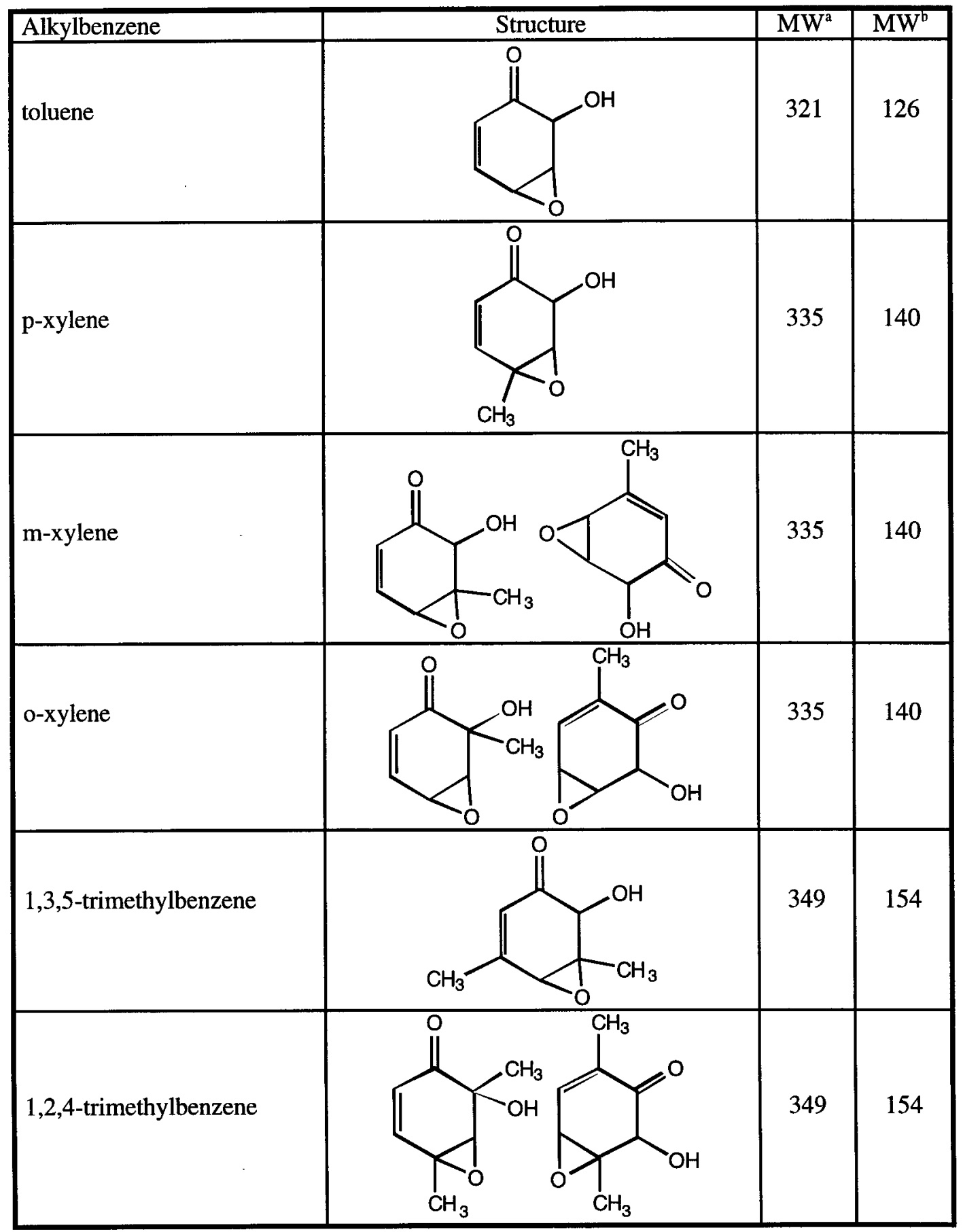


Table 6.26:

Structure X Type Epoxy Carbonyls and Saturated Dicarbonyl Co-products Formed from Six Alkylbenzenes

\begin{tabular}{|c|c|c|c|}
\hline Alkylbenzene & Structure & $\mathrm{MW}^{\mathrm{a}}$ & $\mathrm{MW}^{\mathrm{b}}$ \\
\hline toluene & 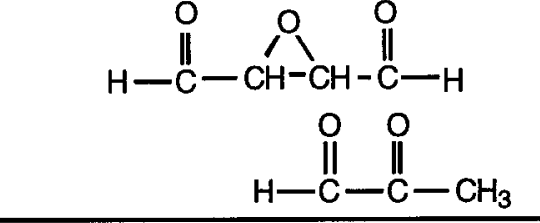 & $\begin{array}{l}490 \\
462\end{array}$ & $\begin{array}{l}100 \\
72\end{array}$ \\
\hline p-xylene & 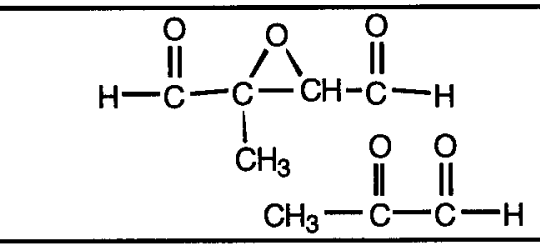 & $\begin{array}{l}504 \\
462\end{array}$ & $\begin{array}{l}114 \\
72\end{array}$ \\
\hline m-xylene & 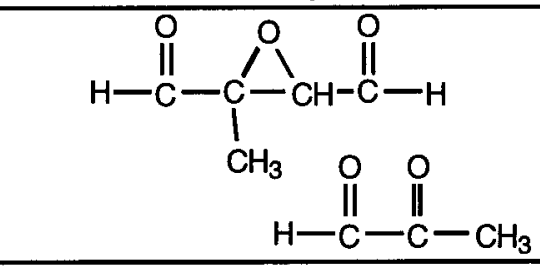 & $\begin{array}{l}504 \\
462\end{array}$ & $\begin{array}{l}114 \\
72\end{array}$ \\
\hline o-xylene & 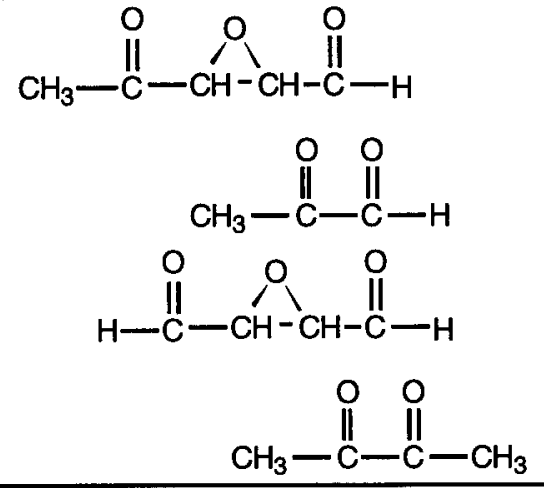 & $\begin{array}{r}504 \\
462 \\
490 \\
476\end{array}$ & $\begin{array}{c}114 \\
72 \\
100 \\
86\end{array}$ \\
\hline 1,3,5-trimethylbenzene & 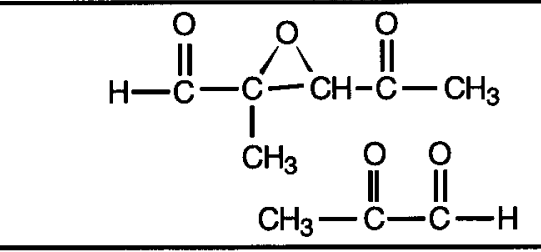 & $\begin{array}{r}518^{*} \\
462\end{array}$ & $\begin{array}{l}128 \\
72\end{array}$ \\
\hline 1,2,4-trimethylbenzene & 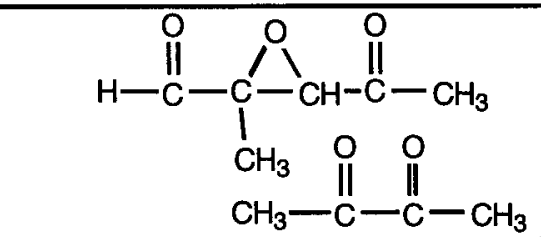 & $\begin{array}{l}504 \\
476\end{array}$ & $\begin{array}{l}100 \\
86\end{array}$ \\
\hline
\end{tabular}

* not observed 


\section{Chapter 7}

\section{Airshed Modeling}

\subsection{Emissions Inventory Improvements}

\subsubsection{Area Source Emissions}

The Urban Airshed Model and its preprocessors use data originating in many different places. North Carolina Modeling Domain data included emissions data from industrial point sources, traffic data from transportation demand models, vehicle emissions data from an emission factor model such as EPA's MOBILE model, road locations from Geographic Information Systems (GIS) software, nonroad mobile calculations, area source calculations, and temperature and wind field data from National Weather Service and private weather stations.

The Emissions Preprocessor System within the UAM is written in FORTRAN and is responsible for converting the county-based emissions inventory from a base year to a gridbased, hourly emissions inventory for the days being modeled. Coordinates of point sources and link-based mobile sources are specified in the inventory. However, exact locations of other emissions sources are not known. These sources are spatially allocated to grid cells using surrogates such as population and water. It is frequently true that these surrogates are incomplete or wrongly specified for many domains. For example, the "water" surrogate (representing a river) has often been found to suddenly stop at a county line, yet continue in the next county.

In our UAM studies, we developed a relational database management techniques applied them to emissions inventory and spatial surrogate files for the North Carolina Modeling Domain [23]. Application of this code to QA work on emissions inventory files resulted in many refinements of the spatial allocation of emissions.

Working with emissions files in a relational database management system (RDBMS) has many advantages over continuing to work in FORTRAN. When writing programs in FORTRAN and other third-generation computer languages, the file structure, method of data access, and input and output functions must be explicitly written in each program. 
Although the main processing portion of any individual program may be only a few lines long, the entire program may be many pages long. All this code must be debugged and tested for accuracy. However, when writing programs using an RDBMS, the data structure is defined once in a centralized data dictionary, and the RDBMS itself handles the method of data access and input and output processing. Some RDBMS languages even include "high level functions" such as TOTAL and COUNT functions, eliminating the need to explicitly write such code every time it is needed. Also, an RDBMS inherently has the ability to relate data in different files in the database, simplifying lookup functions and file comparisons. Ad hoc reports may be quickly generated using an English-like query language, allowing people other than computer programmers access to data for analysis.

To investigate the quality of emissions data and spatial surrogates, these data files were loaded into a PROGRESS database. Query programs then searched the emissions inventory files for missing and duplicate data. Quality assurance was also performed on the spatial surrogate file provided by EPA. Query programs operated on the emissions and the spatial surrogate file in the database, performing many of the tasks of UAM's Emissions Preprocessor System 2.07 (EPS). Then, using the spatial surrogates, emissions using each spatial surrogate were allocated to the appropriate grid cells in the modeling domain. These gridded emission files for each surrogate were then exported to SAS/GRAPH for spatial analysis.

The total gridded emissions may be correct, but may be in the wrong grid cells. The gridded emissions may be in the correct grid cells, but the total gridded emissions may be incorrect if the total fractional surrogates do not correctly sum to 1 .

Both types of errors were found in the North Carolina surrogate file. When allocating emissions using the original urban and water surrogates, the surrogates were zero for some counties, resulting in lost emissions during the gridding process of EPS. Also, the surrogates apparently originated from older land-use data bases using a very coarse grid size, resulting in over-smoothing of the emisions field and artifically spreading of emissions beyond the emissions sources.

\subsubsection{Mobile Source Emissions}

Preparing motor vehicle emission inventories for use in photochemical modeling can be time-consuming and cumbersome. To simplify the calculations, current methods often ignore the variability of traffic flow characteristics and environmental conditions over space and time. The manpower and resources necessary to represent this variability using current methods is prohibitive, and therefore, its effect on predicted emissions and air quality has not been adequately explored.

As part of this project and in cooperation with another project at UNC, we have addressed these problems by [24]:

- Implementing the current emission estimation methodology in a relational database management system to produce inventories in a more flexible and efficient manner 
- Incorporating information from a UTPS-type transportation demand model to improve the spatial and temporal resolution of vehicle activity in the emission calculations.

The first task has resulted in a SAS-based package known as ProcMobl 1.0, which:

- Predicts future traffic volumes based on linear regression of historical data

- Applies trip-averaged emission factors from EPA's MOBILE 5a model

- Chemically speciates the emissions for representation in UAM

- Temporally allocates the emissions by hour

- spatially allocates the emissions to UAM grid celis.

ProcMobl 1.0 calculates emissions for individual roadway links or for entire counties. The system includes specific quality control checks and facilitates flexible reporting of calculation results.

The current EPA methodology implemented in ProcMobl 1.0 estimates vehicle emissions based on an average emission per vehicle mile traveled. This approach ignores the fact that emissions vary according to whether the vehicle is starting a trip, is in the middle of a trip, has just ended a trip, or is parked. These modes of vehicle operation are distributed differently in space and time. For example, in the morning, a residential suburb may experience many trip starts while a central business district may experience many trip ends. This sort of spatial and temporal variability may be important to the formation of urban ozone, but it is not captured by the current emission calculation methodology.

Building on ProcMobl 1.0, we developed ProcMobl 2.0 to represent the spatial and temporal variability of emissions based on four operating modes (starts, stabilized, ends, and parked). ProcMobl 2.0 uses linear regression models to represent the relationships between speed and ambient temperature and the emissions for each operating mode as predicted by MOBILE 5a. The emissions are spatially and temporally allocated using the information generated by an UTPS-type transportation demand model.

To evaluate the effects of increased resolution, motor vehicle emission inventories were developed for the Charlotte, NC, metropolitan area.

In a subsequent paper, we used these different inventories in UAM simulations of a Charlotte, North Carolina ozone episode. These results were reported at an International Specialty Conference [25]. This paper is attached as an appendix.

\subsection{Process Analysis}

As part of this project (and others) we have developed a method called integrated process rate analysis, (IPRA), or just "process analysis," which allows us to fully explain a model's prediction. We have applied this method to static smog chamber simulations [26], to 
trajectory models [1,27-29], to regional Eulerian models [31,32], and most recently to the Urban Airshed Model [33].

\subsubsection{The Model's Processes}

Most air quality models create output files of just species concentrations over space and time. In process analysis we focus on the change caused by each major physical and chemical process in the model, and thus our method requires additional model outputs. The processes that we need separately quantified at each time step are:

Chemistry: To perform a complete system analysis of the chemical transformations, the integrated reaction rates, or IRRs, of each chemical reaction in the model are needed. The reaction rate and IRR for a second order reaction is defined as

$$
\begin{aligned}
r_{g} & =k_{g} M_{i} M_{j} \\
\Delta r_{g} & =\int_{t_{1}}^{t_{2}} r_{g} \mathrm{~d} t,
\end{aligned}
$$

where $M_{i}$ and $M_{j}$ are the mixing ratios of reactants in reaction $g$, and $k_{g}$ is the reaction rate constant. In addition, for other types of process analysis, we need the net change in each species' mixing ratio caused just by the chemistry, $\Delta M_{C}$. This value and other similar values are obtained by integrating the rate of change of each process separately rather than summing all rates of change and then integrating once, that is,

$$
\Delta M_{p}=\left.\int_{t_{1}}^{t_{2}} \frac{\mathrm{d} M}{\mathrm{~d} t}\right|_{p} \mathrm{~d} t,
$$

and $p$ indicates the process.

Horizontal transport: The change in each species' mixing ratio caused by advection, $\Delta M_{H}$, from cell to cell by winds must be quantified and

$$
\Delta M_{H}=\Delta M_{H_{N}}+\Delta M_{H_{E}}+\Delta M_{H_{S}}+\Delta M_{H_{W}}
$$

where the subsubscripts indicate the direction of transport from the north, east, south, or west into the cell.

Vertical transport: The change in each species caused by advection and diffusion of material, $\Delta M_{V}$, from above and below each cell must be quantified.

Emissions: The change in each species caused by emissions, $\Delta M_{E}$, into each cell must be quantified. It is also useful to distinguish low level emissions from elevated emissions. 


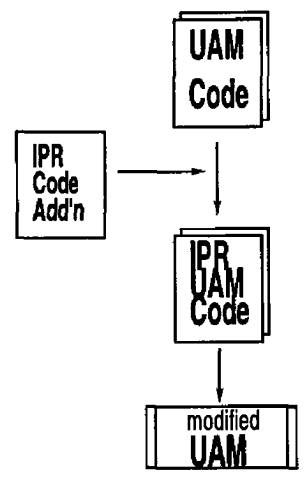

Figure 7.1: Air quality models must be modified to include new code to output the process rates.

Deposition: The change in each species caused by deposition of material on surfaces, $\Delta M_{D}$, must be quantified.

Initial: The concentration of each species at the beginning of the time step, $M_{i}$, is also needed, i.e., the initial species concentrations for the time step.

It is clear that

$$
M_{f}=M_{i}+\Delta M_{C}+\Delta M_{H}+\Delta M_{V}+\Delta M_{E}+\Delta M_{D},
$$

where $M_{f}$ is the final concentration for each time step. Obviously, some of the terms on the right-hand-side will be negative and some will be positive, so at any given time different processes are driving the species' mixing ratios up and some are driving it down. In an analysis of mixing ratio predictions, it is not possible to explain the mixing ratio time series by just examining the mixing ratios. Therefore, in the IPRA method, all of the additional output described above is subjected to further processing by several auxiliary or post-simulation programs. The code changes needed and the auxiliary programs will be briefly described next.

\subsubsection{The Code Changes}

To perform process analysis, minor additions (less than 500 lines) must be made to the solver codes in each air quality model to be analyzed and a new executable version of the model must be produced (see Figure 7.1). These changes have already been made to the regulatory version and to our CRAY vectorized version of UAM-IV, to the High Resolution RADM, and are currently being made to the UAM-V. In making these changes, a small amount of additional code is added at the end of each of the process routines in the model [34]. The purpose of this new code is to perform a simple integration of the rate of each process that effects species concentrations over each model solution time step and, depending upon a user command, to either accumulate these integrated rates for an hour before writing them 


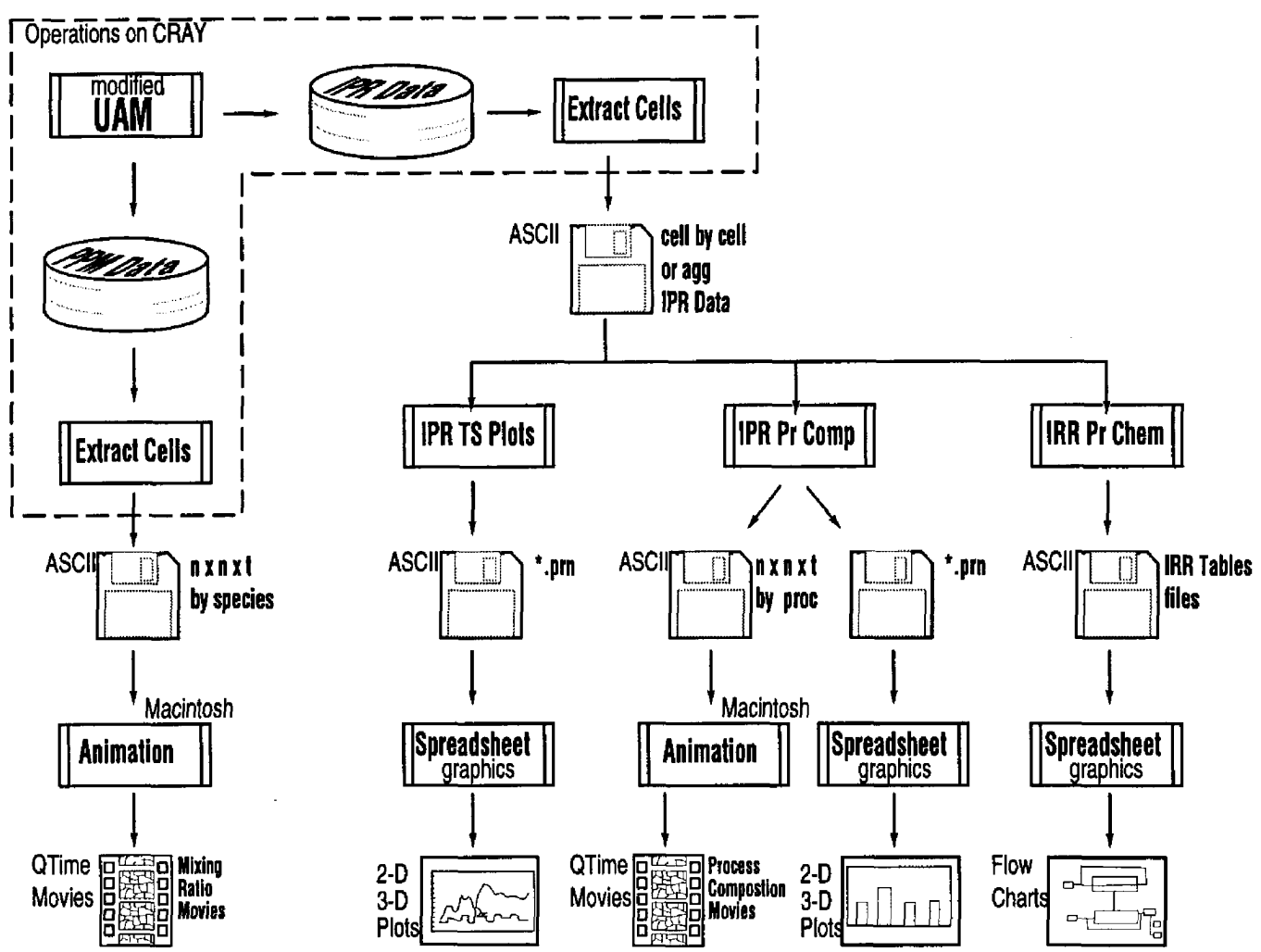

Figure 7.2: Schematic of a 'complete' process analysis for a model scenario run.

out, or to write them out each time step. Thus, the model's output will consist of both the usual species concentration data and the new IPR data (see Figure 7.2). These additional model calculations use the process rates of change that the model already computes, and thus, place only a very small additional computational burden on the system. An abbreviated example of the extract of one time-step output from an IPR file is shown in Tables 7.1 and 7.2 .

For an environmental chamber analysis or for a Lagrangian air quality model analysis, the total output file consists of a set of values like those in Tables 7.1 and 7.2, usually one set for each hour. Note that some of the items in the IPR file are not needed for these simple types of analysis (e.g, the horizontal transport terms) and in these cases these items are written out as a field of zeros. In other cases, some species are computed by a steady-state approximation and are not transported from cell to cell. These species therefore appear with small initial values and have zero rates for many physical processes. For an Eulerian model, such a set as in the tables would be written for each cell of the model, resulting in a large amount of additional output-nearly a doubling the output of the model if all cells are written out. For this reason, the changes made to the UAM allow rectangular "regions" to be selected for process analysis output. Furthermore, some types of process analysis (e.g, the process compositional analyses) require output data at every advective time step ( 6 minfor 
Table 7.1: The First Part of the Integrated Process Rate Output: The Integrated Reaction Rates of Each Chemical Reaction in the Model for One Time Step.

\begin{tabular}{|c|c|}
\hline \multicolumn{2}{|c|}{ "IR/MB FOR UAM-IV" } \\
\hline Time $=$ & $\begin{array}{l}\text { 11. Dark = } \\
\text { Int rate. }\end{array}$ \\
\hline$\{1\}$ & $1.28502 \mathrm{E}-01$ \\
\hline$\{2\}$ & $3.08729 \mathrm{E}-01$ \\
\hline i 3$\}$ & $1.06341 \mathrm{E}-01$ \\
\hline ( 4$\}$ & $3.61750 E-06$ \\
\hline ( 5$)$ & $6.07917 \mathrm{E}-07$ \\
\hline ( 6 ) & $1.33169 \mathrm{E}-07$ \\
\hline 7) & $9.16786 \mathrm{E}-04$ \\
\hline ( 8$)$ & $1.71110 \mathrm{E}-01$ \\
\hline$\{9\}$ & $1.02786 \mathrm{E}-02$ \\
\hline$(10)$ & $8.84278 E-03$ \\
\hline & $\cdots$ \\
\hline (24) & $1.60628 \mathrm{E}-05$ \\
\hline (25) & 1. $61543 \mathrm{E}-12$ \\
\hline - & $\cdots \cdot$ \\
\hline (81) & $4.34745 E-04$ \\
\hline (82) & $0.00000 \mathrm{E}+00$ \\
\hline 83$\}$ & $0.00000 \mathrm{E}+00$ \\
\hline 84) & $2.74100 \mathrm{E}-06$ \\
\hline 85$\}$ & $9.53471 \mathrm{E}-05$ \\
\hline 86$)$ & $0.00000 \mathrm{E}+00$ \\
\hline
\end{tabular}

Table 7.2:. The Second Part of the Integrated Process Rate Output: The Integrated Process Rates of Each Physical and Chemical Process in the Model for One Time Step.

\begin{tabular}{|c|c|c|c|c|c|c|}
\hline $\begin{array}{l}\int_{0}^{\text {specles }} \\
0_{0} \text { olp }\end{array}$ & $\begin{array}{l}\text { Intt cone } \\
0.100000 \mathrm{E}-19 \\
0.100000 \mathrm{E}-19\end{array}$ & $\begin{array}{l}\text { Gas Chem } \\
0.000000 \mathrm{E}+00 \\
0.000000 \mathrm{E}+00\end{array}$ & $\begin{array}{l}\text { West Trans } \\
0.000000 \mathrm{E}+00 \\
0.000000 \mathrm{E}+00\end{array}$ & $\begin{array}{l}\text { East Trans } \\
0.000000 \mathrm{E}+00 \\
0.000000 \mathrm{E}+00\end{array}$ & $\begin{array}{l}\text { South Trans } \\
0.000000 \mathrm{E}+00 \\
0.000000 \mathrm{E}+00\end{array}$ & $\begin{array}{l}\text { North Trahs } \\
0.000000 \mathrm{E}+00 \\
0.000000 \mathrm{E}+00\end{array}$ \\
\hline - & - & - & - & - & - & - \\
\hline $\begin{array}{l}\text { "PAR } \\
\text { "TOE }\end{array}$ & $\begin{array}{l}0.604878 \mathrm{E}-01 \\
0.127839 \mathrm{E}-02\end{array}$ & $\begin{array}{c}-0.561872 \mathrm{E}-02 \\
-0.387130 \mathrm{E}-03 \\
\end{array}$ & $\begin{array}{c}0.445677 E-01 \\
0.839104 E-0.3\end{array}$ & $\begin{array}{c}-0.473587 \mathrm{E}-01 \\
-0.936106 \mathrm{E}-03 \\
\end{array}$ & $\begin{array}{c}0.487423 \mathrm{E}-03 \\
0.102265 \mathrm{E}-04 \\
.\end{array}$ & $\begin{array}{c}0.636204 \mathrm{E}-02 \\
0.1030 .55 \mathrm{E}-03 \\
-\end{array}$ \\
\hline $\begin{array}{l}\text { "ISOP } \\
\text { " CLBR }\end{array}$ & $\begin{array}{l}0.176950 \mathrm{E}-04 \\
0.993197 \mathrm{E}+00\end{array}$ & $\begin{array}{r}-0.242456 \mathrm{~B}-03 \\
0.000000 \mathrm{E}+00\end{array}$ & $\begin{array}{l}0.340836 E-04 \\
0.823980 E+00\end{array}$ & $\begin{array}{l}-0.370586 \mathrm{E}-04 \\
-0.829784 \mathrm{E}+00\end{array}$ & $\begin{array}{l}0.930089 \mathrm{E}-07 \\
0.868804 \mathrm{E}-02\end{array}$ & $\begin{array}{l}0.382198 \varepsilon-05 \\
0.115545 E+00\end{array}$ \\
\hline i & & & & & & \\
\hline ! Species & Titrate & Ver Trans & Low lev em $1 \mathrm{~s}$ & E1ev PT emis & Deposition & Final cone \\
\hline OO1D & $\begin{array}{l}0.000000 \mathrm{E}+00 \\
0.00000 \mathrm{E}+00\end{array}$ & $\begin{array}{l}0.000000 \mathrm{E}+00 \\
0.000000 \mathrm{E}+00\end{array}$ & $\begin{array}{l}0.000000 \mathrm{~B}+00 \\
0.00000 \mathrm{E}+00\end{array}$ & $\begin{array}{l}0.000000 \mathrm{E}+00 \\
0.000000 \mathrm{E}+00\end{array}$ & $0.000000 \mathrm{E}+$ & $0.100000 \mathrm{E}-19$ \\
\hline - & - & - & - & - & - & - \\
\hline "PAR & $0.000000 \mathrm{E}+00$ & $-0.150652 E-01$ & $0.699708 \mathrm{E}-02$ & $0.101726 \mathrm{E}-04$ & $0.290276 \mathrm{E}-14$ & $0.508696 \mathrm{E}-01$ \\
\hline "TOL & $0.000000 \mathrm{E}+00$ & $-0.409817 \mathrm{E}-03$ & $0.324779 E-03$ & $0.000000 \mathrm{E}+00$ & $0.551037 \mathrm{E}-16$ & $0.822499 \mathrm{E}-03$ \\
\hline - & - & - & - & - & - & - \\
\hline "ISOP & $0.000000 \mathrm{E}+00$ & $-0.543736 \mathrm{E}-04$ & $0.304680 \mathrm{E}-03$ & $0.00 .0000 \mathrm{E}+00$ & $0.147928 \mathrm{E}-17$ & $0.264846 \mathrm{E}-04$ \\
\hline - CLBR & $0.000000 \mathrm{E}+00$ & $-0.113493 \mathrm{E}+00$ & $0.000000 E+00$ & $0.000000 \mathrm{E}+00$ & $0.515579 \mathrm{E}-13$ & $0.998133 \mathrm{E}+00$ \\
\hline
\end{tabular}

the UAM), while the rest use values accumulated for $1 \mathrm{~h}$.

For efficiency in storage and for speed during the simulation, the IPR data are written as binary data in a model-specific format. At the present time, a post-simulation program, EXTRACT, is used to select cells and times from the binary file and to produce ASCII files that are portable to other computing platforms such as PCs. Although the hourly output for 0500-1900 LDT for a single cell in the ASCII format is only 152 kilobytes, the "every-time-step" output needed for the process compositional analysis is 1.8 megabytes per cell for the same time period. Typically a 6 by 6 cell region is analyzed which results in having to transfer and store 65 megabytes of data. Therefore, we are considering changing the EXTRACT program to output HDF data sets as an alternative to the ASCII format. HDF, or hierarchal data format, was invented at the National Center for Supercomputing Applications for exchanging data among different applications. It can also exchange binary data among different computer platforms, e.g., CRAY's and PC's. 


\subsubsection{The Process Analyses}

As shown in Figure 7.2, there are currently four basic types of process analyses:

1. Movies of lumped process magnitude fields, which will be described in more detail below, show by color in each model cell, for each hour, the total new radical production, and in another movie the odd-oxygen production, and in a third movie the total termination product formation (i.e., the beginning, middle, and end of the chemical cycles). Movies of species concentration fields, show $\mathrm{O}_{3}, \mathrm{O}_{\mathrm{x}}, \mathrm{NO}, \mathrm{NO}_{2}, \mathrm{CO}$, and $\mathrm{VOC}$ mixing ratios in each cell each hour. These movies are useful combined in a spatial and temporal analysis. These will be described in more detail below. These movies were produced by data visualization software on Macintosh computers in combination with Apple's QuickTime movie extensions for both Macintosh and MS-Windows on DOS PCs. Each movie is small-about 350 kilobytes-and can be easily played by anyone with MicroSoft Windows.

2. IPRA time series plots that show a time series of the mixing ratio of selected species (e.g., $\mathrm{NO}, \mathrm{NO}_{2}, \mathrm{O}_{3}, \mathrm{CO}$, and total VOCs) along with the time series of the change produced each hour by chemistry, net horizontal transport, net vertical transport, elevated and low level emissions, and deposition. These plots have been typically produced by common PC spreadsheet programs using ASCII text files that are imported into the spreadsheet program.

3. IPRA process composition plots and movies. In this analysis, the composition of a selected species in a given cell at a particular time is expressed in terms of the following sources:

(a) Initial-here-the fraction or mixing ratio that was initially present in the target cell (and still remains)

(b) Initial-other-the fraction or mixing ratio that was initially present in some other cell and was subsequently transported to the target cell

(c) Emitted-here-the fraction or mixing ratio that was emitted into the target cell (and still remains)

(d) Emitted-other-the fraction or mixing ratio that was emitted into some other cell and was subsequently transported to the target cell

(e) Chemistry-here-the fraction or mixing ratio that was chemically formed in the target cell (and still remains)

(f) Chemistry-other - the fraction or mixing ratio that was chemically formed in some other cell and was subsequently transported to the target cell

(g) Boundary-horizontal - the fraction or mixing ratio that was transported through a N, S, E, or W domain boundary face and was subsequently transported to the target cell 
(h) Boundary-vertical - the fraction or mixing ratio that was transported through a top or bottom boundary face and was subsequently transported to the target cell.

The simplest form of this output is an area chart for the composition in the target cell over time. Movies, one for each process (initial, emission, chemistry, vertical boundary, and horizontal boundary), which show the spatial and temporal distribution of the process contribution to a selected target cell are the most complex form of output for this analysis. Note that it is possible to reverse this analysis and report the contribution of a particular source to all affected cells. The data processing for this analysis has typically occurred on large memory PCs with visualization via PC spreadsheet tools.

4. Integrated Reaction Rate or IRRMB process analysis is applied to just the chemical transformation change. It is usually presented in the form of time series, piecharts, flowcharts, and systems diagrams. These outputs include:

(a) Time series of new radical strength and pie charts of source composition

(b) Time series and average hydroxyl radical chain length, or number of times each hydroxyl radical is used before being lost; time series and average NO chain length, or number of times each $\mathrm{NO}$ is oxidized to $\mathrm{NO}_{2}$ before being lost as a nitrogen product

(c) The amount of VOC consumed by hydoxyl radicals, by $\mathrm{O}_{3}$, or by photolysis reactions, and the average number of NO-to- $\mathrm{NO}_{2}$ conversions per VOC reacted

(d) The amount of $\mathrm{O}_{3}$ produced by each $\mathrm{VOC}$ reacted

(e) The total odd-oxygen production, the average $\mathrm{O}_{3}$ yield per $\mathrm{NO}_{2}$ photolysis, and the total amount of $\mathrm{O}_{3}$ produced

(f) The $\mathrm{VOC}$ and $\mathrm{NO}_{x}$ propagation factors and the distribution of termination products

(g) The amount of $\mathrm{O}_{x}$ and $\mathrm{O}_{3}$ formed by each VOC in the system

(h) Detailed VOC and nitrogen mass balances.

PCs or workstations were used to do the data processing for this analysis, which has resulted in a collection of 12 ASCII tables being produced. Selected data from these tables are manually entered into spreadsheets or onto plots produced via PC/Mac Drawing programs.

Example output from some of these analyses will be briefly described below.

\subsubsection{Example Analyses Results}

The purpose of this section is to demonstrate our recent success in unraveling problems and finding errors in applications of air quality models. We have conducted this work as part 


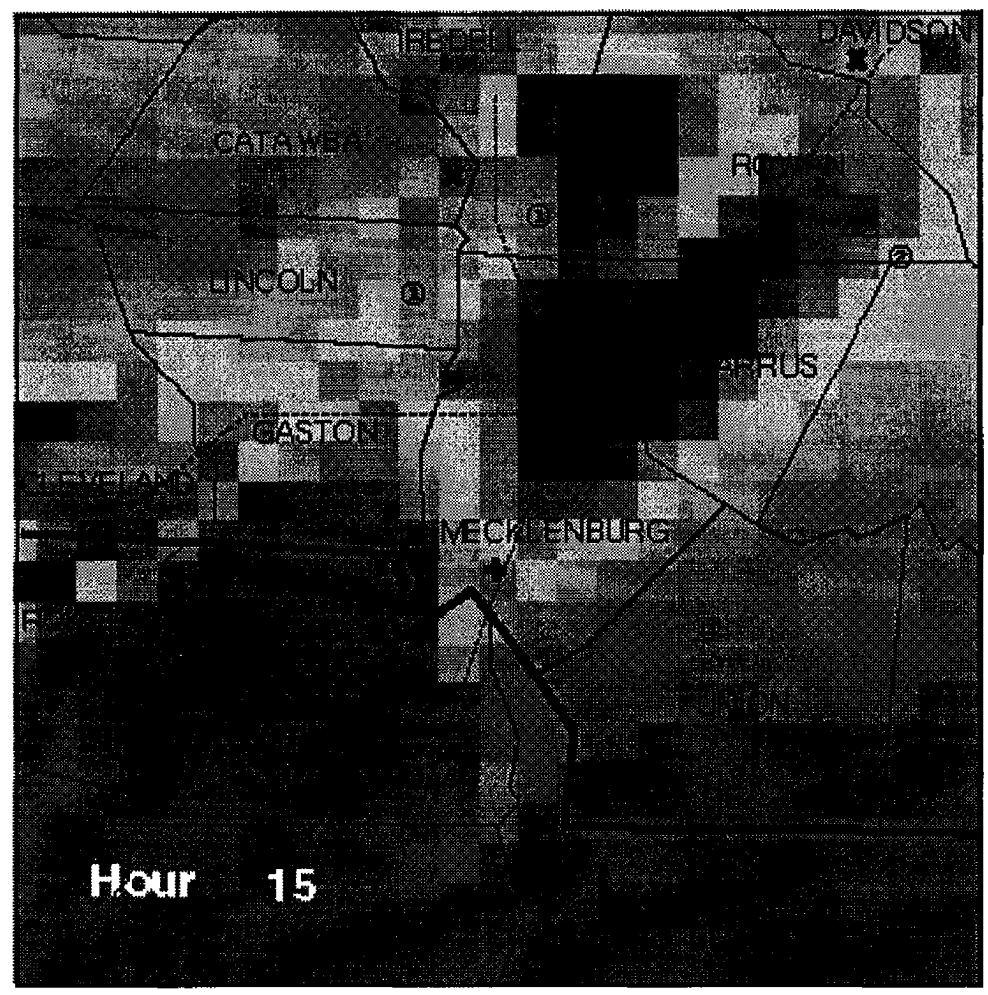

Figure 7.3:. One frame from Day Two of a UAM movie of ozone mixing ratios for a 1988 Charlotte, North Carolina episode. The darkness is not proportional to $\mathrm{O}_{3}$ in this figure and deep blue (for no $\mathrm{O}_{3}$ ) and bright red (for $\mathrm{O}_{3}$ equal to or exceeding $120 \mathrm{ppb}$ ) both show as dark colors.

of our work on a EPA cooperative agreement; work with the North Carolina Department of Environment, Health, and Natural Resources on the state's request for redesignation for Charlotte North Carolina; and work for this DOE project.

\section{Movies of Concentrations}

We cannot show you movies here, but they are available for Macintosh and MS Windows 3.1 computers with color monitors having at least VGA resolution and 256 colors. The QuickTime Movie viewer software is available free from Apple Computer's WWW Site and example UAM movies of concentration fields and selected process magnitude fields are available free from Jeffries' WWW and ftp site at $f t p:$ / / airsite. unc . edu.

The movies have one frame for each hour of a 24-h simulation and play in 24 s. Often of more value is the ability to stop the movie play and "rock back and forth" over a series of frames to gain insight into what changed over the time interval. Figure 7.3 is a grayscale rendition of one frame from the $\mathrm{O}_{3}$ mixing ratio movie for day two of a 1988 Charlotte, North Carolina episode basecase simulation. Although the color transformations to grayscale are not linear, this frame still shows the essential features of the UAM movies: 
- The total view is 25 by 25 cells centered on downtown Charlotte;

- The cell size is 5 by 5 kilometers and is clearly visible in the figure

- County lines are marked in black and the NC-SC state line is marked in bold black

- the dotted lines are the approximate locations of two Interstate highways; the one that runs diagonal is I85 and the one that runs N-S is I77

- the legend for map symbols is:

- The three +-marks are the sites of the monitoring stations in Charlotte ( $\mathrm{S}$ to $\mathrm{N}$, Arrowwood, Plaza, and County line)

- The four $\boldsymbol{*}$-marks are the sites of Duke Power's fossil fuel power plants

- The (1)-mark in Lincoln county is the future site of Duke Power's gas turbine station for meeting peak power demand (this was not present in the 1988 base case, but was in the future 1999 and 2005 cases)

- The (1)-mark in Iredell county is a natural gas pumping station

The (2)-marks are smaller low level $\mathrm{NO}_{x}$ sources.

The movies are 385 by 385 pixels and two movies can be played side by side on a standard VGA monitor. On a computer with large monitors, as many as four movies can be played at the same time. By comparing the movie frames of species mixing ratios (e.g., $\mathrm{O}_{3}$ ) with movie frames of lumped processes, such as total odd oxygen production per hour per cell, one can see spatial and temporal relationships that would be difficult to achieve any other way. For example, by showing total odd oxygen production locations a few hours before the peak $\mathrm{O}_{3}$ time, we can determine the most important sources that should be controlled to reduce the $\mathrm{O}_{3}$ maximum.

\section{IPRA Time Series}

Figure 7.4 shows process analysis time series plots for $\mathrm{NO} \mathrm{NO}_{2}, \mathrm{VOC}$, and $\mathrm{O}_{3}$ for a Charlotte UAM scenario in 1987. These are probably the outputs that are the most useful to nonmodelers. In this example, the IPR data values were aggregated for a $6 \times 6$ cell region that included both the Arrowwood and Plaza monitors for this analysis. The 1987 episode was very stagnant and the UAM was having problems predicting accurate $\mathrm{O}_{3}$ mixing ratios in areas south of the center city; e.g., see the large late increase in $\mathrm{O}_{3}$ in Figure 7.4. The measured peak maximum for this day was $131 \mathrm{ppb}$ (see Fig. 7.6)

Nitric Oxide: In the NO processes plot, we see that emissions in the $6 \times 6$ area increased the NO mixing ratio by $57 \mathrm{ppb}$ over the time period 500-1900 LDT and only $4.6 \mathrm{ppb}$ came from elevated sources. Chemistry processes were the major consumer of NO, removing $45 \mathrm{ppb}$ NO over the day. Vertical transport was an important process for NO in the morning hours, but decreased to small values during the afternoon when the NO mixing ratio was being driven to low values by the chemistry processes. 

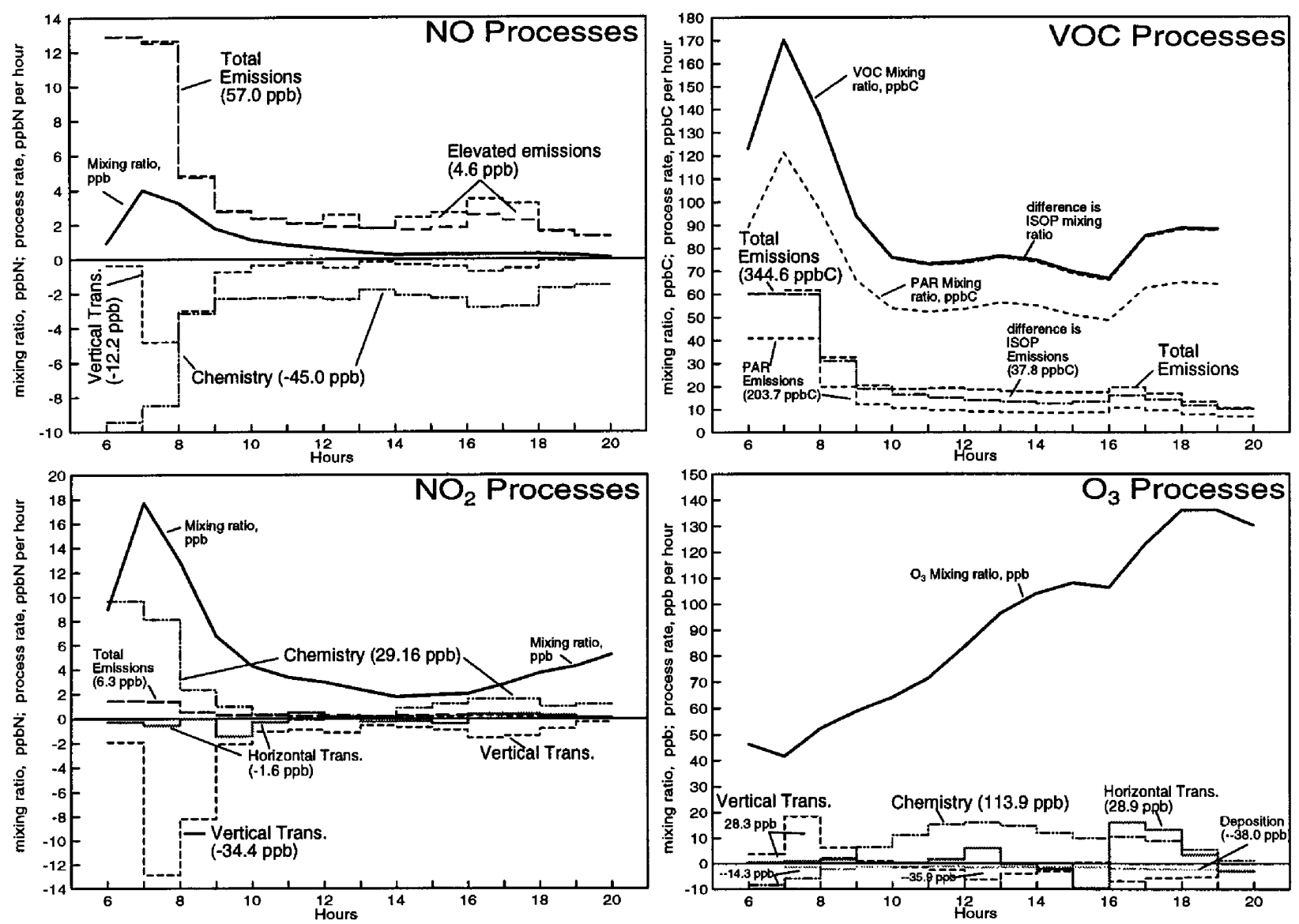

Figure 7.4: Process time series for Charlotte 1987 episode.

Nitrogen Dioxide: In the $\mathrm{NO}_{2}$ processes plot, we see that $\mathrm{NO}_{2}$ emissions were a much smaller contributor to $\mathrm{NO}_{x}$, only $6.3 \mathrm{ppb}$. Chemistry contributed the large majority of the $\mathrm{NO}_{2}, 29.6 \mathrm{ppb}$, but note that the increase in $\mathrm{NO}_{2}$ was less than the NO decrease because of chemistry. Vertical transport of $\mathrm{NO}_{2}$ removed $34 \mathrm{ppb}$ of $\mathrm{NO}_{2}$ from layer one. In keeping with the view that this was a relatively stagnant episode, horizontal transport of $\mathrm{NO}_{2}$ was very low, only $-1.6 \mathrm{ppb}$.

VOCs: In the VOC process plot in Figure 7.4, we see that total emissions were $344.6 \mathrm{ppbC}$. Combined with the $\mathrm{NO}_{\mathrm{x}}$ emissions, this gives a morning peak emissions VOC-to- $\mathrm{NO}_{\mathrm{x}}$ ratio of 5.4:1. The model VOC-to-NO $\mathrm{N}_{\mathrm{x}}$ ratio based on mixing ratios, however, was 7.7:1. The difference was due to carryover VOC from the day and night before. Note that the isoprene emissions into the $6 \times 6$ grid were $37.8 \mathrm{ppbC}$, yet its contribution to the $\mathrm{VOC}$ mixing ratio was almost undetectable. Another time series process analysis not shown here revealed that chemical loss accounted for $70 \%$ and that vertical transport accounted for $29 \%$ of the isoprene emissions. Note that in the middle of the day, isoprene emissions were about 


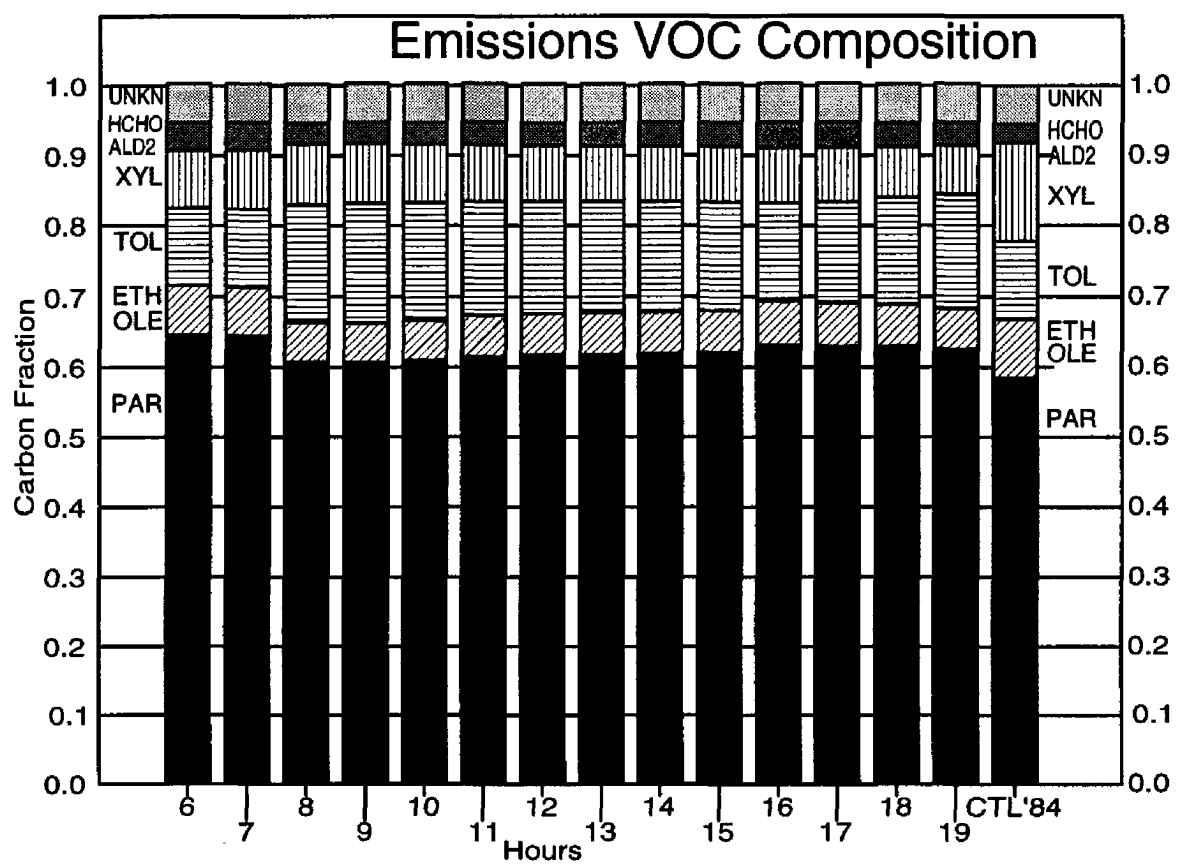

Figure 7.5:. Model Predicted and Observed VOC composition in 6 by $65-\mathrm{km}$ cells near downtown Charlotte North Carolina in 1987 UAM scenario. The bars labeled with hours are the model's composition, the last bar on right is the average from EPA ambient VOC canister sampling program. Note the difference in toluene and xylene.

one-fourth of the total VOC emissions. If isoprene is removed from the total VOC emissions, the anthropogenic-VOC-to-NO ratio would be $4.7: 1$, which is a factor of 1.6 lower than that based on the mixing ratios. This shows some of the difficulty in attempting to compare model predictions with ambient observations and blaming the differences on the emissions inventory.

Before we leave the issue of VOC emissions, let us illustrate another problem that process analysis found in the the UAM inputs. Figure 7.5 shows a bar chart of VOC composition for each hour in the 1987 Charlotte UAM scenario. At the right end of the chart, we show the average composition that was determined from a 6-9 a.m. canister sampling program (ambient observations) that was conducted in Charlotte in 1984 as part of a larger EPA program. Although there is remarkably good agreement for the classes paraffins, olefins, and aromatics, within the aromatics class there is a large difference: the TOL to XYL ratio in the model is backwards compared to the observations. In other words, the model's TOL was about twice the model's XYL. This disagreement prompted us to conduct a detailed examination of TOL-to-XYL ratios in the emissions input files, which led to the discovery that the speciation for all the area surface coatings and solvents were computed with a single species profile by EPS 2.0, the EPA-approved UAM emissions processor. Subsequent investigation of this profile revealed that it was based on only four paint samples from Los Angeles that were taken in the late 1970s and these samples were very high in xylene and very low in toluene. EPA's Emissions Inventory Group has been informed of this error and 


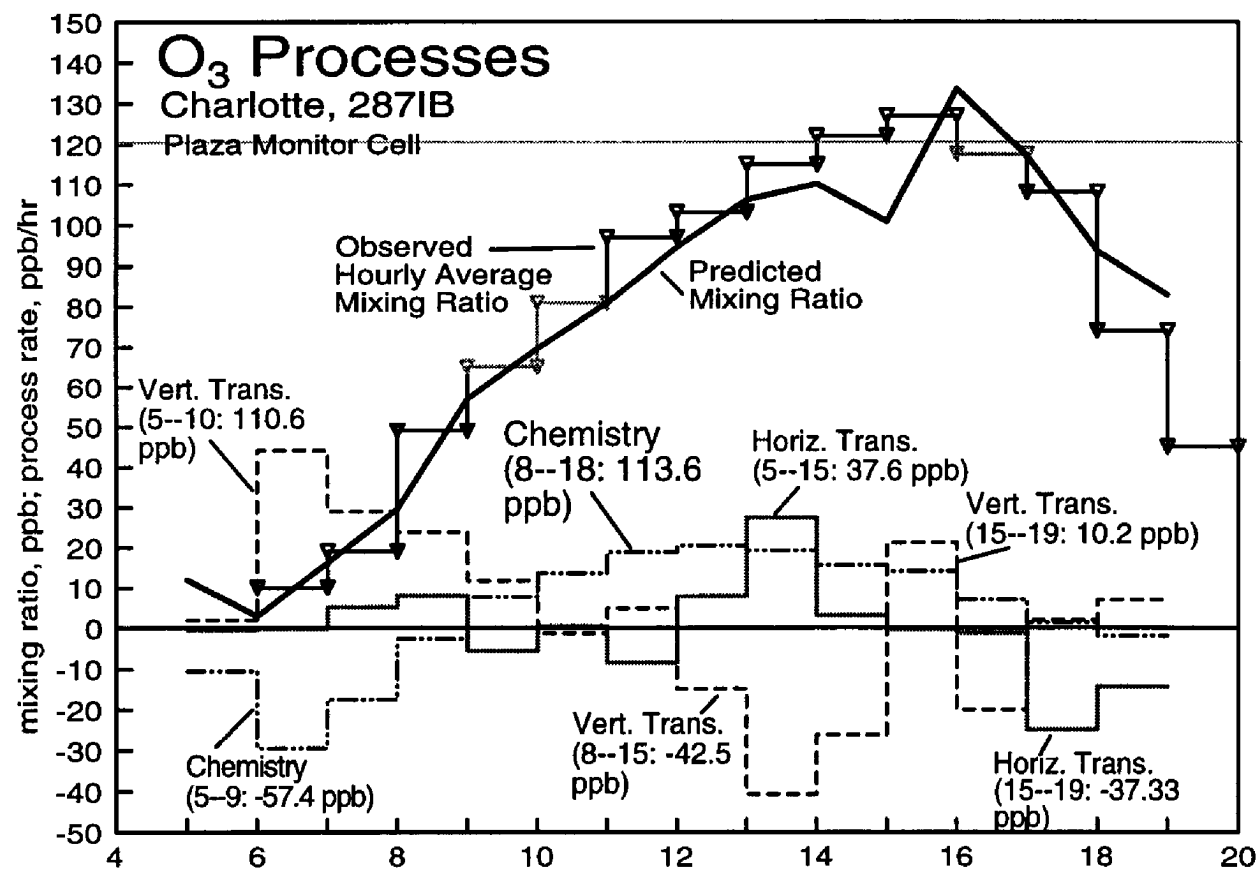

Figure 7.6: UAM predicted and observed $\mathrm{O}_{3}$ mixing ratio and the change caused by each process in the UAM.

they are now creating new speciation profiles based on newer data.

Ozone: In the $\mathrm{O}_{3}$ process plot in Figure 7.4, we see that the early morning source of $\mathrm{O}_{3}$ was vertical transport from aloft. Chemistry was a negative contributor to $\mathrm{O}_{3}$ during this time period as the freshly emitted NO titrated the downward mixed $\mathrm{O}_{3}$. After $0900 \mathrm{LDT}$, chemistry became a producer of $\mathrm{O}_{3}$, and remained positive until sunset, producing a total of $113.9 \mathrm{ppb}$ of $\mathrm{O}_{3}$. After chemistry started to produce $\mathrm{O}_{3}$, vertical transport out of the layer one cell became a loss process. Deposition of $\mathrm{O}_{3}$ was a significant loss process, consuming about $38 \mathrm{ppb}$ of $\mathrm{O}_{3}$. Until 1600 LDT, horizontal transport of $\mathrm{O}_{3}$ was negligible, but at $1600 \mathrm{LDT}$ in this scenario, horizontal transport brought $29 \mathrm{ppb}$ of $\mathrm{O}_{3}$ into this $6 \times 6$ cell area that already had the highest $\mathrm{O}_{3}$ mixing ratio in the model. This additional $\mathrm{O}_{3}$ increased the $6 \times 6$ cell's $\mathrm{O}_{3}$ mixing ratio from $105 \mathrm{ppb}$ to $135 \mathrm{ppb}$. This was in dramatic contrast to the observed values reported by the two monitors located within the $6 \times 6$ cells, which showed decreases of $\mathrm{O}_{3}$ during this time period (see Figure 7.6). Although horizontal transport was increasing the cell's mixing ratio, vertical transport was removing less than half the increase and chemical production was still increasing the mixing ratio by about $20 \mathrm{ppb}$. Individual cells within the $6 \times 6$ region showed even more dramatic effects-as much as an $80 \mathrm{ppb}$ increase in $\mathrm{O}_{3}$ from 1600 to 1700 LDT, producing a peak $\mathrm{O}_{3}$ mixing ratio of $170 \mathrm{ppb}$ (see Figure 7.7).

Because process analysis plots made it clear that horizontal transport was responsible for this large increase, the incorrect behavior of the model was traced to problems in the 
Diagnostic Windfield Model (DWM). Inappropriate interpolation of the hourly monitored wind data was producing artificial convergence. Numerous experiments with the DWM were unable to resolve this problem. Eventually, it was necessary to use manual "objective analysis" of the wind data to obtain any reasonable predictions.

Other Wind Problems Figure 7.6 shows a process time series plot for $\mathrm{O}_{3}$ for a single surface cell (the one containing the $\mathrm{Plaza}_{3}$ monitor-the middle cross in Figure 7.3) and it reveals another problem we have repeatedly observed in UAM simulations-a switch in the direction of vertical transport when there is a major change in wind direction in the UAM. In this plot, observe that vertical transport was first positive into the lower layer during the morning hours as the mixing height was rising and $\mathrm{O}_{3}$ from aloft was being brought down. Once the cell began to produce $\mathrm{O}_{3}$ chemically (after 1000 LDT) vertical transport became strongly negative, carrying the higher concentration $\mathrm{O}_{3}$ in the lowest cell upward; i.e., until the hour 1500-1600 LDT in which suddenly vertical transport was a source of $\mathrm{O}_{3}$ in the lowest cell. At 1600 LDT, the vertical transport once again returned to its former nature. This one hour of positive vertical $\mathrm{O}_{3}$ transport caused a significant "glitch" in the $\mathrm{O}_{3}$ mixing ratio time series, resulting in the over-prediction and a predicted excedance of the NAAQS for this cell. What happened during the hour starting at 1500? The wind, which in the UAM is held constant for each hour and is only changed on the hour, made a major change in direction at 1500 LDT. Every UAM scenario we have run in which the wind made a large shift in direction in the afternoon has exhibited this vertical transport transient behavior. Because the winds in each layer of UAM are independent of each other, and the model attempts to conserve mass by means of a calibration gas tracer species, the rapid shift in winds from one time step of the model to the next results in an artifact in vertical transport to compensate for the dynamic imbalance in the model.

\section{IPRA Process Composition}

Figure 7.7 shows a "process composition time series" plot for $\mathrm{O}_{3}$ in a single cell (the one containing the Arrowwood $\mathrm{O}_{3}$ monitor) in the 287FC UAM simulation for Charlotte, North Carolina. By "process composition" we mean that we have effectively "colored" the $\mathrm{O}_{3}$ by the type of process that gave rise to the $\mathrm{O}_{3}$ that is still in the cell at a particular time. At the beginning of the analysis time, all the $\mathrm{O}_{3}$ in the cell would be from the "initial process". As time proceeds and the meteorological processes cause vertical cell growth and horizontal mass flow, this "initial $\mathrm{O}_{3}$ " decreases and $\mathrm{O}_{3}$ with other process origins are brought into the cell. For example, in the top plot of Fig. 7.7 we see that vertical transport from layer two into the layer one cell began to increase the cell's $\mathrm{O}_{3}$ at $0600 \mathrm{LST}$ (at the same time, this decreased the mixing ratio of the "initial $\mathrm{O}_{3}$ " because of dilution). Also at the same time, $\mathrm{O}_{3}$ that originated somewhere to the west of the Arrowwood cell was being transported into the Arrowwood cell. At $0700 \mathrm{LST}$, transport also began to contribute $\mathrm{O}_{3}$ from the north of the Arrowwood cell. We also see from the plot that after 1500 LST, there was a very large 

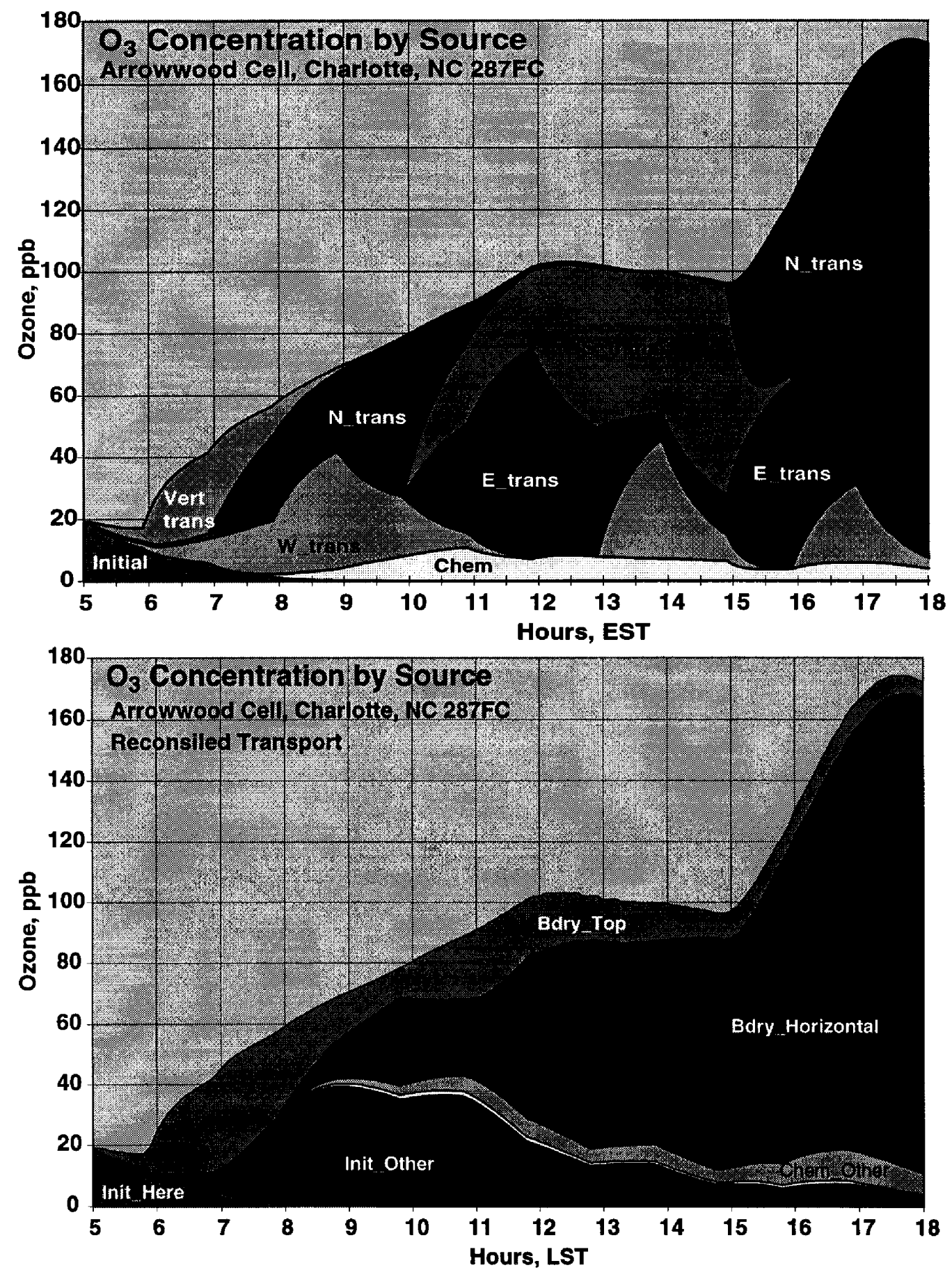

Figure 7.7:. Process composition times series for $\mathrm{O}_{3}$ in Episode 287FC Charlotte, North Carolina UAM simulation. TOP: Sources of $\mathrm{O}_{3}$ by process in Arrowwood Cell; BOTTOM: Sources of $\mathrm{O}_{3}$ by process in Arrowwood Cell with transport sources replaced by original sources. 
amount of $\mathrm{O}_{3}$ transported from the north which was responsible for the large and rapid rise in $\mathrm{O}_{3}$ from about $100 \mathrm{ppb}$ to over $170 \mathrm{ppb}$.

These process composition plots are possible because each cell in an Eulerian model is well-mixed. Therefore, they can be treated as a continuous stirred tank reactor (CSTR), which is a chemical engineering term for a common type of flow-through reactor. In a CSTR, just as in an Eulerian model cell, mass flows into the reactor volume, mass flows out of the reactor volume, and reactions occur in the volume. By performing a mass balance on the cell and using the mass changes by process from the IPR file for the cell as a function of time, we can develop the fraction of mass in a cell that was accumulated from each source (details of how to carry out these calculations are available in [35]). By keeping such a process composition on every cell and by replacing the transported-in mass with the process composition of the up-flow cell, that is, resolve the mass of material transported in a cell into its process composition by applying to it the composition of the material in the cell from which the transport is occurring, thus, eliminating all transport terms. When such calculations are carried out at each timestep, we can obtain a new process composition time series plot that looks like the bottom plot in Figure 7.7.

In the bottom plot, there is no mass labeled with transport directions. Instead the original process origins of the transported masses have been identified and these have been collected into groups of origin. For example, "Init_Other" in the plot represents $\mathrm{O}_{3}$ that was initial $\mathrm{O}_{3}$ in one of the other $6 x 6$ cells at the start of the analysis. "Bdry_Top" represents $\mathrm{O}_{3}$ that came through the layer one top boundary in any of the $6 x 6$ cells and "Bdry_Horizontal" represents $\mathrm{O}_{3}$ that came from outside the $6 \times 6$ cells.

This latter plot shows that until after 1200 a large amount of $\mathrm{O}_{3}$ in this cell came from $\mathrm{O}_{3}$ that was initially present in the $6 x 6$ region at the start of the day or from $\mathrm{O}_{3}$ that was aloft ("old $\mathrm{O}_{3}$.")

In Figure 7.8 we take a different view of this same $\mathrm{O}_{3}$. These "bar" plots show the spatial distribution of the origins of the $\mathrm{O}_{3}$ that was in the Arrowwood cell at the indicated times. For this plot, the identity of the the original process that created the $\mathrm{O}_{3}$ in the other cells has been neglected. Here, we are only interested in the cell location where the $\mathrm{O}_{3}$ originated, not the process that created it. Thus, at $0700,55 \%$ of the $\mathrm{O}_{3}$ in the Arrowwood cell originated in the Arrowwood cell (and from Figure 7.7 we know that this would have been from initial $\mathrm{O}_{3}$ and from $\mathrm{O}_{3}$ transported vertically down into the Arrowwood cell. The rest of the $\mathrm{O}_{3}$ in the Arrowwood cell had come from the north and west as shown by the height of the bars to the west and north of the Arrowwood cell. By 0830, the spatial origins of the $\mathrm{O}_{3}$ in the Arrowwood cell included most of the $6 \times 6$ cell region to the west and north, and significant amounts of $\mathrm{O}_{3}$ had started to come from regions outside the $6 x 6$ cells (e.g., there was $7.2 \%$ of the Arrowwood cell's $\mathrm{O}_{3}$ from the west and $4.4 \%$ from the north of the $6 \times 6$ cell area). 


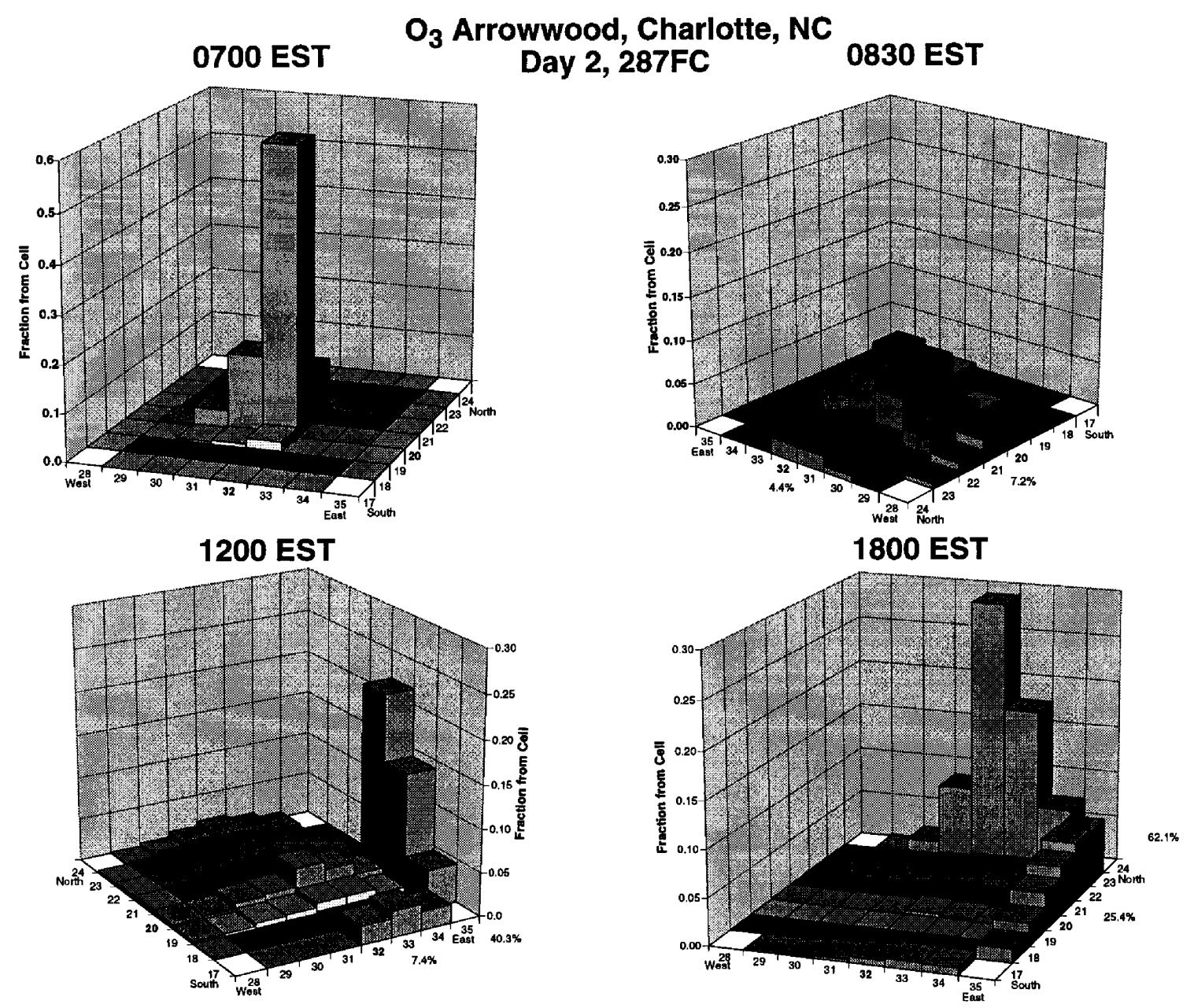

Figure 7.8:. Process composition spatial contribution to a single cell's $\mathrm{O}_{3}$ in Episode 287FC Charlotte, NC UAM simulation. Each 3-D plot shows the contribution of all processes in each cell of a $6 \times 6$ cell area and from outside the $6 \times 6$ cell area to Arrowwood cell's $\mathrm{O}_{3}$ at the hour indicated. At hour $1200 \mathrm{LST}$, for example, $49 \%$ of the $O_{3}$ in the Arrowwood cell was from processes that occurred in one of the $6 \times 6$ cells and the rest was from transport from outside the $6 x 6$ cell region. For the latter, $40.5 \%$ was transported from the east and $7.4 \%$ was transported from the south.

\section{IRR Chemical Process Analysis}

This type of analysis is applied to the chemical transformations within model cells or aggregated model cells using the IRR data from the chemical reaction mechanism included in the air quality model. Details on how this analysis works are in the literature [1,26-28]. Basically we have adopted an abstract or systems approach, recognizing two inter-acting cycles (the $\mathrm{OH}$ radical cycle and the NO oxidation cycle (see Figure 7.9), within a positive feedback loop (the photolysis of $\mathrm{O}_{3}$ provides about one-half of the new radicals in the system). Radicals are accounted for via initiation, propagation, and termination processes. Oxides of 


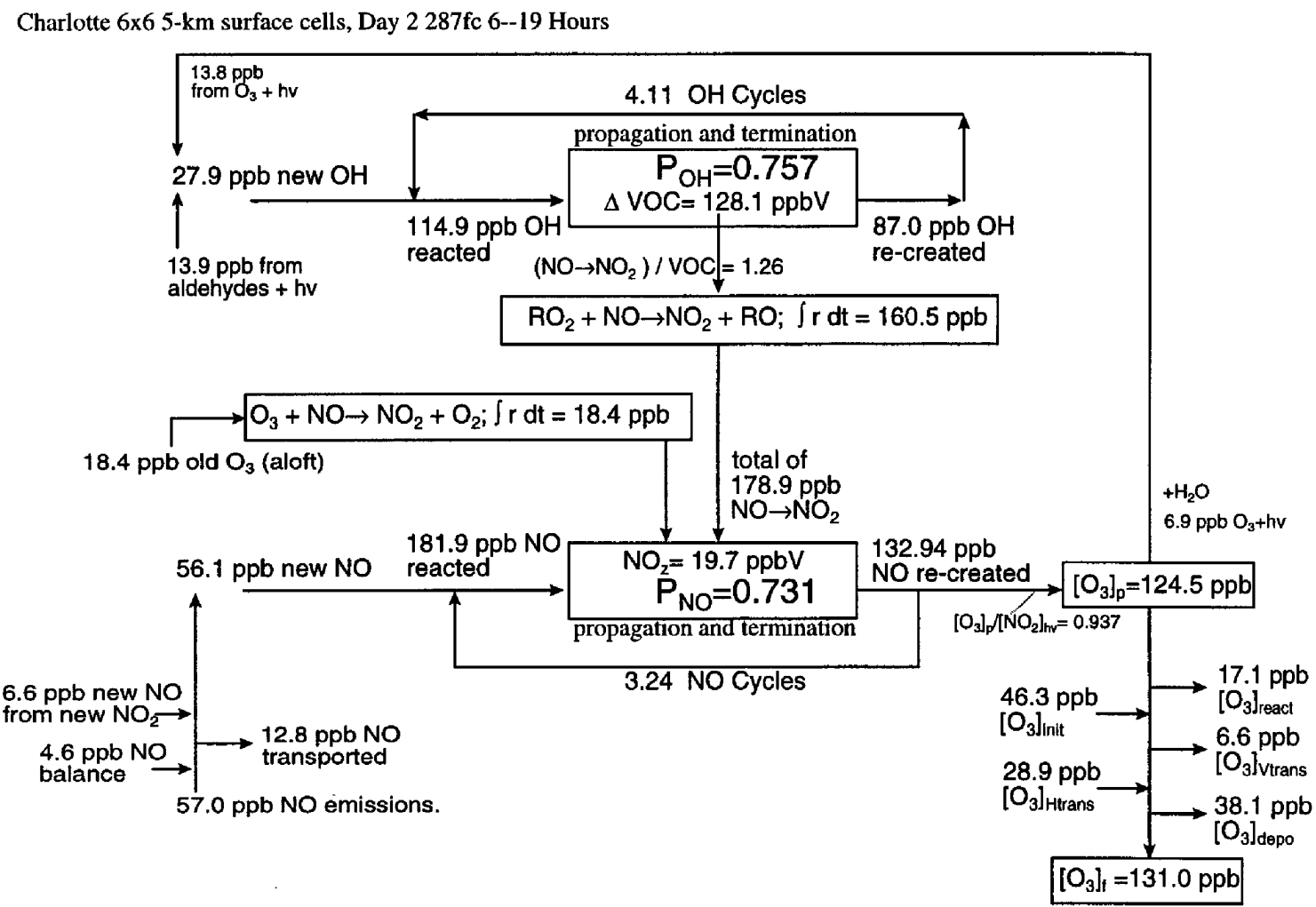

Figure 7.9:. The ${ }^{\circ} \mathrm{OH}$ and $\mathrm{NO}_{x}$ Cycles from Integrated Reaction Rates for $6 x 6$ Cell Area in UAM Scenario $287 \mathrm{FC}$ for Charlotte, NC.

nitrogen are accounted for via emissions, oxidation, and terminal product production. The two cycles are coupled via odd-production by reactions such as

$$
\begin{aligned}
& \mathrm{HO}_{2}+\mathrm{NO} \longrightarrow \mathrm{NO}_{2}+\mathrm{OH} \\
& \mathrm{RO}_{2}+\mathrm{NO} \longrightarrow \mathrm{NO}_{2}+\mathrm{RO},
\end{aligned}
$$

which is necessary for both the radical and nitrogen cycles to continue.

This systems process view introduces several important new parameters that can be used to classify and compare the chemistries of different scenarios for the same model, the same scenario for different models, or different locations, scenarios, and models. Some of these new parameters are:

- new radical source strength-new radicals are formed by photolysis of organic (mostly aldheydes) and inorganic (mostly $\mathrm{O}_{3}$ ) species that are both present initially and are formed via the oxidation cycles. We have found that for urban simulations, about half the radicals come from organic and about half come from inoganic sources. That is, $\mathrm{O}_{3}$ is very important in producing more $\mathrm{O}_{3}$ (see details in [27]). In Figure 7.9 there New York City in a RADM simulation, there was $34.1 \mathrm{ppb}$ new $\mathrm{OH}$ produced.

- $\mathrm{OH}$ propagation factor and chain length-each new radical created above is used, recreated, and used again in a chain oxidation process. We measure the fraction of 


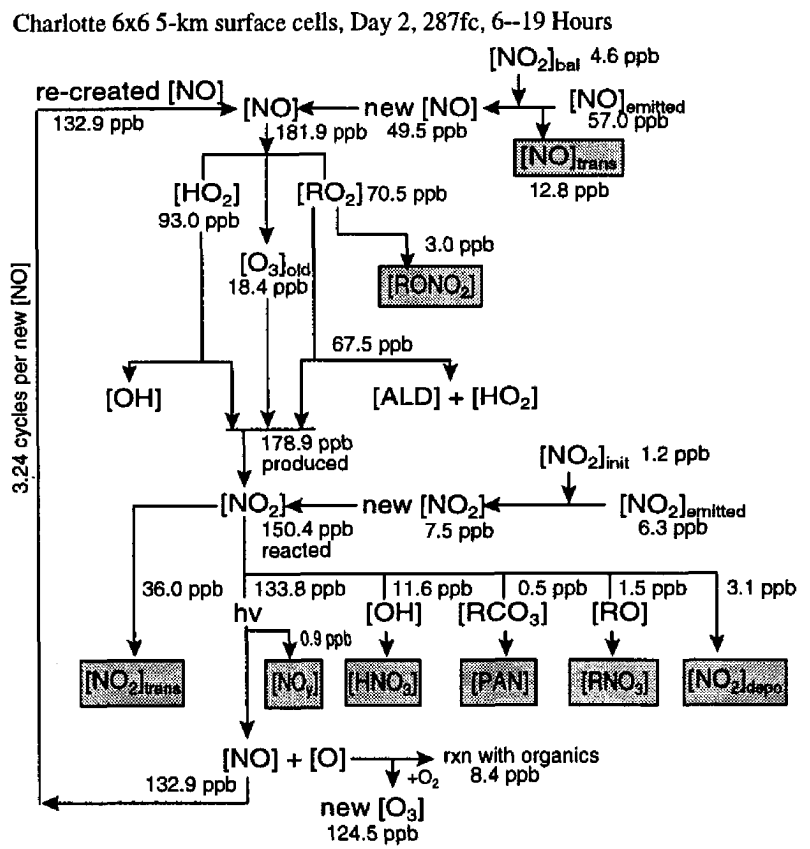

Figure 7.10:. The NO Cycle and Mass Balance from Integrated Reaction Rates for $6 x 6$ Cell Area in UAM Scenario 287FC for Charlotte, NC.

$\mathrm{OH}$ radicals that make it all the way through the chain each cycle; this is called the $\mathrm{OH}$-propagation factor. We have found that this is usually about 0.75 regardless of chemical mechanism; e.g., in the New York City RADM simulation $\mathbf{P}_{r}$ was 0.747 giving 3.96 cycles per $\mathrm{OH}$. The throughput for the CB4 mechanism in Charlotte translates into 4.11 cycles for each $\mathrm{OH}$ before being lost in termination. The reacted VOC in Charlotte for a $36^{2} \mathrm{~km}$ area was $128.1 \mathrm{ppbV}$, where as for New York City for RADM for a $20^{2} \mathrm{~km}$ area this was $112.9 \mathrm{ppb}$.

- NO propagation factor and chain length-similar to $\mathrm{OH}$-propagation factor, each $\mathrm{NO}$ is oxidized to $\mathrm{NO}_{2}$, and re-created via photolysis to be reoxidized again. Some reactions remove $\mathrm{NO}$ and $\mathrm{NO}_{2}$, leading to less than $100 \%$ throughput. We have found that in most models this factor is about $0.73-0.78$, or each NO is used about 3.2 to 4.4 times before being lost. New NO (or NO emissions) in the $36^{2} \mathrm{~km}$ area in Charlotte were $56 \mathrm{ppb}$ for the day, whereas in New York City for a $20^{2} \mathrm{~km}$ area they were $47.9 \mathrm{ppb}$.

- NO oxidations per VOC consumed - this is the total number of NO's oxidized divided by the total amount of primary VOC reacted and is a measure of the "reactivity" of the VOCs in the model. This value was 1.84 for the RADM mechanism in New York City for fresh VOC emissions, but as shown in Figure 7.9 the value was only 1.26 for the CB4 mechanism in Charlotte. This was traced to the fact that on this second day of simulation in a stagnant episode, about one-fourth of the reacted VOC was older, aged VOC, which had a much shorter oxidation chain length and therefore fewer NO-to- $\mathrm{NO}_{2}$ 
conversions per chain.

- $\mathrm{O}_{3}$ produced per $\mathrm{NO}_{2}$ photolysis-not all of the atomic oxygen that is produced when $\mathrm{NO}_{2}$ photolyzes is converted into $\mathrm{O}_{3}$. In the New York City RADM simulation this fraction was 0.909 , compared to 0.937 in the UAM Charlotte simulation.

Except for methane, the products of the low-reactivity-compounds' reactions are not strong sources of new radicals; for example, $\mathrm{OH}+\mathrm{CO}$ produces $\mathrm{CO}_{2}$ and the alkanes produce longer chain aldehydes that have low photolysis rates. This is in contrast to products of the other high-reactivity-compounds in the model mixture-olefins, xylene, and emitted aldehydes - which are prolific sources of new radicals. So, in Figure 7.11, the low-reactive, higher-concentration species were the source of most of the $\mathrm{O}_{3}$, while the high-reactive, lower-concentration species were the source of most of the new radicals. This is why when the so-called low reactivity compounds are irradiated by themselves, the produce little $\mathrm{O}_{3}$. The alkanes (e.g., PARs), for example, produce aldehydes that are very slow at photolyzing; thus, alone with $\mathrm{NO}_{\mathbf{x}}$ these do not produce much ozone. In the presence of another species' $\mathrm{HCHO}$, however, the alkanes can produce significant $\mathrm{O}_{3}$. Thus, there is a synergistic interaction among the different VOC species in a mixture. Because in urban areas there is always a large amount of $\mathrm{CO}$ and methane that can easily utilize any $\mathrm{OH}$ radicals introduced, the source of new radicals becomes the most important factor for the production of ozone. At present, the aldehydes and dialdehydes either emitted, or produced when emitted VOCs are reacted-and maybe even left over from the day before-are the most important factors in explaining the origins of urban ozone.

From this brief comparison of IRR process parameters we can see that the downtown area of Charlotte, North Carolina has chemistry just as intense as the center of New York City. A major difference, however, is that Charlotte only has only one area with this intensity, whereas the next area downwind in New York City was similar to the one described here and the one after that too. Thus, in Charlotte chemical processes decrease rapidly with distance from the center city, whereas in New York City these same processes are sustained at high levels over a much larger distance.

Figure 7.11 shows another type of output that can be produced by IRR/MB analysis. We have developed a set of history lists that are maintained on each reaction. Using these, we can "replace" intermediates that are formed as products in the reaction of the primary VOCs with the products of the reactions of the intermediates, thus eliminating the intermediate species. In this way we can obtain an accurate assessment of the contribution of each primary VOC to the total $\mathrm{O}_{3}$ production as is shown in the figure. In every case where we have performed this analysis, $\mathrm{CO}$, methane, and the paraffins-the least reactive species-have accounted for more than half the $\mathrm{O}_{3}$ produced.

For example, on the second day of an UAM simulation of a 1987 episode in Charlotte, one-fourth of the $\mathrm{O}_{3}$ was formed by reactions of $\mathrm{CO}$ and methane $\left(\mathrm{CH}_{4}\right)$, and another onefourth by reactions of the alkanes (PAR) in the mixture (see Figure 7.11, bottom). Methane has been considered essentially "unreactive" and CO is rarely discussed as a major source of urban ozone. Another one-forth of the ozone on Day 2 of this simulation was formed 
Table 7.3:. $\mathrm{NO}_{\mathrm{y}}$ Mass Balance from Integrated Reaction Rates for $6 x 6$ Cell Area in UAM Scenario 287FC for Charlotte. Units are parts per billion (ppbN). These results show a $4.8 \mathrm{ppb}$ gain in $\mathrm{NO}_{\mathrm{y}}$ mass over the course of the simulation. The error does not arise in the chemistry solver, but must be due to errors in the transport solver.

\begin{tabular}{|c|c|c|c|c|c|c|c|c|c|}
\hline \multirow{2}{*}{ Process } & \multicolumn{2}{|c|}{ No } & \multicolumn{2}{|c|}{$\mathrm{NO}_{2}$} & \multicolumn{2}{|c|}{$\mathrm{NO} y-\mathrm{NO}$} & \multicolumn{2}{|c|}{ NOy } & \multirow{2}{*}{ sum } \\
\hline & Sources & Sinks & Sources & Sinks & Sources & Sinks & Sources & Sinks & \\
\hline Initial Conc. & 0.93 & & 8.97 & & 7.18 & & 17.08 & 0.00 & 17.08 \\
\hline Emissions & 57.01 & & 6.28 & & 0.00 & & 63.29 & 0.00 & 63.29 \\
\hline $\begin{array}{l}\text { Net Horizontal Trans } \\
\text { Net Vertical Trans }\end{array}$ & & $\begin{array}{r}-0.63 \\
-12.17\end{array}$ & & $\begin{array}{r}-1.66 \\
-34.36\end{array}$ & $\begin{array}{l}1.44 \\
2.86\end{array}$ & & $\begin{array}{l}1.44 \\
2.86\end{array}$ & $\begin{array}{r}-2.28 \\
-46.53\end{array}$ & $\begin{array}{r}-0.84 \\
-43.68\end{array}$ \\
\hline Chemistry & 132.94 & -182.48 & 185.08 & -147.32 & 19.21 & -7.44 & 337.23 & -337.23 & 0.00 \\
\hline Deposition & & -0.06 & & -3.12 & & -13.59 & 0.00 & -16.77 & -16.77 \\
\hline Final Conc & & -0.11 & & -5.27 & & -9.31 & 0.00 & -14.69 & -14.69 \\
\hline $\begin{array}{l}\text { Totals } \\
\text { Source-Sink }\end{array}$ & $\begin{array}{r}190.87 \\
-4\end{array}$ & -195.44 & $\begin{array}{r}200.34 \\
8 .\end{array}$ & $\begin{array}{l}-191.73 \\
\end{array}$ & $\begin{array}{r}30.68 \\
0\end{array}$ & -30.34 & 421.90 & $9^{-417.51}$ & $\frac{4.39}{4.39}$ \\
\hline
\end{tabular}

from oxygenated compounds ( $\mathrm{HCHO}, \mathrm{RCHO}, \mathrm{MGLY}$, and PAN) left over from the oxidation of VOC emissions from the day before (this was a very stagnant case). Thus, the compounds that are commonly described as "reactive" (i.e., OLE, TOL, XYL, and the "natural" hydrocarbons, including isoprene) actually account for only about one-fourth of the chemical ozone production. This apparent contradiction-least reactive, most ozone produced-can be explained by recognizing that hydroxyl $(\mathrm{OH})$ radicals will react with any Voc proportional to its rate constant times its concentration. Most people have focused on the species rate constant and subsequent chemistry when declaring a species to be "reactive." In the scenario discussed here (see top pie chart in Figure 7.11), nearly two-thirds of the reacted carbon was $\mathrm{CO}$, methane, and alkanes; this was because their higher concentrations relative to the concentrations of the so-called "reactive" VOCs in the urban environment compensates for their lower reaction rate constants for reaction with $\mathrm{OH}$. Thus, the "less reactive" but much higher concentration VOCs compete very effectively for the available $\mathrm{OH}$ radicals and contribute significantly to urban ozone production.

This is due to large mass in the urban area, whereas the so-called most reactive species have small emitted masses and are rapidly consumed before producing much $\mathrm{O}_{3}$. The radicals produced by these so-called reactive species are what really do the work using the large mass of $\mathrm{CO}$, methane, and paraffins.

Finally, Table 7.3 shows a disturbing result of a full mass balance for $\mathrm{NO}_{\mathrm{y}}$ for this case. The total $\mathrm{NO}_{\mathrm{y}}$ sources plus initial $\mathrm{NO}_{\mathrm{y}}$ exceed the total $\mathrm{NO}_{\mathrm{y}}$ sinks plus final $\mathrm{NO}_{\mathrm{y}}$ by $4.39 \mathrm{ppb}$. We had expected the chemistry solver in UAM, which uses steady-state approximations, to be at fault, but to our surprise the chemistry process was balanced within $0.005 \mathrm{ppbN}$. Instead, the problem lies somewhere within the transport processes. A similar problem showed up in a St. Louis UAM simulation. EPA has given SAI, the UAM authors, a task order to investigate this problem. 


\section{Total VOC Reacted $=128.1 \mathrm{ppbV}$}
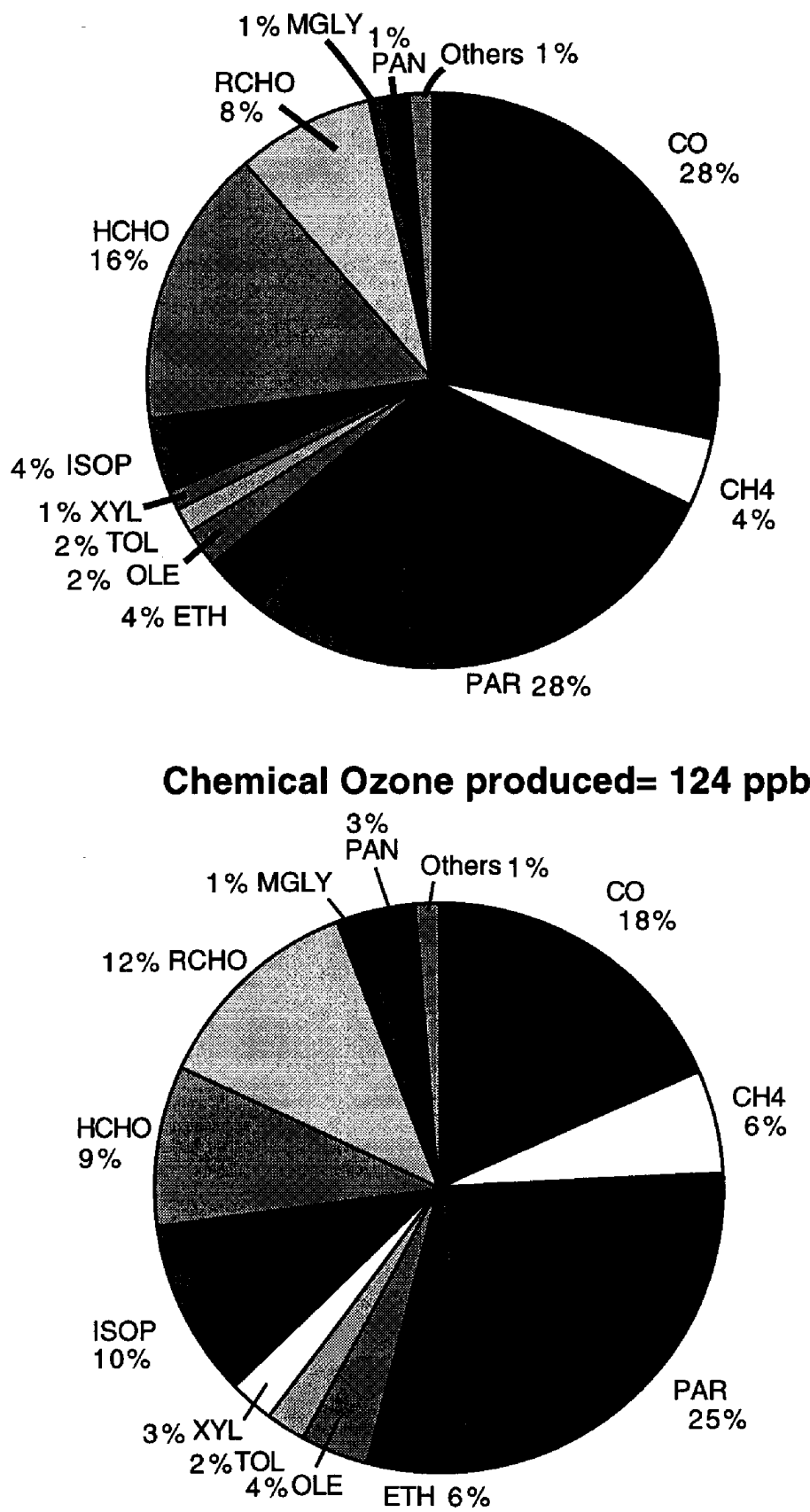

Figure 7.11:. The distribution of reacted voc and ozone produced in an Urban Airshed Model simulation of Charlotte, NC in 1987. The so-called "least reactive" vocs create more than half of the chemically produced ozone, because most of the reacted voc are these compounds due to their larger concentrations in urban areas. 


\section{Bibliography}

[1] Jeffries, H. E. "Photochemical Air Pollution," Chapter 9 in Composition, Chemistry, and Climate of the Atmosphere, Ed. H.B. Singh, Van Nostand-Reinhold, New York, ISBN 0-44201264-0, 1995.

[2] Atkinson, Roger, Aschmann, S. M., and Arey, J., "Formation of Ring-Retaining Products from the OH Radical-Initiated Reactions of o-, m-, and p-Xylene," Int. J. Chem. Kinet., 23, p77-97, 1991.

[3] Bierbach, A., Barnes, Ian, Becker, K.H., and Wiesen, E. (1994), "Atmospheric Chemistry of unsaturated carbonyls," Envr. Sci. Technol. 28, 715-729.

[4] Jeffries, H.E., Sexton, K.G., "The Relative Ozone Forming Potential of MethanolFueled Vehicle Emissions and Gasoline-Fueled Vehicle Emissions in Outdoor Smog Chambers," bound report prepared for Timothy Belian, Coordinating Research Council, Atlanta, GA, Final Report CRC Project No ME-1, Jan. 1995. Also available at ftp://airsite.unc.edu/pdfs/ese_unc/jeffries/reports/crcme1.

[5] Jeffries, H.E., Gery, M., Carter, W.P.L., "Protocols for Evaluating Oxidant Mechanisms For Urban and Regional Models," bound report Prepared for Marcia Dodge, U.S. Environmental Protection Agency, Research Triangle Park, N.C., EPA-600/R-92/112, 1992.

[6] Yu, J,Jeffries, H.E., La Lacheur, R.M., "Identifying Airborne Carbonyl Compounds In The Atmospheric Oxidation Products of Isoprene by Their PFBHA Oximes Using Gas Chromatography /Ion Trap Mass Spectrometry," Environ. Sci. Technol. 29(8), 1923, 1995.

[7] Cancilla, D. A.; Hee, S. S. Q. J. of Chromatography, 1, 627, 1992.

[8] Cancilla, D. A.; Chou, C. C.; Barthel, R.; Hee, S. S. Q., J. of AOAC Inter, 75, 842, 1992.

[9] Glaze, W. H.; Koga, M.; Cancilla, D., Environ. Sci. Technol., 23, 838, 1989.

[10] Le Lacheur, R. M.; Sonnenberg, L. B.; Singer, P. C ; Christman, R. F.; Charles. M. J., Environ. Sci. Technol., 27, 2745, 1993.

[11] Klotz, B.G., Bierbach, A., Barnes, I., and Becker, K.H., "Atmospheric Chemistry of Muconaldehydes," Envr. Sci. Technol. 29, 2322-2332, 1995

[12] Tuazon, E. C., Atkinson, R., Leod, H. M., Blermann, H. W., Winer,A. M., Carter, W. P. L., and Pitts, Jr., J. N., "Yields of Glyoxal and Methylglyoxal from the NOx - Air Photooxidations of Toluene and m- and p-Xylene," Envr. Sci. Technol. 18, 981-984, 1984 
[13] Bartolotti, L. and Edney, E. O., "Density Function Theory Derived Intermediates from the OH Initiated Atmospheric Oxidation of Toluene," Chem. Phys. Letters, 245, 119-122, 1995.

[14] McCann, J., Choi, E., Yamasaki, E., and Ames, B. N. ,"Detection of Carcinogens as mutagens in the Salmonella/microsome test: Assay of 300 Chemicals", Proc. Nat. Acad. Sci. USA, 72, 5135-5139, 1975.

[15] Knaap, G.A.C., Voogd, C. E., and Kramers, P. G. N., "Comparison of Mutagenic Potency of 2-Chlorethanol, 2-Bromoethanol, 1,2-epoxybutane, Epichlorhydrin and Glycidaldehyde in Klebsiella pneumoniae, Drosophila melanogaster and L51789 mouse Lymphoma Cells", Mutat. Res., 101, 199-208, 1982.

[16] Van Duuren, B. L., Langseth, L., Orris, L., Baden, M., and Kuschner, M., "Carcinogenicity of Epoxides, Lactones and Peroxy Compounds. V. Subcutaneous Injection in Rats", J. Natl. Cancer Inst., 39, 1213-1216 (1967).

[17] Golding, B. T., Kennedy, G., and Watson, W. P., "Structure Determination of Adducts from the Reaction of (R)-Glycidaldehyde and Guanosine", Carcinogenesis, 11, 865-868, 1990.

[18] Hemminki, K., "Nucleic Acid Adducts of Chemical Carcinogens and Mutagens", Arch. Toxicol., 52, 249-285 (1983).

[19] Segerback, D., "DNA Alkylation by Ethylene Oxide and Some Mono-substituted Epoxides", in DNA Adducts: Identification and Biological Significance, ed by Hemminki, K., Dipple, A., Shuker, D.E.G., Kadlubar, F. F., Segerback, D. and Bartsch, H., International Agency for Research on Cancer, Lyon. pp37-47, 1994.

[20] Ehrenberg, L., and Hussain, S., "Genetic Toxicity of Some Important Epoxides", Mutat. Res., 86, 1-113, 1981.

[21] Shepson, P.B., Kleindienst, T. E., Edney, E. O., Namie, G. R., Pittman, J. H., Cupitt, L. T., and Claxton, L. D., "The Mutagenic Activity of Irradiated Toluene $/ \mathrm{NO}_{x} / \mathrm{H}_{2} \mathrm{O} /$ Air Mixtures", Environ. Sci. Technol., 19, 249-255, 1985.

[22] Shiraishi, F., Hashimoto, S., and Bandow, H., "Induction of Sister-Chromated Exchanges in Chinese Hamster V79 Cells by Exposure to the Photochemical Reaction Products of Toluene Plus $\mathrm{NO}_{2}$ in the Gas Phase", Mutation Res., 173, 135-139, 1986.

[23] Cawlfield, J.E., “Using a Relational Database Management System with Emissions Inventory and Surrogate Files in North Carolina," Proceedings of the International Specialty Conference on Computing in Environmental Management, Air and Waste Management Association, 1995.

[24] Keating, T., Voyzey, J., Chaudhary, C., Brandmeyer-Cawlfield, J.E. and Jeffries, H.E., "Improving the Spatial and Temporal Resolution of Motor Vehicle Emission Inventories." presented at Vehicle Emissions and Air Quality Symposium, Center for Transportation Studies, University of Minnesota, Minneapolis July 26-28, 1995. 
[25] Keating, T., Cawlfield, J.E., Voyzey, J. Chaudhary, C., and Jeffries, H.E., "The Sensitivity of Urban Ozone Predictions to Assumptions in the Motor Vehicle Emission Inventory," presented at International Specialty Conference The Emission Inventory: Programs and Progress, Research Triangle Park, NC, October 11 - 13, 1995.

[26] Jeffries, H. E., "A Different View of Ozone Formation", Fuel Reformulation, 3(1): 57; ISSN: 1062-3744,1993.

[27] Tonnesen, S., Jeffries, H.E., "Inhibition of Odd Oxygen Production in Carbon Bond IV and Generic Reaction Set Mechanisms," Atmos. Environ,28:(7) 1339-1349, 1994.

[28] Jeffries, H.E., Tonnesen, S., "A Comparison of Two Photochemical Reaction Mechanisms Using a Mass Balance Process Analysis," Atmos. Environ., 28(18):2991-3003, 1994.

[29] Tonnesen, S., Jeffries, H.E., "The Contribution of Biogenic Hydrocarbons, Carbon Monoxide, Methane, and VOCs to Ozone Production in 39 Urban Areas," available at ftp://airsite.unc.edu/pdfs/ese_unc/jeffries/ipradocs/39cities.pdf.

[30] Tonnesen, S., Jeffries, H.E., "Analysis of Uncertainty in the Use of Incremental Reactivity for Hydrocarbon Reactivity Adjustment Factors," available at ftp://airsite.unc.edu/pdfs/ese_unc/jeffries/ipradocs/increact.pdf.

[31] Jang, J.C., Jeffries, H.E., Byun, D., Pleim, J.E., "Sensitivity of Ozone to Model Grid Resolution: Part I. Application of High-Resolution Regional Acid Deposition Model," Atmos. Environ., 29(21):3085,1995.

[32] Jang, J.C., Jeffries, H. E., Tonnesen, S., "Sensitivity of Ozone to Model Grid Resolution: Part II. Detailed Process Analysis for Ozone Chemistry," Atmos. Environ., 29(21):3114,1995.

[33] Wang, Z., Langstaff, J., and Jeffries, H.E., "Application of Integrated Process Analysis to Investigate Urban Airshed Model Sensitivity to Speciation in VOC Emissions Data," A\&WMA 88th Annual Meeting \& Exhibition, San Antonio, TX, June 18 - 23, 1995.

[34] Wang, Z. "Summary of Modifications Made to the Regulatory Version of the Urban Airshed Model to Include IPRA Output," UNC Environemental Modeling Laboratory Technical Note, 1995.

[35] Jeffries, H.E., "How to Track Origins of $\mathrm{NO}_{y}$ Mass in Eulerian Models", UNC Environmental Modeling Laboratory Technical Report, 1993 (available at $\mathrm{ftp}$ : / / airsite.unc . edu.

[36] Jeffries, H.E., Sexton, K.G., Kamens, R.M., Holleman, M.S., "Outdoor Smog Chamber Experiments To Test Photochemical Models: Phase II." Environmental Protection Agency, EPA600/3-85/029, March 1985.

[37] Jeffries, H.E.,Sexton, K.G., Holleman, M.S. "Outdoor Smog Chamber Experiments: Reactivity of Methanol Exhaust Part II: Quality Assurance and Data Processing System Description," Environmental Protection Agency Report, EPA-460/3-85/009b, September 1985. 
[38] Sexton, K.G., Jeffries, H.E., Arnold, J.R., Kale, T.L., Kamens, R.M., "Validation Data For Photochemical Mechanisms," Environmental Protection Agency Report, EPA-600/3-87/003, January 1987.

[39] Murdoch, R.W., Shores, R.C., Wright, R.S., Yeager, W.M., "Technical Systems Audit of University of North Carolina Smog Chamber Laboratory," bound report prepared for Coordinating Research Council, Research Triangle Institute, Research Triangle Park, NC, RTI/5462/001-01F, 70 pages, April, 1993. Also availalble at ftp://airsite.unc.edu/pdfs/ese_unc/jeffries/reports/qa/. 


\section{Appendix A}

\section{Chamber Facilities}

\section{A.1 The UNC Outdoor Chamber}

Location. The UNC Ambient Air Research Facility is off-campus, approximately 32 kilometers from the University of North Carolina at Chapel Hill. It is in the adjacent county, Chatham County, and is approximately 10 kilometers from the small town of Pittsboro in an isolated rural area. Chatham County is one of the most rural, least industralized counties in North Carolina and is heavily wooded. These conditions contribute to low background in the air used to fill the chambers. The background concentrations of $\mathrm{NO}_{\mathrm{x}}$ and nonmethane hydrocarbons at this site are usually less than $5 \mathrm{ppb}$ and less than $80 \mathrm{ppbC}$. More importantly, the air exhibits very low reactivity in the chamber.

Materials. The chamber surfaces are Fluorinated Ethylene Propylene (FEP) Teflon. The film's transmission in the UV and visible regions of the solar spectrum is excellent, and it has only a few absorption bands in the IR, a property necessary to reduce the "greenhouse effect". It has a very low permeability for most chemical species and can be heat-sealed to form large durable panels. For this application its worst property is its ability to hold a static charge for long periods of time. Type A film, $0.13 \mathrm{~mm}$ thick is used.

Physical Design. Inlet and outlet doors, stirring fans, manifolds, and other fittings come in through a solid floor. The sides are free for light entry. The floor of the chamber is elevated approximately $1.2 \mathrm{~m}$ to allow for easy access under the chamber.

The design is an A-frame $9.14 \mathrm{~m}$ wide, $12.10 \mathrm{~m}$ long and $6.10 \mathrm{~m}$ high at the peak on plywood floor $1.22 \mathrm{~m}$ above the ground. Wooden beams, $5.08 \mathrm{~cm}$ by $20.32 \mathrm{~cm}$, located on edge at $99.1 \mathrm{~cm}$ centers form an exterior framework on top of the plywood floor. Continuous $16.46 \mathrm{~m}$ lengths of film are attached to the inside by aluminum u-channels, screwed firmly to the wooden beams, thus compressing the film against the external support. 


\section{A.2 The Teflon Bag Reactor Chamber}

UNC has an indoor chamber that uses 500-liter Teflon bags exposed at constant temperature $\left(25^{\circ} \mathrm{C}\right)$ to constant irradiation from a mixture of black lamps and sunlamps placed on both sides of the bag in a aluminum foil-lined box. We call this system the Teflon Bag Reactor (TBR). The TBR is flushed and filled with clean air from a 250 liters $/ \mathrm{min}$ Addco Clean Air Generator. In a typical TBR experiment, $10 \mathrm{ppmC}$ of organic compound, $10 \mathrm{ppm}$ of NO, and about $10 \mathrm{ppm}$ of hydrogen peroxide are added to the filling stream. After the initial conditions has been established, the lights are turned on for up to $3 \mathrm{~h}$ of irradiation. 


\section{Appendix B}

\section{UAM Ozone Sensitivities}




\title{
The Sensitivity of Urban Ozone Predictions \\ to Assumptions in the Motor Vehicle Emission Inventory
}

\author{
Terry J. Keating, Jo Ellen Brandmeyer-Cawlfield, John Voyzey, \\ Ashraf Chaudhary, and Harvey Jeffries \\ Department of Environmental Sciences and Engineering \\ CB 7400, Rosenau Hall \\ University of North Carolina \\ Chapel Hill, NC 27599-7400 \\ terry_keating@unc.edu
}

\author{
Presented at \\ The Emission Inventory: Programs \& Progress \\ An AWMA/EPA Specialty Conference \\ October 11-13, 1995 \\ Research Triangle Park, NC
}

\begin{abstract}
This study tested the sensitivity of the Urban Airshed Model's (UAM) ozone predictions to three assumptions in the motor vehicle emission calculations: trip average emission factor, average daily temperature, and uniform speed by road classification. These assumptions were tested by preparing a series of motor vehicle modeling inventories using conventional methodology, disaggregated emission factors, hourly temperature profiles, and speeds by road. These inventories were then merged with the area, point, biogenic, and nonroad mobile inventory for a 1988 ozone episode in Charlotte, North Carolina, and used in UAM simulations.

Spatially and temporally allocating the disaggregated emissions to trip starts, trip ends, and trip mileage increased both the maximum gridded hourly emissions and the variability of gridded hourly emissions. VOC and $\mathrm{CO}$ showed a larger change than $\mathrm{NO}_{\mathrm{x}}$. This reallocation moved emissions from major highway corridors to the central business district. Varying the temperature by hour had little effect on emissions. Assigning speeds to individual roads using output from a travel demand model decreased $\mathrm{NO}_{\mathrm{x}}$ and significantly increased VOC emissions. For this ozone episode, peak ozone changed by less than $3 \mathrm{ppb}$, but spatial ozone changes were more significant. Effect of this disaggregation methodology may be greater in other urban areas or future year scenarios with different VOC to $\mathrm{NO}_{\mathrm{x}}$ ratios. Also, the disaggregation methodology may be more important for $\mathrm{CO}$ calculations than for ozone.
\end{abstract}

\section{INTRODUCTION}

The conventional method for estimating motor vehicle emissions embodied in EPA's MOBILE emission factor model $[1,2]$ and inventory guidelines [3] has been widely criticized as being inaccurate and simplistic $[4,5,6]$. Many of the criticisms arise from the conventional method's 
reliance on highly-aggregated data and unrepresentative assumptions which fail to accurately characterize the spatial and temporal variability of vehicle emissions. Current research efforts are attempting to develop more realistic characterizations of driver behavior, vehicle performance, traffic flow characteristics, and environmental conditions [7]. While these efforts will improve our ability to estimate motor vehicle emissions in the future, it is not clear what effect these efforts will have on our ability to predict ambient concentrations of secondary pollutants, such as urban ozone.

In this paper, we evaluate the sensitivity of urban ozone concentrations predicted by the Urban Airshed Model (UAM) [8] to changes in the assumptions in the motor vehicle emissions estimates. We focus on three assumptions of the conventional methodology:

1) the use of a trip-averaged emission factor

2) the use of a daily average temperature

3) the use of a uniform speed for all roadways of a particular functional class

These assumptions are challenged by using data from a travel demand model to:

1) separate the emissions components associated with different segments of a vehicle trip

2) vary ambient temperature by hour of the day

3) vary vehicle speeds by individual roadway link according to the traffic volume and road capacity

The impact of these assumptions is described in terms of changes in the spatial and temporal distribution of emissions of nitrogen oxides $\left(\mathrm{NO}_{\mathrm{x}}\right)$ and volatile organic compounds $(\mathrm{VOC})$ and UAM-predicted ozone concentrations for one meteorological episode in Charlotte, North Carolina.

\section{METHODS}

\section{Automation of the Inventory Development}

Preparing motor vehicle emission inventories for use in photochemical modeling can be timeconsuming and cumbersome. Before exploring the impact of different assumptions in the motor vehicle emissions inventory, we needed an efficient system for developing the motor vehicle emission inputs. To meet this need, we developed ProcMobl, a flexible computer package for calculating mobile source emissions and preparing UAM inputs. Using the relational database management features of SAS, ProcMobl

1) predicts future traffic volumes based on linear regression of historical data

2) applies trip-averaged or component-specific emission factors from EPA's MOBILE 5 a model

3) chemically speciates $\mathrm{NO}_{\mathrm{x}}$ and VOC for representation in UAM

4) temporally allocates daily average emissions by hour

5) spatially allocates emissions from counties or roadway links to grid cells

ProcMobl automates calculation and data manipulation steps and replaces UAM's Emission Preprocessor System [9] for on-road mobile emissions. ProcMobl also facilitates quality assurance checks and report generation.

\section{Conventional Emission Estimation Method}

Using the conventional method, emissions may be estimated on the basis of a link (road segment) or geographical area (county). Typically, the link approach represents travel along major 
thoroughfares. The area, or nonlink, approach represents travel between major thoroughfares along local streets. Both the link and nonlink calculations are based on the following simple equation:

\begin{tabular}{|c|c|c|c|c|}
\hline & & $\begin{array}{l}\text { Vehicle } \\
\text { Activity }\end{array}$ & * $\begin{array}{c}\text { Emission } \\
\text { Factor }\end{array}$ & $\begin{array}{c}\text { Speciation } \\
\text { Factor }\end{array}$ \\
\hline
\end{tabular}

The terms of equation (1) are defined below.

Vehicle Activity. Vehicle activity is conventionally defined in units of average daily vehicle miles traveled (VMT), which is the product of average daily traffic volume and roadway length. In the link calculation, the VMT for each link is used. In the nonlink calculation, the VMT for the entire area is distributed over each functional road class. In both calculations, the VMT is distributed over a characteristic vehicle type mix and assigned a characteristic speed according to road class.

Emission Factor. The emission factors, in units of grams of pollutant per VMT, are generated using the MOBILE 5a model. A separate emission factor is generated for each road class, vehicle type, pollutant $\left(\mathrm{CO}, \mathrm{NO}_{\mathrm{x}}, \mathrm{VOC}\right)$, and control program (e.g., inspection and maintenance program, gasoline volatility controls) combination. In our evaluation, a constant daily average ambient temperature was assumed in the conventional method calculations.

Speciation Factor. Speciation factors are used to convert the emissions from grams of pollutant to moles of the chemical species represented in the photochemical model. Thus, grams of $\mathrm{NO}_{\mathrm{X}}$ are converted into moles of $\mathrm{NO}$ and $\mathrm{NO}_{2}$, and grams of VOC are converted into moles of UAM Carbon Bond species. Separate sets of speciation factors are applied to the exhaust and evaporative emissions from each vehicle type.

Temporal Factor. Temporal factors are used to allocate the average daily VMT to each hour of the day. Different hourly distributions are assigned according to road class.

Gridding Factor. Finally, gridding factors are used to assign emissions from the link or area to the appropriate grid cells used by the photochemical model. For links, the gridding factor is defined as the fraction of the link length within each grid cell. In the nonlink calculation, the gridding factor is based on some surrogate for the spatial distribution of traffic. In our evaluation, population was the spatial surrogate. The nonlink gridding factors, therefore, represented the fraction of the total county population that fell within each grid cell.

\section{Disaggregated Estimation Method}

Vehicle emissions vary according to whether the vehicle is starting a trip, is in the middle of a trip, has just ended a trip, or is parked. Figure 1 presents a hypothetical time-series profile of VOC emissions associated with a single vehicle trip. Before the trip, low levels of emissions are generated by the vehicle. These emissions are known as diurnal emissions, caused by the evaporation of fuel due to ambient temperature increases, and resting losses, caused by the evaporation of fuel seeping through fuel lines and gaskets. When the vehicle is started, it produces a large spike of emissions which quickly tapers off as the vehicle warms up. The size and shape of the spike depends on whether the vehicle is cold upon ignition or is already hot. During the trip, the mass of emissions varies as the vehicle accelerates and decelerates. After the vehicle 


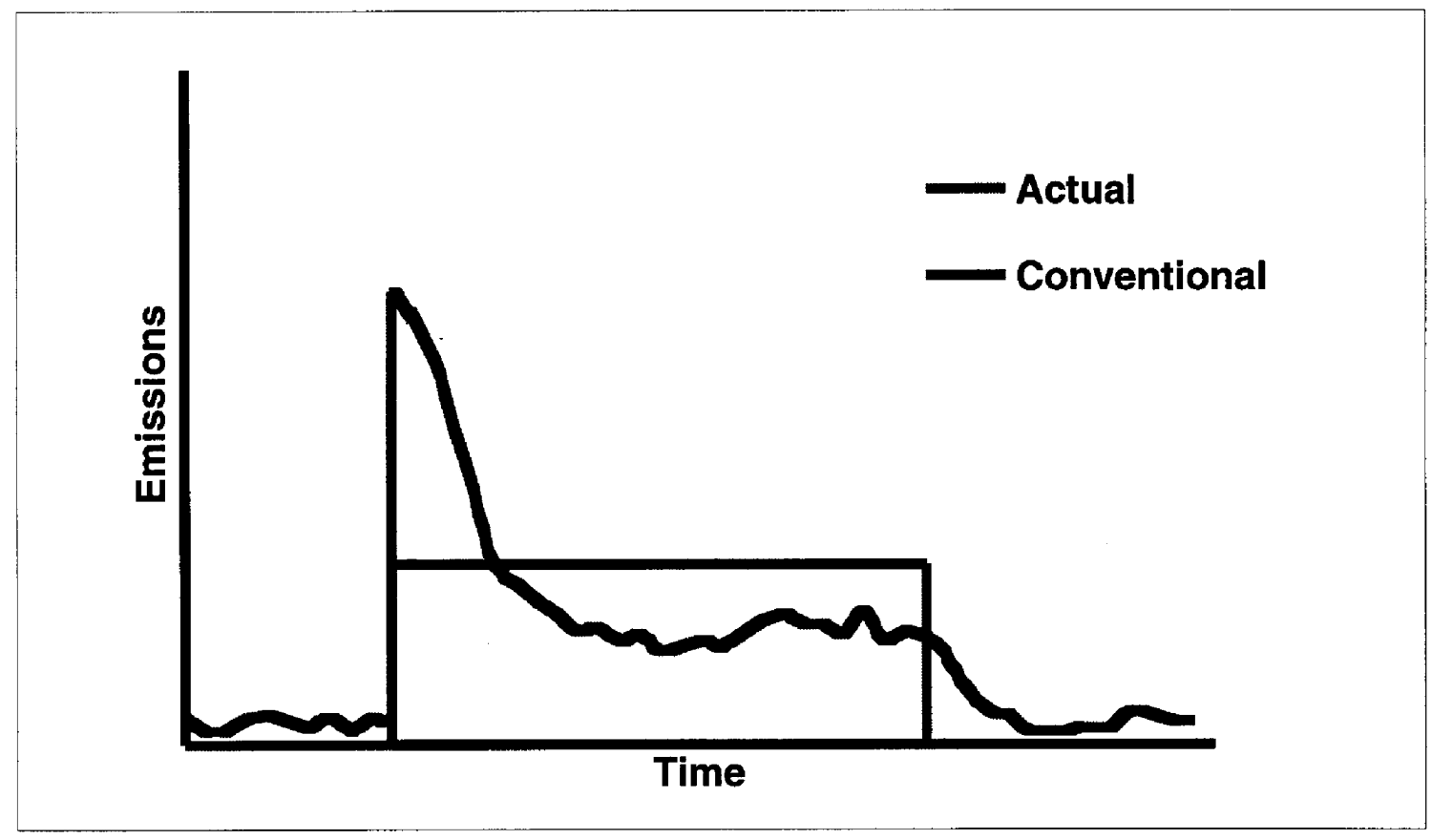

Figure 1. The conventional representation of emissions from a vehicle trip.

stops, fuel in the hot engine evaporates producing hot soak emissions which decrease as the engine cools down.

The conventional emissions estimation methodology described above does not attempt to represent this variability of emissions between vehicle operating modes. Instead, the sum of the emissions which occur over the entire trip (and a weighted fraction of diurnal and resting emissions) are spread out over the length of the trip by the grams per mile emission factor, also shown in Figure 1. This uniform rate approach may approximate the overall mass of emissions well (i.e., equal areas under the two curves), but it ignores the fact that the various modes of vehicle operation are distributed differently in space and time. For example, during a morning commute, a residential suburb may experience many trip starts while a central business district may experience many trip ends. Using the conventional link calculation approach, the start and end emissions would not be assigned to either the suburb or the central business district. Instead, they would be spread out along the roadways between the two. While this dislocation of emissions may not be important for calculating the total mass of emissions from an urban area, it may be important to the formation of urban ozone.

To represent the variability of vehicle emissions due to operating mode, we divided the vehicle emission profile into four segments: start, stabilized, end, and nontrip. These segments are shown in relation to our hypothetical VOC emissions profile in Figure 2. The terms in Equation (1) for each of these segments are defined below.

Vehicle Activity. To disaggregate the various segments of the vehicle trip, we used information from a UTPS-type four-step travel demand model [10] which is widely used in transportation planning. 


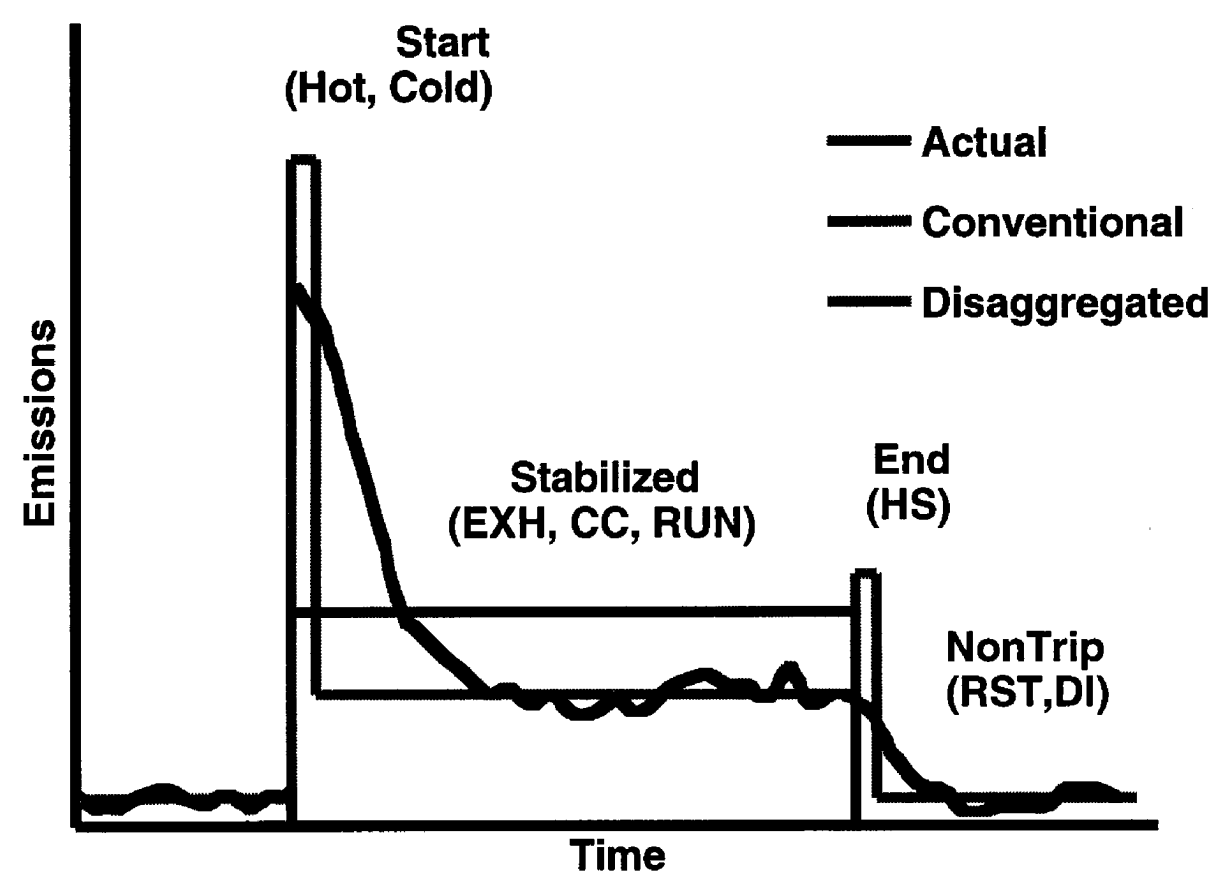

Figure 2. The disaggregated representation of emissions from a vehicle trip.

For the stabilized emissions, the calculation is almost identical to the link approach described above. We defined vehicle activity as the average daily VMT for each roadway link as predicted by the travel demand model. Characterizing vehicle activity for the other trip segments was not as straightforward.

To calculate the emissions associated with vehicle starts and ends, we used the travel demand model to predict where vehicle trips begin and end. In the travel demand modeling process, the modeling domain is divided into traffic analysis zones. Using census data to extrapolate from the information collected in a home survey, the travel demand model estimates the number of trips which originate in one zone and end in another. The result of this process, known as a trip table, is a matrix with the origination zone on one axis and the destination zone on the other. For our purposes, we only needed to know the row and column totals of the matrix indicating the total number of starts and ends in each zone. The travel demand model may generate a separate trip table for different types of trips. In our evaluation, six types of trips were identified: home-based work trips, home-based other trips, non-home-based trips, airport trips, truck and taxi trips, and externally-based trips (with respect to the study area). Thus, for start and end emissions, we defined vehicle activity as the number of starts or ends of trips of a particular type within each traffic analysis zone.

Diurnal and resting loss evaporative emissions comprise the nontrip related emissions associated with parked vehicles. It is not possible to estimate the total population of vehicles from traffic observations or the standard travel demand model. Therefore, we estimated the total mass of nontrip related emissions using total county VMT and the grams per mile emission factors generated by the MOBILE model. We then used the population and employment in each traffic analysis zone and the trip table information to spatially distribute the emissions over the county, 
using population and home-based trips as a surrogate for cars parked at home, and employment and work-based trips as a surrogate for cars parked at work.

Emission Factor. Instead of using the default trip-averaged emission factor, we used the component emission factors generated by the MOBILE model to calculate emissions from each of the four trip segments. Instead of fixing vehicle speeds by road class and using a single daily average temperature, we wanted to allow the speed to vary by link and the temperature to vary by hour. Therefore, we ran the MOBILE model with a range of critical speed and temperature inputs. We fit these model outputs with separate regression models for each emission component and vehicle type. These regression models were then used to generate emission factors for speed and temperature conditions in our simulations.

The stabilized segment emission factors consist of the stabilized $\mathrm{CO}, \mathrm{NO}_{\mathrm{x}}$, and $\mathrm{VOC}$ exhaust emissions and crankcase and running loss VOC evaporative emissions. To represent the effect of speed and temperature on the stabilized emission factors, we fit the product of emission factor times speed with a polynomial involving both speed and temperature.

Emission factors for the incremental contribution of cold and hot starts to $\mathrm{CO}, \mathrm{NO}_{\mathrm{x}}$, and VOC exhaust were calculated by subtracting the cold and hot start components from the stabilized component as predicted by the MOBILE model for a vehicle speed of $19.5 \mathrm{mph}$ and a temperature of $75^{\circ} \mathrm{F}$, which are the conditions of the Federal Test Procedure (FTP). The resulting grams per mile emission factor was converted to grams per start by multiplying by 3.59 miles, the distance covered in the start portion of the FTP [11].

For trip ends, the MOBILE model generates a separate hot soak emission factor for VOC in grams per trip end.

As discussed above, nontrip emissions were estimated using the grams per mile emission factors for the diurnal and resting loss VOC evaporative components. The MOBILE model generates a separate grams per mile emission factor for resting losses. For the diurnal emissions, however, the MOBILE model output contains a mileage-weighted diurnal emission factor in grams per day. Therefore, we modified the MOBILE model to output the grams per mile diurnal factor before the mileage weighting was applied.

Speciation Factor. Speciation for the individual trip segments was performed the same as in the conventional method with separate speciation profiles applied to the evaporative and the exhaust emissions of each vehicle type.

Temporal Factor. Instead of using hourly profiles associated with different road classes, the daily emissions for each of the trip segments were distributed over the day using hourly profiles of traffic observed in different regions of the county. An hourly profile of average ambient temperature across the study area was also used to calculate the emissions in each hour.

For start emissions, a third temporal profile was applied enabling the ratio of cold to hot starts to vary over the day [12]. Separate cold to hot profiles were applied to each trip type.

Separate hourly profiles were applied to the diurnal and resting loss components of the non-trip emissions. The resting loss component was assumed to be distributed uniformly over the entire day. The diurnal emissions were assigned to each hour of the day according to the fraction of the 
daily temperature rise occurring in that hour. Thus, diurnal emissions were zero in hours during which the ambient temperature remained constant or declined.

Gridding Factor. The four segments of vehicle emissions were spatially allocated to the UAM grid. Stabilized emissions were assigned in the same manner as in the conventional link calculation: A factor was calculated representing the fraction of each in each grid cell.

For the start, end, and non-trip emissions, gridding was performed by assigning all of the emissions associated with a particular zone to the cell in which the zone centroid was located. This crude assumption was adequate because the traffic analysis zones were much smaller than the $5 \mathrm{x}$ $5 \mathrm{~km}$ grid cells used in our UAM simulations.

\section{The Comparison}

To evaluate the sensitivity of urban ozone production to the different assumptions and methods described above, we conducted four simulations of a particular meteorological episode which occurred on June 16-18, 1988, in Charlotte, North Carolina. This episode is one of three episodes modeled by the State of North Carolina for purposes of their ozone State Implementation Plan (SIP) [13].

Four sets of motor vehicle emissions inputs were developed for a $25 \times 25-5 \mathrm{~km}$ grid cell domain centered over Charlotte. ${ }^{1}$

1) Scenario $C$, the conventional method

2) Scenario $D$, the disaggregated method

3) Scenario $T$, the disaggregated method with hourly temperatures

4) Scenario $S$, the disaggregated method with hourly temperatures and link-specific speeds

Scenario $\mathrm{C}$ is an implementation of the conventional methodology using the same assumptions as the State of North Carolina in their SIP modeling. In this scenario, the emissions associated with traffic on major thoroughfares were calculated on a link basis. All other emissions were calculated on a county basis.

In Scenario D, the link calculations for Mecklenburg County, the county in which Charlotte is located, were replaced by the four disaggregated trip segments described above. However, the same vehicle speed and ambient temperature assumptions were used as in Scenario $\mathrm{C}$. The emissions in the other counties in the domain were calculated using the conventional methods.

Scenario $\mathrm{T}$ is identical to Scenario $\mathrm{D}$ except that the ambient temperature was allowed to vary by hour.

Scenario $S$ is identical to Scenario $T$ except that the speeds on the links were assigned by the travel demand model as opposed to uniformly set by road class.

\footnotetext{
${ }^{1}$ A fifth scenario $(\mathrm{H})$ was performed using the conventional method and the VMT predicted from HPMS observations, as opposed to VMT predicted by a travel demand model used in Scenario C. For Mecklenburg County in 1988, HPMS predicted a total VMT of 9,366,090 [13]. The VMT predicted by the travel demand model was $14,663,589$ [10]. Scenario $\mathrm{H}$ also used a less dense link network than in Scenario C.
} 
Each of the four mobile input files were substituted into the UAM inputs used by the State of North Carolina and executed.

In addition, the impact of grid size on the effect of the disaggregation of emissions was tested by preparing inputs for Scenarios C and D for a domain of $125 \times 125-1 \mathrm{~km}$ grid cells, referred to as Scenarios C1 and D1.

\section{RESULTS}

\section{Emission Impacts}

The overall impact on the emission inventory of changing the assumptions between the four scenarios is summarized in Table 1. Comparing Scenarios $\mathrm{C}$ and $\mathrm{D}$, the disaggregation of the link emissions did not significantly change the total mass of emissions of $\mathrm{NO}_{\mathrm{x}}$ and slightly increased the total mass of VOC. For both pollutants, disaggregation increased the maximum emissions in any one cell in any one hour, and the effect was much stronger for VOC than for $\mathrm{NO}_{\mathrm{x}}$. Disaggregation also increased the variability of VOC emissions as measured by the variance of the hourly emissions in each cell.

Varying the ambient temperature by hour in Scenario $\mathrm{T}$ had little effect on the total mass or distribution of $\mathrm{NO}_{\mathrm{x}}$ emissions. For VOC, the temperature variations had a small impact: decreasing the total mass and increasing the maximum cell.

Changing the speed assumptions in Scenario $\mathrm{S}$ had opposite impacts on $\mathrm{VOC}$ and $\mathrm{NO}_{\mathrm{x}}$. For $\mathrm{NO}_{\mathrm{x}}$, the total mass and maximum cell decreased by a small amount. For VOC, the total mass and maximum cell increased significantly.

The spatial redistribution of emissions due to the disaggregation is shown in Figure 3. This figure shows the difference between Scenarios D and C in terms of the mass of VOC assigned to each cell over the entire day. Negative values indicate areas from which the disaggregation removed emissions, and positive values indicate areas to which the disaggregation added emissions. There is a detectable movement of emissions away from the major highway corridors and into the central business district. The spatial redistribution due to disaggregation was even more dramatic when the grid size was reduced to $1 \mathrm{~km}$ grid cells in scenarios $\mathrm{Cl}$ and $\mathrm{D} 1 .^{2}$

\section{Ozone Impacts}

The impact of the various assumptions on predicted ozone concentrations is summarized in Table 2. In all four scenarios, the location and timing of the peak ozone concentration did not change on either day of interest due to the changes in the motor vehicle emissions. The height and breadth of the peak, however, did change somewhat. Moving from Scenario C to D, the disaggregation did increase the peak concentrations on both days. Allowing the temperature to vary by hour had practically no impact on either day. Although changing the speed assumptions significantly

\footnotetext{
${ }^{2}$ The total mass predicted for Scenario $\mathrm{H}$ was $14 \%$ and $25 \%$ lower for $\mathrm{NO}_{\mathrm{x}}$ and VOC, respectively, as compared to Scenario C, corresponding to the $36 \%$ decrease in VMT. The maximum grid cell decreased by $33 \%$ and $61 \%$ for $\mathrm{NO}_{\mathrm{x}}$ and VOC, respectively, due to the decrease in the density of the link network.
} 
Table 1. Impact of Disaggregation, Temperature, Speed, and Grid Resolution on Emissions.

\begin{tabular}{|c|c|c|c|c|c|c|c|c|}
\hline & & \multicolumn{6}{|c|}{ Scenario } & \multirow[t]{2}{*}{ Units } \\
\hline & & $\mathrm{C}$ & $\mathrm{D}$ & $\mathrm{T}$ & $S$ & $\mathrm{C} 1$ & D1 & \\
\hline \multicolumn{2}{|c|}{ Grid Cell Size } & 25 & 25 & 25 & 25 & 1 & 1 & $\mathrm{~km}^{2}$ \\
\hline \multirow{3}{*}{$\mathrm{CO}$} & Total Mass & 638 & 684 & 687 & 934 & 580 & 626 & tons \\
\hline & Max Cell & 6047 & 8278 & 8272 & 9465 & 536 & 848 & $\mathrm{lbs} / \mathrm{hr} / \mathrm{cell}$ \\
\hline & Variance & 4 & 5 & 5 & 11 & 0 & 0 & $\left(10^{3} \mathrm{lbs} / \mathrm{hr} / \mathrm{cell}\right)^{2}$ \\
\hline \multirow{3}{*}{ NOx } & Total Mass & 87 & 86 & 87 & 84 & 83 & 83 & tons \\
\hline & Max Cell & 878 & 998 & 1000 & 875 & 100 & 101 & lbs/hr/cell \\
\hline & Variance & 7017 & 7356 & 7382 & 6067 & 31 & 31 & $(\mathrm{lbs} / \mathrm{hr} / \mathrm{cell})^{2}$ \\
\hline \multirow{3}{*}{ VOC } & Total Mass & 86 & 89 & 88 & 136 & 77 & 81 & tons \\
\hline & Max Cell & 811 & 1148 & 1204 & 1532 & 70 & 148 & $\mathrm{lbs} / \mathrm{hr} / \mathrm{cell}$ \\
\hline & Variance & 6507 & 8706 & 9062 & 28304 & 16 & 22 & $(\mathrm{lbs} / \mathrm{hr} / \mathrm{cell})^{2}$ \\
\hline
\end{tabular}

Table 2. Impact of Disaggregation, Temperature, and Speed on Ozone Concentrations.

\begin{tabular}{|c|c|c|c|c|c|c|}
\hline & & & \multicolumn{4}{|c|}{ Scenario } \\
\hline & & & $\mathrm{C}$ & $\mathrm{D}$ & $\mathrm{T}$ & $S$ \\
\hline \multirow{4}{*}{$\begin{array}{c}\text { June } 16 \\
1988\end{array}$} & Max Cell & $(\mathrm{ppb})$ & 128.7 & 130.7 & 130.5 & 129.8 \\
\hline & Grid Cell Hours & $>120 \mathrm{ppb}$ & 48 & 55 & 54 & 45 \\
\hline & Grid Cell Hours & $>100 \mathrm{ppb}$ & 909 & 930 & 929 & 942 \\
\hline & Grid Cell Hours & $>80 \mathrm{ppb}$ & 4194 & 4194 & 4194 & 4229 \\
\hline \multirow{4}{*}{$\begin{array}{c}\text { June } 17 \\
1988\end{array}$} & Max Cell & $(\mathrm{ppb})$ & 130.5 & 132.6 & 132.4 & 130.8 \\
\hline & Grid Cell Hours & $>120 \mathrm{ppb}$ & 37 & 44 & 42 & 34 \\
\hline & Grid Cell Hours & $>100 \mathrm{ppb}$ & 406 & 419 & 418 & 415 \\
\hline & Grid Cell Hours & $>80 \mathrm{ppb}$ & 2874 & 2906 & 2908 & 2923 \\
\hline
\end{tabular}




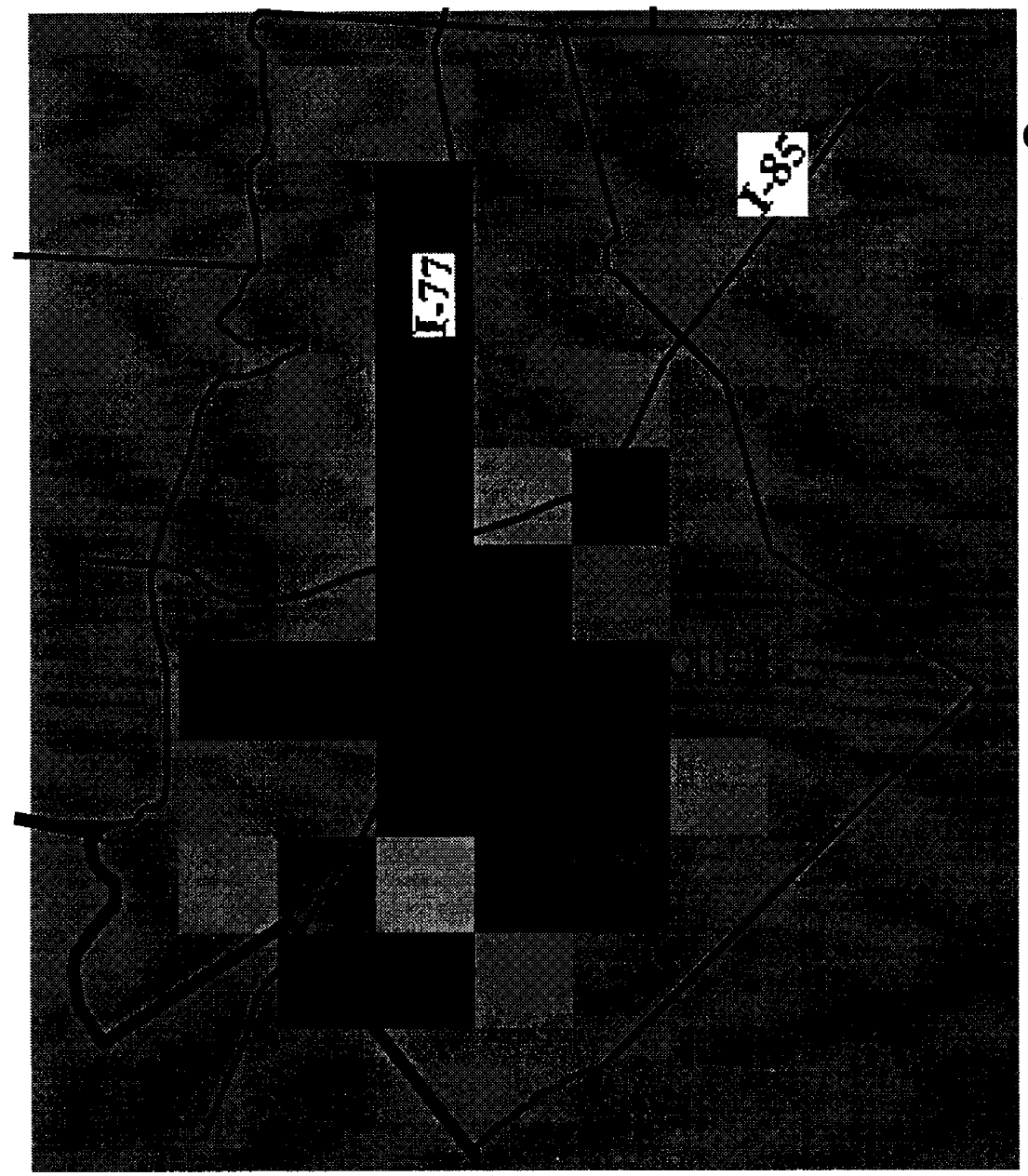

\section{Change in VOC} due to Disaggregation

(change: $1 \mathrm{bs} /$ day/gridcell)

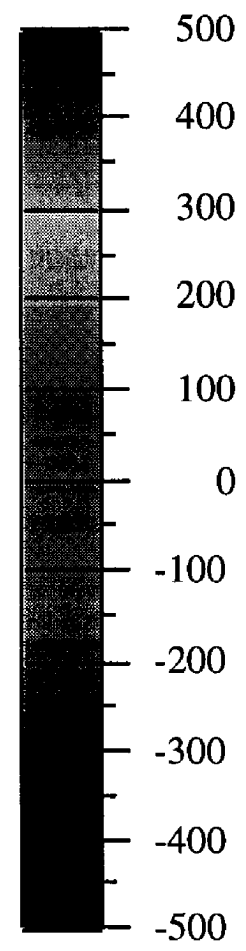

Figure 3. The change in daily VOC emissions due to disaggregating portions of th e vehicle trip. Note the decrease in emissions along the Interstate-77 corridor and the increase in emissions in the central business district. 
increased emissions of VOC, the peak ozone concentration decreased. This effect is attributable to the slight decrease in NOX emissions and the NOX-limited nature of the Charlotte area [13]. ${ }^{3}$

\section{CONCLUSIONS}

We tested the impact of three assumptions inherent in the conventional motor vehicle emission estimation method: the trip averaged emission factor, the daily average temperature, and uniform speeds by road class. Varying the ambient temperature had little impact on either emission estimates or ozone predictions. Disaggregating the emission factor and varying speeds on individual links had significant impacts on the distribution and mass of emissions, particularly for $\mathrm{CO}$ and VOC. The spatial redistribution due to disaggregation was even more significant at higher grid resolutions. However, the impact of these changes on ozone predictions was small, primarily due to the $\mathrm{NO}_{\mathrm{x}}$-limited nature of the Charlotte area in 1988. Effects on ozone concentrations in scenarios with different VOC to $\mathrm{NO}_{\mathrm{x}}$ ratios may be more significant. Furthermore, disaggregation may have a significant impact on predictions of $\mathrm{CO}$ concentrations.

While the conventional methods for estimating motor vehicle emissions have been criticized as being unrealistic and unrepresentative, this work demonstrates that, under some conditions, major efforts to refine the emission estimates will have little impact on air quality predictions, at least at the current resolution of air quality models. Under different conditions, however, these changes in the emissions estimates may produce very significant air quality impacts.

\section{ACKNOWLEDGMENTS}

The authors would like to acknowledge the assistance of Joseph McLelland, Charlotte Department of Transportation; Behshad Norowzi and Laura Boothe, NCDEHNR Air Quality Section; Jeff Vukovich and Don Olerud, MCNC Environmental Programs; and GeGe Coleman, ITRE GIS group. Support was provided by the Center for Transportation and the Environment in cooperation with the North Carolina Department of Transportation and the University of North Carolina Institute for Transportation Research and Education. CTE is an activity of North Carolina State University. Additional support was provided by the National Renewable Energy Laboratory under contract to the U.S. Department of Energy.

\section{REFERENCES}

1. Office of Mobile Sources; Programmer's Guide to MOBILE4, EPA-AA- TEB-89-02;

U.S. Environmental Protection Agency: Ann Arbor, 1989.

2. Office of Mobile Sources; Mobile 5a Update to Chapter 2; U.S. Environmental Protection Agency: Ann Arbor, March 26, 1993.

3. Office of Mobile Sources; Procedures for Emission Inventory Preparation, Volume IV: Mobile Sources, EPA/450/4-81/026D-REV; U.S. Environmental Protection Agency, Ann Arbor, 1992.

\footnotetext{
${ }^{3}$ The peak ozone concentration predicted for Scenario H was $124.6 \mathrm{ppb}$ on both days.
} 
4. U.S. General Accounting Office; Air Pollution: Reliability of EPA's Mobile Source Emission Model Could Be Improved, GAO/RCED-90-138. 8; Washington, DC, 1990.

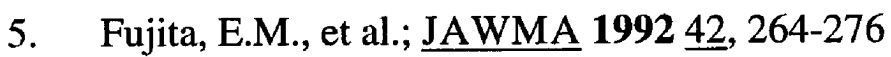

6. Beaton, S.P., Bishop, G.A., Stedman, D.H.; Science 1995 268, 991.

7. Cadle, S.H., Carlock, M., Cullen, K., Gorse, R.A., Knapp, K.T., Lawson, D.R.; JAWMA $1994 \underline{44}, 1180-1187$.

8. Office of Air Quality Planning and Standards; User's Guide for the Urban Airshed Model, EPA/450/4-90-007; U.S. Environmental Protection Agency, Research Triangle Park, 1990.

9. Office of Air Quality Planning and Standards; User's Guide for the Urban Airshed Model, Volume IV: User's Manual for the Emissions Preprocessor System 2.0, EPA/450/4-90007D(R); U.S. Environmental Protection Agency, Research Triangle Park, 1992.

10. Charlotte Department of Transportation; 2005 Transportation Plan Technical Report; Transportation Planning Division; Charlotte, NC, 1992.

11. Office of Mobile Sources; FTP Test Program Driving Cycles; U.S. Environmental Protection Agency: Ann Arbor, November 9, 1993.

12. Venigalla, M.M. A Network Assignment Based Approach to Modeling Mobile Source Emissions, Ph.D. Dissertation; University of Tennessee, Knoxville, 1994.

13. North Carolina Department of Environment, Health, and Natural Resources; Supplement to the Redesignation Demonstration and Maintenance Plan for The Charlotte/Gastonia Ozone Nonattainment Area; Division of Environmental Management, Raleigh, NC, December 16, 1994. 


\section{UNC's Response to Comments on DOE Report}

\section{Review 1}

How appropriate is the 54-component SynURB smog chamber mixture for representing today's composition of urban VOC? The actual conditions today are quite likely different.

The UNC SynURB mixture is based on the most comprehensive and best QA'ed urban VOC data available. More than 4 years of data from 41 cities over the period 1984 to 1988 were analyzed for up to 300 species by a single investigator at EPA using a single instrument and calibration set. UNC analyzed and further QA'ed more than 1000 canister samples from this set. To quote from the CRC Final Report (copies obtainable from

ftp: //airsite.unc.edu/pdfs/ese_unc/jeffries/reports/crcme1/pdfs/*), which described the creation of this mixture:

In determining the VOC speciation for the average urban mixture, we examined several VOC speciation data bases: $i$ ) the US EPA National Ambient Volatile Organic Compounds Data Base, i i) the 1990 Southern Oxidant Study Atlanta VOC and aldehyde data base, $\mathrm{i}$ i i ) the Southern California Air Quality Study (SCAQS) VOC and aldehyde data base, i $v$ ) the US EPA Five-Cities Aldehyde Study, and $v$ ) the US EPA Ambient 6-9 AM Urban Hydrocarbon Study. In a previous project, UNC had extensively quality assured the data reported in the last study named above. These data were produced by Bill Lonnemann using canisters collected in 29 cities over the period 1984-1986. For this study, we obtained from Lonnemann additional ambient data VOC data for the years 1987-1988 and integrated this with the data from the previous study resulting in a data set that spanned 1984-1988 and included 41 cities. The 1987-1988 data included extensive multiple analyses of canisters and multiple measurements of certain compounds with different analytical instruments. These permitted us to conduct a series of quality assurance analysis and to request Lonnemann to provide additional scrutiny for any questions that were raised. More than 1000 canisters were analyzed by us to produce an average urban composition having 200 different organic species.

We believe that this Lonnemann data set is the best urban VOC composition data base available. This set was compared with the SCAQS data base, for example, and it was determined that, while there was good agreement for the largest species present (e.g., propane, iso-pentane, and toluene) there was significant disagreement (up to a factor of two) for most species. This was explained by the fact that many fewer species were measured in the SCAQS data, e.g., only 27 species account for $80 \%$ of the carbon reported in the SCAQS data, while 48 species are needed in the EPA data set. After we 
determined this, we chose not to do any further analysis with the SCAQS data.

For part of the Lonnemann data set, additional data were obtained in an EPA 5-site ambient aldehyde study. The 240 measured three-hour aldehyde concentrations in the five cities were carefully matched with the VOC canisters which were taken at the same time at the same location so that reliable carbon fractions for the aldehydes could be obtained. In addition, we examined real-time FTIR data from the 1990 Atlanta field program, which confirmed the magnitudes of formaldehyde in the morning before the mixing height starts to rise. While the SCAQS data showed similar levels of formaldehyde and acetaldehyde as the values used here, these data reported very high fractions (e.g., up to $8 \%$ for $\mathrm{C}_{6}$ ketones) of some compounds. As these data were quite uncertain at the time we were formulating our mixtures, and because the SCAQS VOC composition data were highly suspect, and because the EPA 5-cities study was carefully matched with the VOC canister data, we elected to use only the EPA data for the aldehyde fractions to be included in the urban mixture.

The purpose of producing the SynURB mixture was to move from using three species (as Carter has done) or even 20 species VOC mixtures in smog chamber studies to a mixture that 1 ) was much more representative, 2) was much more complex, 3) was very easy to analyze, and 4) was very reproducible. In constructing the mixture, we did cluster analysis on the different city compositions and we did find that different cities have slightly different compositional signatures. For example, some cities were clearly "oil and gas" producing cities while others were clearly "car"-only cities. The biggest variable among the different cities was the aromatic/olefin fraction. SynURB was based on the median composition of all cities.

We must stress, however, that the purpose of the smog chamber runs is not to "simulate" a city, but to provide an extremely well characterized and reproducible chemical and physical (e.g., radiation and temperature) environment in which to generate quality species concentration observations to challenge predicted concentrations from photochemical reaction mechanisms. This role of the smog chamber data was clearly illustrated in Figure 1.1 on page 5 of this final report. One first must, as a necessary but not sufficient condition, obtain agreement between the theoretical predictions of the photochemical reaction mechanisms and chamber observations, where only chemistry is responsible for the concentration changes, before one can claim that an urban air quality model using the mechanism would be capable of predicting the effects of fuel changes on ozone in future scenarios with different urban compositions. To further create as stressful a test as possible, we use the side-by-side experiment to create two different conditions under the same physical environment, measured with the same instruments, and require that an acceptable model must agree with both sides at the same time. 
It is not as important that the mixture composition match closely the current ambient conditions of a particular city or national average. What is most important is that the range of composition variation in the experiments match or exceed the expected variation in the atmosphere.

We believe that there is no better high quality ambient $\mathrm{HC}$ dataset from a large number of cities representing current conditions than the one we used.

In addition, we wanted to be able to compare the results of this work with the previous work conducted with methanol/gasoline fuels which also used the UNC SynURB mixture. Also, the SynURB mixture has been used to generate test data in other EPA sponsored work including TVA and CSIRO in Sydney, Australia, permitting a comparison of performance across different chambers, operated by different groups.

The SynURB mixture appears to be photochemically "hot;" it is more reactive than any of the vehicle emissions mixtures that were investigated.

The SynURB mix reflects ambient measurements containing emissions from area and industry emission sources, as well as automobile emissions from "real" vehicles. The emissions from different fuels and automobiles on dynamometers represents a more controlled source of data as would be expected from a new technology. The modern fuels (especially the alcohol based fuels) are designed to be unreactive. Compared to SynIAG, the SynURB mix has a larger fraction of aromatics reflecting solvent use, and the SynIAG has larger fraction of benzene and toluene reflecting the industry average composition of gasoline. The SynIAG has a larger alkane fraction with a larger fraction of nbutane reflecting the evaporative contribution. The alkene fraction of SynIAG has a larger ethylene fraction. Similar statements can be said about the methanol fuel; it has a large alkane fraction with a large butane fraction. The ethanol fuel emissions have large fractions of relatively unreactive components such as ethane, butane, pentane, ethene, benzene and toluene. All of these characteristics would tend to make the "pure" fuel compositions somewhat less reactive than the SynURB mixture.

When adding synthetic emissions mixtures to SynURB, a major consequence is that the highly reactive SynURB mixture is diluted. Is this really an accurate representation of the processes believed to occur when using conventional and alternative-fueled vehicles?

The experimental design is to consider the reactivity of the SynURB mixture as an atmospheric benchmark (i.e., a reasonable estimate of national urban reactivity, a reasonable base composition containing all of the basic $\mathrm{HC}$ classes in a reasonable distribution, certainly a reasonable challenge for a chemical mechanism to simulate). The design then compares the reactivity of 
$50 \%$ substitution of the SynURB with either the SynIAG or alternative fuels. The $50 \%$ substitution is not an expected estimate of actual regulatory policy or actual usage but a level that shows what changes in reactivity one can expect from a more than reasonable level of substitution and change in atmospheric composition, and provides the chemical mechanisms with a wider range of composition change than is actually expected anytime soon. This level of composition change provides for an excellent test for the chemical mechanisms. The experimental design then allows for comparisons of different substitution mixtures such as URB/IAG versus URB/E85.

What is the basis for the composition of the SynCNG mixture? Does it come from testing just three vehicles, as is implied by Fig 2.2? What was the composition of the CNG fuel used in these emissions tests?

The CNG emissions composition data is from the Federal Test Procedure (FTP) results from three OEM 1992 Sierra pickup trucks (low mileage 4000-6000, PAS conversion, tests numbers 780, 291, and 259, conducted between March and May of 1993). These data were obtained by Southwest Research Institute under DOE/NREL funding and were supplied to us for producing the test mixture for the chamber experiments. The fuel was identified only as CNG.

Why are the two SynE85 compositions shown in Fig 2.3 so different from each other? Is this a reflection of different vehicle technologies between the two data sets? This large variation in emissions composition suggests that caution must be exercised when drawing conclusions about ozone impacts from use of alternative fuels.

We do know that there is some difference in technologies between the two data sets. Both sources of the emissions data are considered reliable and were specified for the program. We decided to treat the two sets as the basis for the range of variation in the ethanol fuel emissions and test with both compositions. The usefulness of the two compositions is to show that the chemical mechanisms are able to simulate the reactivity of both mixtures, and that there is no concern about "tuning" to a particular mixture reactivity.

It is somewhat confusing to show the original smog chamber experimental plan in the Executive Summary page 22 but not the final plan that was actually executed. Perhaps both plans could be included.

In the first case we were discussing the conceptual design of the program and in the other case we wish to show what we achieved. To show both would be too much detail in the Executive Summary. 
The HC/NOx ratios used in each experiment should be indicated on the smog chamber plots shown in the Exec Summary.

The NOx levels are always the same (0.33), so the VOC is a direct indicator of the $\mathrm{HC} / \mathrm{NOx}$ ratio. Thus, 4.5, 6.0, and 9.0 $\mathrm{HC} / \mathrm{NOx}$ are merely 1.5, 2.0, and 3.0 ppmC, which are already labeled on the plots.

What is the basis for the SynLPG composition used in this study?

The basis for the SynLPG composition is FTP dynamometer data from the EPA, Research Triangle Park, NC and Southwest Research Institute, San Antonio, Texas. The EPA vehicle was stated to be more representative of standard LPG vehicles, and was specified to be the direct source of the composition data for the design of our SynLPG (J.A.W.M.A. V45, 770-777, 1995). The composition data is from three replicate FTP tests for a dedicated LPG, 1993 Ford F150 Pick-up with a $5.0 \mathrm{~L} \mathrm{V-8,} \mathrm{with} \mathrm{about} 60,000$ miles, using a fuel specified as LPG1 (94.5\% propane, $3.8 \%$ ethane, $0.97 \%$ isobutane, $0.6 \%$ propene, $0.13 \%$ other). The SRI data was compared and used as supporting data. The SRI vehicle was a converted1993 Chevy Lumina with a 3.1 L V-6 and about 5700 miles, equipped with a catalyst (not the same as used for gasoline), selected because of its effectiveness on methane. The conversion kit was a Mogas ECOLO with Autotronics oxygen-sensor feedback, very basic with no adaptive learn. The test fuel was commercially available LPG, composition : propane $91.4 \%$, propylene $6.1 \%$, ethane $2.0 \%$, butane and higher $0.4 \%$, methane $0.1 \%$.

It is implied on page 23 that two E85 compositions were used because of the large difference in acetaldehyde levels between the two. To explore this issue, it would seem preferable to use a single base composition, and to vary acetaldehyde concentration in a series of sensitivity tests.

Because there are several other important differences between the two mixtures besides the difference in acetaldehyde, both were used in the program. The acetaldehyde fraction appeared to be the most obvious difference in the two compositions. The NIPER data had only a $2 \%$ aromatic fraction compared to the $12.7 \%$ fraction in the EPA data. Also the NIPER had an alkane fraction approximately twice that of the EPA data.

The experimental finding that there was very little effect of temperature upon ozone formation in these smog chamber experiments seems very surprising. Isn't it well 
established that temperature has a strong positive effect on ozone formation in actual urban atmospheres? Could this finding be a "smog chamber artifact" which results from the static conditions or some other factor related to the manner in which these experiments were conducted? Similarly, could the conclusion on page 31 that the CB4 mechanism "exhibits an incorrect and overly sensitive temperature dependence for ozone formation " be limited to these experimental smog chamber conditions?

Ambient ozone levels do show a general trend with temperature such that higher temperatures tend to be associated with higher ozone levels, but a wide range of specific temperatures can be associated with a single ozone level. This is not a cause and effect relationship, but one in which temperature is a surrogate for other meteorological factors. For specific individual compounds like nbutane, temperature does have a strong positive effect on ozone formation. This is due to the large temperature effect on the radical unimolecular decomposition rate. This is already included in the CB4 mechanism. Our observations are definitely not due to "smog-chamber-artifacts." We have QA/QC data that shows that the temperature data are reliable. The only chamber effect which can account for the higher temperatures before 1994 is the wall particulate discussed in the report.

Remember that the methane/NOx experiments were easily simulated with CB4 regardless of the chamber temperature. "Chamber artifacts," if they exist, should show up in these experiments, but they are easily simulated with out any special assumptions. N-butane experiments, not shown in the report, do show a strong temperature effect in the UNC chamber. It is only the complex SynURB mixture that does not show the strong temperature effect. CB4 does not reproduce the observed results and therefore CB4 is clearly incorrect in this temperature range for the complex SynURB mixture. Note that this mixture was not used in the formulation and testing of CB4, only much simpler mixtures and virtually no mixtures with aromatics. Clearly it is CB4 that is at fault here and not the "real" observations.

The figure of the O-()pentaflurobenzyl oxime of acetaldehyde shown on page 36 is somewhat difficult to understand-at least when presented in shades of gray. I would prefer a depiction of the chemical equation, showing reactants and products, as on page 183.

This was due to the poor reproduction of the copies sent out to reviews. The better copies show excellent gray scale shading.

In the many tables of initial smog chamber conditions that are shown in Chapter 3, it would be helpful to include an indication of the HC/NOx ratios. For instance, this would show clearly that different $H C / N O x$ ratios were used on the two sides of the chamber on 15-Sep-94 (see Table 3.20 on page 100). 
See comment above.

It is mentioned on page 97 that "Even with no initial acetaldehyde, the E85 mixture was more reactive than the IAG with aldehyde." What is the explanation for this?

Ethanol reacts with $\mathrm{OH}$ to produce acetaldehyde.

The smog chamber experiment on 28-Sep-94 was meant to investigate the impact of a large ethanol fraction in the SynE85 mixture. Yet, it appears from the initial conditions shown in Table 3.21 (page 102) that very little additional ethanol was included-maybe a $15 \%$ increase. Am I missing something here?

The ethanol fraction is already large in the original composition. The ethanol was increased $19 \%$ (to us this seems large) without adjusting the remaining initial conditions, resulting in an additional $11 \%$ in carbon compared to the other side. We were interested in determining how sensitive the experimental results were to reasonable, but non-trivial changes in composition such as this increase in ethanol.

The issues of reactivity and reactivity scales are mentioned frequently in this report. It may be useful to show the specific reactivity of all the synthetic VOC mixtures used in this study, as computed with the most common reactivity scale, the Carter MIR scale. Comparing these computed reactivity figures with the experimental results observed under a variety of smog chamber conditions would emphasize the severe limitations of a fixed reactivity scale.

We don't see any value in applying scales derived from trajectory modeling to static chamber conditions.

The identification of numerous new carbonyl products from the photochemical oxidation of aromatics is very encouraging! Could the authors speculate as to how much of the "missing carbon" that is usually associated with reaction of toluene and xylenes can now be accounted for? Have any of the triones or epoxycarbonyls which are identified in this work ever been observed before in photochemical experiments with these aromatics?

Two new papers are now in print in Atmos. Environment (1997) that describe time series for many of these species and provide an estimate of the relative importance of the various pathways. The epoxide and trione observations are totally new with this project. 


\section{REVIEW 2}

A major finding of this work is that temperature variations have only a small effect on the ozone formation. I wonder why other groups such as UCR, GM and Johnson have seen a rather large effect of temperature. What this means in my opinion is that we still do not understand the size of or the reason for the temperature effect on urban ozone formation.

The statement above is not a correct representation of what we reported. We reported that for the SynURB mixture, at temperatures between 80 and 105 degrees Fahrenheit there appeared to be a compensating mechanism between the nitrogen products and the ozone production such that little dependence of ozone on temperature was observed. We certainly have observed strong ozone dependence on temperature for $\mathrm{n}$-butane and for other simple mixtures. Also note that CB4 did correctly predict the methane/NOx experiments in the UNC Chamber. We certainly do not understand the nitrogen products that are produced in this system and we believe based on the time series of the NOxanalyzer that it is these compounds that are causing this temperature response.

I was surprised that only five characterization experiments were performed, especially when the Teflon film was renewed and some chamber parameters were changed. Also, it would be useful to include a Table of all runs, the average temperature, and some measure of the light intensity such as the NO2 photolysis or the integrated Eppleymeasured UV flux.

Five characterization experiments were performed as part of this project. More characterization experiments were performed but they were for other projects and were not reported in the DOE report. These are available as part of the entire UNC Chamber Data Base. Further, the chamber characterization experiments did not produce any surprising results and there was a great desire to complete as much of the planned experimental program as possible, which we were not able to do due to the weather.

\section{REVIEW 3}

In many of the NOx-VOC-air irradiations, the "NO2" has not been consumed (admittedly, this may not really NO2) and the $\mathrm{O} 3$ has not reached a maximum by the time the experiment is terminated. Therefore, many of the conclusions are drawn from the relatively initial stages of the photoxidations where the rate of formation of ozone is dominant, and not from the maximum amounts of ozone formed.

These assertions are wrong. The "NO2" data after the NO2 peak is definitely not NO2 and except by modeling one has no idea how much NO2 
remains. The smog chamber experiments are full day experiments. All experiments "reach their" maximum ozone under these conditions because the sun sets. This is quite different than constant light indoor chambers where letting the experiment run longer produces more ozone.

How can the reviewer say that ozone formed 14 hours into to an experiment is "relatively initial stage of the photoxidations"?

The conclusions stated on page 148 concerning the temperature effect predicted by the Carbon Bond 4 model are extremely important and surely have important implications for airshed modeling. I urge the authors to publish their research and conclusions concerning the effects of temperature on ozone formation in irradiated NOx-VOC-air mixtures, and comparison of these results with the CB4 chemical mechanism.

Considered.

As noted above, the derivatization method used is providing important information as to the reaction products formed. At present, this method appears to be largely qualitative; its extension to obtain quantitative concentration data is important and should be continued.

See comments above. 


\section{REPORT DOCUMENTATION PAGE}

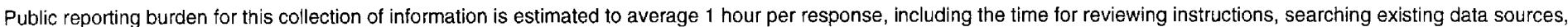

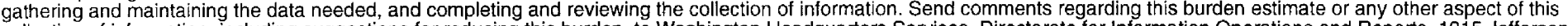

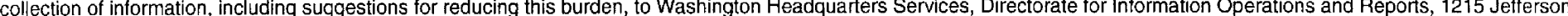

Davis Highway, Suite 1204, Arlington, VA 22202-4302, and to the Office of Management and Budget, Paperwork Reduction Project (0704-0188), Washington, DC 20503.

\begin{tabular}{|l|l|l}
\hline 1. AGENCY USE ONLY (Leave blank) & $\begin{array}{l}\text { 2. REPORT DATE } \\
\text { July } 1998\end{array}$ & $\begin{array}{l}\text { 3. REPORT TYPE AND DATES COVERED } \\
\text { Subcontract report }\end{array}$ \\
\hline
\end{tabular}

\section{TITLE AND SUBTITLE}

5. FUNDING NUMBERS

Atmospheric Photochemistry Studies of Pollutant Emissions from Transportation Vehicles Operating on Alternative Fuels

6. AUTHOR(S)

H. Jeffries, K. Sexton, and J. Yu

\section{PERFORMING ORGANIZATION NAME(S) AND ADDRESS(ES)}

Department of Environmental Sciences and Engineering

School of Public Health

University of North Carolina

Chapel Hill, NC 27599-7400

\section{SPONSORING/MONITORING AGENCY NAME(S) AND ADDRESS(ES)}

National Renewable Energy Laboratory

1617 Cole Boulevard

Golden, CO 80401-3393

(C) XAT-3-11153-01

(TA) FU704230

8. PERFORMING ORGANIZATION REPORT NUMBER

10. SPONSORING/MONITORING AGENCY REPORT NUMBER

NREUSR-540-21426

\section{SUPPLEMENTARY NOTES}

\section{2a. DISTRIBUTION/AVAILABILITY STATEMENT}

National Technical Information Service

U.S. Department of Commerce

5285 Port Royal Road

Springtield, VA 22161 12b. DISTRIBUTION CODE

UC-1504

\section{ABSTRACT (Maximum 200 words)}

This project was undertaken with the goal of improving our ability to predict the changes in urban ozone resulting from the widespread use of alternative fuels in automobiles. This report presents the results in detail. It is clear that no single approach is adequate for determining the potential atmospheric benefits of alternative fuel use: both experimental and modeling studies are needed. In addition, fundamental advances are needed to improve scientific understanding of reactivity.

\section{SUBJECT TERMS}

Alternative fuels, transportation fuels, air quality, emissions, urban airshed modeling

17. SECURITY CLASSIFICATION OF REPORT

\section{SECURITY CLASSIFICATION} OF THIS PAGE

\section{SECURITY CLASSIFICATION} OF ABSTRACT
15. NUMBER OF PAGES

290

16. PRICE CODE

20. LIMITATION OF ABSTRACT 